NUREG/CR-5513

Received by OSTI

EGG-2592

Vol. 2

APR 181990

Accident Management Information Needs

Appendices A - F

Prepared by D. J. Hanson, L. W. Ward,

W. R. Nelson and O. R. Meyer

Idaho National Engineering Laboratory EG\&G Idaho, Inc.

Prepared for

U.S. Nuclear Regulatory Commission 


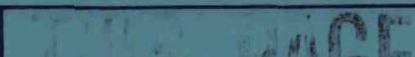

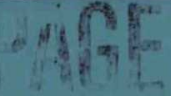

AVAILABILITY NOTICE

Availability of Reference Materials Cited in NRC Publications

Most documents clted in NRC publications will be avallable from one of the following sources:

1. The NRC Public Document Room, 2120 L Street, NW, Lower Level, Washington, DC 20555

2. The Superintendent of Documents, U.S. Government Printing Office, P.O. Box 37082 , Washington, DC 20013-7082

3. The National Technical Information Service, Springfield, VA 22161

Although the listing that follows represents the majority of documents cited in NRC publications, it is not Intended to be exhaustive.

Referenced documents avallable for Inspection and copying for a fee from the NRC Public Document Room include NRC correspondence and Internal NRC memoranda; NRC Office of Inspection and Enforcement bulletins, circulars, Information notices, inspection and investigation notices; Licensee Event Reports; vendor reports and correspondence; Commission papers; and applicant and licensee documents and correspondence.

The following documents in the NUREG serles are available for purchase from the GPO Sales Program: formal NRC staff and contractor reports, NRC-sponsored conference proceedings, and NRC booklets and brochures. Also avallable are Regulatory Guides, NRC regulations in the Code of Federal Regulations, and Nuclear Regulatory Commission Issuances.

Documents avallable from the National Technical Information Service include NUREG series reports and technical reports prepared by other federal agencies and reports prepared by the Atomic Energy Commission, forerunner agency to the Nuclear Regulatory Commission.

Documents avallable from public and special technical libraries include all open literature items, such as books, Journal and periodical articles, and transactions. Federal Register notices, federal and state legislation, and congressional reports can usually be obtained from these llbraries.

Documents such as theses, dissertations, forelgn reports and translations, and non-NRC conference proceedings are avallable for purchase from the organization sponsoring the publication cited.

Single coples of NRC draft reports are avaliable free, to the extent of supply, upon written request to the Office of Information Resources Management, Distribution Section, U.S. Nuclear Regulatory Commission, Washington, DC 20555.

Coples of Industry codes and standards used in a substantive manner in the NRC regulatory process are maintained at the NRC Library, 7920 Norfolk Avenue, Bethesda, Maryland, and are avallable there for reference use by the public. Codes and standards are usually copyrighted and may be purchased from the originating organization or. If they are American National Standards, from the American National Standards Institute, 1430 Broadway, New York, NY 10018.

\section{DISCLAIMER NOTICE}

This report was prepared as an account of work sponsored by an agency of the United States Government. Neither the United States Government nor any agency thereof, or any of their employees, makes any warranty, expresed or implied, or assumes any legal liability of responsibility for any third party's use, or the results of such use, of any information, apparatus, product or process disclosed in this report, or represents that its use by such third party would not infringe privately owned rights. 


\title{
Accident Management Information Needs
}

\author{
Appendices A - F
}

Manuscript Completed: March 1990

Date Published: April 1990

Prepared by

D. J. Hanson, L. W. Ward,

W.R. Nelson and O. R. Meyer

\section{DISCLAIMER}

This report was prepared as an account of work sponsored by an agency of the United States Government. Neither the United States Government nor any agency thereof, nor any of their employees, makes any warranty, express or implied, or assumes any legal liability or responsibility for the accuracy, completeness, or usefulness of any information, apparatus, product, or process disclosed, or represents that its use would not infringe privately owned rights. Reference herein to any specific commercial product, process, or service by trade name, trademark, manufacturer, or otherwise does not necessarily constitute or imply its endorsement, recommendation, or favoring by the United States Government or any agency thereof. The views and opinions of authors expressed herein do not necessarily state or reflect those of the United States Government or any agency thereof.

Idaho National Engineering Laboratory

Managed by the U.S. Department of Energy

EG\&G Idaho, Inc.

P.O. Box 1625

Idaho Falls, ID 83415

Prepared for

Division of Systems Research

Office of Nuclear Regulatory Research

U.S. Nuclear Regulatory Commission

Washington, DC 20555

NRC FIN B5723

Under DOE Contract No. DE-AC07-761D0-1570 


\section{DISCLAIMER}

This report was prepared as an account of work sponsored by an agency of the United States Government. Neither the United States Government nor any agency Thereof, nor any of their employees, makes any warranty, express or implied, or assumes any legal liability or responsibility for the accuracy, completeness, or usefulness of any information, apparatus, product, or process disclosed, or represents that its use would not infringe privately owned rights. Reference herein to any specific commercial product, process, or service by trade name, trademark, manufacturer, or otherwise does not necessarily constitute or imply its endorsement, recommendation, or favoring by the United States Government or any agency thereof. The views and opinions of authors expressed herein do not necessarily state or reflect those of the United States Government or any agency thereof. 


\section{DISCLAIMER}

Portions of this document may be illegible in electronic image products. Images are produced from the best available original document. 
APPENDIX A

INFORMATION-NEEDS TABLES FOR A

PW WITH A LARGE, DRY CONTAINMENT

$A-1 / A-2$ 


\section{APPENDIX A}

\section{INFORMATION-NEEDS TABLES FOR A PWR WITH A LARGE, DRY CONTAINMENT}

The tables contained in this Appendix A describe the information needs for a pressurized water reactor (PWR) with a large, dry containment. To identify these information needs, the branch points in the safety objective trees were examined to decide what information is necessary to (a) determine the status of the safety functions in the plant, i.e., whether the safety functions are being adequately maintained within predetermined limits, (b) identify plant behavior (mechanisms) or precursors to this behavior which indicate that a challenge to plant safety is occurring or is imminent, and (c) select strategies that will prevent or mitigate this plant behavior and monitor the implementation and effectiveness of these strategies. The information needs for the challenges to the safety functions are not examined since the summation of the information needs for all mechanisms associated with a challenge comprise the information needs for the challenge itself.

In the tables, the rows correspond to five levels of information which were derived from the levels of the safety objective trees. The first section (row) of the table contains the information needs that relate to the safety function. This section is used to describe the information needed to determine whether the safety function is being maintained within the accepted safety limits. The second section (row) of the table displays information to identify a specific mechanism that may be a challenge to a safety function. Two different categories of information are important for identifying mechanisms: indicators and precursors. The indicators include information that identifies when a mechanism is actually occurring and challenging a safety function. The precursor information identifies whether a mechanism would be expected to occur in the future based on currently available information.

The final three categories (rows) relate to strategy selection and evaluation. The Selection Criteria category identifies the information needed to determine which strategies should be selected for a given situation, including consideration of the plant conditions under which the strategy can operate and be effective. The Strategy Initiation category gives the information needed for the operating staff to determine whether a strategy has been implemented as intended. The Strategy Effectiveness row describes the information needed to determine whether the strategy is having its intended effect; that is, whether implementation of the strategy is having a beneficial effect on the status of the safety function that is being challenged.

The respective columns in the table format include the identified information needs, the sources of the information categorized as to how well they represent the information needs, and the existing measurements that could supply the needed information. The information sources are subdivided into those that are considered to be either direct or indirect. A direct information source is one that can be used to provide information that will positively determine the presence or absence of a specific condition on the safety objective tree. For example, if the safety function addresses pressure control, a pressure measurement is a direct information source for understanding challenges to the safety function. An indirect information source can be used to infer the needed information, but there may be conditions in which the information source may provide ambiguous results. For example, core exit temperature readings may provide reasonable information for fuel cladding temperatures for some system conditions, but would not provide an accurate indication for other combinations of system flow and fluid conditions.

Development of the input to the rows and columns required the expertise of personnel with diverse backgrounds. A team of personnel with operations, instrumentation, and severe accident experience were used to produce the tables. 
TABLE A1.1. PREVENT CORE DISPERSAL FROM VESSEL (V)--INADEQUATE INVENTORY MECHANISM (V1A1)

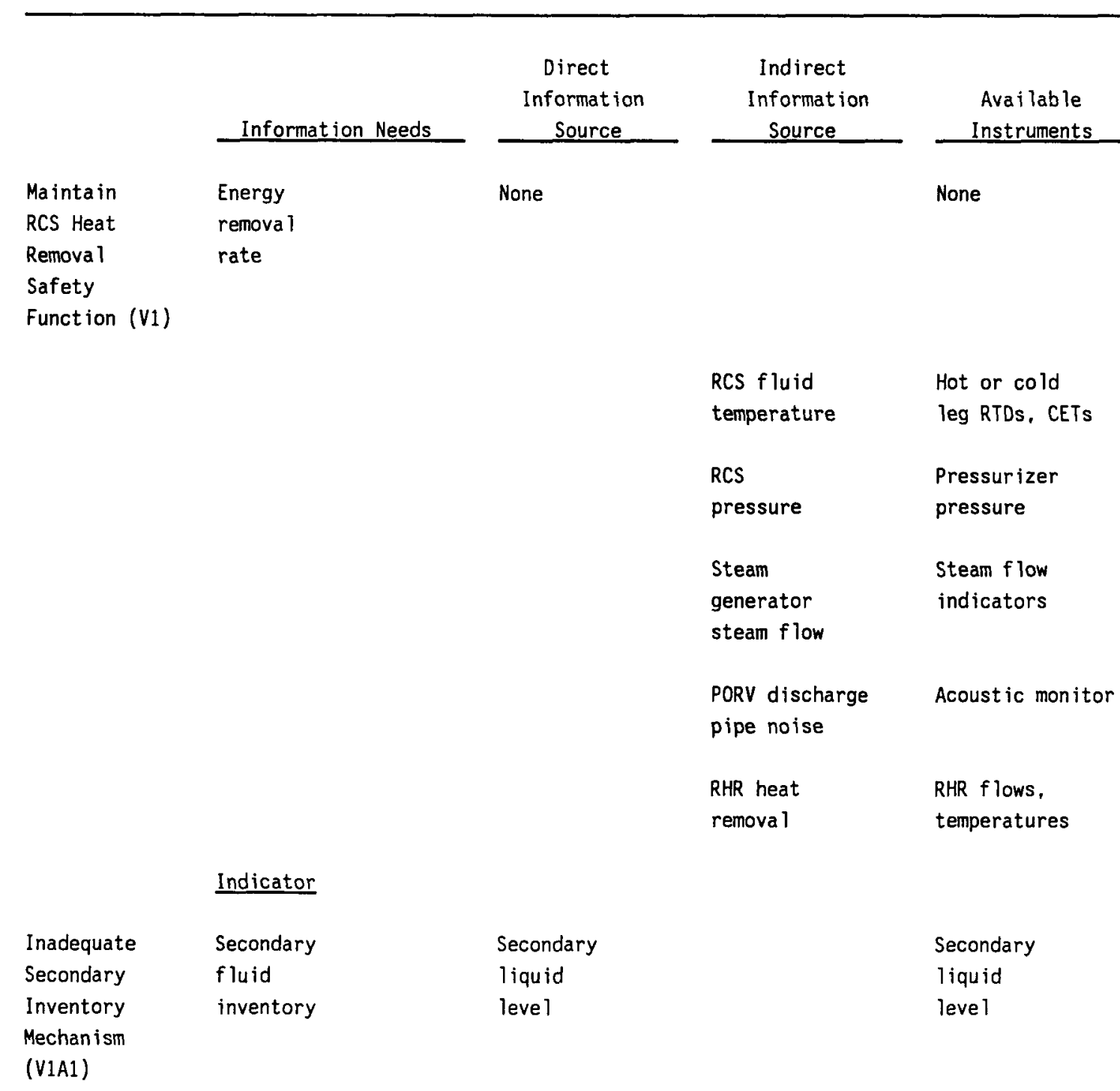


TABLE A1.1. (cont inued)

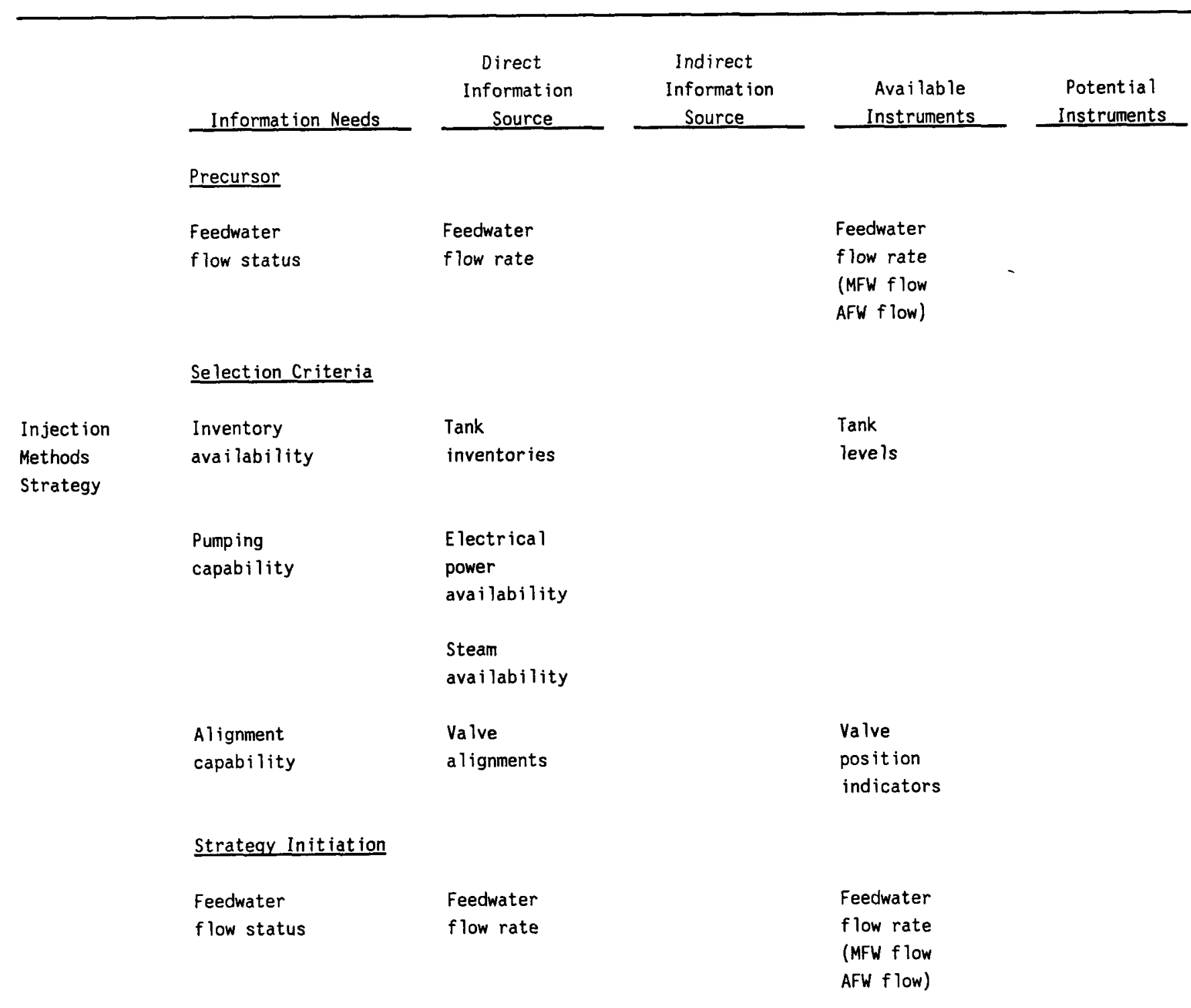


TABLE A1.1. (cont inued)

\begin{tabular}{|c|c|c|c|c|c|}
\hline & Information Needs & $\begin{array}{c}\text { Direct } \\
\text { Information } \\
\text { Source } \\
\end{array}$ & $\begin{array}{c}\text { Indirect } \\
\text { Information } \\
\text { Source } \\
\end{array}$ & $\begin{array}{c}\text { Available } \\
\text { Instruments } \\
\end{array}$ & $\begin{array}{c}\text { Potential } \\
\text { Instruments }\end{array}$ \\
\hline & $\begin{array}{l}\text { Strategy Initiation } \\
\text { Injection water } \\
\text { inventory } \\
\text { (decreasing) }\end{array}$ & $\begin{array}{l}\text { tinued) } \\
\text { Tank } \\
\text { inventory }\end{array}$ & & $\begin{array}{l}\text { Tank leve I } \\
\text { SI Tanks RWST }\end{array}$ & \\
\hline & $\begin{array}{l}\text { Strategy Effectivene } \\
\text { RCS fluid } \\
\text { temperature }\end{array}$ & $\begin{array}{l}\text { RCS fluid } \\
\text { temperature }\end{array}$ & & $\begin{array}{l}\text { Hot or cold } \\
\text { leg RTDs, CETs }\end{array}$ & \\
\hline & $\begin{array}{l}\text { Secondary } \\
\text { fluid } \\
\text { inventory }\end{array}$ & $\begin{array}{l}\text { Secondary } \\
\text { liquid } \\
\text { leve1 }\end{array}$ & & $\begin{array}{l}\text { Secondary } \\
\text { liquid } \\
\text { level }\end{array}$ & \\
\hline $\begin{array}{l}\text { Inventory } \\
\text { Sources } \\
\text { Strategy }\end{array}$ & $\begin{array}{l}\text { Selection Criteria } \\
\text { Inventory } \\
\text { avai lability }\end{array}$ & $\begin{array}{l}\text { Tank } \\
\text { inventories }\end{array}$ & & $\begin{array}{l}\text { Tank } \\
\text { leve } 1 \\
\text { (SIT RWST) }\end{array}$ & \\
\hline & $\begin{array}{l}\text { Pumping } \\
\text { capability }\end{array}$ & $\begin{array}{l}\text { Electric } \\
\text { power } \\
\text { availability } \\
\text { Steam } \\
\text { availability }\end{array}$ & & & \\
\hline
\end{tabular}


TABLE Al.1. (cont inued)

\begin{tabular}{|c|c|c|c|c|}
\hline Information Needs & $\begin{array}{c}\text { Direct } \\
\text { Information } \\
\text { Source } \\
\end{array}$ & $\begin{array}{l}\text { Indirect } \\
\text { Information } \\
\text { Source } \\
\end{array}$ & $\begin{array}{c}\text { Ava i lab le } \\
\text { Instruments }\end{array}$ & $\begin{array}{c}\text { Potential } \\
\text { Instruments }\end{array}$ \\
\hline \multicolumn{5}{|c|}{ Selection Criteria (cont inued) } \\
\hline $\begin{array}{l}\text { Alignment } \\
\text { capability }\end{array}$ & $\begin{array}{l}\text { Valve } \\
\text { alignments }\end{array}$ & & $\begin{array}{l}\text { Valve position } \\
\text { indicators }\end{array}$ & \\
\hline \multicolumn{5}{|l|}{ Strategy Initiation } \\
\hline $\begin{array}{l}\text { Injection water } \\
\text { inventory } \\
\text { (decreasing) }\end{array}$ & $\begin{array}{l}\text { Tank } \\
\text { inventory }\end{array}$ & & $\begin{array}{l}\text { Tank } \\
\text { leve } 1 \\
\text { (SIT RWST) }\end{array}$ & \\
\hline $\begin{array}{l}\text { Feedwater } \\
\text { flow status }\end{array}$ & $\begin{array}{l}\text { Feedwater } \\
\text { flow rate }\end{array}$ & & $\begin{array}{l}\text { Feedwater } \\
\text { f low rate } \\
\text { (MFW flow } \\
\text { AFW flow) }\end{array}$ & \\
\hline \multicolumn{5}{|c|}{ Strategy Effectiveness } \\
\hline $\begin{array}{l}\text { RCS fluid } \\
\text { temperature }\end{array}$ & $\begin{array}{l}\text { RCS fluid } \\
\text { temperature }\end{array}$ & & $\begin{array}{l}\text { Hot or cold } \\
\text { leg RTDs, CETs }\end{array}$ & \\
\hline Secondary & Secondary & & Secondary & \\
\hline $\begin{array}{l}\text { fluid } \\
\text { inventory }\end{array}$ & $\begin{array}{l}\text { liquid } \\
\text { level }\end{array}$ & & $\begin{array}{l}\text { liquid } \\
\text { leve } 1\end{array}$ & \\
\hline
\end{tabular}


TABLE A1.2. PREVENT CORE DISPERSAL FROM VESSEL (V)--INADEQUATE PRESSURE CONTROL MECHANISM (V1A2)

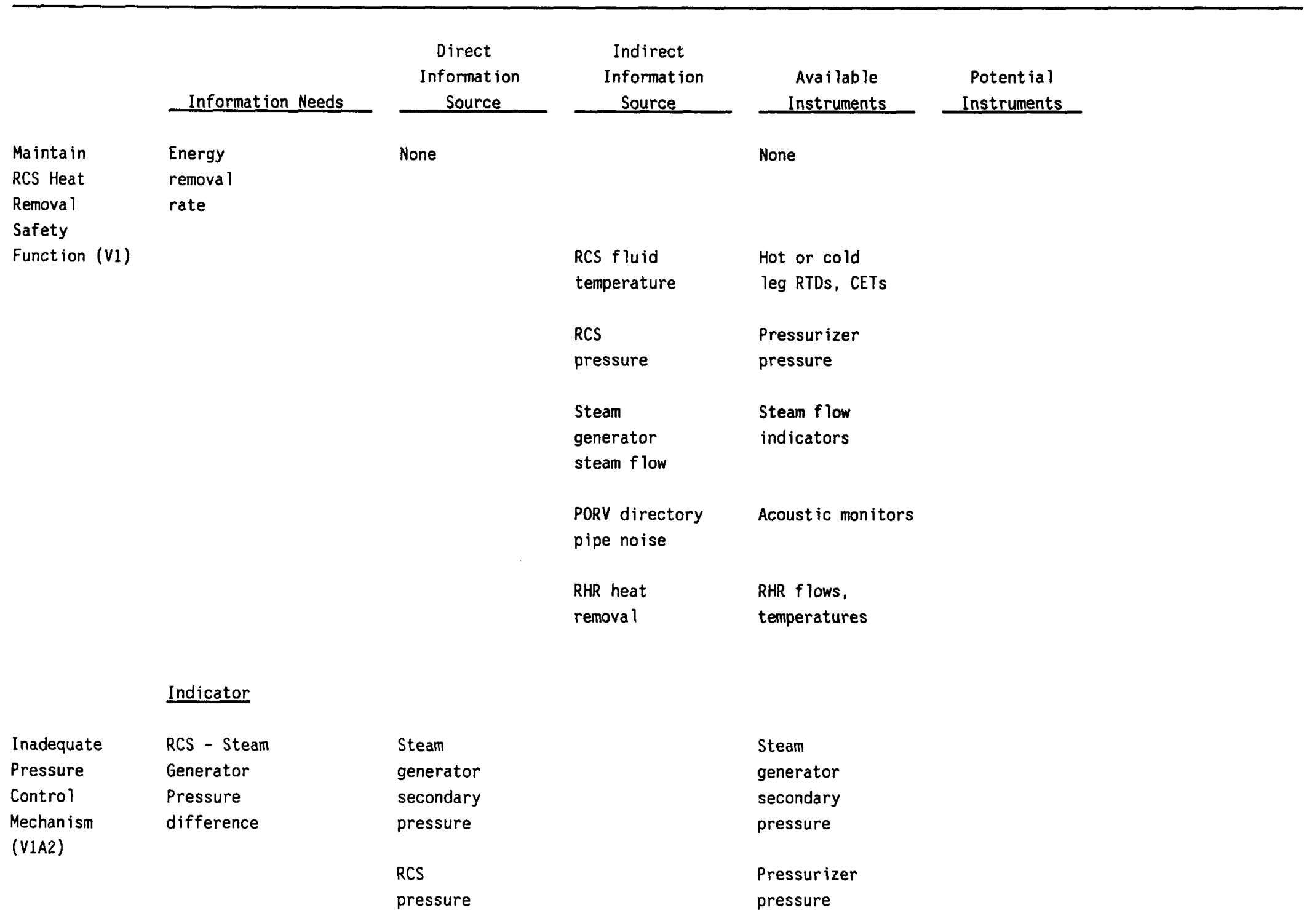


TABLE Al.2. (cont inued)

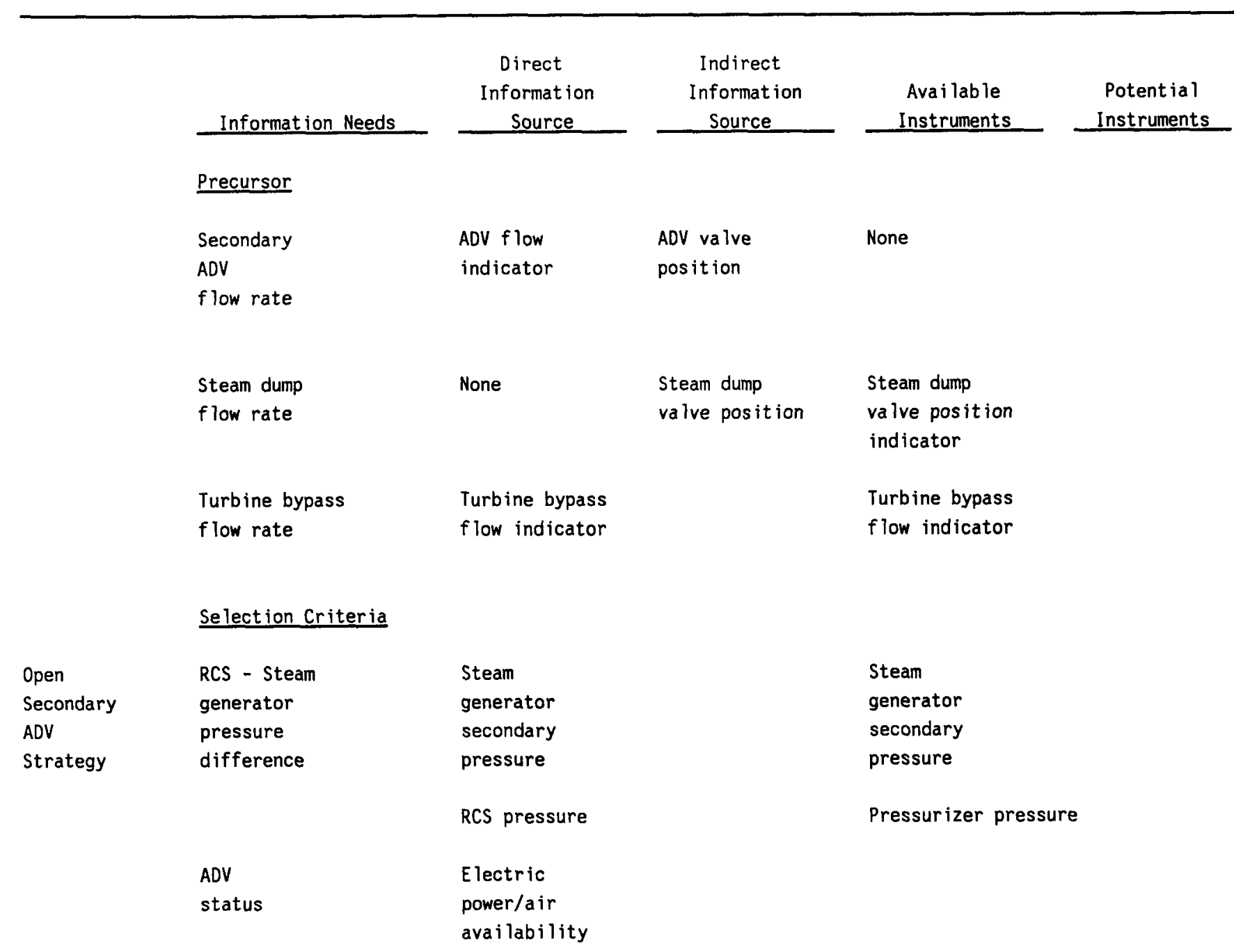


TABLE A1.2. (cont inued)

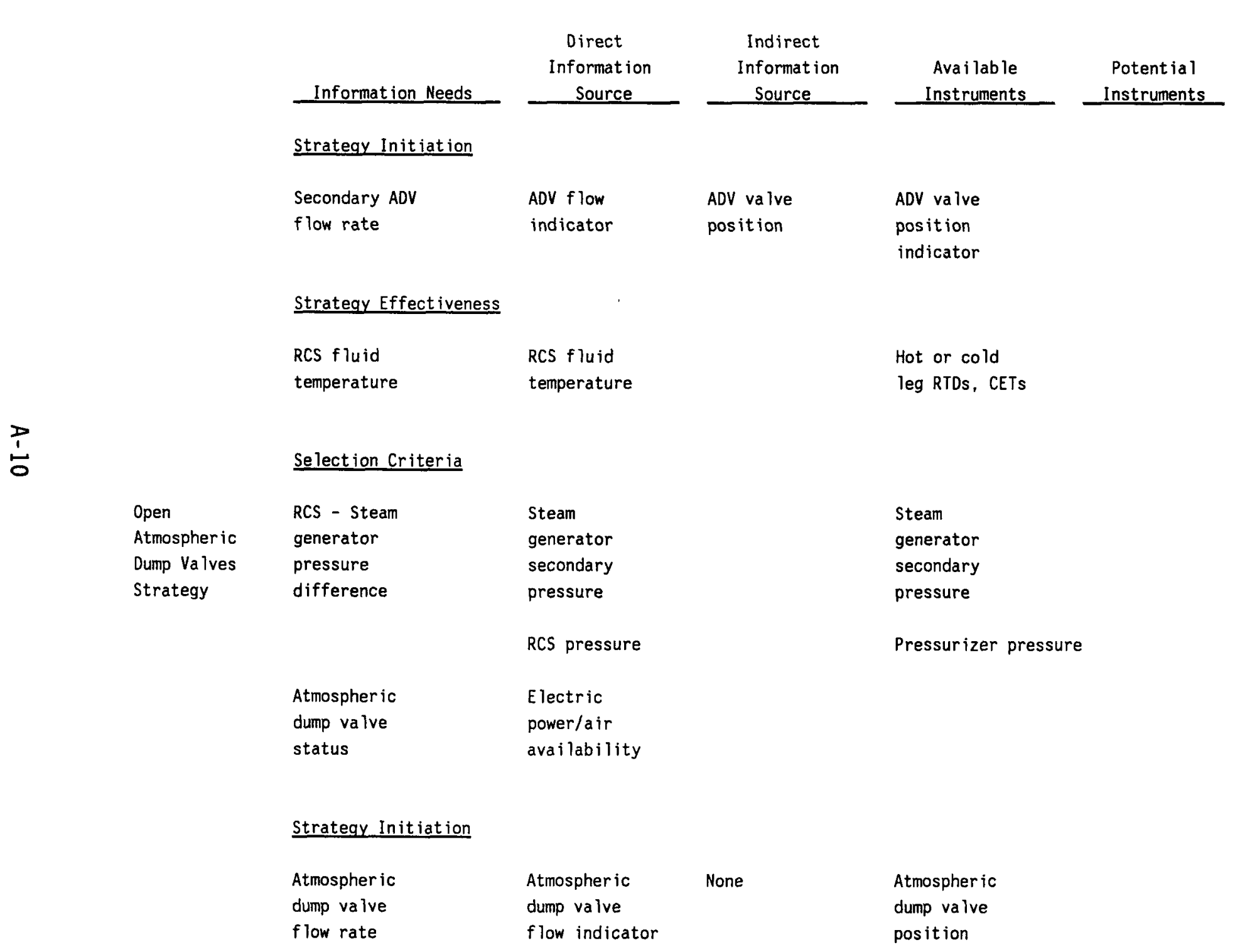


TABLE A1.2. (continued)

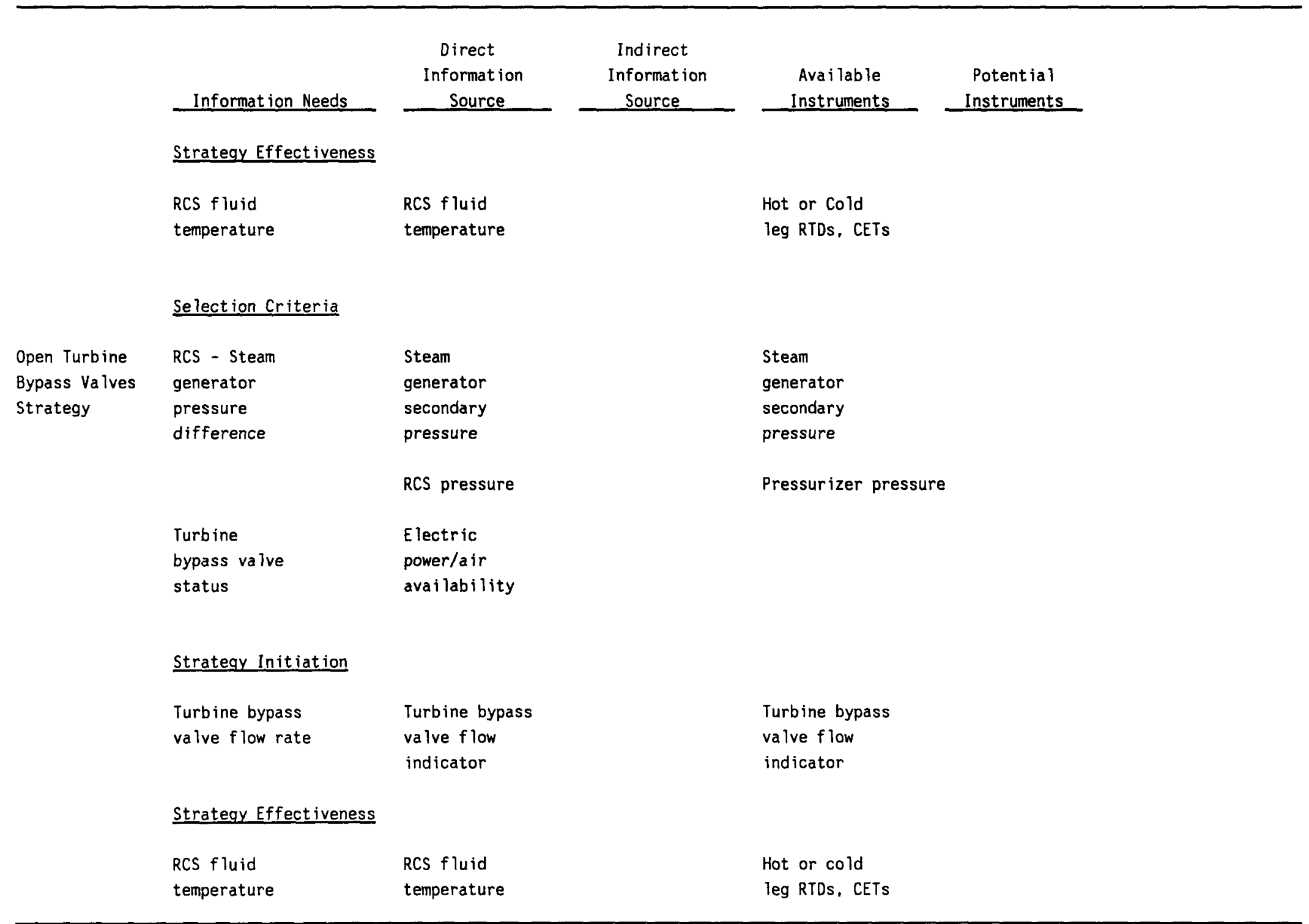


TABLE A1.3. PREVENT CORE DISPERSAL FROM VESSEL (V)--INADEQUATE RCS ENERGY TRANSPORT MECHANISM (VIA3)

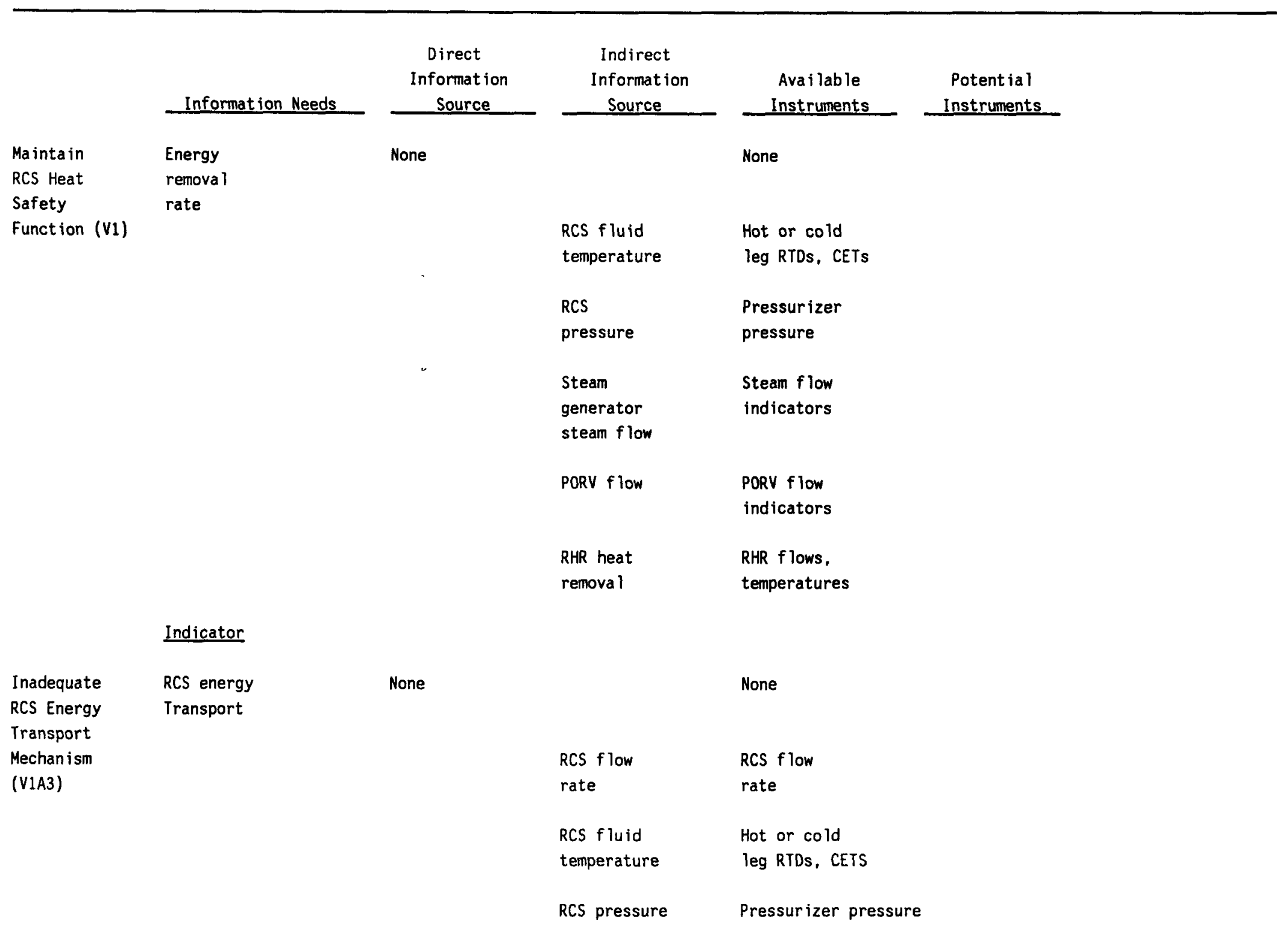


TABLE A1.3. (cont inued)

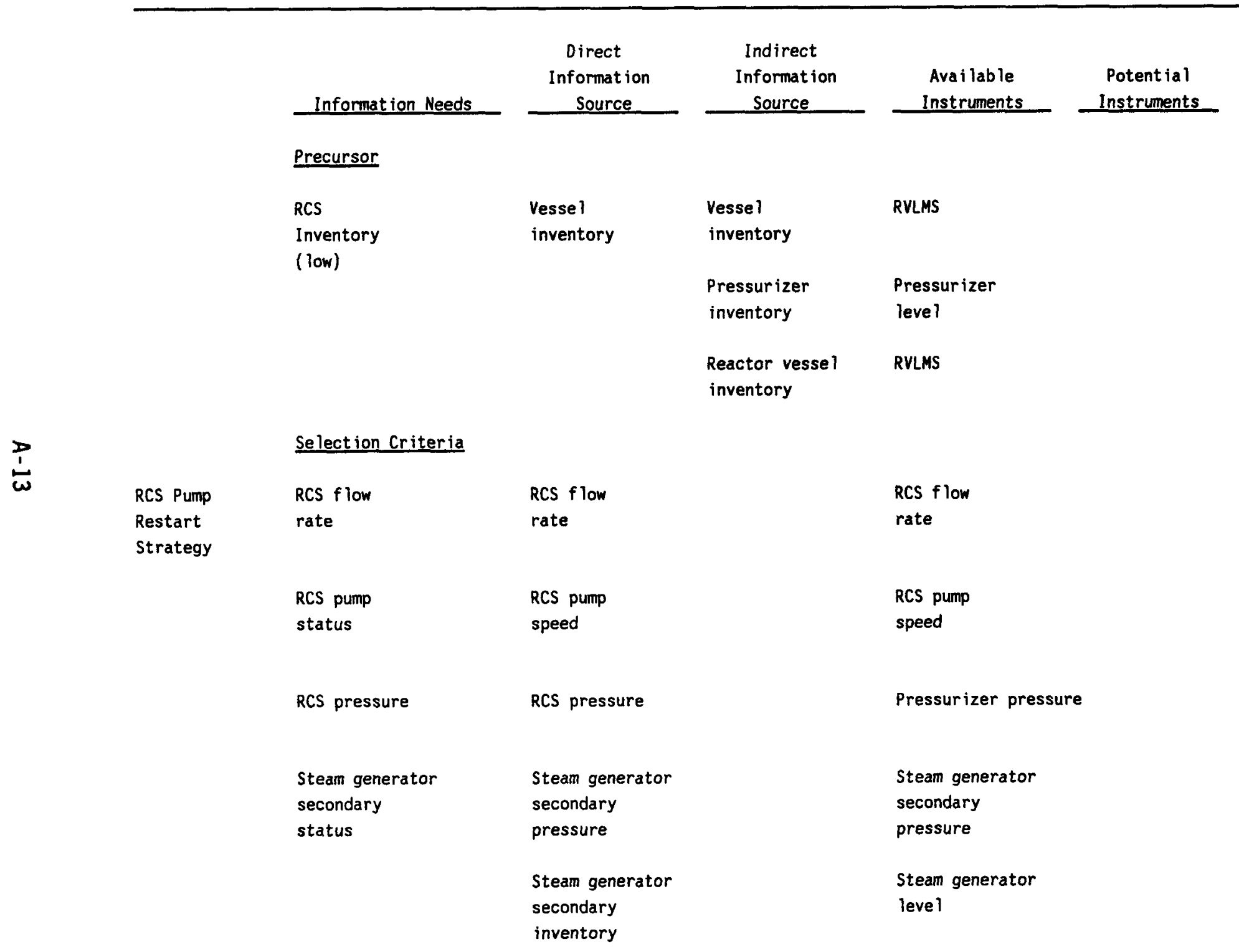


TABLE A1.3. (cont inued)

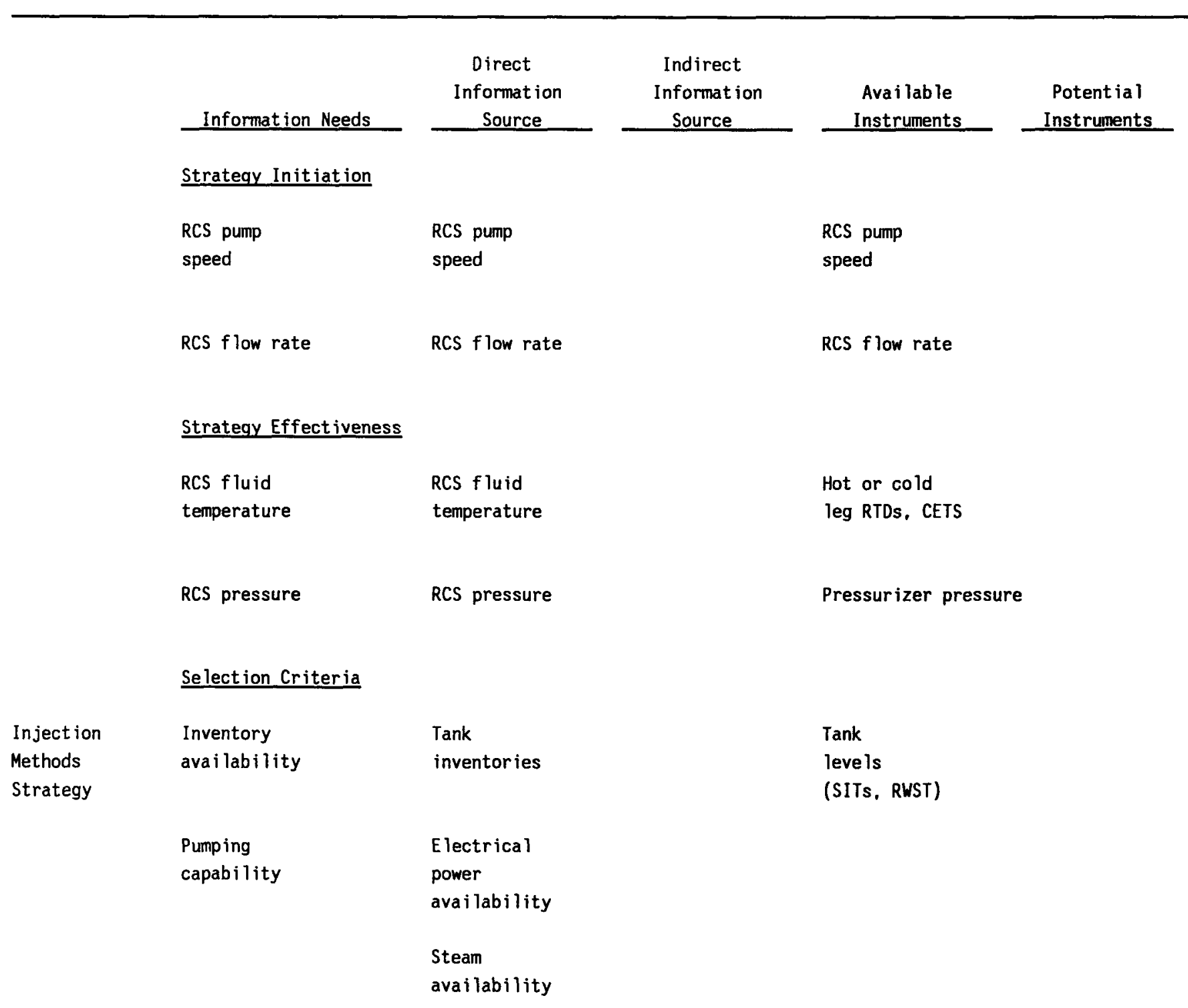


TABLE A1.3. (cont inued)

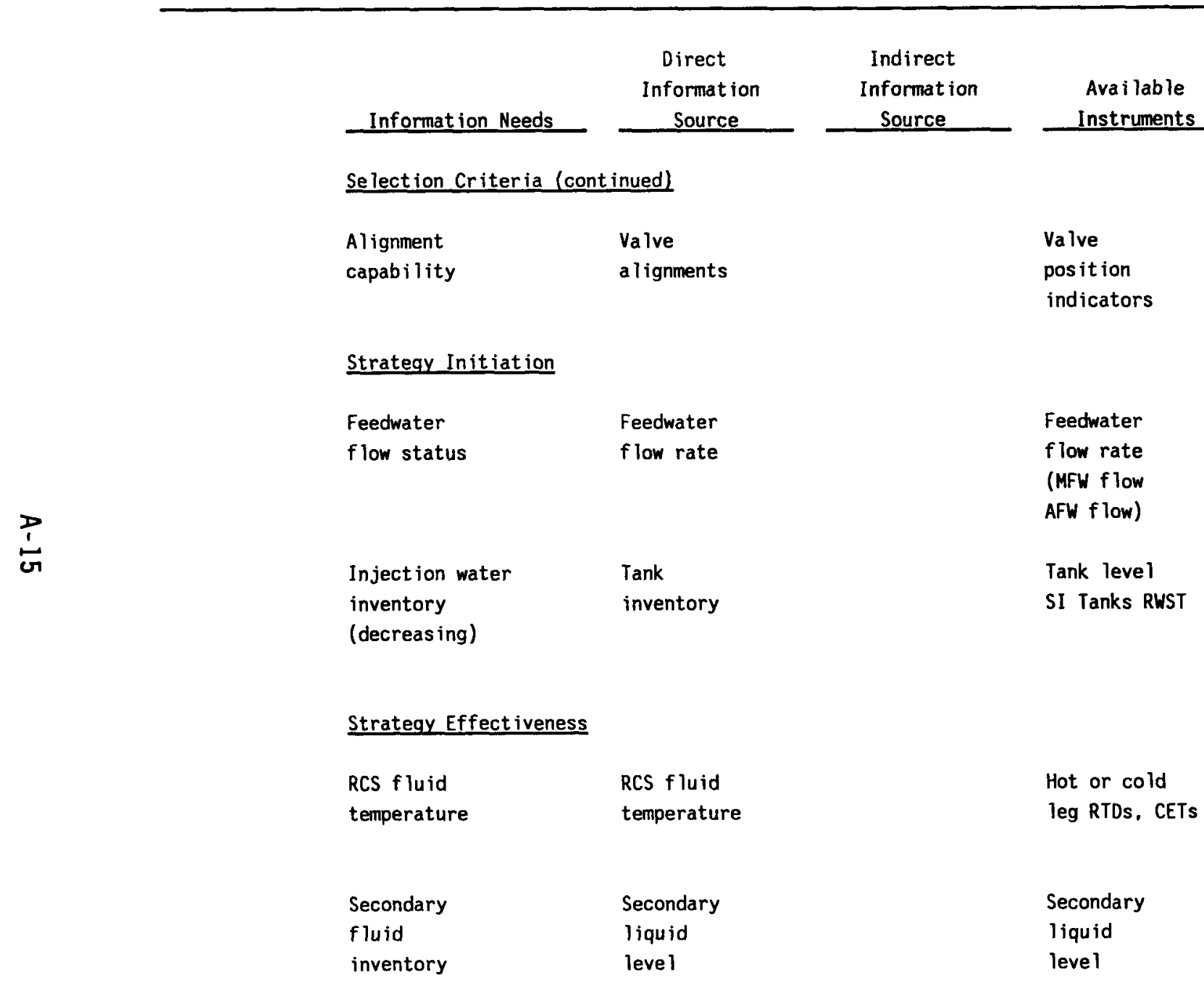


TABLE A1.3. (continued)

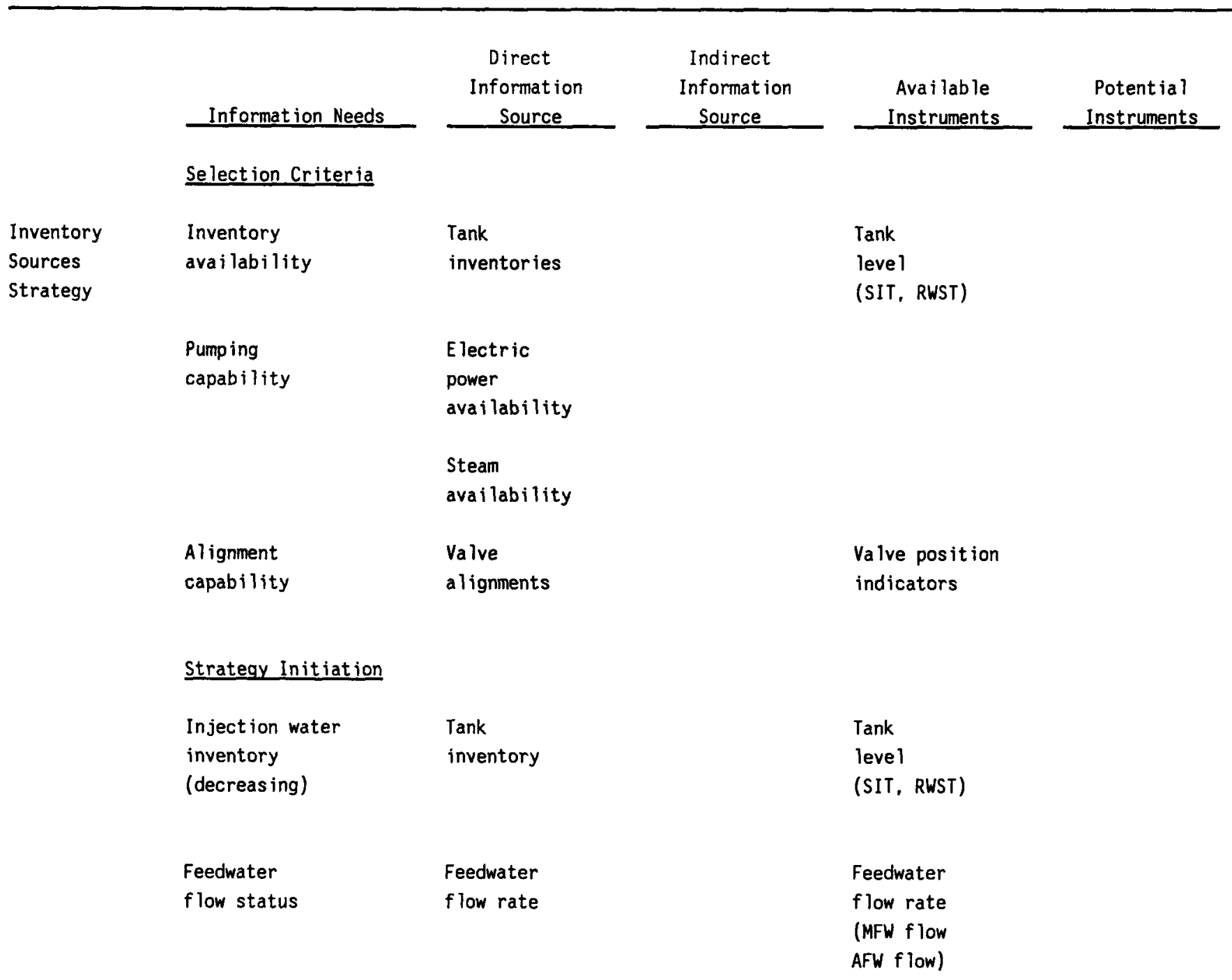


TABLE A1.3. (continued)

\begin{tabular}{|c|c|c|c|c|}
\hline Informat ion Needs & $\begin{array}{c}\text { Direct } \\
\text { Information } \\
\text { Source } \\
\end{array}$ & $\begin{array}{c}\text { Indirect } \\
\text { Information } \\
\text { Source } \\
\end{array}$ & $\begin{array}{c}\text { Ava i lable } \\
\text { Instruments }\end{array}$ & $\begin{array}{c}\text { Potential } \\
\text { Instruments }\end{array}$ \\
\hline Strategy Effectiveness & & & & \\
\hline $\begin{array}{l}\text { RCS fluid } \\
\text { temperature }\end{array}$ & $\begin{array}{l}\text { RCS fluid } \\
\text { temperature }\end{array}$ & & $\begin{array}{l}\text { Hot or cold } \\
\text { leg RTDs, CETs }\end{array}$ & \\
\hline Secondary & Secondary & & Secondary & \\
\hline fluid & liquid & & Tiquid & \\
\hline inventory & leve 1 & & level & \\
\hline
\end{tabular}


TABLE A1.4. PREVENT CORE DISPERSAL FROM VESSEL (V)--RESTRICTED RCS BLEED MECHANISM (V1B1)

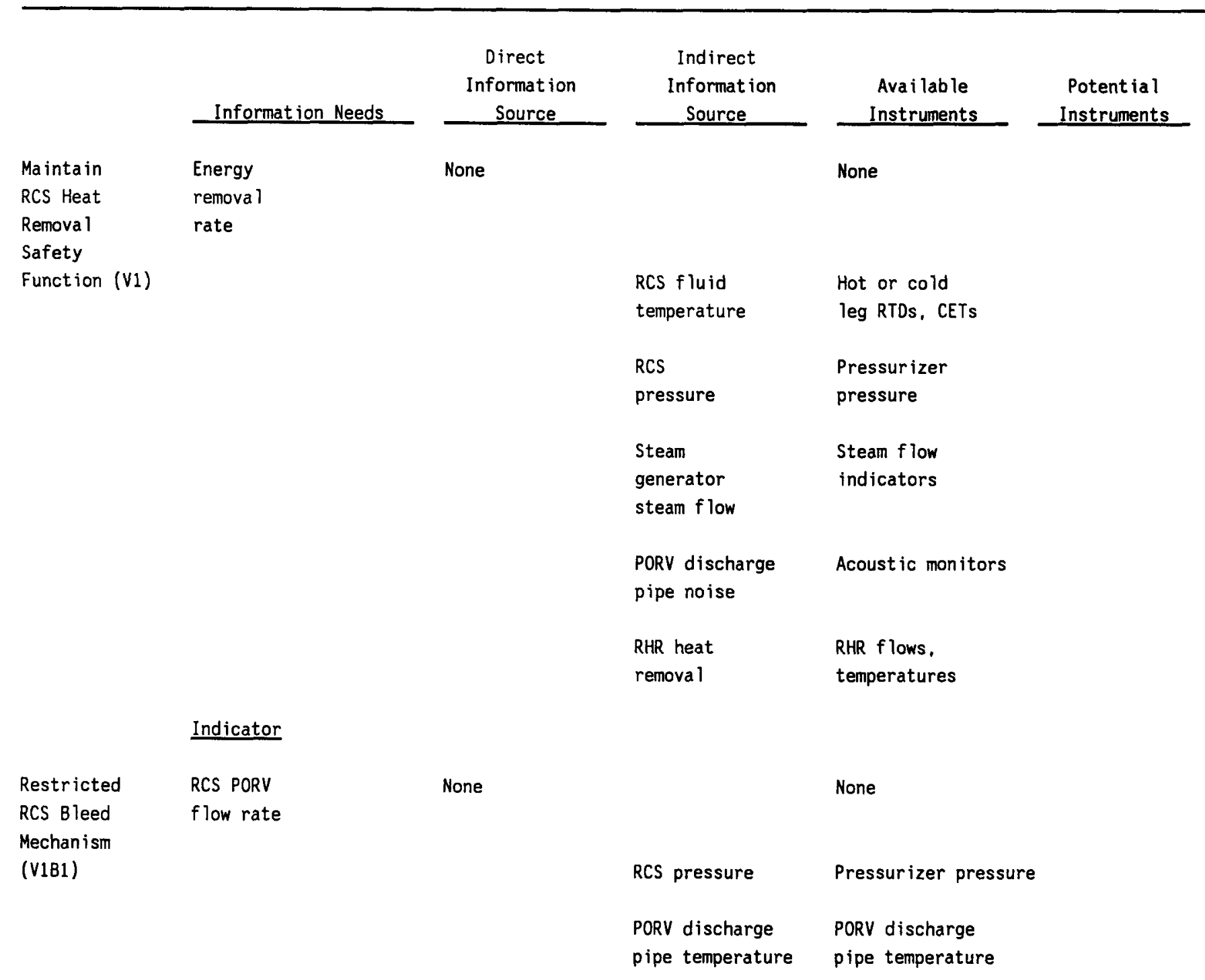


TABLE A1.4. (cont inued)

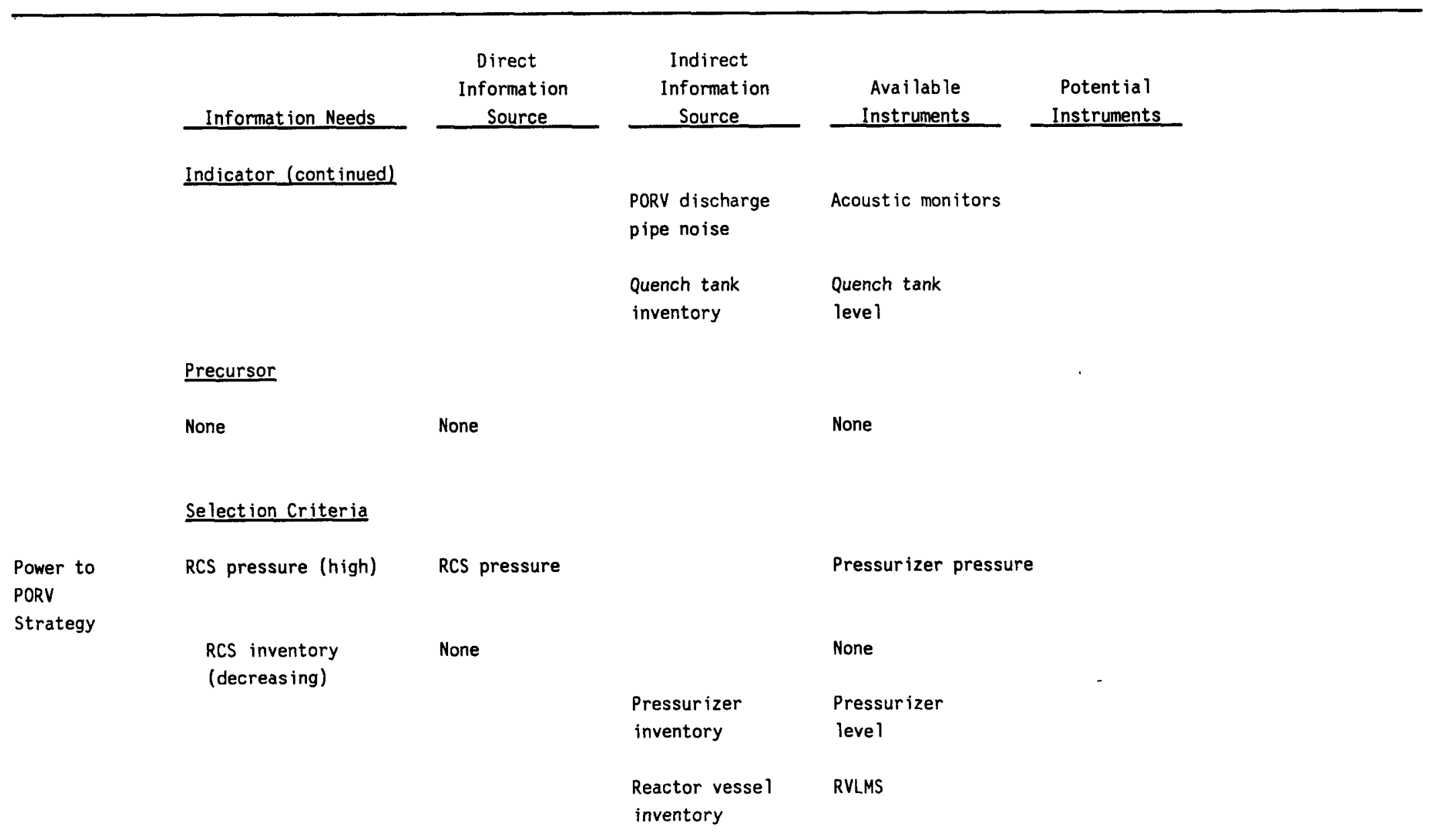


TABLE A1.4. (cont inued)

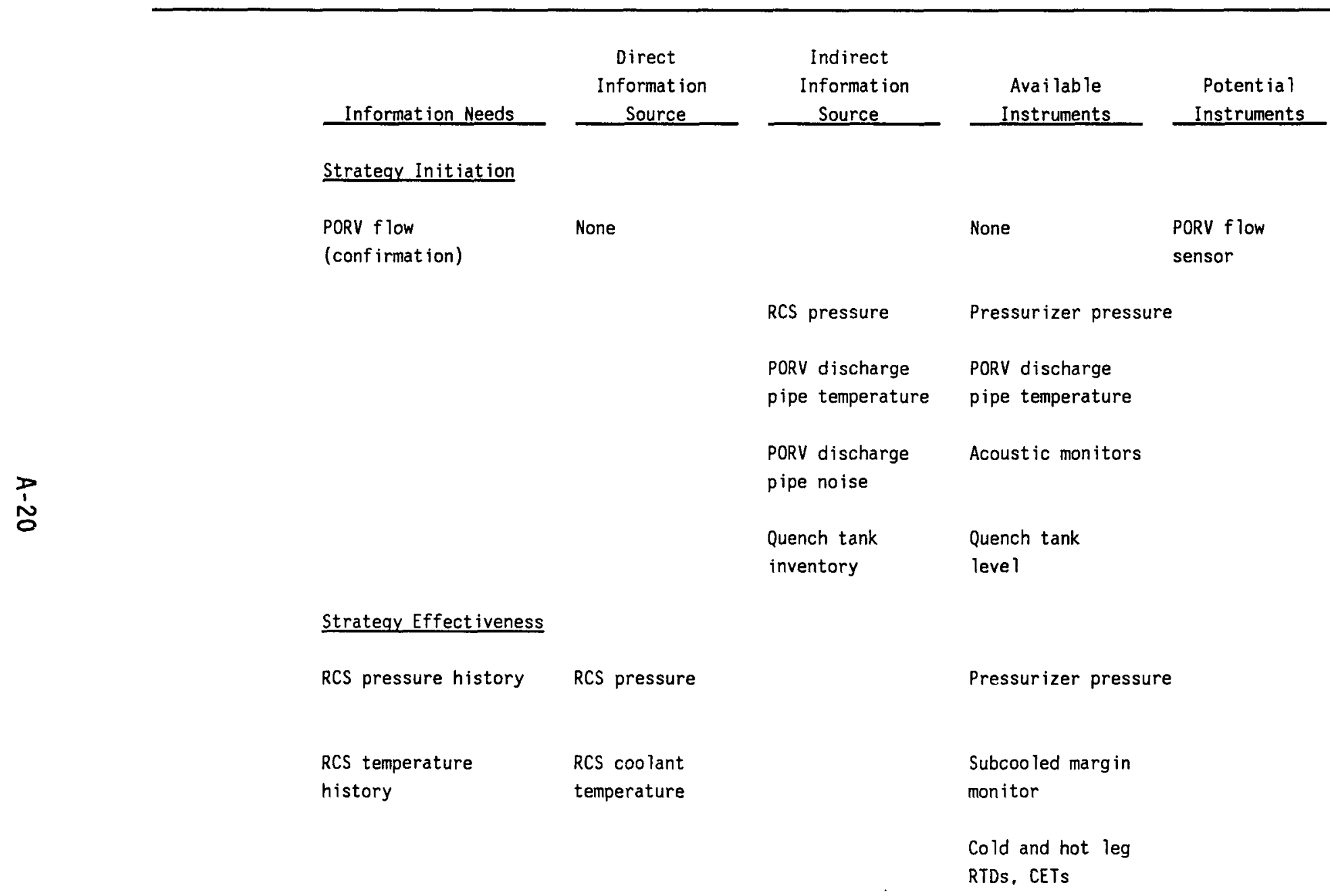


TABLE A1.4. (cont inued)

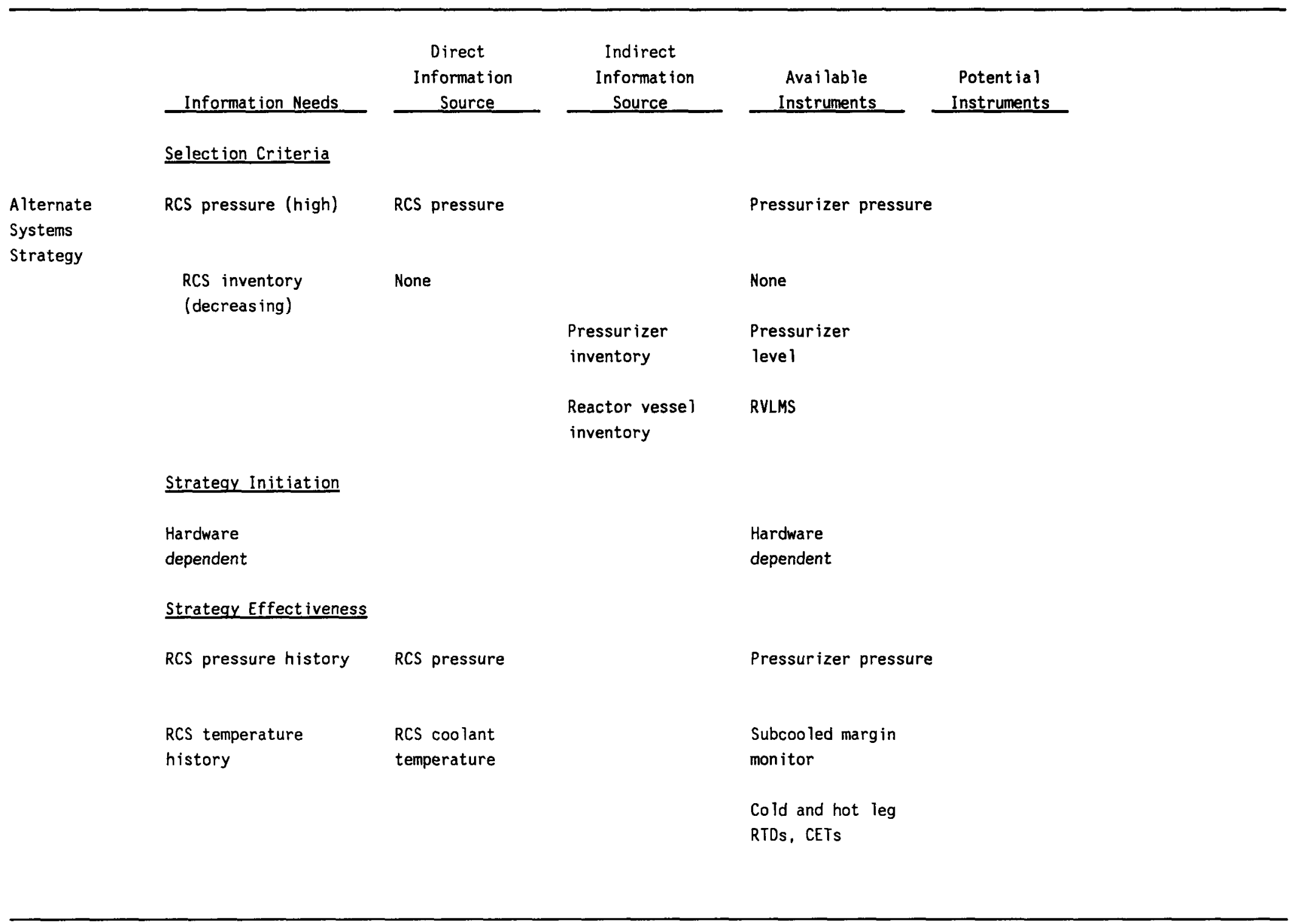


TABLE A1.5. PREVENT CORE DISPERSAL FROM VESSEL (V)--RHR SYSTEMS INOPERABLE (V1B2)

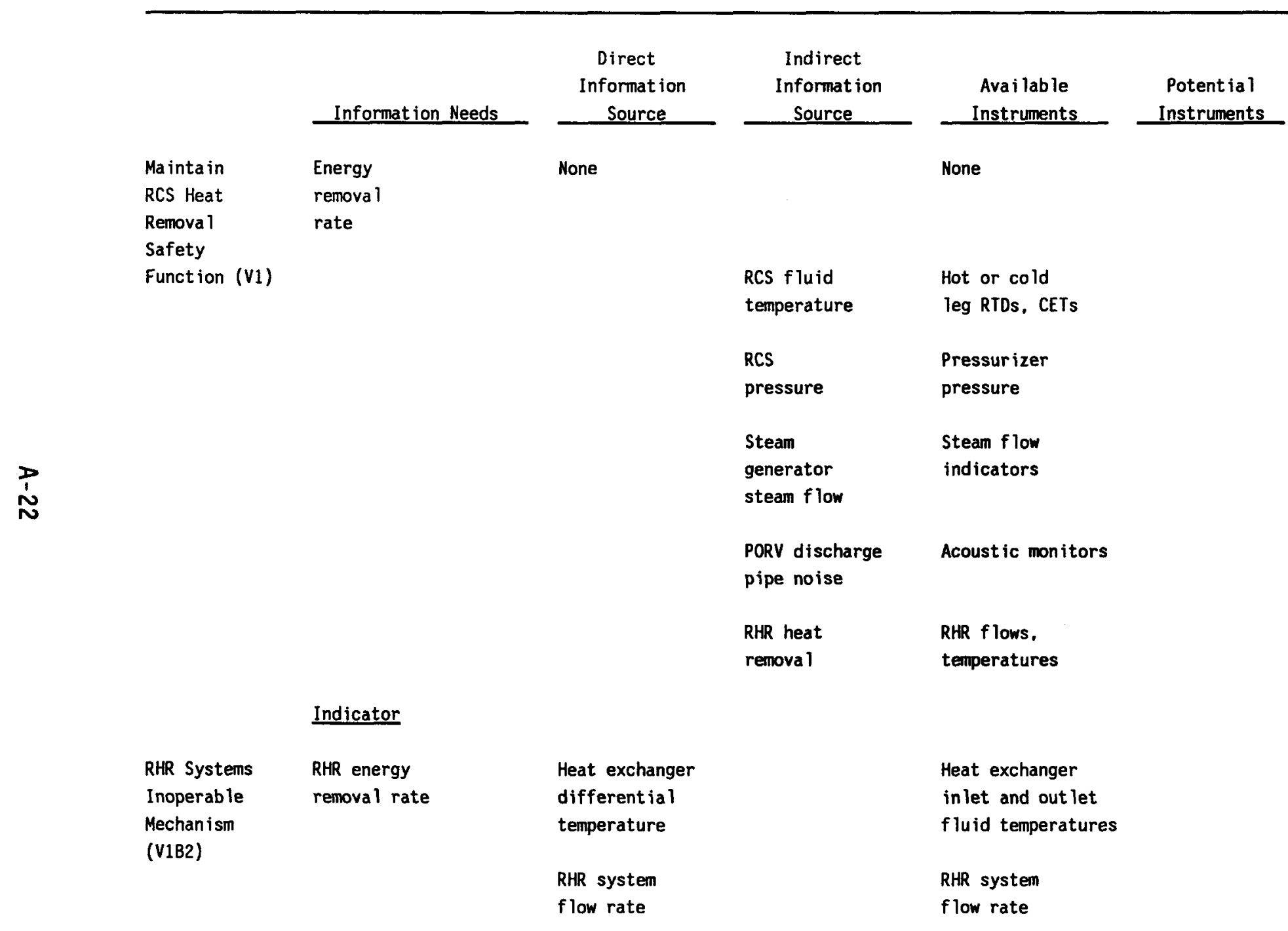


TABLE A1.5. (cont inued)

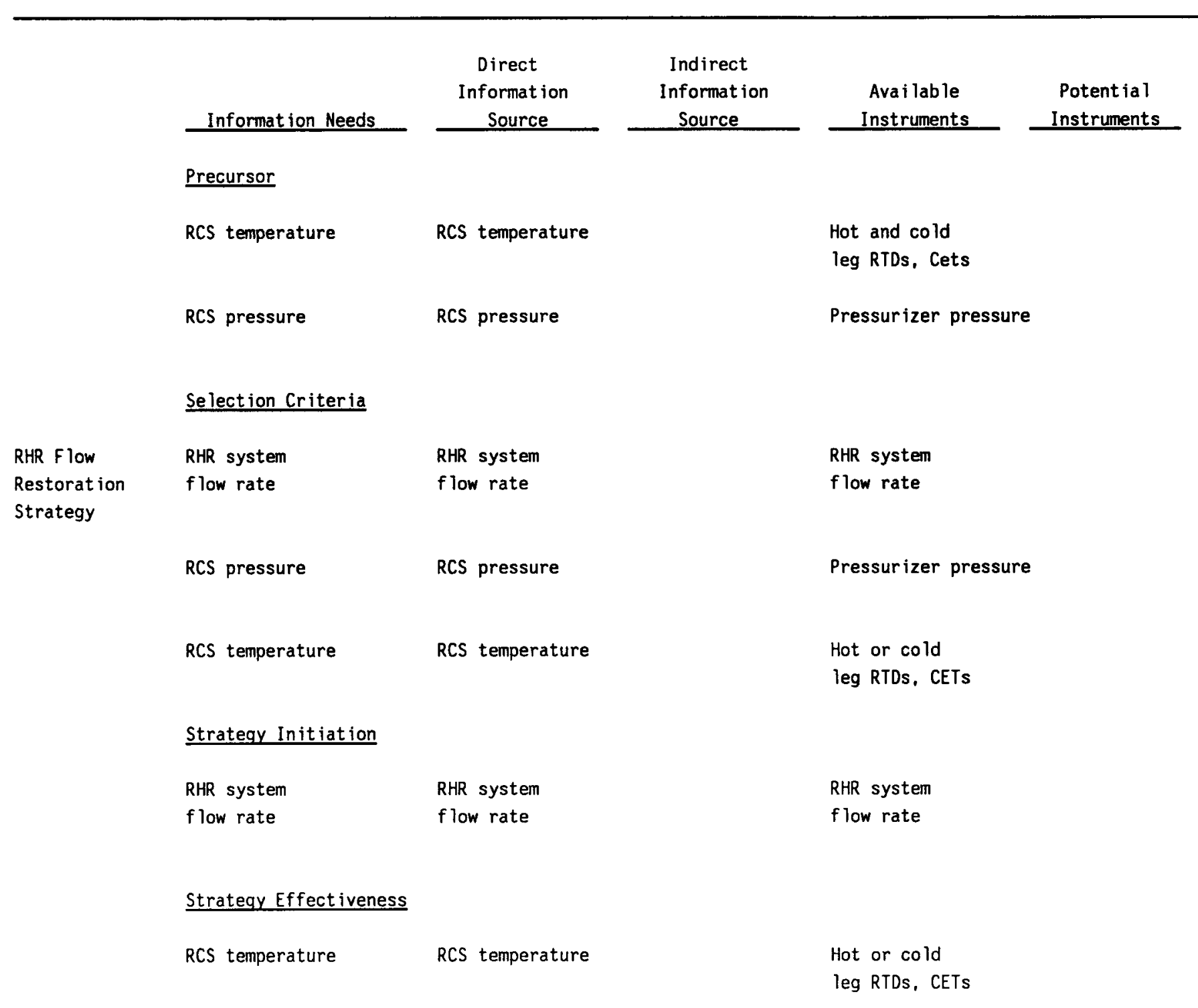


TABLE A1.5. (cont inued)

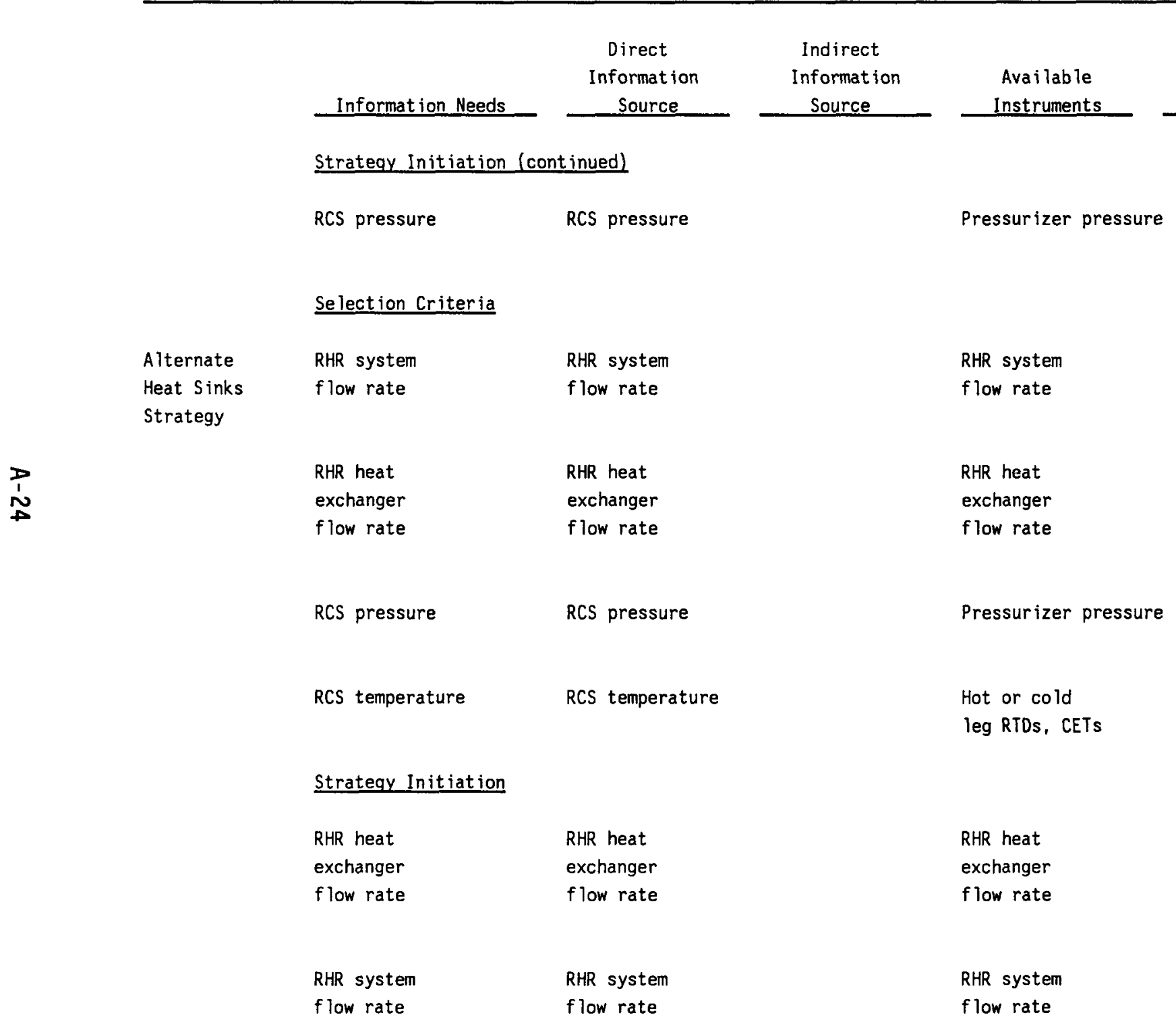


TABLE A1.5. (cont inued)

\begin{tabular}{|c|c|c|c|c|}
\hline Information Needs & $\begin{array}{c}\text { Direct } \\
\text { Information } \\
\text { Source } \\
\end{array}$ & $\begin{array}{c}\text { Indirect } \\
\text { Informat ion } \\
\text { Source }\end{array}$ & $\begin{array}{c}\text { Ava i lable } \\
\text { Instruments } \\
\end{array}$ & $\begin{array}{c}\text { Potential } \\
\text { Instruments }\end{array}$ \\
\hline \multicolumn{5}{|l|}{ Strategy Effect iveness } \\
\hline RCS temperature & RCS temperature & & $\begin{array}{l}\text { Hot or cold } \\
\text { leg RTDs, CETs }\end{array}$ & \\
\hline RCS pressure & RCS pressure & & Pressurizer pre & \\
\hline
\end{tabular}


TABLE A1.6. PREVENT CORE DISPERSAL FROM VESSEL (V)--SCRAM FAILURE MECHANISM (V2A1)

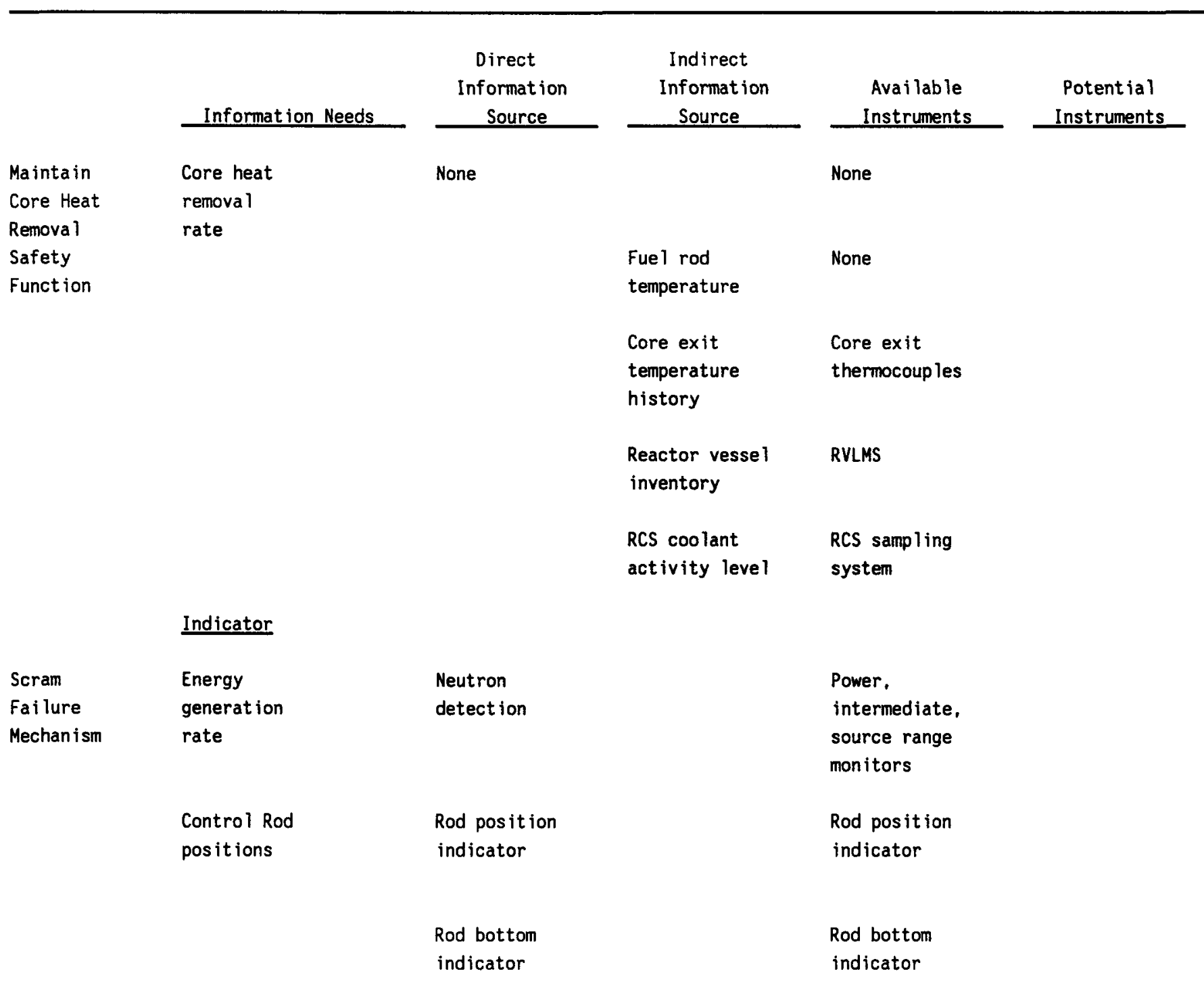


TABLE A1.6. (cont inued)

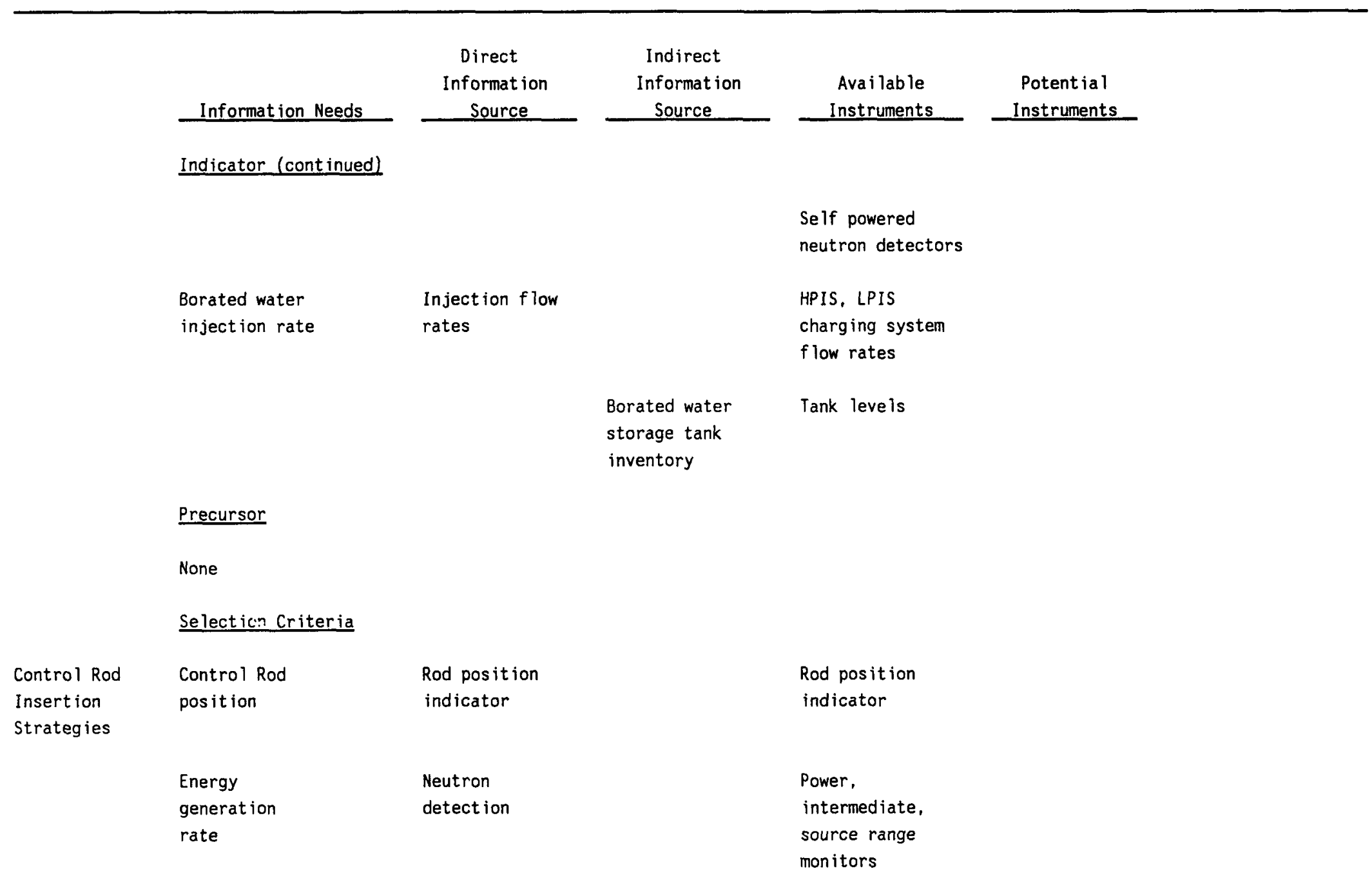


TABLE A1.6. (cont inued)

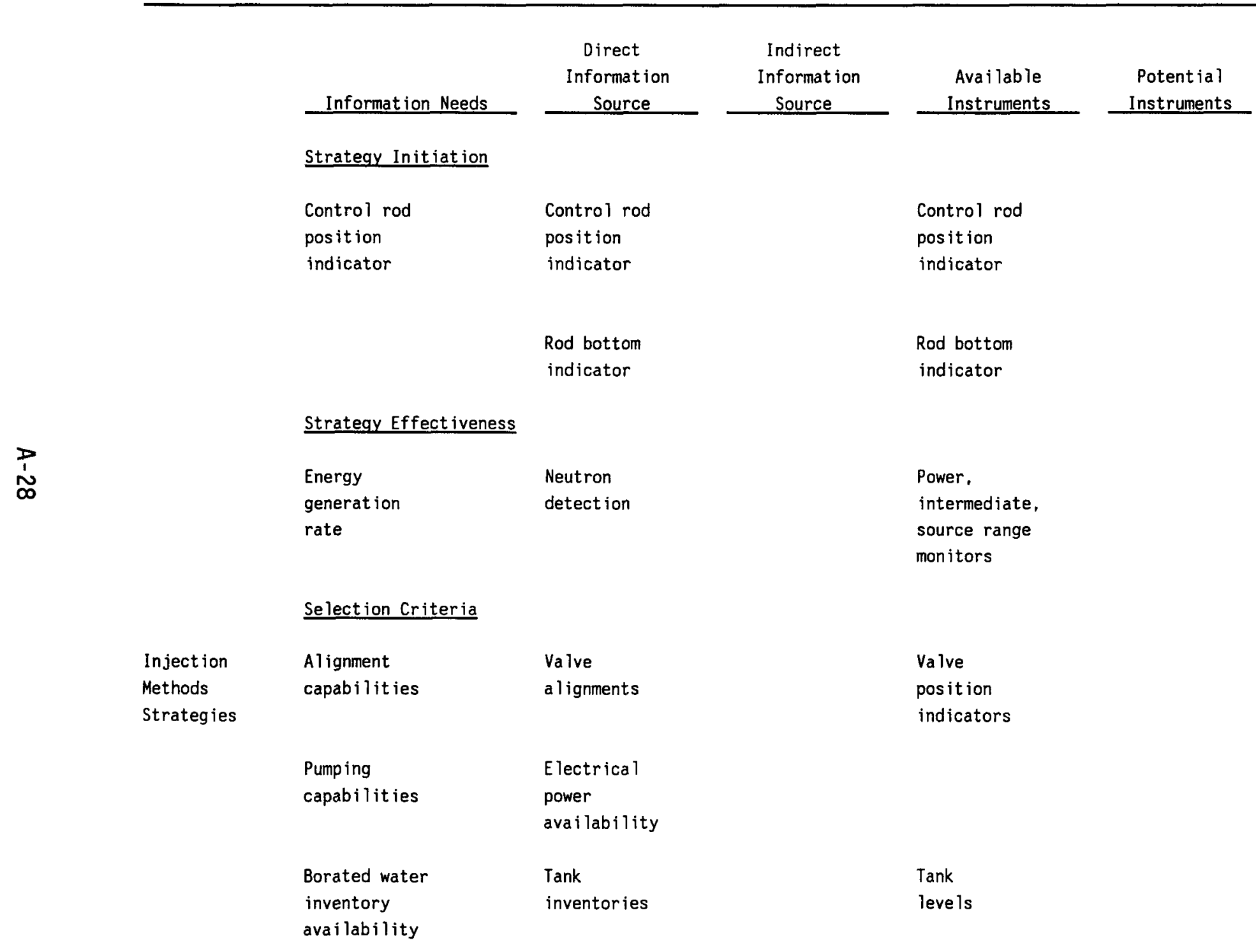


TABLE A1.6. (cont inued)

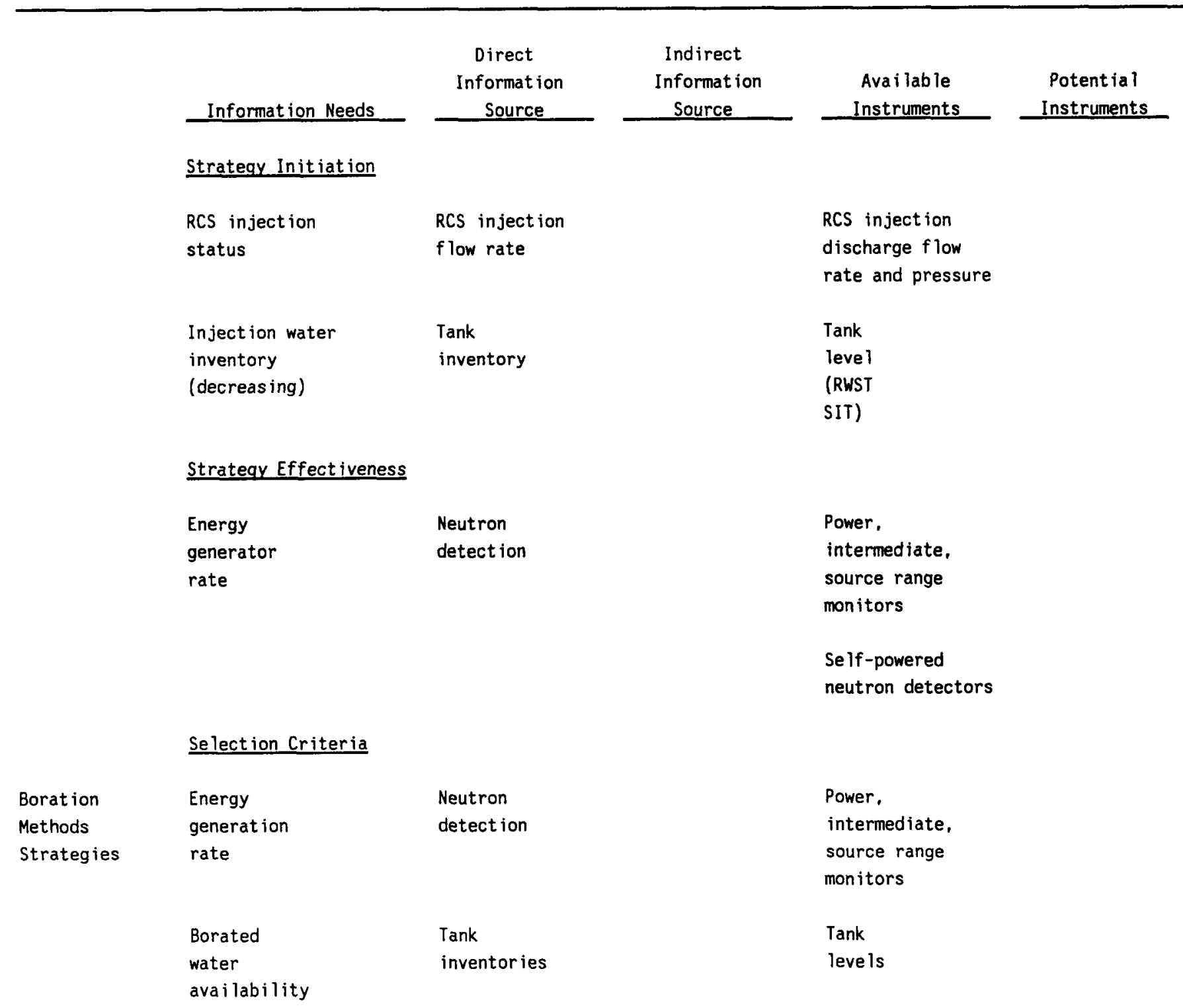


TABLE Al.6. (cont inued)

\begin{tabular}{|c|c|c|c|c|}
\hline & Direct & Indirect & & \\
\hline Informat ion Needs & $\begin{array}{c}\text { Information } \\
\text { Source }\end{array}$ & $\begin{array}{c}\text { Information } \\
\text { Source }\end{array}$ & $\begin{array}{l}\text { Ava i lable } \\
\text { Instruments }\end{array}$ & $\begin{array}{c}\text { Potential } \\
\text { Instruments }\end{array}$ \\
\hline
\end{tabular}

Se lection Criteria (cont inued)

$\begin{array}{ll}\text { Injection } & \text { Injection } \\ \text { system } & \text { system } \\ \text { capabilities } & \text { status }\end{array}$

Strategy Initiation

RCS injection RCS injection

status flow rate

Strategy Effectiveness

Energy

generator

rate

Neutron

detection
RCS injection

discharge flow

rate and pressure

Power

intermediate,

source range

monitors

Self-powered

neutron detectors 
TABLE A1.7. PREVENT CORE DISPERSAL FROM VESSEL (V)--RECRITICALITY (V2A2)

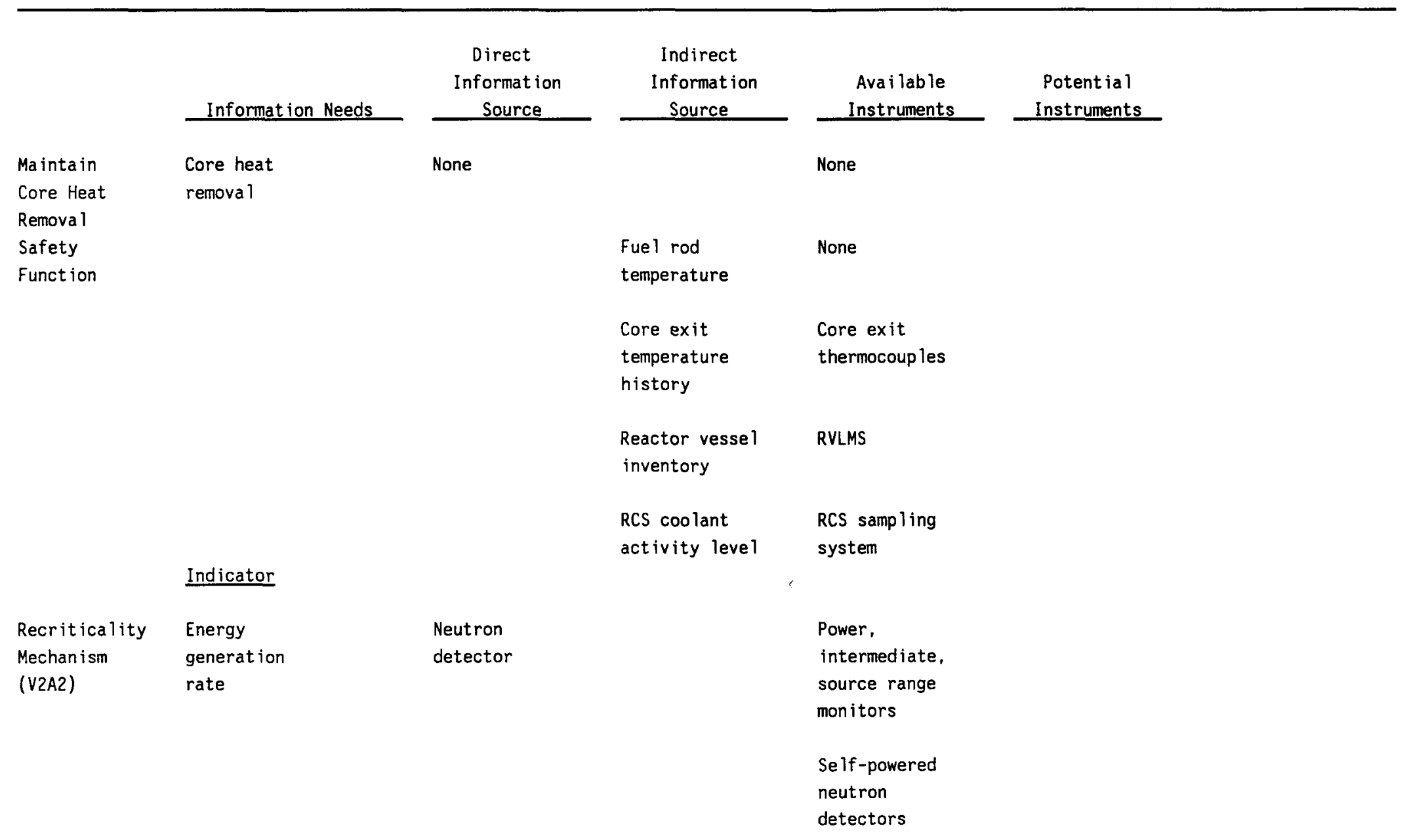


TABLE A1.7. (cont inued)

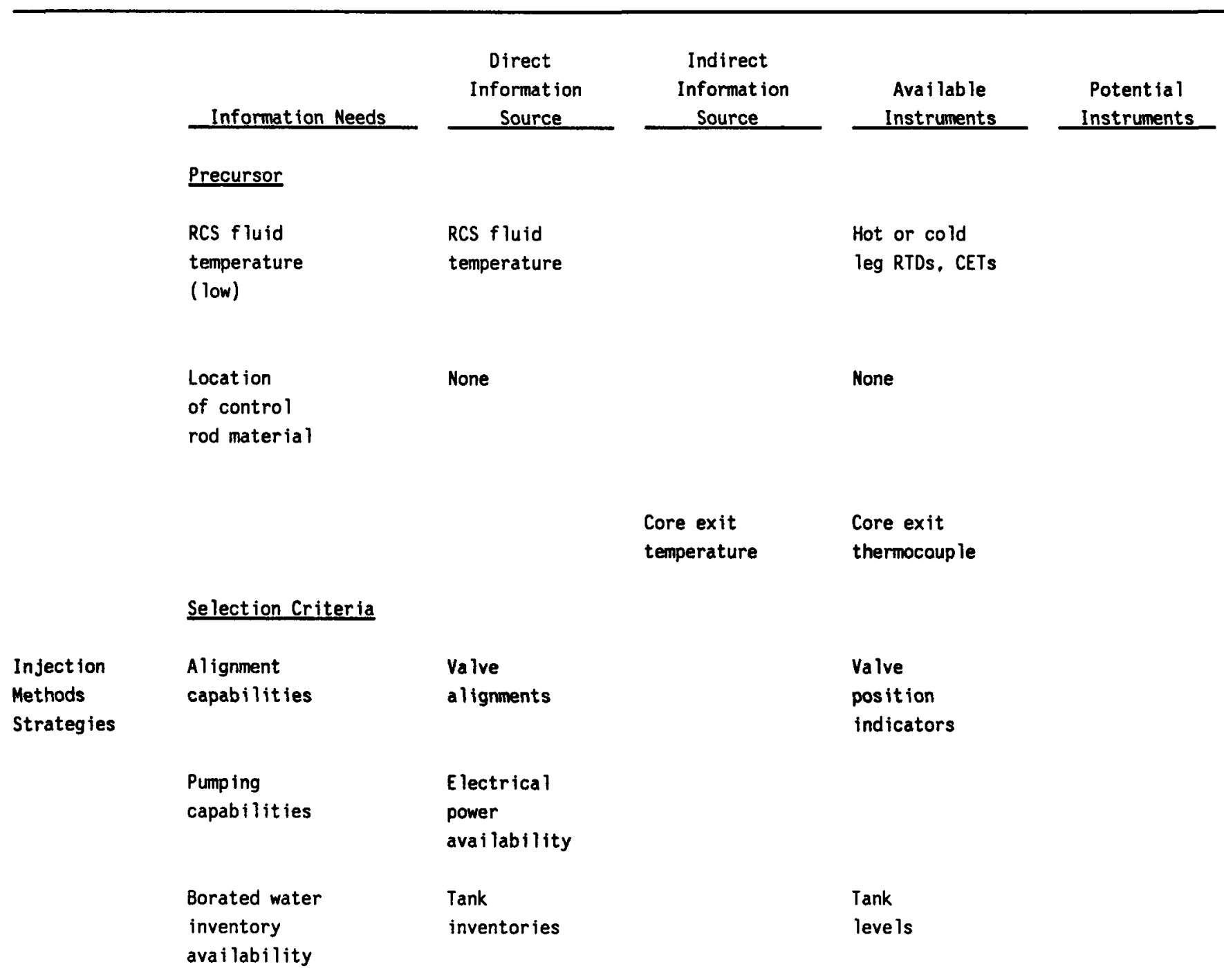


TABLE A1.7. (cont inued)

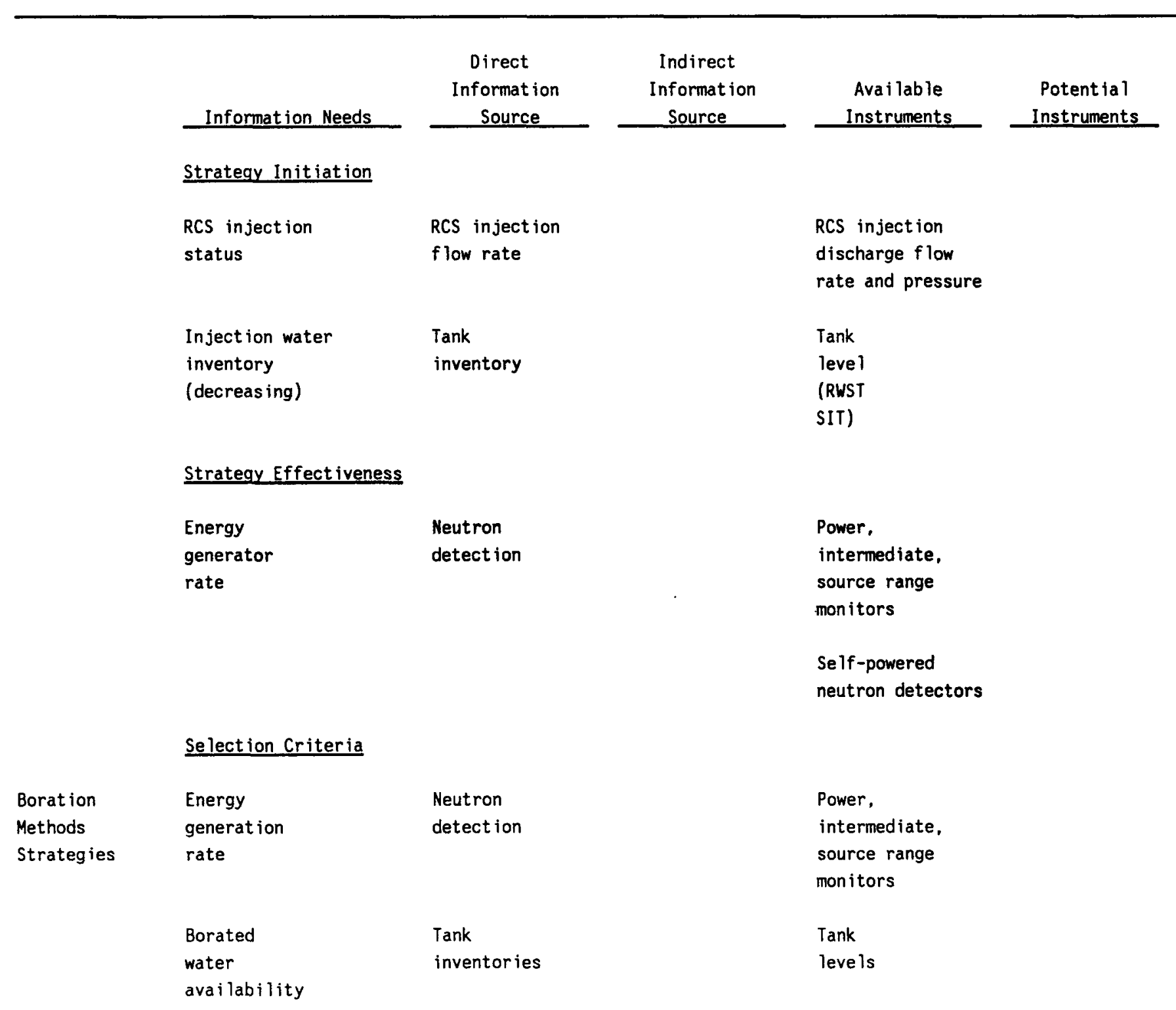


TABLE A1.7. (cont inued)

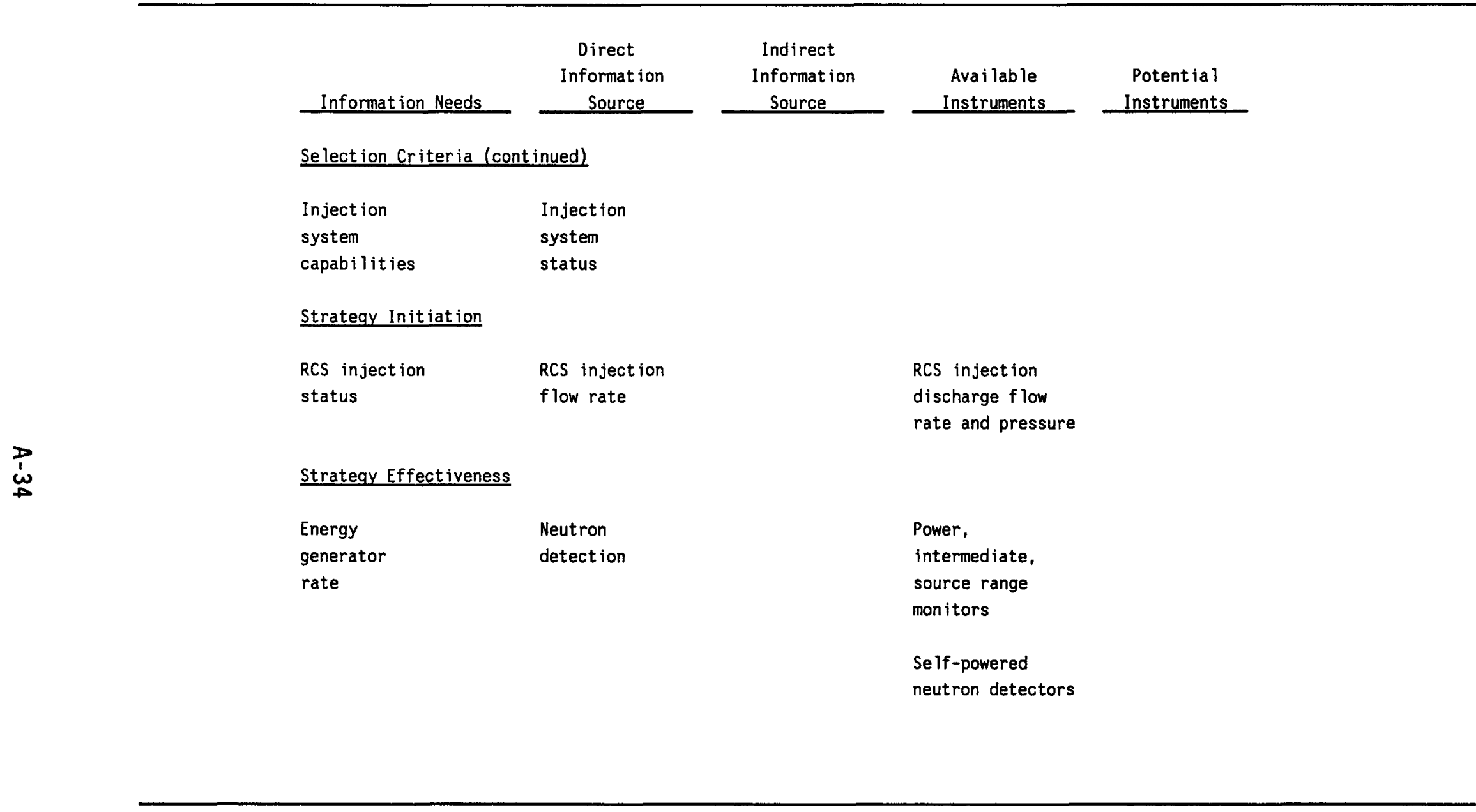


TABLE A1.8. PREVENT CORE DISPERSAL FROM VESSEL (V)--CIRCULATION FAILURE MECHANISM (VZA3)

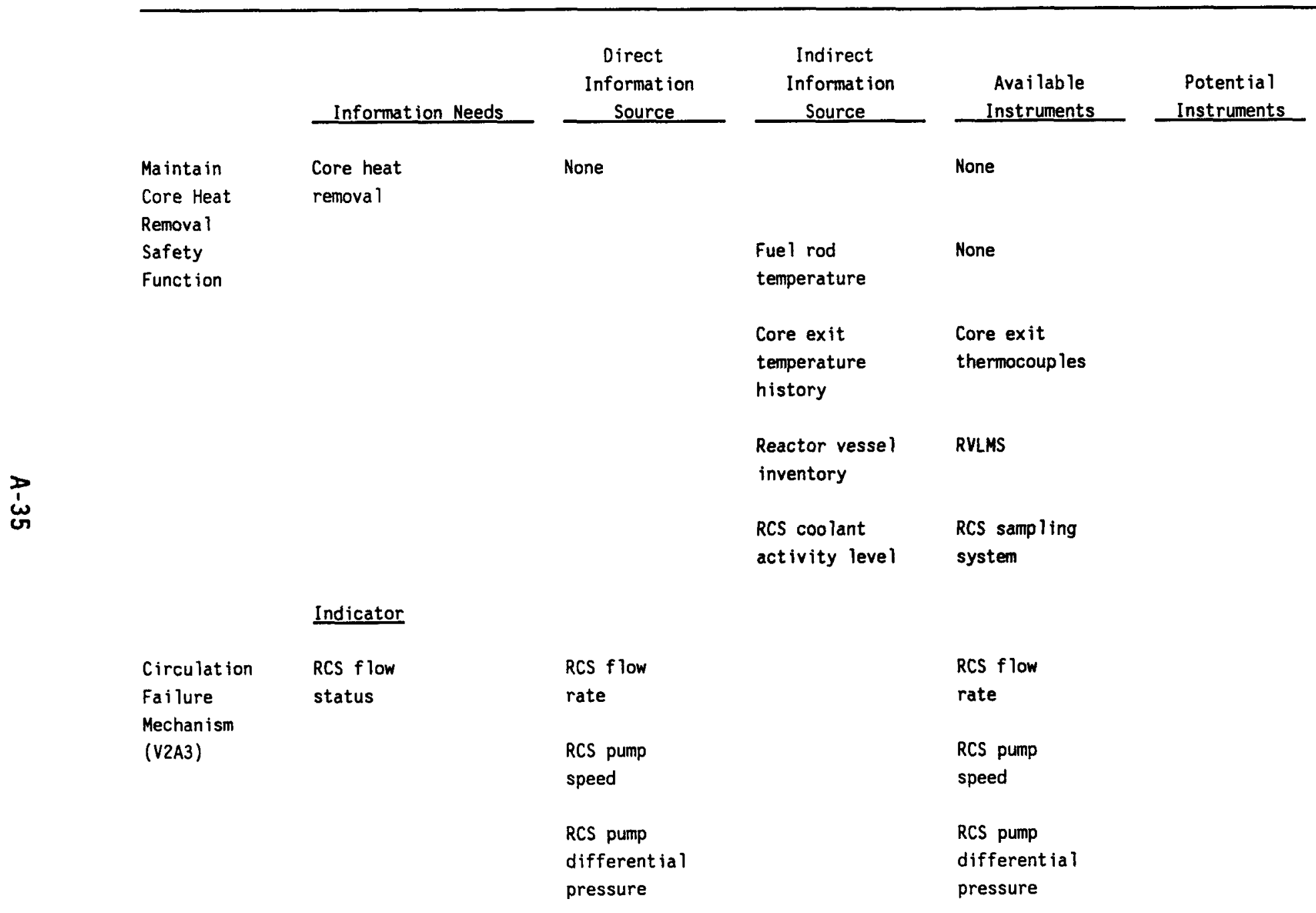


TABLE A1.8. (cont inued)

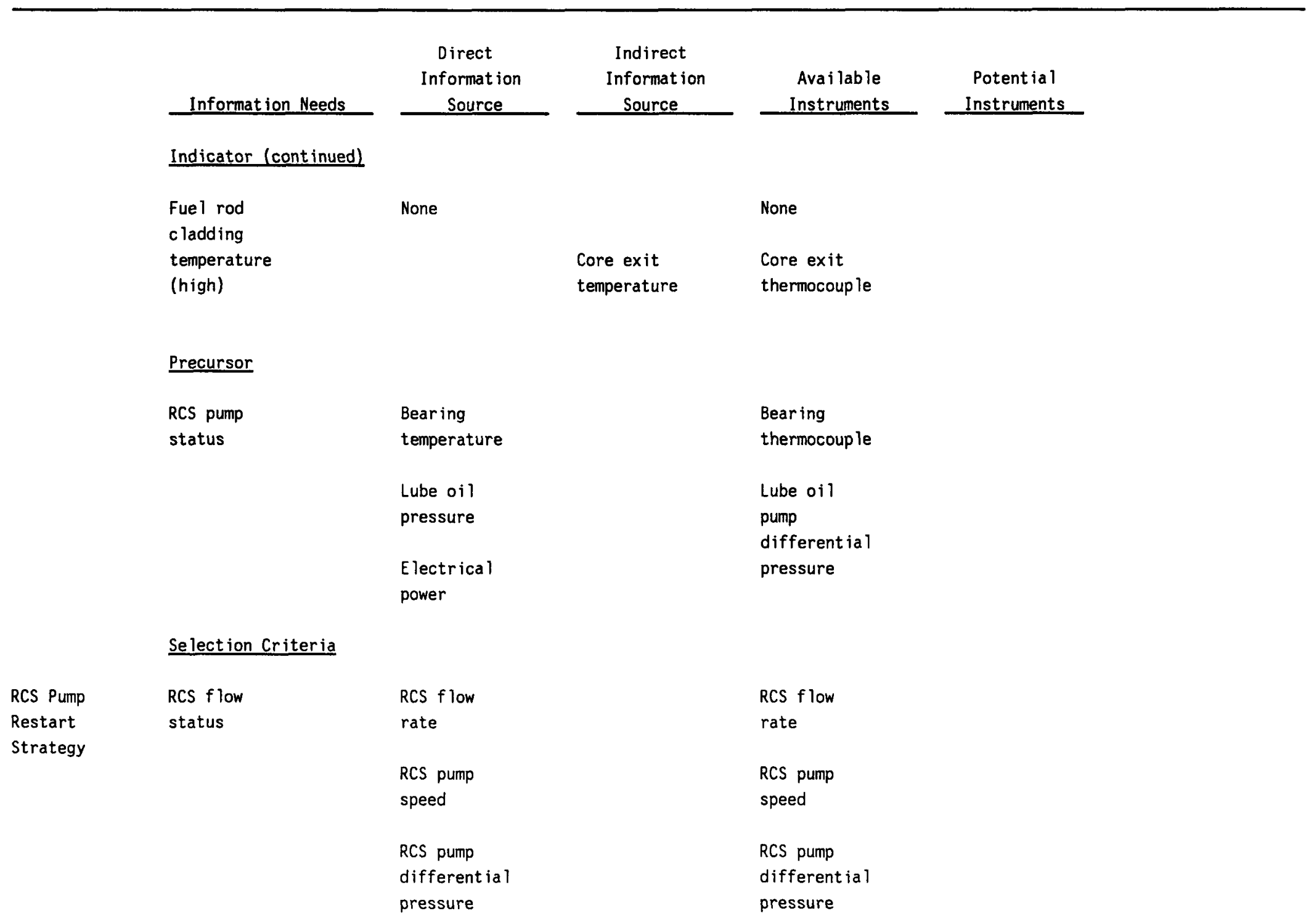


TABLE A1.8. (cont inued)

\begin{tabular}{|c|c|c|c|c|}
\hline Information Needs & $\begin{array}{c}\text { Direct } \\
\text { Information } \\
\text { Source } \\
\end{array}$ & $\begin{array}{c}\text { Indirect } \\
\text { Information } \\
\text { Source }\end{array}$ & $\begin{array}{c}\text { Available } \\
\text { Instruments }\end{array}$ & $\begin{array}{l}\text { Potential } \\
\text { Instruments }\end{array}$ \\
\hline
\end{tabular}

Fuel rod None
cladding

None

None

temperature

Core exit Core exit

temperature thermocouple

Strategy Initiation

$\begin{array}{lll}\begin{array}{l}\text { RCS flow } \\ \text { status }\end{array} & \text { RCS flow } & \text { RCS flow } \\ \text { rate } & \text { rate }\end{array}$

Strategy Effectiveness

$\begin{array}{lll}\begin{array}{l}\text { RCS flow } \\ \text { status }\end{array} & \begin{array}{l}\text { RCS flow } \\ \text { rate }\end{array} & \begin{array}{l}\text { RCS flow } \\ \text { rate }\end{array} \\ \begin{array}{l}\text { Fuel rod } \\ \text { cladding } \\ \text { temperature }\end{array} & \text { None } & \text { None } \\ & & \begin{array}{l}\text { Core exit } \\ \text { temperature }\end{array}\end{array}$


TABLE A1.9. PREVENT CORE DISPERSAL FROM VESSEL (V)--INADEQUATE RCS INVENTORY (V2A4)

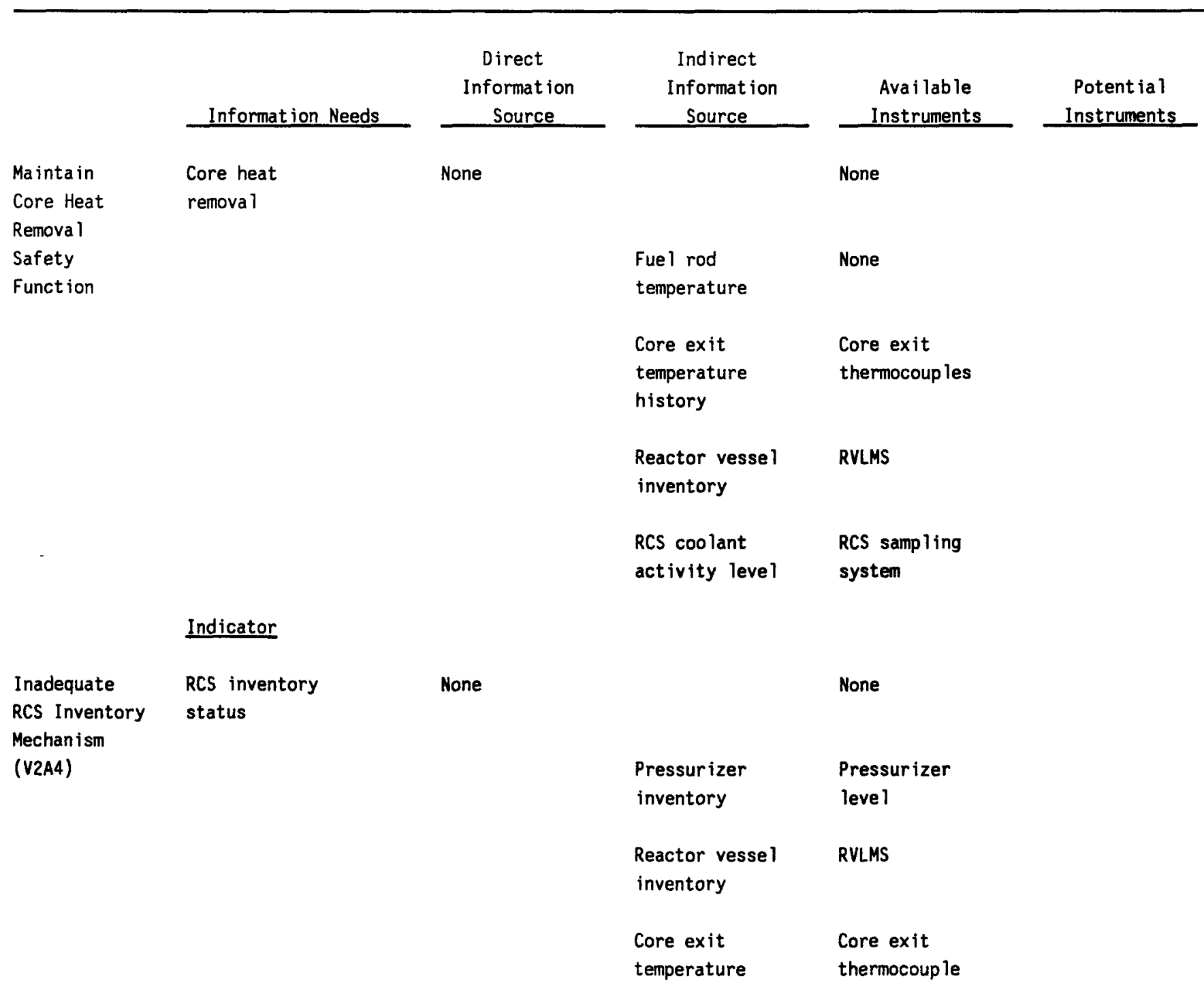


TABLE A1.9. (cont inued)

\begin{tabular}{|c|c|c|c|}
\hline Informat ion Needs & $\begin{array}{c}\text { Direct } \\
\text { Information } \\
\text { Source } \\
\end{array}$ & $\begin{array}{c}\text { Indirect } \\
\text { Information } \\
\text { Source } \\
\end{array}$ & $\begin{array}{r}\text { Available } \\
\text { Instrument }\end{array}$ \\
\hline \multicolumn{4}{|l|}{ Indicator (cont inued) } \\
\hline $\begin{array}{l}\text { Conta inment } \\
\text { sump inventory } \\
\text { (increasing) }\end{array}$ & $\begin{array}{l}\text { Containment } \\
\text { sump level }\end{array}$ & & $\begin{array}{l}\text { Containment } \\
\text { sump level }\end{array}$ \\
\hline \multicolumn{4}{|l|}{ Precursor } \\
\hline $\begin{array}{l}\text { RCS pressure } \\
\text { (low) }\end{array}$ & RCS pressure & & $\begin{array}{l}\text { Pressurizer } \\
\text { pressure }\end{array}$ \\
\hline $\begin{array}{l}\text { Injection } \\
\text { flow rate }\end{array}$ & $\begin{array}{l}\text { Injection } \\
\text { flow rate }\end{array}$ & & $\begin{array}{l}\text { Injection } \\
\text { flow rate }\end{array}$ \\
\hline
\end{tabular}

\section{Selection Criteria}

RCS Injection

Methods

Strategies
RCS pressure history RCS pressure

RCS inventory

(trend)

None
Pressurizer pressure

None

Pressurizer Pressurizer level

inventory

Reactor vesse $7 \quad$ RVLMS

inventory
Potential

Instruments 
TABLE A1.9. (cont inued)

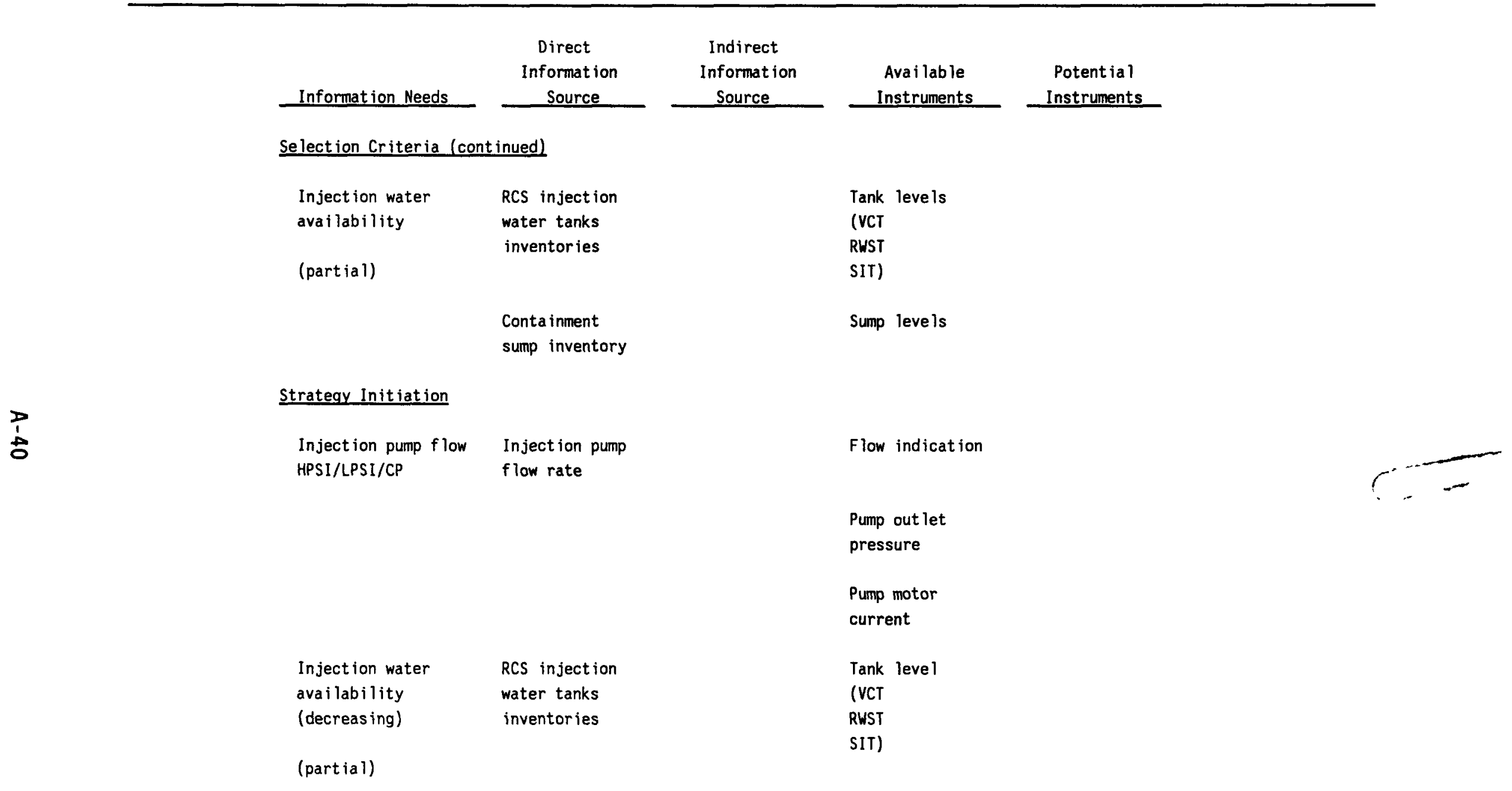


TABLE A1.9. (cont inued)

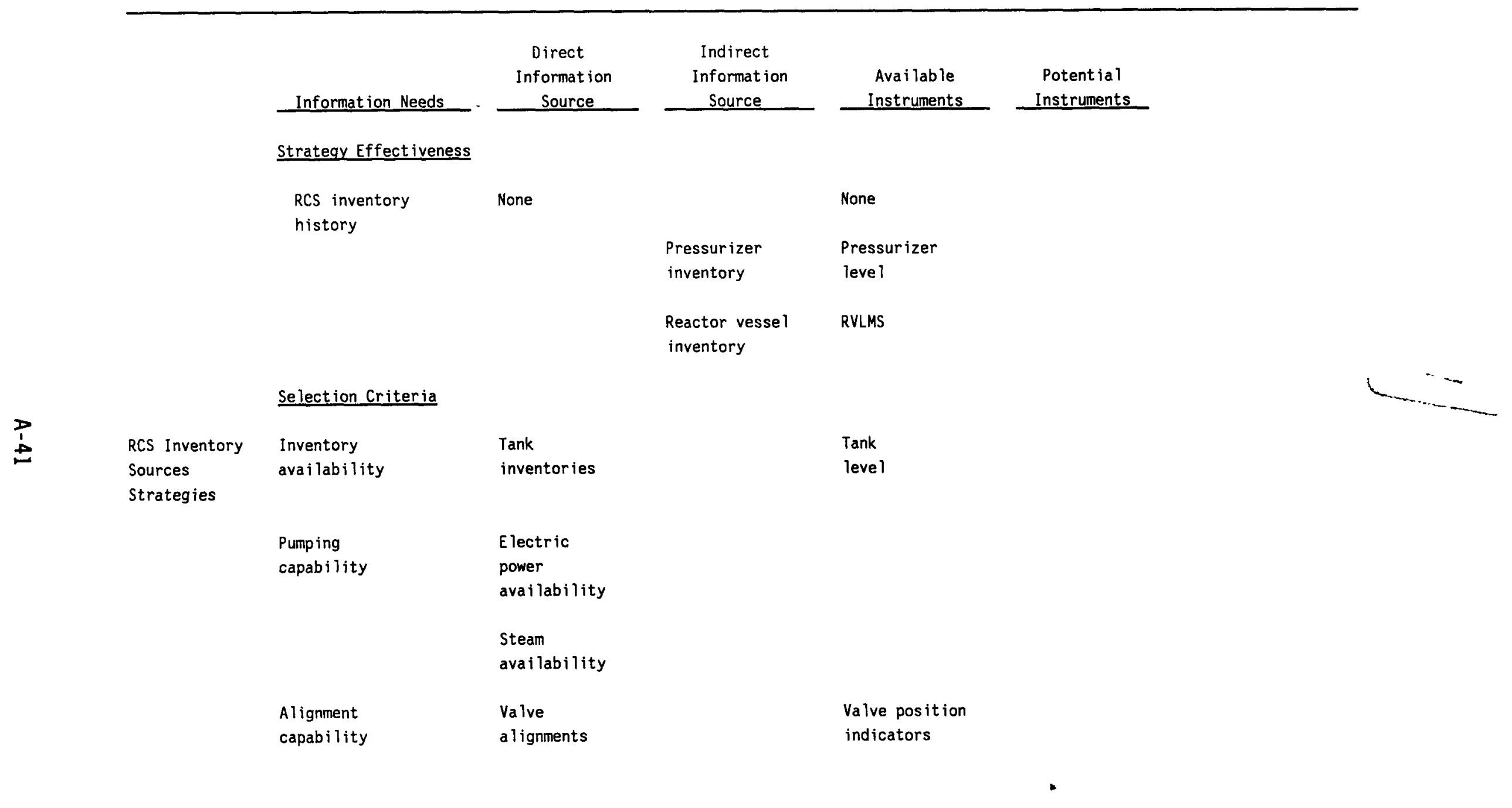


TABLE A1.9. (cont inued)

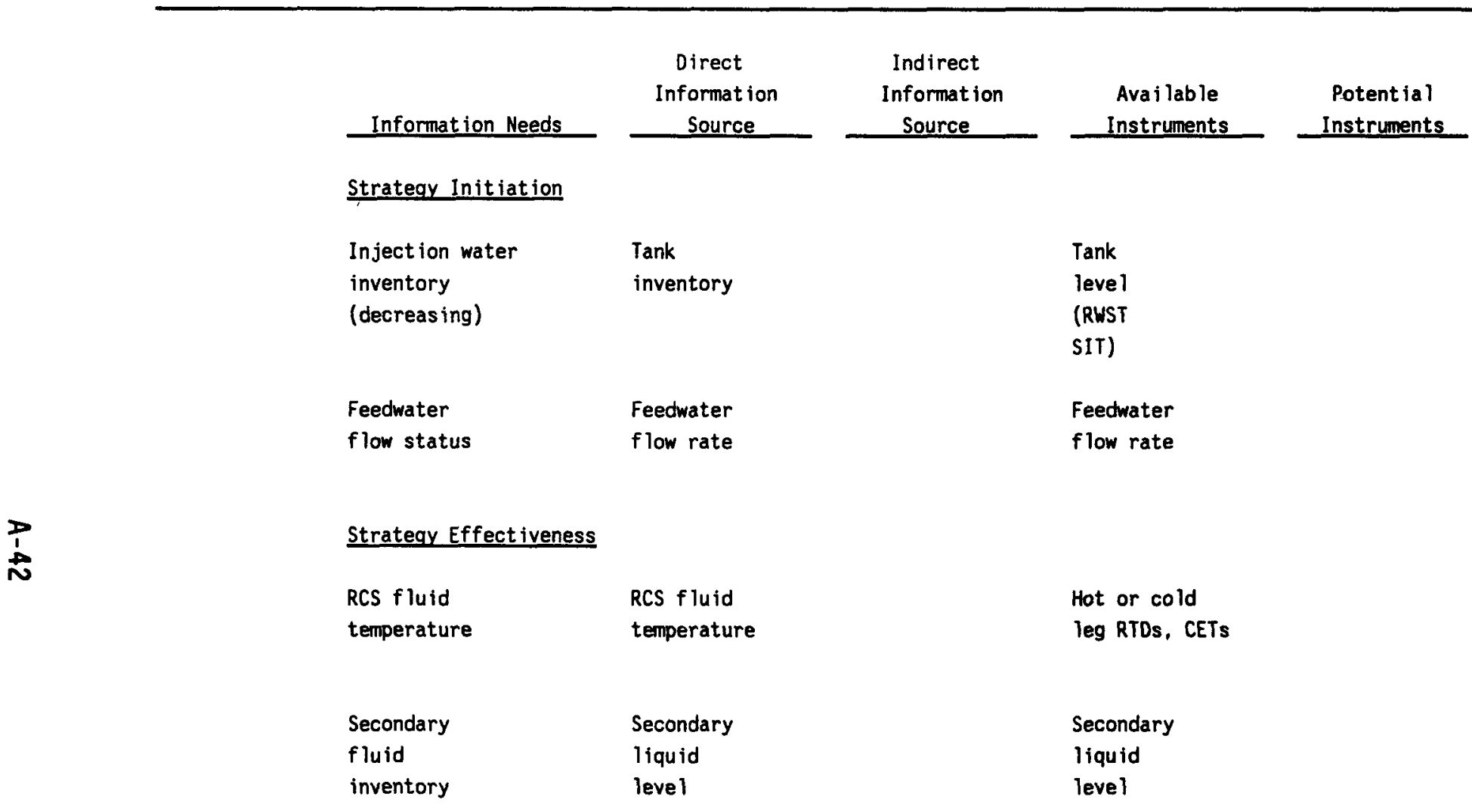


TABLE A1.10. CHANGE IN CORE GEOMETRY MECHANISM (V2B1)

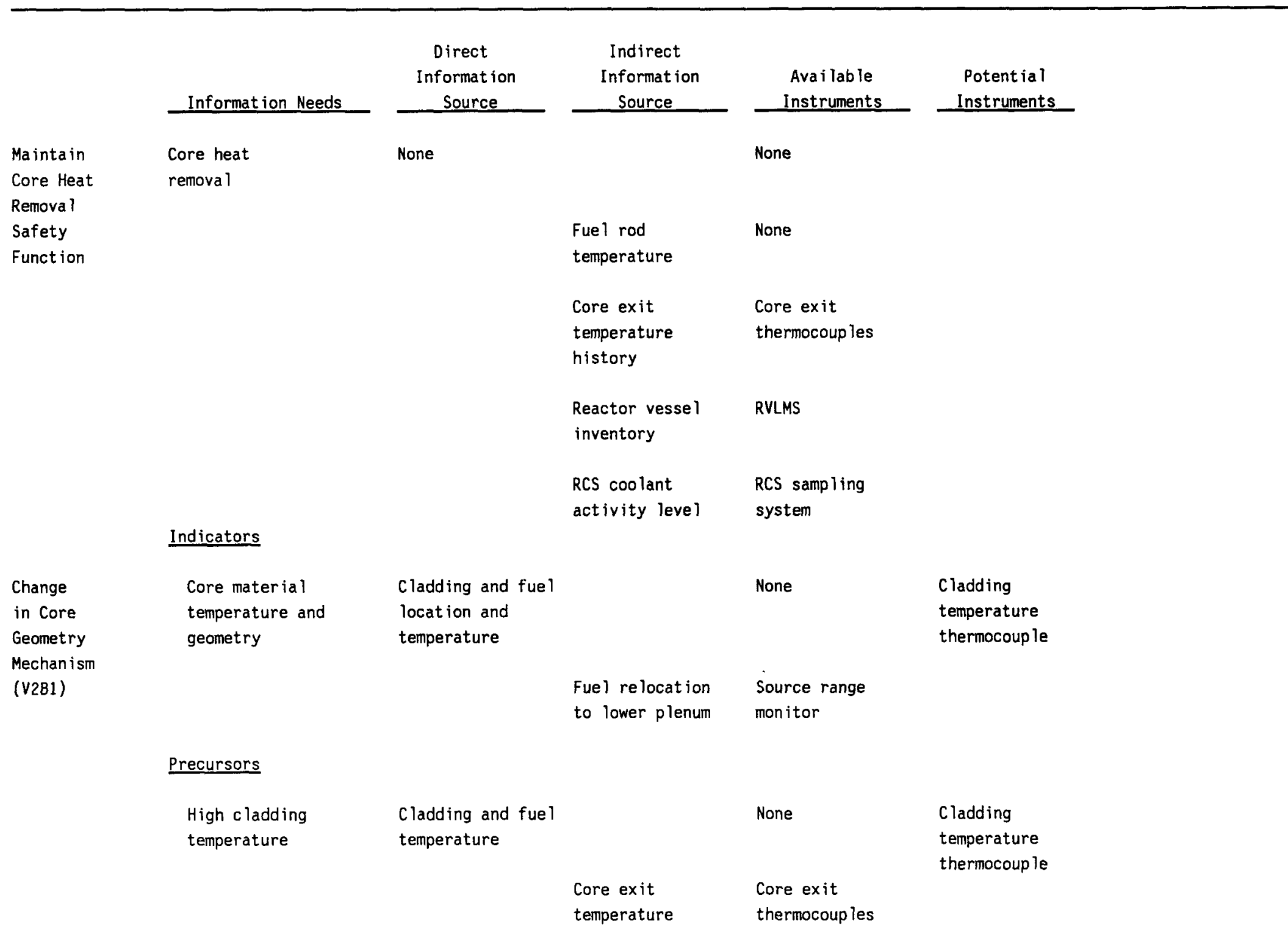


TABLE A1.10. (continued)

\begin{tabular}{|c|c|c|c|c|}
\hline & Information Needs & $\begin{array}{c}\text { Direct } \\
\text { Information } \\
\text { Source } \\
\end{array}$ & $\begin{array}{c}\text { Indirect } \\
\text { Information } \\
\text { Source } \\
\end{array}$ & $\begin{array}{l}\text { Avai lable } \\
\text { Instruments }\end{array}$ \\
\hline & \multicolumn{4}{|l|}{ Precursors (cont inued) } \\
\hline & & & $\begin{array}{l}\text { Cladding } \\
\text { temperature }\end{array}$ & SPNDS \\
\hline & & & $\begin{array}{l}\text { Coolant activity } \\
\text { level }\end{array}$ & $\begin{array}{l}\text { RCS sampling } \\
\text { system }\end{array}$ \\
\hline & \multicolumn{4}{|l|}{ Selection Criteria } \\
\hline $\begin{array}{l}\text { RCS Pump } \\
\text { Restart }\end{array}$ & $\begin{array}{l}\text { RCS flow } \\
\text { status }\end{array}$ & \multicolumn{2}{|l|}{$\begin{array}{l}\text { RCS flow } \\
\text { rate }\end{array}$} & $\begin{array}{l}\text { RCS flow } \\
\text { rate }\end{array}$ \\
\hline & & \multicolumn{2}{|l|}{$\begin{array}{l}\text { RCS pump } \\
\text { speed }\end{array}$} & $\begin{array}{l}\text { RCS pump } \\
\text { speed }\end{array}$ \\
\hline & & \multicolumn{2}{|l|}{$\begin{array}{l}\text { RCS pump } \\
\text { differential } \\
\text { pressure }\end{array}$} & $\begin{array}{l}\text { RCS pump } \\
\text { differential } \\
\text { pressure }\end{array}$ \\
\hline & $\begin{array}{l}\text { Fuel rod } \\
\text { cladding } \\
\text { temperature }\end{array}$ & \multicolumn{2}{|l|}{ None } & None \\
\hline & & & $\begin{array}{l}\text { Core exit } \\
\text { temperature }\end{array}$ & $\begin{array}{l}\text { Core exit } \\
\text { thermocouple }\end{array}$ \\
\hline & \multicolumn{4}{|l|}{ Strategy Initiation } \\
\hline & $\begin{array}{l}\text { RCS flow } \\
\text { status }\end{array}$ & \multicolumn{2}{|l|}{$\begin{array}{l}\text { RCS flow } \\
\text { rate }\end{array}$} & $\begin{array}{l}\text { RCS flow } \\
\text { rate }\end{array}$ \\
\hline
\end{tabular}


TABLE A1.10. (cont inued)

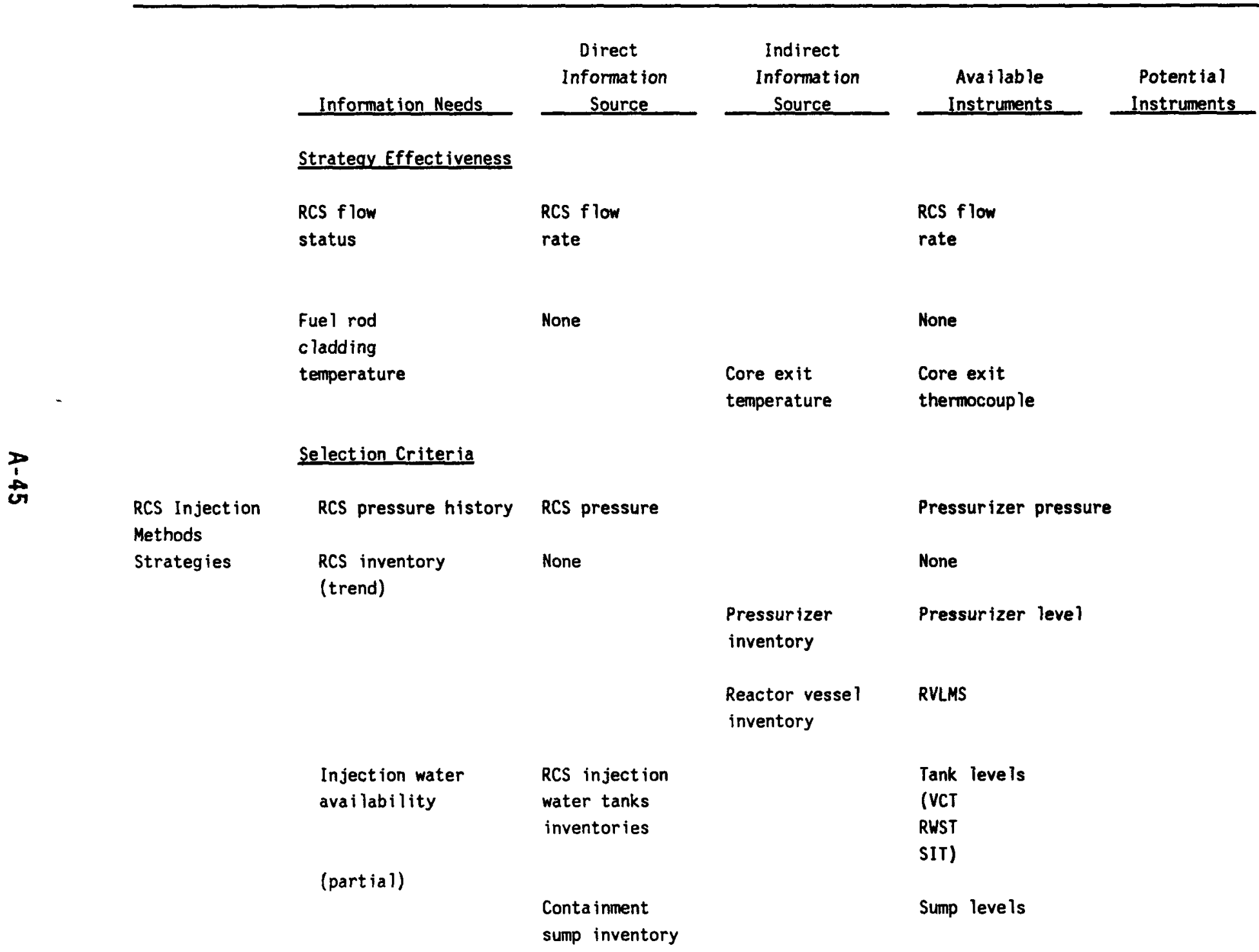


TABLE A1.10. (cont inued)

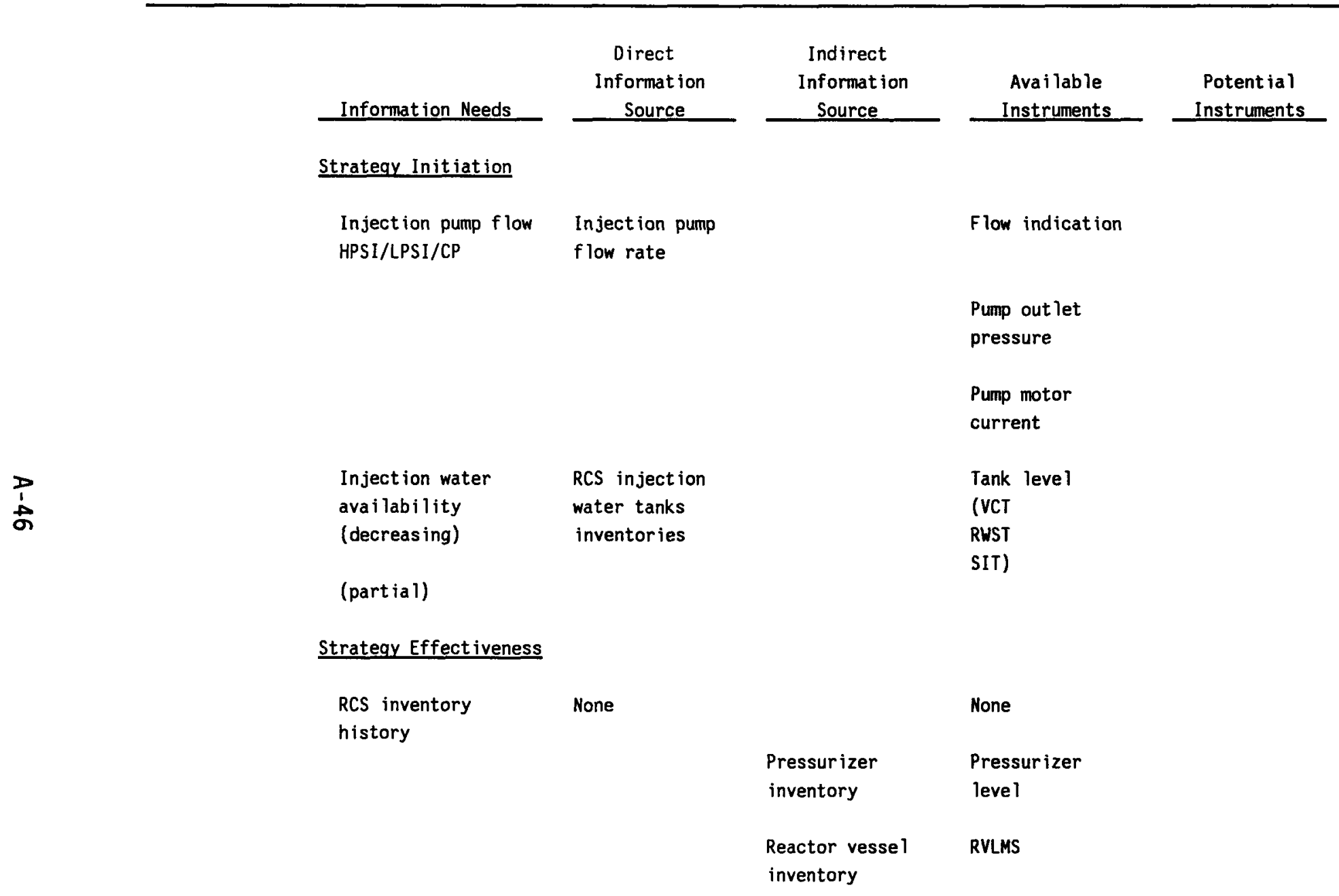


TABLE A1.10. (cont inued)

\begin{tabular}{|c|c|c|c|c|c|}
\hline & Information Needs & $\begin{array}{c}\text { Direct } \\
\text { Information } \\
\text { Source } \\
\end{array}$ & $\begin{array}{l}\text { Indirect } \\
\text { Information } \\
\text { Source } \\
\end{array}$ & $\begin{array}{c}\text { Ava i lable } \\
\text { Instruments } \\
\end{array}$ & $\begin{array}{l}\text { Potential } \\
\text { Instruments }\end{array}$ \\
\hline & Selection Criteria & & & & \\
\hline \multirow[t]{7}{*}{$\begin{array}{l}\text { RCS Inventory } \\
\text { Sources } \\
\text { Strategies }\end{array}$} & $\begin{array}{l}\text { Inventory } \\
\text { ava i lability }\end{array}$ & $\begin{array}{l}\text { Tank } \\
\text { inventories }\end{array}$ & & $\begin{array}{l}\text { Tank } \\
\text { leve l } \\
\text { (RWST } \\
\text { SIT) }\end{array}$ & \\
\hline & $\begin{array}{l}\text { Pumping } \\
\text { capability }\end{array}$ & $\begin{array}{l}\text { Electric } \\
\text { power } \\
\text { availability }\end{array}$ & & & \\
\hline & & $\begin{array}{l}\text { Steam } \\
\text { availability }\end{array}$ & & & \\
\hline & $\begin{array}{l}\text { Alignment } \\
\text { capability }\end{array}$ & $\begin{array}{l}\text { Valve } \\
\text { alignments }\end{array}$ & & $\begin{array}{l}\text { Valve position } \\
\text { indicators }\end{array}$ & \\
\hline & Strategy Initiation & & & & \\
\hline & $\begin{array}{l}\text { Injection water } \\
\text { inventory } \\
\text { (decreasing) }\end{array}$ & $\begin{array}{l}\text { Tank } \\
\text { inventory }\end{array}$ & & $\begin{array}{l}\text { Tank } \\
\text { leve I } \\
\text { (RWST } \\
\text { SIT) }\end{array}$ & \\
\hline & $\begin{array}{l}\text { Feedwater } \\
\text { flow status }\end{array}$ & $\begin{array}{l}\text { Feedwater } \\
\text { f low rate }\end{array}$ & & $\begin{array}{l}\text { Feedwater } \\
\text { flow rate }\end{array}$ & \\
\hline
\end{tabular}


TABLE A1.10. (cont inued)

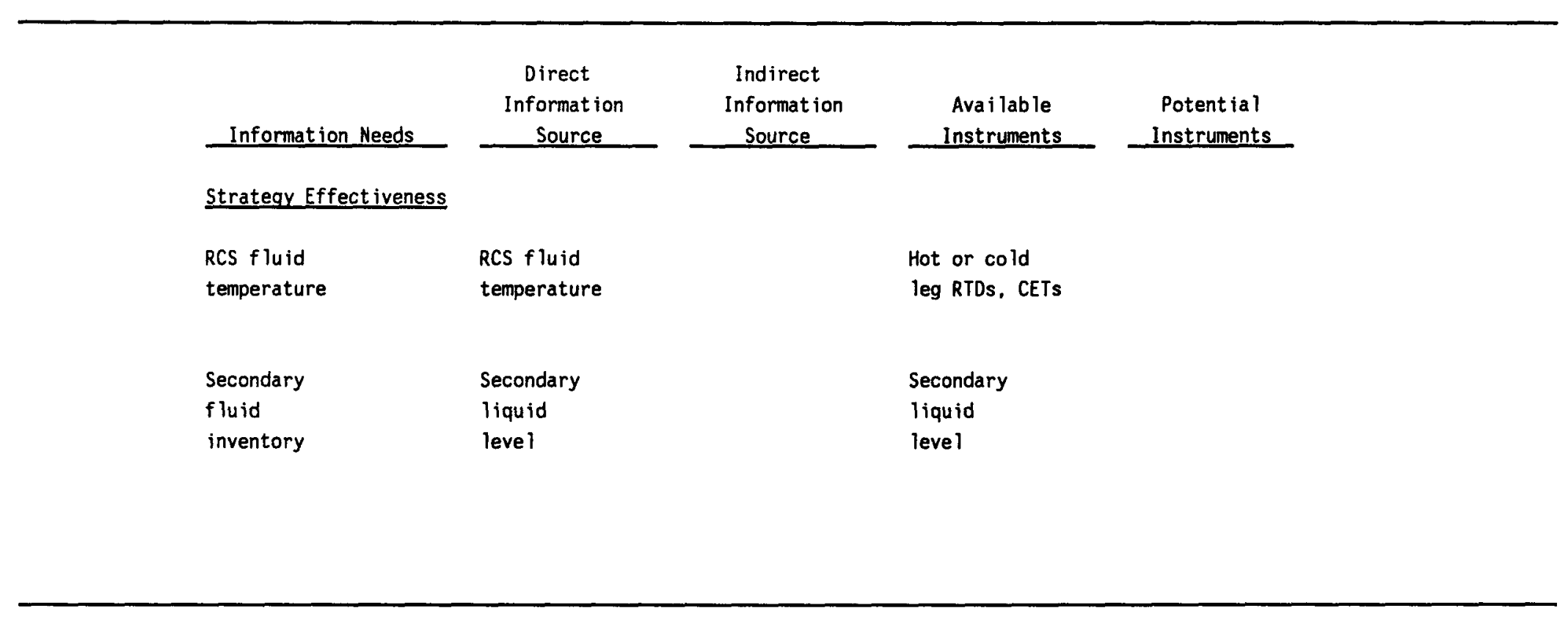


TABLE A1.11. PREVENT VESSEL FAILURE (V) - COOLABLE RELOCATION MECHANISM (V3A1)

\begin{tabular}{|c|c|c|c|c|c|}
\hline & Information Needs & $\begin{array}{c}\text { Direct } \\
\text { Information } \\
\text { Source }\end{array}$ & $\begin{array}{c}\text { Indirect } \\
\text { Information } \\
\text { Source } \\
\end{array}$ & $\begin{array}{c}\text { Available } \\
\text { Instruments }\end{array}$ & $\begin{array}{c}\text { Potential } \\
\text { Instruments } \\
\end{array}$ \\
\hline \multirow{5}{*}{$\begin{array}{l}\text { Maintain } \\
\text { vessel boundary } \\
\text { safety } \\
\text { function (V3) }\end{array}$} & Vessel integrity & $\begin{array}{l}\text { Vesse } 1 \text { visual } \\
\text { observation }\end{array}$ & & None & $\begin{array}{l}\text { Camera for } \\
\text { vessel }\end{array}$ \\
\hline & & & & & observation \\
\hline & & & Vessel wall & None & Vesse 1 \\
\hline & & & temperature & & $\begin{array}{l}\text { temperature } \\
\text { sensor }\end{array}$ \\
\hline & & & RCS pressure & Pressurizer pre & \\
\hline
\end{tabular}

Indicators

$\begin{array}{lll} & \begin{array}{l}\text { Coolable } \\ \text { relocation }\end{array} & \begin{array}{l}\text { Vesse } 1 \\ \text { integrity }\end{array} \\ & \text { mechanism } & \\ \frac{1}{6} & \text { (V3A1) } & \end{array}$

Vesse 1 visual
observation

\begin{tabular}{|c|c|c|}
\hline & None & $\begin{array}{l}\text { Camera for } \\
\text { vesse } 7\end{array}$ \\
\hline $\begin{array}{l}\text { Vessel wal1 } \\
\text { temperature }\end{array}$ & None & $\begin{array}{l}\text { Vesse } 1 \\
\text { temperature } \\
\text { sensor }\end{array}$ \\
\hline
\end{tabular}

\section{Precursors}

$\begin{array}{lll}\begin{array}{l}\text { Core relocation } \\ \text { status }\end{array} & \text { None } & \text { None } \\ & \begin{array}{l}\text { Ration levels } \\ \text { outside core } \\ \text { region }\end{array} & \begin{array}{l}\text { Source range } \\ \text { monitor }\end{array} \\ & \begin{array}{l}\text { RCS pressure } \\ \text { (rise) }\end{array} & \text { Pressurizer pressure } \\ \text { Cold leg } & \text { Cold leg RTD } \\ \end{array}$


TABLE A1.11. (cont inued)

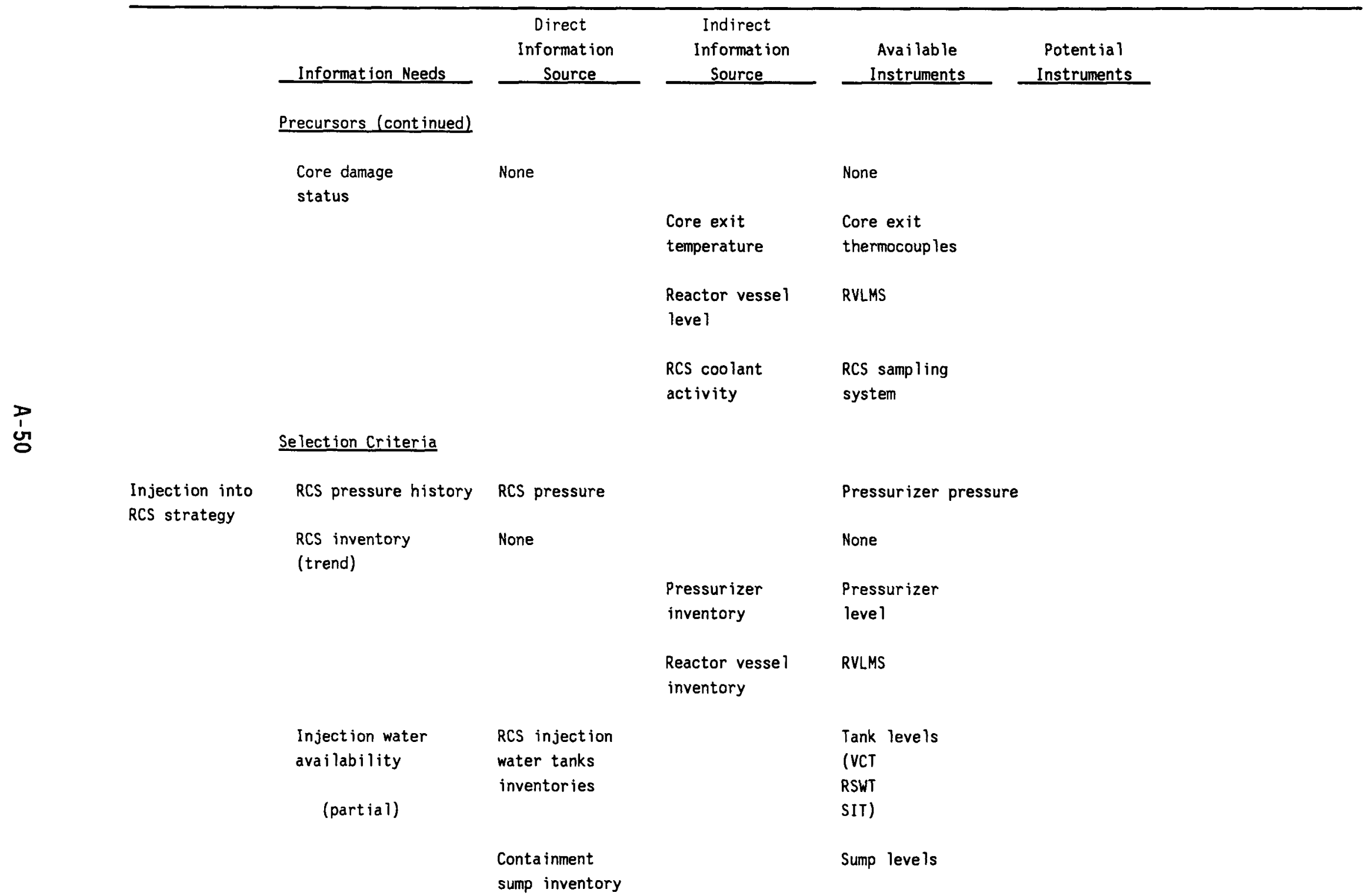


TABLE A1.11. (continued)

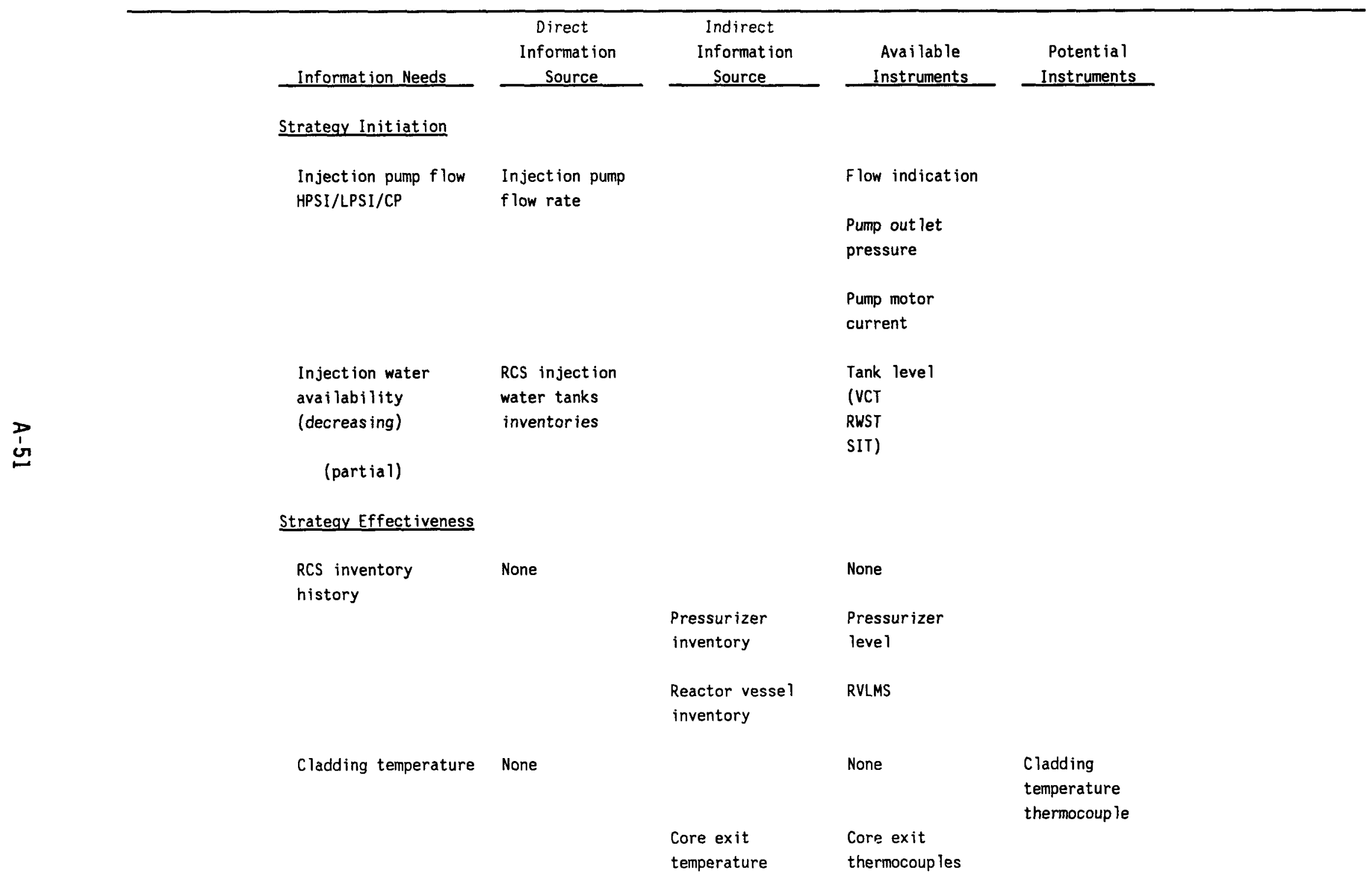


TABLE A1 11 (cont inued)

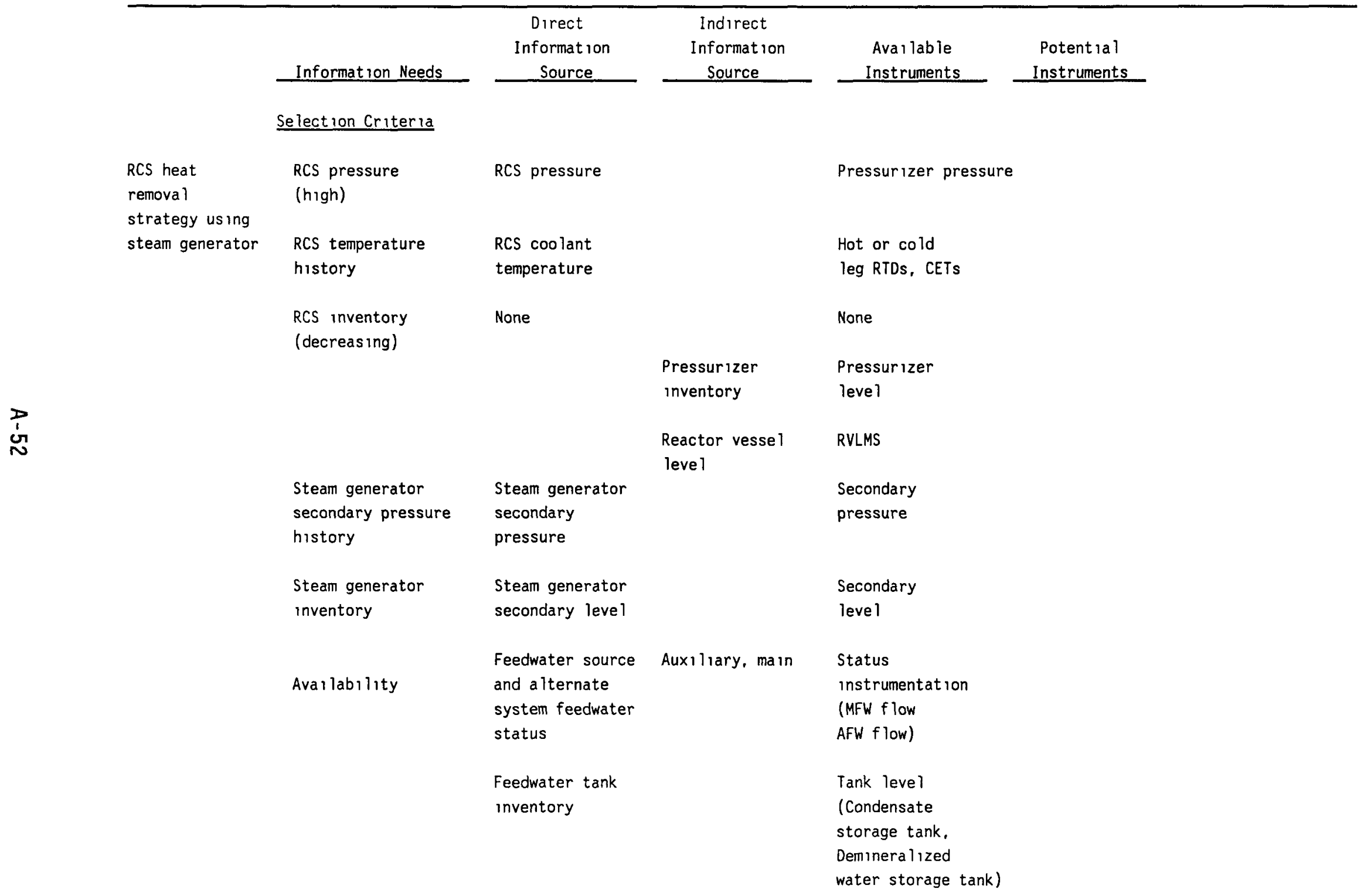


TABLE A1.11. (cont inued)

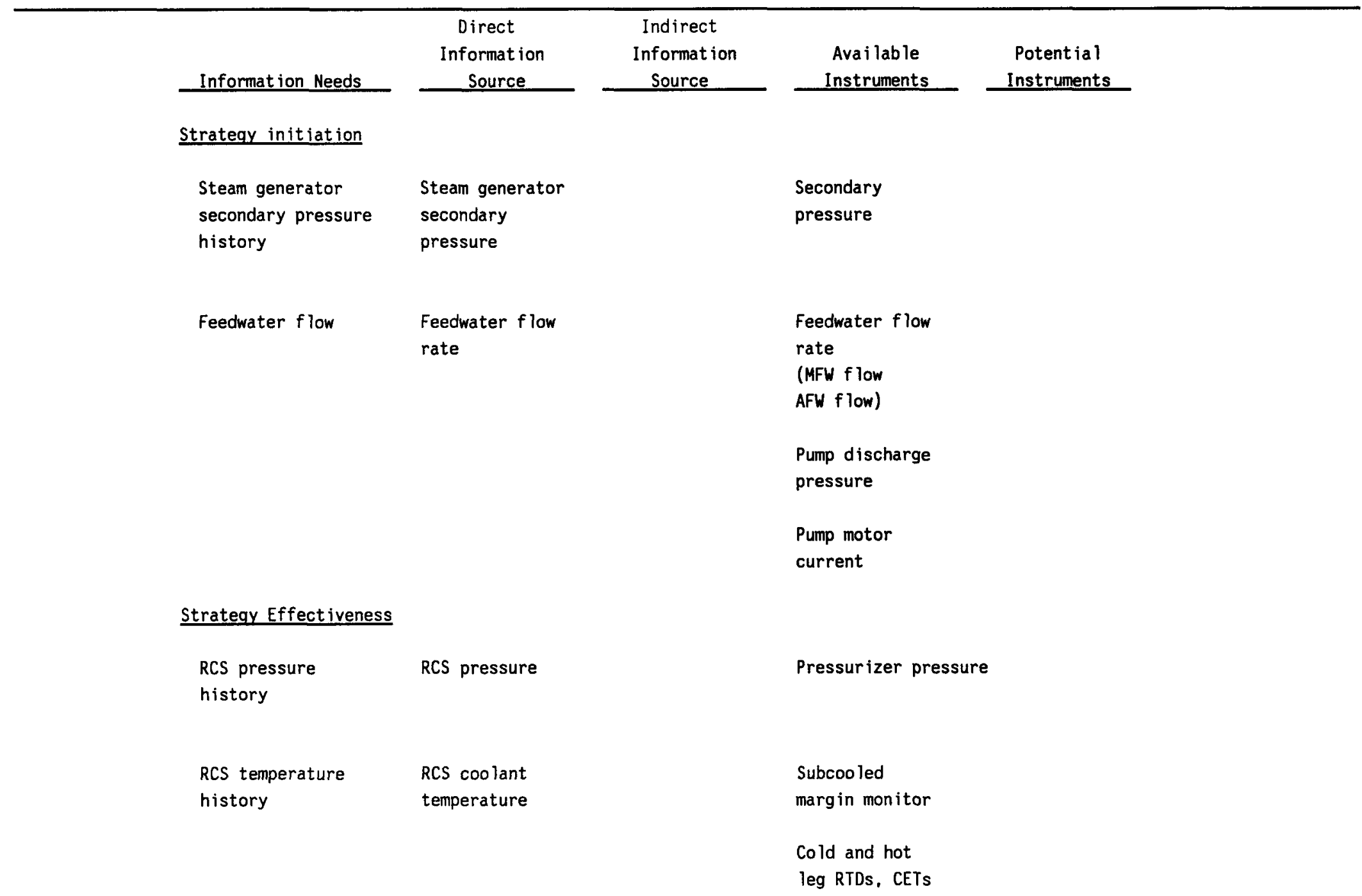


TABLE A1.11. (cont inued)

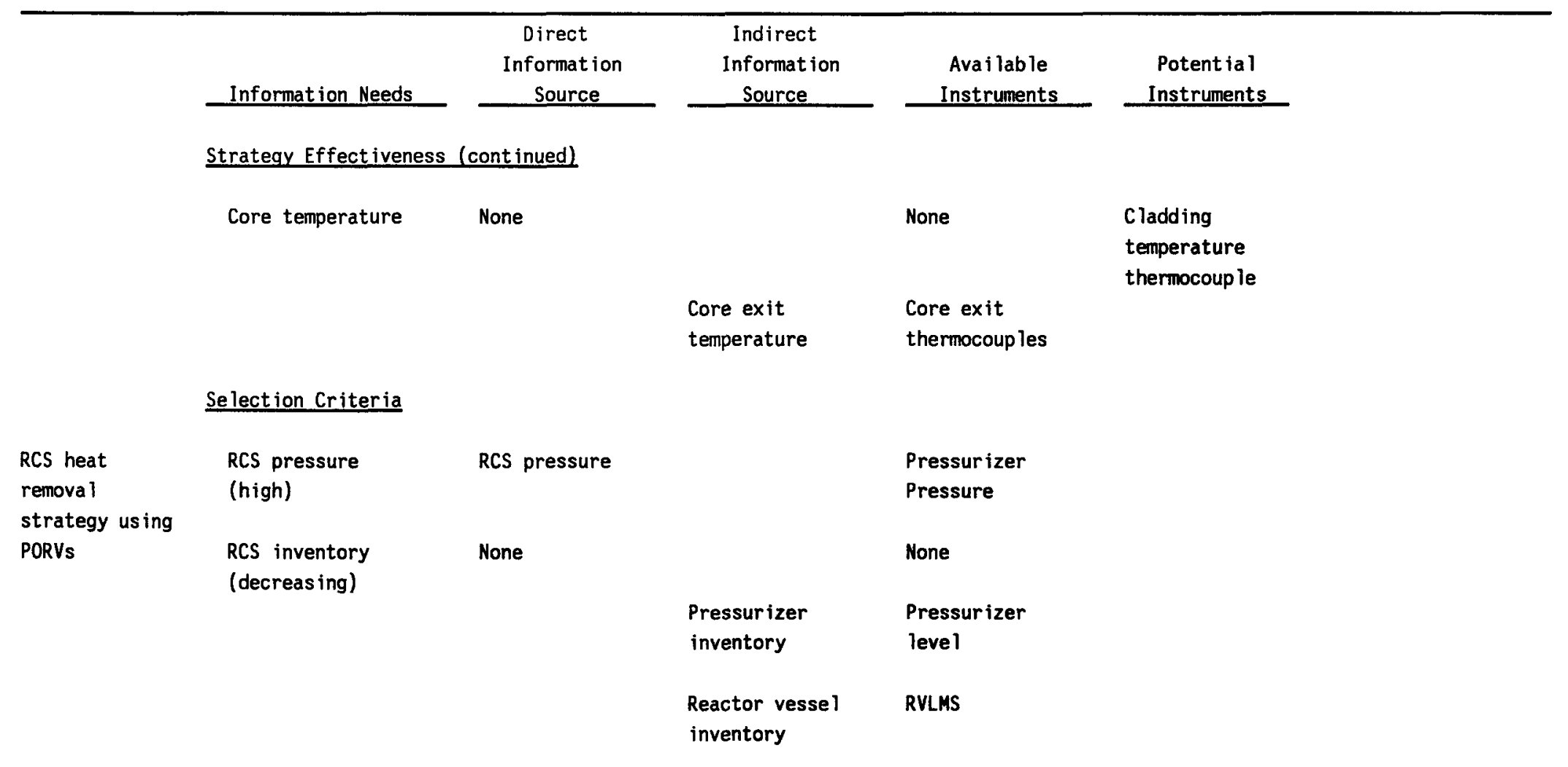


TABLE A1.11. (cont inued)

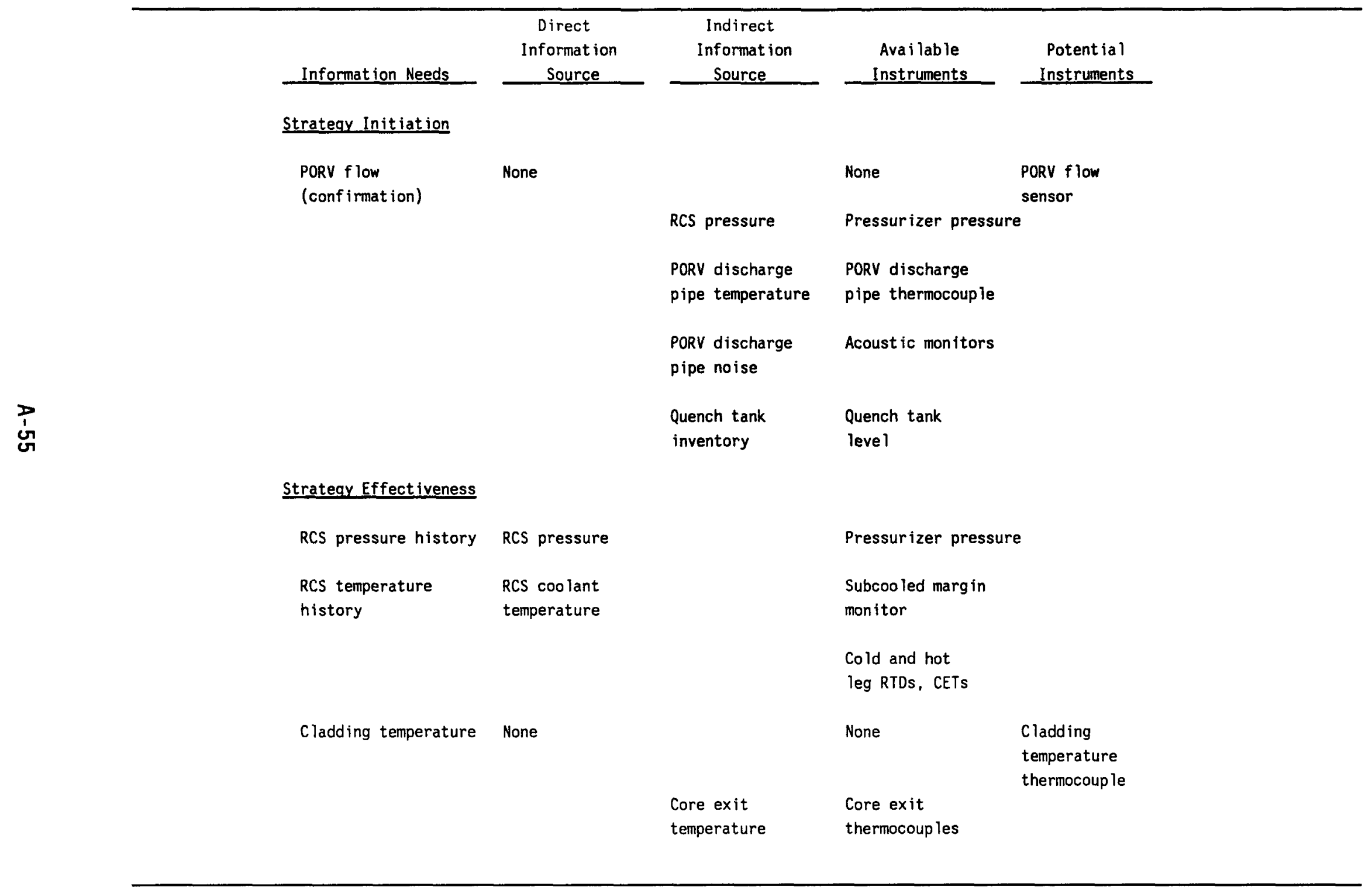


TABLE A2.1. PREVENT CONTAINMENT FAILURE (C)--INSUFFICIENT ENERGY REMOVAL MECHANISM (C1A1)

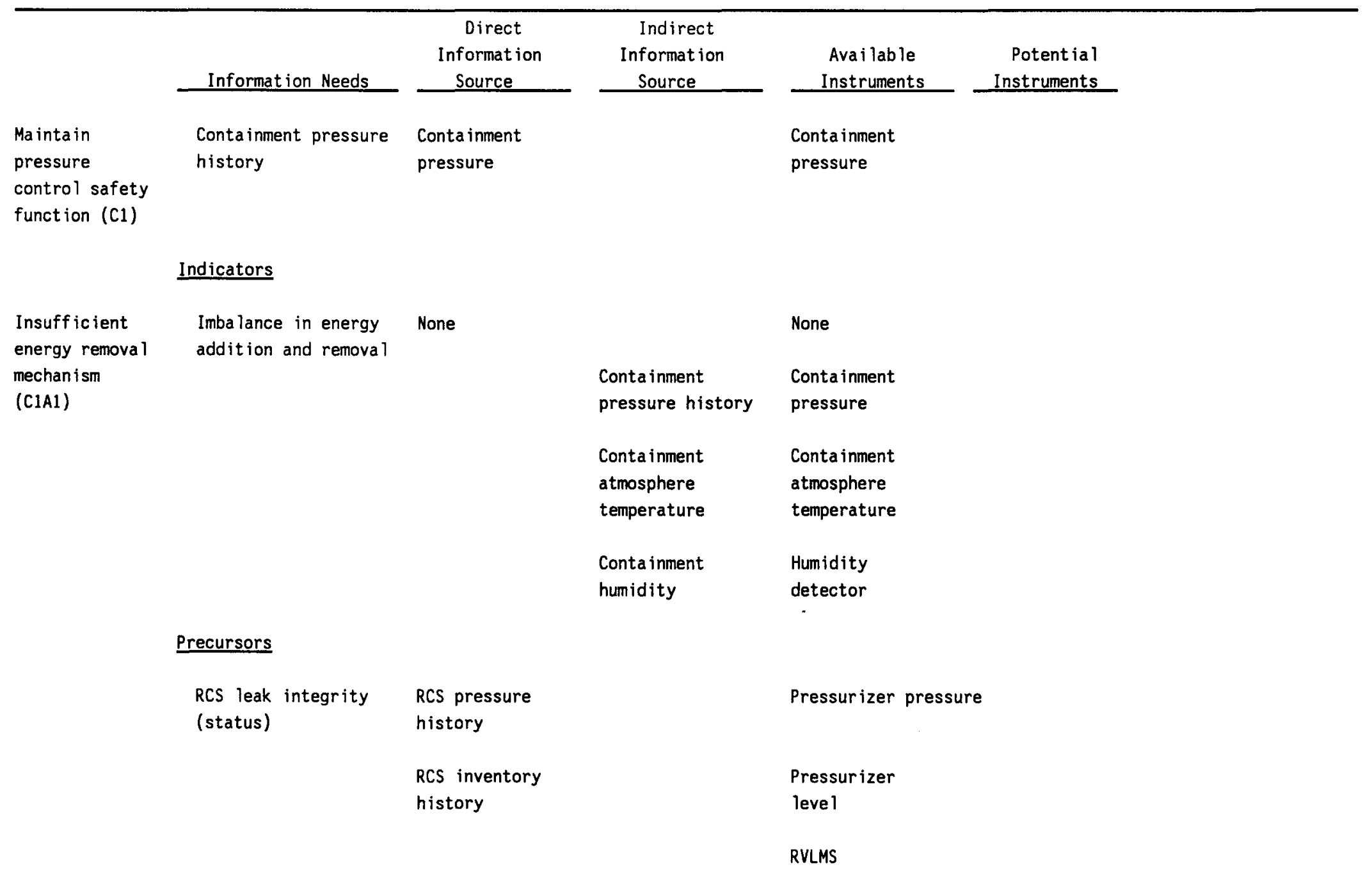


TABLE A2.1. (cont inued)

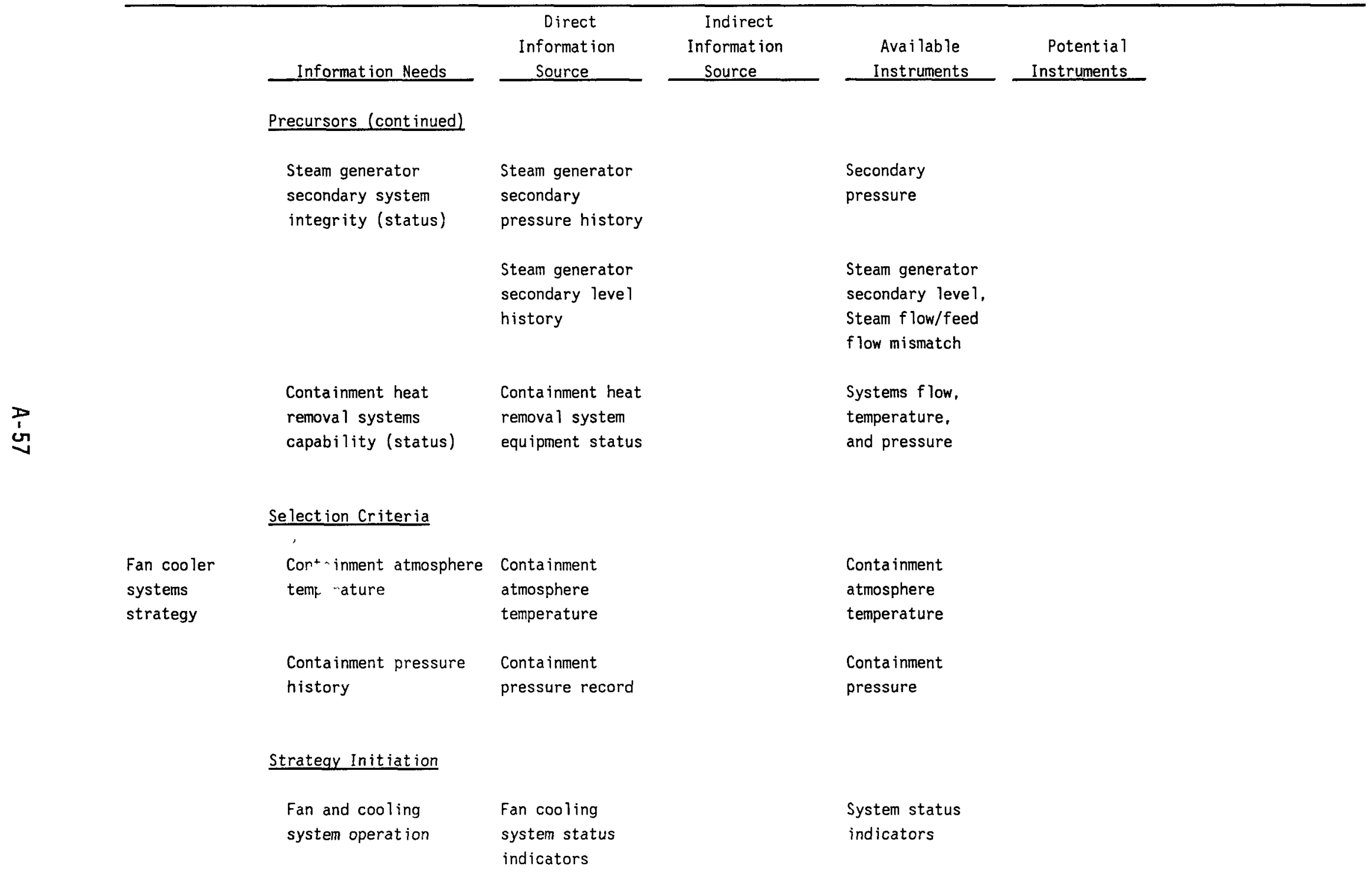


TABLE A2.1. (cont inued)

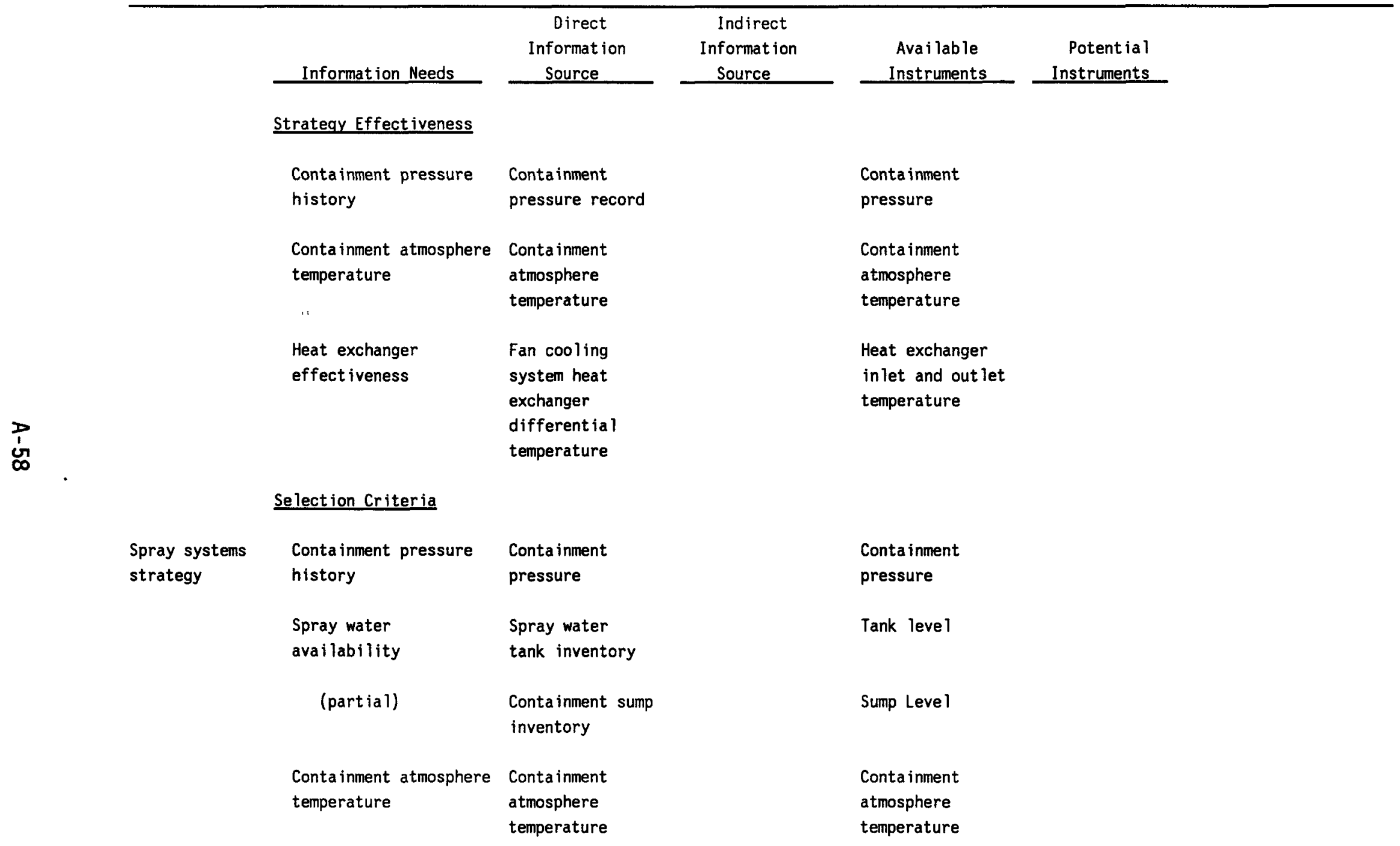


TABLE A2.1. (cont inued)

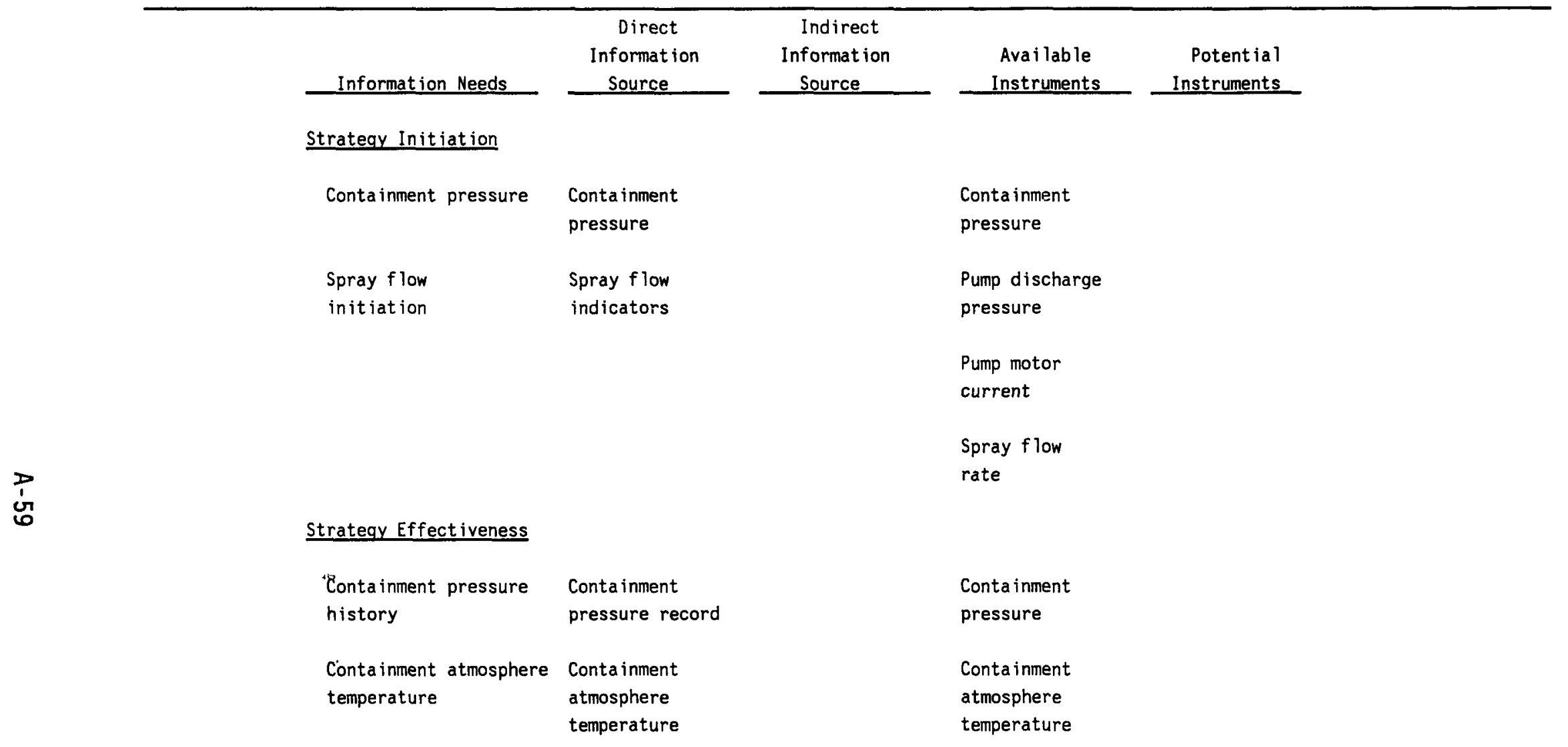


TABLE A2.1. (cont inued)

\begin{tabular}{|c|c|c|c|c|c|}
\hline & Informat ion Needs & $\begin{array}{c}\text { Direct } \\
\text { Information } \\
\text { Source } \\
\end{array}$ & $\begin{array}{l}\text { Indirect } \\
\text { Information } \\
\text { Source }\end{array}$ & $\begin{array}{c}\text { Available } \\
\text { Instruments }\end{array}$ & $\begin{array}{r}\text { Potential } \\
\text { Instruments } \\
\end{array}$ \\
\hline & Selection Criteria & & & & \\
\hline \multirow[t]{8}{*}{ Vent strategy } & $\begin{array}{l}\text { Containment pressure } \\
\text { history }\end{array}$ & $\begin{array}{l}\text { Conta inment } \\
\text { pressure }\end{array}$ & & $\begin{array}{l}\text { Containment } \\
\text { pressure }\end{array}$ & \\
\hline & $\begin{array}{l}\text { Presence of radiation } \\
\text { in conta inment }\end{array}$ & $\begin{array}{l}\text { Containment } \\
\text { radiation leve } 1\end{array}$ & & $\begin{array}{l}\text { Containment } \\
\text { radiation } \\
\text { monitors }\end{array}$ & \\
\hline & $\begin{array}{l}\text { Conta inment } \\
\text { humidity }\end{array}$ & $\begin{array}{l}\text { Conta inment } \\
\text { humidity }\end{array}$ & & $\begin{array}{l}\text { Humidity } \\
\text { detectors }\end{array}$ & \\
\hline & Strategy Initiation & & & & \\
\hline & $\begin{array}{l}\text { Vent system } \\
\text { initiation }\end{array}$ & $\begin{array}{l}\text { Vent system } \\
\text { flow rate }\end{array}$ & & & $\begin{array}{l}\text { Vent system } \\
\text { flow } \\
\text { measurement }\end{array}$ \\
\hline & Strategy Effectiveness & & & & \\
\hline & $\begin{array}{l}\text { Containment pressure } \\
\text { history }\end{array}$ & $\begin{array}{l}\text { Containment } \\
\text { pressure record }\end{array}$ & & $\begin{array}{l}\text { Containment } \\
\text { pressure }\end{array}$ & \\
\hline & $\begin{array}{l}\text { Containment atmosphere } \\
\text { temperature }\end{array}$ & $\begin{array}{l}\text { Conta inment } \\
\text { atmosphere } \\
\text { temperature }\end{array}$ & & $\begin{array}{l}\text { Containment } \\
\text { atmosphere } \\
\text { temperature }\end{array}$ & \\
\hline
\end{tabular}


TABLE A2.2. PREVENT CONTAINMENT FAILURE (C)--NONCONDENSABLE GAS BUILDUP MECHANISM (C1A2)

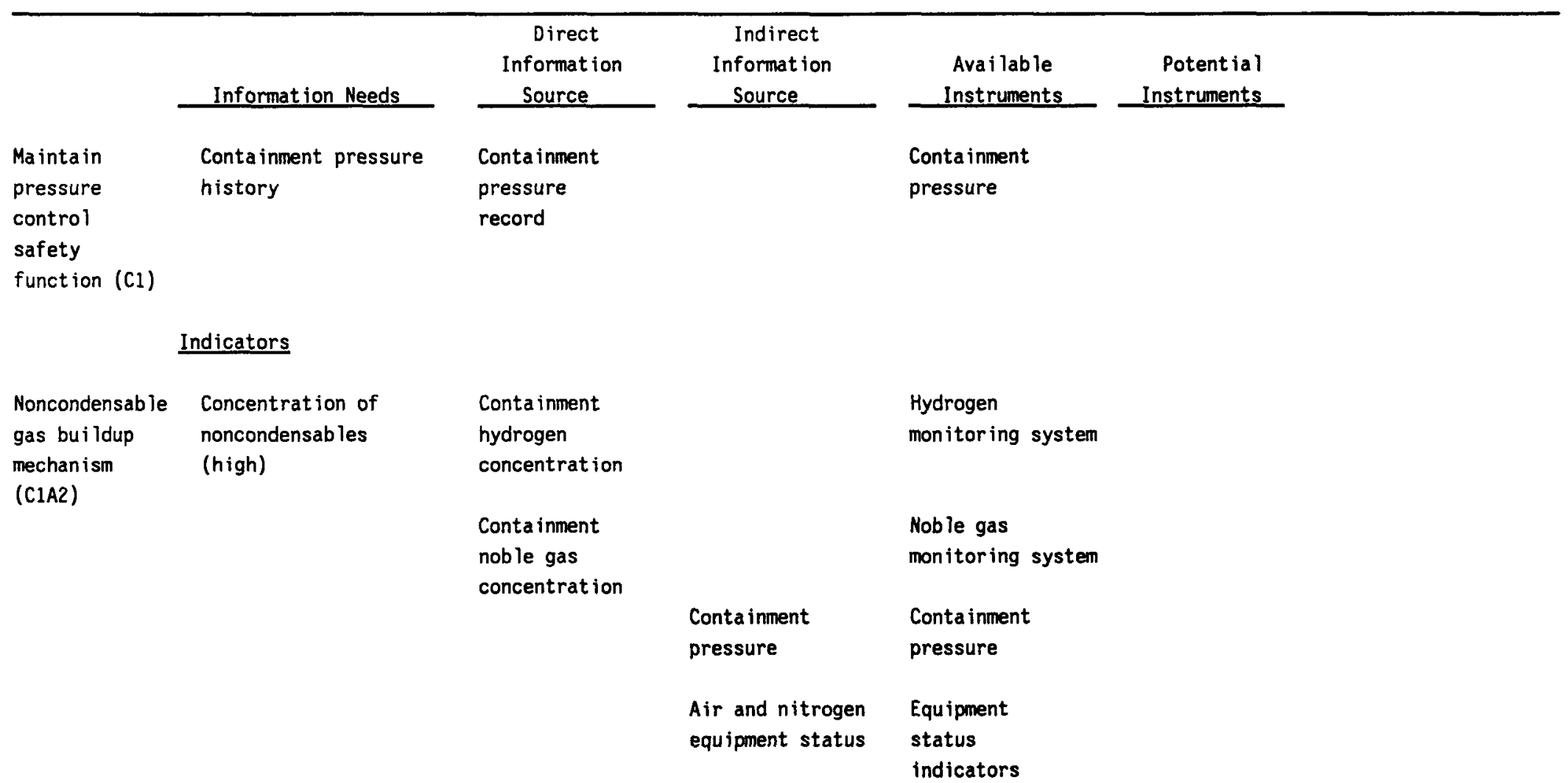

Precursors

RCS leak integrity (status)
RCS pressure

history

RCS inventory history
RCS pressure

Pressurizer

leve 1

RVLMS 
TABLE A2.2. (cont inued)

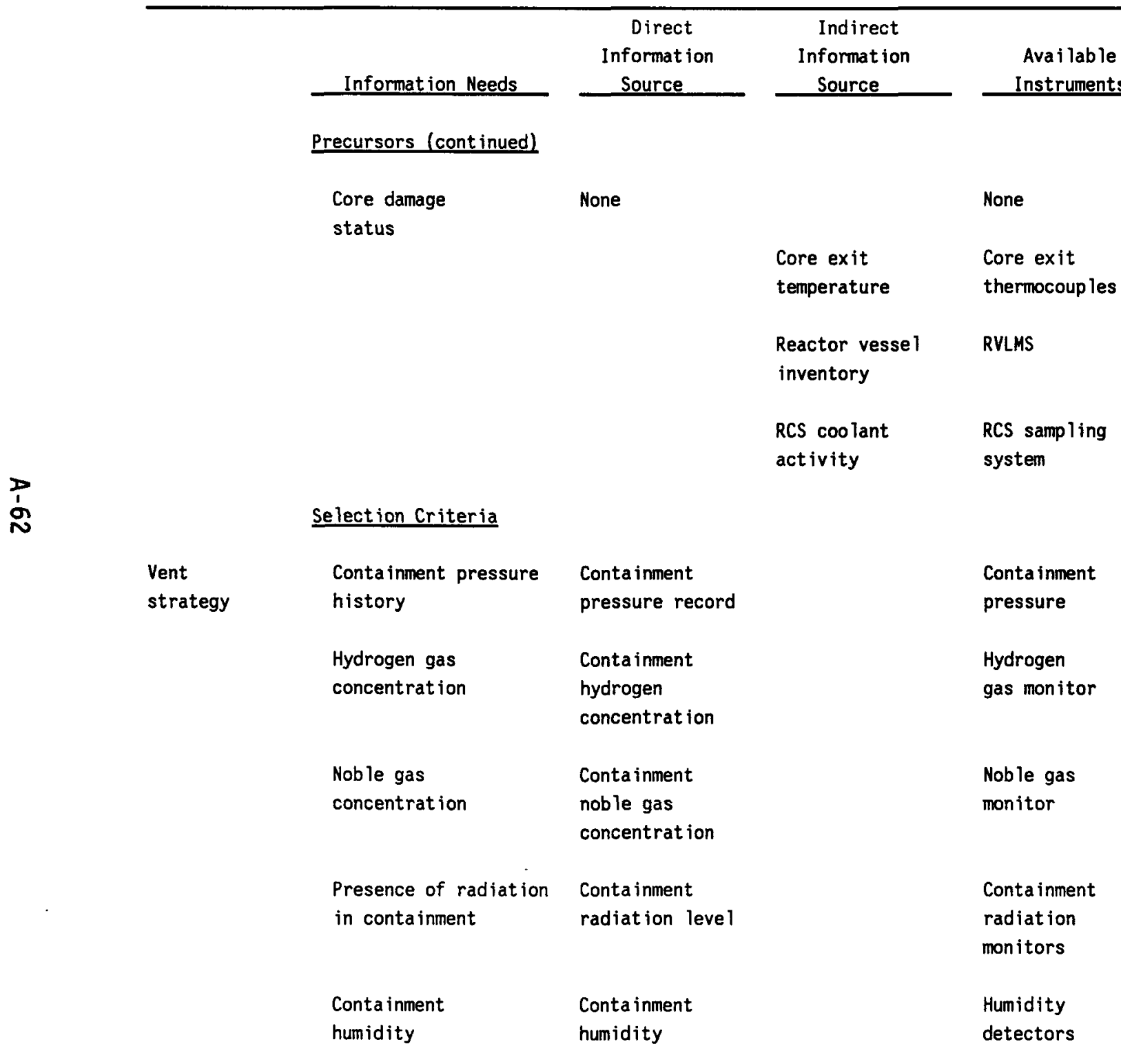


TABLE A2.2. (cont inued)

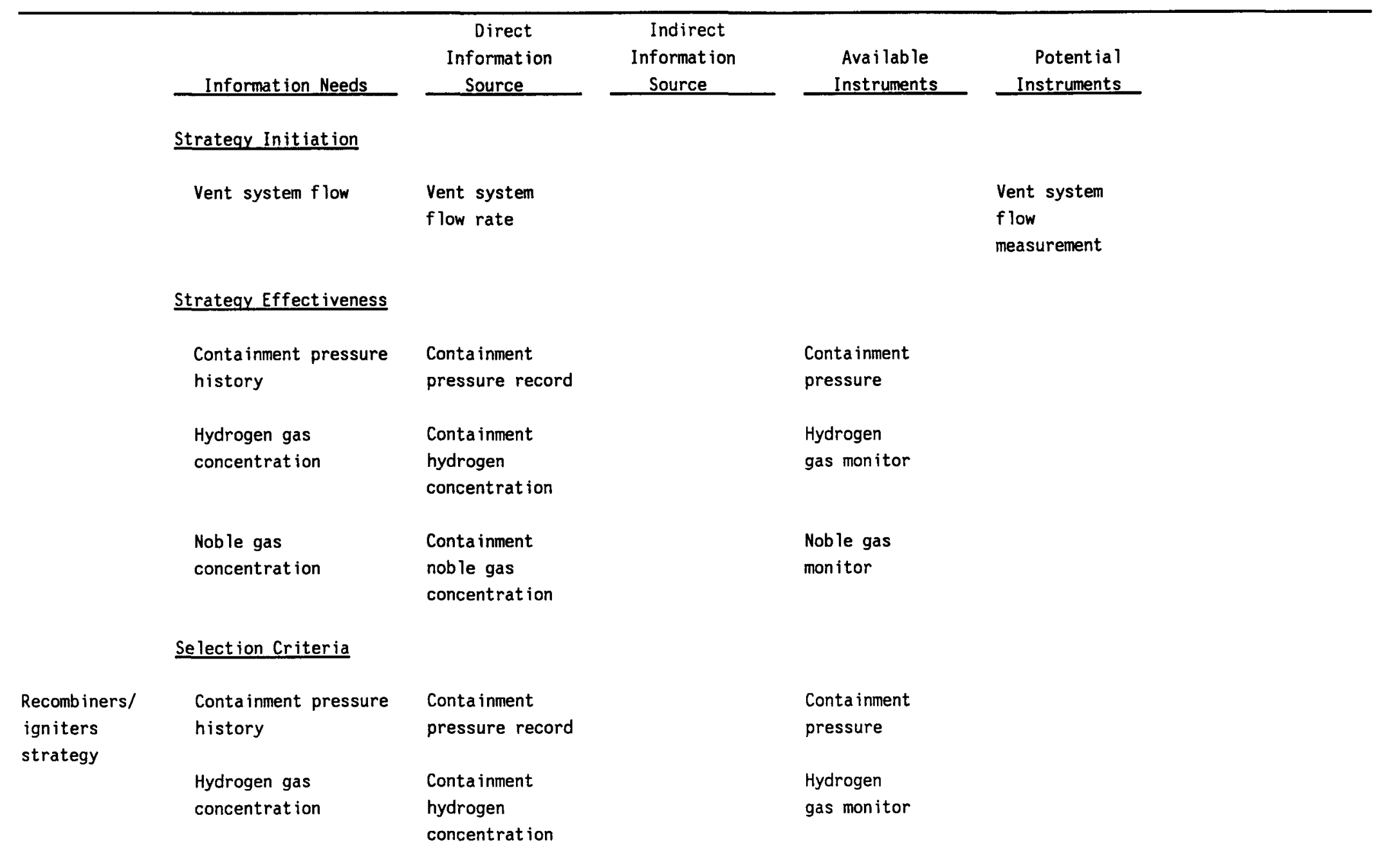


TABLE A2.2. (continued)

\begin{tabular}{|c|c|c|c|c|}
\hline Information Needs & $\begin{array}{c}\text { Direct } \\
\text { Informat ion } \\
\text { Source } \\
\end{array}$ & $\begin{array}{c}\text { Indirect } \\
\text { Information } \\
\text { Source }\end{array}$ & $\begin{array}{c}\text { Available } \\
\text { Instruments } \\
\end{array}$ & $\begin{array}{r}\text { Potential } \\
\text { Instruments } \\
\end{array}$ \\
\hline \multicolumn{5}{|l|}{ Strategy Initiation } \\
\hline $\begin{array}{l}\text { Initiation of } \\
\text { recombiners/ } \\
\text { igniters }\end{array}$ & $\begin{array}{l}\text { Recombiners/ } \\
\text { igniters } \\
\text { equipment status }\end{array}$ & & $\begin{array}{l}\text { Status } \\
\text { indicators }\end{array}$ & \\
\hline $\begin{array}{l}\text { Hydrogen gas } \\
\text { concentration }\end{array}$ & $\begin{array}{l}\text { Conta inment } \\
\text { hydrogen } \\
\text { concentration }\end{array}$ & & $\begin{array}{l}\text { Hydrogen } \\
\text { gas monitor }\end{array}$ & \\
\hline $\begin{array}{l}\text { Conta inment pressure } \\
\text { history }\end{array}$ & $\begin{array}{l}\text { Conta inment } \\
\text { pressure record }\end{array}$ & & $\begin{array}{l}\text { Conta inment } \\
\text { pressure }\end{array}$ & \\
\hline
\end{tabular}


TABLE A2.3. PREVENT CONTAINMENT FAILURE (C)--DIRECT CONTAINMENT HEATING MECHANISM (C1B1)

\begin{tabular}{|c|c|c|c|c|c|}
\hline & Information Needs & $\begin{array}{c}\text { Direct } \\
\text { Information } \\
\text { Source }\end{array}$ & $\begin{array}{c}\text { Indirect } \\
\text { Information } \\
\text { Source } \\
\end{array}$ & $\begin{array}{l}\text { Available } \\
\text { Instruments }\end{array}$ & $\begin{array}{r}\text { Potential } \\
\text { Instruments } \\
\end{array}$ \\
\hline $\begin{array}{l}\text { Maintain } \\
\text { pressure } \\
\text { control } \\
\text { safety } \\
\text { function (C1) }\end{array}$ & $\begin{array}{l}\text { Containment pressure } \\
\text { history }\end{array}$ & $\begin{array}{l}\text { Containment } \\
\text { pressure }\end{array}$ & & $\begin{array}{l}\text { Containment } \\
\text { pressure }\end{array}$ & \\
\hline
\end{tabular}

\section{Indicators}

\begin{tabular}{|c|c|c|c|}
\hline $\begin{array}{l}\text { Direct } \\
\text { containment }\end{array}$ & $\begin{array}{l}\text { Containment pressure } \\
\text { history }\end{array}$ & $\begin{array}{l}\text { Containment } \\
\text { Pressure record }\end{array}$ & $\begin{array}{l}\text { Containment } \\
\text { pressure }\end{array}$ \\
\hline $\begin{array}{l}\text { mechan ism } \\
\text { (C1B1) }\end{array}$ & $\begin{array}{l}\text { Containment } \\
\text { temperature history }\end{array}$ & $\begin{array}{l}\text { Conta inment } \\
\text { atmosphere } \\
\text { temperature }\end{array}$ & $\begin{array}{l}\text { Conta inment } \\
\text { thermocoup les }\end{array}$ \\
\hline & $\begin{array}{l}\text { Containment } \\
\text { radiation level }\end{array}$ & $\begin{array}{l}\text { Containment } \\
\text { radiation leve } 1\end{array}$ & $\begin{array}{l}\text { Containment } \\
\text { radiation monitor }\end{array}$ \\
\hline
\end{tabular}

\section{Precursors}

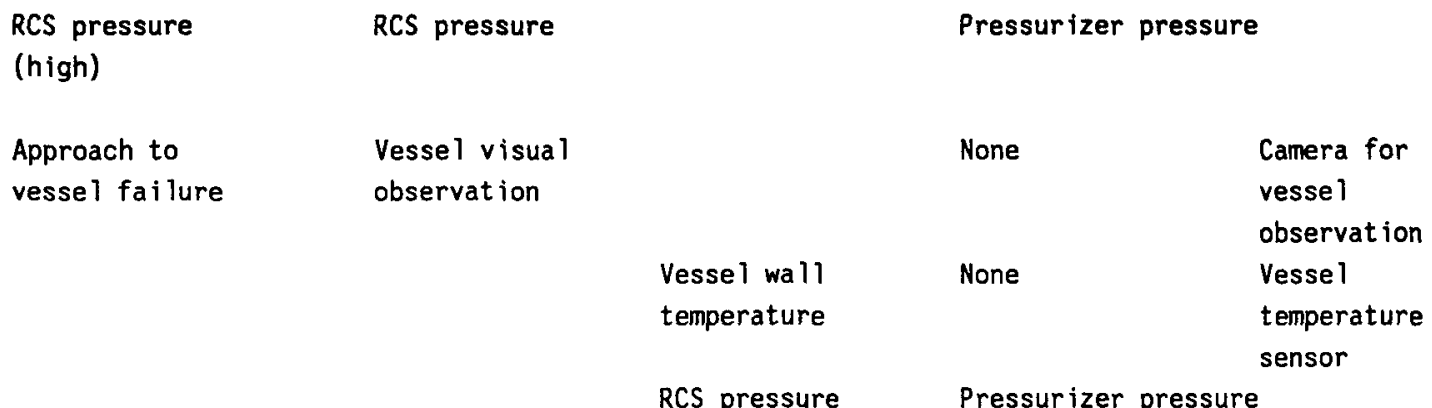


TABLE A2.3. (continued)

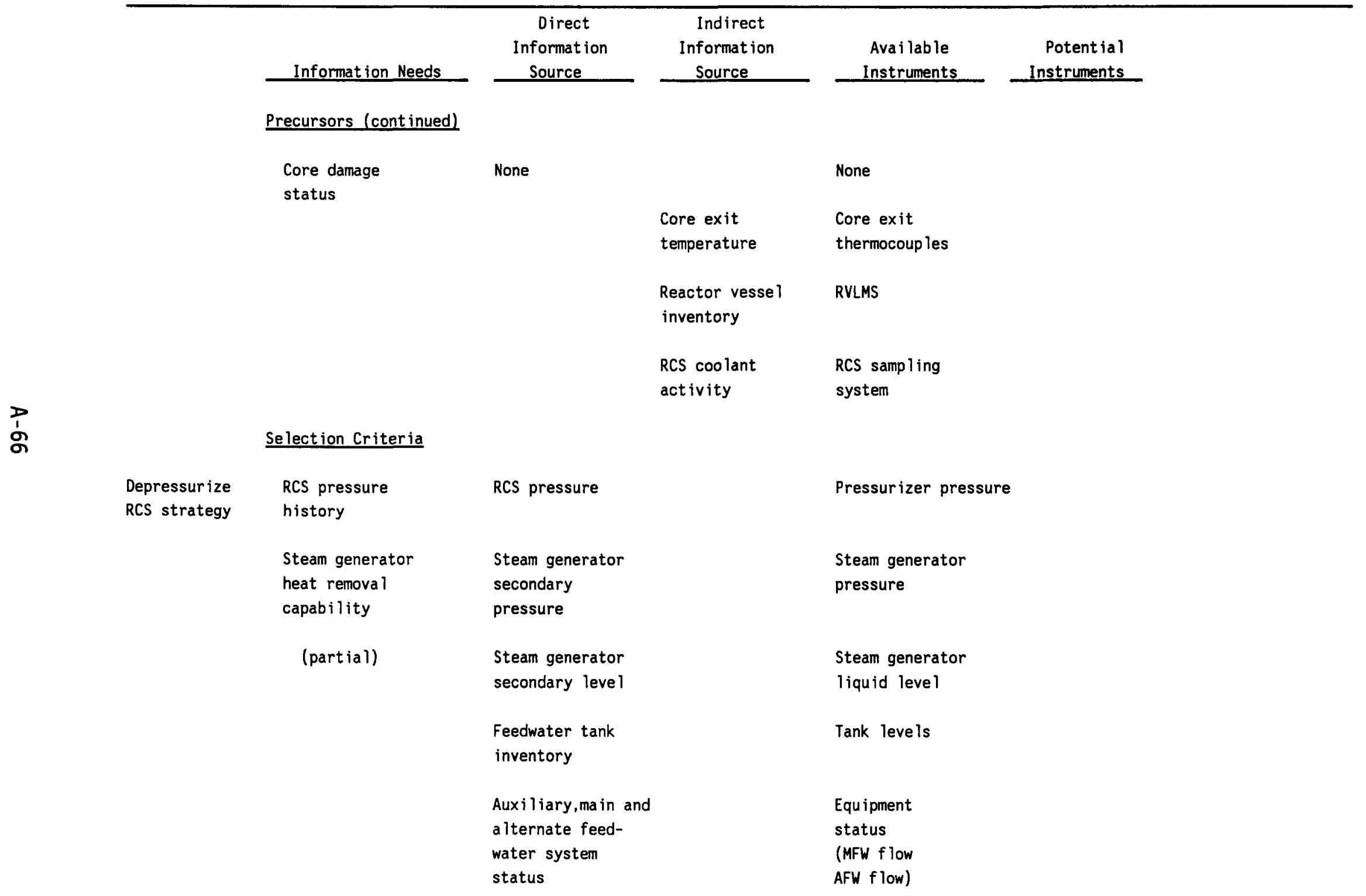


TABLE A2.3. (cont inued)

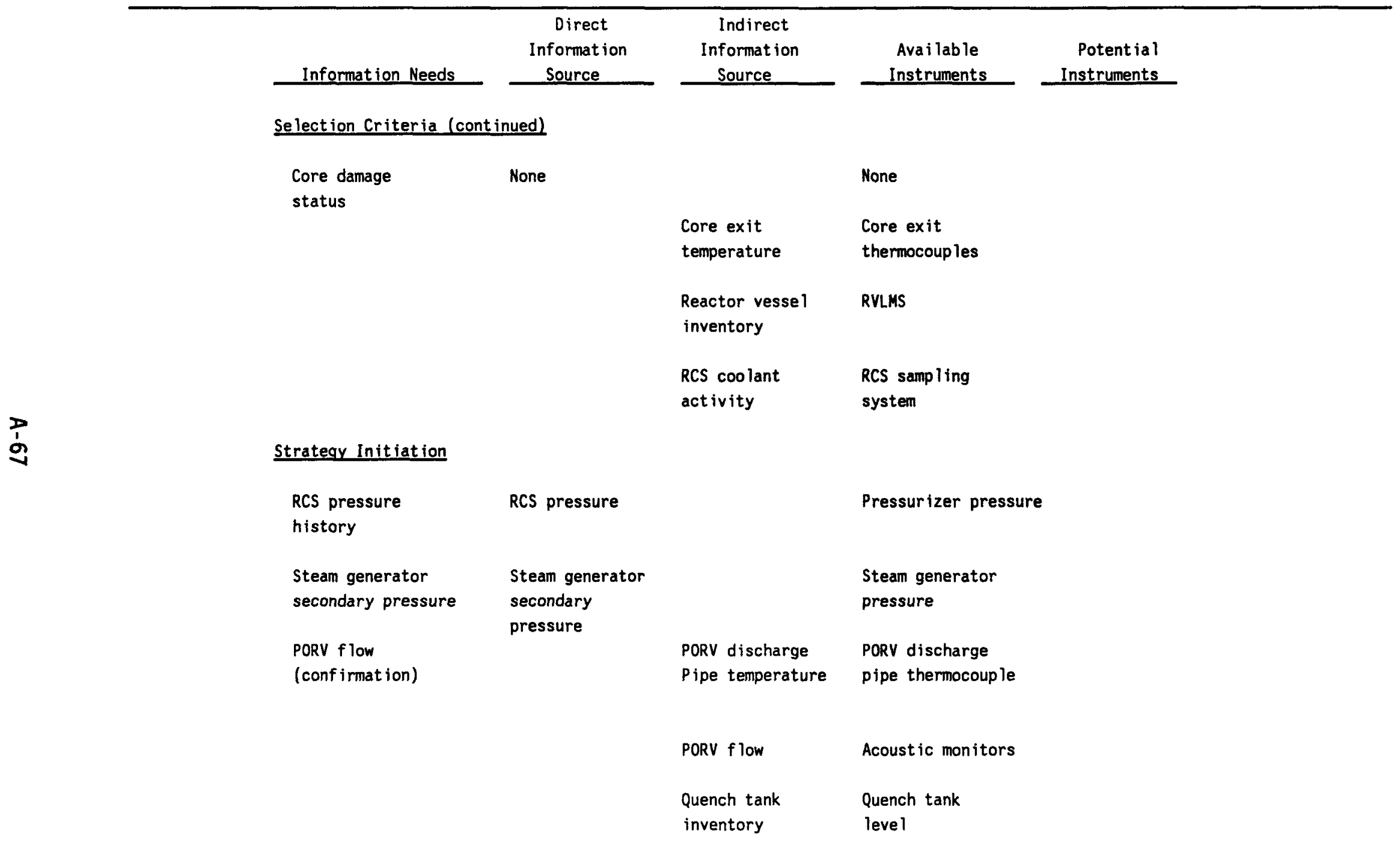


TABLE A2.3. (cont inued)

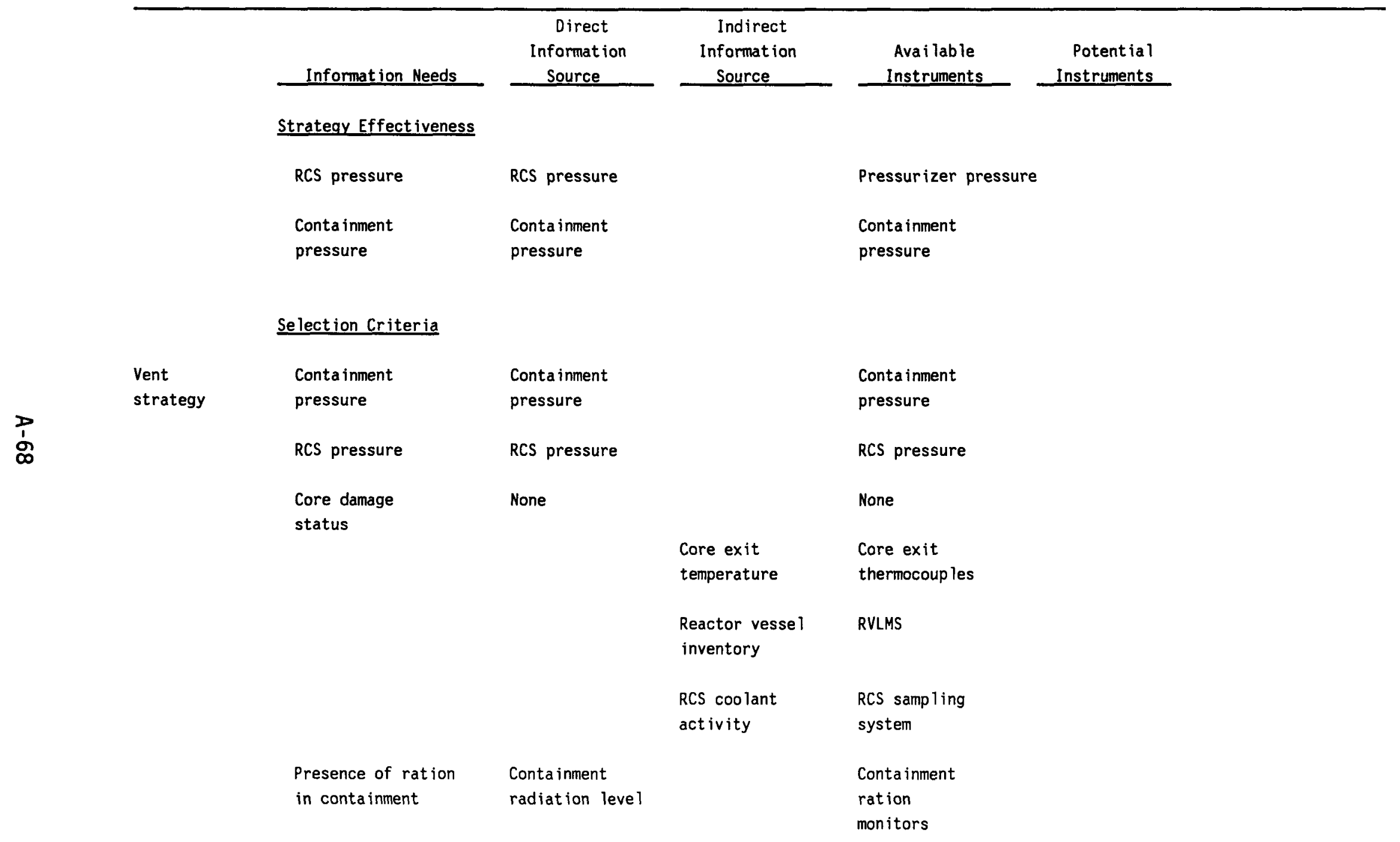


TABLE A2.3. (cont inued)

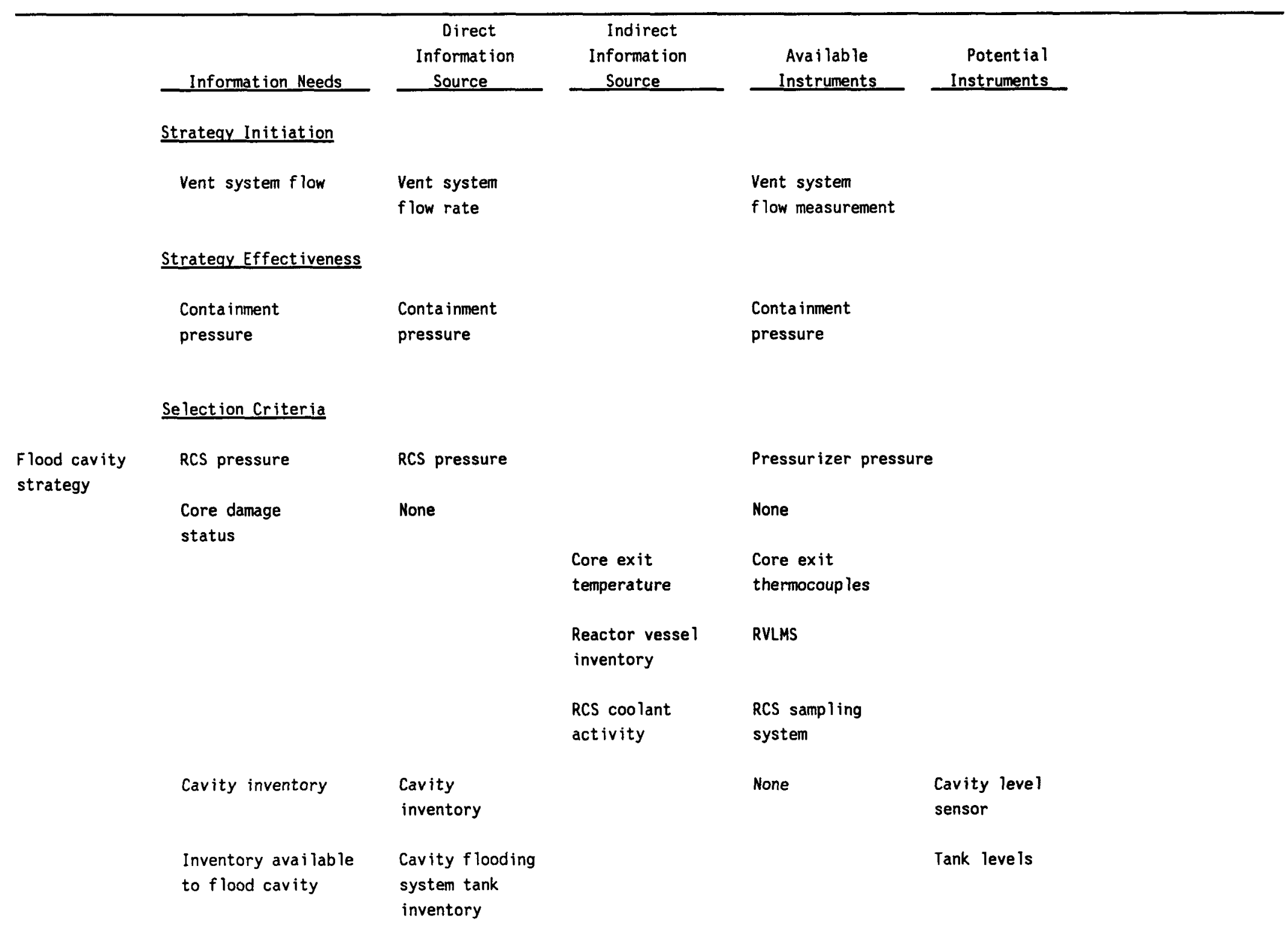


TABLE A2.3. (cont inued)

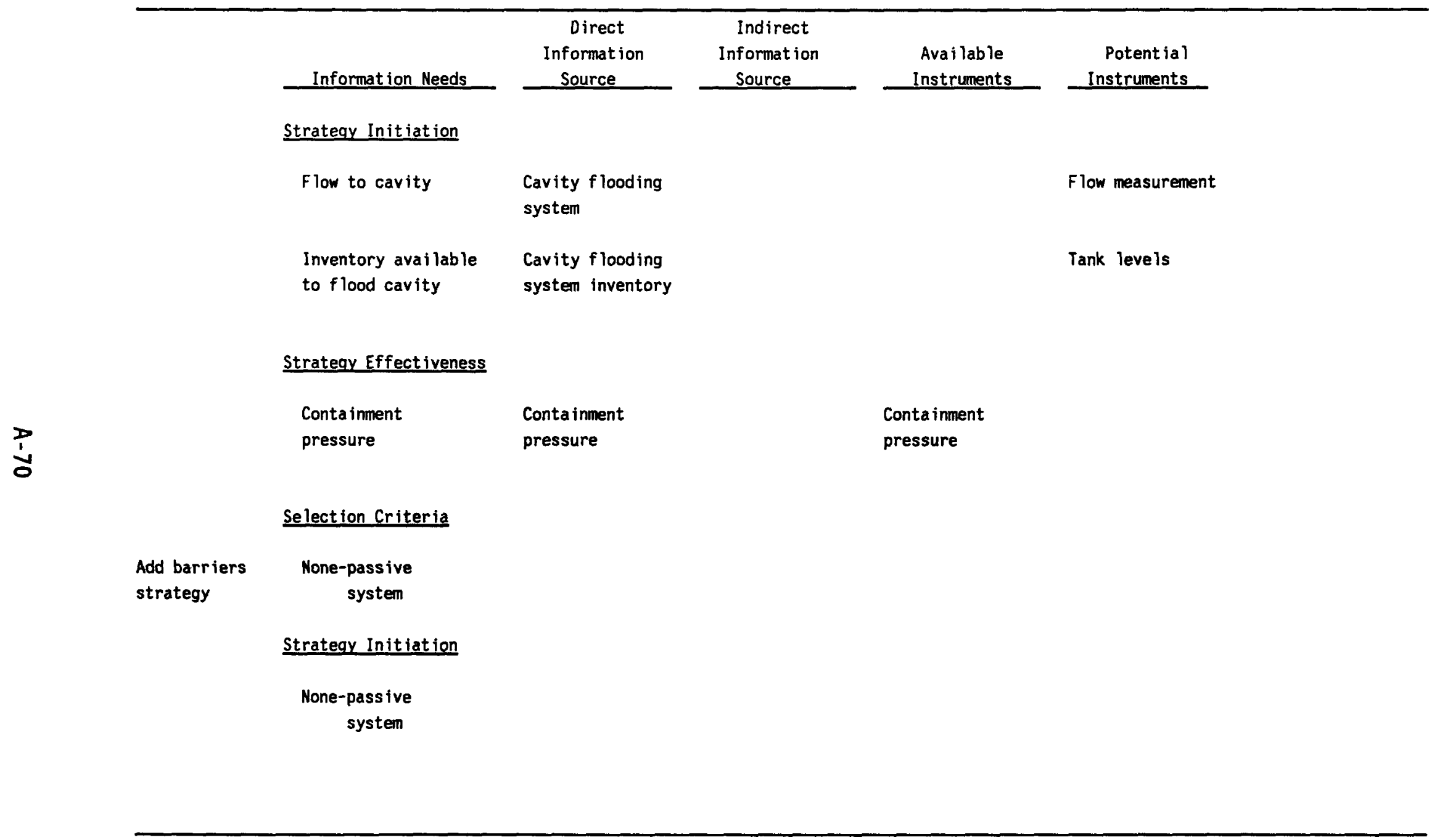


TABLE A2.4, PREVENT CONTAINMENT FAILURE (C)--COMBUSTABLE GAS DETONATION MECHANISM (C1B2)

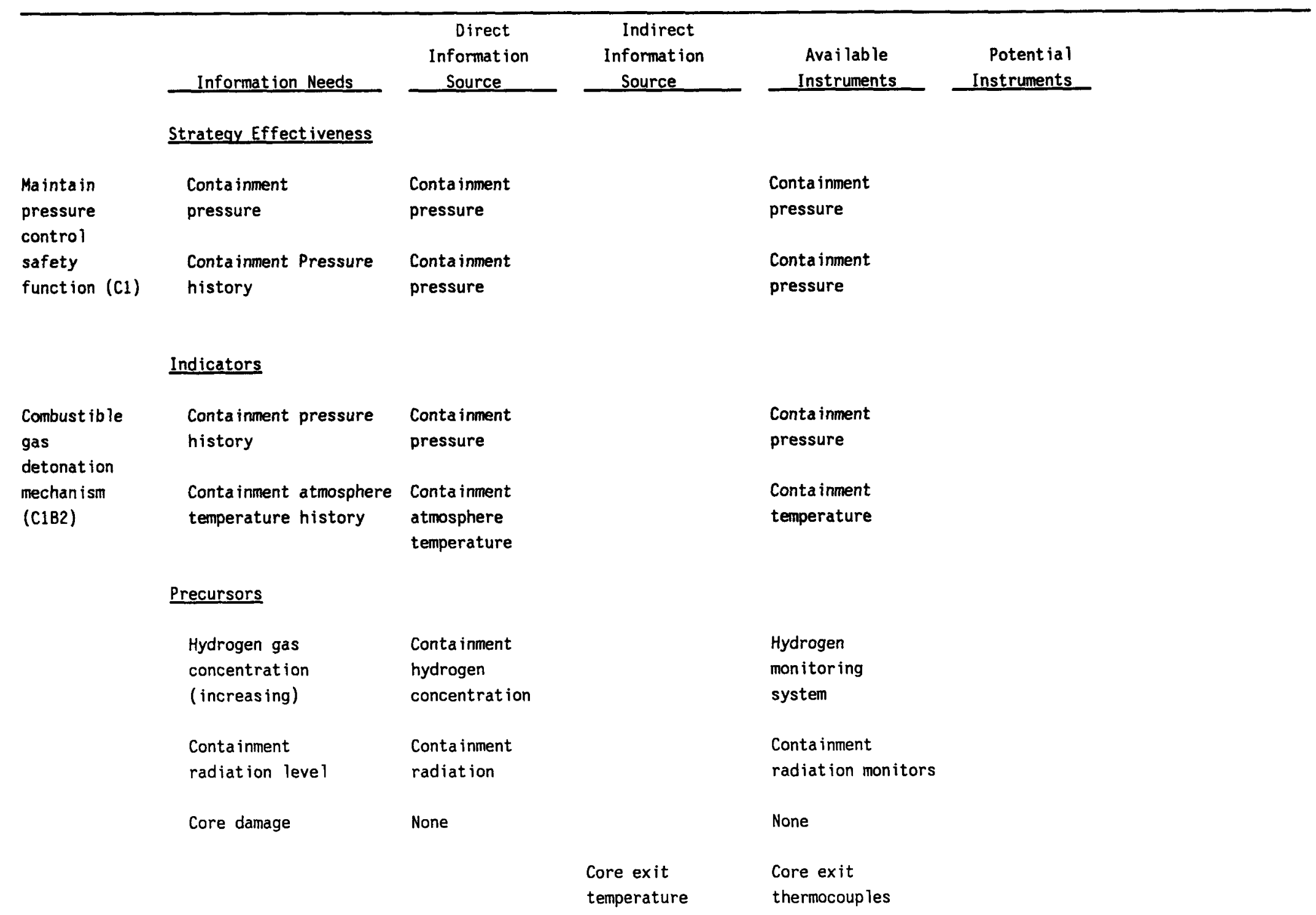


TABLE A2.4. (cont inued)

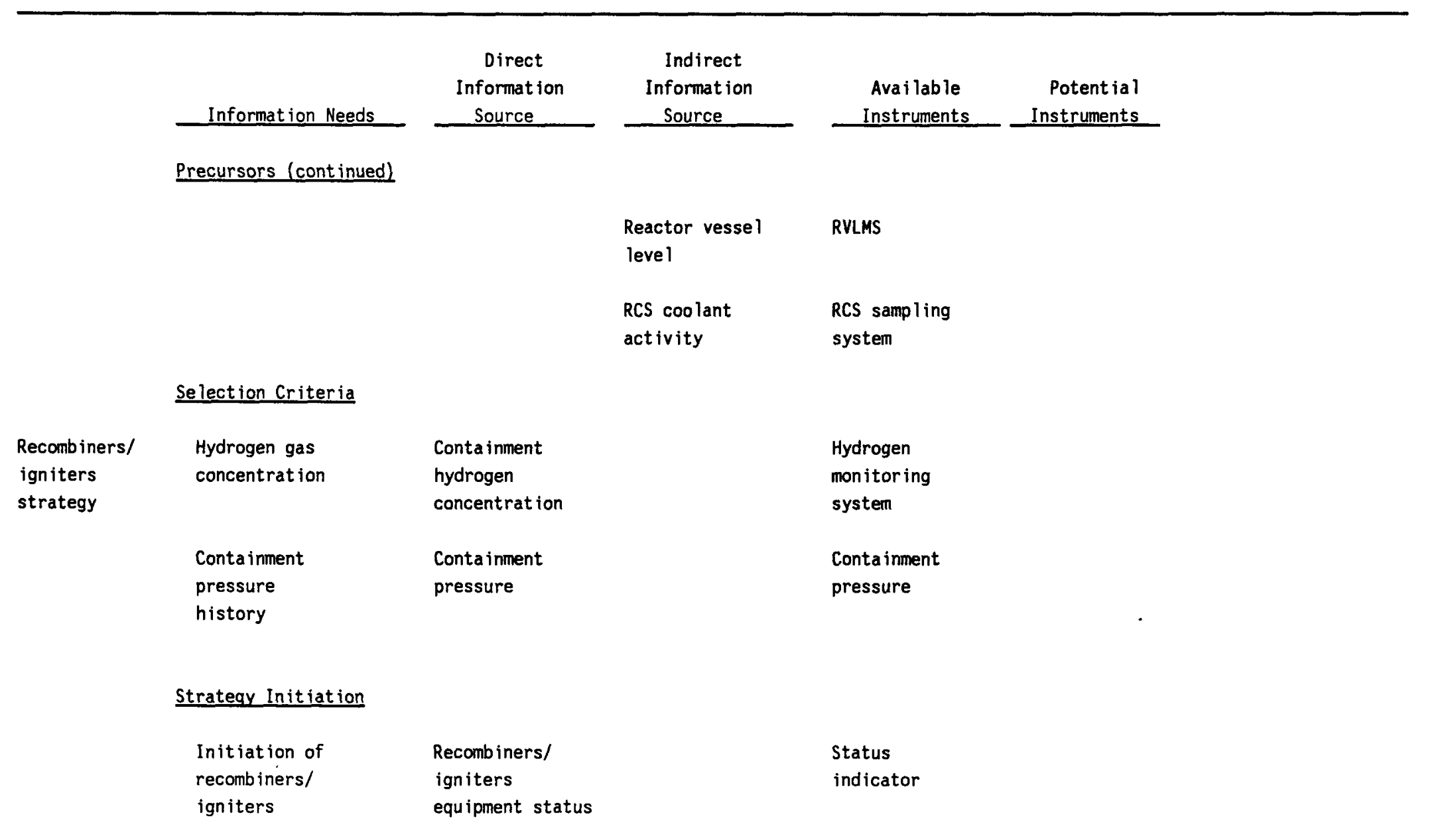


TABLE A2.4. (cont inued)

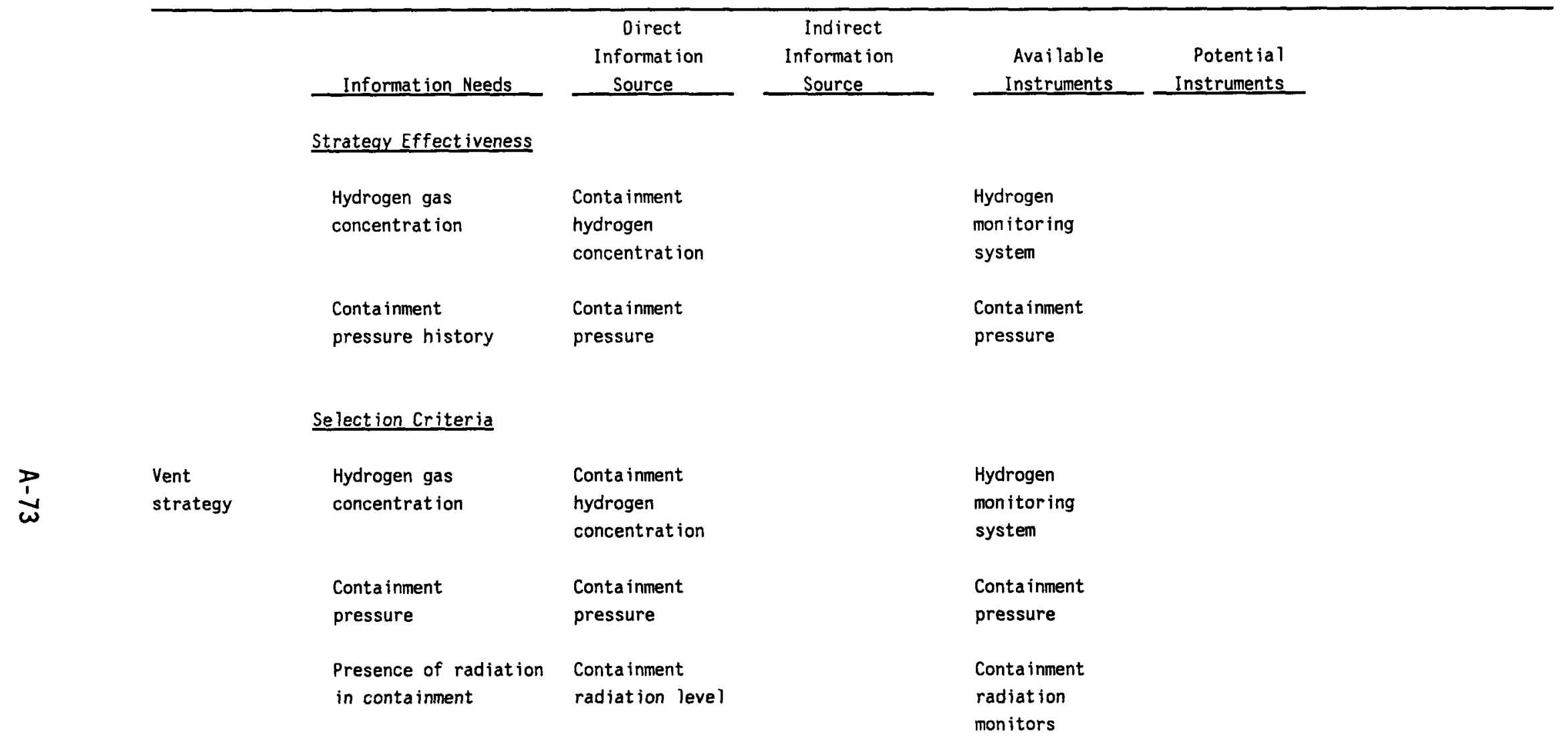


TABLE A2.4. (cont inued)

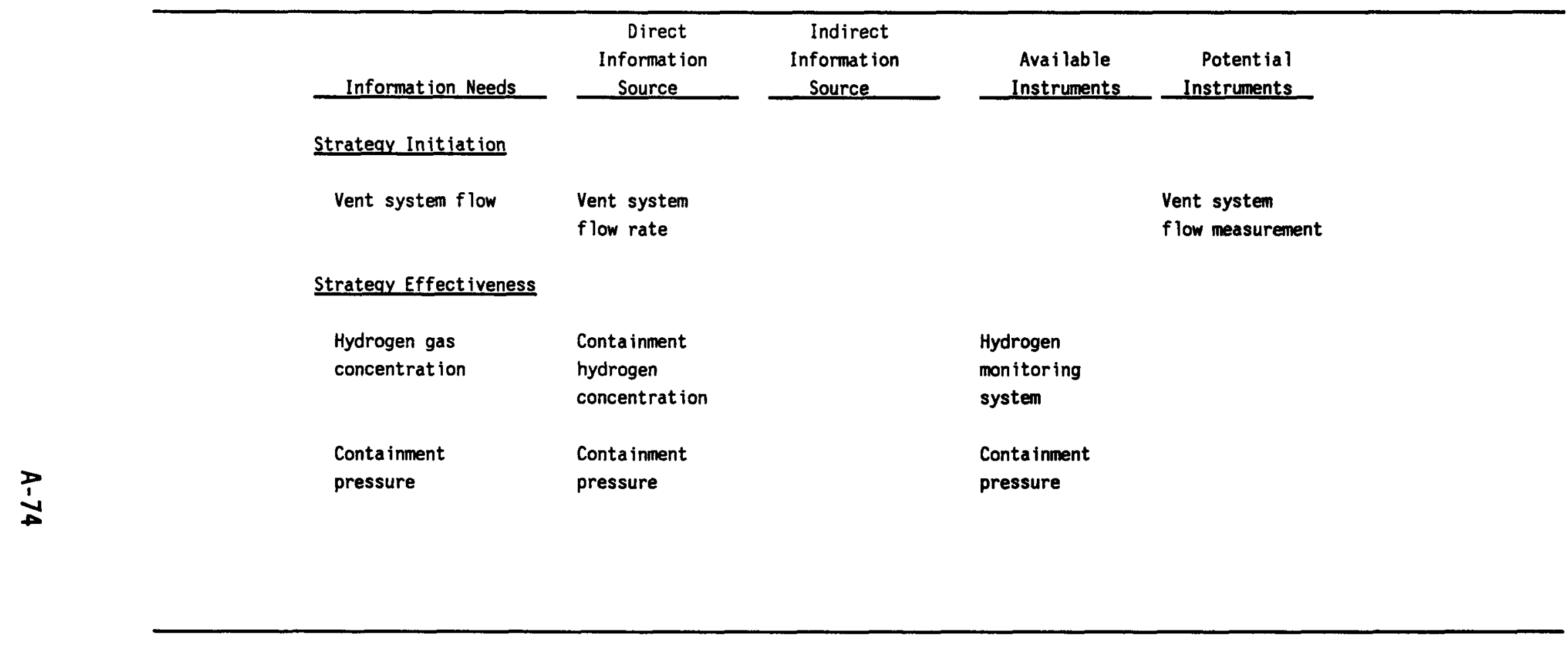


TABLE A2.5. PREVENT CONTAINMENT FAILURE (C)--STEAM EXPLOSION MECHANISM (C1B3)

\begin{tabular}{|c|c|c|c|c|c|}
\hline \multirow{3}{*}{$\begin{array}{l}\text { Mainta in } \\
\text { pressure } \\
\text { control safety } \\
\text { function (C1) }\end{array}$} & Informat ion Needs & $\begin{array}{c}\text { Direct } \\
\text { Information } \\
\text { Source } \\
\end{array}$ & $\begin{array}{c}\text { Indirect } \\
\text { Information } \\
\text { Source } \\
\end{array}$ & $\begin{array}{c}\text { Ava i lable } \\
\text { Instruments } \\
\end{array}$ & $\begin{array}{r}\text { Potential } \\
\text { Instruments } \\
\end{array}$ \\
\hline & $\begin{array}{l}\text { Containment pressure } \\
\text { history }\end{array}$ & $\begin{array}{l}\text { Containment } \\
\text { pressure }\end{array}$ & & $\begin{array}{l}\text { Containment } \\
\text { pressure }\end{array}$ & \\
\hline & Indicators & & & & \\
\hline \multirow{9}{*}{$\begin{array}{l}\text { Steam } \\
\text { explosion } \\
\text { mechan ism } \\
\text { (C1B3) }\end{array}$} & $\begin{array}{l}\text { Containment pressure } \\
\text { history }\end{array}$ & None & $\begin{array}{l}\text { Containment } \\
\text { pressure }\end{array}$ & $\begin{array}{l}\text { Containment } \\
\text { pressure }\end{array}$ & \\
\hline & $\begin{array}{l}\text { Containment atmosphere } \\
\text { temperature history }\end{array}$ & None & $\begin{array}{l}\text { Conta inment } \\
\text { atmosphere } \\
\text { temperature }\end{array}$ & $\begin{array}{l}\text { Containment } \\
\text { thermocouples }\end{array}$ & \\
\hline & $\begin{array}{l}\text { Containment } \\
\text { radiation level }\end{array}$ & None & $\begin{array}{l}\text { Containment } \\
\text { radiation level }\end{array}$ & $\begin{array}{l}\text { Containment } \\
\text { radiation } \\
\text { monitors }\end{array}$ & \\
\hline & Precursors & & & & \\
\hline & Vesse 1 integrity & $\begin{array}{l}\text { Vessel visual } \\
\text { observation }\end{array}$ & & None & $\begin{array}{l}\text { Camera for } \\
\text { vesse } 1 \\
\text { observation }\end{array}$ \\
\hline & & & $\begin{array}{l}\text { Vessel wall } \\
\text { temperature }\end{array}$ & None & $\begin{array}{l}\text { Vessel } \\
\text { temperature } \\
\text { sensor }\end{array}$ \\
\hline & & & $\begin{array}{l}\text { RCS (vesse 1) } \\
\text { pressure }\end{array}$ & $\begin{array}{l}\text { Pressurizer } \\
\text { pressure }\end{array}$ & \\
\hline & $\begin{array}{l}\text { Core relocation } \\
\text { status }\end{array}$ & None & & None & \\
\hline & & & $\begin{array}{l}\text { Radiation levels } \\
\text { outside core } \\
\text { region }\end{array}$ & $\begin{array}{l}\text { Source range } \\
\text { monitors }\end{array}$ & \\
\hline
\end{tabular}


TABLE A2.5. (cont inued)

\begin{tabular}{|c|c|c|c|c|c|}
\hline & Information Needs & $\begin{array}{c}\text { Direct } \\
\text { Information } \\
\text { Source }\end{array}$ & $\begin{array}{c}\text { Indirect } \\
\text { Information } \\
\text { Source } \\
\end{array}$ & $\begin{array}{c}\text { Ava i lab le } \\
\text { Instruments }\end{array}$ & $\begin{array}{r}\text { Potent ia } 1 \\
\text { Instruments } \\
\end{array}$ \\
\hline & Precursors (cont inued) & & & & \\
\hline & $\begin{array}{l}\text { Core damage } \\
\text { status }\end{array}$ & None & & None & \\
\hline & & & $\begin{array}{l}\text { Core exit } \\
\text { temperature }\end{array}$ & $\begin{array}{l}\text { Core exit } \\
\text { thermocouples }\end{array}$ & \\
\hline & & & $\begin{array}{l}\text { Reactor vessel } \\
\text { level }\end{array}$ & RVLMS & \\
\hline & & & $\begin{array}{l}\text { RCS coolant } \\
\text { activity }\end{array}$ & RCS sampling & \\
\hline & Water in Cavity & $\begin{array}{l}\text { Cavity } \\
\text { inventory }\end{array}$ & None & None & $\begin{array}{l}\text { Cavity level } \\
\text { sensor }\end{array}$ \\
\hline & Selection Criteria & & & & \\
\hline \multirow{5}{*}{$\begin{array}{l}\text { Eliminate } \\
\text { water } \\
\text { strategy }\end{array}$} & $\begin{array}{l}\text { Cavity } \\
\text { inventory }\end{array}$ & $\begin{array}{l}\text { Cavity } \\
\text { inventory }\end{array}$ & & None & $\begin{array}{l}\text { Cavity leve } 1 \\
\text { sensor }\end{array}$ \\
\hline & $\begin{array}{l}\text { Core damage } \\
\text { status }\end{array}$ & None & & None & \\
\hline & & & $\begin{array}{l}\text { Core exit } \\
\text { temperature }\end{array}$ & $\begin{array}{l}\text { Core exit } \\
\text { thermocouples }\end{array}$ & \\
\hline & & & $\begin{array}{l}\text { Reactor vesse } 1 \\
\text { inventory }\end{array}$ & RVLMS & \\
\hline & & & $\begin{array}{l}\text { RCS coolant } \\
\text { activity }\end{array}$ & $\begin{array}{l}\text { RCS sampling } \\
\text { system }\end{array}$ & \\
\hline
\end{tabular}


TABLE A2.5. (cont inued)

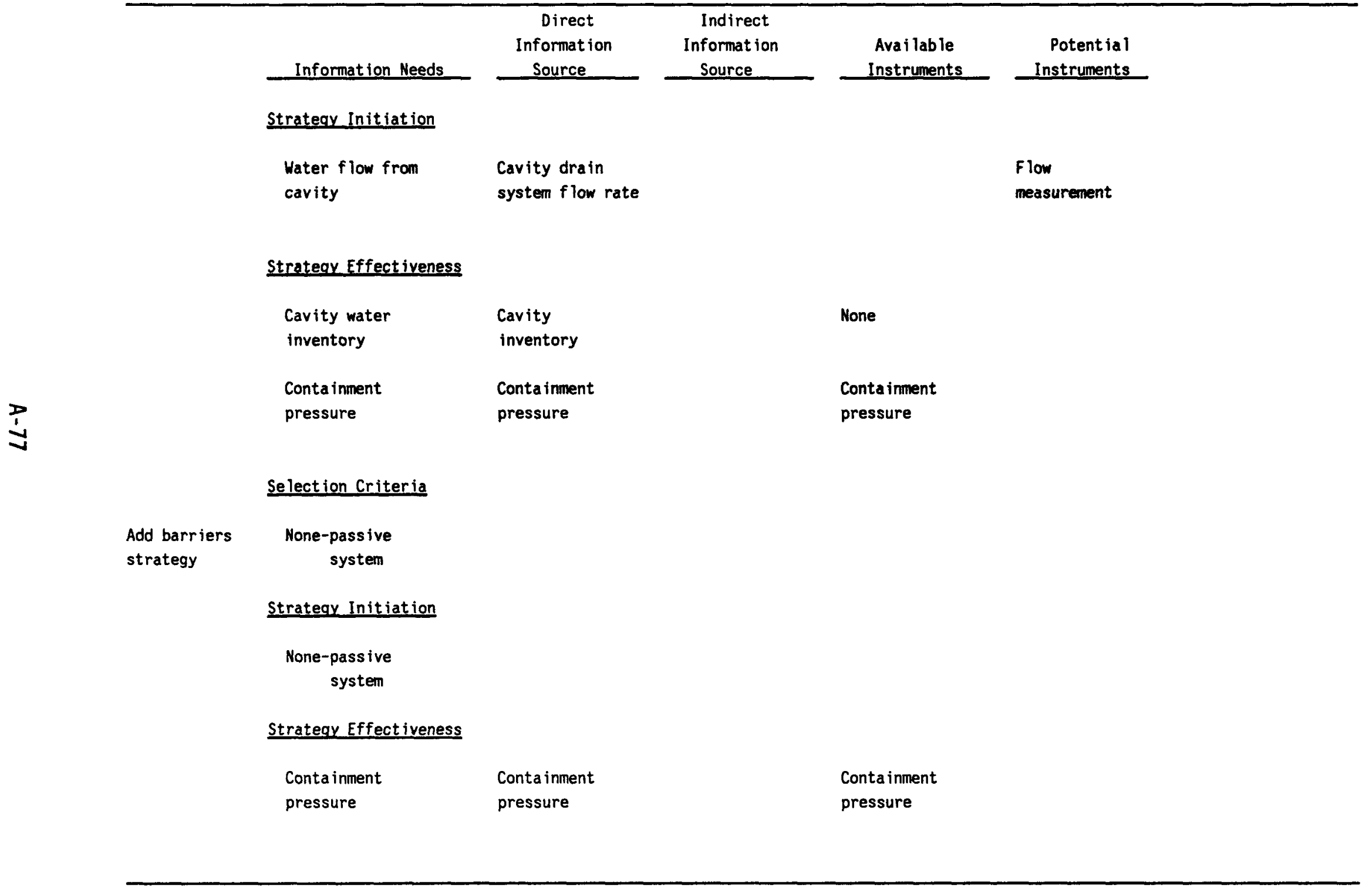


TABLE A2.6. PREVENT CONTAINMENT FAILURE (C)--ENERgY ADOITION AT VESSEL FAILURE MECHANISM (C1B4)

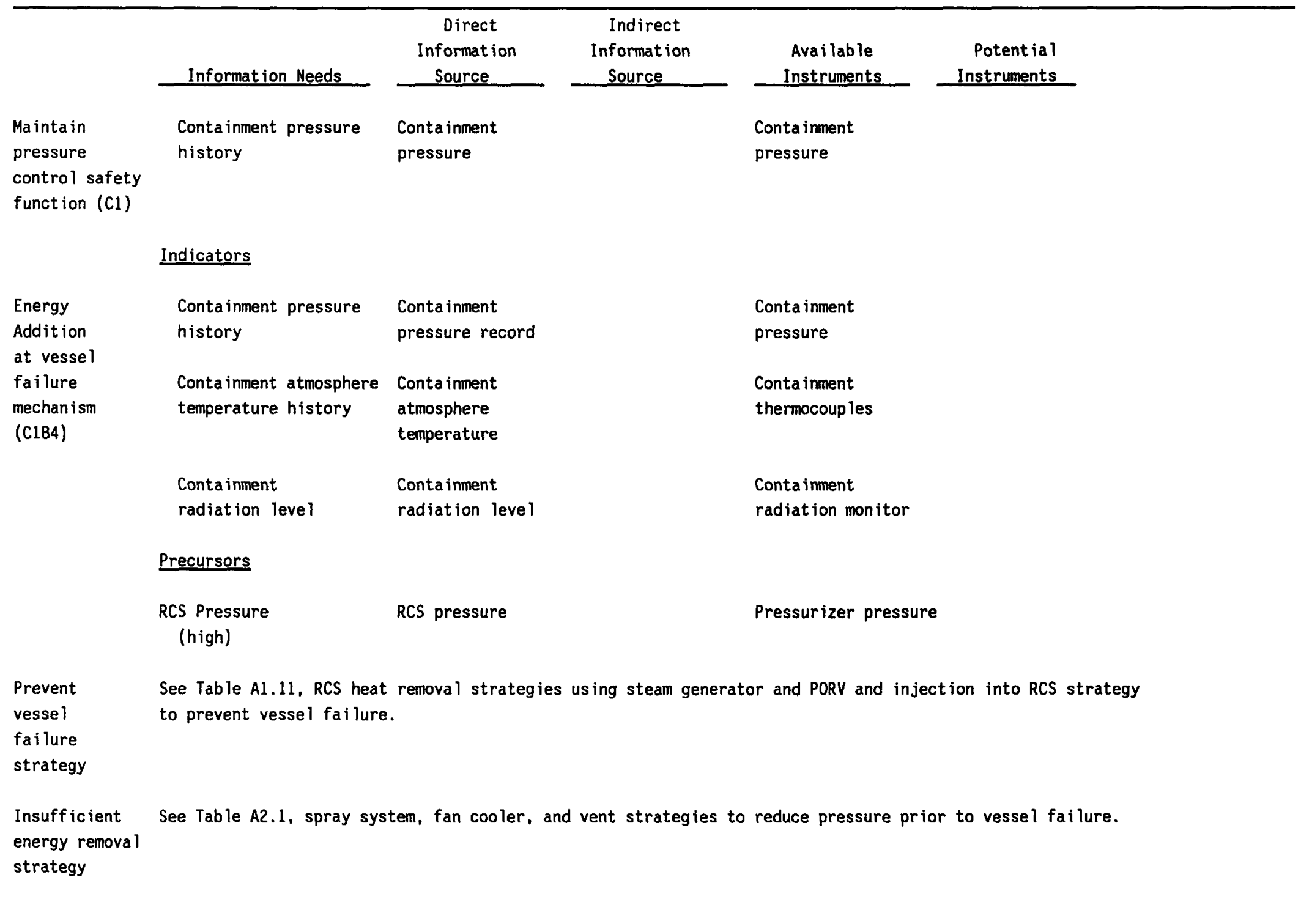




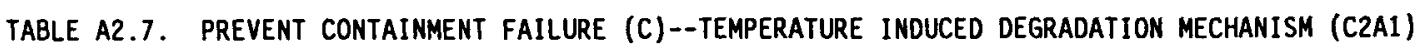

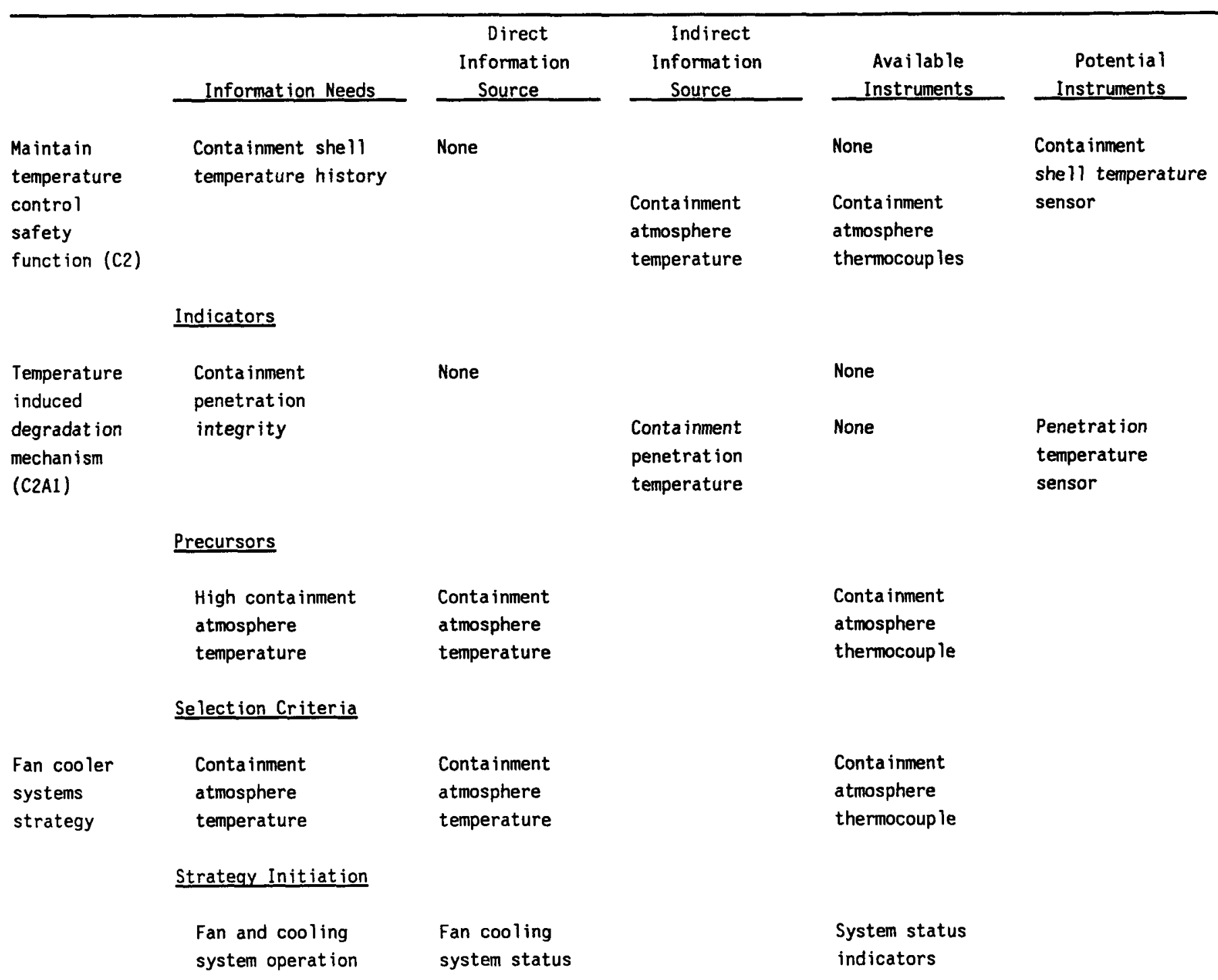


TABLE A2.7. (cont inued)

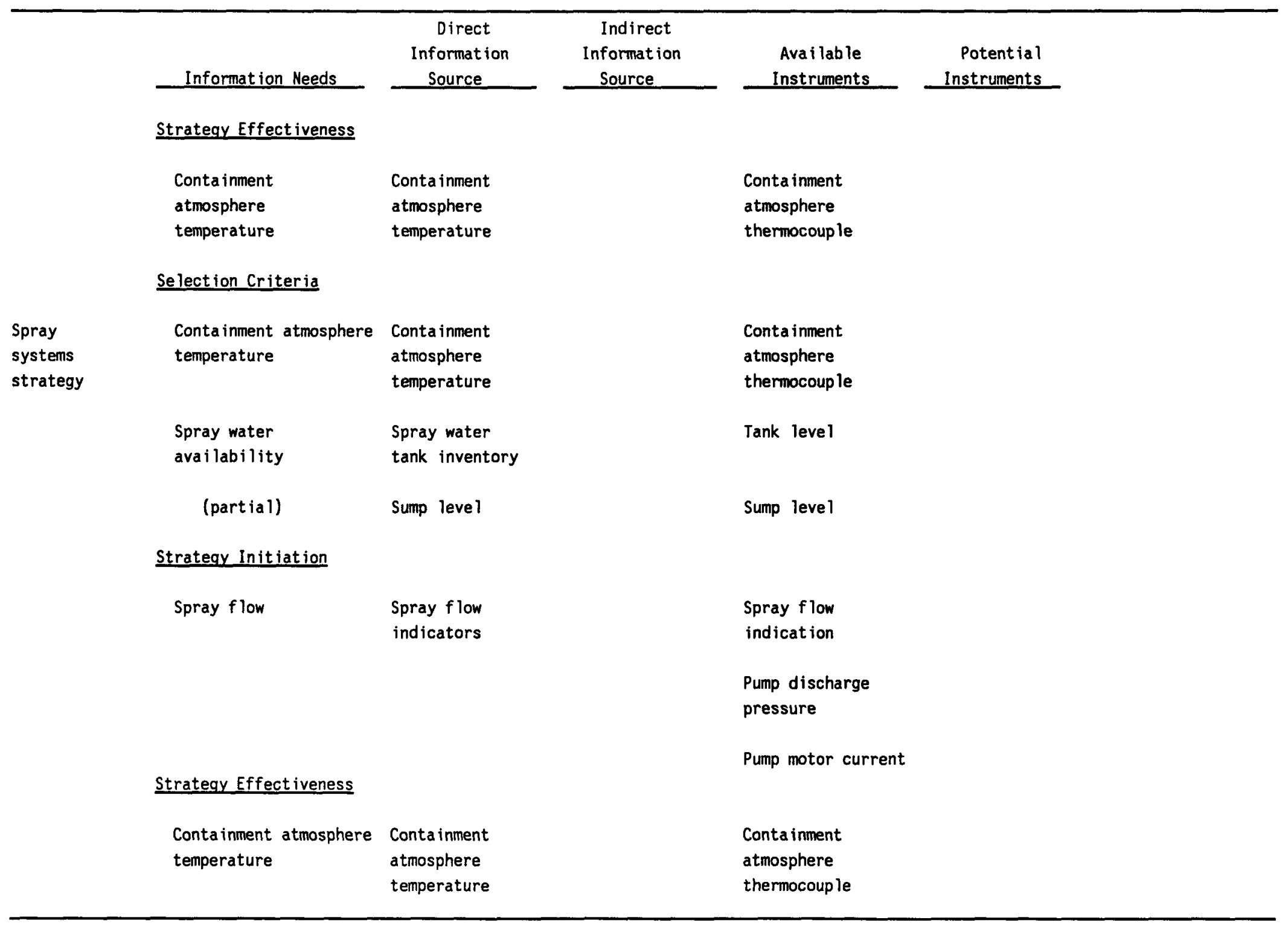


TABLE A2.8. PREVENT CONTAINMENT FAILURE (C)--CORE/CONCRETE INTERACTION MECHANISM (C2B1)

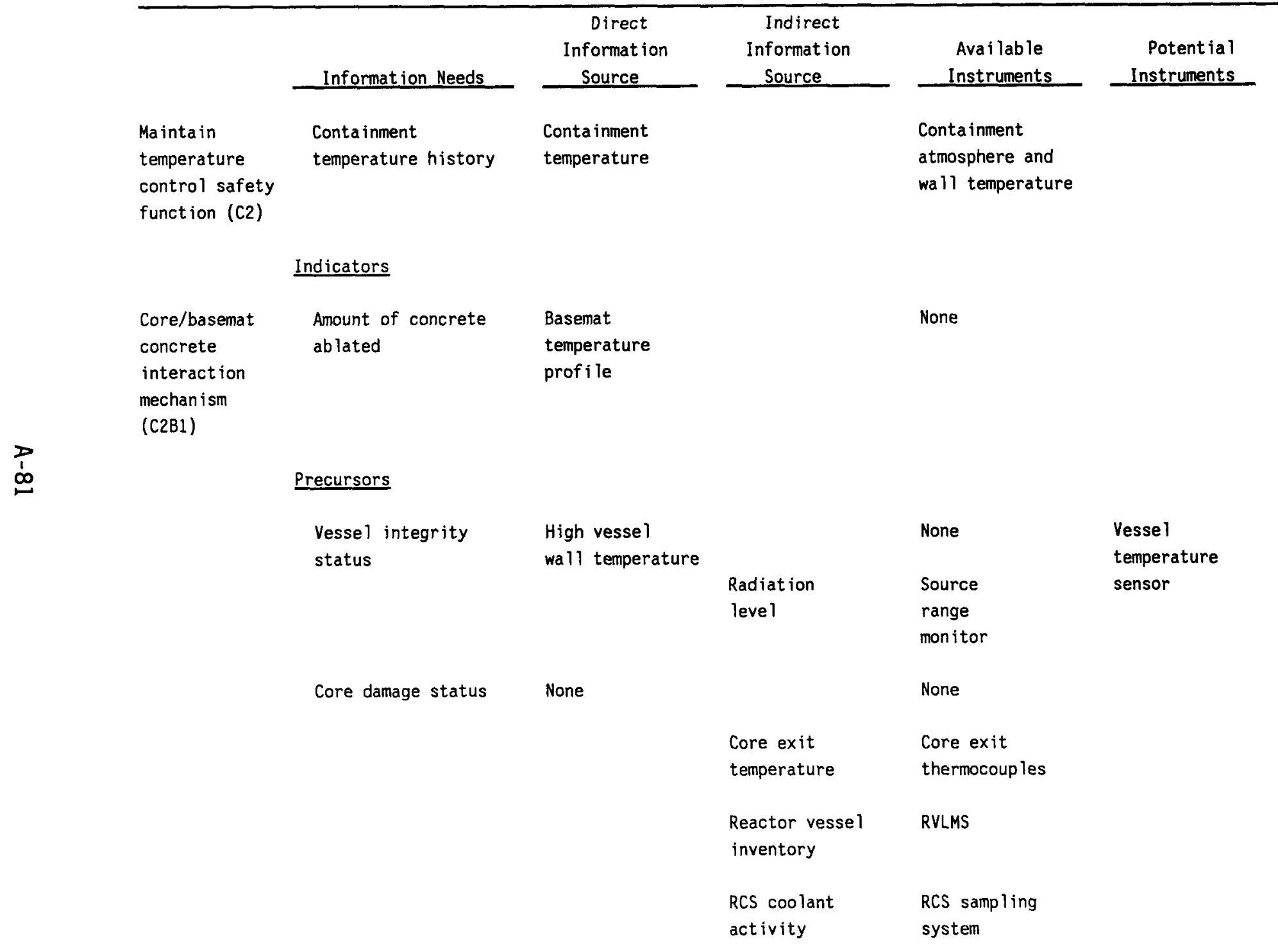


TABLE A2.8. (cont inued)

\begin{tabular}{|c|c|c|c|c|c|}
\hline & Information Needs & $\begin{array}{l}\text { Direct } \\
\text { Information } \\
\text { Source } \\
\end{array}$ & $\begin{array}{c}\text { Indirect } \\
\text { Information } \\
\text { Source } \\
\end{array}$ & $\begin{array}{l}\text { Ava i lable } \\
\text { Instruments }\end{array}$ & $\begin{array}{r}\text { Potentia } 1 \\
\text { Instruments } \\
\end{array}$ \\
\hline & Selection Criteria & & & & \\
\hline \multirow{9}{*}{$\begin{array}{l}\text { F lood } \\
\text { cavity } \\
\text { strategy }\end{array}$} & Core damage status & None & & None & \\
\hline & & & $\begin{array}{l}\text { Core exit } \\
\text { temperature }\end{array}$ & $\begin{array}{l}\text { Core exit } \\
\text { thermocouples }\end{array}$ & \\
\hline & & & $\begin{array}{l}\text { Reactor vessel } \\
\text { level }\end{array}$ & RVLMS & \\
\hline & & & $\begin{array}{l}\text { RCS coolant } \\
\text { activity }\end{array}$ & RCS sampling & \\
\hline & Cavity inventory & Cavity inventory & & None & $\begin{array}{l}\text { Cavity level } \\
\text { sensor }\end{array}$ \\
\hline & $\begin{array}{l}\text { Inventory available } \\
\text { to flood cavity }\end{array}$ & $\begin{array}{l}\text { Cavity flooding } \\
\text { system tank } \\
\text { inventory }\end{array}$ & & & Tank levels \\
\hline & Strategy Initiation & & & & \\
\hline & Flow to cavity & $\begin{array}{l}\text { Cavity flooding } \\
\text { system flow rate }\end{array}$ & & & Flow measurement \\
\hline & $\begin{array}{l}\text { Inventory available } \\
\text { to flood cavity }\end{array}$ & $\begin{array}{l}\text { Cavity flooding } \\
\text { system tank } \\
\text { inventory }\end{array}$ & & & Tank levels \\
\hline
\end{tabular}


TABLE A2.8. (cont inued)

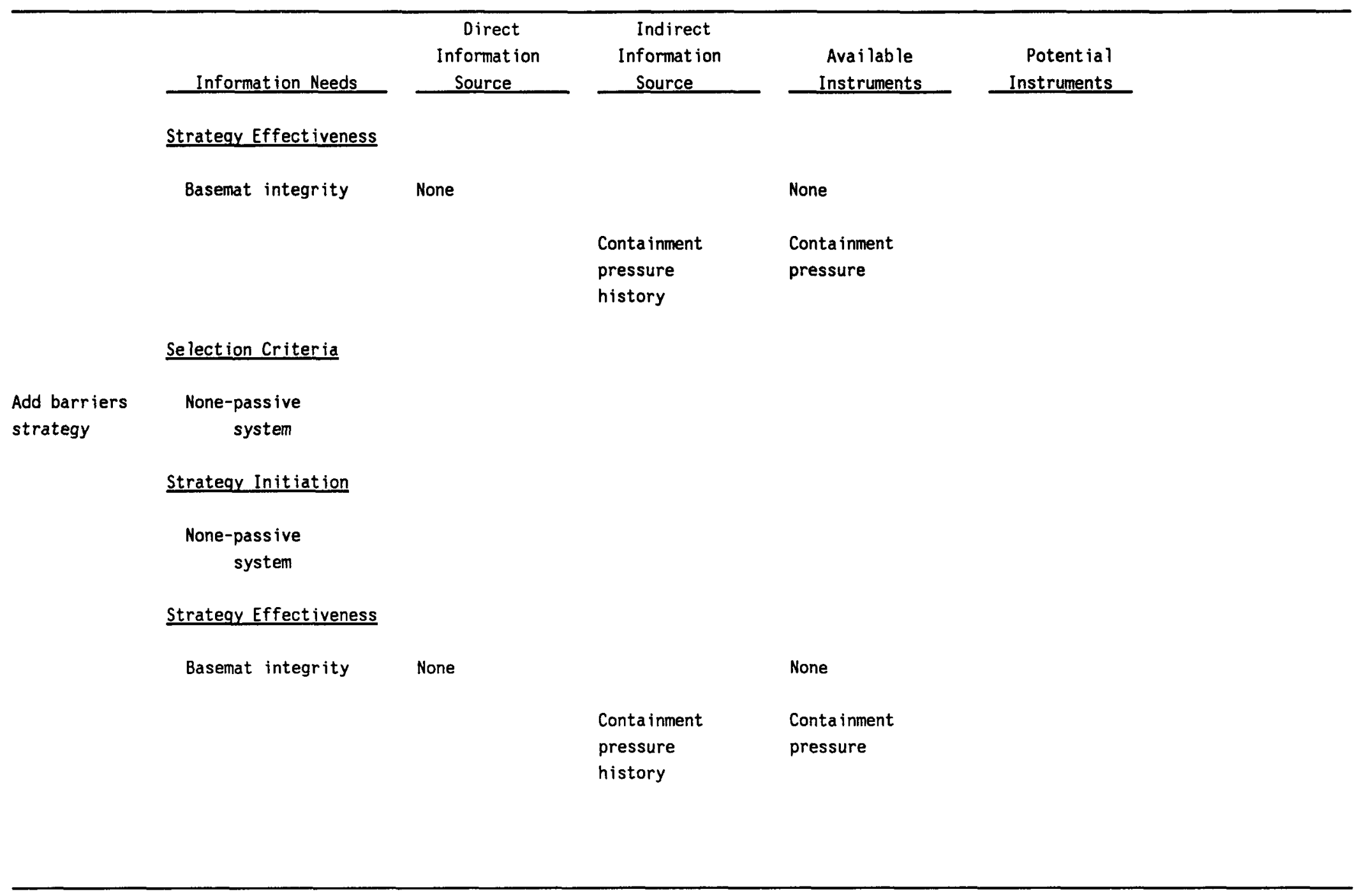


TABLE A2.9. PREVENT CONTAINMENT FAILURE (C)--FAILURE TO ISOLATE MECHANISM (C3A1)

\begin{tabular}{|c|c|c|c|c|c|}
\hline & Information Needs & $\begin{array}{c}\text { Direct } \\
\text { Information } \\
\text { Source } \\
\end{array}$ & $\begin{array}{c}\text { Indirect } \\
\text { Information } \\
\text { Source } \\
\end{array}$ & $\begin{array}{c}\text { Ava ilable } \\
\text { Instruments } \\
\end{array}$ & $\begin{array}{r}\text { Potential } \\
\text { Instruments }\end{array}$ \\
\hline \multirow{6}{*}{$\begin{array}{l}\text { Mainta in } \\
\text { integrity } \\
\text { safety } \\
\text { function (C1) }\end{array}$} & $\begin{array}{l}\text { Conta inment } \\
\text { leak rate }\end{array}$ & None & & None & \\
\hline & & & $\begin{array}{l}\text { Containment } \\
\text { pressure } \\
\text { history }\end{array}$ & $\begin{array}{l}\text { Containment } \\
\text { pressure }\end{array}$ & \\
\hline & & & $\begin{array}{l}\text { Radiation leve } 1 \\
\text { outside } \\
\text { containment }\end{array}$ & $\begin{array}{l}\text { Radiation } \\
\text { monitoring } \\
\text { system }\end{array}$ & \\
\hline & $\begin{array}{l}\text { Conta inment } \\
\text { Leak location }\end{array}$ & None & & None & \\
\hline & & & $\begin{array}{l}\text { Radiation } \\
\text { location } \\
\text { outside } \\
\text { Containment }\end{array}$ & $\begin{array}{l}\text { Radiation } \\
\text { monitoring } \\
\text { system }\end{array}$ & \\
\hline & Indicators & & & & \\
\hline \multirow{3}{*}{$\begin{array}{l}\text { Failure } \\
\text { to isolate } \\
\text { mechan ism } \\
\text { (C3A1) }\end{array}$} & $\begin{array}{l}\text { Containment isolation } \\
\text { system failure } \\
\text { location }\end{array}$ & $\begin{array}{l}\text { Containment } \\
\text { isolation } \\
\text { system status }\end{array}$ & & $\begin{array}{l}\text { Conta inment } \\
\text { isolation } \\
\text { system status }\end{array}$ & \\
\hline & & & $\begin{array}{l}\text { Containment } \\
\text { pressure } \\
\text { history }\end{array}$ & $\begin{array}{l}\text { Containment } \\
\text { pressure }\end{array}$ & \\
\hline & & & $\begin{array}{l}\text { Radiation leve } 1 \\
\text { outside } \\
\text { containment }\end{array}$ & $\begin{array}{l}\text { Radiation } \\
\text { monitoring } \\
\text { system }\end{array}$ & \\
\hline
\end{tabular}


TABLE A2.9. (cont inued)

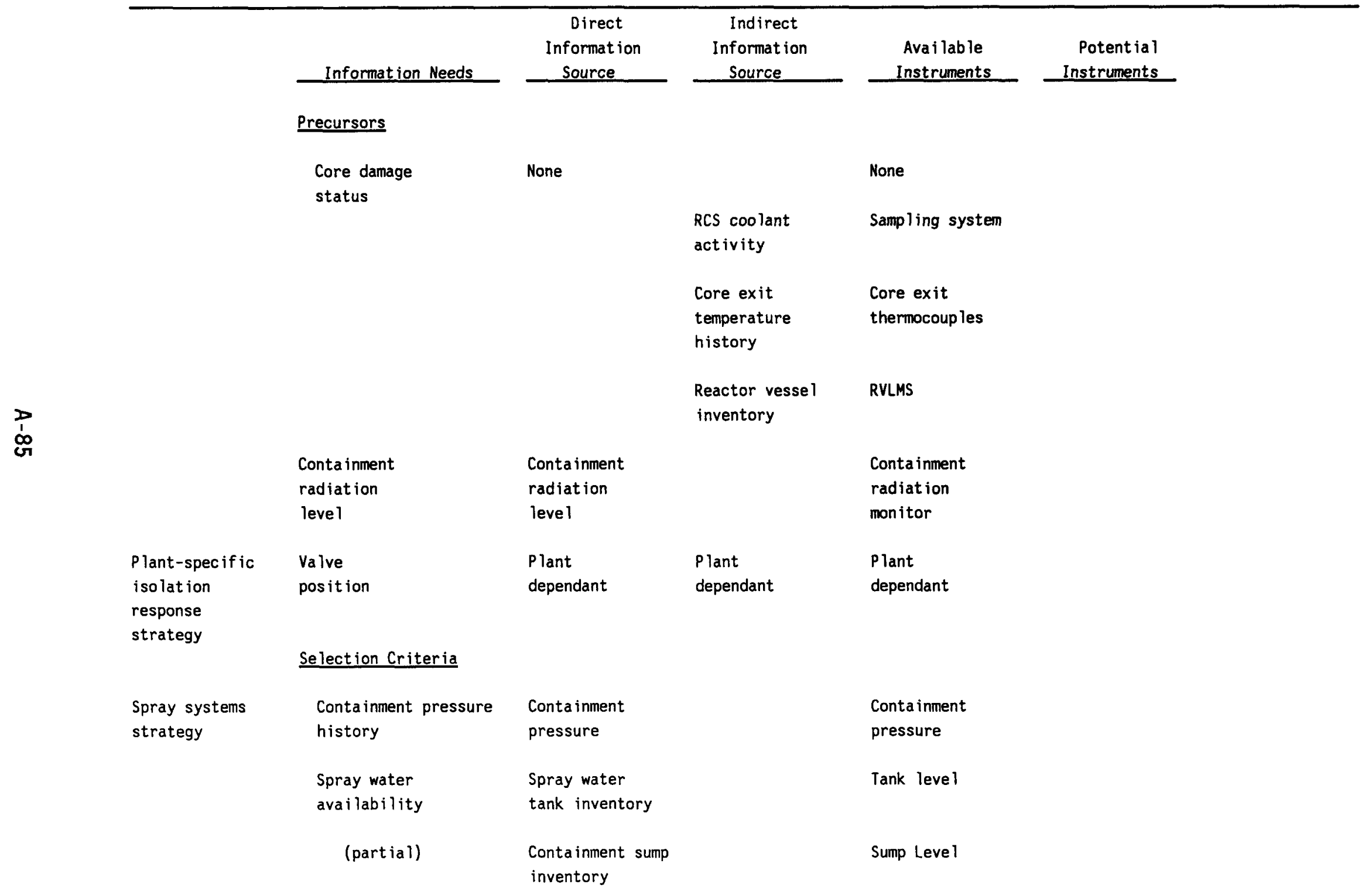


TABLE A2.9. (cont inued)

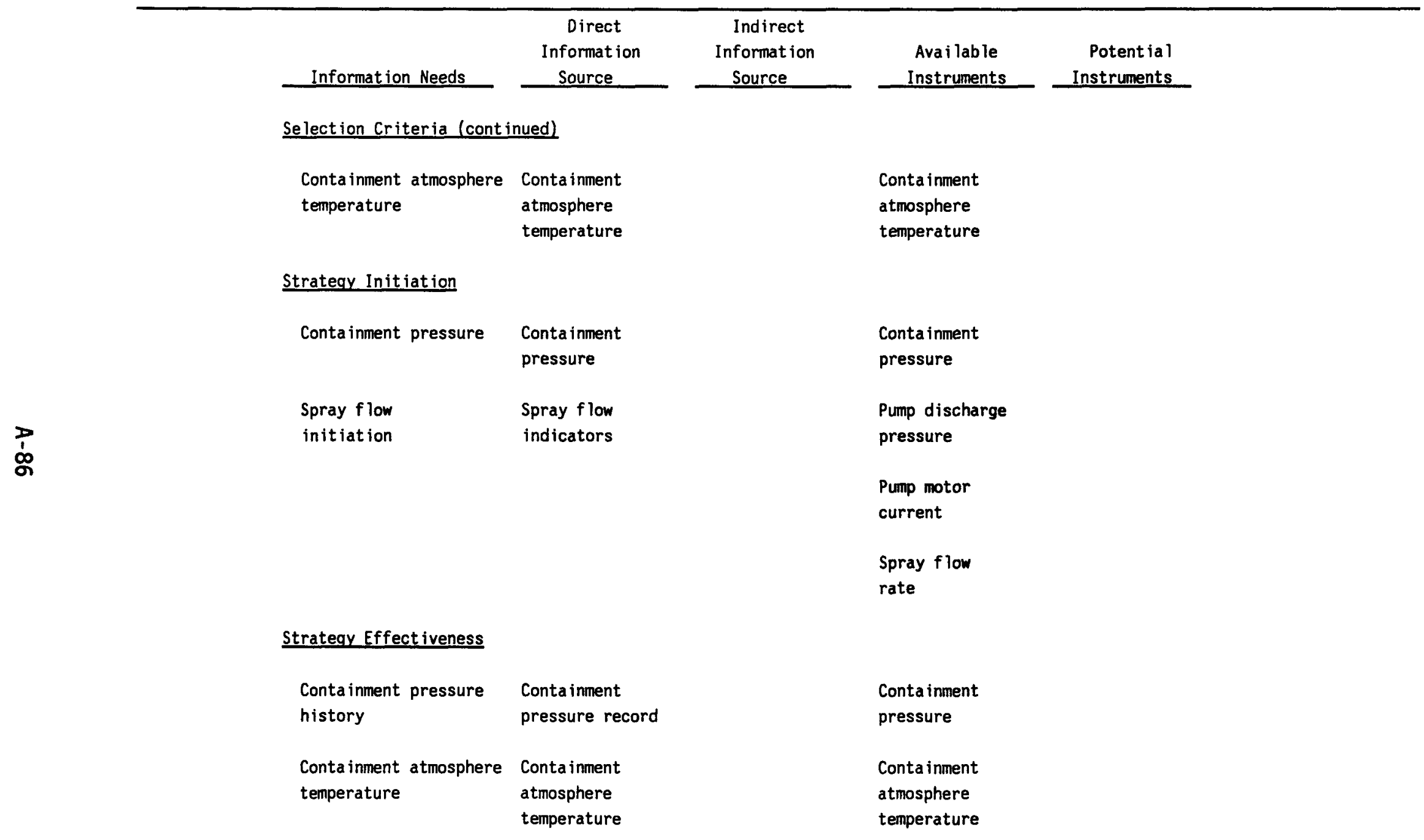


TABLE A2.9. (cont inued)

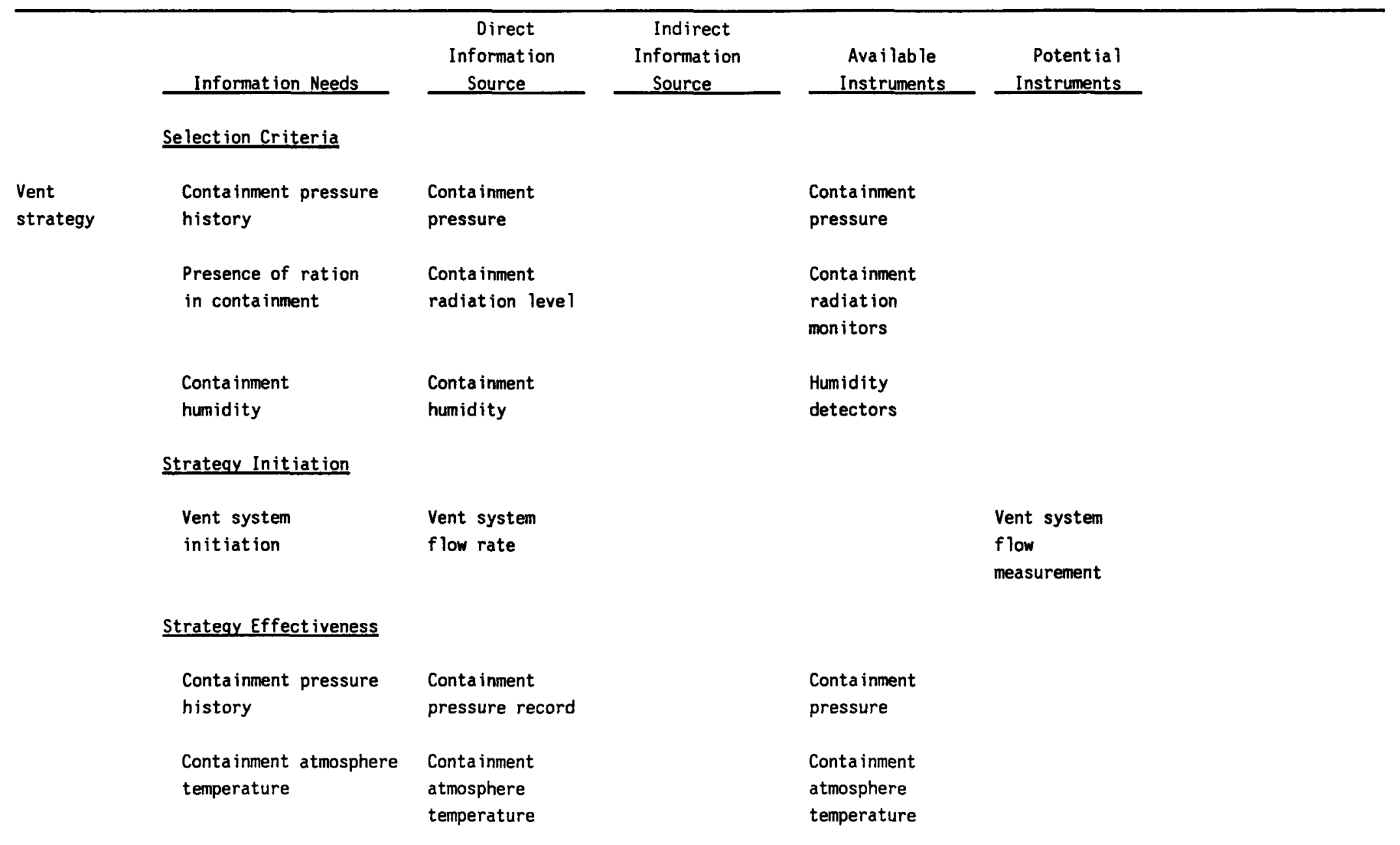


TABLE A2.10. PREVENT CONTAINMENT FAILURE (C)--FAILURE AFTER ISOLATION MECHANISM (C3A2)

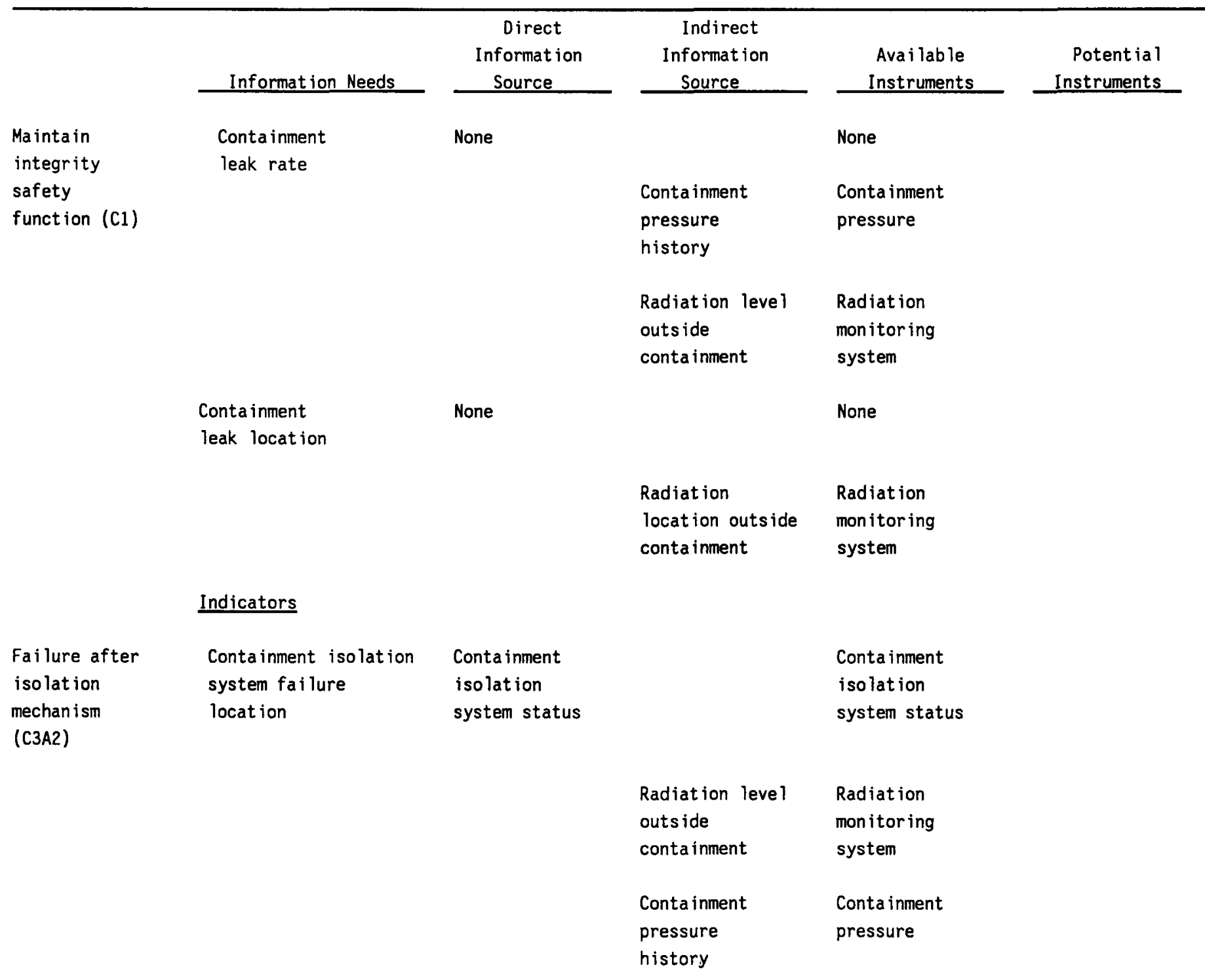


TABLE A2.10. (cont inued)

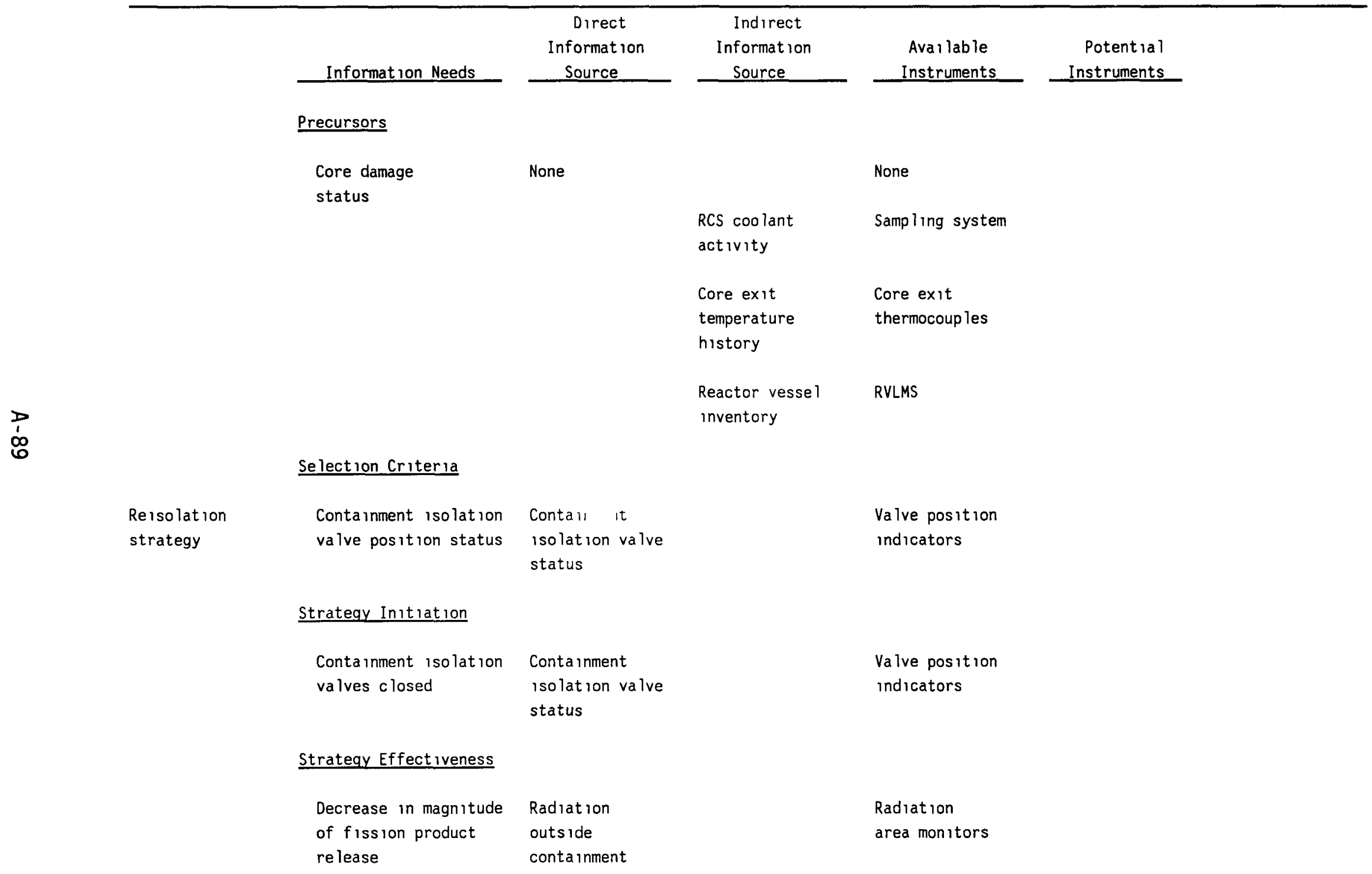


TABLE A2.10. (cont inued)

\begin{tabular}{|c|c|c|c|c|c|}
\hline & Information Needs & $\begin{array}{l}\text { Direct } \\
\text { Information } \\
\text { Source } \\
\end{array}$ & $\begin{array}{c}\text { Indirect } \\
\text { Information } \\
\text { Source } \\
\end{array}$ & $\begin{array}{l}\text { Available } \\
\text { Instruments }\end{array}$ & $\begin{array}{r}\text { Potential } \\
\text { Instruments } \\
\end{array}$ \\
\hline & election Criteria & & & & \\
\hline \multirow[t]{3}{*}{$\begin{array}{l}\text { Depressurize } \\
\text { containment } \\
\text { strategy }\end{array}$} & $\begin{array}{l}\text { Reisolation not } \\
\text { possible }\end{array}$ & & $\begin{array}{l}\text { Containment } \\
\text { isolation valve }\end{array}$ & $\begin{array}{l}\text { Valve position } \\
\text { indicators }\end{array}$ & \\
\hline & $\begin{array}{l}\text { Fission produsts in } \\
\text { containment } \\
\text { atmosphere }\end{array}$ & $\begin{array}{l}\text { Containment } \\
\text { radiation leve } 1\end{array}$ & & $\begin{array}{l}\text { Radiation } \\
\text { monitoring system }\end{array}$ & \\
\hline & $\begin{array}{l}\text { Conta inment } \\
\text { pressure }\end{array}$ & $\begin{array}{l}\text { Containment } \\
\text { pressure }\end{array}$ & & $\begin{array}{l}\text { Conta inment } \\
\text { pressure }\end{array}$ & \\
\hline
\end{tabular}

See Table A2.1 for spray, fan cooler, and venting strategies.

\section{Strategy Effectiveness}

$\begin{array}{lll}\text { Decrease in } & \text { Radiation } & \text { Radiation } \\ \text { magnitude of } & \text { outside } & \text { area monitors } \\ \text { fission product } & \text { containment } & \\ \text { release } & & \end{array}$


TABLE A2.10. (cont inued)

\begin{tabular}{|c|c|c|c|c|c|}
\hline & Information Needs & $\begin{array}{c}\text { Direct } \\
\text { Information } \\
\text { Source } \\
\end{array}$ & $\begin{array}{c}\text { Indirect } \\
\text { Information } \\
\text { Source } \\
\end{array}$ & $\begin{array}{l}\text { Available } \\
\text { Instruments }\end{array}$ & $\begin{array}{r}\text { Potential } \\
\text { Instruments } \\
\end{array}$ \\
\hline \multirow{5}{*}{$\begin{array}{l}\text { Flood } \\
\text { containment } \\
\text { leak strategy }\end{array}$} & $\begin{array}{l}\text { Leak point can be } \\
\text { located and flooded }\end{array}$ & $\begin{array}{l}\text { Containment } \\
\text { leak location }\end{array}$ & & & $\begin{array}{l}\text { Visual or } \\
\text { camera }\end{array}$ \\
\hline & Strategy Initiation & & & & \\
\hline & $\begin{array}{l}\text { Flow to leak } \\
\text { location }\end{array}$ & $\begin{array}{l}\text { Flow in leak } \\
\text { flooding system }\end{array}$ & & & Flow meter \\
\hline & Strategy Effectiveness & & & & \\
\hline & $\begin{array}{l}\text { Decrease in } \\
\text { magnitude of } \\
\text { fission product } \\
\text { release }\end{array}$ & $\begin{array}{l}\text { Radiation } \\
\text { outside } \\
\text { containment }\end{array}$ & & $\begin{array}{l}\text { Radiation } \\
\text { monitors }\end{array}$ & \\
\hline
\end{tabular}


TABLE A2.11. MItIGATE FISSION PRODUCT RELEASE (F)--STEAM GENERATOR TUBE RUPTURE FAILURE MECHANISM (C3B1)

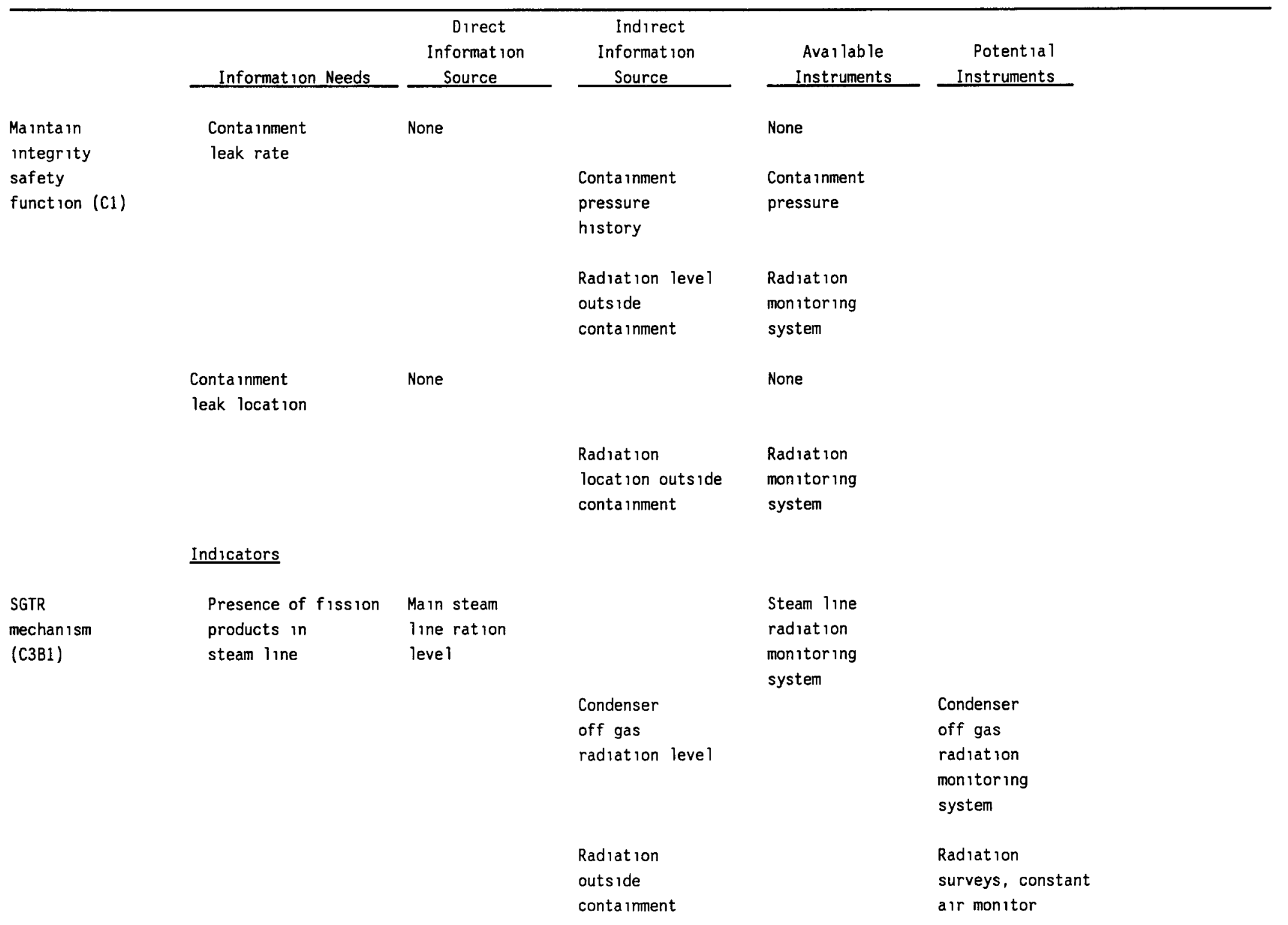


TABLE A2.11. (cont inued)

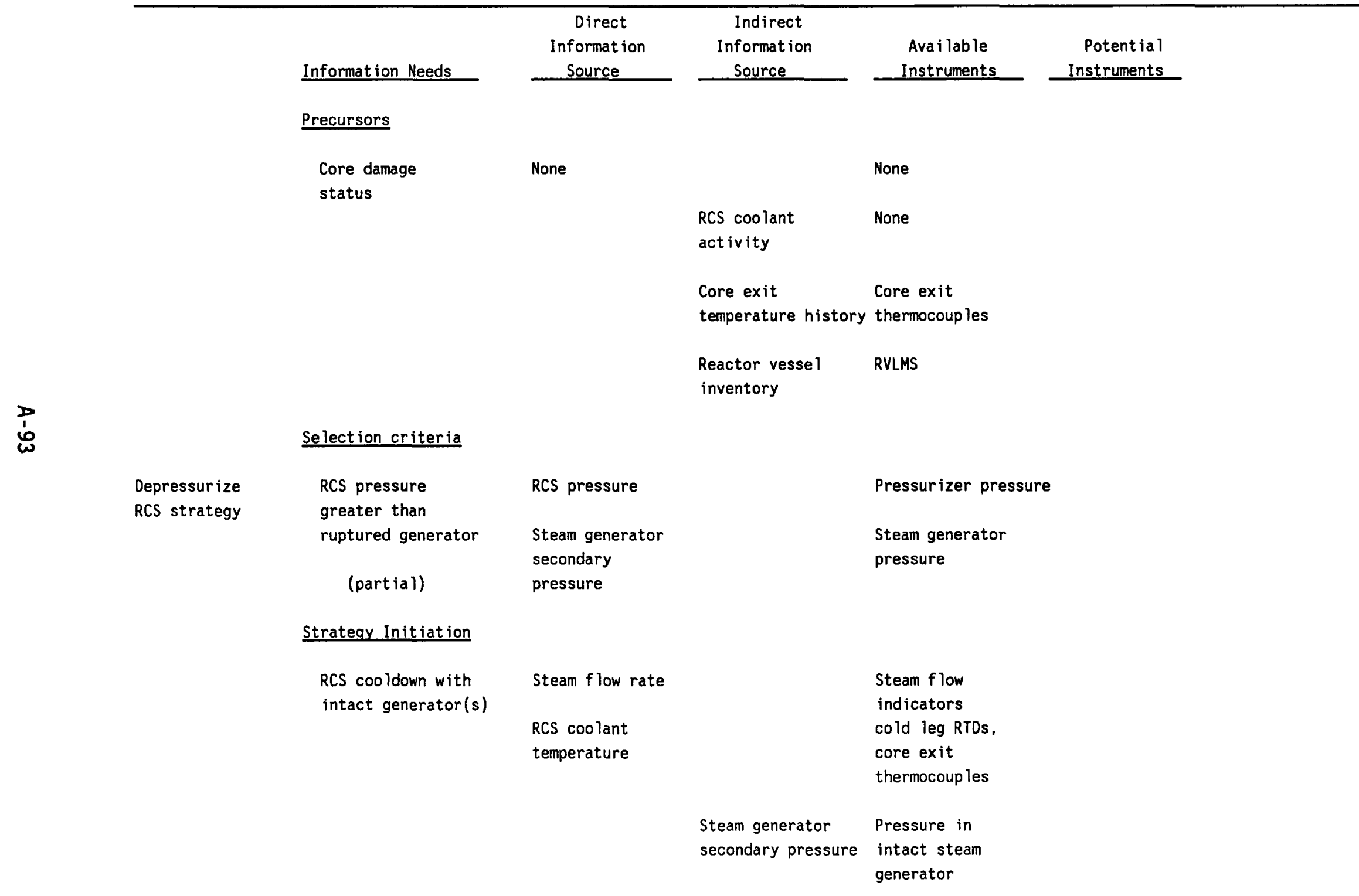


TABLE A2.11. (cont inued)

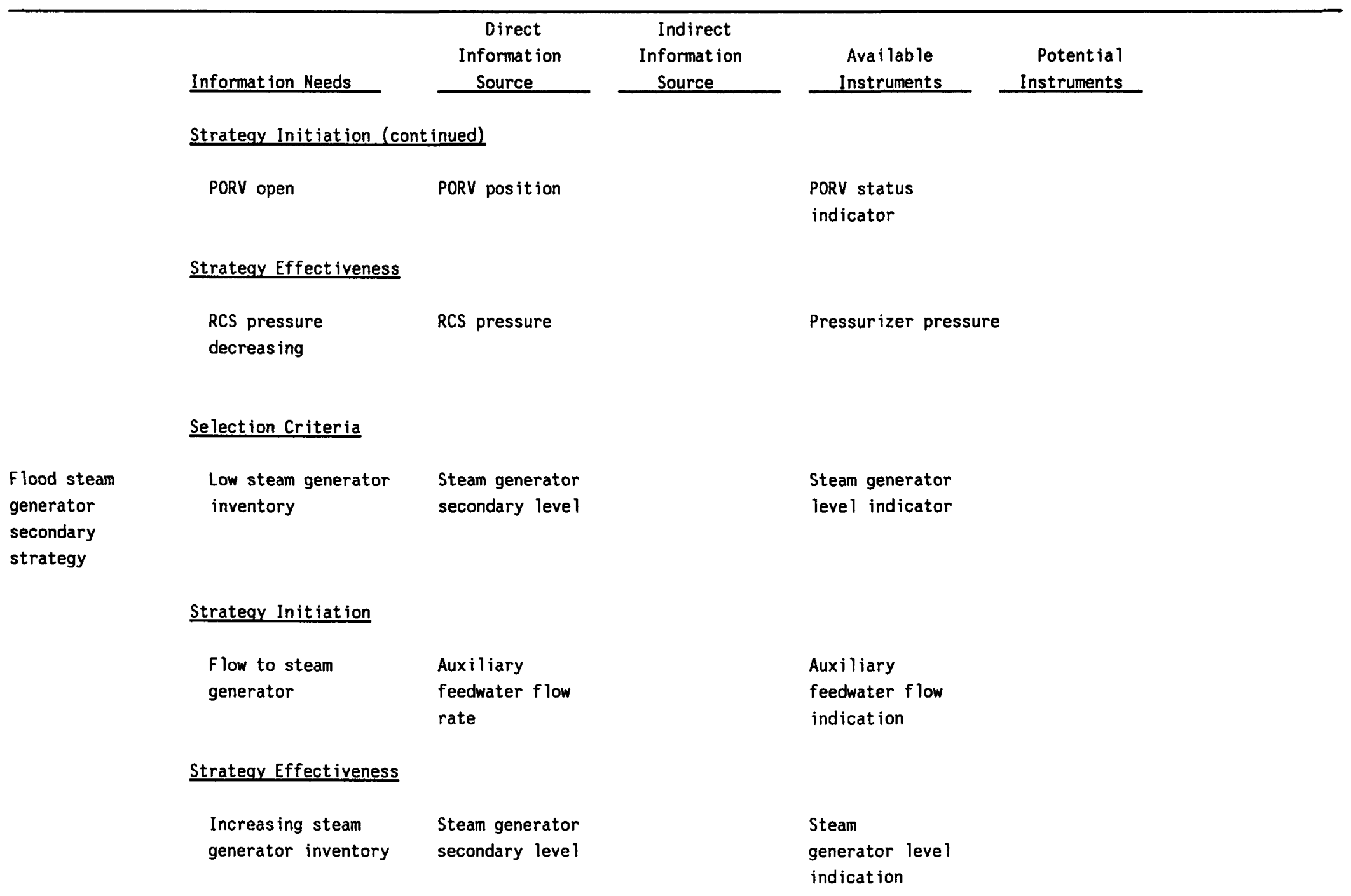


TABLE A2.12. PREVENT CONTAINMENT FAILURE (C)--ISLOCA MECHANISM (C3B2)

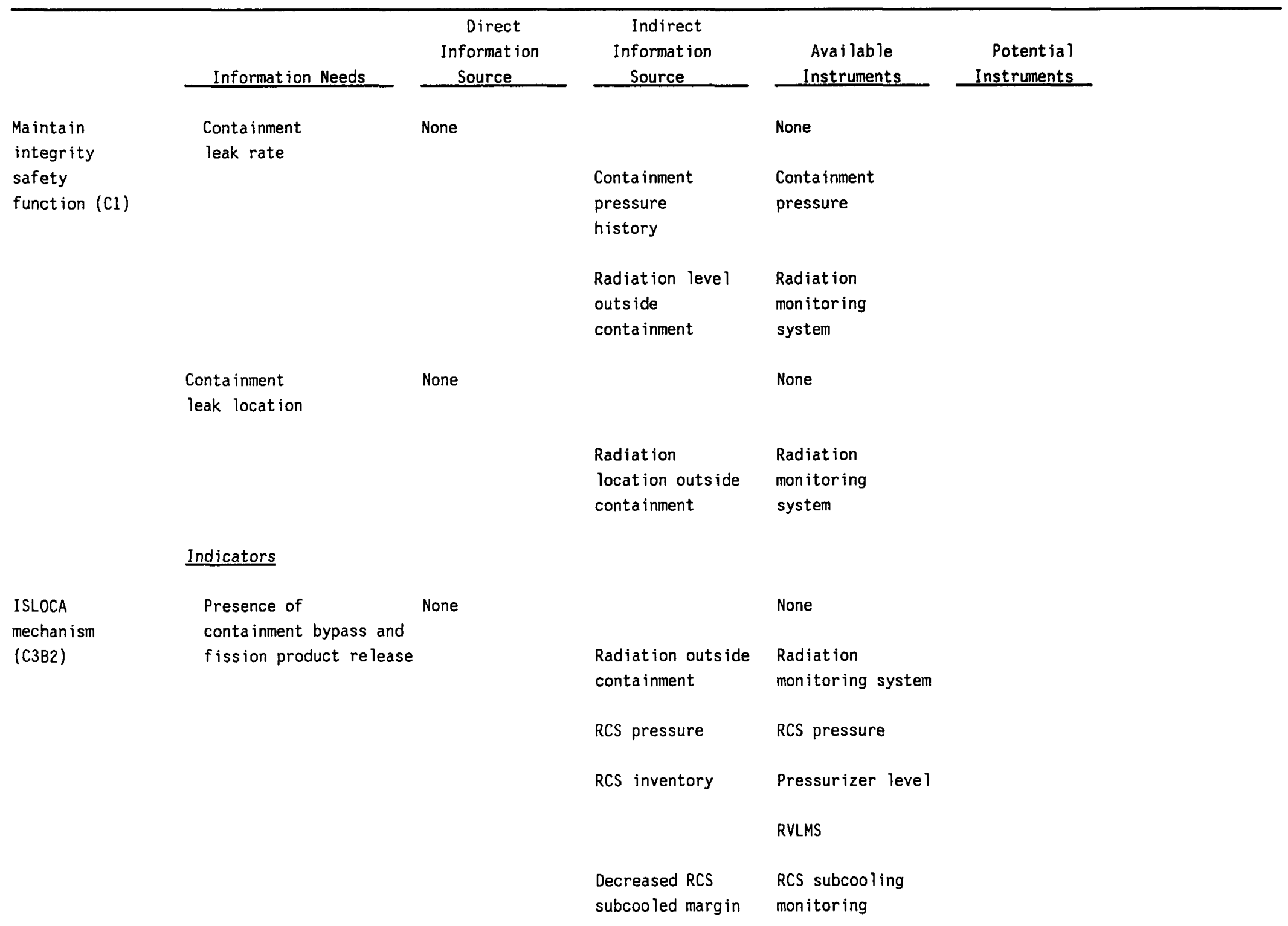


TABLE A2.12. (cont inued)

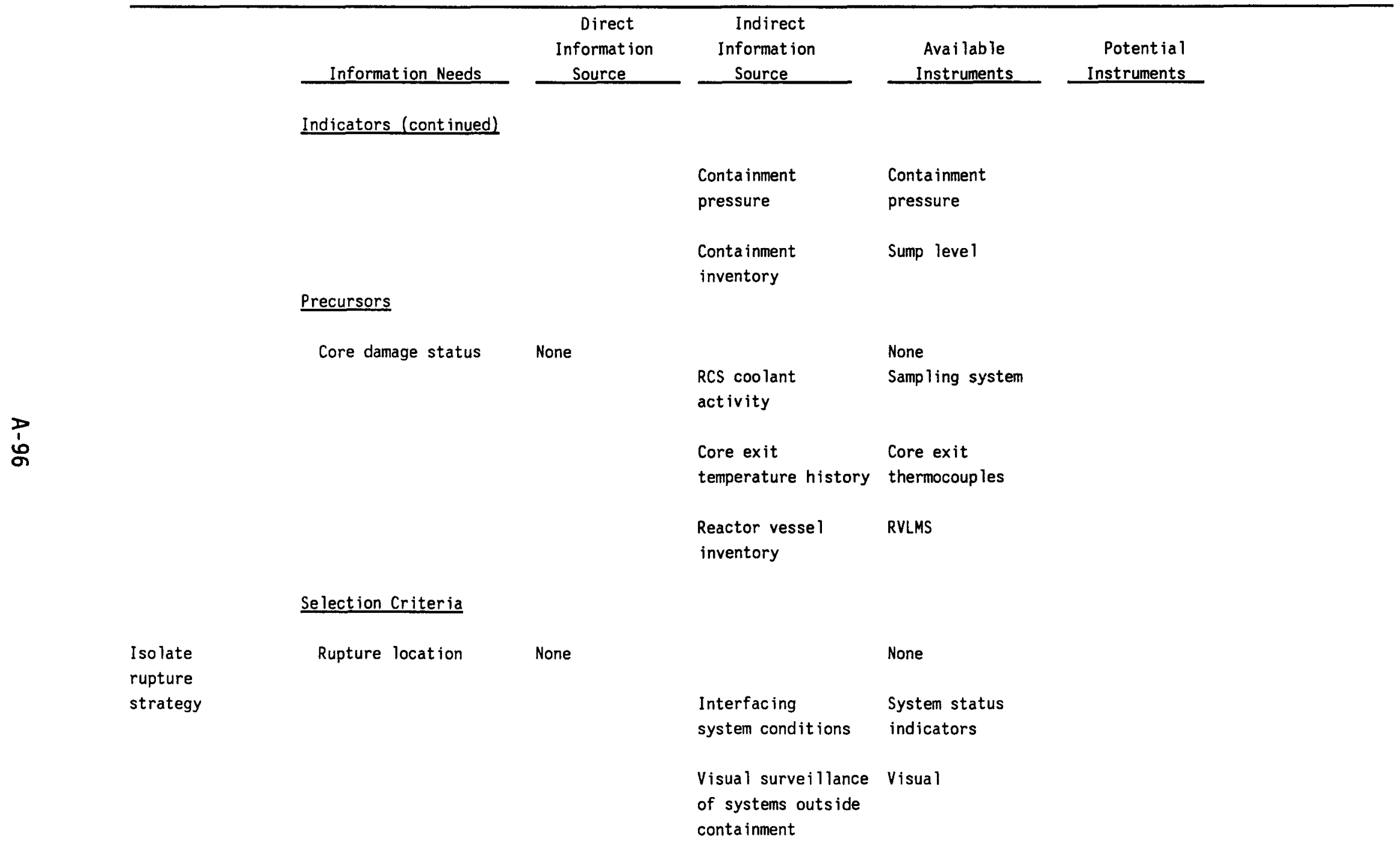


TABLE A2.12. (cont inued)

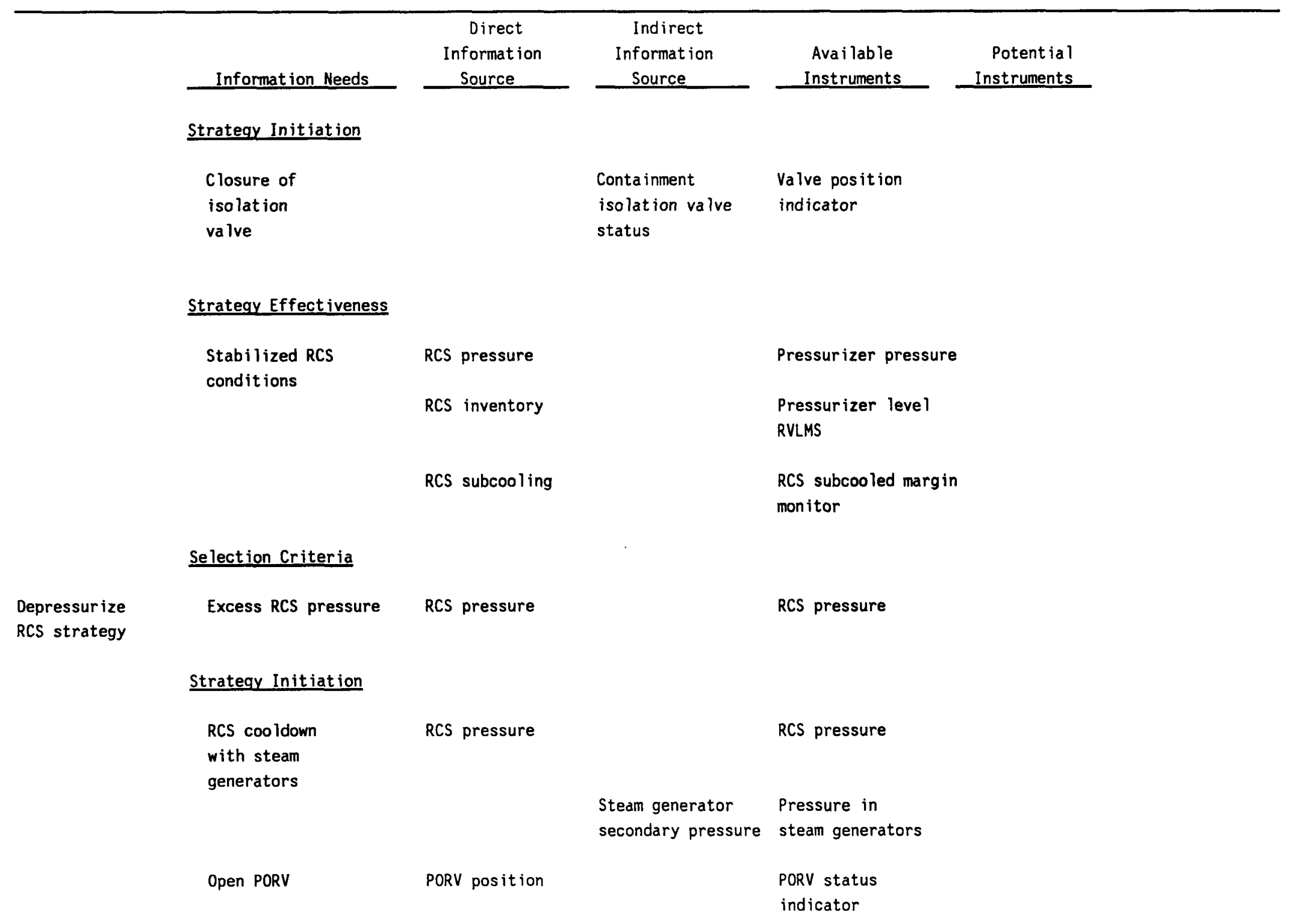


TABLE A2.12. (cont inued)

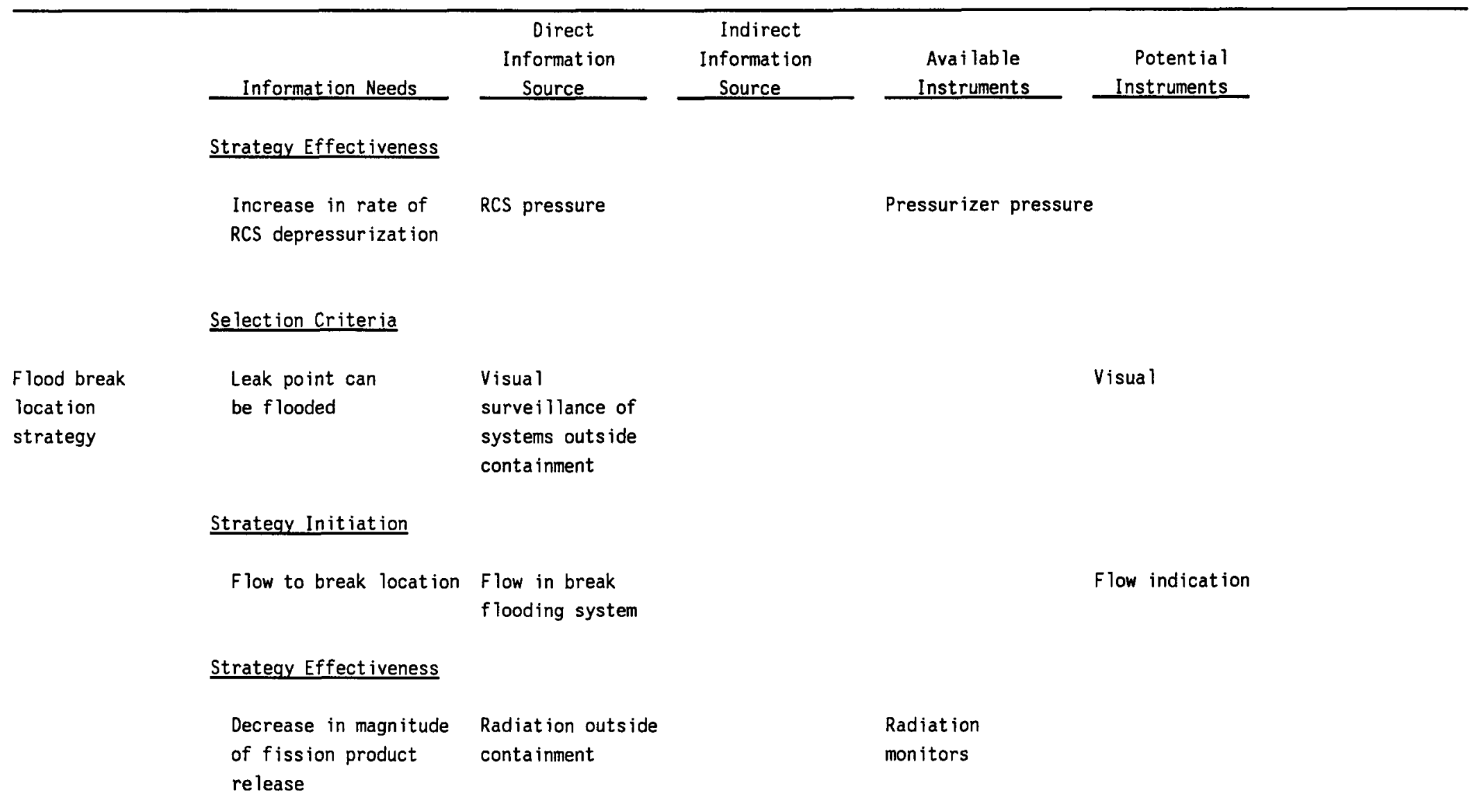


TABLE A2.13. PREVENT CONTAINMENT FAILURE (C)--STEAM EXPLOSION MISSILE MECHANISM (C3C1)

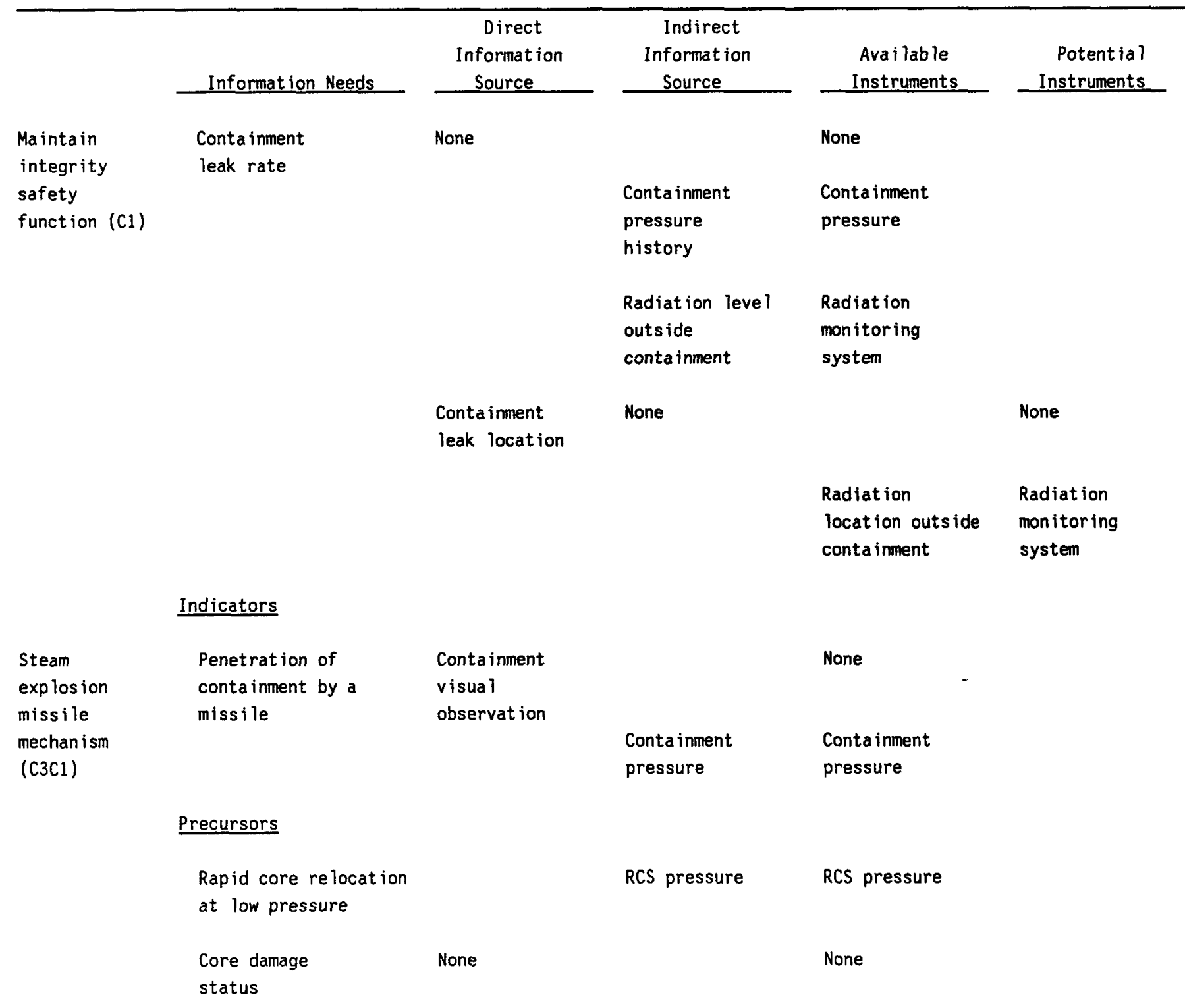


TABLE A2.13. (cont inued)

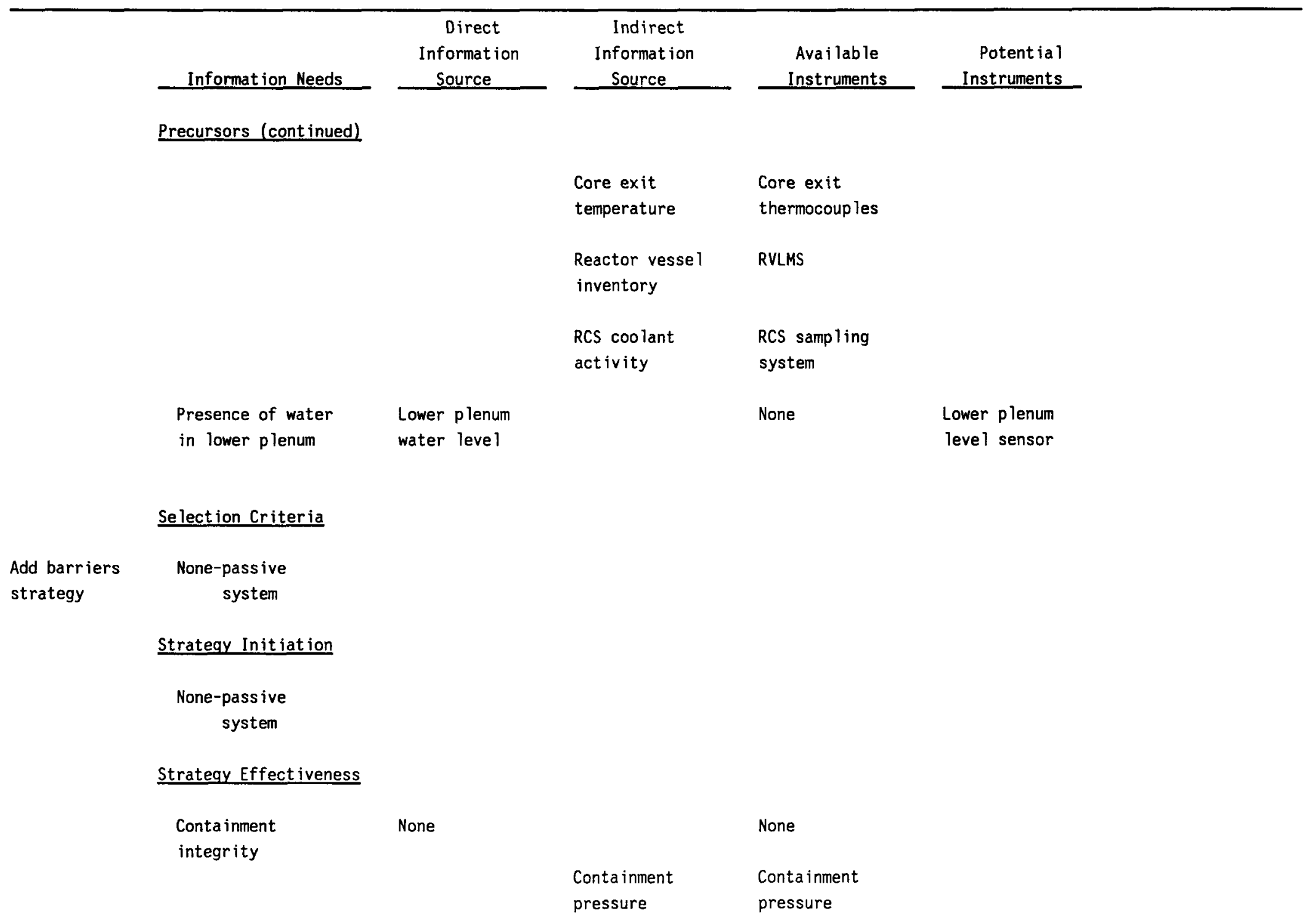


TABLE A2.13. (cont inued)

\begin{tabular}{|c|c|c|c|c|c|}
\hline & Information Needs & $\begin{array}{c}\text { Direct } \\
\text { Information } \\
\text { Source } \\
\end{array}$ & $\begin{array}{c}\text { Indirect } \\
\text { Information } \\
\text { Source } \\
\end{array}$ & $\begin{array}{c}\text { Available } \\
\text { Instruments }\end{array}$ & $\begin{array}{r}\text { Potentia } 1 \\
\text { Instruments } \\
\end{array}$ \\
\hline & \multicolumn{5}{|c|}{ Strategy Effectiveness (cont inued) } \\
\hline & & & $\begin{array}{l}\text { Radiation } \\
\text { outs ide } \\
\text { conta inment }\end{array}$ & $\begin{array}{l}\text { Radiation } \\
\text { monitoring } \\
\text { system }\end{array}$ & \\
\hline & Selection Criteria & & & & \\
\hline \multirow{7}{*}{$\begin{array}{l}\text { Dry vesse } 1 \\
\text { strategy }\end{array}$} & Core damage status & None & & None & \\
\hline & & & $\begin{array}{l}\text { Core exit } \\
\text { temperature }\end{array}$ & $\begin{array}{l}\text { Core Exit } \\
\text { thermocouples }\end{array}$ & \\
\hline & & & $\begin{array}{l}\text { Reactor vessel } \\
\text { inventory }\end{array}$ & RVLMS & \\
\hline & & & $\begin{array}{l}\text { RCS coolant } \\
\text { activity }\end{array}$ & $\begin{array}{l}\text { RCS sampling } \\
\text { system }\end{array}$ & \\
\hline & $\begin{array}{l}\text { Lower plenum } \\
\text { inventory }\end{array}$ & $\begin{array}{l}\text { Lower plenum } \\
\text { water level }\end{array}$ & & None & $\begin{array}{l}\text { Lower plenum } \\
\text { level sensor }\end{array}$ \\
\hline & Strategy Initiation & & & & \\
\hline & $\begin{array}{l}\text { Lower plenum } \\
\text { inventory }\end{array}$ & $\begin{array}{l}\text { Lower plenum } \\
\text { water level }\end{array}$ & & None & $\begin{array}{l}\text { Lower plenum } \\
\text { level sensor }\end{array}$ \\
\hline
\end{tabular}


TABLE A2.13. (cont inued)

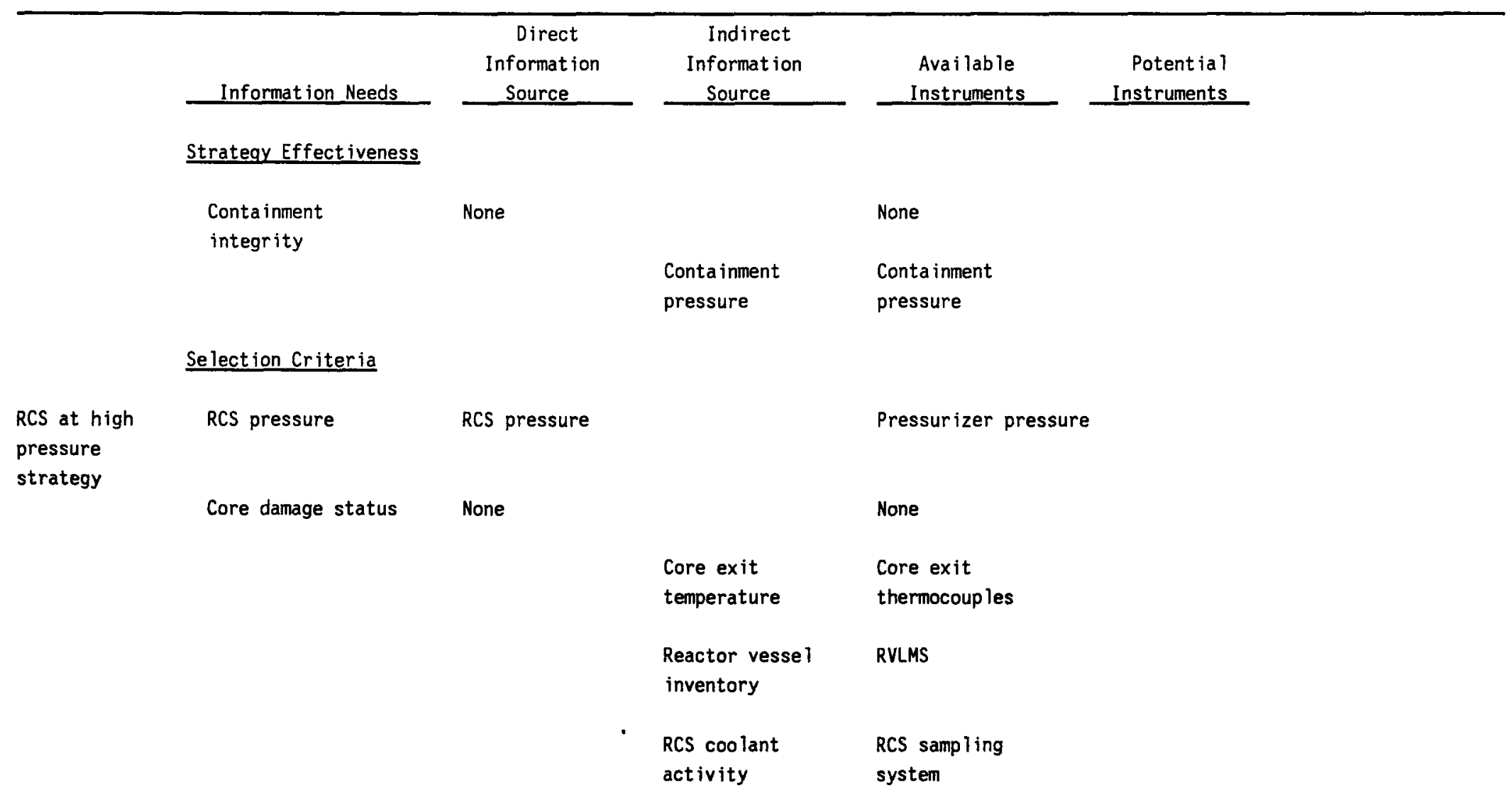


TABLE A2.13. (cont inued)

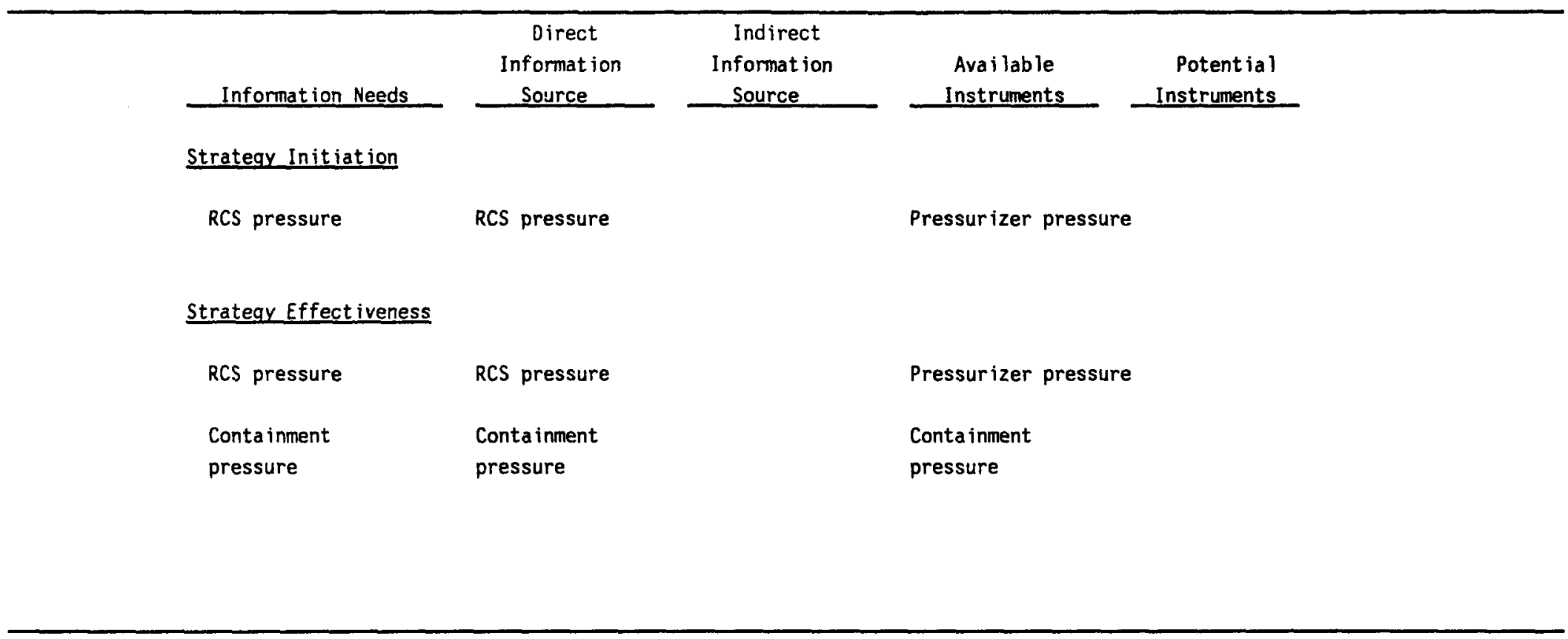


TABLE A2.14. PREVENT CONTAINMENT FAILURE (C)--HYOROGEN DETONATION MISSILE MECHANISM (C3C2)

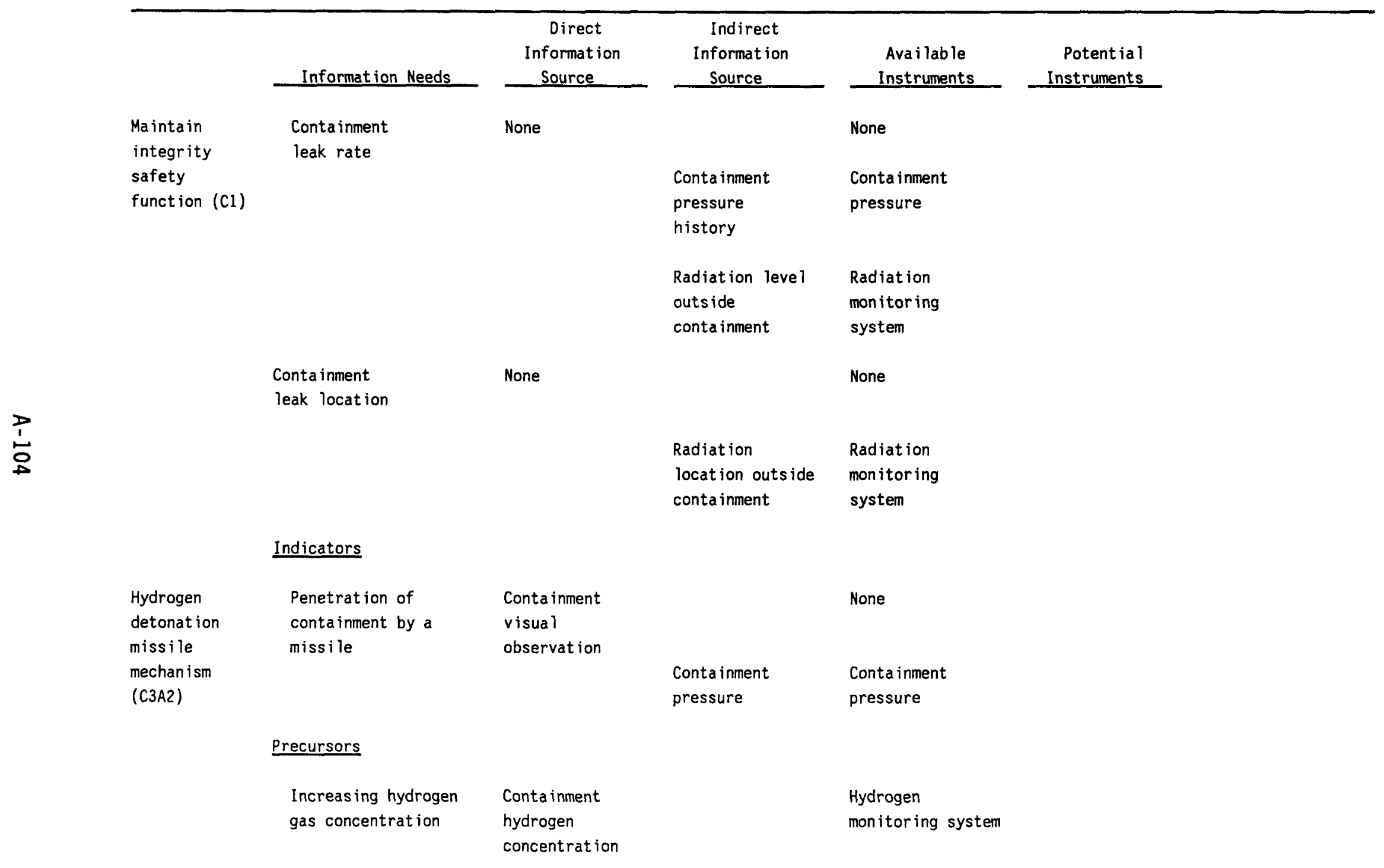


TABLE A2.14. (cont inued)

\begin{tabular}{|c|c|c|c|c|c|}
\hline & Information Needs & $\begin{array}{c}\text { Direct } \\
\text { Information } \\
\text { Source }\end{array}$ & $\begin{array}{c}\text { Indirect } \\
\text { Information } \\
\text { Source } \\
\end{array}$ & $\begin{array}{c}\text { Available } \\
\text { Instruments } \\
\end{array}$ & $\begin{array}{r}\text { Potential } \\
\text { Instruments } \\
\end{array}$ \\
\hline & Precursors (cont inued) & & & & \\
\hline & Core damage status & None & & None & \\
\hline & & & $\begin{array}{l}\text { Core exit } \\
\text { temperature }\end{array}$ & $\begin{array}{l}\text { Core exit } \\
\text { thermocouples }\end{array}$ & \\
\hline & & & $\begin{array}{l}\text { Reactor vesse } 1 \\
\text { inventory }\end{array}$ & RVLMS & \\
\hline & & & $\begin{array}{l}\text { RCS coolant } \\
\text { activity }\end{array}$ & $\begin{array}{l}\text { RCS sampling } \\
\text { system }\end{array}$ & \\
\hline & Selection Criteria & & & & \\
\hline $\begin{array}{l}\text { Add barriers } \\
\text { strategy }\end{array}$ & $\begin{array}{c}\text { None-passive } \\
\text { system }\end{array}$ & & & & \\
\hline & Strategy Initiation & & & & \\
\hline & $\begin{array}{c}\text { None-passive } \\
\text { system }\end{array}$ & & & & \\
\hline
\end{tabular}


TABLE A2.14. (cont inued)

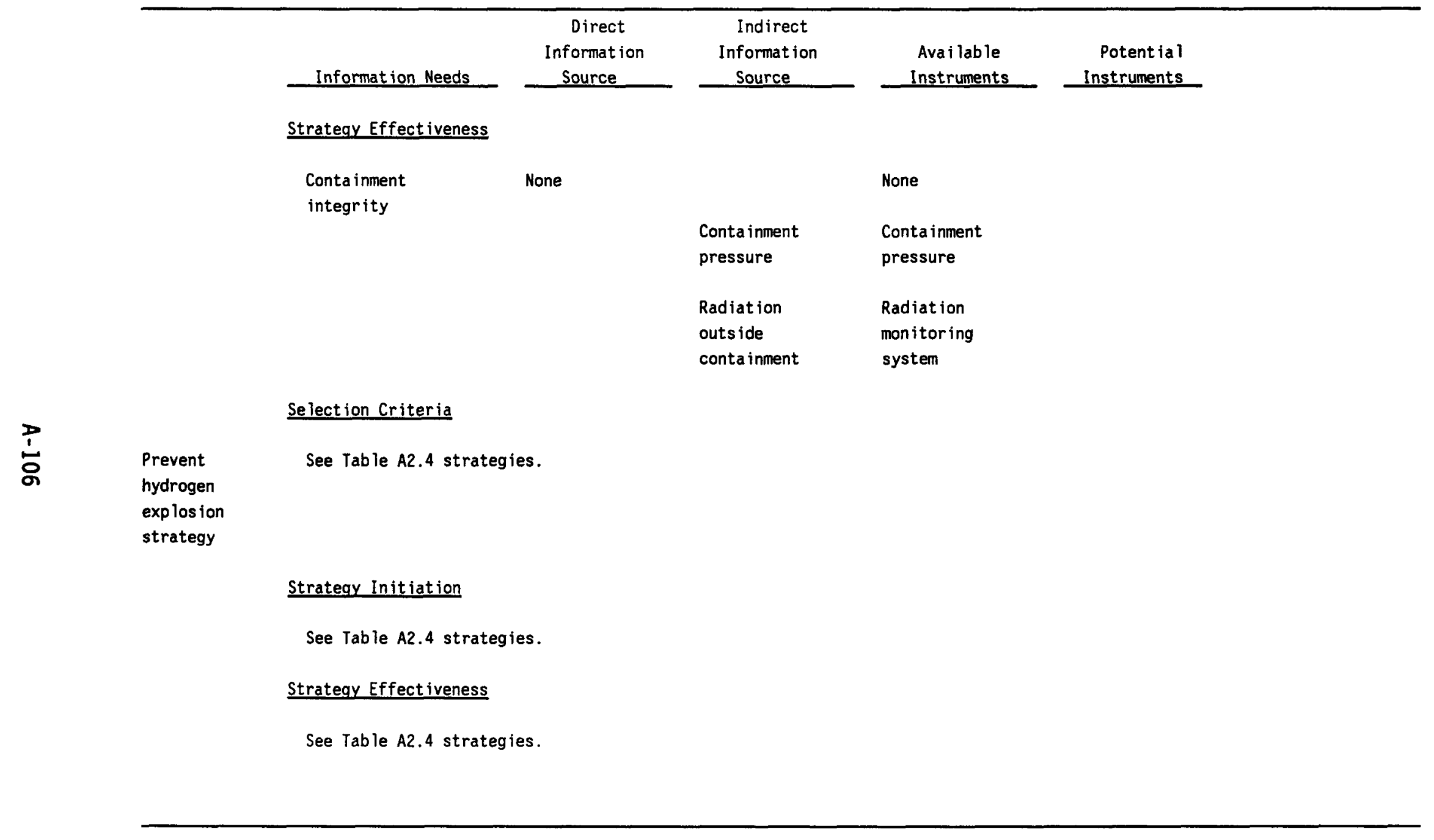


TABLE A3.1. MITIGATE FISSION PROOUCT RELEASE FROM CONTAINMENT (F)--AEROSOL DISPERSION MECHANISM (F1A1)

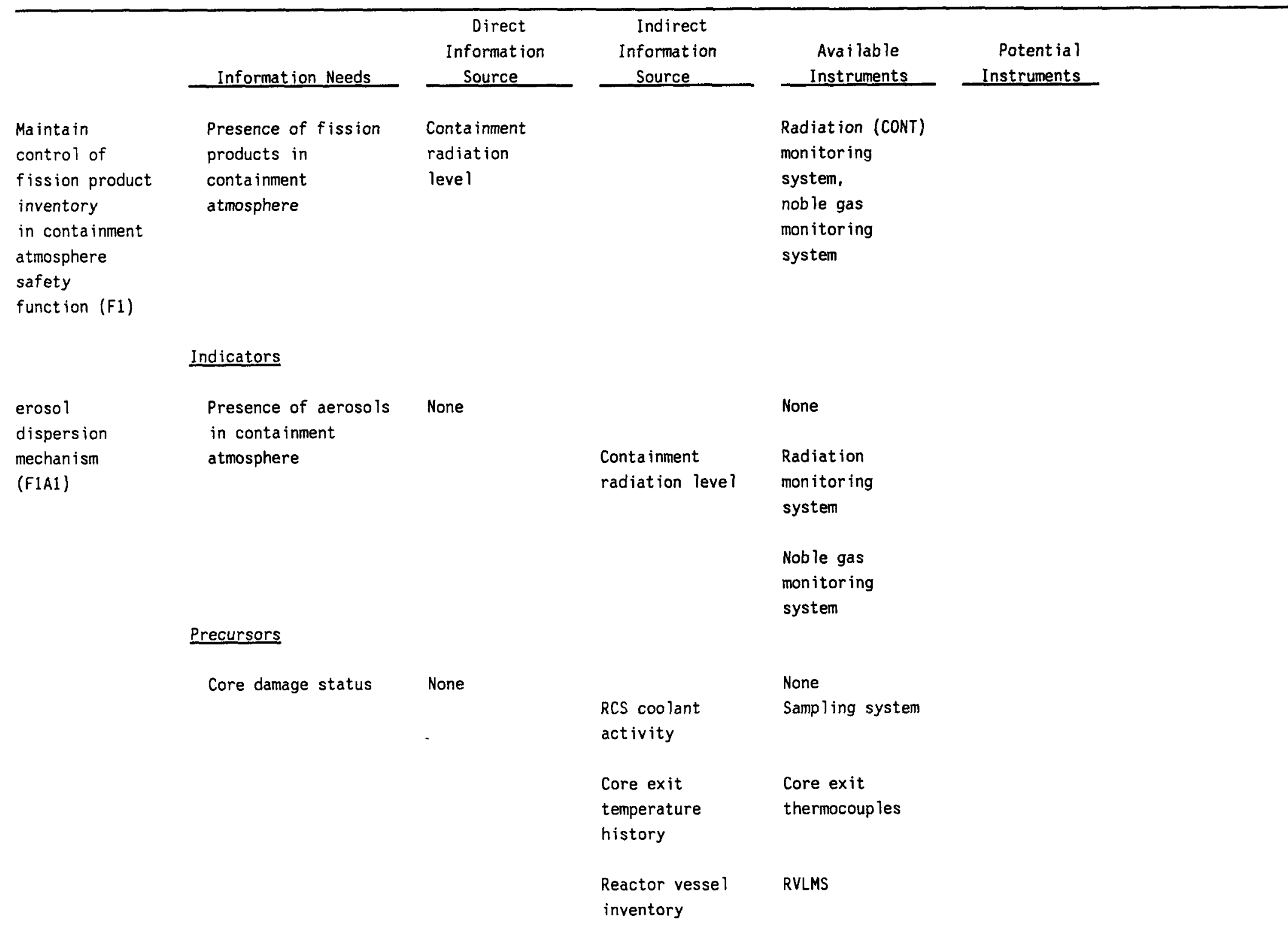


TABLE A3.1. (continued)

\begin{tabular}{|c|c|c|c|c|c|}
\hline & Information Needs & $\begin{array}{c}\text { Direct } \\
\text { Information } \\
\text { Source }\end{array}$ & $\begin{array}{c}\text { Indirect } \\
\text { Information } \\
\text { Source }\end{array}$ & $\begin{array}{l}\text { Available } \\
\text { Instruments }\end{array}$ & $\begin{array}{r}\text { Potential } \\
\text { Instruments }\end{array}$ \\
\hline & Selection Criteria & & & & \\
\hline \multirow{10}{*}{$\begin{array}{l}\text { Spray/ } \\
\text { additives } \\
\text { strategy }\end{array}$} & $\begin{array}{l}\text { Fission products } \\
\text { in containment }\end{array}$ & None & & None & \\
\hline & & & Conta inment & Radiation & \\
\hline & & & radiation & monitoring & \\
\hline & & & leve 1 & system & \\
\hline & Strategy Initiation & & & & \\
\hline & Flow in spray system & Spray flow & & Spray flow & \\
\hline & Strategy Effectiveness & & & & \\
\hline & $\begin{array}{l}\text { Decreased containment } \\
\text { radiation level }\end{array}$ & None & & None & \\
\hline & & & $\begin{array}{l}\text { Containment } \\
\text { radiation level }\end{array}$ & $\begin{array}{l}\text { Radiation } \\
\text { monitoring } \\
\text { system }\end{array}$ & \\
\hline & Selection Criteria & & & & \\
\hline \multirow[t]{4}{*}{$\begin{array}{l}\text { Filter system } \\
\text { strategy }\end{array}$} & $\begin{array}{l}\text { Fission product } \\
\text { in containment }\end{array}$ & None & & None & \\
\hline & atmosphere & & $\begin{array}{l}\text { Containment } \\
\text { radiation level }\end{array}$ & $\begin{array}{l}\text { Radiation } \\
\text { monitoring system }\end{array}$ & \\
\hline & Strategy Initiation & & & & \\
\hline & $\begin{array}{l}\text { Activation of filter } \\
\text { fans }\end{array}$ & $\begin{array}{l}\text { Filter fan } \\
\text { system status }\end{array}$ & & Fan status & \\
\hline
\end{tabular}


TABLE A3.1. (continued)

\begin{tabular}{|c|c|c|c|c|c|}
\hline & Informat ion Needs & $\begin{array}{c}\text { Direct } \\
\text { Information } \\
\text { Source } \\
\end{array}$ & $\begin{array}{c}\text { Indirect } \\
\text { Information } \\
\text { Source } \\
\end{array}$ & $\begin{array}{l}\text { Ava i lable } \\
\text { Instruments }\end{array}$ & $\begin{array}{r}\text { Potent ia } 1 \\
\text { Instruments } \\
\end{array}$ \\
\hline & $\begin{array}{l}\text { Strategy Effectiveness } \\
\text { Decreased containment } \\
\text { radiation level }\end{array}$ & & $\begin{array}{l}\text { Containment } \\
\text { radiation level }\end{array}$ & $\begin{array}{l}\text { Radiation monitoring } \\
\text { system }\end{array}$ & \\
\hline $\begin{array}{l}\text { Chemical } \\
\text { reaction } \\
\text { strategy }\end{array}$ & $\begin{array}{l}\text { Selection Criteria } \\
\text { None-passive } \\
\text { system }\end{array}$ & & & & \\
\hline & $\begin{array}{c}\text { Strategy Initiation } \\
\text { None-passive } \\
\text { system }\end{array}$ & & & & \\
\hline & Strategy Effectiveness & & & & \\
\hline & $\begin{array}{l}\text { Low containment } \\
\text { radiation level }\end{array}$ & None & & None & \\
\hline & & & $\begin{array}{l}\text { Conta inment } \\
\text { radiation leve } 1\end{array}$ & $\begin{array}{l}\text { Radiation } \\
\text { monitoring } \\
\text { system }\end{array}$ & \\
\hline
\end{tabular}


TABLE A3.2. MITIGATE FISSION PRODUCT RELEASE FROM CONTAINMENT (F)--GASEOUS DISPERSION MECHANISM (F1A2)

\begin{tabular}{|c|c|c|c|c|c|}
\hline & Information Needs & $\begin{array}{c}\text { Direct } \\
\text { Information } \\
\text { Source }\end{array}$ & $\begin{array}{c}\text { Indirect } \\
\text { Information } \\
\text { Source } \\
\end{array}$ & $\begin{array}{c}\text { Available } \\
\text { Instruments }\end{array}$ & $\begin{array}{r}\text { Potential } \\
\text { Instruments } \\
\end{array}$ \\
\hline \multirow[t]{2}{*}{$\begin{array}{l}\text { Maintain } \\
\text { control of } \\
\text { fission product } \\
\text { inventory } \\
\text { in containment } \\
\text { atmosphere } \\
\text { safety } \\
\text { function (F1) }\end{array}$} & $\begin{array}{l}\text { Presence of fission } \\
\text { products in } \\
\text { containment } \\
\text { atmosphere }\end{array}$ & $\begin{array}{l}\text { Containment } \\
\text { radiation } \\
\text { level }\end{array}$ & & $\begin{array}{l}\text { Radiation (CONT) } \\
\text { monitoring } \\
\text { system, } \\
\text { noble gas } \\
\text { monitoring } \\
\text { system }\end{array}$ & \\
\hline & Indicators & & & & \\
\hline \multirow[t]{6}{*}{$\begin{array}{l}\text { Gaseous } \\
\text { dispersion } \\
\text { mechanism } \\
(\text { F1A2) }\end{array}$} & $\begin{array}{l}\text { Presence of fission } \\
\text { product gases in } \\
\text { containment } \\
\text { atmosphere }\end{array}$ & None & $\begin{array}{l}\text { Containment } \\
\text { radiation levels }\end{array}$ & $\begin{array}{l}\text { None } \\
\text { Radiation } \\
\text { monitoring } \\
\text { system }\end{array}$ & \\
\hline & Precursors & & & $\begin{array}{l}\text { Noble gas } \\
\text { monitoring system }\end{array}$ & \\
\hline & $\begin{array}{l}\text { Core damage } \\
\text { status }\end{array}$ & None & & None & \\
\hline & & & $\begin{array}{l}\text { RCS coolant } \\
\text { activity }\end{array}$ & Sampling system & \\
\hline & & & $\begin{array}{l}\text { Core exit } \\
\text { temperature } \\
\text { history }\end{array}$ & $\begin{array}{l}\text { Core exit } \\
\text { thermocoup les }\end{array}$ & . \\
\hline & & & $\begin{array}{l}\text { Reactor vesse } 1 \\
\text { inventory }\end{array}$ & RVLMS & \\
\hline
\end{tabular}


TABLE A3.2. (cont inued)

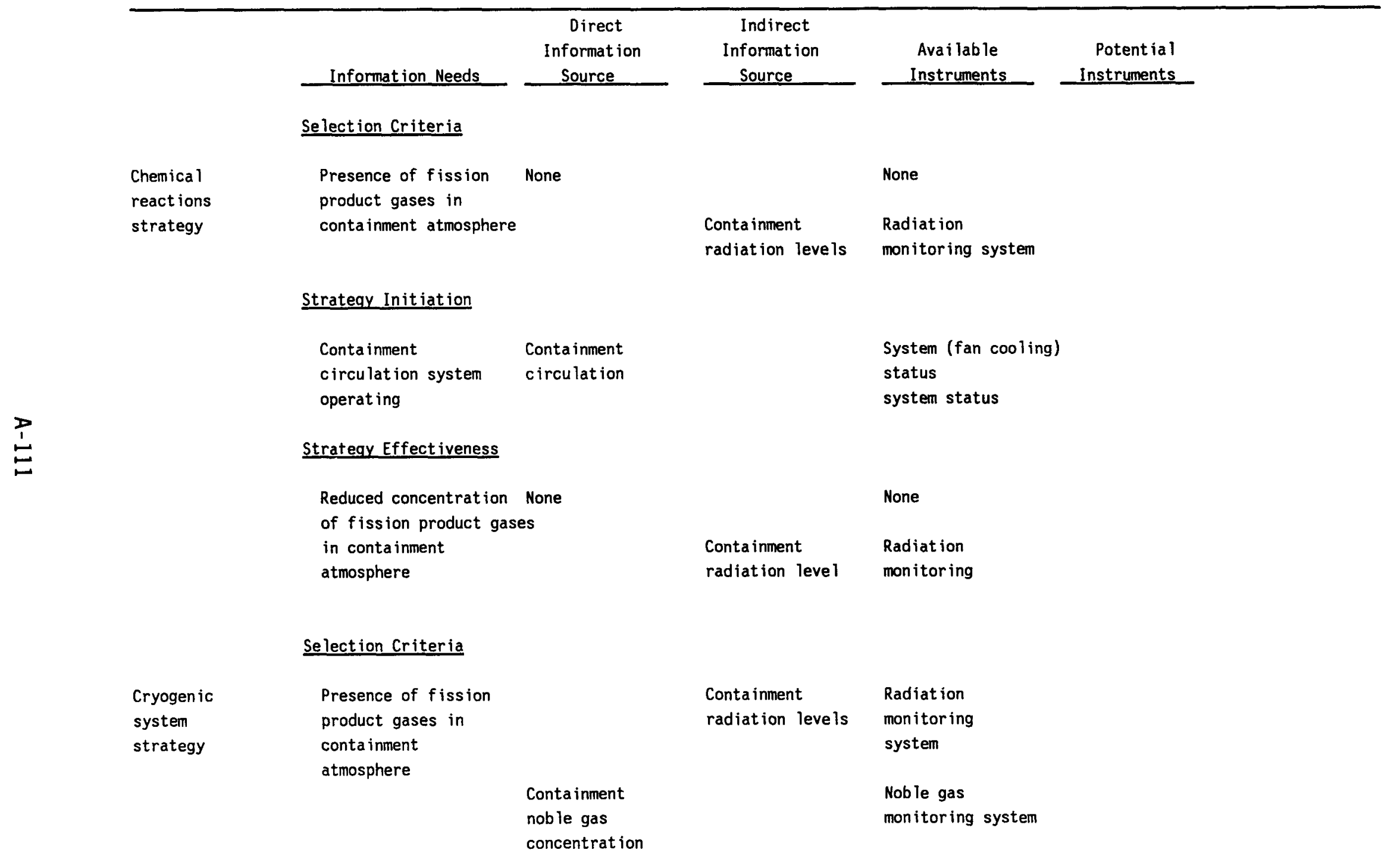


TABLE A3.2. (cont inued)

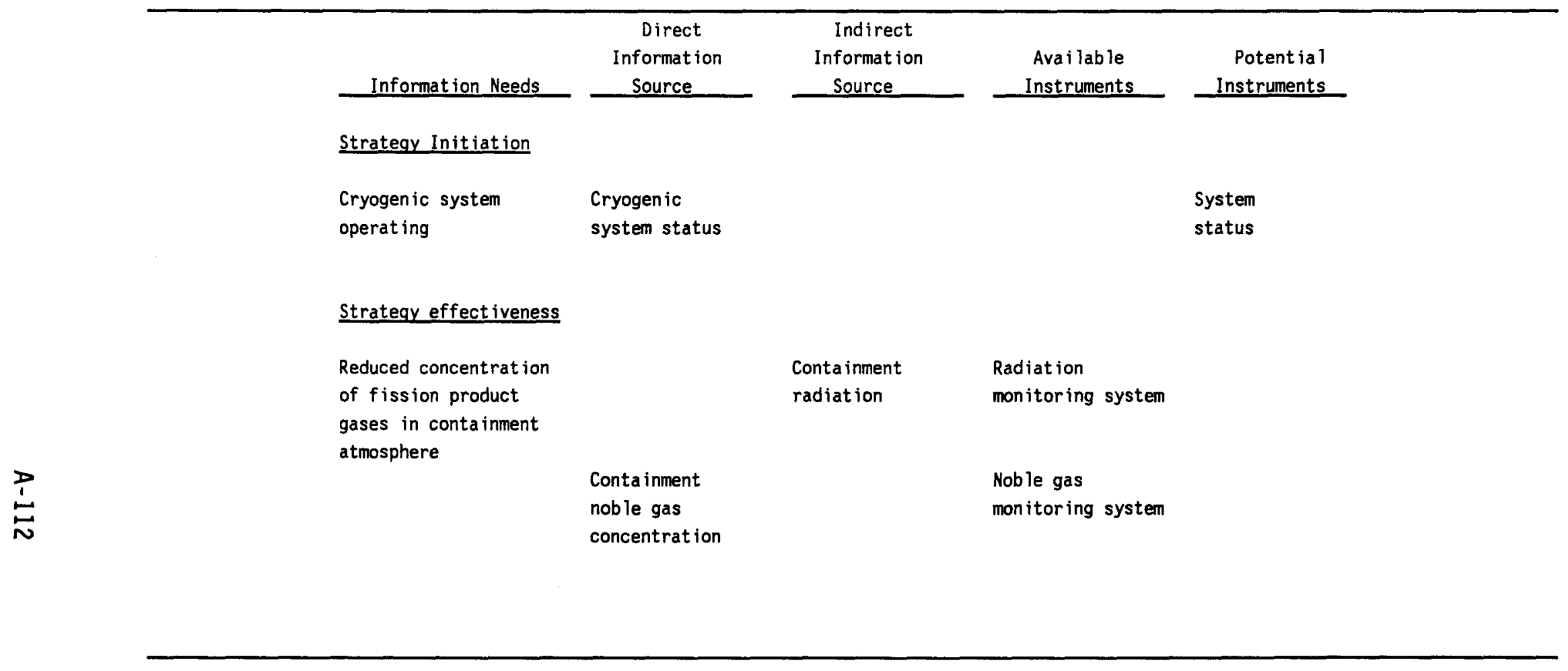


TABLE A3.3. MITIGATE FISSION PRODUCT RELEASE (F)--pH TO0-LOW MECHANISM (F2A1)

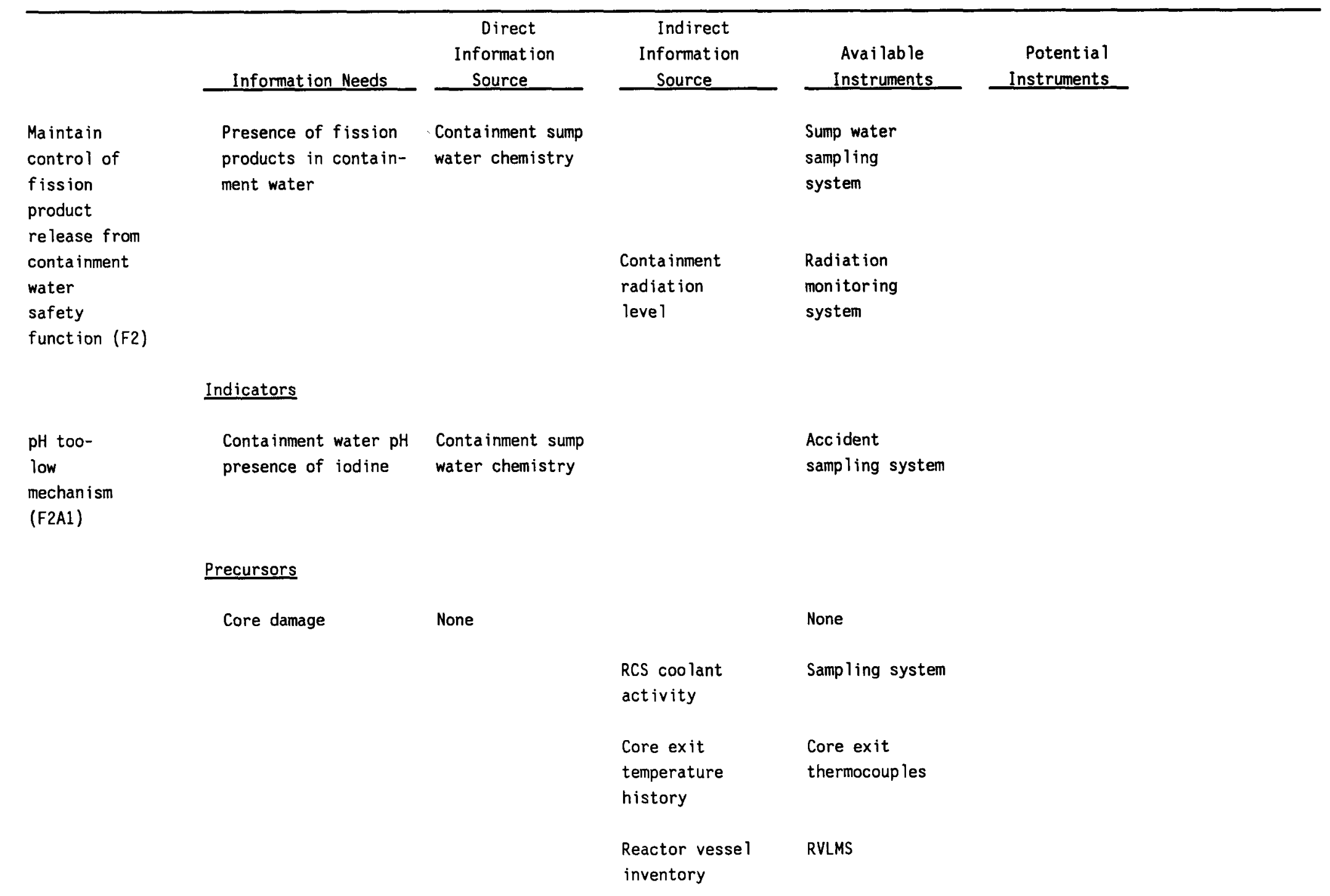


TABLE A3.3. (cont inued)

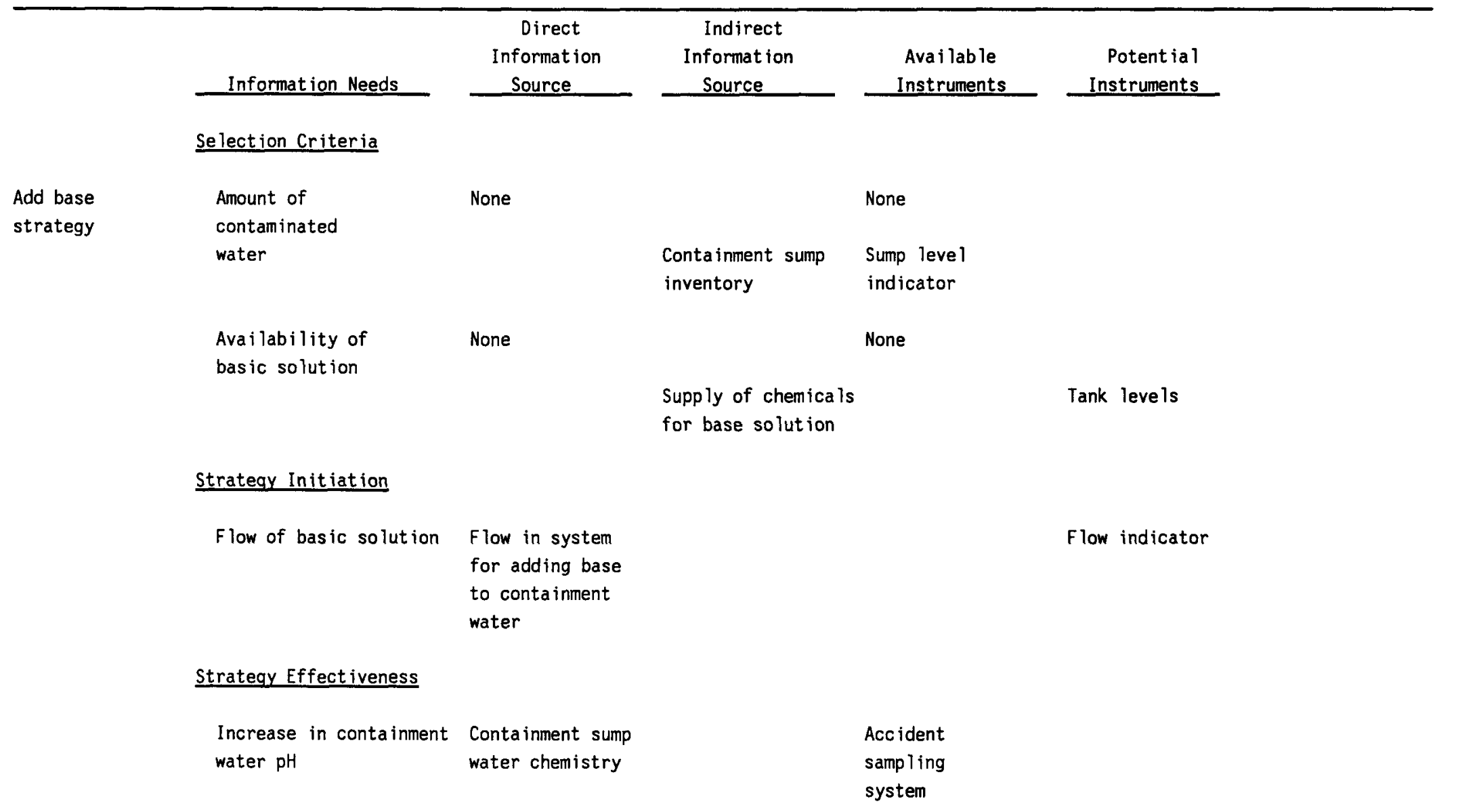


TABLE A3.3. (continued)

\begin{tabular}{|c|c|c|c|c|c|}
\hline & Information Needs & $\begin{array}{c}\text { Direct } \\
\text { Information } \\
\text { Source } \\
\end{array}$ & $\begin{array}{c}\text { Indirect } \\
\text { Information } \\
\text { Source } \\
\end{array}$ & $\begin{array}{c}\text { Available } \\
\text { Instruments }\end{array}$ & $\begin{array}{r}\text { Potential } \\
\text { Instruments } \\
\end{array}$ \\
\hline & Selection Criteria & & & & \\
\hline \multirow[t]{7}{*}{$\begin{array}{l}\text { Dilution } \\
\text { strategy }\end{array}$} & $\begin{array}{l}\text { Amount of } \\
\text { contaminated }\end{array}$ & None & & None & \\
\hline & water & & $\begin{array}{l}\text { Containment sump } \\
\text { inventory }\end{array}$ & $\begin{array}{l}\text { Sump level } \\
\text { indicator }\end{array}$ & \\
\hline & $\begin{array}{l}\text { Inventory of dilution } \\
\text { liquid }\end{array}$ & $\begin{array}{l}\text { Dilution system } \\
\text { tank leve } 1\end{array}$ & & & Level indicator \\
\hline & Strategy Initiation & & & & \\
\hline & $\begin{array}{l}\text { Flow of dilution } \\
\text { liquid to conta inment } \\
\text { waste }\end{array}$ & $\begin{array}{l}\text { Flow in dilution } \\
\text { system }\end{array}$ & & & Flow indicator \\
\hline & Strategy Effectiveness & & & & \\
\hline & $\begin{array}{l}\text { Increase in containment } \\
\text { water } \mathrm{pH}\end{array}$ & $\begin{array}{l}\text { Containment sump } \\
\text { water chemistry }\end{array}$ & & $\begin{array}{l}\text { Accident } \\
\text { sampling system }\end{array}$ & \\
\hline
\end{tabular}


TABLE A3.4. MITIGATE FISSION PRODUCT RELEASE FROM CONTAINMENT (F)--RIOLYSIS MECHANISM (F23A2)

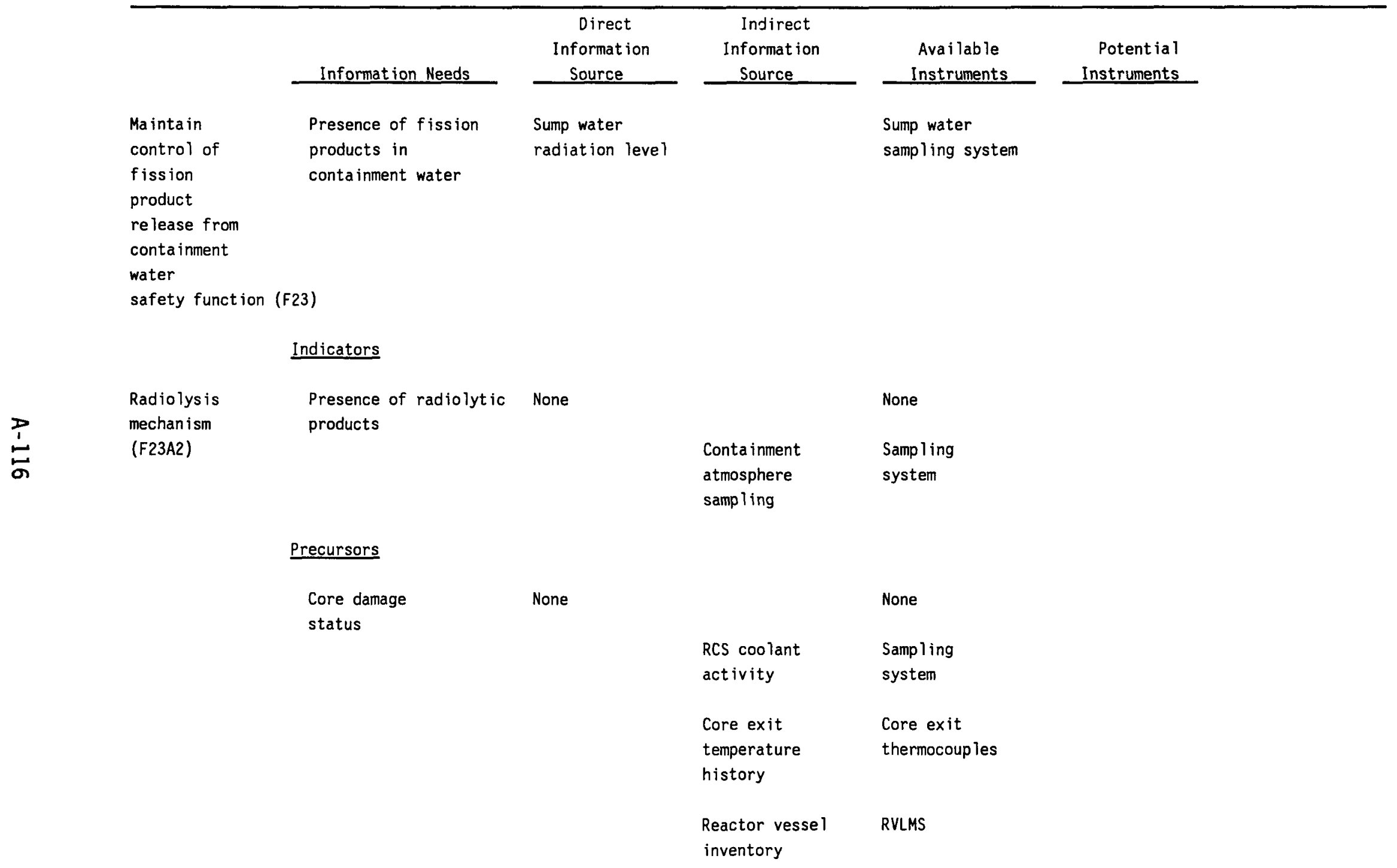


TABLE A3.4. (continued)

\begin{tabular}{|c|c|c|c|c|c|}
\hline & Information Needs & $\begin{array}{c}\text { Direct } \\
\text { Information } \\
\text { Source }\end{array}$ & $\begin{array}{c}\text { Indirect } \\
\text { Information } \\
\text { Source } \\
\end{array}$ & $\begin{array}{c}\text { Ava lable } \\
\text { Instruments }\end{array}$ & $\begin{array}{r}\text { Potential } \\
\text { Instruments } \\
\end{array}$ \\
\hline & Precursors (continued) & & & & \\
\hline & $\begin{array}{l}\text { Water available } \\
\text { for radiolysis }\end{array}$ & None & & None & \\
\hline & & & $\begin{array}{l}\text { Reactor vesse } 1 \\
\text { leve } 1\end{array}$ & RVLMS & \\
\hline & & & Cavity inventory & None & \\
\hline & Selection Criteria & & & & \\
\hline $\begin{array}{l}\text { Dilution } \\
\text { strategy }\end{array}$ & $\begin{array}{l}\text { Availability of } \\
\text { dilution water }\end{array}$ & $\begin{array}{l}\text { Dilution system } \\
\text { tank level }\end{array}$ & & & $\begin{array}{l}\text { Tank level } \\
\text { Indicators }\end{array}$ \\
\hline & Strategy Initiation & & & & \\
\hline & $\begin{array}{l}\text { Flow of water to } \\
\text { damaged core }\end{array}$ & $\begin{array}{l}\text { Injection pump } \\
\text { flow }\end{array}$ & & & Flow indicator \\
\hline & Strategy Effectiveness & & & & \\
\hline & Water level above core & $\begin{array}{l}\text { Reactor vesse] } \\
\text { inventory }\end{array}$ & & RVLMS & \\
\hline
\end{tabular}


TABLE A3.5. MITIGATE FISSION PRODUCT RELEASE FROM CONTAINMENT (F)--EXCESSIVE WATER TEMPERATURE MECHANISM (F2A3)

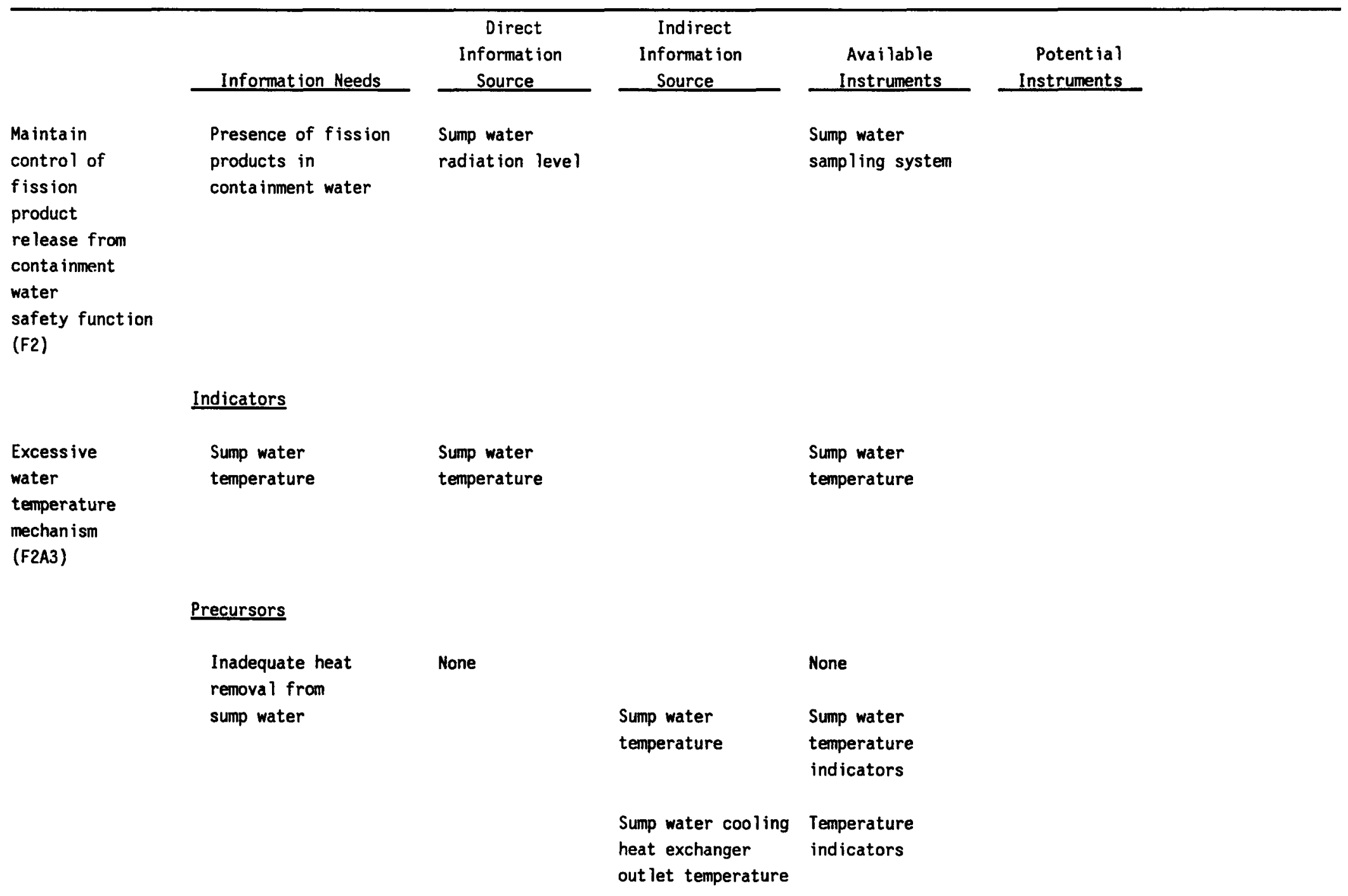


TABLE A3.5. (cont inued)

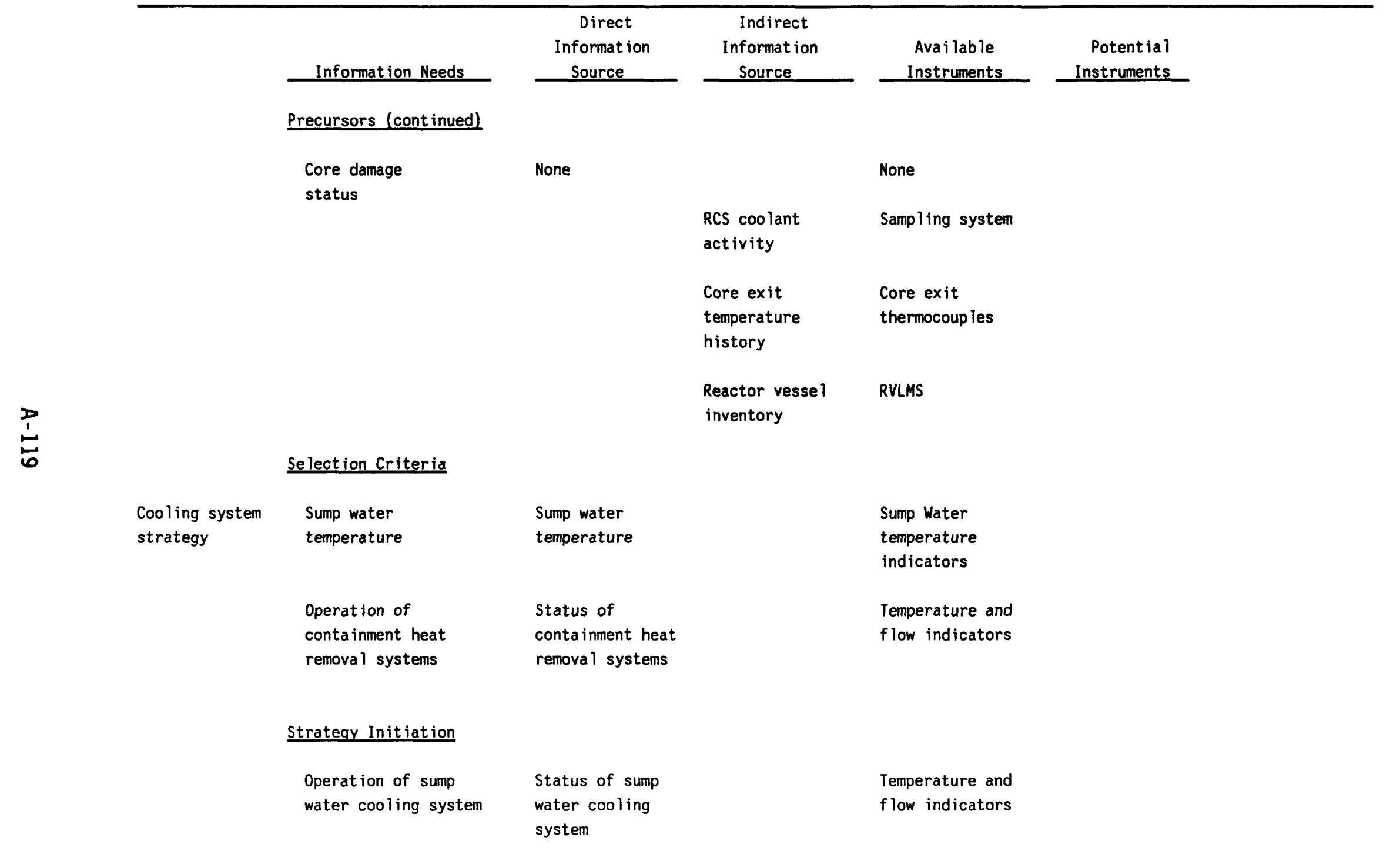


TABLE A3.5. (cont inued)

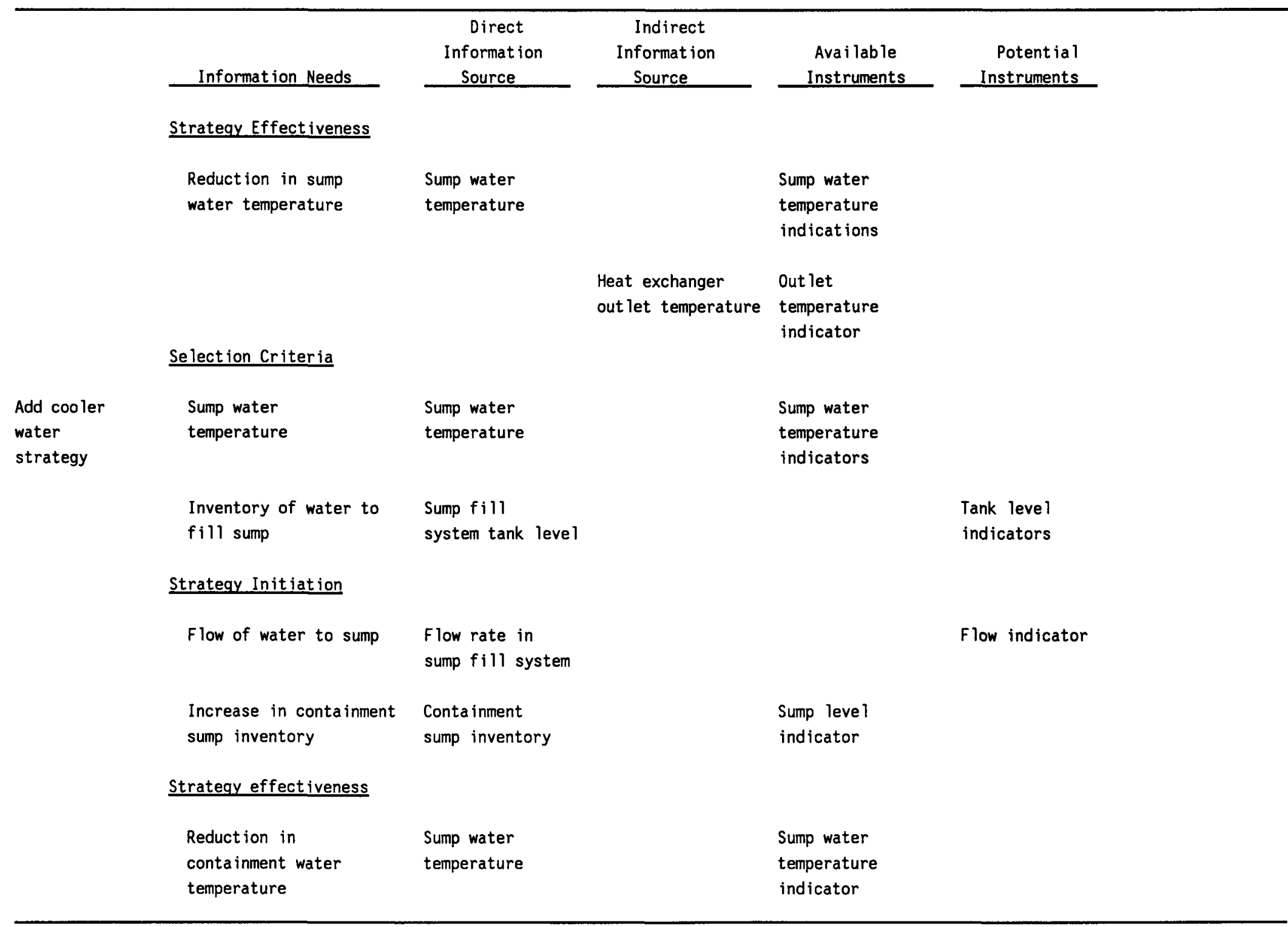


APPENDIX B

PLANT-SPECIFIC MEASUREMENTS COMPARED TO REGULATORY GUIDE 1.97

B- $-1 / b-2$ 


\section{APPENDIX B \\ PLANT-SPECIFIC MEASUREMENTS COMPARED TO REGULATORY GUIDE 1.97}

The tables contained in Appendix B were developed to summarize the availability of plant-specific measurements for accident management and the requirements for their qualification. Tables B1, B2, and B3 list the existing measurements according to that contained in Regulatory Guide 1.97. Regulatory Guide 1.97 specifies a method acceptable to the NRC staff for providing instrumentation to monitor plant variables during and following an accident in a lightwater-cooled nuclear power plant. Regulatory Guide 1.97 specifies three different categories of qualification:

Category 1 provides for full qualification, redundancy, and continuous real-time display and requires onsite (standby) power. Category 2 provides for qualification but is less stringent in that is does not (of itself) include seismic qualification, redundancy, or continuous display and requires only a high-reliability power source (not necessarily standby power). Category 3 is the least stringent. It provides for high-quality commercial-grade equipment that requires only offsite power.
Table B1 in Appendix B lists those existing measurements from Category 1 as specified in Regulatory Guide 1.97. Similarly, Tables B2 and B3 list existing measurements from Category 2 and Category 3, respectively. These tables represent a Combustion Engineering 2700 Mwt plant such as Calvert Cliffs Units 1 and 2 or Millstone Unit 2. Columns are provided in these tables to list information regarding current requirements for environmental qualification for the specific measurement, and the location of the sensor (in the reactor, auxiliary building, containment, turbine building, etc.). It is important to note that representative containment harsh environments include a maximum pressure of $60 \mathrm{psia}$ and a maximum temperature of $300^{\circ} \mathrm{F}$ and are generally associated with the design basis loss-of-coolant accident. Some instruments are required for other design basis (DB) accidents which do not include harsh environments. To identify the two potential environments, these requirements are denoted as DBA Harsh and DBA non-Harsh in the table. This information will be used to determine the survivability of the instruments in the environment that can be expected during a severe accident sequence. 
TABLE B1. PLANT-SPECIFIC MEASUREMENTS COMPARED TO REgULATORY GUIDE 1.97--CATEGORY 1

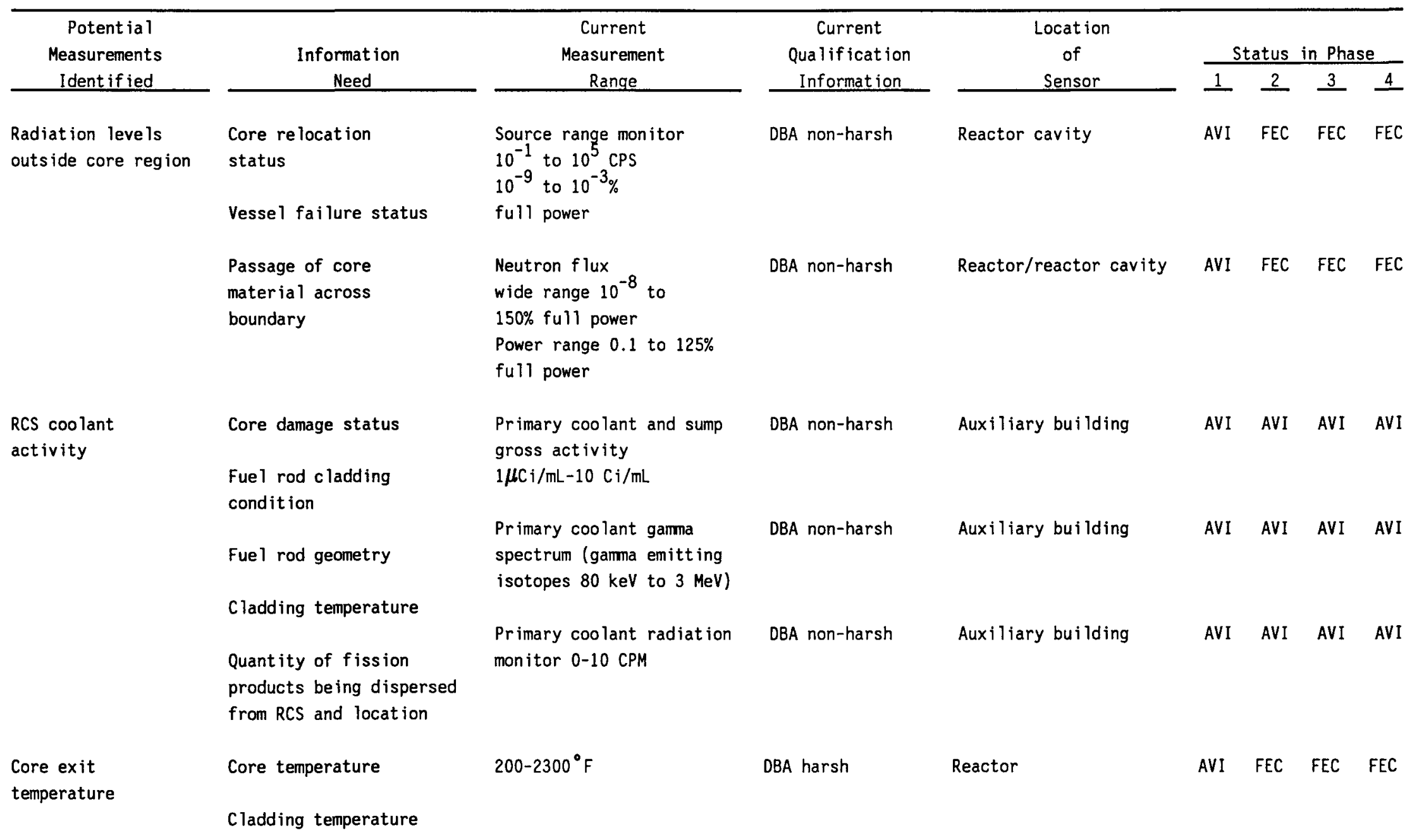

\section{Core damage status}


TABLE B1. (continued)

\begin{tabular}{|c|c|c|c|c|c|c|c|c|}
\hline $\begin{array}{c}\text { Potential } \\
\text { Measurements }\end{array}$ & Information & $\begin{array}{c}\text { Current } \\
\text { Measurement }\end{array}$ & $\begin{array}{c}\text { Current } \\
\text { Qualification }\end{array}$ & $\begin{array}{l}\text { Location } \\
\text { of }\end{array}$ & \multicolumn{4}{|c|}{ Status in Phase } \\
\hline Ident ified & Need & Range & Information & Sensor & 1 & 2 & 3 & 4 \\
\hline & $\begin{array}{l}\text { Cladding oxidation } \\
\text { status }\end{array}$ & & & & & & & \\
\hline & $\begin{array}{l}\text { Fuel rod cladding } \\
\text { condition }\end{array}$ & & & & & & & \\
\hline $\begin{array}{l}\text { Reactor vessel } \\
\text { inventory }\end{array}$ & Water level above core & $\begin{array}{l}0-100 \% \text { level above core } \\
\text { (RVLMS) }\end{array}$ & DBA harsh & Reactor & AVI & FEC & FEC & FEC \\
\hline & RCS inventory & & & & & & & \\
\hline & $\begin{array}{l}\text { RCS leak integrity } \\
\text { (status) }\end{array}$ & & & & & & & \\
\hline & Core damage status & & & & & & & \\
\hline & Cladding temperature & & & & & & & \\
\hline & Core temperature & & & & & & & \\
\hline & $\begin{array}{l}\text { Water available for } \\
\text { radiolysis }\end{array}$ & & & & & & & \\
\hline $\begin{array}{l}\text { Pressurizer } \\
\text { inventory }\end{array}$ & RCS inventory & $\begin{array}{l}0-360 \text { in. } \\
0-100 \% \\
\text { pressurizer level }\end{array}$ & DBA harsh & Conta inment and RCS & AVI & FEC & FEC & $\begin{array}{l}\text { FEC } \\
\text { (T\&P) }\end{array}$ \\
\hline & $\begin{array}{l}\text { RCS leak integrity } \\
\text { (status) }\end{array}$ & & & & & & & \\
\hline $\begin{array}{l}\text { Coolant hydrogen } \\
\text { concentration }\end{array}$ & Cladding oxidation & $\begin{array}{l}\text { Primary coolant and sump } \\
\text { Dissolved hydrogen: Post- } \\
\text { accident RCS } 0-10 \% \text { volume; } \\
\text { Post-accident sump } 0-100 \%\end{array}$ & DBA harsh & Auxiliary building & AVI & AVI & AVI & AVI \\
\hline
\end{tabular}


TABLE B1. (cont inued)

\begin{tabular}{|c|c|c|c|c|c|c|c|c|}
\hline $\begin{array}{l}\text { Potential } \\
\text { Measurements }\end{array}$ & Information & $\begin{array}{l}\text { Current } \\
\text { Measurement }\end{array}$ & $\begin{array}{c}\text { Current } \\
\text { Qualification }\end{array}$ & $\begin{array}{l}\text { Location } \\
\text { of }\end{array}$ & & atus & $n$ Phas & \\
\hline Identified & Need & Range & Information & Sensor & 1 & 2 & 3 & 4 \\
\hline \multirow[t]{11}{*}{ RCS pressure } & RCS pressure & $0-4000$ psig & DBA harsh & Containment and RCS & AVI & FEC & FEC & $\begin{array}{l}\text { FEC } \\
(T \& P)\end{array}$ \\
\hline & PORV flow conf irmation & & & & & & & \\
\hline & $\begin{array}{l}\text { Rate of RCS } \\
\text { depressurization }\end{array}$ & & & & & & & \\
\hline & Relationship to & & & & & & & \\
\hline & $\begin{array}{l}\text { Core relocation } \\
\text { status }\end{array}$ & & & & & & & \\
\hline & Vesse 1 integrity & & & & & & & \\
\hline & RCS leak integrity & & & & & & & \\
\hline & Approach to vessel failure & & & & & & & \\
\hline & $\begin{array}{l}\text { RCS boundary } \\
\text { failure and fission } \\
\text { product release }\end{array}$ & & & & & & & \\
\hline & $\begin{array}{l}\text { Lower head failure and } \\
\text { fission product release }\end{array}$ & & & & & & & \\
\hline & $\begin{array}{l}\text { RCS pressure greater } \\
\text { than ruptured generator }\end{array}$ & & & & & & & \\
\hline
\end{tabular}


TABLE B1. (cont inued)

\begin{tabular}{|c|c|c|c|c|c|c|c|c|}
\hline $\begin{array}{l}\text { Potential } \\
\text { Measurements }\end{array}$ & Information & $\begin{array}{l}\text { Current } \\
\text { Measurement }\end{array}$ & $\begin{array}{c}\text { Current } \\
\text { Qualification }\end{array}$ & $\begin{array}{l}\text { Location } \\
\text { of }\end{array}$ & \multicolumn{3}{|c|}{ Status in Phase } & \\
\hline Identified & Need & Range & Information & Sensor & 1 & 2 & 3 & 4 \\
\hline & $\begin{array}{l}\text { Containment bypass } \\
\text { and fission product } \\
\text { release } \\
\text { Stabilized RCS conditions } \\
\text { (V-sequence isolation) } \\
\text { RCS cooldown with } \\
\text { steam generators }\end{array}$ & & & & & & & \\
\hline $\begin{array}{l}\text { Containment } \\
\text { isolation valve }\end{array}$ & $\begin{array}{l}\text { Containment isolation } \\
\text { valve position status }\end{array}$ & $\begin{array}{l}\text { Isolation valve position } \\
\text { closed-open }\end{array}$ & DBA harsh & $\begin{array}{l}\text { Containment-auxiliary } \\
\text { building }\end{array}$ & AVI & AVI & AVI & FEC \\
\hline & $\begin{array}{l}\text { Conta inment isolation } \\
\text { valves closed }\end{array}$ & & & & & & & \\
\hline & Reisolation not possible & & & & & & & \\
\hline & $\begin{array}{l}\text { Closure of isolation valve } \\
\text { (V-sequence isolation) }\end{array}$ & & & & & & & \\
\hline & $\begin{array}{l}\text { Isolation failure and } \\
\text { fission product release }\end{array}$ & & & & & & & \\
\hline $\begin{array}{l}\text { Conta inment sump } \\
\text { inventory }\end{array}$ & $\begin{array}{l}\text { Injection water } \\
\text { availability }\end{array}$ & $\begin{array}{l}\text { Narrow range } 0-30 \mathrm{in.} \\
\text { Wide range } 0-120 \mathrm{in.} \\
\text { (Containment floor } \\
+9 \mathrm{in} .-600.000 \text { gallon } \\
\text { level }+4 \mathrm{ft} \text { ) }\end{array}$ & $\begin{array}{l}\text { Narrow range } \\
\text { not qual if ied } \\
\text { DBA harsh }\end{array}$ & Conta inment & AVI & AVI & AVI & FEC \\
\hline
\end{tabular}


TABLE B1. (cont inued)

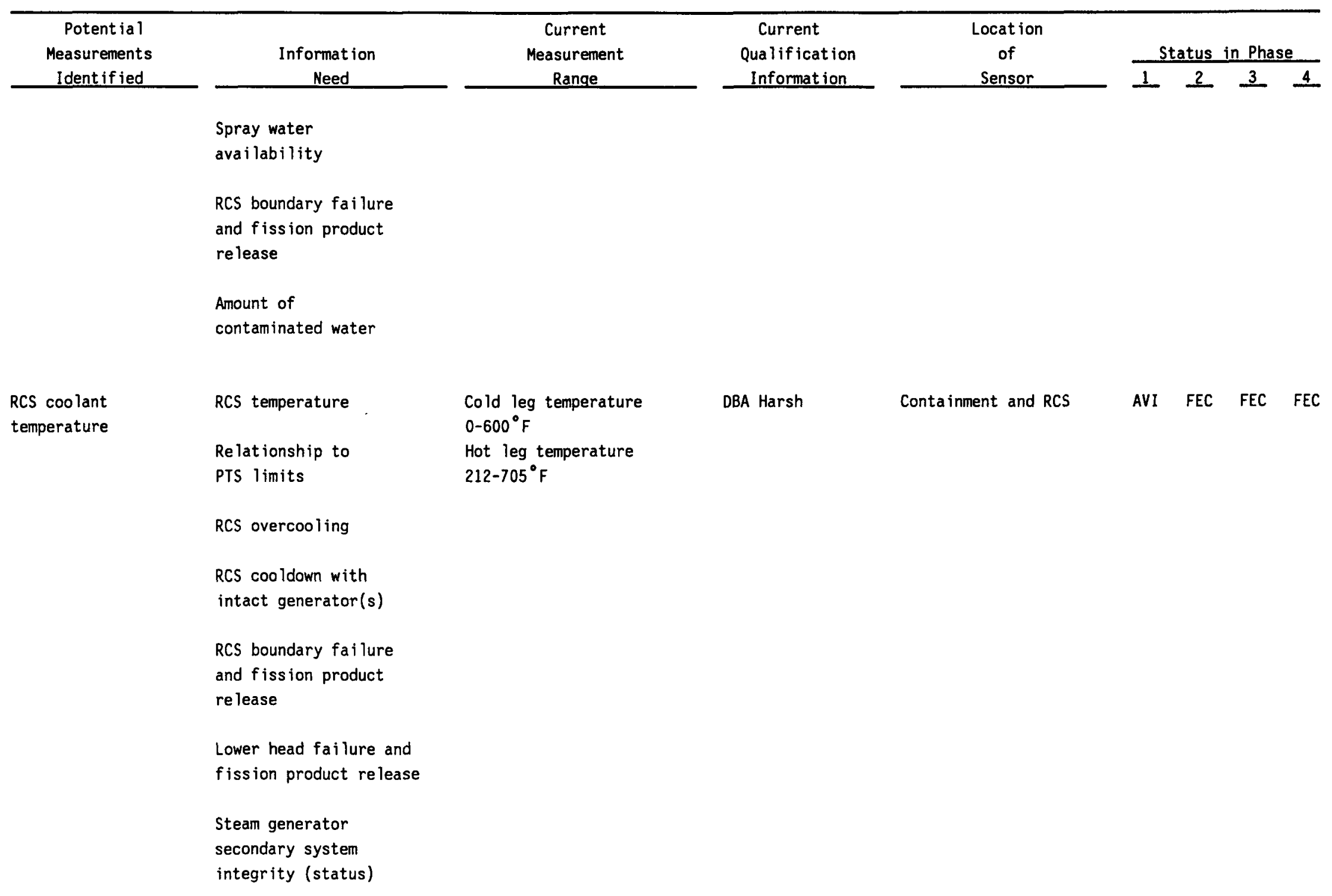


TABLE B1. (cont inued)

\begin{tabular}{|c|c|c|c|c|c|c|c|c|}
\hline $\begin{array}{c}\text { Potential } \\
\text { Measurements }\end{array}$ & Information & $\begin{array}{c}\text { Current } \\
\text { Measurement }\end{array}$ & $\begin{array}{l}\text { Current } \\
\text { Qualification }\end{array}$ & $\begin{array}{l}\text { Location } \\
\text { of }\end{array}$ & & \multicolumn{3}{|c|}{ Status in Phase } \\
\hline Identified & Need & Range & Information & Sensor & 1 & 2 & 3 & 4 \\
\hline Cold leg temperature & Core relocation status & $0-600^{\circ} \mathrm{F}$ & DBA harsh & Containment and RCS & AVI & FEC & FEC & FEC \\
\hline \multirow[t]{3}{*}{$\begin{array}{l}\text { Steam generator } \\
\text { secondary level }\end{array}$} & $\begin{array}{l}\text { Steam generator } \\
\text { inventory }\end{array}$ & $\begin{array}{l}\text { Tubesheet to separators } \\
-401 \text { in. to }+63.5 \text { in. } \\
\text { top of steam separators }\end{array}$ & DBA harsh & Containment & AVI & AVI & AVI & FEC \\
\hline & $\begin{array}{l}\text { Steam generator } \\
\text { secondary system } \\
\text { integrity (status) }\end{array}$ & $\begin{array}{l}=+391 / 4 \text { in; } \\
\text { Tubesheet is at } 44411 / 16 \\
\text { be low norma l operating } \\
\text { leve? }\end{array}$ & n. & & & & & \\
\hline & $\begin{array}{l}\text { Steam generator heat } \\
\text { removal capability }\end{array}$ & & & & & & & \\
\hline \multirow[t]{2}{*}{$\begin{array}{l}\text { Feedwater tank } \\
\text { inventory }\end{array}$} & $\begin{array}{l}\text { Feedwater source } \\
\text { availability }\end{array}$ & $\begin{array}{l}\text { Condensate storage tank } \\
\text { leve 1, } 1 \text { to } 38 \mathrm{ft} \text { (top) }\end{array}$ & DBA harsh & Yard & AVI & AVI & AVI & AVI \\
\hline & $\begin{array}{l}\text { Stean generator heat } \\
\text { removal capability }\end{array}$ & & & & & & & \\
\hline \multirow[t]{3}{*}{ Containment pressure } & Conta inment pressure & -5 to $150 \mathrm{psig}$ & DBA Harsh & $\begin{array}{l}\text { Auxiliary building } \\
\text { and conta inment }\end{array}$ & AVI & AVI & AVI & AVI \\
\hline & Containment integrity & & & & & & & \\
\hline & $\begin{array}{l}\text { Overpressure } \\
\text { control status } \\
\text { Penetration of } \\
\text { containment by a } \\
\text { missile }\end{array}$ & & & & \multicolumn{4}{|c|}{$\begin{array}{l}\text { Assumes pressure tap } \\
\text { in containment does } \\
\text { not fail due to } \\
\text { containment temperature }\end{array}$} \\
\hline
\end{tabular}


TABLE B1. (cont inued)

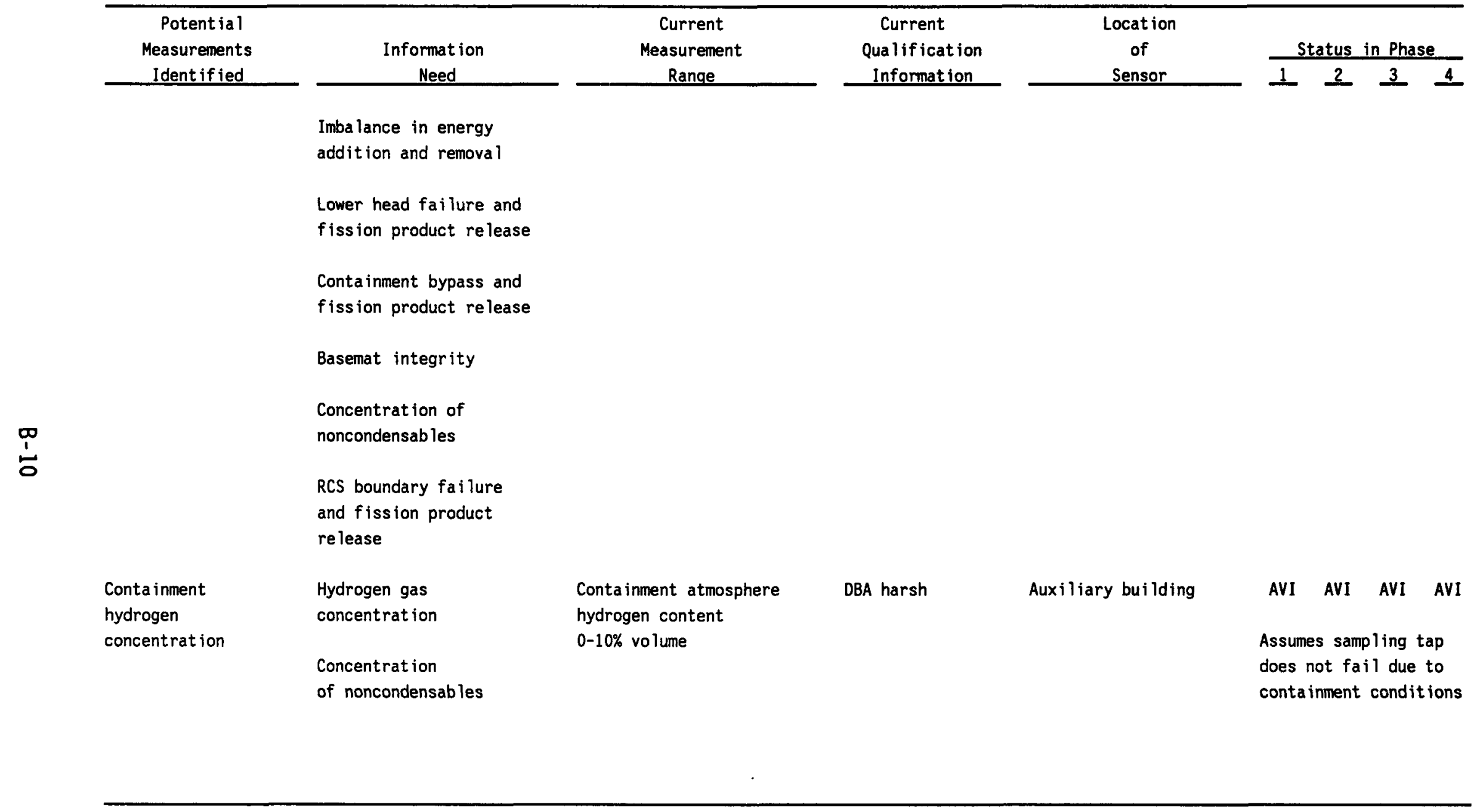


TABLE B2. PLANT-SPECIFIC MEASUREMENTS COMPARED TO REGULATORY GUIDE 1.97--CATEGORY 2

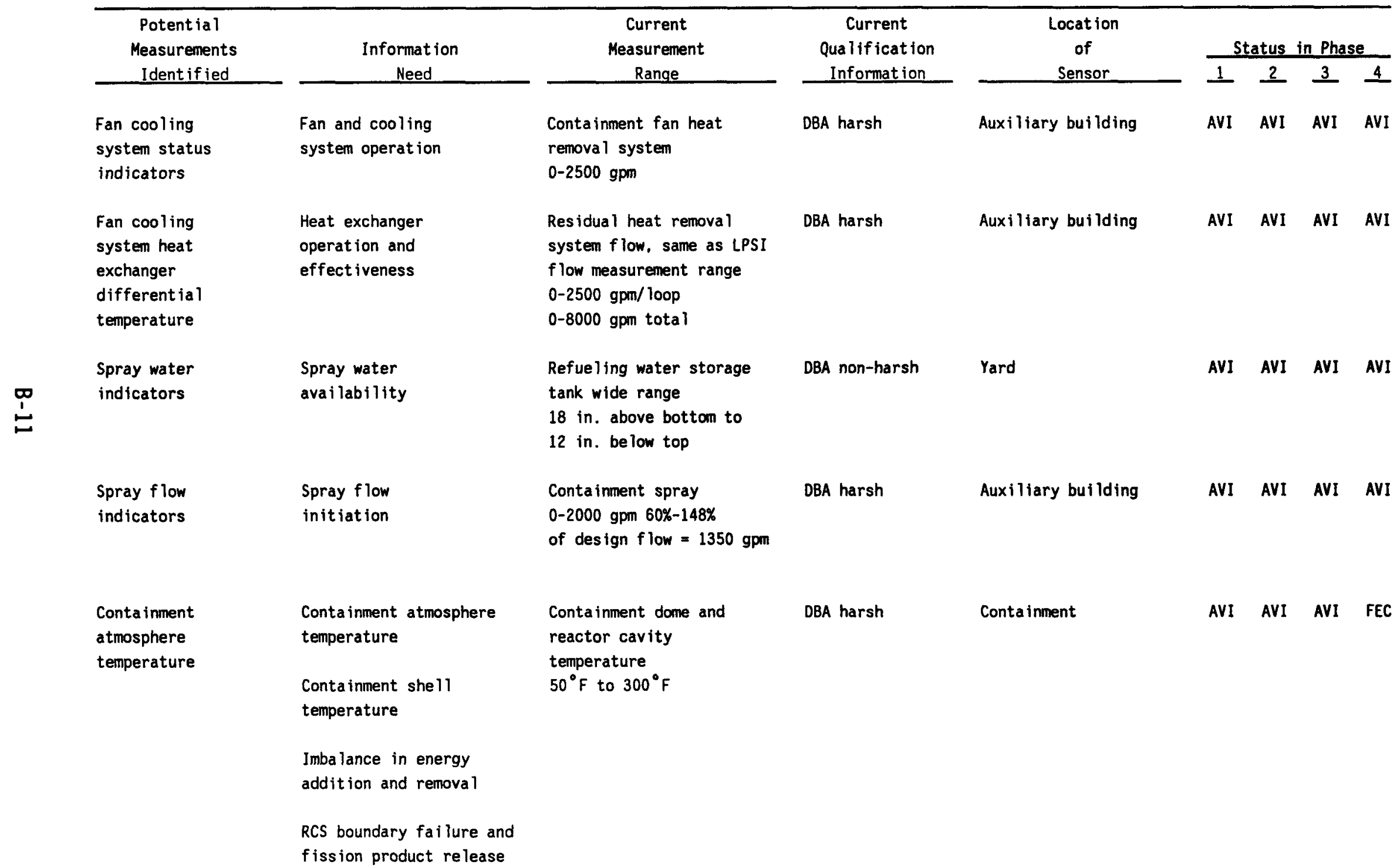


TABLE B2. (cont inued)

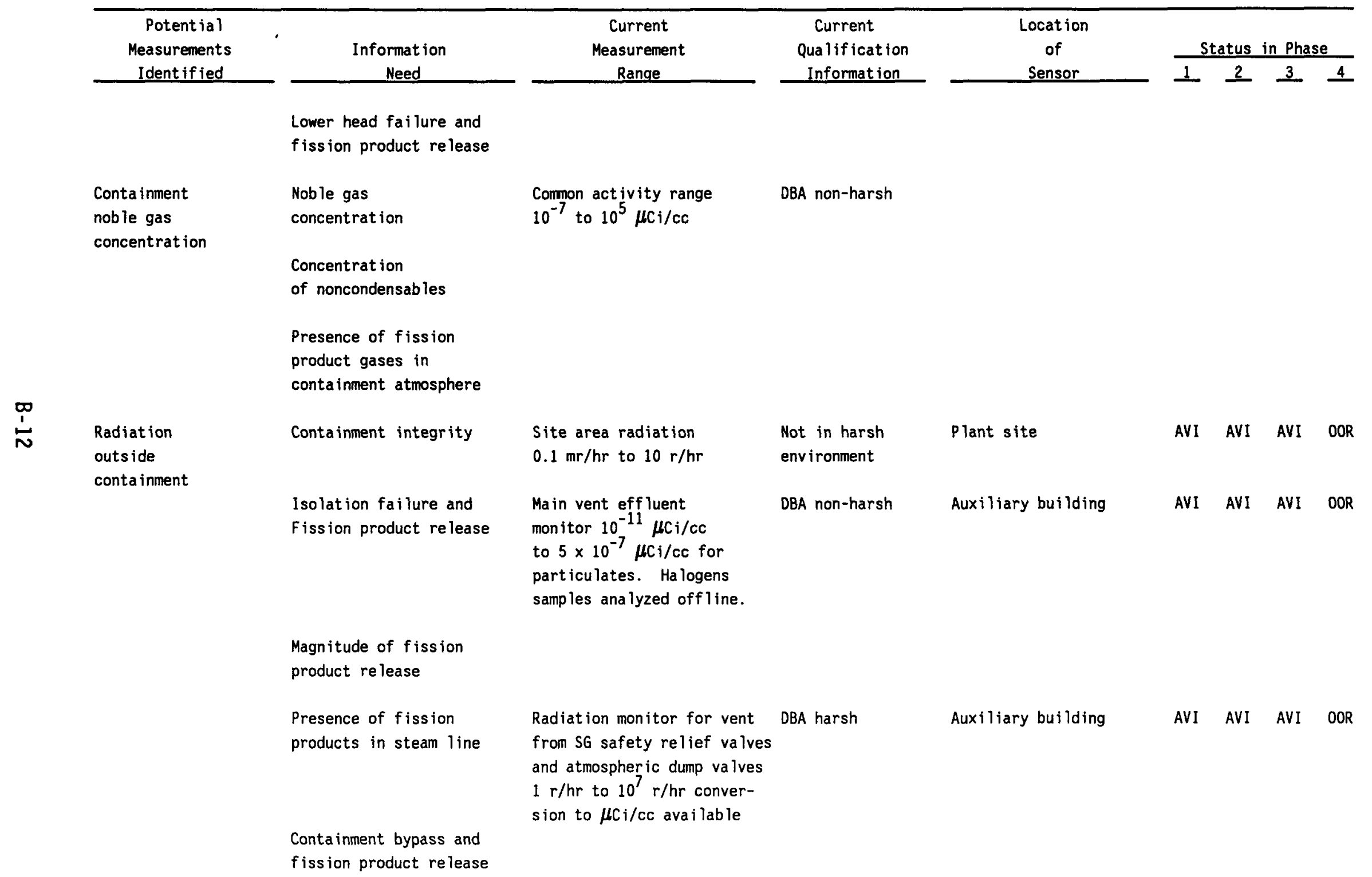


TABLE B2. (cont inued)

\begin{tabular}{|c|c|c|c|c|c|c|c|c|}
\hline $\begin{array}{c}\text { Potential } \\
\text { Measurements }\end{array}$ & Information & $\begin{array}{c}\text { Current } \\
\text { Measurement }\end{array}$ & $\begin{array}{c}\text { Current } \\
\text { Qualification }\end{array}$ & $\begin{array}{l}\text { Location } \\
\text { of }\end{array}$ & \multicolumn{4}{|c|}{ Status in Phase } \\
\hline Ident if ied & Need & Range & Information & Sensor & 1 & 2 & 3 & 4 \\
\hline Injection pump & Injection pump & HPSI $0-300 \mathrm{gpm} / 100 \mathrm{p}$ & DBA harsh & Auxiliary building & AVI & AVI & AVI & AVI \\
\hline flow rate & flow & LPSI $0-2500 \mathrm{gpm} / \mathrm{loop}$ & DBA harsh & Auxiliary building & AVI & AVI & AVI & AVI \\
\hline HPCI/LPSI/CP/ & & $0-8000 \mathrm{gpm}$ total flow & DBA harsh & Auxiliary building & AVI & AVI & AVI & AVI \\
\hline \multirow{2}{*}{$\begin{array}{l}\text { Boric acid charging } \\
\text { flow }\end{array}$} & & $C P \quad 0-140 \mathrm{gpm}$ & DBA harsh & Auxiliary building & AVI & AVI & AVI & AVI \\
\hline & $\begin{array}{l}\text { F low of water to } \\
\text { damaged core }\end{array}$ & $\begin{array}{l}\text { Boric acid makeup pumps } \\
0-140 \mathrm{gpm}\end{array}$ & DBA non-harsh & Auxiliary building & AVI & AVI & AVI & AVI \\
\hline \multirow[t]{4}{*}{$\begin{array}{l}\text { Steam generator } \\
\text { secondary pressure }\end{array}$} & $\begin{array}{l}\text { Steam generator } \\
\text { secondary pressure }\end{array}$ & $0-1200$ psia & DBA harsh & Conta inment & AVI & AVI & AVI & FEC \\
\hline & $\begin{array}{l}\text { Steam generator } \\
\text { removal capability }\end{array}$ & & & & & & & \\
\hline & $\begin{array}{l}\text { RCS pressure greater than } \\
\text { ruptured generator }\end{array}$ & & & & & & & \\
\hline & $\begin{array}{l}\text { RCS cooldown with } \\
\text { steam generator(s) }\end{array}$ & & & & & & & \\
\hline \multirow{2}{*}{$\begin{array}{l}\text { RCS injection } \\
\text { water tanks } \\
\text { inventories }\end{array}$} & $\begin{array}{l}\text { Injection water } \\
\text { availability }\end{array}$ & $\begin{array}{l}\text { Volume control tank } \\
0-120 \text { in. } 0-100 \%\end{array}$ & Non-DBA harsh & Auxiliary building & AVI & AVI & AVI & AVI \\
\hline & $\begin{array}{l}\text { Injection water } \\
\text { inventory }\end{array}$ & $\begin{array}{l}\text { Refueling water } \\
\text { storage tank } \\
\text { wide range } \\
18 \text { in. above bottom to } \\
12 \text { in. below top }\end{array}$ & $\begin{array}{l}\text { DBA } \\
\text { Not in harsh } \\
\text { environment }\end{array}$ & Yard & AVI & AVI & AVI & AVI \\
\hline
\end{tabular}


TABLE B2. (cont inued)

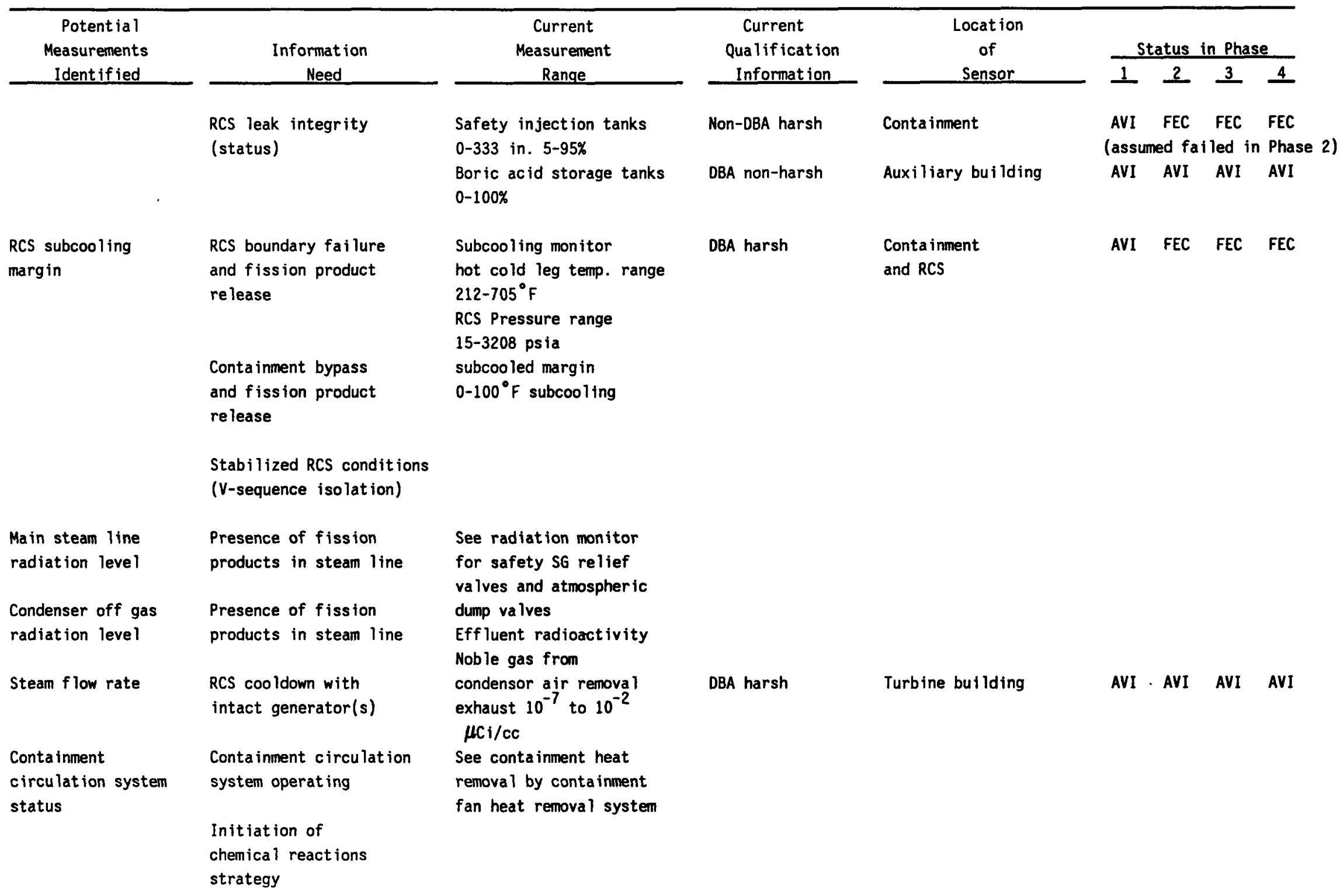


TABLE B2. (cont inued)

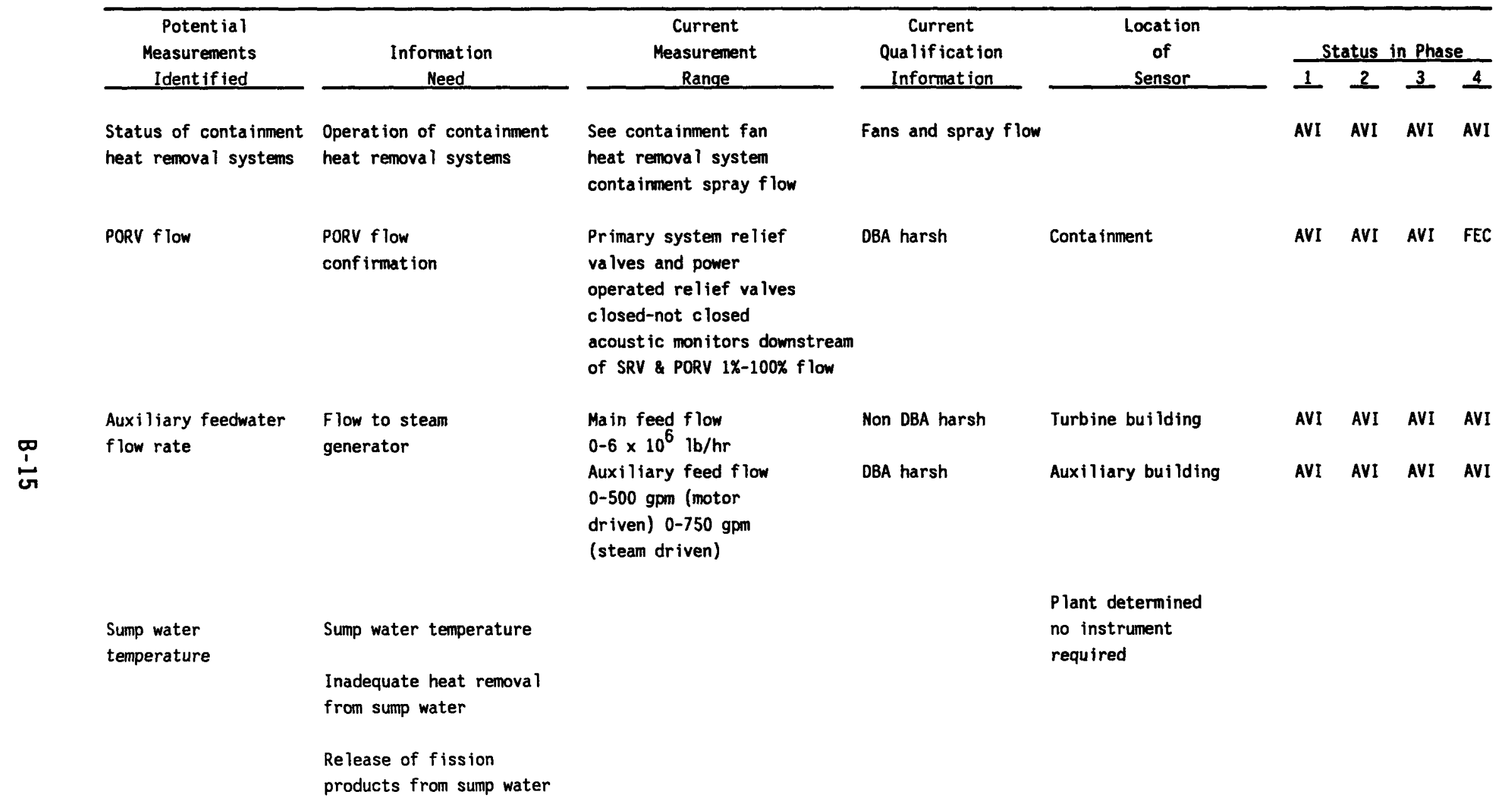


TABLE B2. (cont inued)

\begin{tabular}{|c|c|c|c|c|c|c|c|c|}
\hline $\begin{array}{c}\text { Potential } \\
\text { Measurements }\end{array}$ & Information & $\begin{array}{c}\text { Current } \\
\text { Measurement }\end{array}$ & $\begin{array}{c}\text { Current } \\
\text { Qualification }\end{array}$ & $\begin{array}{c}\text { Location } \\
\text { of }\end{array}$ & \multicolumn{4}{|c|}{ Status in Phase } \\
\hline Ident if ied & Need & Range & Information & Sensor & 1 & 2 & 3 & 4 \\
\hline $\begin{array}{l}\text { Sump water cooling } \\
\text { heat exchanger out let } \\
\text { temperature }\end{array}$ & $\begin{array}{l}\text { Inadequate heat removal } \\
\text { from sump water }\end{array}$ & $\begin{array}{l}\text { Residual heat remova } 1 \\
\text { out let temperature } \\
0-650^{\circ} \mathrm{F}\end{array}$ & OBA harsh & Auxiliary building & AVI & AVI & AVI & AVI \\
\hline & $\begin{array}{l}\text { Trend in sump water } \\
\text { temperature }\end{array}$ & $\begin{array}{l}\text { Residual system flow/ } \\
\text { decay heat removal } \\
\text { flow uses LPSI pump } \\
0-2500 \mathrm{gpm} / \text { pump } \\
0-8000 \mathrm{gpm} \text { total flow }\end{array}$ & DBA harsh & Auxiliary building & AVI & AVI & AVI & AVI \\
\hline Status of containment & Operation of containment & $\begin{array}{l}\text { See containment fan } \\
\text { containment spray flow } \\
0-2000 \mathrm{gpm}\end{array}$ & & & & & & \\
\hline $\begin{array}{l}\text { Status of sump water } \\
\text { cooling system }\end{array}$ & $\begin{array}{l}\text { Operation of sump water } \\
\text { cooling system }\end{array}$ & $\begin{array}{l}60-148 \% \text { design } \\
\text { Design flow }=1350 \mathrm{gpm}\end{array}$ & DBA harsh & Auxiliary building & AVI & AVI & AVI & AVI \\
\hline
\end{tabular}


TABLE B3. PLANT-SPECIFIC MEASUREMENTS COMPARED TO REGULATORY GUIDE 1.97 --CATEGORY 3

\begin{tabular}{|c|c|c|c|c|c|c|c|c|}
\hline Measurements & Information & $\begin{array}{c}\text { Current } \\
\text { Measurement }\end{array}$ & $\begin{array}{c}\text { Current } \\
\text { Qualification }\end{array}$ & $\begin{array}{c}\text { Location } \\
\text { of }\end{array}$ & \multicolumn{4}{|c|}{ Status in Phase } \\
\hline Ident if ied & Need & Range & Information & Sensor & 1 & 2 & 3 & 4 \\
\hline $\begin{array}{l}\text { Feedwater flow } \\
\text { rate }\end{array}$ & $\begin{array}{l}\text { Flow to steam } \\
\text { generator }\end{array}$ & $0-6 \times 10^{6} \mathrm{lb} / \mathrm{hr}$ & Non-DBA non-harsh & Turbine building & FSS & FSS & FSS & FSS \\
\hline & $\begin{array}{l}\text { Feedwater flow } \\
\text { initiation }\end{array}$ & & & & & & & \\
\hline $\begin{array}{l}\text { Quench tank } \\
\text { inventory }\end{array}$ & Quench tank level & $\begin{array}{l}\text { QT level } 6-48 \text { in. } \\
4 \%-96 \%\end{array}$ & Non DBA harsh & Containment & FSS & FSS & FSS & FSS \\
\hline & $\begin{array}{l}\text { Quench tank level } \\
\text { above relief valve } \\
\text { discharge level }\end{array}$ & $\begin{array}{l}\text { QT pressure } 0-100 \text { psig } \\
\text { QT temperature } \\
50-350^{\circ} \mathrm{F}\end{array}$ & & & & & & \\
\hline & $\begin{array}{l}\text { PORV flow } \\
\text { confirmation }\end{array}$ & & & & & & & \\
\hline & $\begin{array}{l}\text { Transport of fission } \\
\text { products through } \\
\text { relief valve }\end{array}$ & & & & & & & \\
\hline & Relief valve flow statu & & & & & & & \\
\hline $\begin{array}{l}\text { Containment } \\
\text { radiation leve } 1 \\
\text { (and location) }\end{array}$ & $\begin{array}{l}\text { Presence of } \\
\text { radiation in } \\
\text { containment }\end{array}$ & $\begin{array}{l}\text { Conta inment area radiation } \\
\text { high range } 1 \text { to } 10^{8} \mathrm{r} / \mathrm{hr} \\
\text { for energy range } 0.1 \text { to } \\
3 \mathrm{MeV}\end{array}$ & Non-DBA harsh & Conta inment & FSS & FSS & FSS & FSS \\
\hline & $\begin{array}{l}\text { Containment radiation } \\
\text { level } \\
\text { Quantity of fission } \\
\text { products being } \\
\text { dispersed from RCS and } \\
\text { location }\end{array}$ & $\begin{array}{l}\text { Common plant vent-noble } \\
\text { gas vent flow rate noble gas } \\
\text { activity range } 10^{-7} \text { to } \\
10^{5} \mu \mathrm{i} \mathrm{i} / \mathrm{cc} \\
\text { Activity plus flow displayed } \\
\text { Activity recorded on trend } \\
\text { recorders }\end{array}$ & Non DBA harsh & Auxiliary building & FSS & FSS & FSS & FSS \\
\hline
\end{tabular}


TABLE B3. (continued)

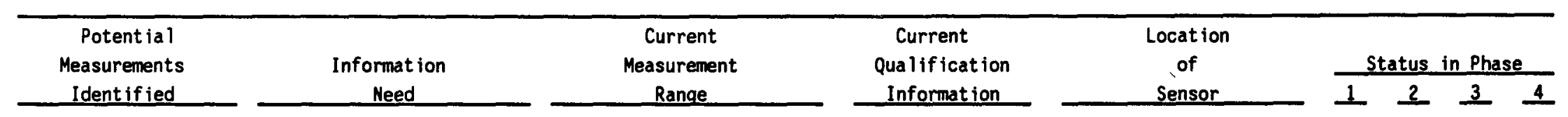

Conta inment

Presence of fission

radiation level (and products in containment

location)

atmosphere

(cont'd)

Presence of fission

products in quench

tank

Presence of fission

product gases in

containment atmosphere

Presence of fission

products in relief

valve discharge flow

RCS boundary failure

and $f$ ission product

release

Lower head failure and

fission product release

Presense of aerosols

in containment

atmosphere

Presence of fission

products in

conta inment water 
TABLE B3. (cont inued)

\begin{tabular}{|c|c|c|c|c|c|c|c|c|}
\hline $\begin{array}{c}\text { Potential } \\
\text { Measurements }\end{array}$ & Information & $\begin{array}{c}\text { Current } \\
\text { Measurement }\end{array}$ & $\begin{array}{l}\text { Current } \\
\text { Qualification }\end{array}$ & $\begin{array}{l}\text { Location } \\
\text { of }\end{array}$ & \multicolumn{4}{|c|}{ Status in Phase } \\
\hline Ident ified & Need & Range & Information & Sensor & 1 & $\underline{2}$ & 3 & 4 \\
\hline $\begin{array}{l}\text { Sump water radiation } \\
\text { level }\end{array}$ & $\begin{array}{l}\text { Presence of fission } \\
\text { products in containment } \\
\text { water }\end{array}$ & $\begin{array}{l}\text { See primary and sump } \\
\text { gross activity }\end{array}$ & & & & & & \\
\hline $\begin{array}{l}\text { Quench tank water } \\
\text { temperature }\end{array}$ & Quench tank temperature & $\begin{array}{l}\text { Quench tank temp } \\
50-350^{\circ} \mathrm{F}\end{array}$ & Non-DBA harsh & Conta inment & FSS & FSS & FSS & FSS \\
\hline & Quench tank cooling & $\begin{array}{l}\text { Quench tank leve ? } \\
0-48 \text { in. a larm }=55 \% \\
26.4 \text { in. }\end{array}$ & & & & & & \\
\hline & $\begin{array}{l}\text { Relief valve flow } \\
\text { status }\end{array}$ & $\begin{array}{l}\text { See safety relief valve } \\
\text { position/flow above }\end{array}$ & & & & & & \\
\hline & $\begin{array}{l}\text { Transport of fission } \\
\text { products through } \\
\text { relief valve }\end{array}$ & & & & & & & \\
\hline Quench tank pressure & Quench tank pressure & $\begin{array}{l}0-100 \text { psig, rupture disc } \\
\text { pressure }=100 \text { psig }\end{array}$ & Non-DBA harsh & Conta inment & FSS & FSS & FSS & FSS \\
\hline
\end{tabular}

Relief valve flow status

Transport of fission

products through

relief valve 
TABLE B3. (cont inued)

\begin{tabular}{|c|c|c|c|c|c|c|c|c|}
\hline $\begin{array}{c}\text { Potential } \\
\text { Measurements }\end{array}$ & Information & $\begin{array}{c}\text { Current } \\
\text { Measurement }\end{array}$ & $\begin{array}{c}\text { Current } \\
\text { Qualification }\end{array}$ & $\begin{array}{l}\text { Location } \\
\text { of }\end{array}$ & \multicolumn{4}{|c|}{ Status in Phase } \\
\hline Identified & Need & Range & Information & Sensor & 1 & 2 & 3 & 4 \\
\hline \multirow[t]{3}{*}{$\begin{array}{l}\text { Conta inment sump } \\
\text { water chemistry }\end{array}$} & Containment water $\mathrm{pH}$ & $\begin{array}{l}\text { Primary coolant and } \\
\text { sump pH } 3-12\end{array}$ & Non-DBA harsh & Auxiliary building & FSS & FSS & FSS & FSS \\
\hline & Presence of iodine & & & & & & & \\
\hline & $\begin{array}{l}\text { Presence of fission } \\
\text { products in containment } \\
\text { water }\end{array}$ & $\begin{array}{l}\text { See primary coolant } \\
\text { and sump gross } \\
\text { activity }\end{array}$ & & & & & & \\
\hline Reactor coolant flow & $\begin{array}{l}\text { Reactor coolant pump } \\
\text { status }\end{array}$ & $\begin{array}{l}\text { Pump motor current } \\
0-400 \text { amps }\end{array}$ & $\begin{array}{l}\text { Non-DBA } \\
\text { Non-harsh }\end{array}$ & Auxiliary building & FSS & FSS & FSS & FSS \\
\hline
\end{tabular}




\section{APPENDIX C}

EXISTING MEASUREMENTS NOT

SPECIFIED IN REGULATORY GUIDE 1.97

$c-1 / c-2$ 


\section{APPENDIX C \\ EXISTING MEASUREMENTS NOT SPECIFIED IN REGULATORY GUIDE 1.97}

The tables contained in Appendix $\mathrm{C}$ list those existing measurements that could be used for accident management that are not specified in Regulatory Guide 1.97. This table includes columns to list the current measurement range of the instrument, the severe accident measurement range, and the location of the sensor. 
TABLE C. EXISTING MEASUREMENTS NOT SPECIFIED IN REGULATORY GUIDE 1.97

\begin{tabular}{|c|c|c|c|c|}
\hline $\begin{array}{l}\text { Existing } \\
\text { Measurements } \\
\text { Identified } \\
\end{array}$ & $\begin{array}{l}\text { Information } \\
\text { Need } \\
\end{array}$ & $\begin{array}{c}\text { Current } \\
\text { Measurement } \\
\text { Range } \\
\end{array}$ & $\begin{array}{l}\text { Potential } \\
\text { Severe Accident } \\
\text { Measurement } \\
\text { Range } \\
\end{array}$ & $\begin{array}{c}\text { Location } \\
\text { of } \\
\text { Sensor } \\
\end{array}$ \\
\hline $\begin{array}{l}\text { Core Temperature } \\
\text { (SPNDS) }\end{array}$ & Core Temperature & Current & Same & Reactor \\
\hline \multirow[t]{3}{*}{ Containment } & $\begin{array}{l}\text { Containment } \\
\text { Humidity }\end{array}$ & $\begin{array}{l}\text { Containment Dome } \\
\text { and Reactor } \\
\text { Cavity } \\
0 \text { to } 100 \%\end{array}$ & Same & $\begin{array}{l}\text { Containment and } \\
\text { Conta inment Cavity }\end{array}$ \\
\hline & $\begin{array}{l}\text { RCS Boundary and } \\
\text { Fission Product } \\
\text { Release }\end{array}$ & & & \\
\hline & $\begin{array}{l}\text { Lower Head Failure } \\
\text { and Fission Product } \\
\text { Release }\end{array}$ & & & \\
\hline $\begin{array}{l}\text { Air and Nitrogen } \\
\text { Equipment Status }\end{array}$ & $\begin{array}{l}\text { Concentration of } \\
\text { Noncondensables } \\
\text { (Air and Nitrogen } \\
\text { Leak Status) }\end{array}$ & & & \\
\hline $\begin{array}{l}\text { Recombiner/ } \\
\text { Igniters Equip- } \\
\text { ment Status }\end{array}$ & $\begin{array}{l}\text { Initiation of } \\
\text { Recombiners/Igniters }\end{array}$ & & & \\
\hline $\begin{array}{l}\text { Auxiliary Spray } \\
\text { Flow }\end{array}$ & $\begin{array}{l}\text { Auxiliary Spray } \\
\text { Flow Initiation }\end{array}$ & $\begin{array}{l}\text { Pressurizer } \\
\text { Auxiliary Spray } \\
\text { Temperature } \\
0-600^{\circ} \mathrm{F}\end{array}$ & Same & Auxiliary Building \\
\hline
\end{tabular}


TABLE C. (cont inued)

-

\begin{tabular}{|c|c|c|c|c|}
\hline & & & Potential & \\
\hline $\begin{array}{l}\text { Potential } \\
\text { Measurements } \\
\text { Ident if ied } \\
\end{array}$ & $\begin{array}{c}\text { Informat ion } \\
\text { Need }\end{array}$ & $\begin{array}{c}\text { Current } \\
\text { Measurement } \\
\text { Range } \\
\end{array}$ & $\begin{array}{c}\text { Severe Accident } \\
\text { Measurement } \\
\text { Range } \\
\end{array}$ & $\begin{array}{l}\text { Location } \\
\text { of } \\
\text { Sensor } \\
\end{array}$ \\
\hline $\begin{array}{l}\text { Auxiliary, main, } \\
\text { and alternate } \\
\text { feedwater system } \\
\text { status }\end{array}$ & $\begin{array}{l}\text { Steam generator heat } \\
\text { removal capability }\end{array}$ & $\begin{array}{l}\text { Main-pump suction flow } \\
0-2000 \mathrm{GPM} \text {, pump suction } \\
\text { pressure } 0-500 \text { psig, steam } \\
\text { and feed flow } 0-6 \times 10^{6} \\
\text { lbs/hr, pump bearing } \\
\text { temperature, pump discharge } \\
\text { pressure } 0-2000 \text { psig, } \\
\text { vibration monitor: } \\
\text { auxiliary-discharge } \\
\text { pressure } 0-2000 \text { psig, } \\
\text { turbine steam pressure } \\
0-1200 \text { psig, pump motor } \\
\text { current } 0-100 \text { amps; } \\
\text { CST level } 0-38 \mathrm{ft} \text {. }\end{array}$ & $\begin{array}{l}\text { Same } \\
\text { Same }\end{array}$ & Turbine building \\
\hline
\end{tabular}




\section{APPENDIX D \\ INFORMATION-NEEDS TABLES FOR A SPECIFIC PWR AND SEVERE ACCIDENT SEQUENCE}

D-1/D-2 


\section{APPENDIX D \\ INFORMATION-NEEDS TABLES FOR A SPECIFIC PWR AND SEVERE ACCIDENT SEQUENCE}

The tables contained in Appendix D present information needs for a specific PWR and for specific severe accident conditions. The instrument availability was determined using the tables in Appendix B and transposed to Tables D1, D2, and D3 of Appendix D to identify the status of the information needs for each of the safety objective trees. Information need status is indicated by the following acronyms:

- ADI - available directly or indirectly
- ADO - available directly only

- AIO - available indirectly only

- NAV - not available.

The status of the information needs is indicated in brackets in the Information Needs column in Tables D1, D2, and D3. 
TABLE D1.1. PREVENT CORE DISPERSAL FROM VESSEL (V)--INADEQUATE INVENTORY MECHANISM (V1A1)

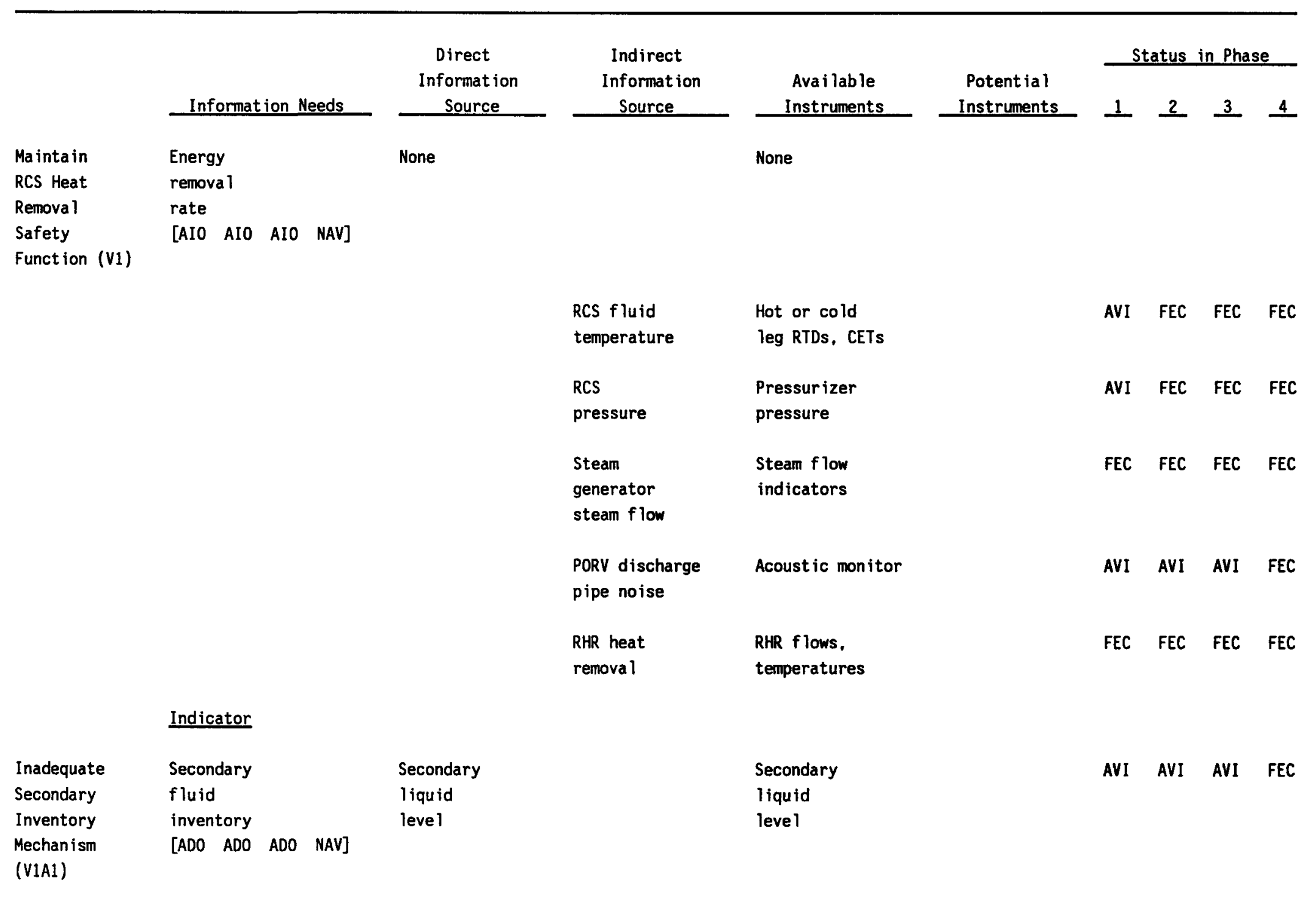


TABLE 01.1. (cont inued)

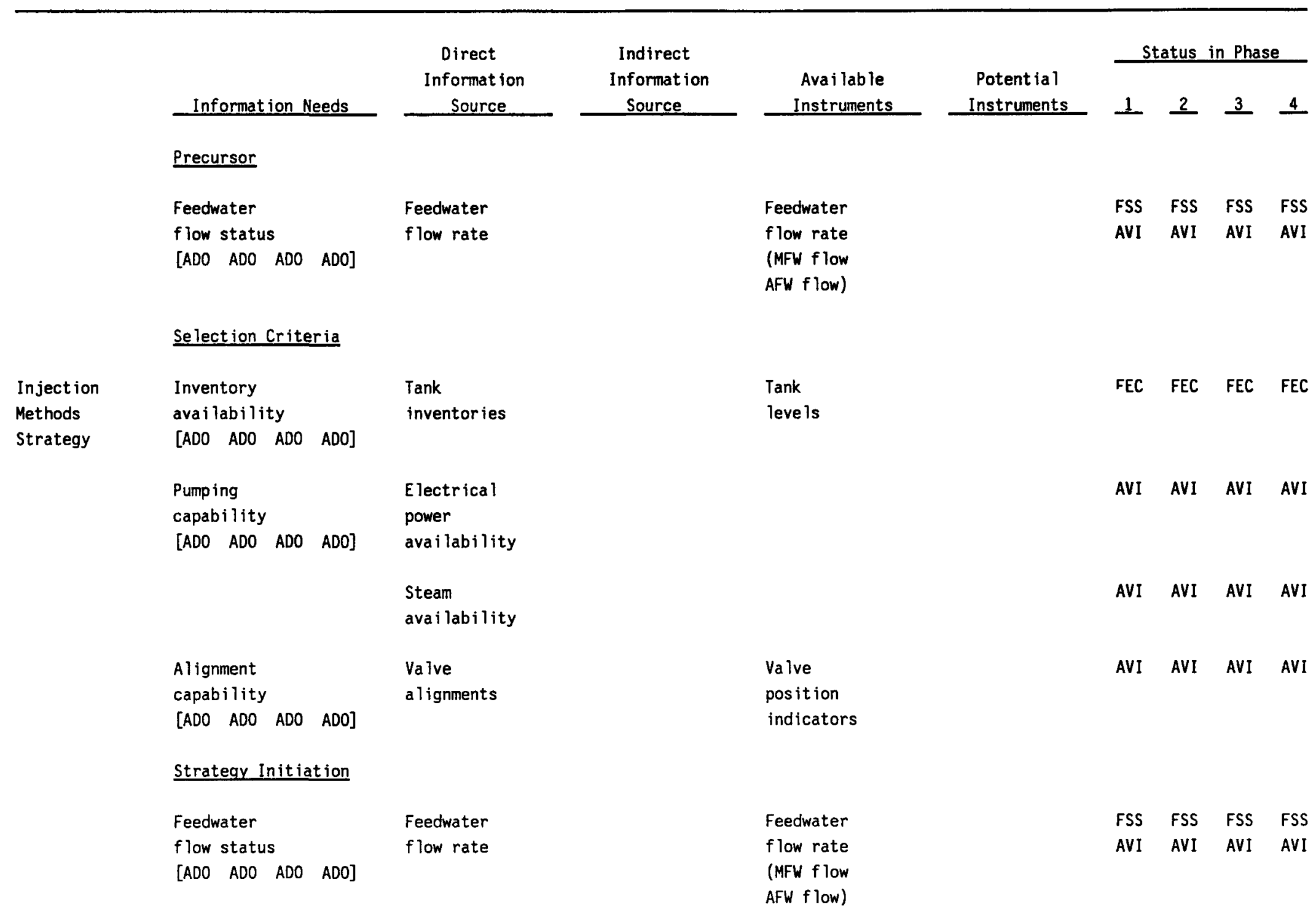


TABLE 01.1. (continued)

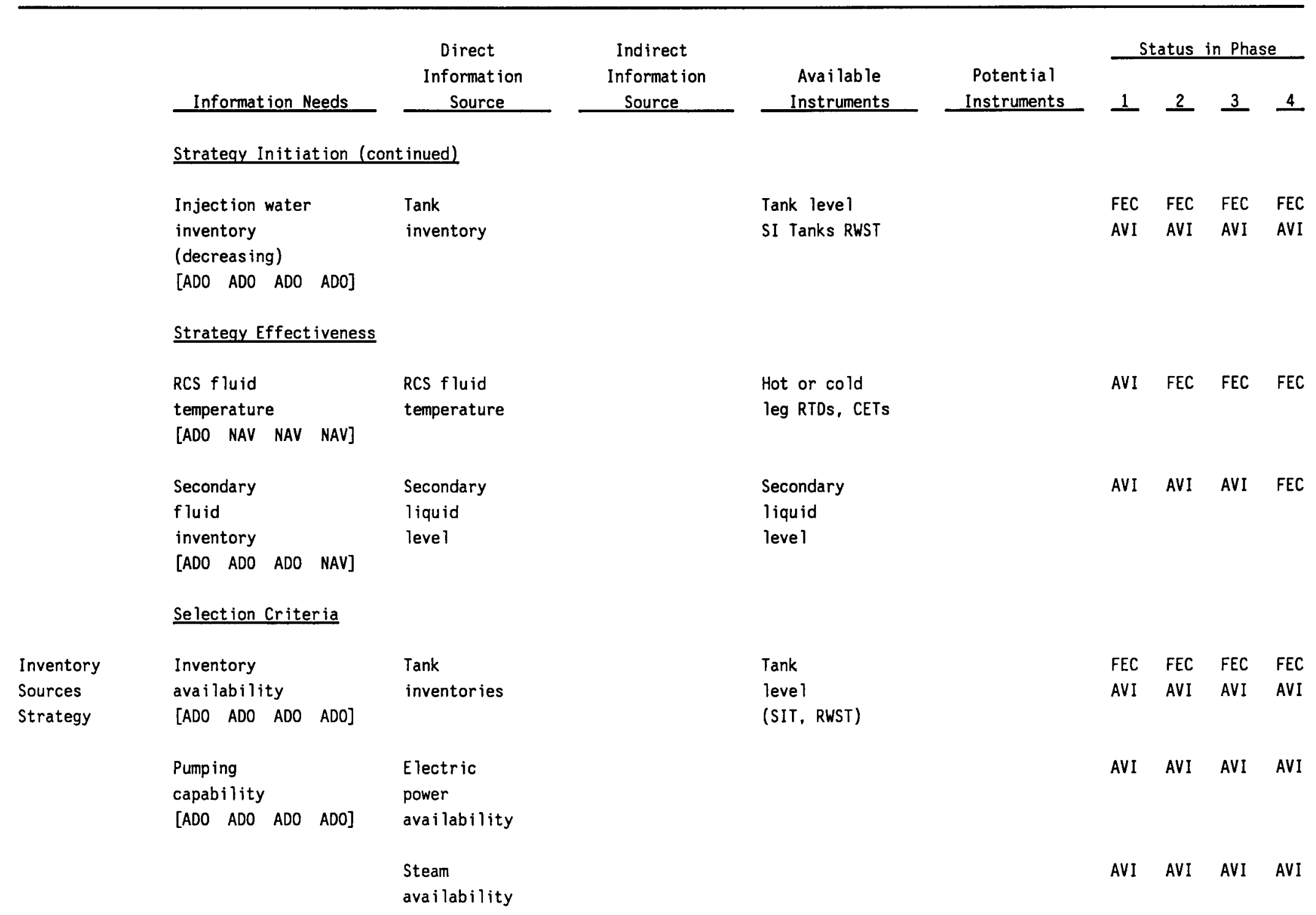


TABLE D1.1. (cont inued)

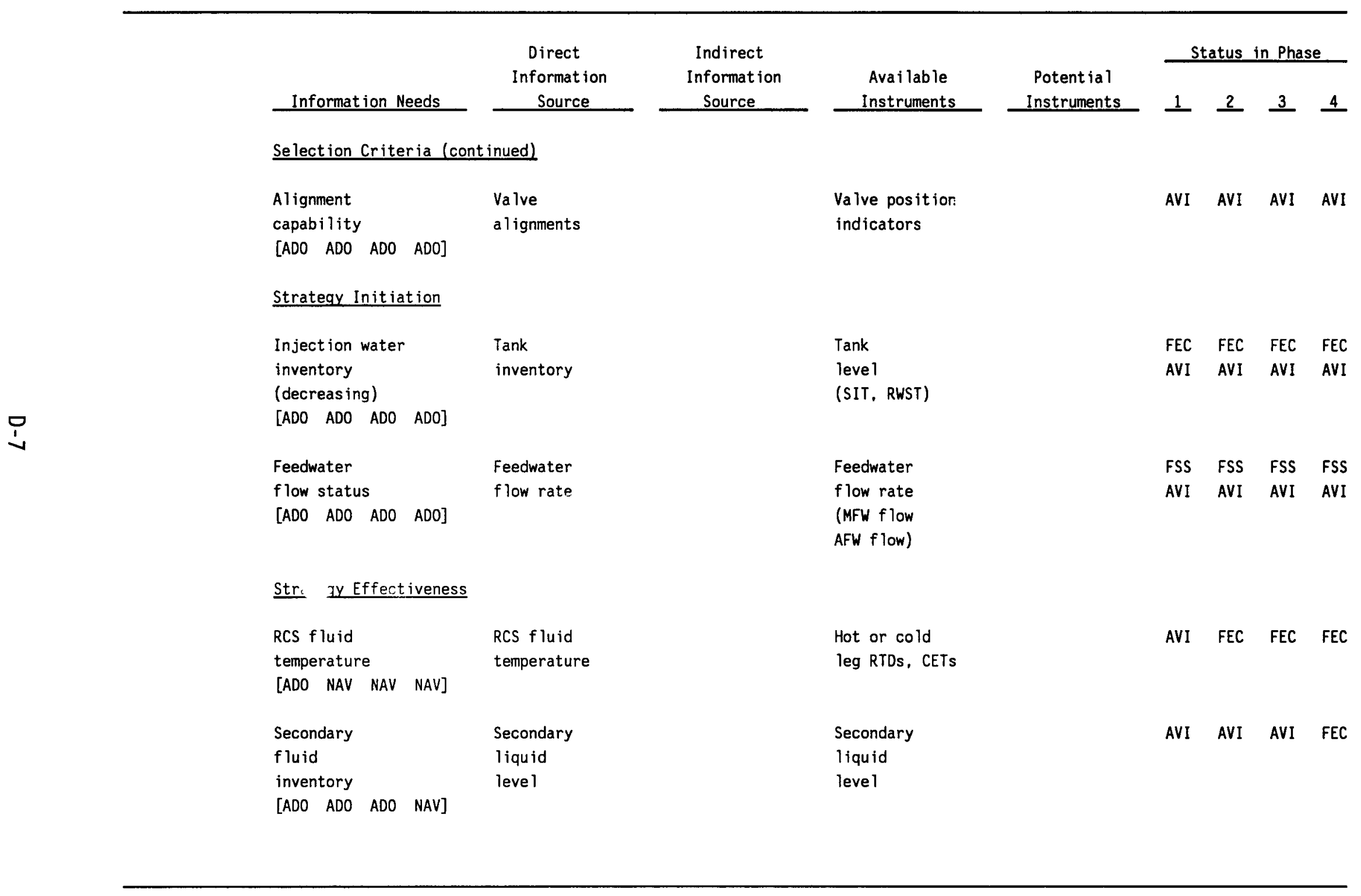


TABLE 01.2. PREVENT CORE DISPERSAL FROM VESSEL (V)--INADEQUATE PRESSURE CONTROL MECHANISM (V1A2)

\begin{tabular}{|c|c|c|c|c|c|c|c|c|c|}
\hline & \multirow[b]{2}{*}{ Information Needs } & \multirow{2}{*}{$\begin{array}{c}\text { Direct } \\
\text { Information } \\
\text { Source } \\
\end{array}$} & \multirow{2}{*}{$\begin{array}{c}\text { Indirect } \\
\text { Information } \\
\text { Source } \\
\end{array}$} & \multirow[b]{2}{*}{$\begin{array}{l}\text { Avai lable } \\
\text { Instruments }\end{array}$} & \multirow[b]{2}{*}{$\begin{array}{l}\text { Potential } \\
\text { Instruments }\end{array}$} & \multicolumn{4}{|c|}{ Status in Phase } \\
\hline & & & & & & 1 & 2 & 3 & 4 \\
\hline $\begin{array}{l}\text { Maintain } \\
\text { RCS Heat } \\
\text { Removal }\end{array}$ & $\begin{array}{l}\text { Energy } \\
\text { removal } \\
\text { rate }\end{array}$ & None & & None & & & & & \\
\hline $\begin{array}{l}\text { Safety } \\
\text { Function (V1) }\end{array}$ & {$\left[\begin{array}{llll}\text { AIO } & \text { NAV NAV NAV] }\end{array}\right.$} & & $\begin{array}{l}\text { RCS fluid } \\
\text { temperature }\end{array}$ & $\begin{array}{l}\text { Hot or cold } \\
\text { leg RTDs, CETs }\end{array}$ & & AVI & FEC & FEC & FEC \\
\hline & & & $\begin{array}{l}\text { RCS } \\
\text { pressure }\end{array}$ & $\begin{array}{l}\text { Pressurizer } \\
\text { pressure }\end{array}$ & & AVI & FEC & FEC & FEC \\
\hline & & & $\begin{array}{l}\text { Steam } \\
\text { generator } \\
\text { steam flow }\end{array}$ & $\begin{array}{l}\text { Steam flow } \\
\text { indicators }\end{array}$ & & FEC & FEC & FEC & FEC \\
\hline & & & $\begin{array}{l}\text { RHR heat } \\
\text { remova } 1\end{array}$ & $\begin{array}{l}\text { RHR flows, } \\
\text { temperatures }\end{array}$ & & AVI & FEC & FEC & FEC \\
\hline & Indicator & & & & & & & & \\
\hline Inadequate & RCS - Steam & Steam & & Steam & & AVI & AVI & AVI & FEC \\
\hline Pressure & Generator & generator & & generator & & & & & \\
\hline Control & Pressure & secondary & & secondary & & & & & \\
\hline Mechanism & difference & pressure & & pressure & & & & & \\
\hline (VIAC) & [AIU NAV NAV NAV] & $\begin{array}{l}\text { RCS } \\
\text { pressure }\end{array}$ & & $\begin{array}{l}\text { Pressurizer } \\
\text { pressure }\end{array}$ & & AVI & FEC & FEC & FEC \\
\hline
\end{tabular}


TABLE D1.2. (cont inued)

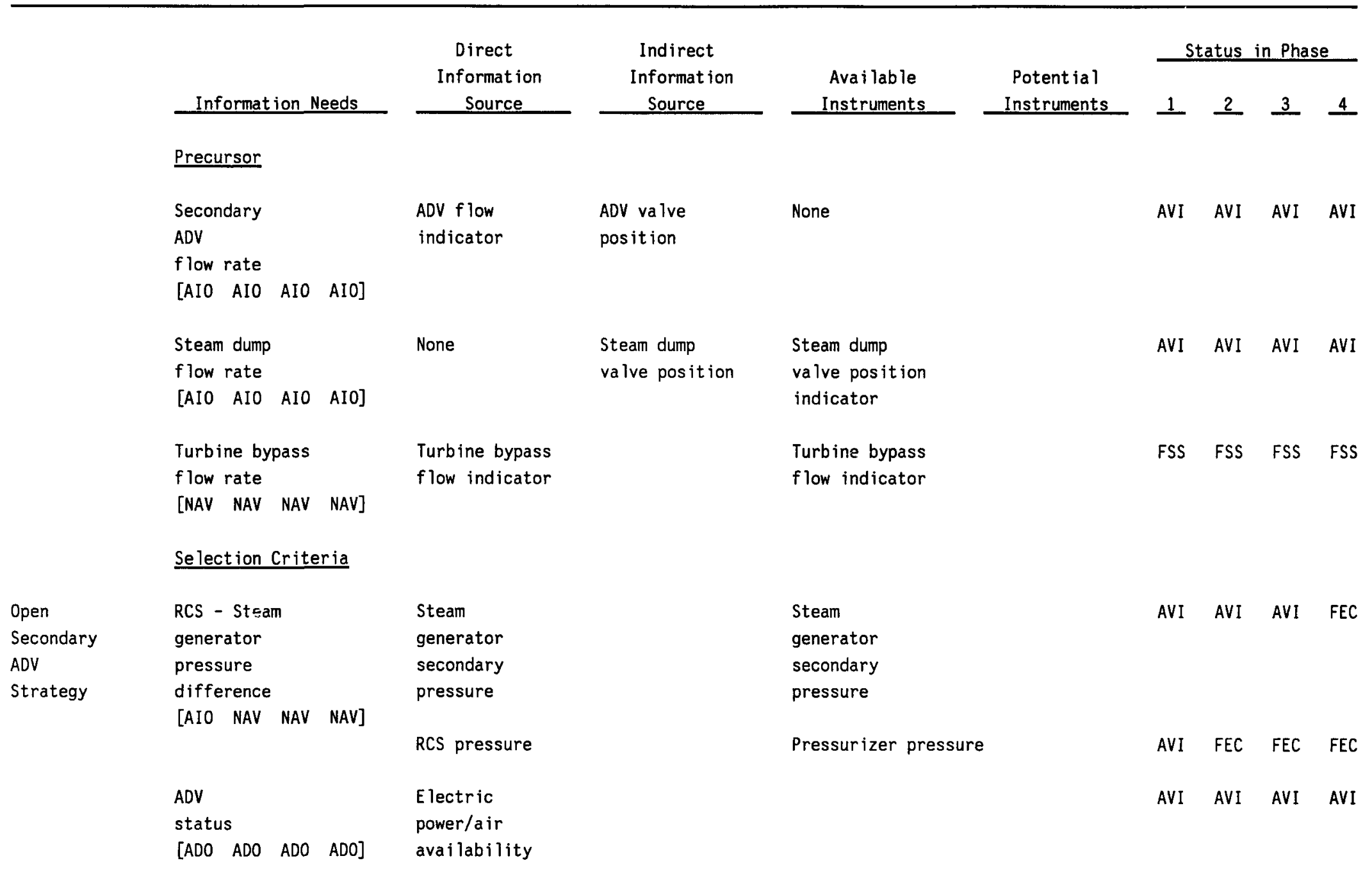


TABLE D1.2. (cont inued)

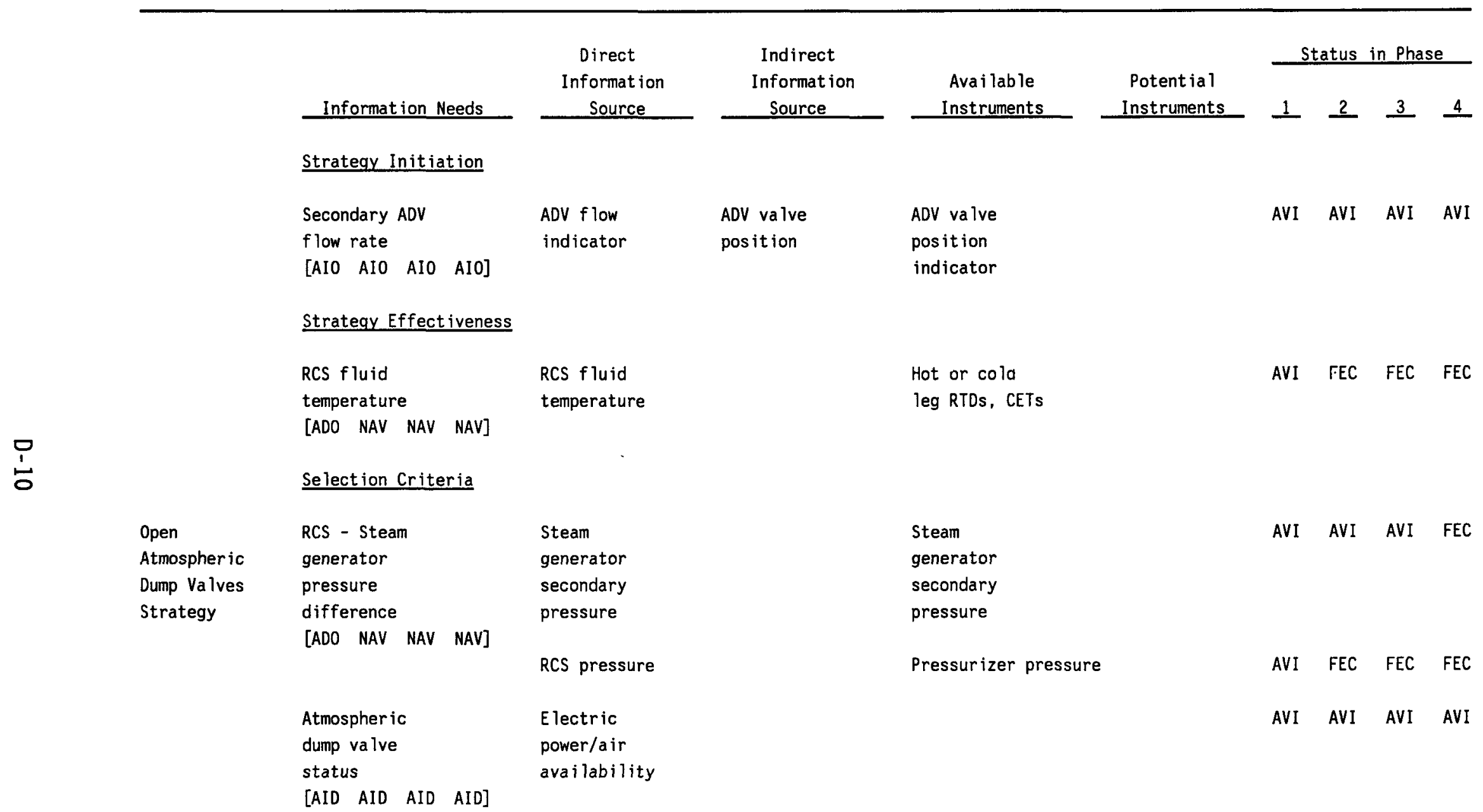


TABLE D1.2. (cont inued)

\begin{tabular}{|c|c|c|c|c|c|c|c|c|c|}
\hline & \multirow[b]{2}{*}{ Information Needs } & \multirow{2}{*}{$\begin{array}{c}\text { Direct } \\
\text { Information } \\
\text { Source } \\
\end{array}$} & \multirow{2}{*}{$\begin{array}{c}\text { Indirect } \\
\text { Informat ion } \\
\text { Source } \\
\end{array}$} & \multirow{2}{*}{$\begin{array}{c}\text { Avai lable } \\
\text { Instruments }\end{array}$} & \multirow{2}{*}{$\begin{array}{c}\text { Potential } \\
\text { Instruments } \\
\end{array}$} & \multicolumn{4}{|c|}{ Status in Phase } \\
\hline & & & & & & 1 & 2 & 3 & 4 \\
\hline & $\begin{array}{l}\text { Atmospheric } \\
\text { dump valve } \\
\text { flow rate }\end{array}$ & $\begin{array}{l}\text { Atmospheric } \\
\text { dump valve } \\
\text { flow indicator }\end{array}$ & None & $\begin{array}{l}\text { Atmospher ic } \\
\text { dump valve } \\
\text { position }\end{array}$ & & AVI & AVI & AVI & AVI \\
\hline & Strategy Effectiveness & & & & & & & & \\
\hline & $\begin{array}{l}\text { RCS fluid } \\
\text { temperature }\end{array}$ & $\begin{array}{l}\text { RCS fluid } \\
\text { temperature }\end{array}$ & & $\begin{array}{l}\text { Hot or Cold } \\
\text { leg RTDs, CETs }\end{array}$ & & AVI & FEC & FEC & FEC \\
\hline & [ADO NAV NAV NAV] & & & & & & & & \\
\hline Strategy & pressure & secondary & & secondary & & & & & \\
\hline & difference & pressure & & pressure & & & & & \\
\hline & {$\left[\begin{array}{llll}A A D O & \text { NAV } & \text { NAV } & \text { NAV }\end{array}\right.$} & & & & & & & & \\
\hline & & RCS pressure & & Pressurizer pressure & & AVI & FEC & FEC & FEC \\
\hline & $\begin{array}{l}\text { Turbine } \\
\text { bypass valve } \\
\text { status }\end{array}$ & $\begin{array}{l}\text { Electric } \\
\text { power/air } \\
\text { availability }\end{array}$ & & & & FSS & FSS & FSS & FSS \\
\hline
\end{tabular}


TABLE D1.2. (cont inued)

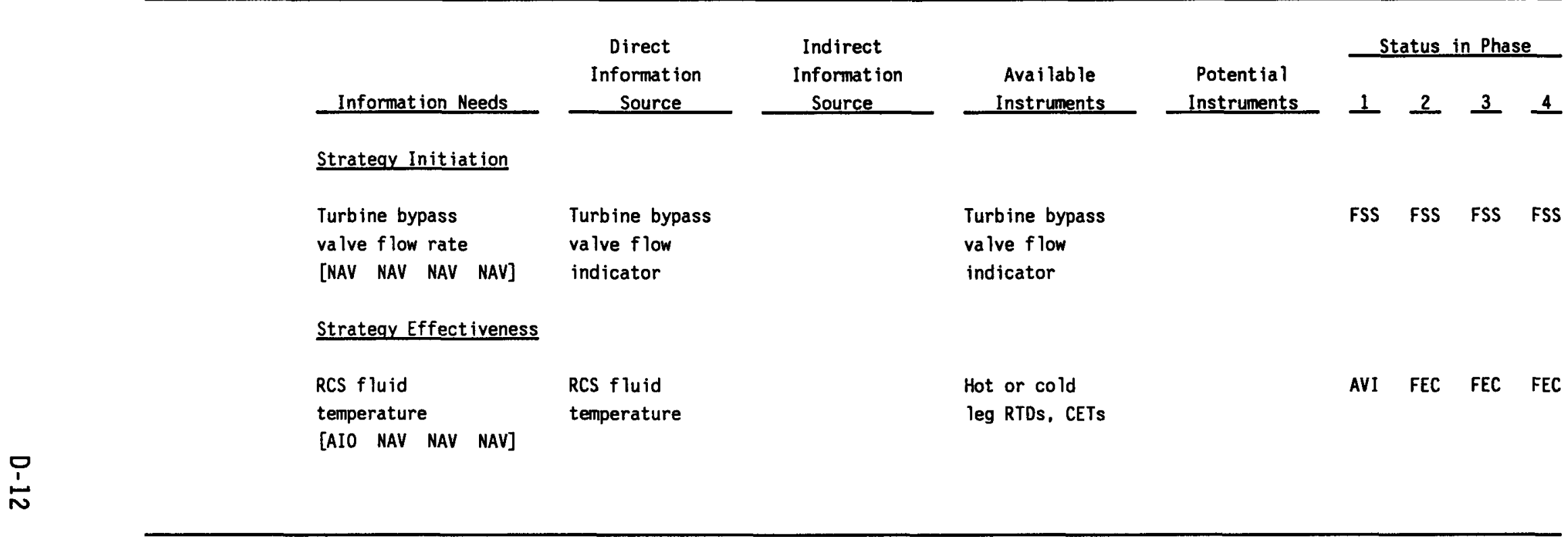


TABLE D1.3. PREVENT CORE DISPERSAL FROM VESSEL (V)--INADEQUATE RCS ENERGY TRANSPORT MECHANISM (V1A3)

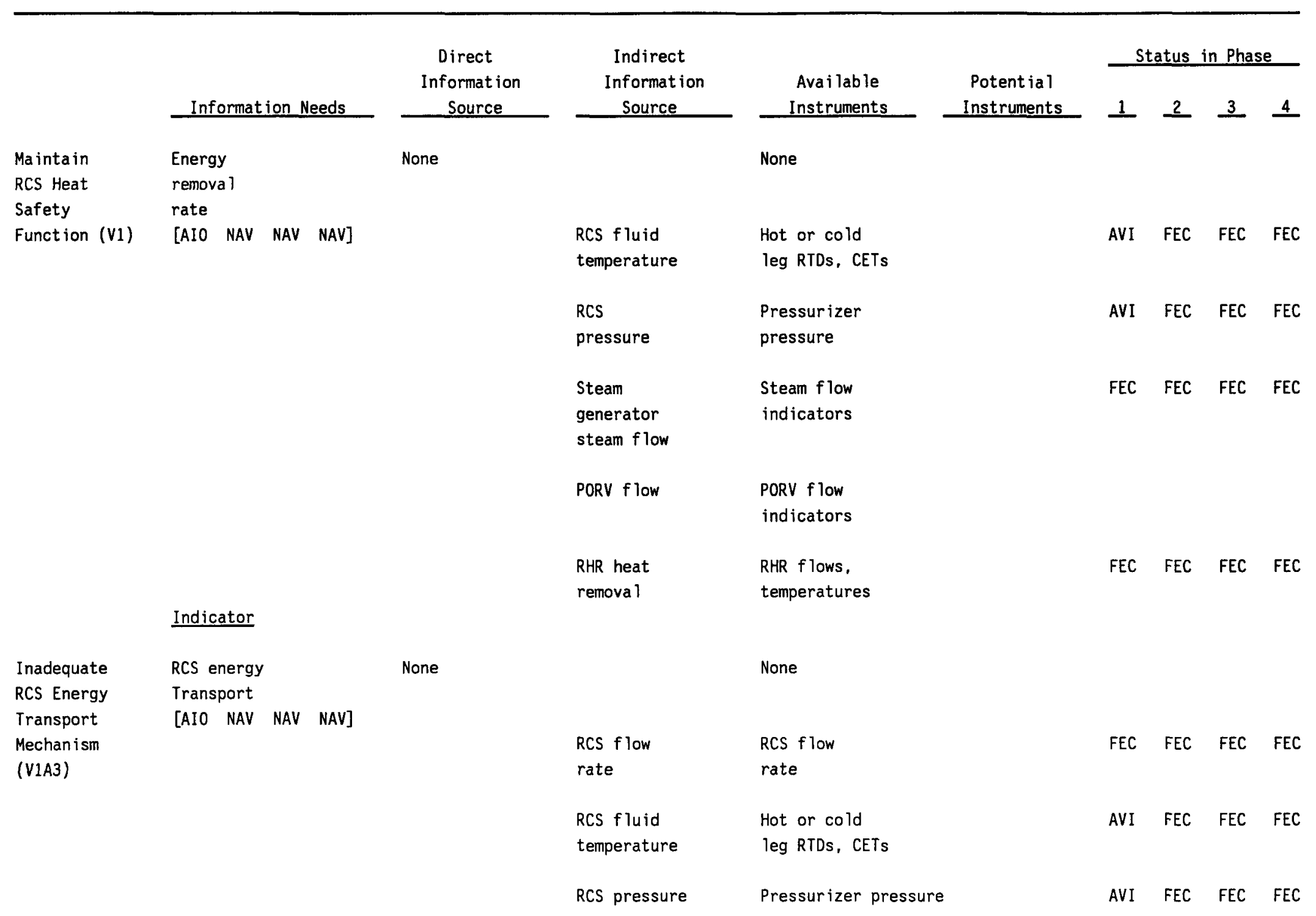


TABLE D1.3. (continued)

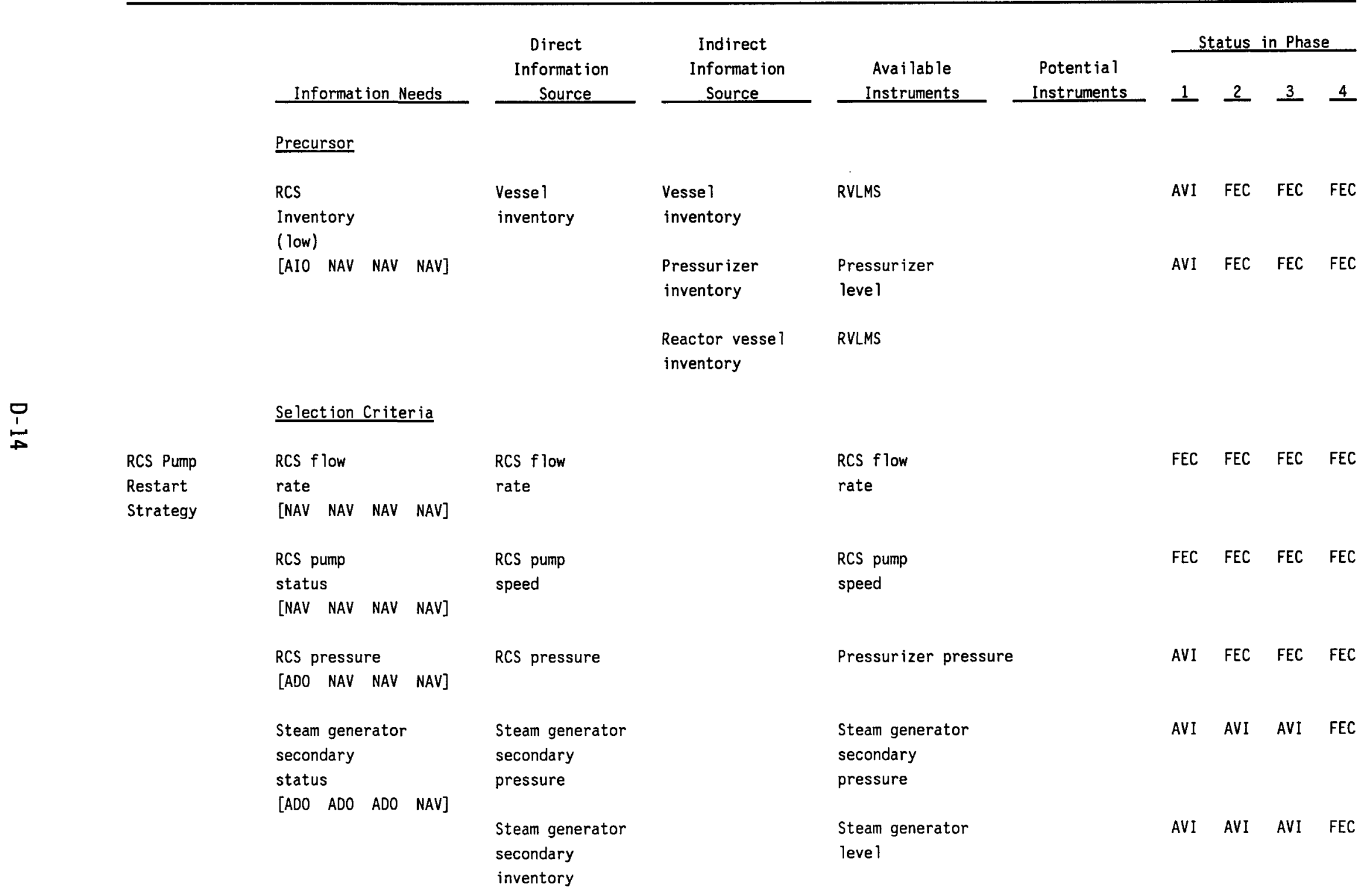


TABLE D1.3. (cont inued)

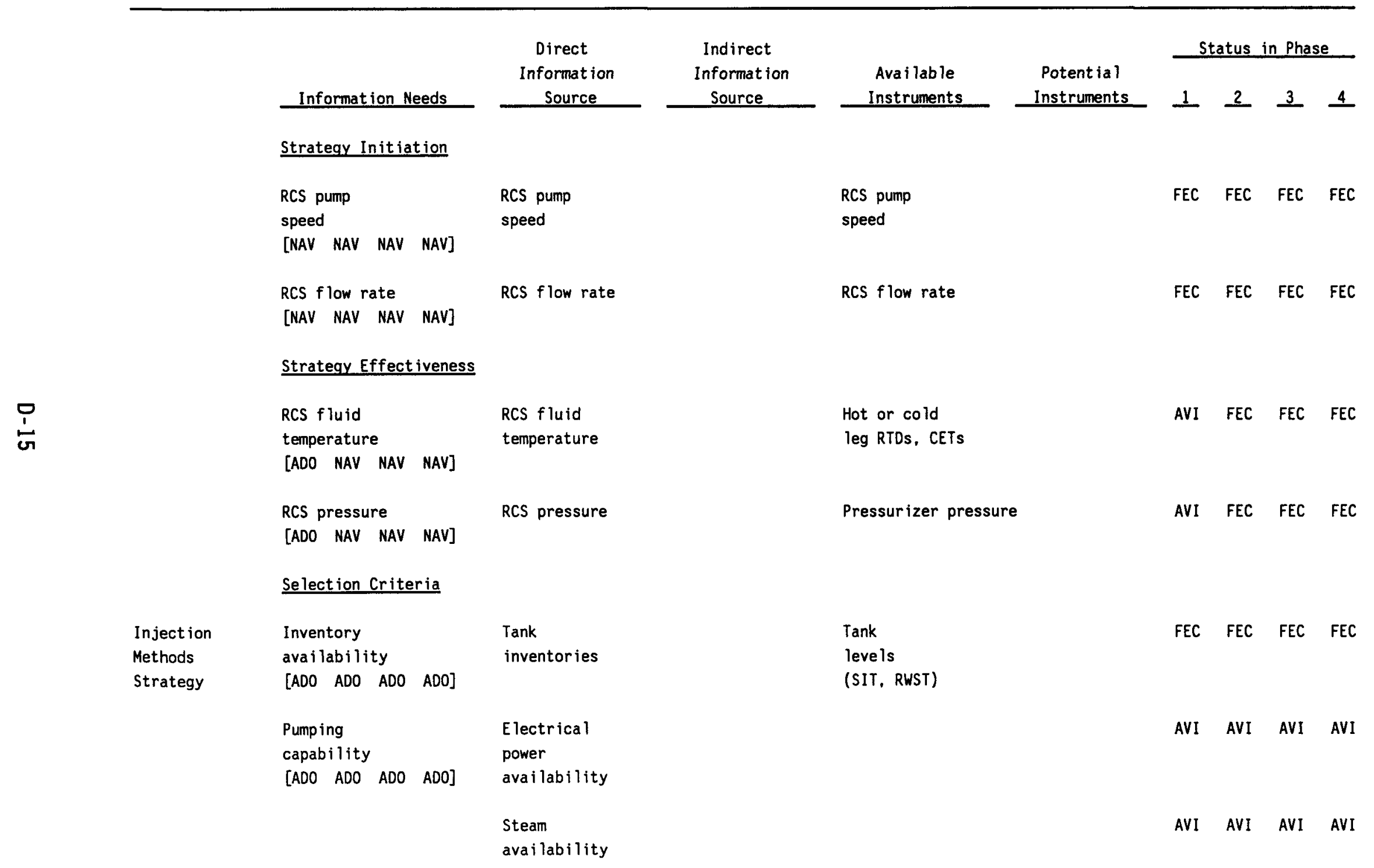


TABLE D1.3. (cont inued)

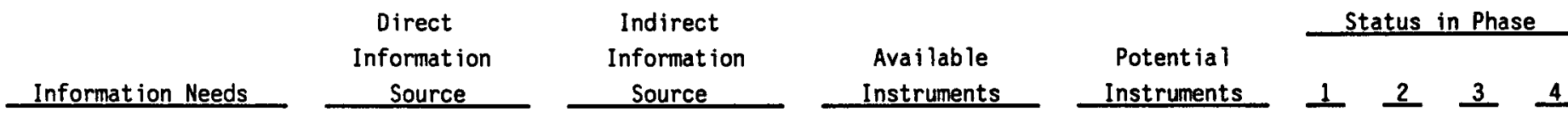

Selection Criteria (continued)

\section{Alignment \\ capability \\ [ADO ADO ADO ADO] \\ Strategy Initiation}

Valve

a lignments

Valve

AVI AVI AVI AVI

position

indicators

$\begin{array}{ll}\text { Feedwater } & \text { Feedwater } \\ \text { flow status } & \text { flow rate }\end{array}$

Feedwater

flow rate

FSS FSS FSS FSS

$\left[\begin{array}{llll}A D O & A D O & A D O & A D O\end{array}\right]$

(MFW flow

AFW flow)

$\begin{array}{ll}\text { Injection water } & \text { Tank } \\ \text { inventory } & \text { inventory } \\ \text { (decreasing) } & \end{array}$

Tank leve I

SI Tanks RWST

FEC FEC FEC

$\left[\begin{array}{llll}A D O & A D O & A D O & A D 0\end{array}\right]$

\section{Strategy Effectiveness}

$\begin{array}{ll}\begin{array}{l}\text { RCS fluid } \\ \text { temperature }\end{array} & \begin{array}{l}\text { RCS fluid } \\ \text { temperature } \\ {[A D O \text { NAV NAV NAV] }}\end{array} \\ & \\ \text { Secondary } & \text { Secondary } \\ \text { fluid } & \begin{array}{l}\text { liquid } \\ \text { level }\end{array} \\ \text { inventory } & \text { laDO ADO ADO NAV] }\end{array}$

Hot or cold

leg RTDs, CETs

AVI FEC FEC FEC

Secondary

liquid

AVI AVI AVI FEC 
TABLE 01.3. (cont inued)

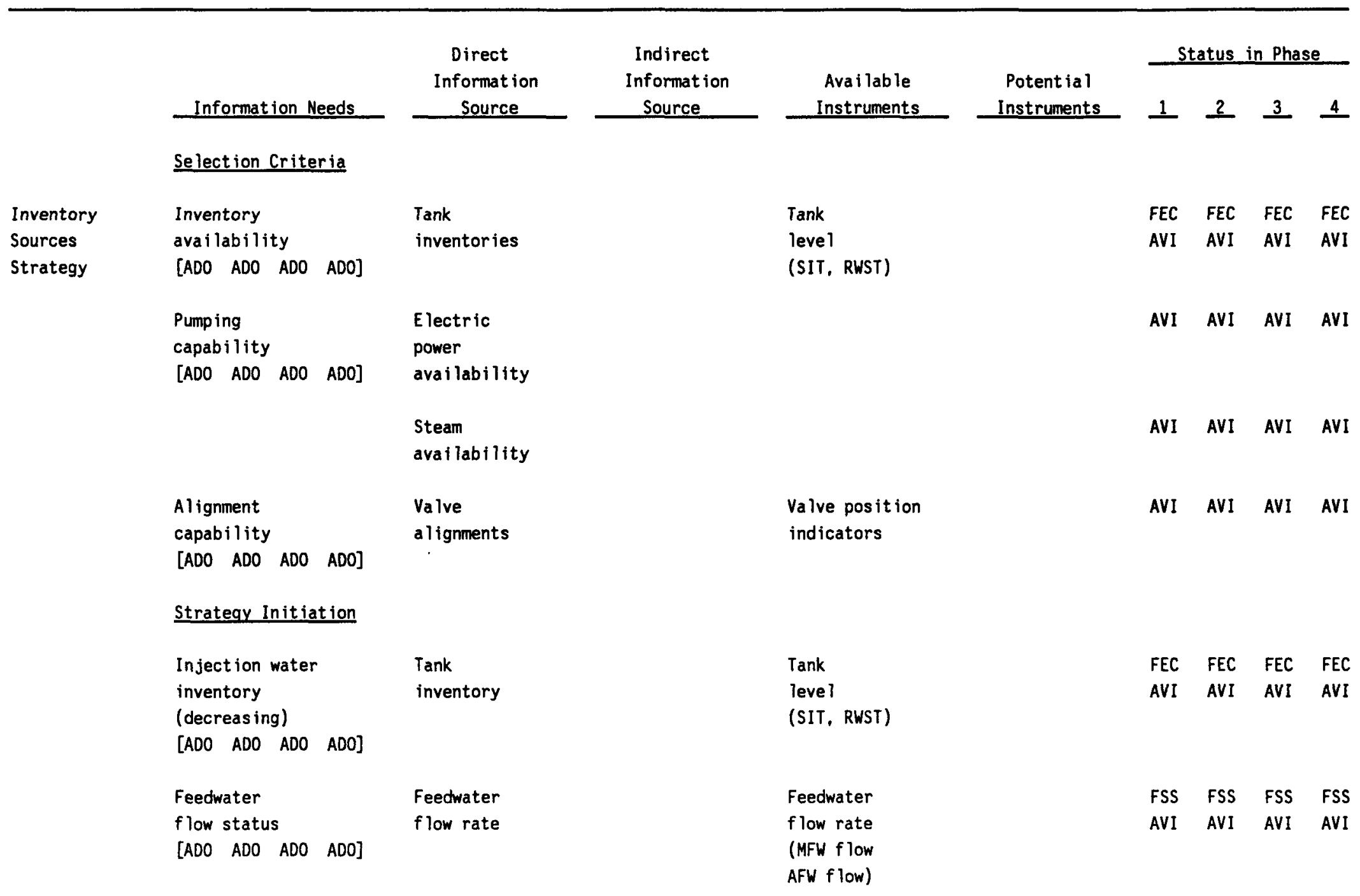


TABLE D1.3. (cont inued)

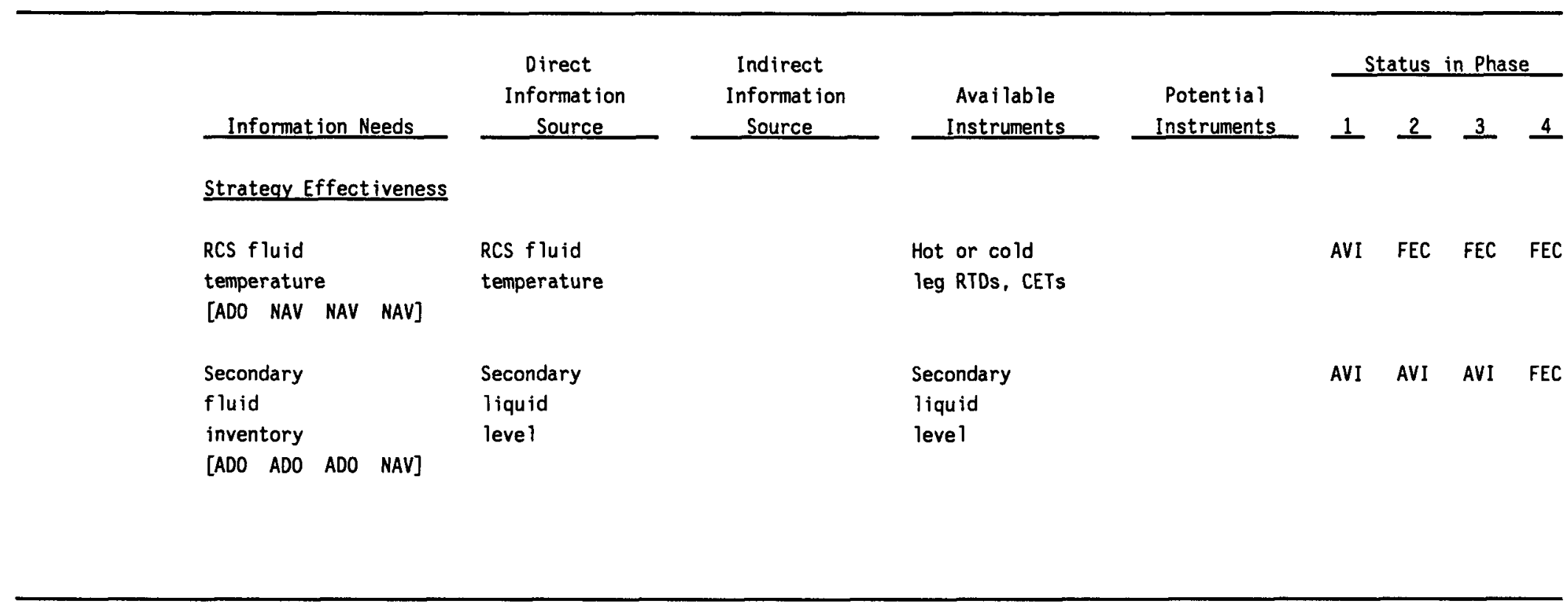


TABLE D1.4. PREVENT CORE DISPERSAL FROM VESSEL (V)--RESTRICTED RCS BLEED MECHANISM (V1B1)

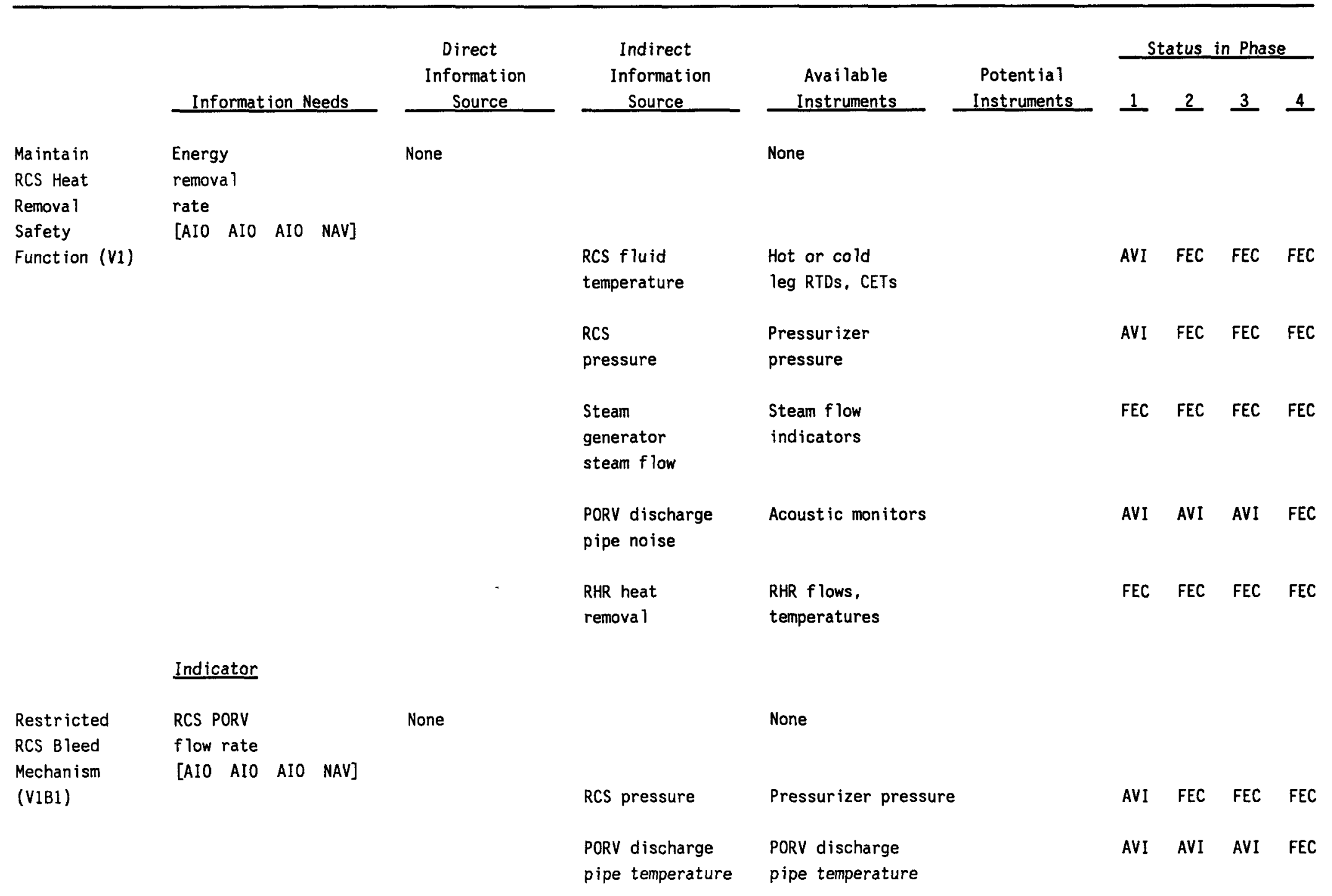


TABLE D1.4. (continued)

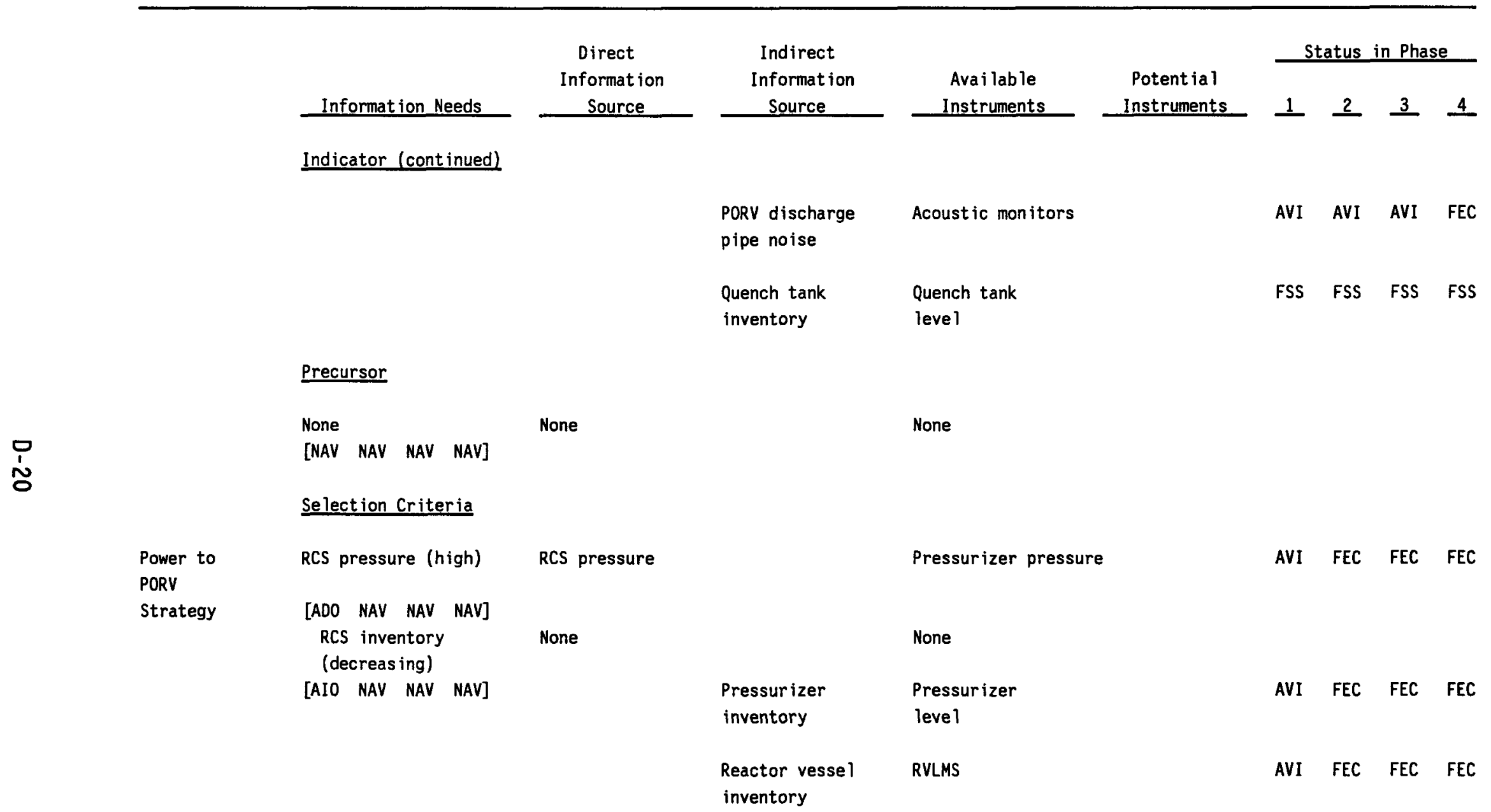


TABLE D1.4. (continued)

\begin{tabular}{|c|c|}
\hline Information Needs & $\begin{array}{c}\text { Direct } \\
\text { Information } \\
\text { Source } \\
\end{array}$ \\
\hline \multicolumn{2}{|l|}{ Strateay Initiation } \\
\hline $\begin{array}{l}\text { PORV f low } \\
\text { (conf irmation) }\end{array}$ & None \\
\hline [AIO AIO AIO NAV] & \\
\hline Strategy Effectiveness & \\
\hline RCS pressure history & RCS pressure \\
\hline [ADO NAV NAV NAV] & \\
\hline $\begin{array}{l}\text { RCS temperature } \\
\text { history }\end{array}$ & $\begin{array}{l}\text { RCS coolant } \\
\text { temperature }\end{array}$ \\
\hline$\left[\begin{array}{llll}A A D O & N A V & N A V & N A V\end{array}\right]$ & \\
\hline
\end{tabular}

\begin{tabular}{|c|c|}
\hline RCS pressure & Pressurizer pressure \\
\hline $\begin{array}{l}\text { PORV discharge } \\
\text { pipe temperature }\end{array}$ & $\begin{array}{l}\text { PORV discharge } \\
\text { pipe temperature }\end{array}$ \\
\hline $\begin{array}{l}\text { PORV discharge } \\
\text { pipe noise }\end{array}$ & Acoust ic monitors \\
\hline $\begin{array}{l}\text { Quench tank } \\
\text { inventory }\end{array}$ & $\begin{array}{l}\text { Quench tank } \\
\text { leve l }\end{array}$ \\
\hline
\end{tabular}

PORV flow

sensor

$\begin{array}{lllll}\text { Pressurizer pressure } & \text { AVI } & \text { FEC } & \text { FEC } & \text { FEC } \\ \begin{array}{l}\text { Subcooled margin } \\ \text { monitor }\end{array} & \text { AVI } & \text { FEC } & \text { FEC } & \text { FEC } \\ \begin{array}{l}\text { Cold and hot leg } \\ \text { RTDs, CETs }\end{array} & \text { AVI } & \text { FEC } & \text { FEC } & \text { FEC } \\ \end{array}$


TABLE 01.4. (continued)

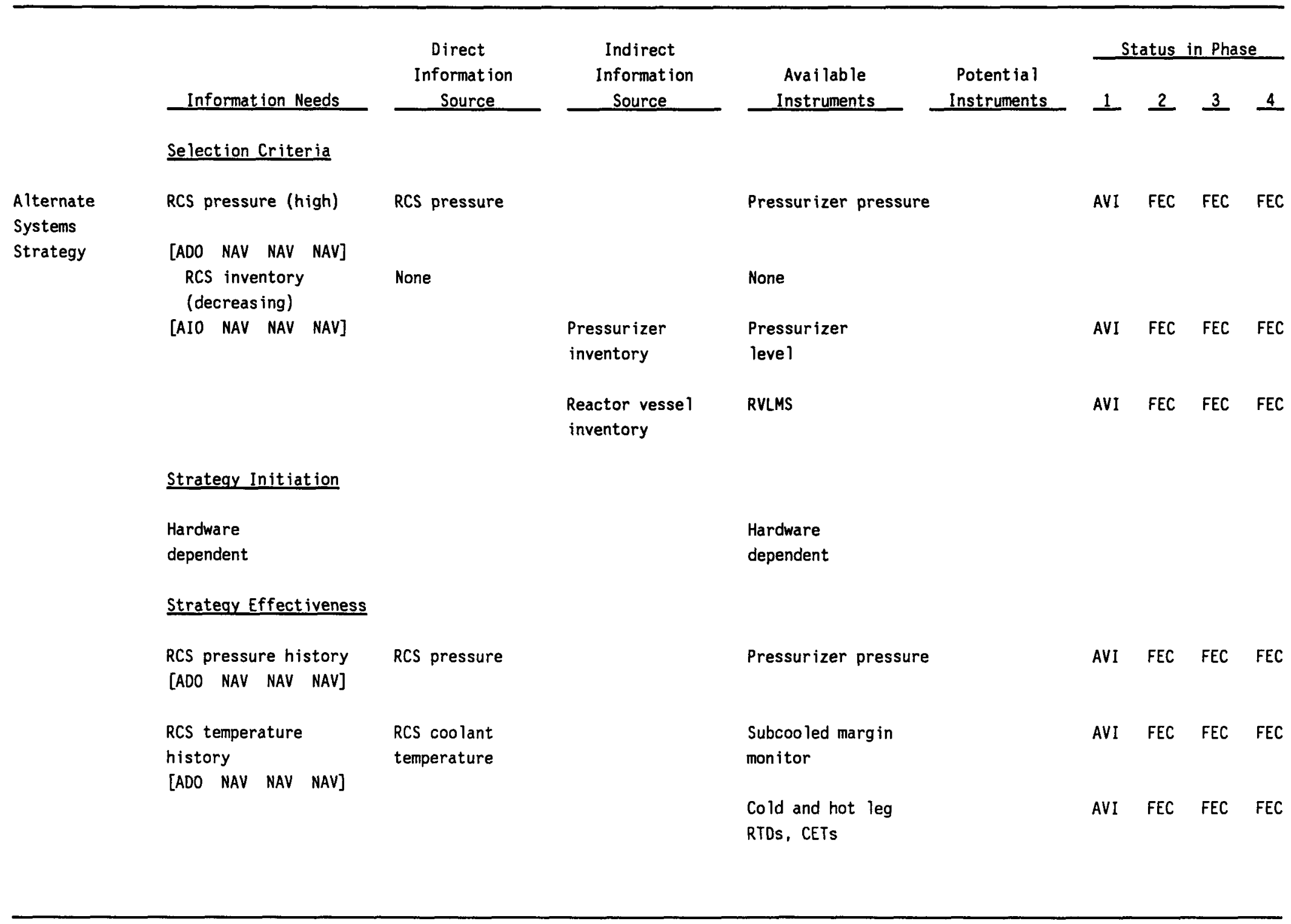


TABLE D1.5. PREVENT CORE DISPERSAL FROM VESSEL (V)--RHR SYSTEMS INOPERABLE (V1B2)

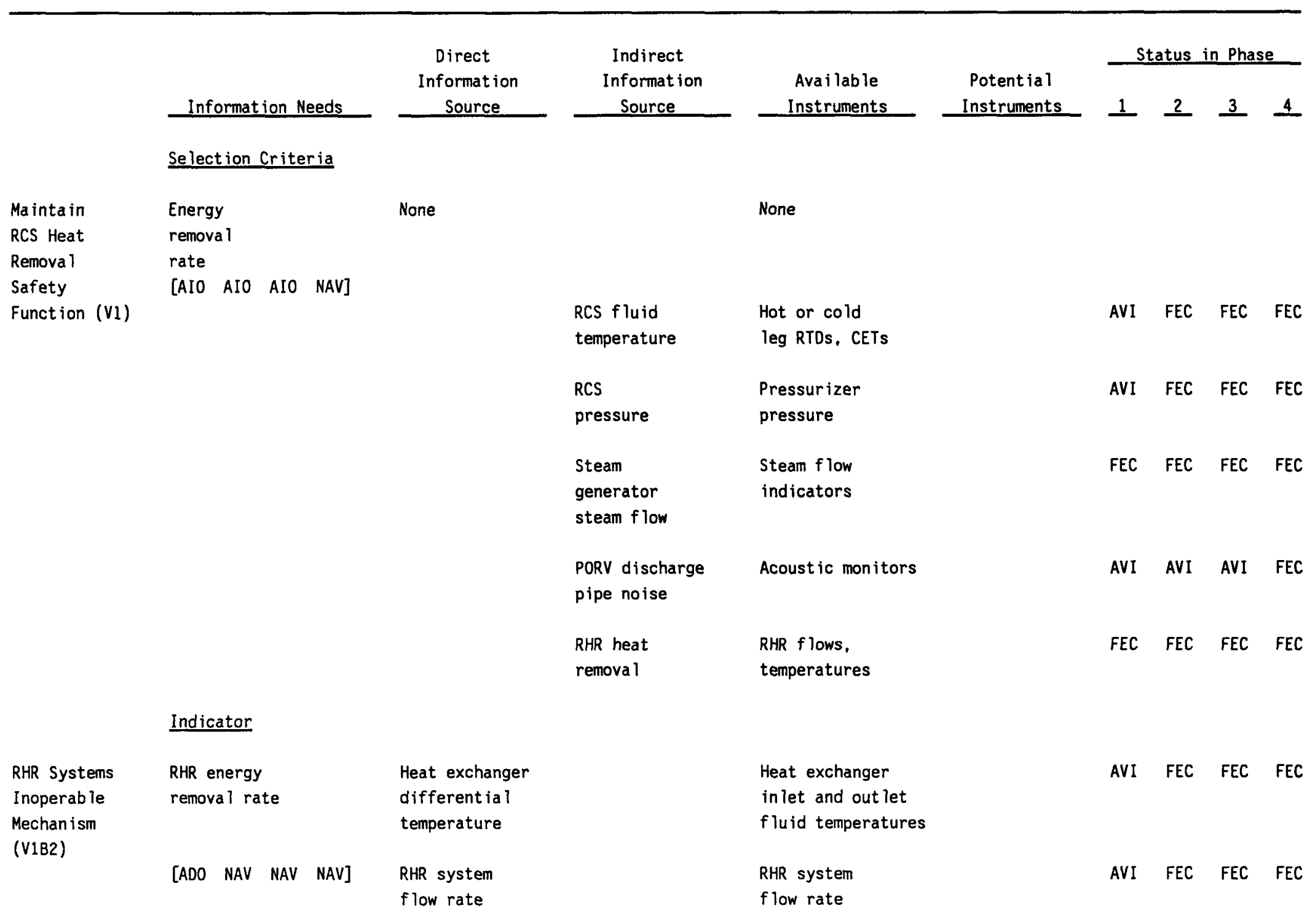


TABLE 01.5. (cont inued)

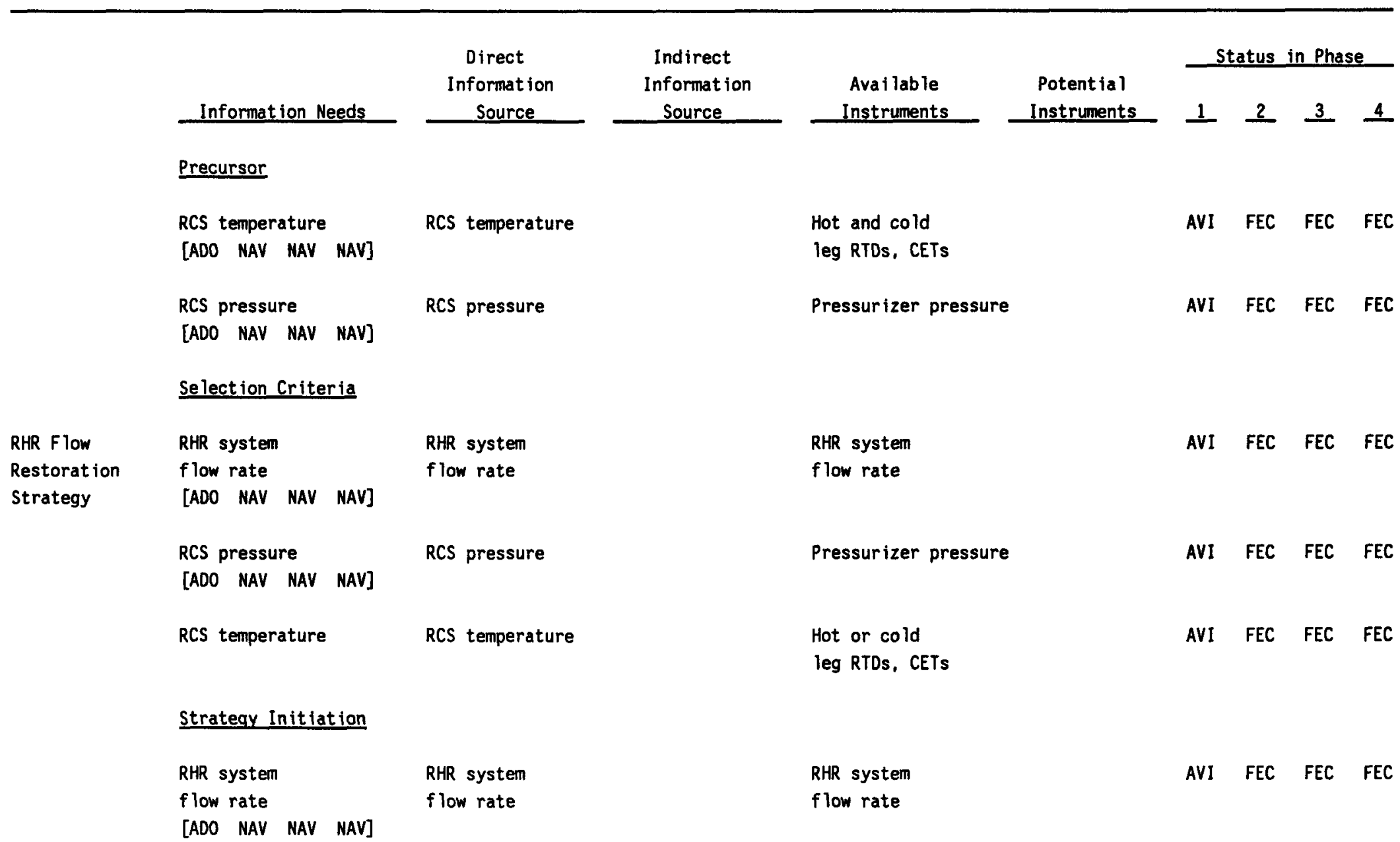


TABLE 01.5. (cont inued)

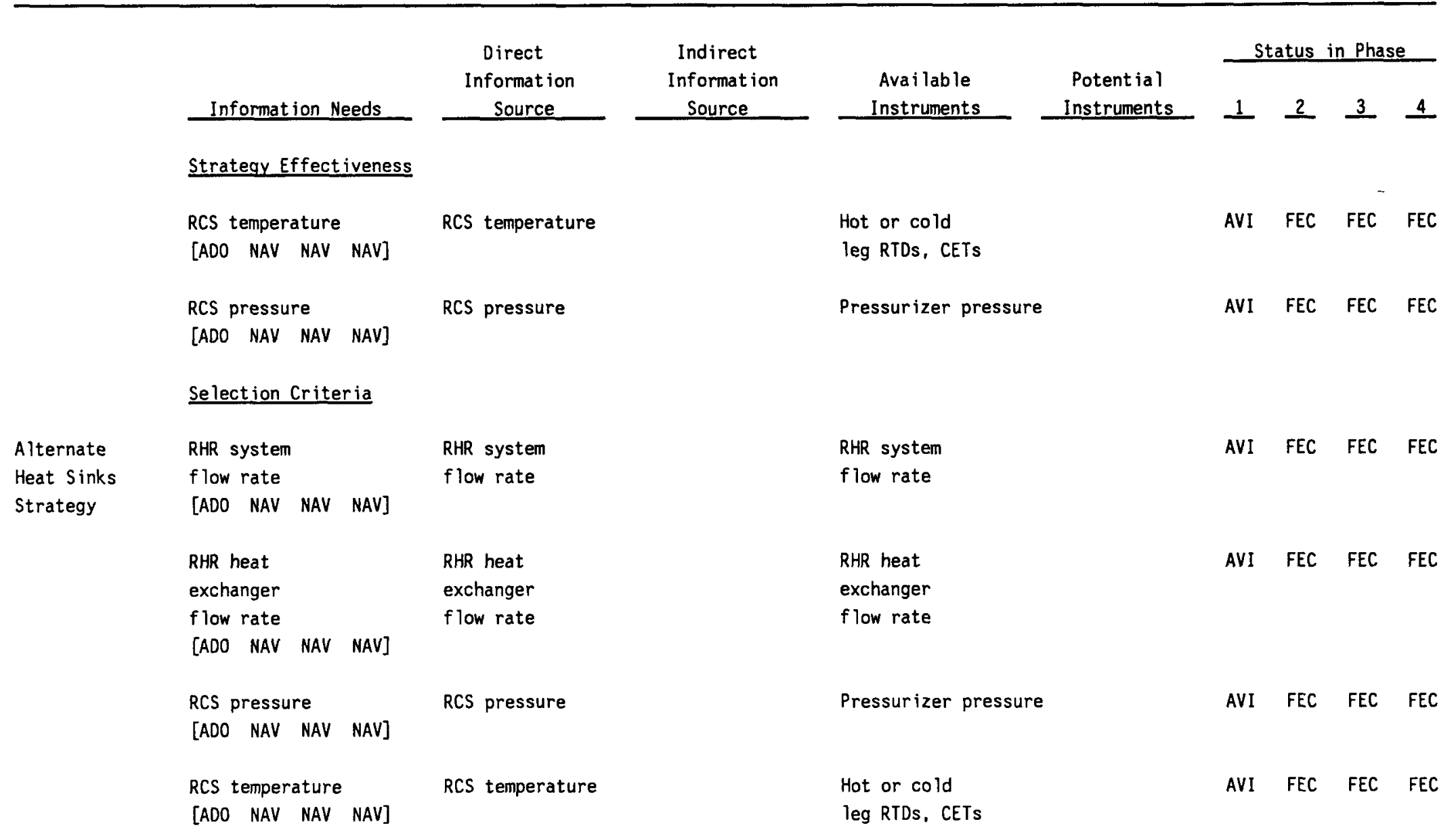


TABLE 01.5. (cont inued)

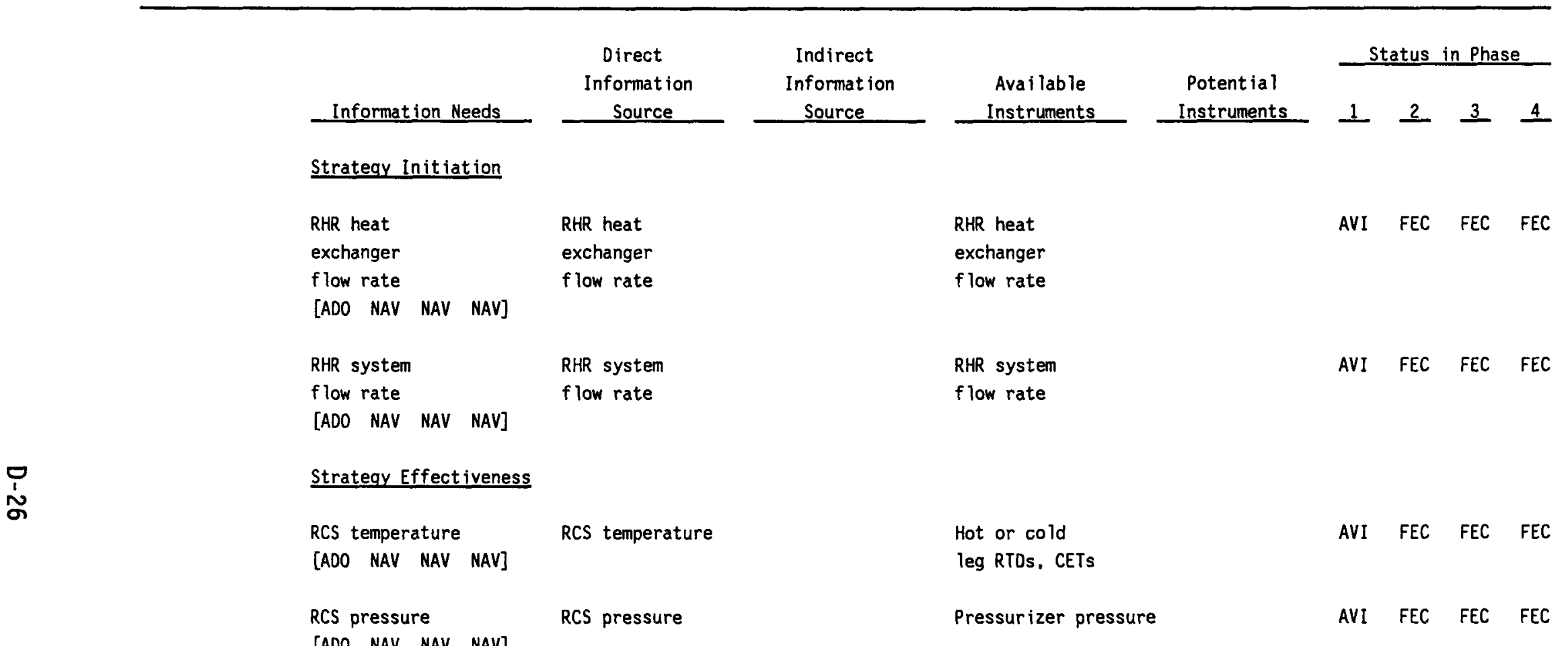


TABLE D1.6. PREVENT CORE DISPERSAL FROM VESSEL (V)--SCRAM FAILURE MECHANISM (V2A1)

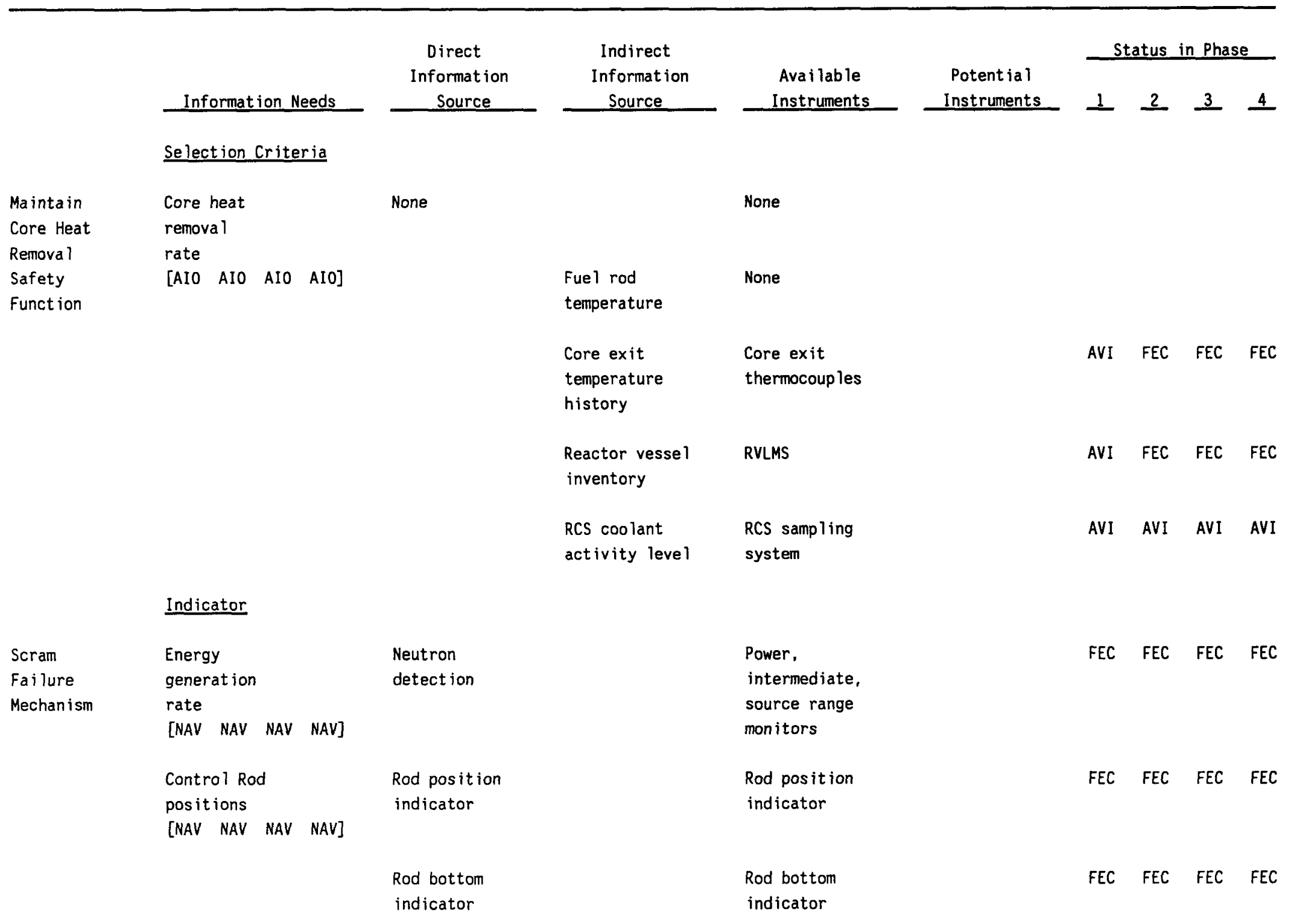


TABLE D1.6. (cont inued)

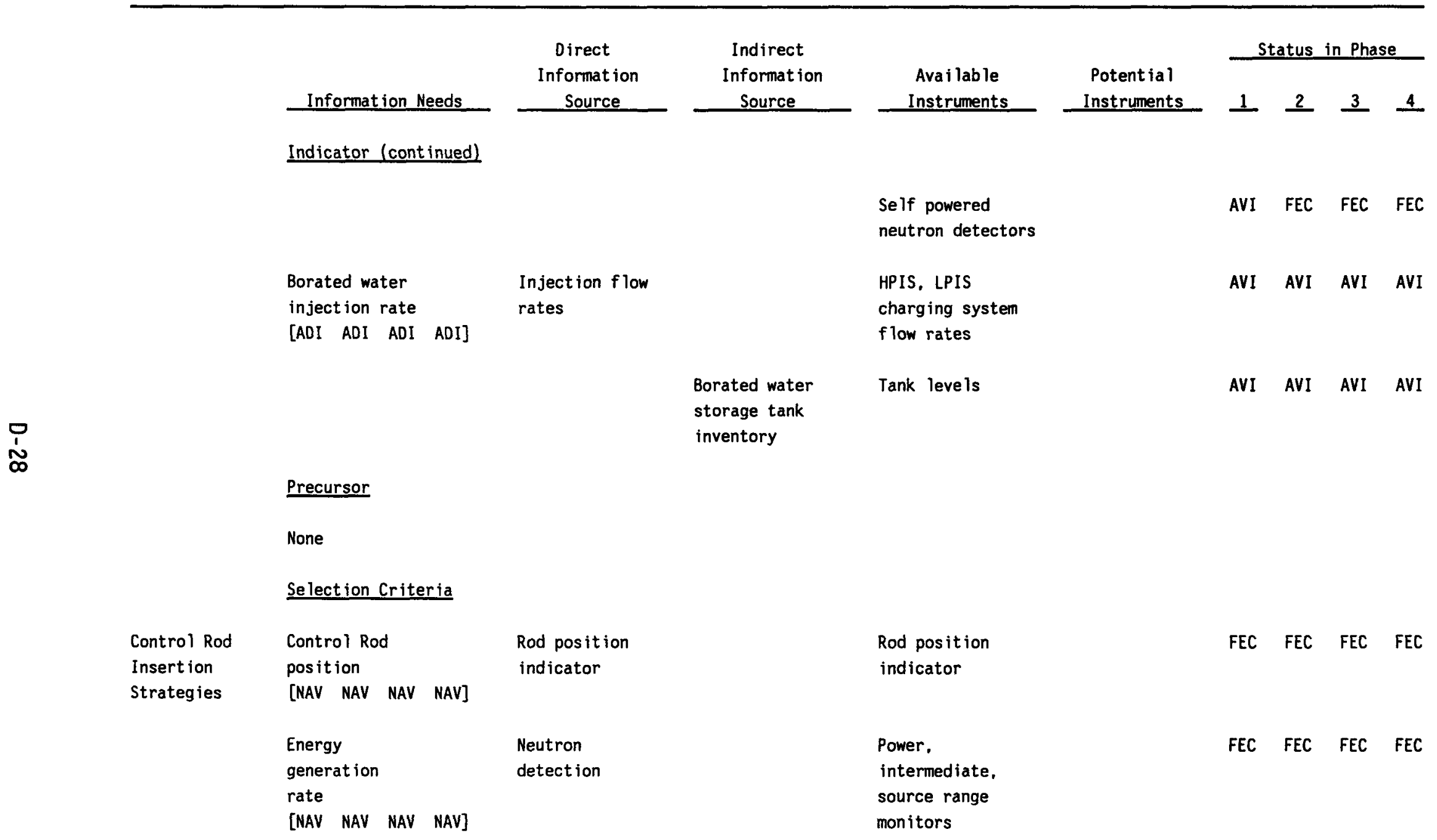


TABLE D1.6. (continued)

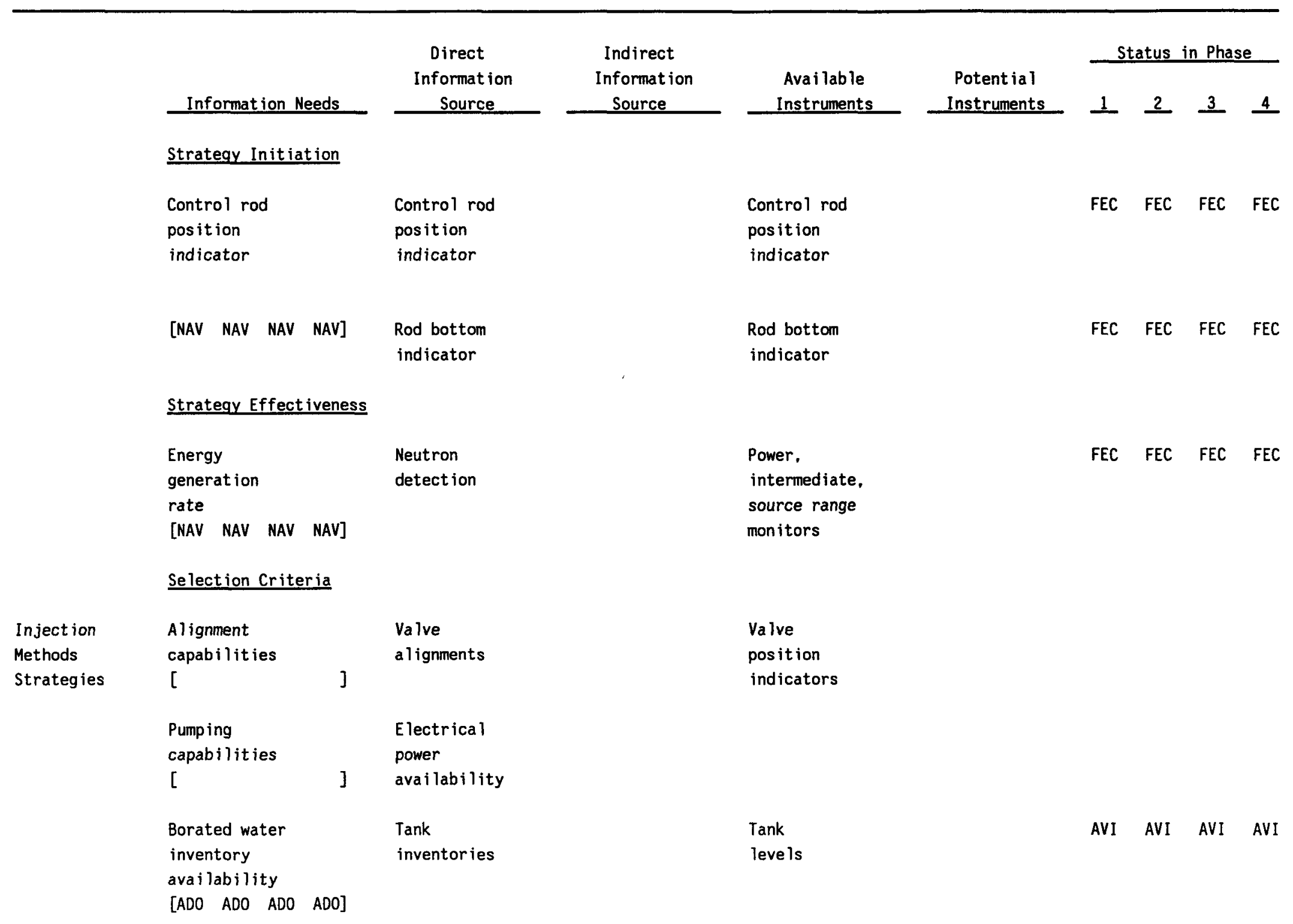


TABLE D1.6. (cont inued)

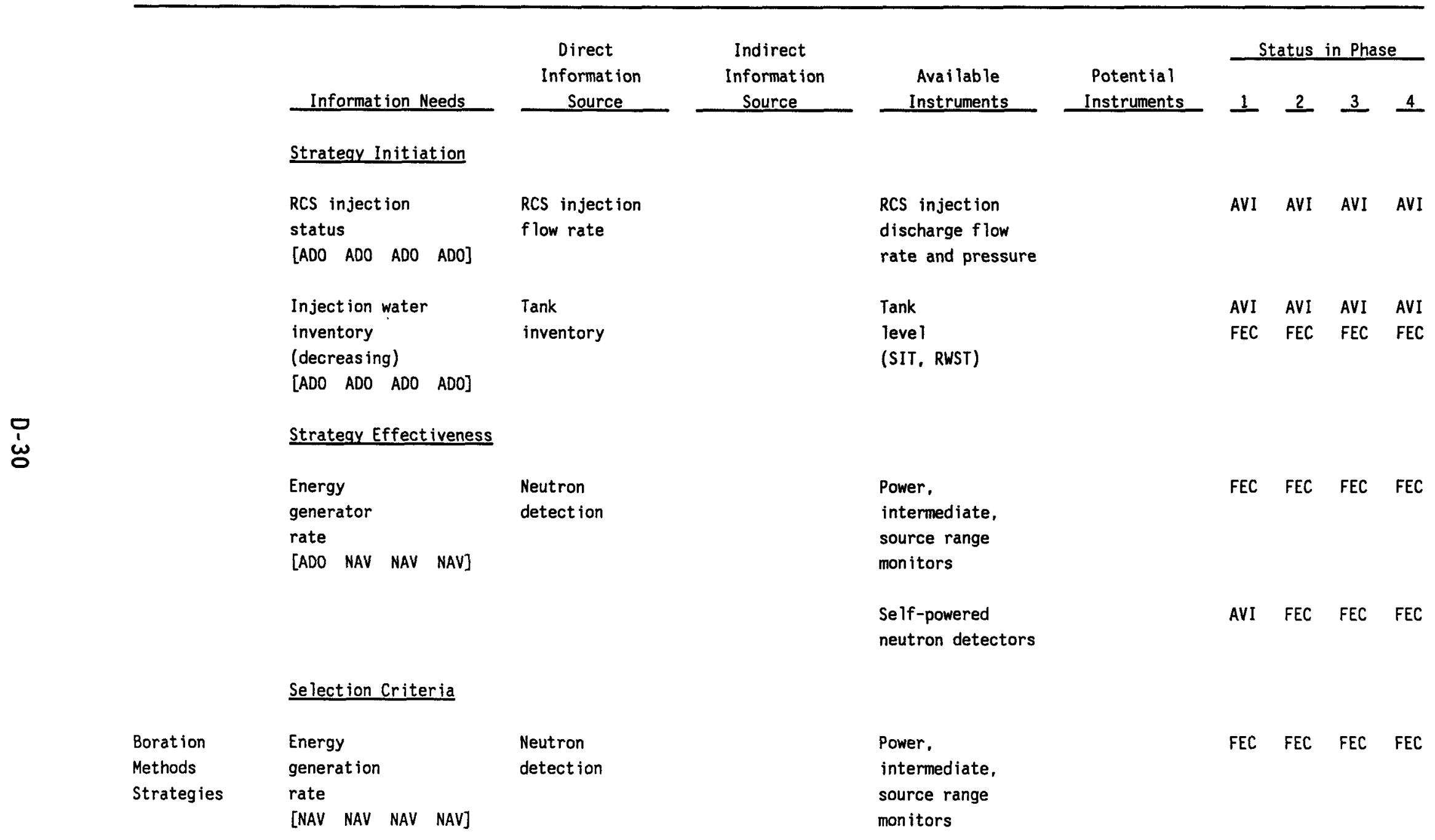


TABLE D1.6. (cont inued)

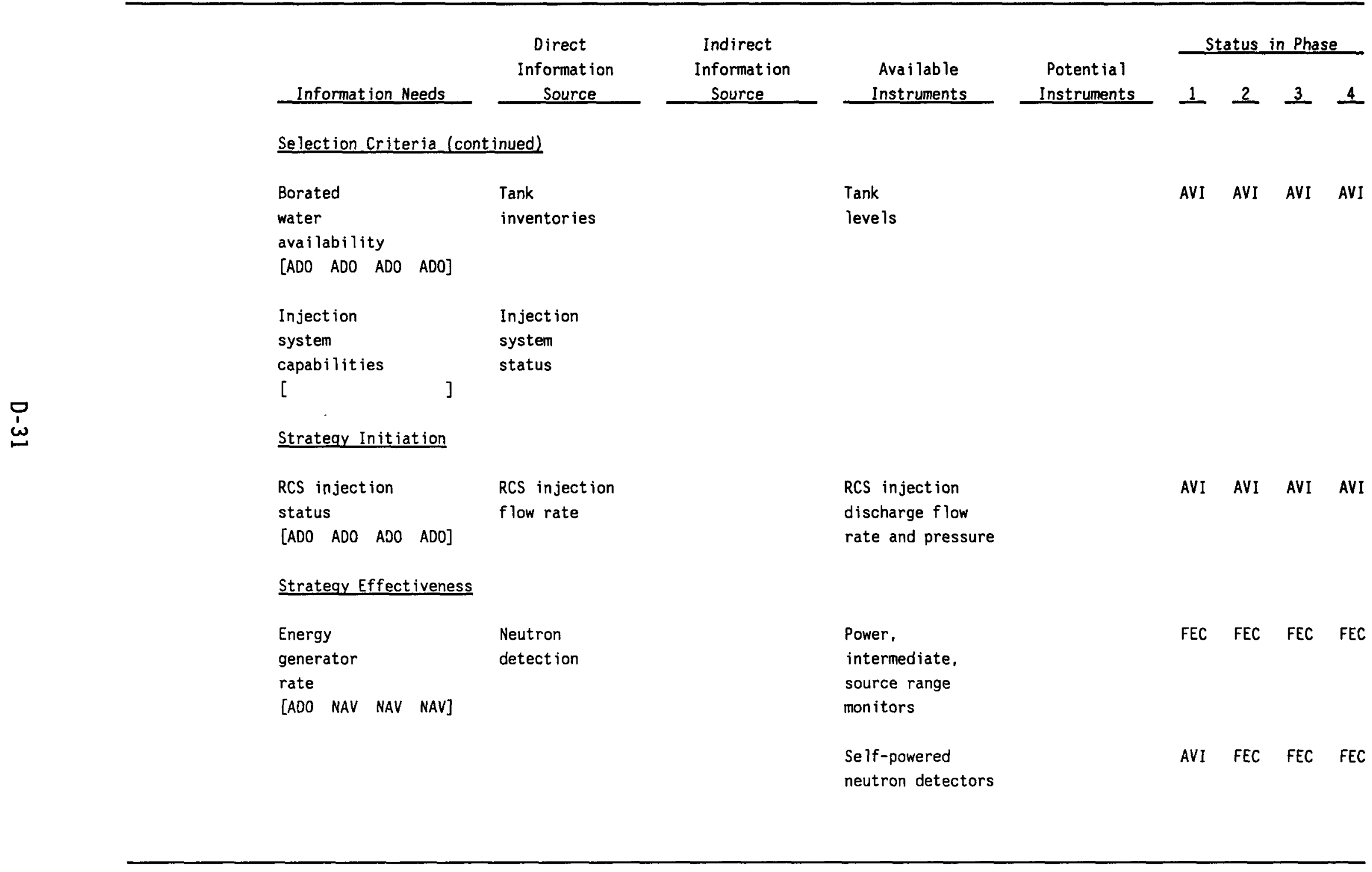


TABLE 01.7. PREVENT CORE DISPERSAL FROM VESSEL (V)--RECRITICALITY (VZA2)

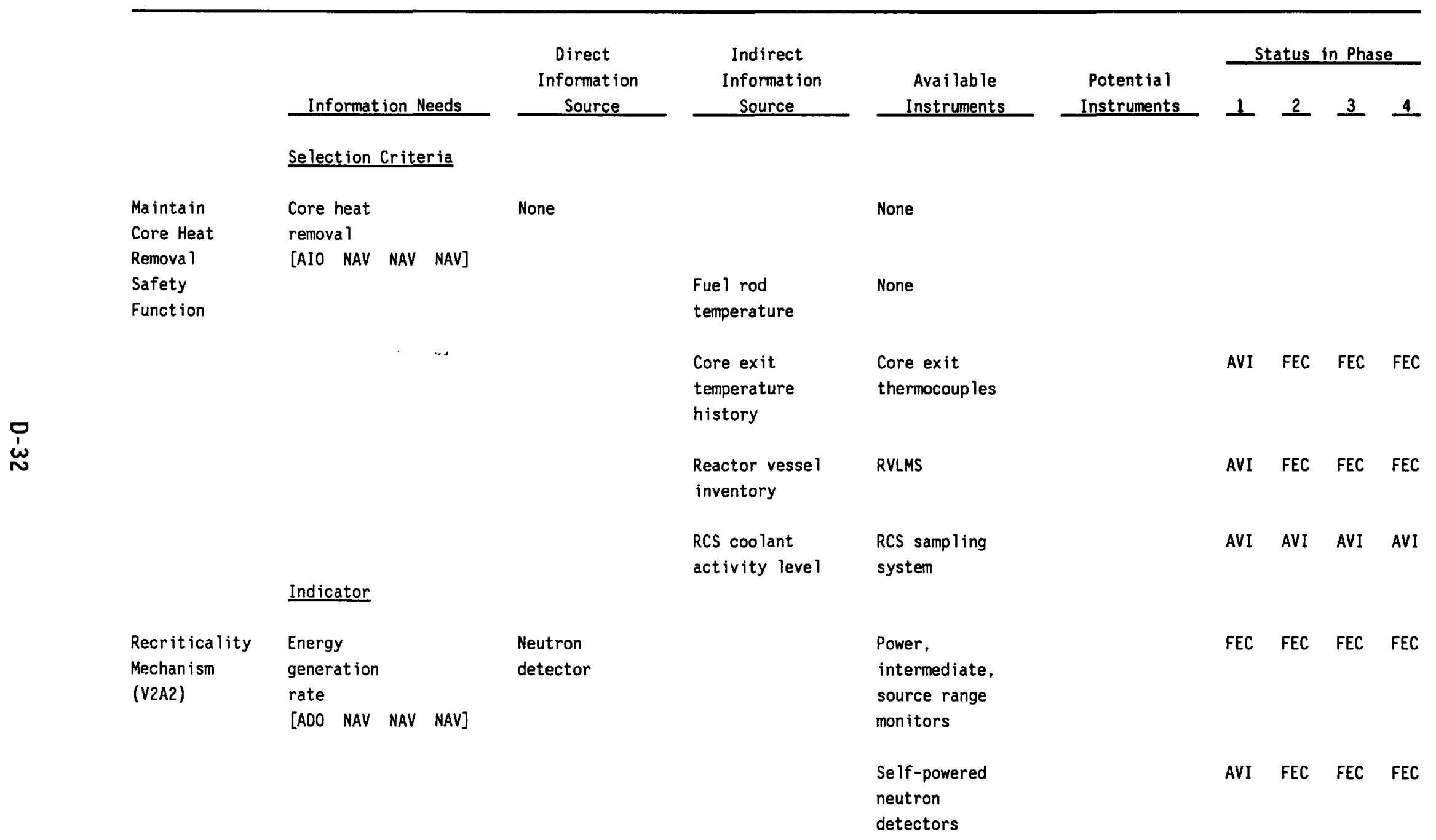


TABLE D1.7. (cont inued)

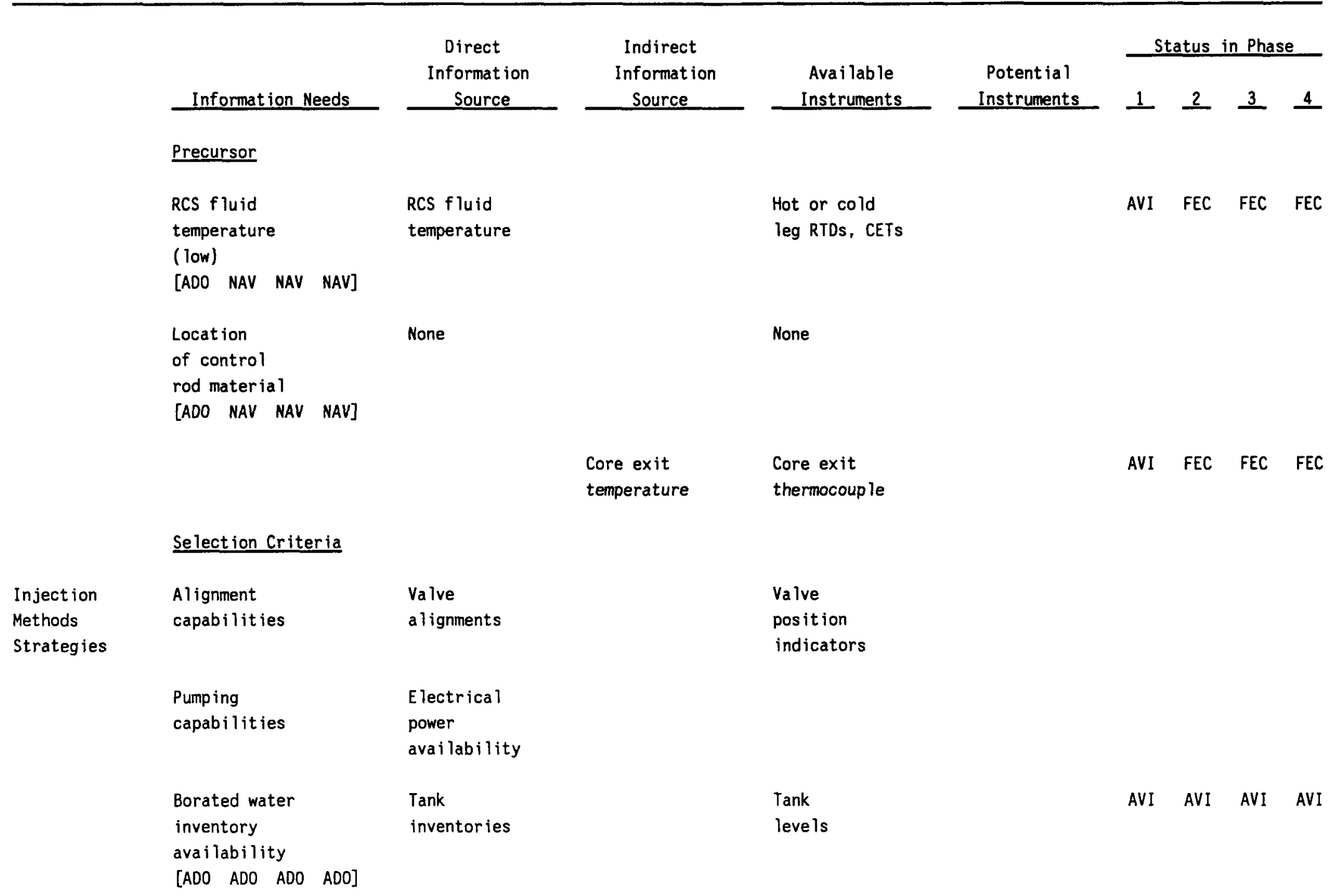


TABLE D1.7. (cont inued)

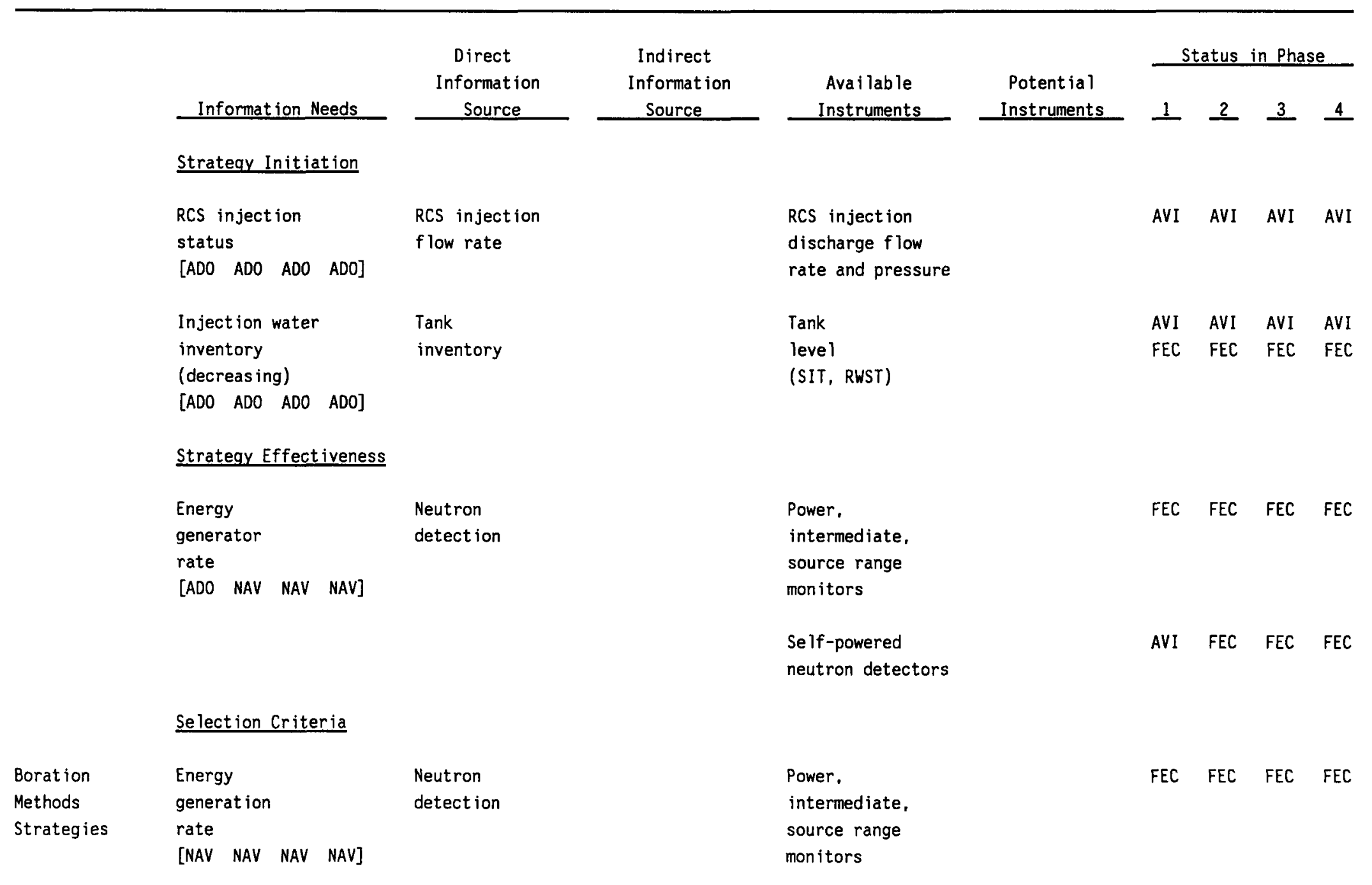


TABLE D1.7. (cont inued)

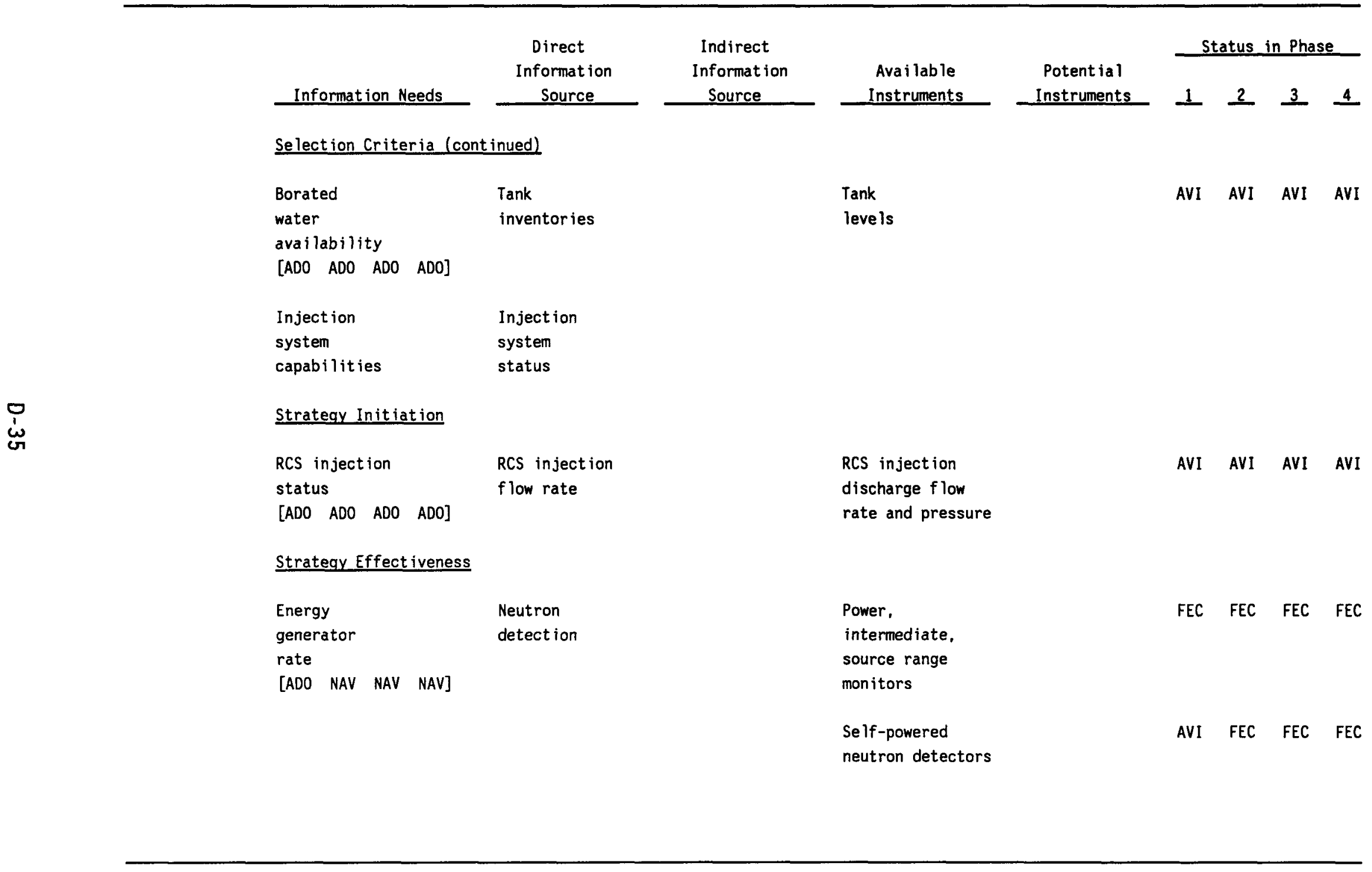


TABLE 01.8. PREVENT CORE DISPERSAL FROM VESSEL (V)--CIRCULATION FAILURE MECHANISM (V2A3)

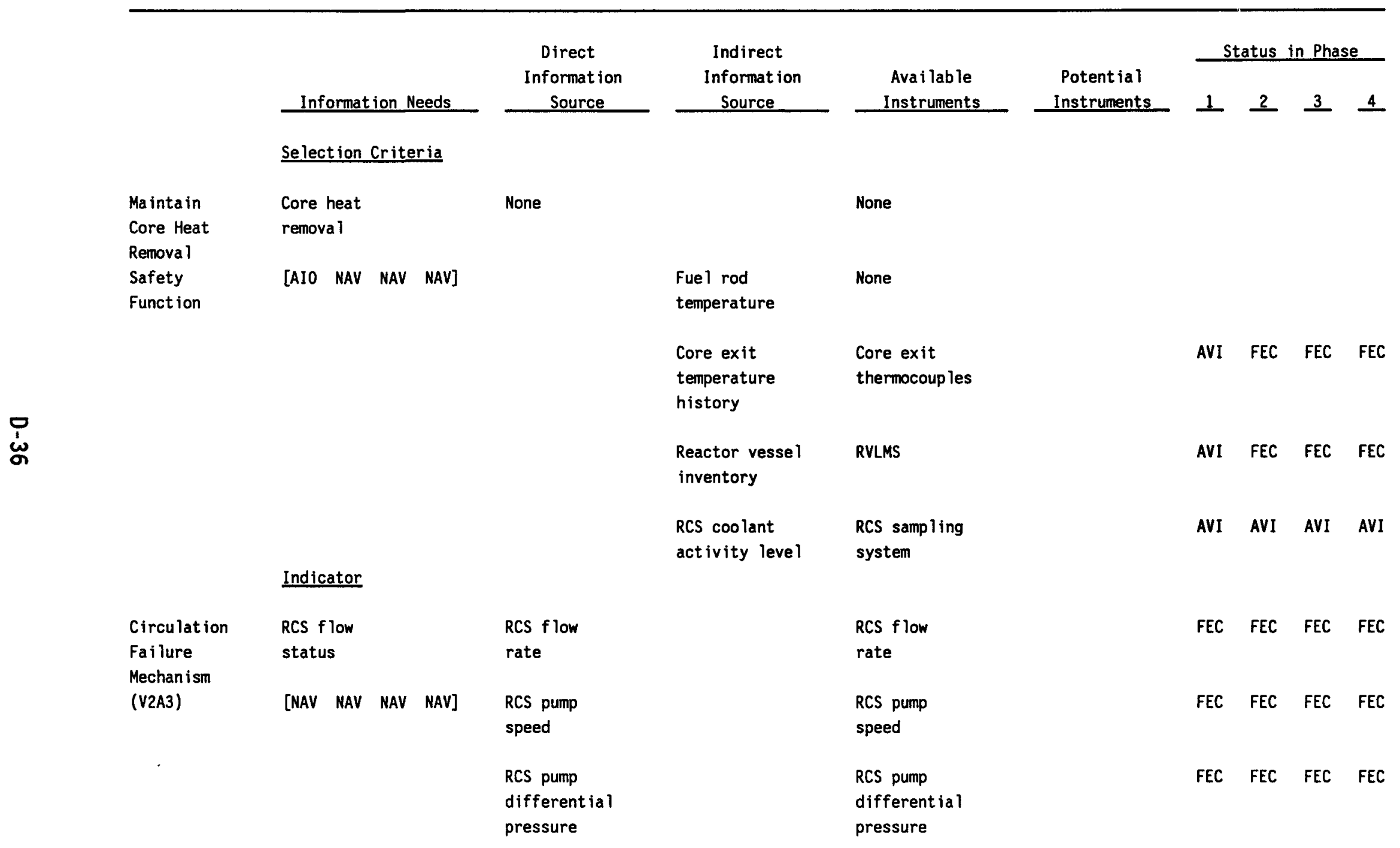


TABLE D1.8. (cont inued)

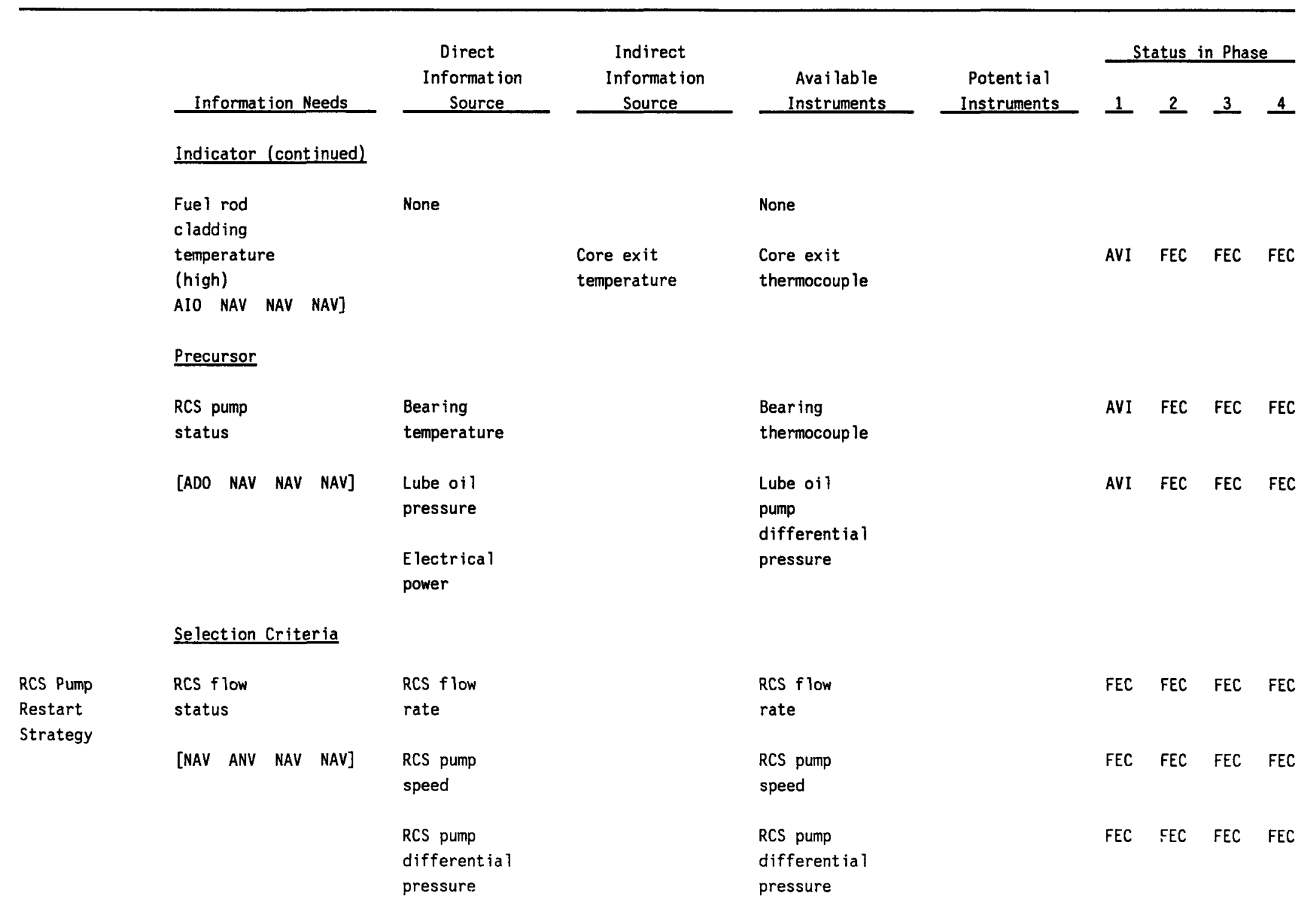


TABLE D1.8. (cont inued)

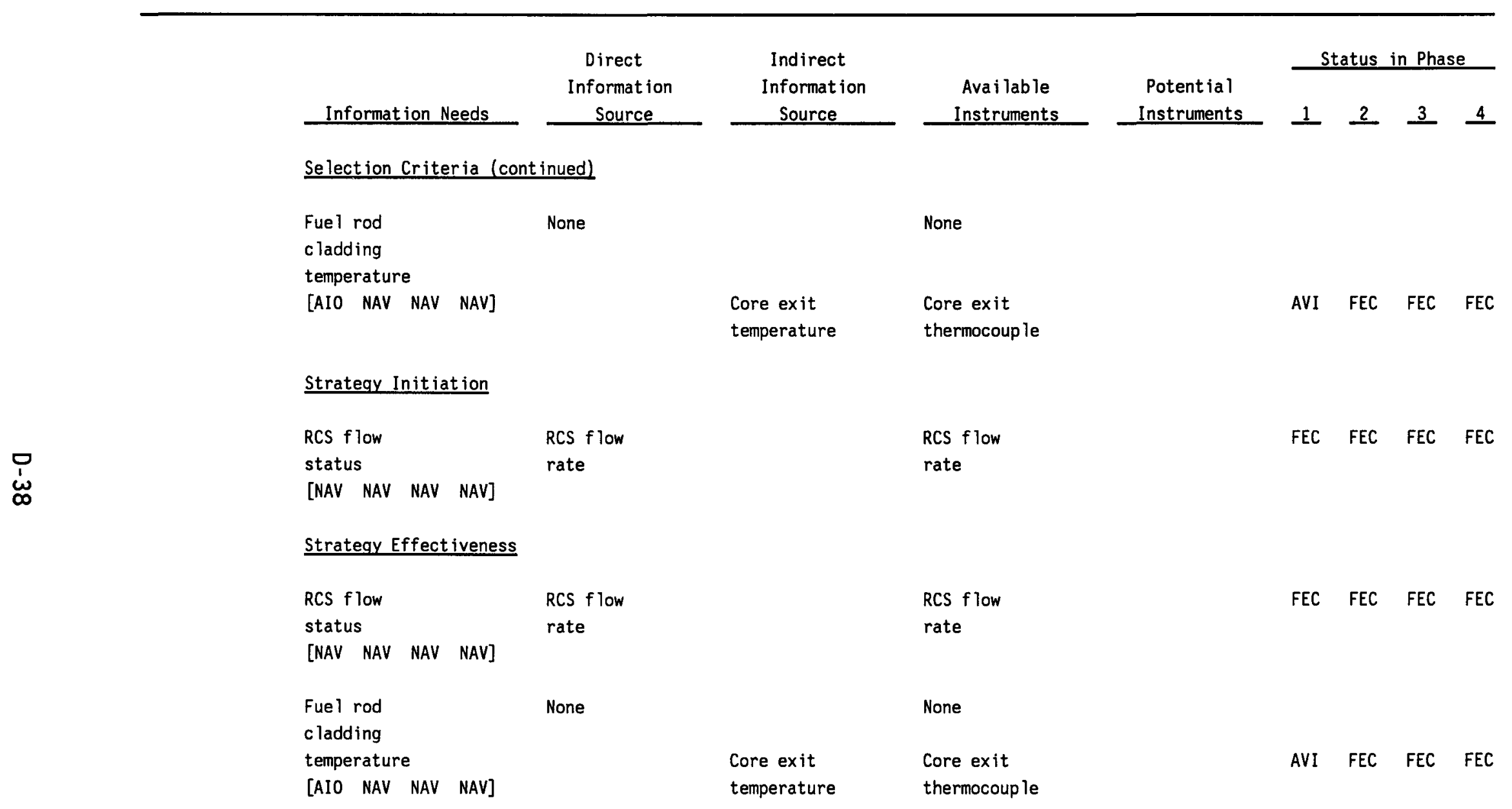


TABLE D1.9. PREVENT CORE DISPERSAL FROM VESSEL (V)--INADEQUATE RCS INVENTORY (V2A4)

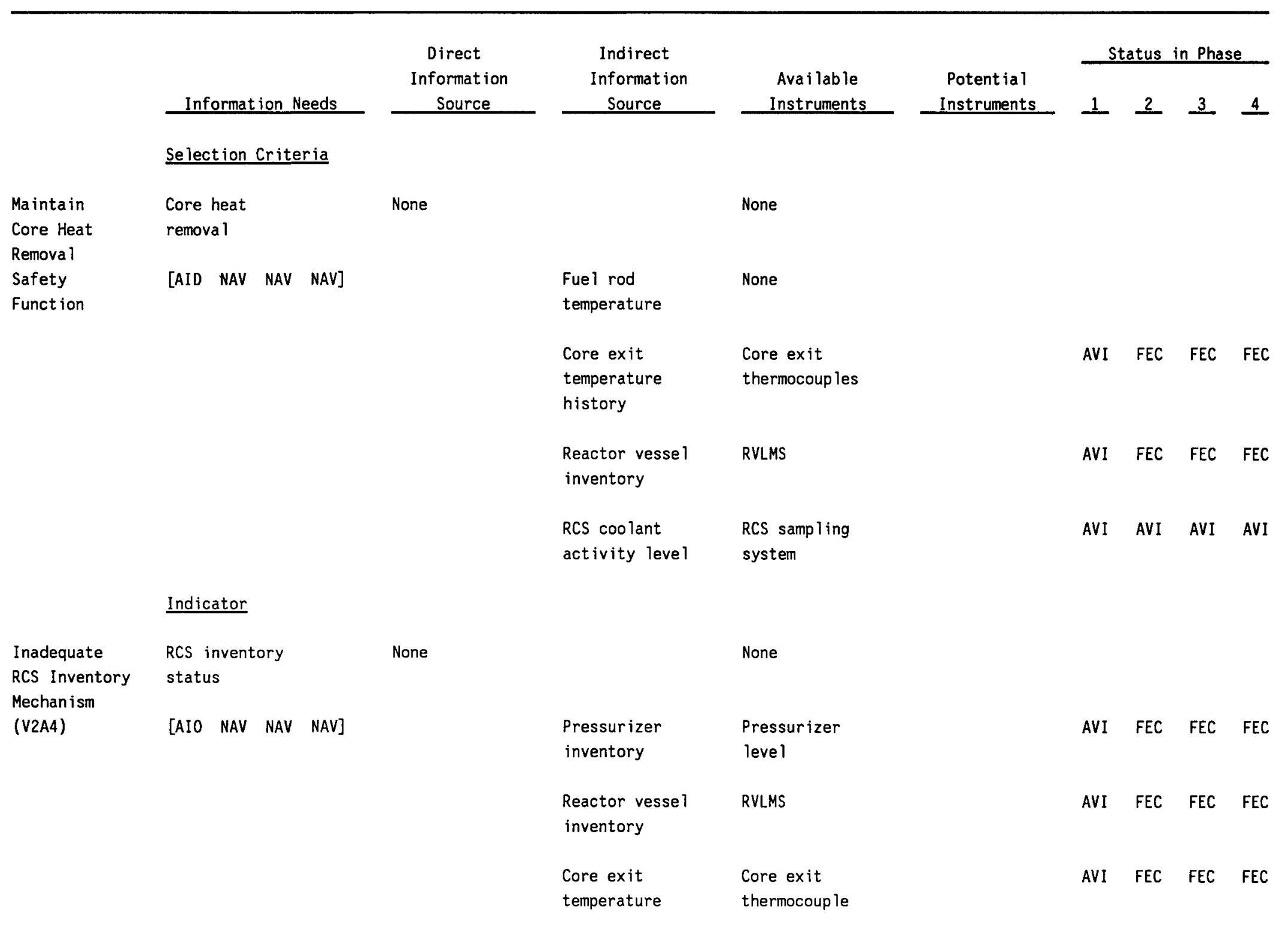


TABLE D1.9. (cont inued)

\begin{tabular}{|c|c|c|c|c|c|c|c|c|c|}
\hline & \multirow[b]{2}{*}{ Information Needs } & \multirow{2}{*}{$\begin{array}{c}\text { Direct } \\
\text { Information } \\
\text { Source } \\
\end{array}$} & \multirow{2}{*}{$\begin{array}{c}\text { Indirect } \\
\text { Informat ion } \\
\text { Source } \\
\end{array}$} & \multirow{2}{*}{$\begin{array}{c}\text { Avai lable } \\
\text { Instruments }\end{array}$} & \multirow{2}{*}{$\begin{array}{l}\text { Potential } \\
\text { Instruments }\end{array}$} & \multicolumn{4}{|c|}{ Status in Phase } \\
\hline & & & & & & 1 & 2 & 3 & 4 \\
\hline & $\begin{array}{l}\text { Conta inment } \\
\text { sump inventory } \\
\text { (increasing) }\end{array}$ & $\begin{array}{l}\text { Conta inment } \\
\text { sump leve } 1\end{array}$ & & $\begin{array}{l}\text { Conta inment } \\
\text { sump level }\end{array}$ & & AVI & AVI & AVI & FEC \\
\hline & \multicolumn{9}{|l|}{ Precursor } \\
\hline & $\begin{array}{l}\text { RCS pressure } \\
\text { (low) }\end{array}$ & RCS pressure & & \multirow{2}{*}{\multicolumn{2}{|c|}{$\begin{array}{l}\text { Pressurizer } \\
\text { pressure }\end{array}$}} & AVI & FEC & FEC & FEC \\
\hline & {$\left[\begin{array}{llll}A A D O & N A V & N A V & N A V\end{array}\right]$} & & & & & & & & \\
\hline & \multicolumn{9}{|l|}{ Selection Criteria } \\
\hline RCS Injection & $\begin{array}{l}\text { RCS pressure history } \\
\text { [ADO NAV NAV NAV] }\end{array}$ & RCS pressure & & \multicolumn{2}{|l|}{ Pressurizer pressure } & AVI & FEC & FEC & FEC \\
\hline Strategies & $\begin{array}{l}\text { RCS inventory } \\
\text { (trend) }\end{array}$ & None & & \multicolumn{2}{|l|}{ None } & & & & \\
\hline & [AIO NAV NAV NAV] & & $\begin{array}{l}\text { Pressurizer } \\
\text { inventory }\end{array}$ & \multicolumn{2}{|l|}{ Pressurizer leve 1} & AVI & FEC & FEC & FEC \\
\hline & & & $\begin{array}{l}\text { Reactor vesse } 1 \\
\text { inventory }\end{array}$ & \multicolumn{2}{|l|}{ RVLMS } & AVI & FEC & FEC & FEC \\
\hline
\end{tabular}


TABLE D1.9. (cont inued)

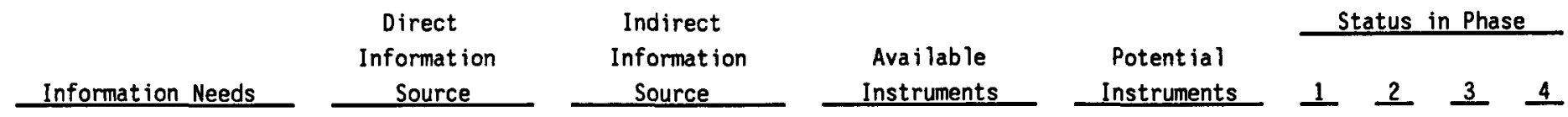

Selection Criteria (cont inued)

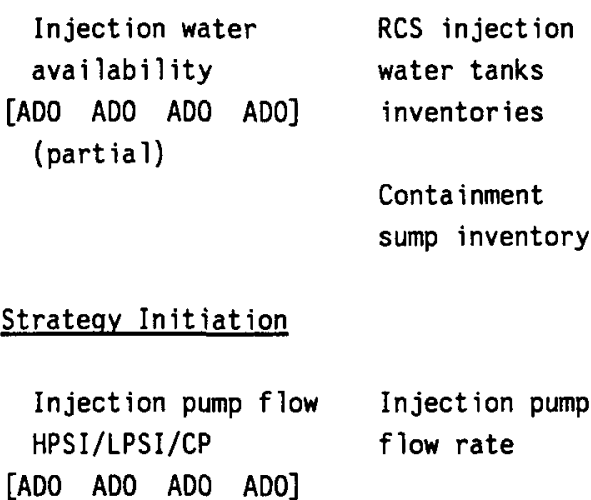

Tank leve $1 \mathrm{~s}$

(SIT, RWST, VCT)

Sump levels

AVI AVI AVI AVI

AVI FEC FEC FEC

AVI FEC FEC FEC

[ADO ADO ADO AD0]

$\begin{array}{ll}\text { Injection water } & \text { RCS injection } \\ \text { availability } & \text { water tanks } \\ \text { (decreasing) } & \text { inventories } \\ {[A D O A D O A D O A D O]} & \\ \text { (partial) } & \end{array}$

AVI AVI AVI AVI

Pump out let

AVI AVI AVI AVI

pressure

Pump motor

AVI AVI AVI AVI

current

Tank leve I

(SIT, RWST, VCT)

AVI AVI AVI AVI
AVI FEC FEC FEC 
TABLE D1.9. (cont inued)

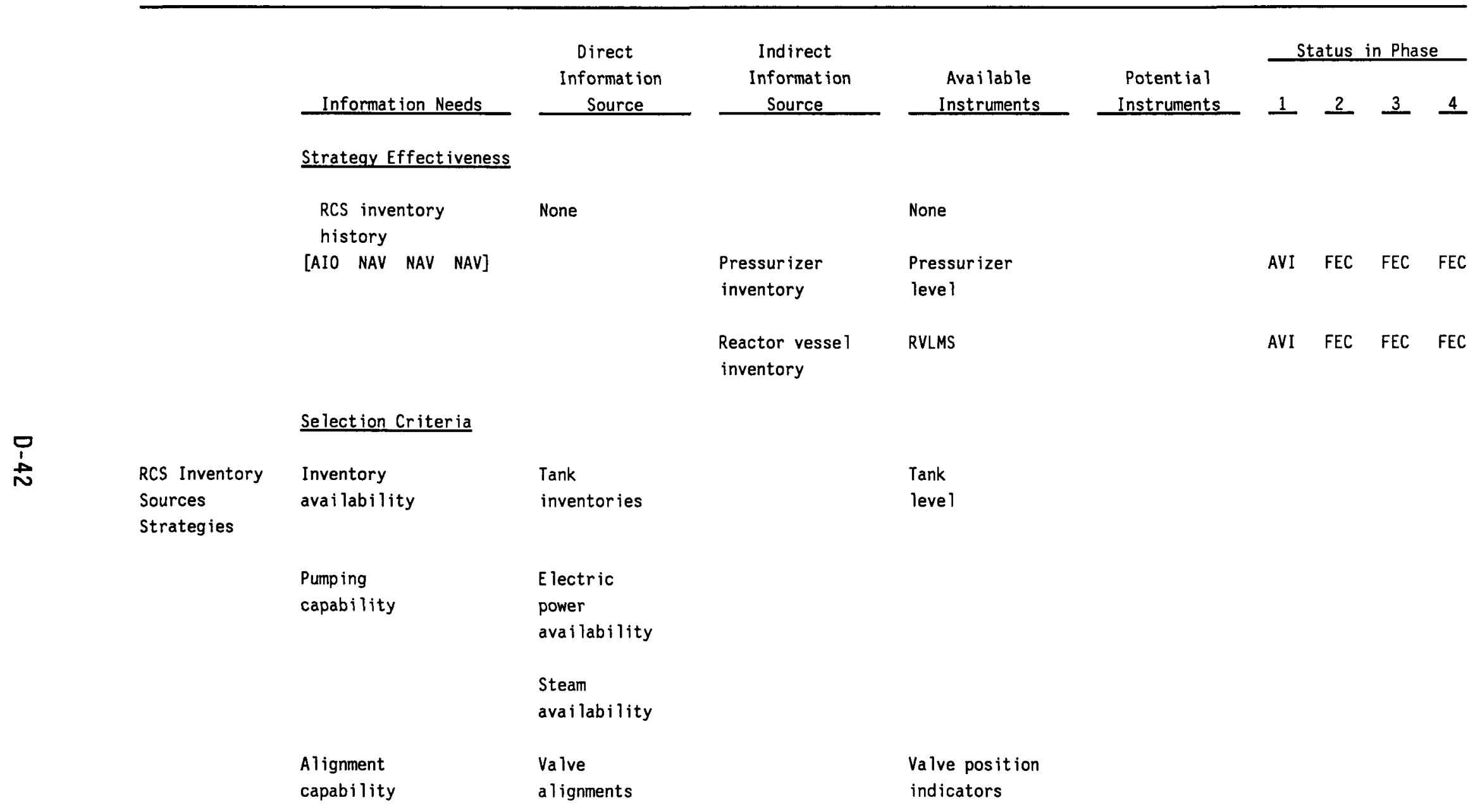


TABLE D1.9. (cont inued)

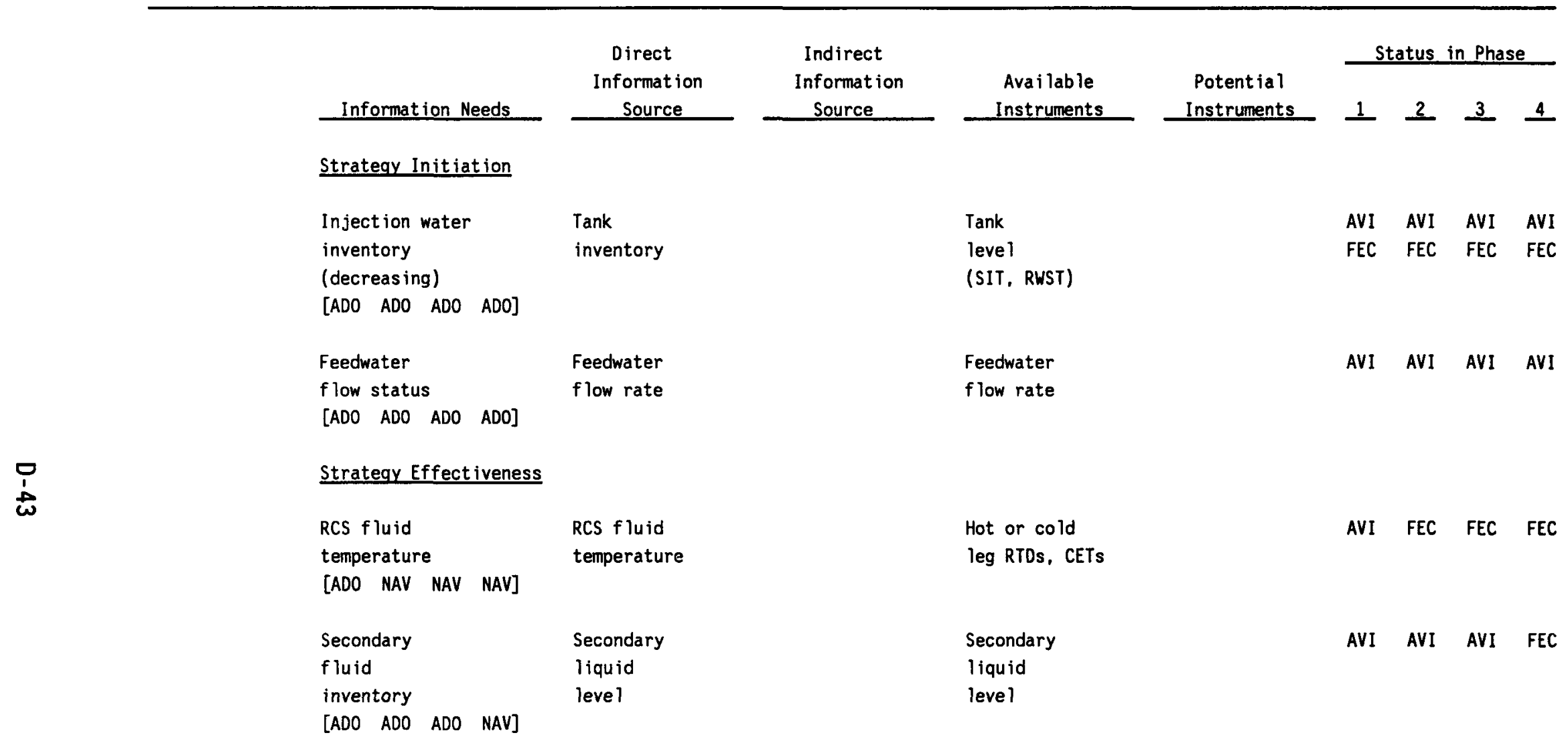


TABLE D1.10. CHANGE IN CORE GEOMETRY MECHANISM (V2B1)

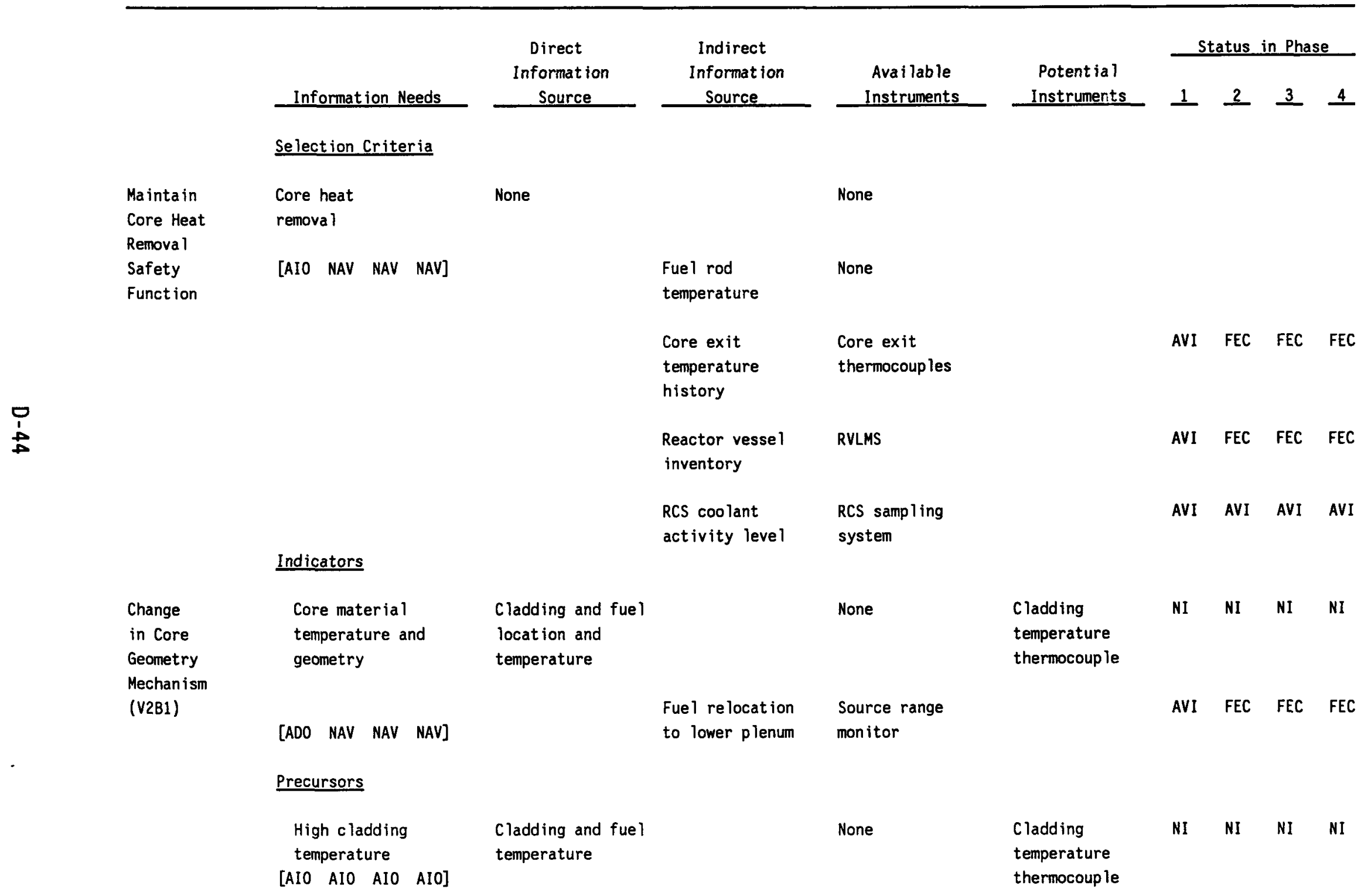


TABLE D1.10. (cont inued)

\begin{tabular}{|c|c|c|c|c|c|c|c|c|c|}
\hline & & Direct & & & & \multicolumn{4}{|c|}{ Status in Phase } \\
\hline & Information Needs & $\begin{array}{c}\text { Information } \\
\text { Source }\end{array}$ & $\begin{array}{c}\text { Information } \\
\text { Source }\end{array}$ & $\begin{array}{c}\text { Available } \\
\text { Instruments }\end{array}$ & $\begin{array}{l}\text { Potential } \\
\text { Instruments }\end{array}$ & 1 & 2 & 3 & 4 \\
\hline & \multicolumn{9}{|l|}{ Precursors (cont inued) } \\
\hline & & & $\begin{array}{l}\text { Core exit } \\
\text { temperature }\end{array}$ & $\begin{array}{l}\text { Core exit } \\
\text { thermocoup les }\end{array}$ & & AVI & FEC & FEC & FEC \\
\hline & & & $\begin{array}{l}\text { Cladding } \\
\text { temperature }\end{array}$ & SPNDS & & AVI & FEC & FEC & FEC \\
\hline & & & $\begin{array}{l}\text { Coolant activity } \\
\text { level }\end{array}$ & $\begin{array}{l}\text { RCS sampling } \\
\text { system }\end{array}$ & & AVI & AVI & AVI & AVI \\
\hline & \multicolumn{9}{|l|}{ Selection Criteria } \\
\hline RCS Pump & \multirow{2}{*}{$\begin{array}{l}\text { RCS flow } \\
\text { status }\end{array}$} & RCS flow & & \multirow{2}{*}{\multicolumn{2}{|c|}{$\begin{array}{l}\text { RCS flow } \\
\text { rate }\end{array}$}} & FEC & FEC & FEC & FEC \\
\hline Restart & & rate & & & & & & & \\
\hline & \multirow[t]{2}{*}{ [NAV NAV NAV NAV] } & $\begin{array}{l}\text { RCS pump } \\
\text { speed }\end{array}$ & & $\begin{array}{l}\text { RCS pump } \\
\text { speed }\end{array}$ & & FEC & FEC & FEC & FEC \\
\hline & & $\begin{array}{l}\text { RCS pump } \\
\text { differential } \\
\text { pressure }\end{array}$ & & $\begin{array}{l}\text { RCS pump } \\
\text { differential } \\
\text { pressure }\end{array}$ & & FEC & FEC & FEC & FEC \\
\hline & \multicolumn{2}{|l|}{$\begin{array}{l}\text { Fuel rod } \\
\text { cladding } \\
\text { temperature }\end{array}$} & & None & & & & & \\
\hline & [AIO NAV NAV NAV] & & $\begin{array}{l}\text { Core exit } \\
\text { temperature }\end{array}$ & $\begin{array}{l}\text { Core exit } \\
\text { thermocouple }\end{array}$ & & ANI & FEC & FEC & FEC \\
\hline
\end{tabular}


TABLE 01.10. (continued)

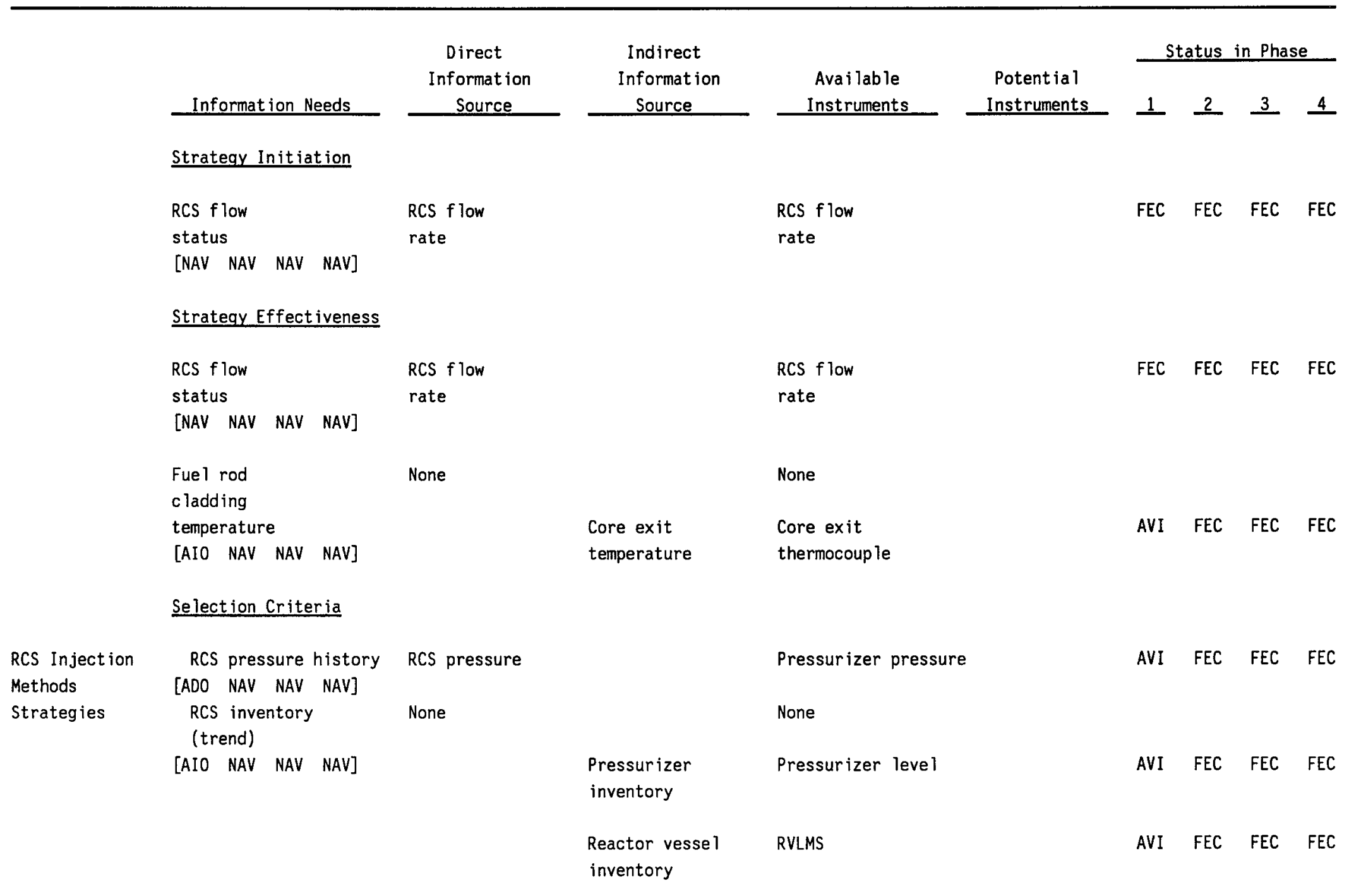


TABLE D1.10. (cont inued)

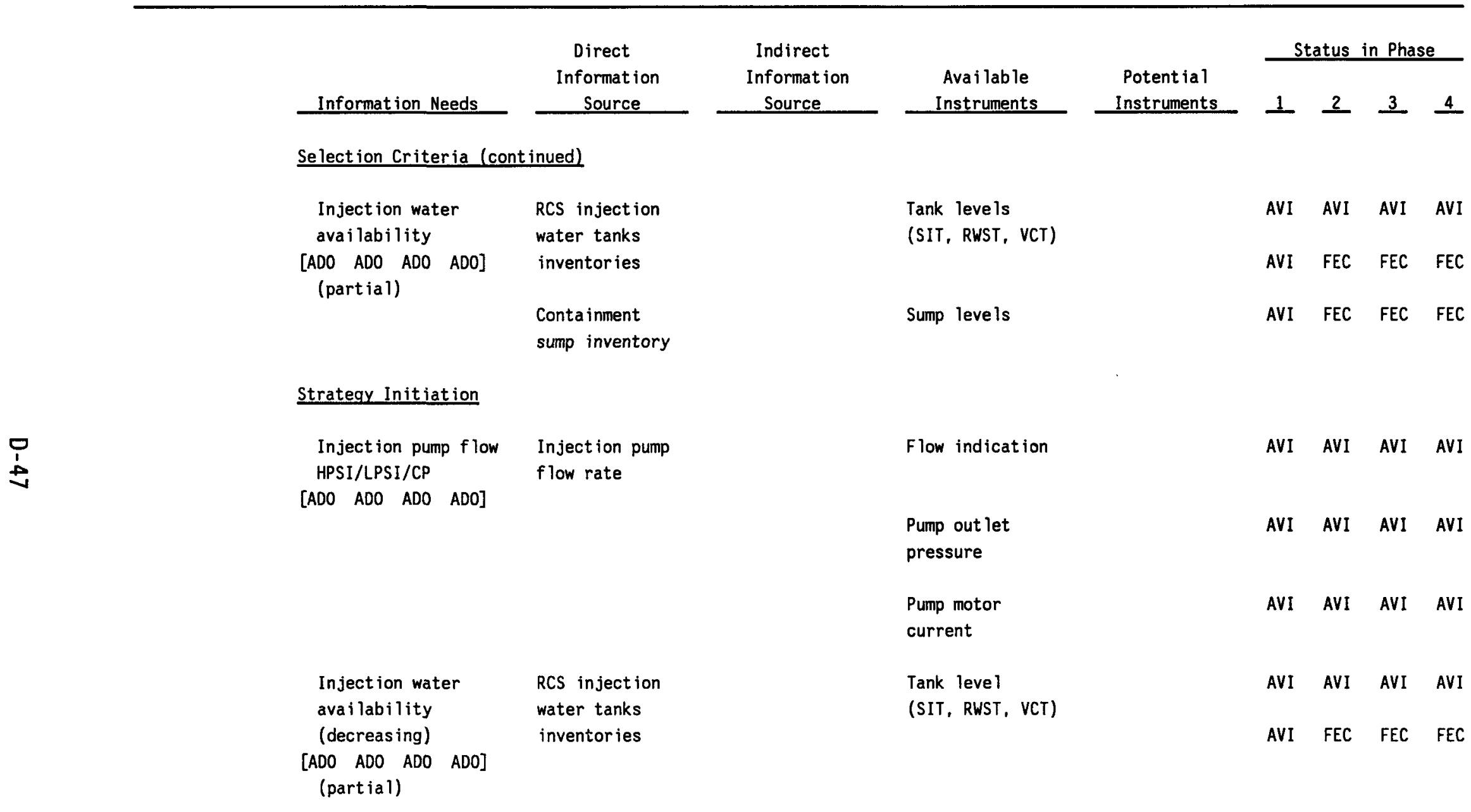


TABLE D1.10. (continued)

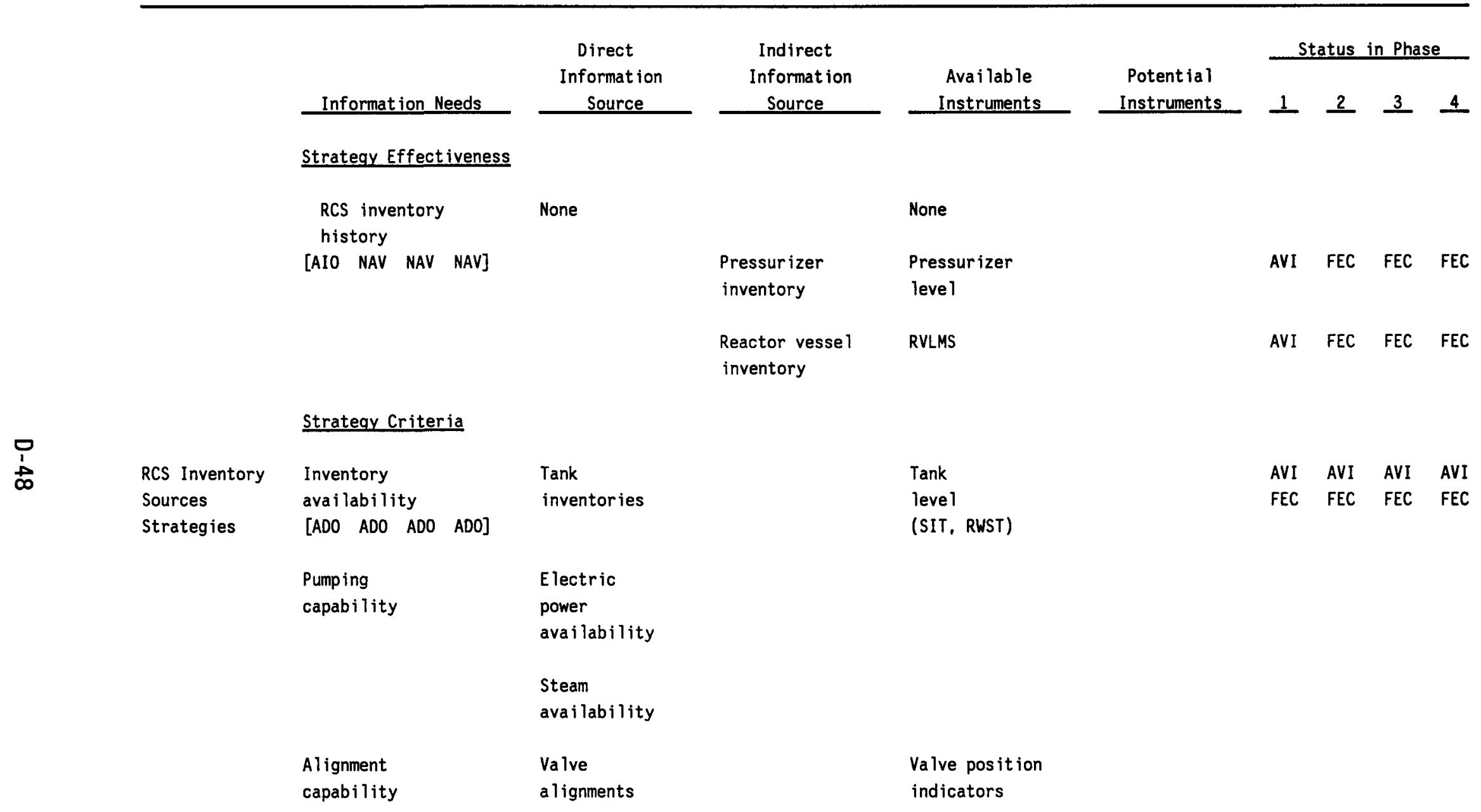


TABLE 01.10. (cont inued)

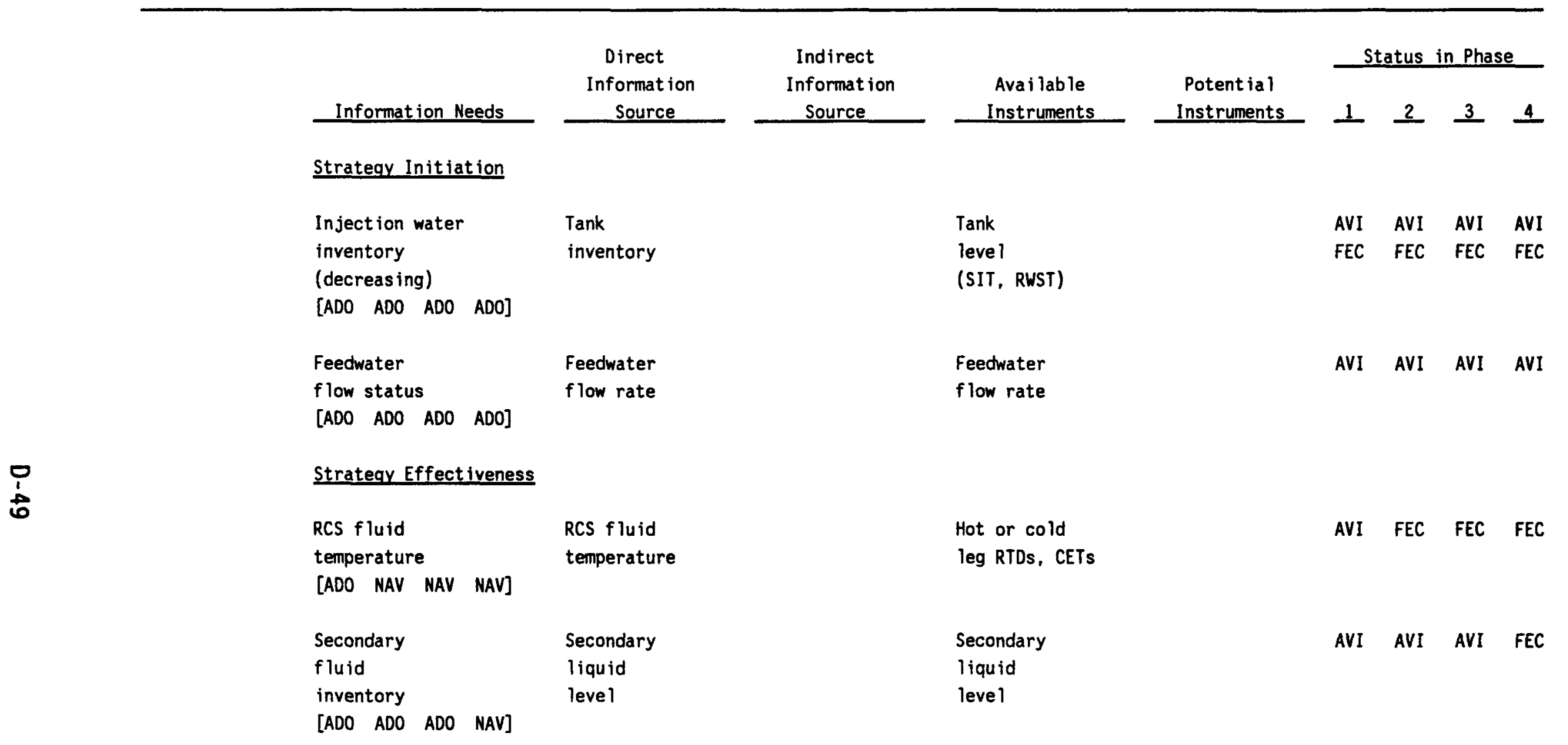


TABLE D1.11. PREVENT VESSEL FAILURE (V) - COOLABLE RELOCATION MECHANISM (V3A1)

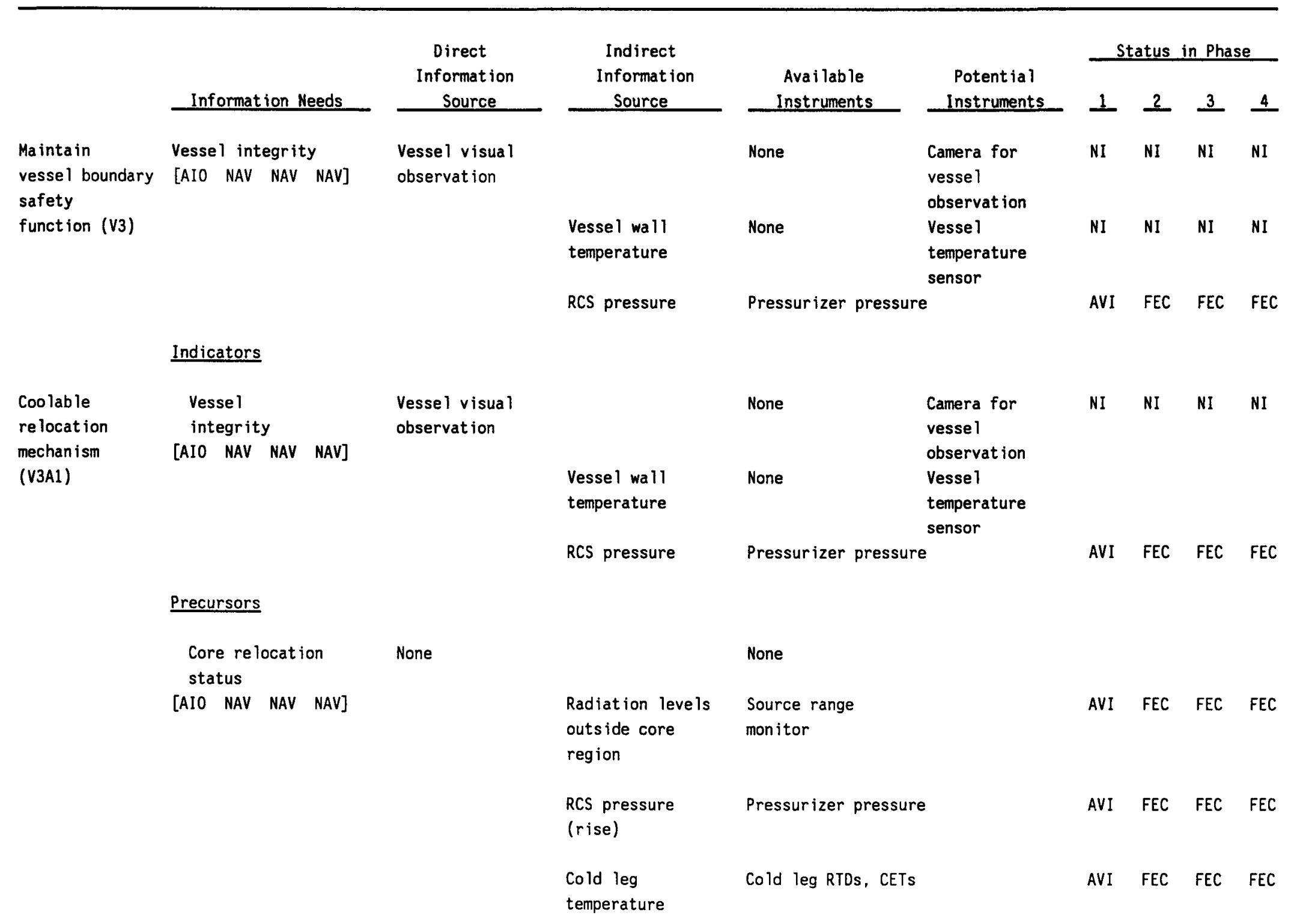


TABLE D1.11. (cont inued)

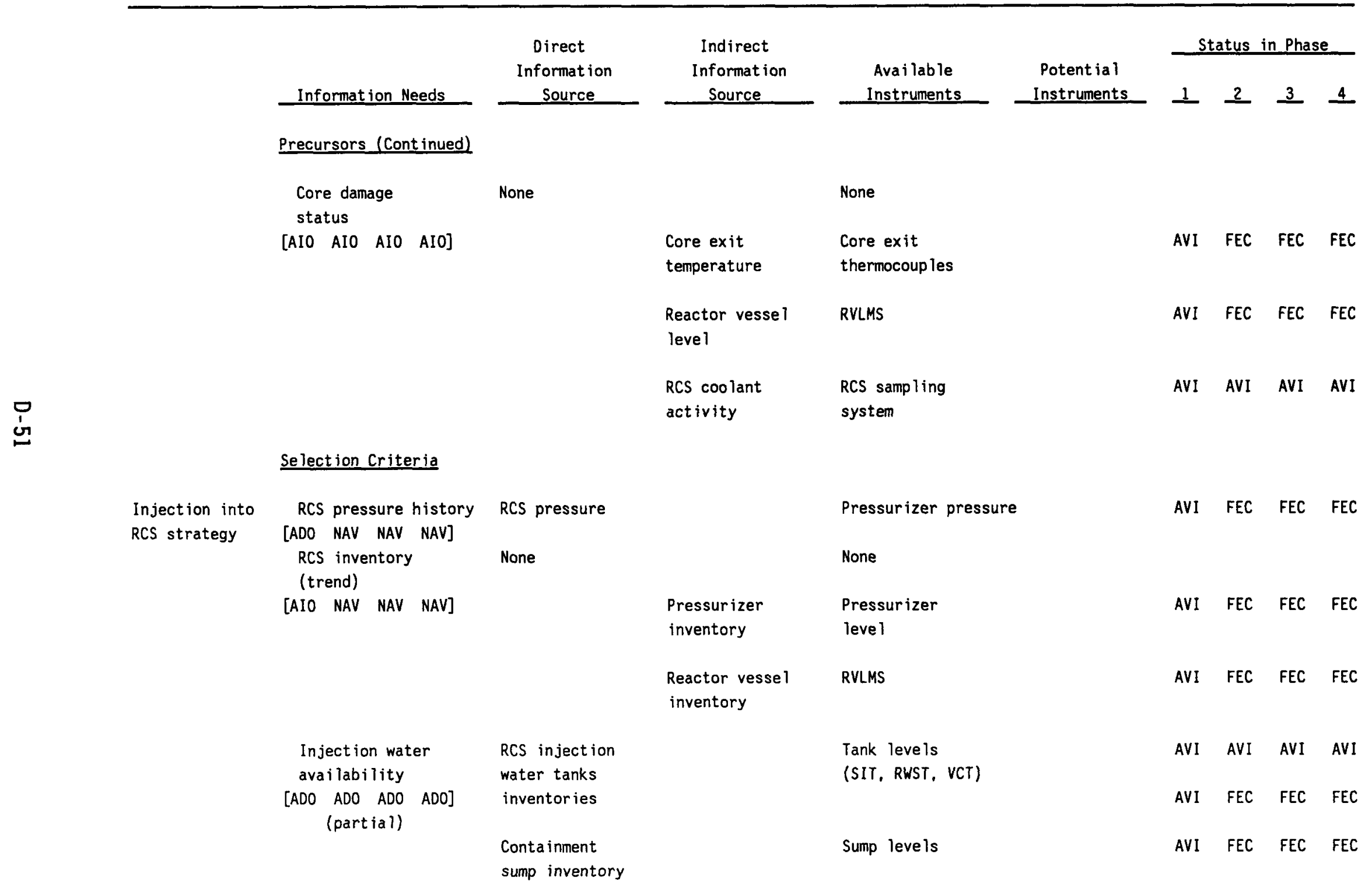


TABLE 01.11. (cont inued)

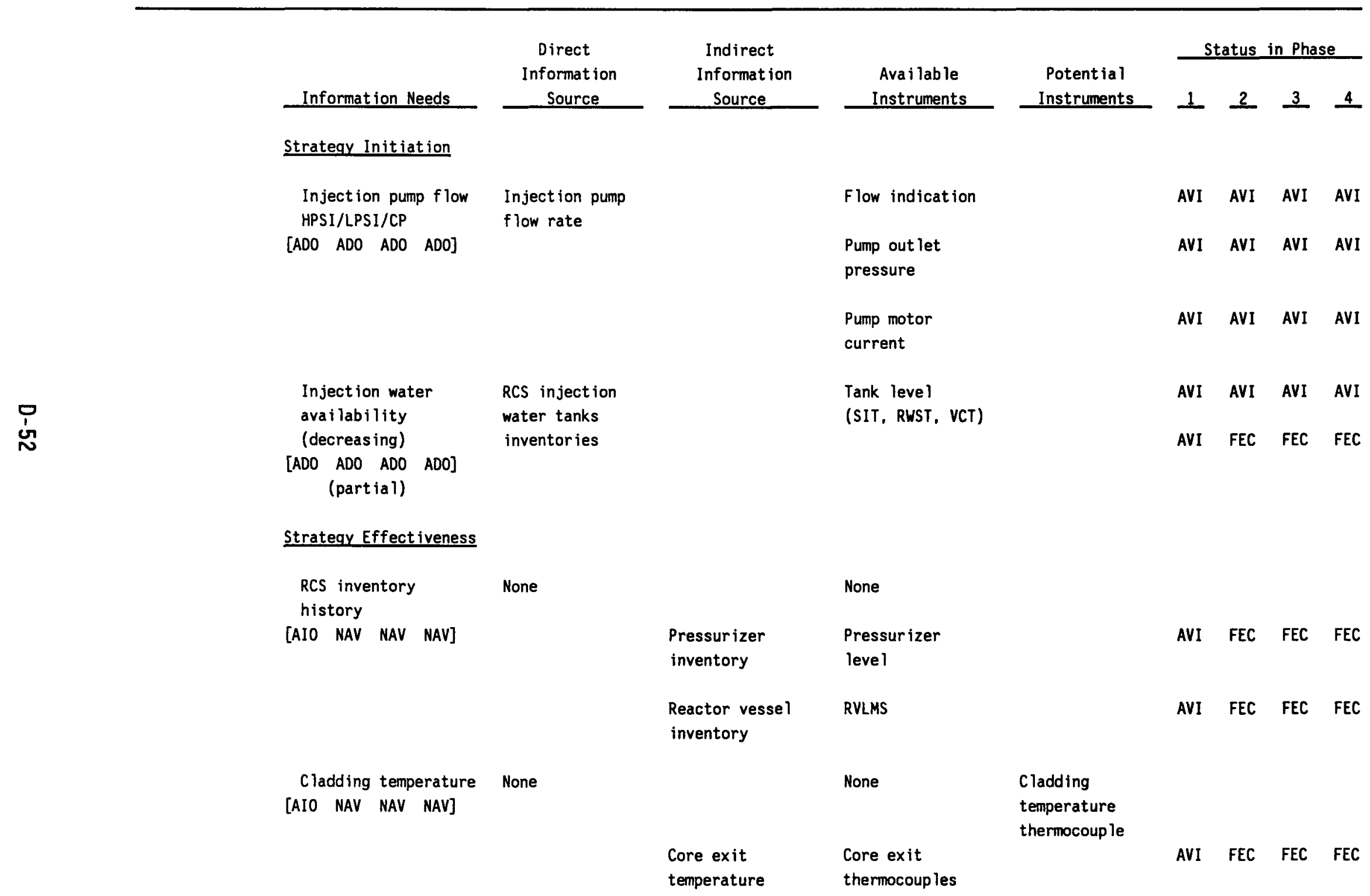


TABLE 01.11. (cont inued)

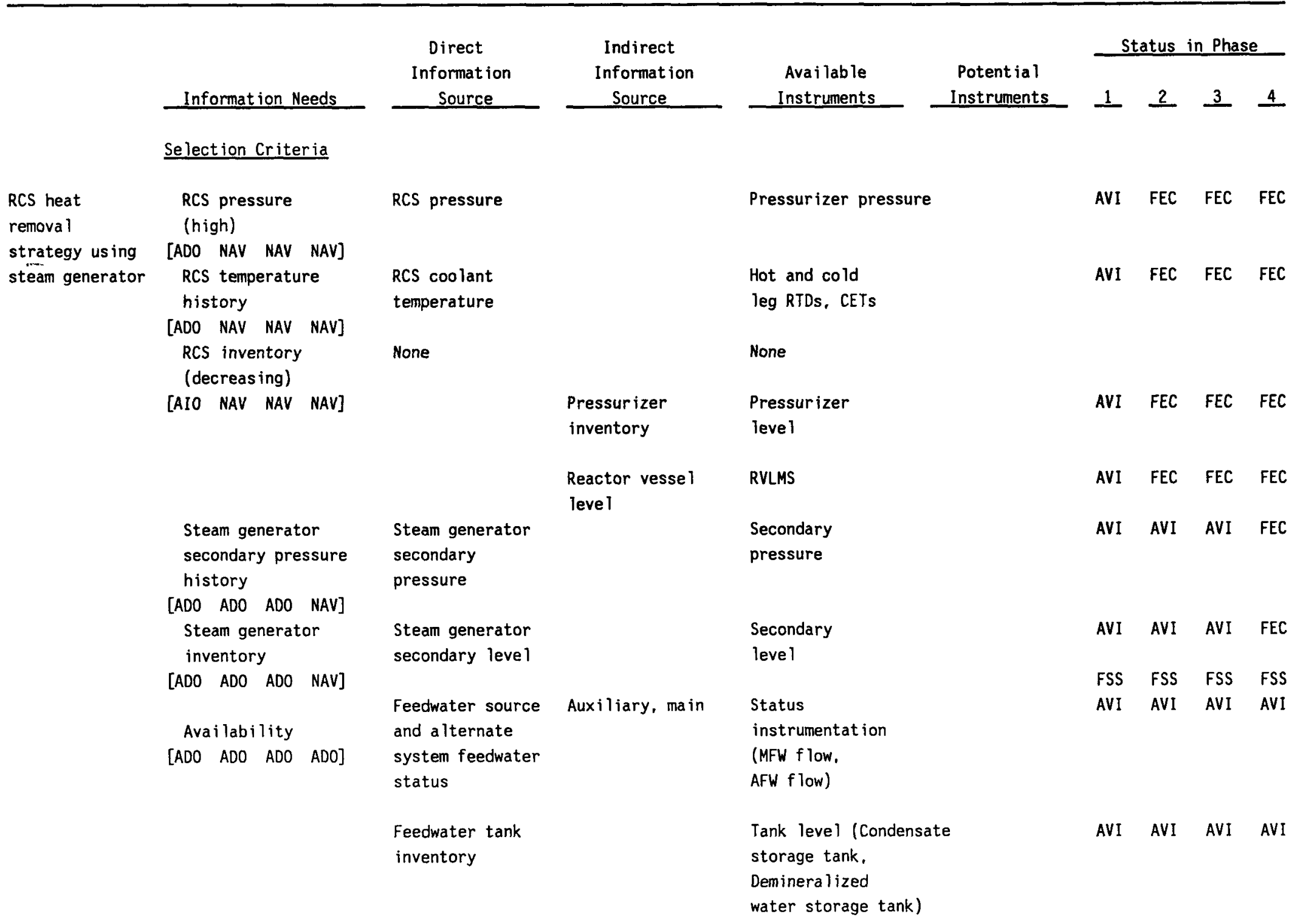


TABLE 01.11. (cont inued)

\begin{tabular}{|c|c|c|c|c|c|c|c|c|}
\hline \multirow[b]{2}{*}{ Information Needs } & \multirow{2}{*}{$\begin{array}{c}\text { Direct } \\
\text { Information } \\
\text { Source } \\
\end{array}$} & \multirow{2}{*}{$\begin{array}{l}\text { Indirect } \\
\text { Informat ion } \\
\text { Source }\end{array}$} & \multirow{2}{*}{$\begin{array}{l}\text { Avai lab le } \\
\text { Instruments }\end{array}$} & \multirow{2}{*}{$\begin{array}{l}\text { Potential } \\
\text { Instruments }\end{array}$} & \multicolumn{4}{|c|}{ Status in Phase } \\
\hline & & & & & 1 & 2 & 3 & 4 \\
\hline \multicolumn{9}{|l|}{ Strategy initiation } \\
\hline $\begin{array}{l}\text { Steam generator } \\
\text { secondary pressure } \\
\text { history }\end{array}$ & \multirow[t]{2}{*}{$\begin{array}{l}\text { Steam generator } \\
\text { secondary } \\
\text { pressure }\end{array}$} & & \multirow{2}{*}{\multicolumn{2}{|c|}{$\begin{array}{l}\text { Secondary } \\
\text { pressure }\end{array}$}} & AVI & AVI & AVI & FEC \\
\hline$\left[\begin{array}{llll}A D O & A D O & A D O & N A V\end{array}\right]$ & & & & & & & & \\
\hline Feedwater flow & \multirow{4}{*}{$\begin{array}{l}\text { Feedwater flow } \\
\text { rate }\end{array}$} & & \multirow{2}{*}{\multicolumn{2}{|c|}{$\begin{array}{l}\text { Feedwater flow } \\
\text { rate } \\
\text { (MFW flow } \\
\text { AFW flow) }\end{array}$}} & FSS & FSS & FSS & FSS \\
\hline$\left[\begin{array}{llll}A D O & A D O & A D O & A D 0\end{array}\right]$ & & & & & AVI & AVI & AVI & AVI \\
\hline & & & \multicolumn{2}{|l|}{$\begin{array}{l}\text { Pump discharge } \\
\text { pressure }\end{array}$} & AVI & AVI & AVI & AVI \\
\hline & & & \multicolumn{2}{|l|}{$\begin{array}{l}\text { Pump motor } \\
\text { current }\end{array}$} & AVI & AVI & AVI & AVI \\
\hline \multicolumn{9}{|l|}{ Strategy Effect iveness } \\
\hline \multirow{2}{*}{$\begin{array}{l}\text { RCS pressure } \\
\text { history } \\
\text { [ADO NAV NAV NAV] }\end{array}$} & \multirow{2}{*}{\multicolumn{2}{|c|}{ RCS pressure }} & \multirow{2}{*}{\multicolumn{2}{|c|}{ Pressurizer pressure }} & AVI & FEC & FEC & FEC \\
\hline & & & & & & & & \\
\hline $\begin{array}{l}\text { RCS temperature } \\
\text { history }\end{array}$ & $\begin{array}{l}\text { RCS coolant } \\
\text { temperature }\end{array}$ & & \multicolumn{2}{|l|}{$\begin{array}{l}\text { Subcooled } \\
\text { marg in monitor }\end{array}$} & AVI & FEC & FEC & FEC \\
\hline$\left[\begin{array}{llll}A D O & \text { NAV } & \text { NAV } & \text { NAV }\end{array}\right]$ & & & $\begin{array}{l}\text { Cold and hot leg } \\
\text { RTDs, CETs }\end{array}$ & & AVI & FEC & FEC & FEC \\
\hline
\end{tabular}


TABLE D1.11. (continued)

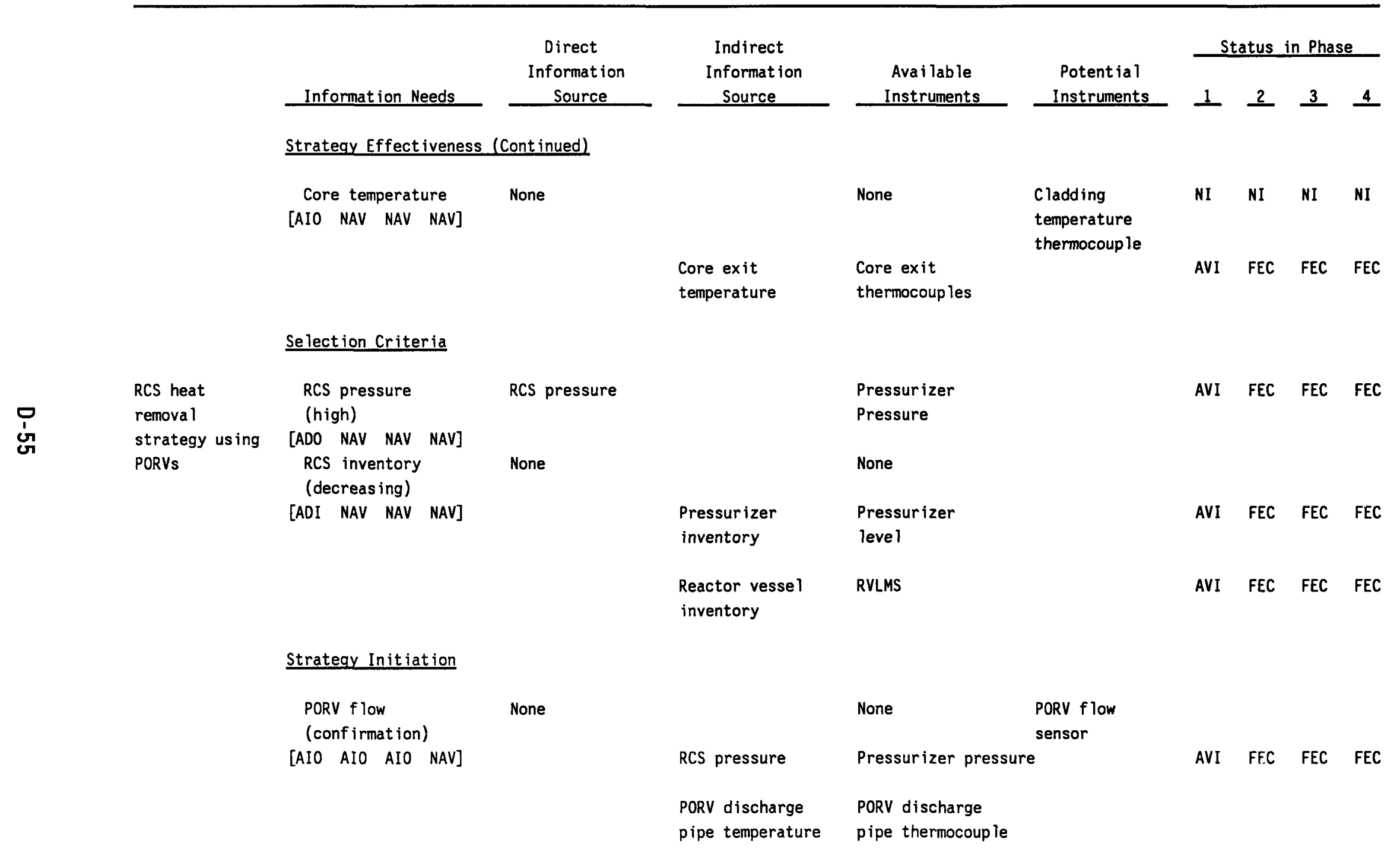




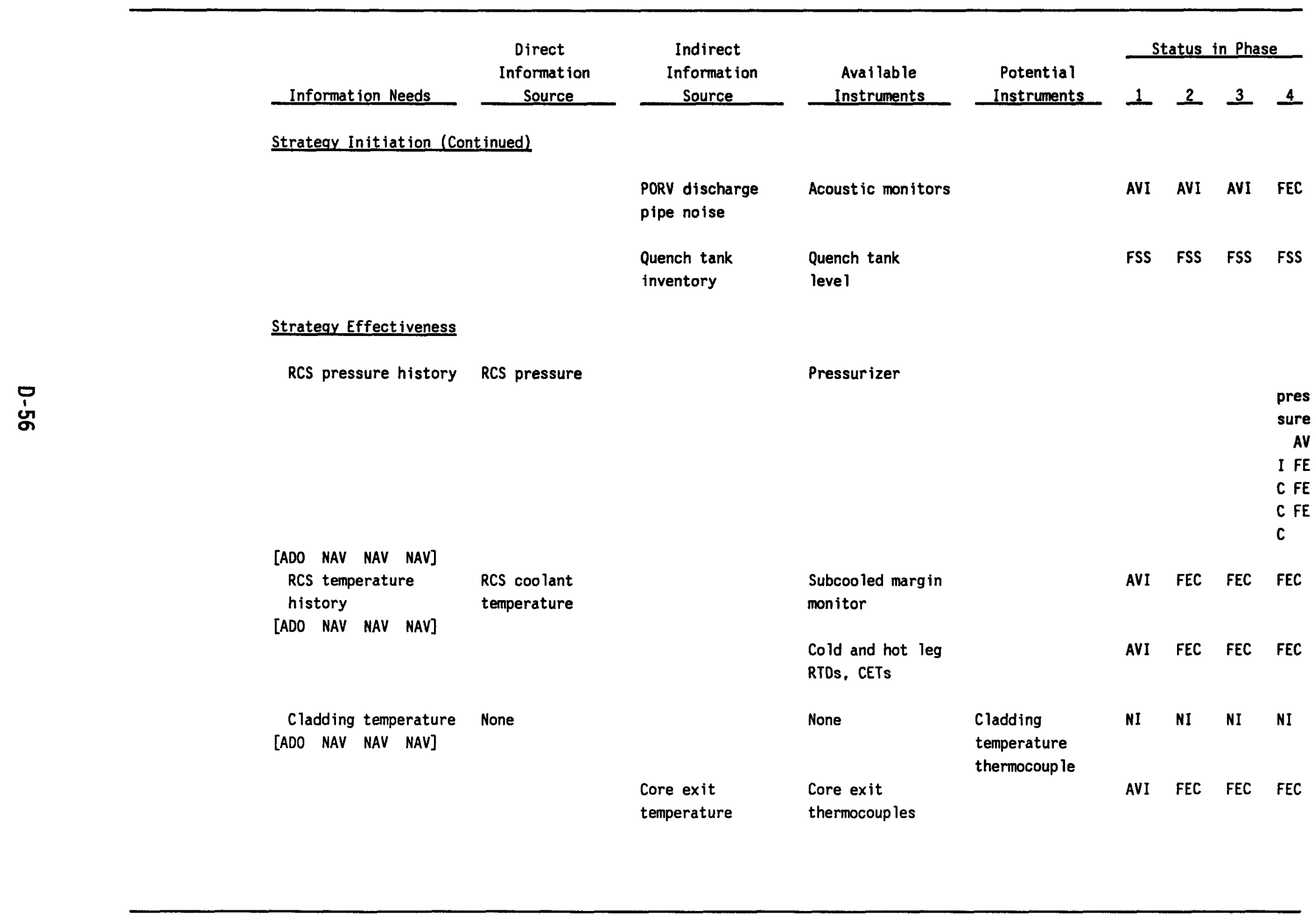


TABLE D1.12. PREVENT VESSEL FAILURE (V)--NONCOOLABLE RELOCATION MECHANISM (V3A2)

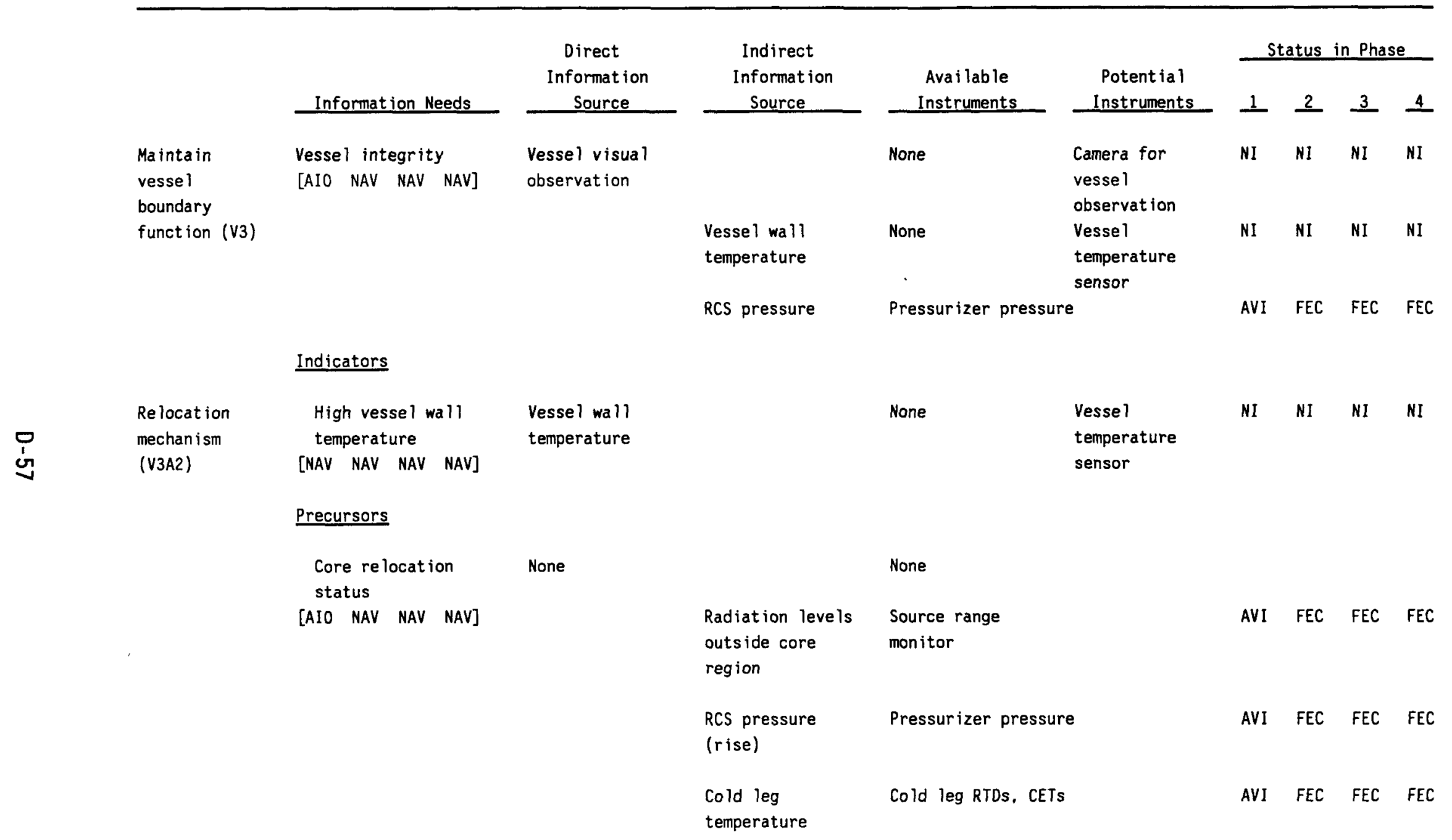


TABLE D1.12. (cont inued)

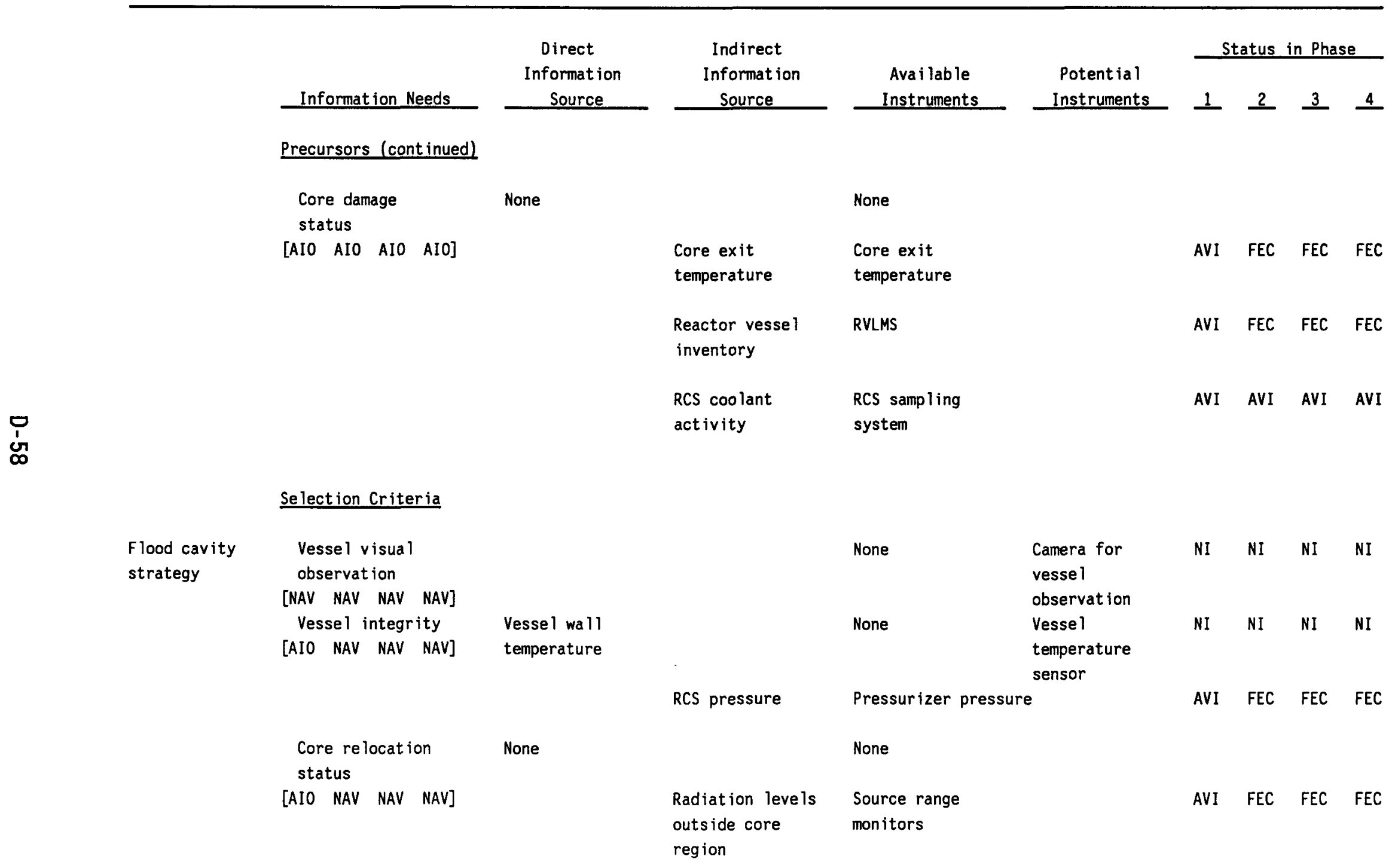


TABLE 01.12. (cont inued)

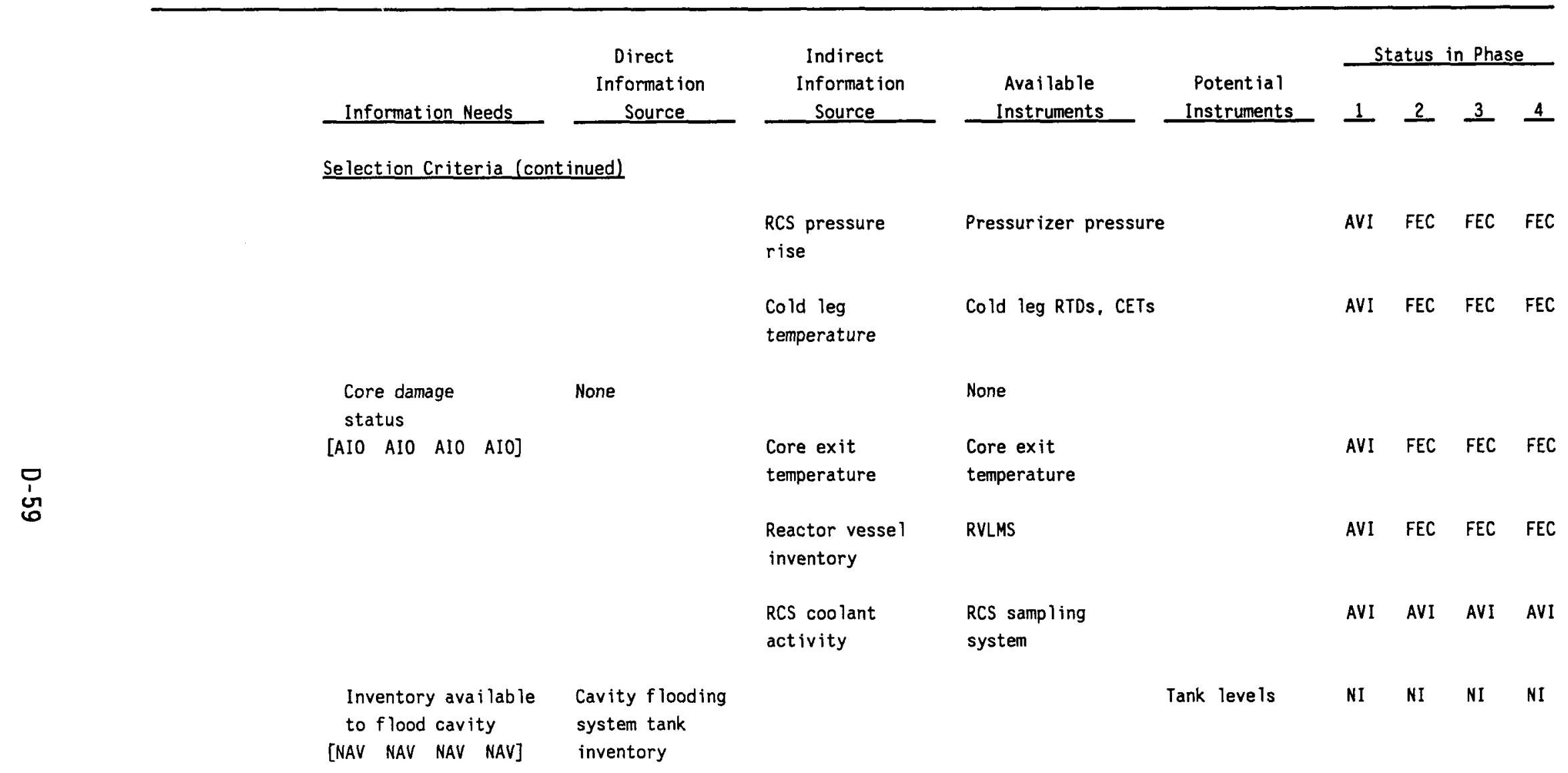


TABLE 01.12. (cont inued)

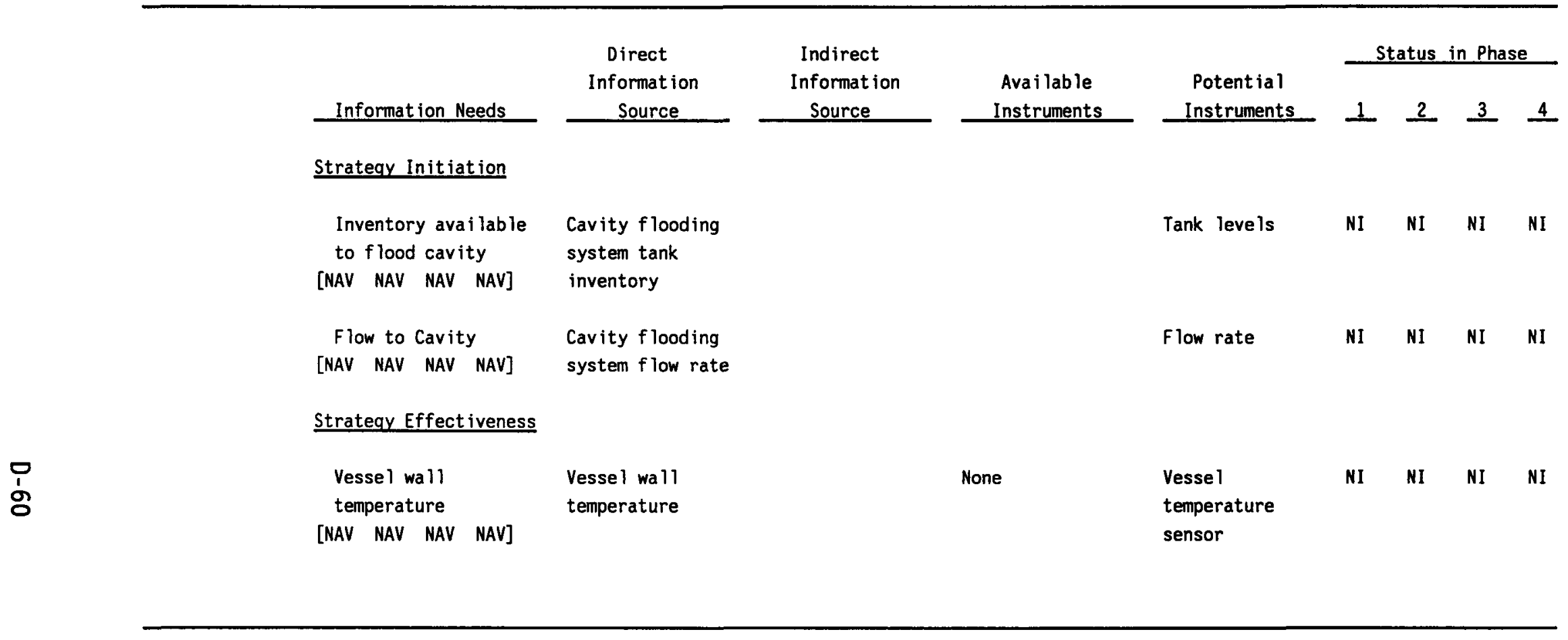


TABLE D2.1. PREVENT CONTAINMENT FAILURE (C)--INSUFFICIENT ENERGY REMOVAL MECHANISM (C1A1)

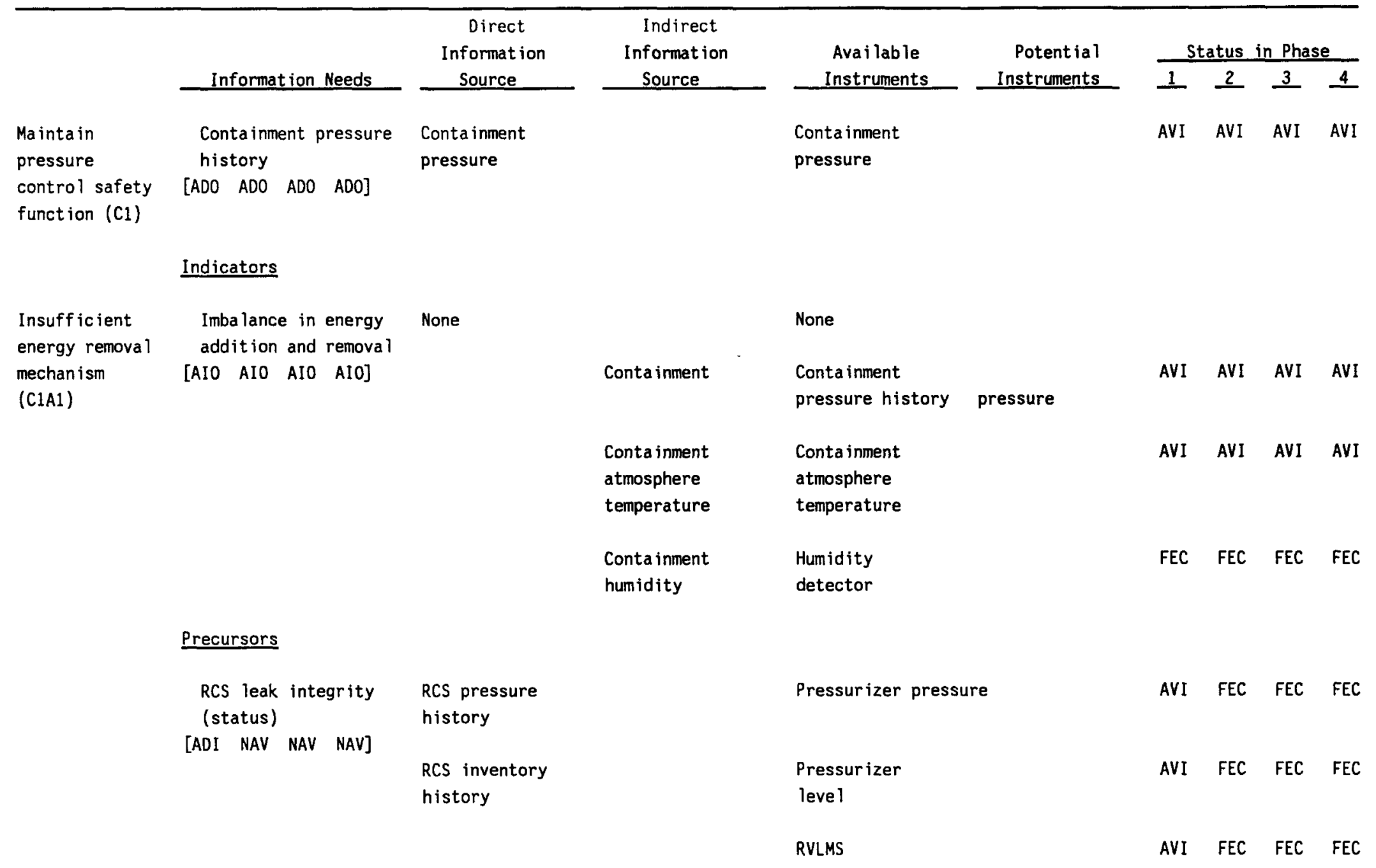


TABLE D2.1. (continued)

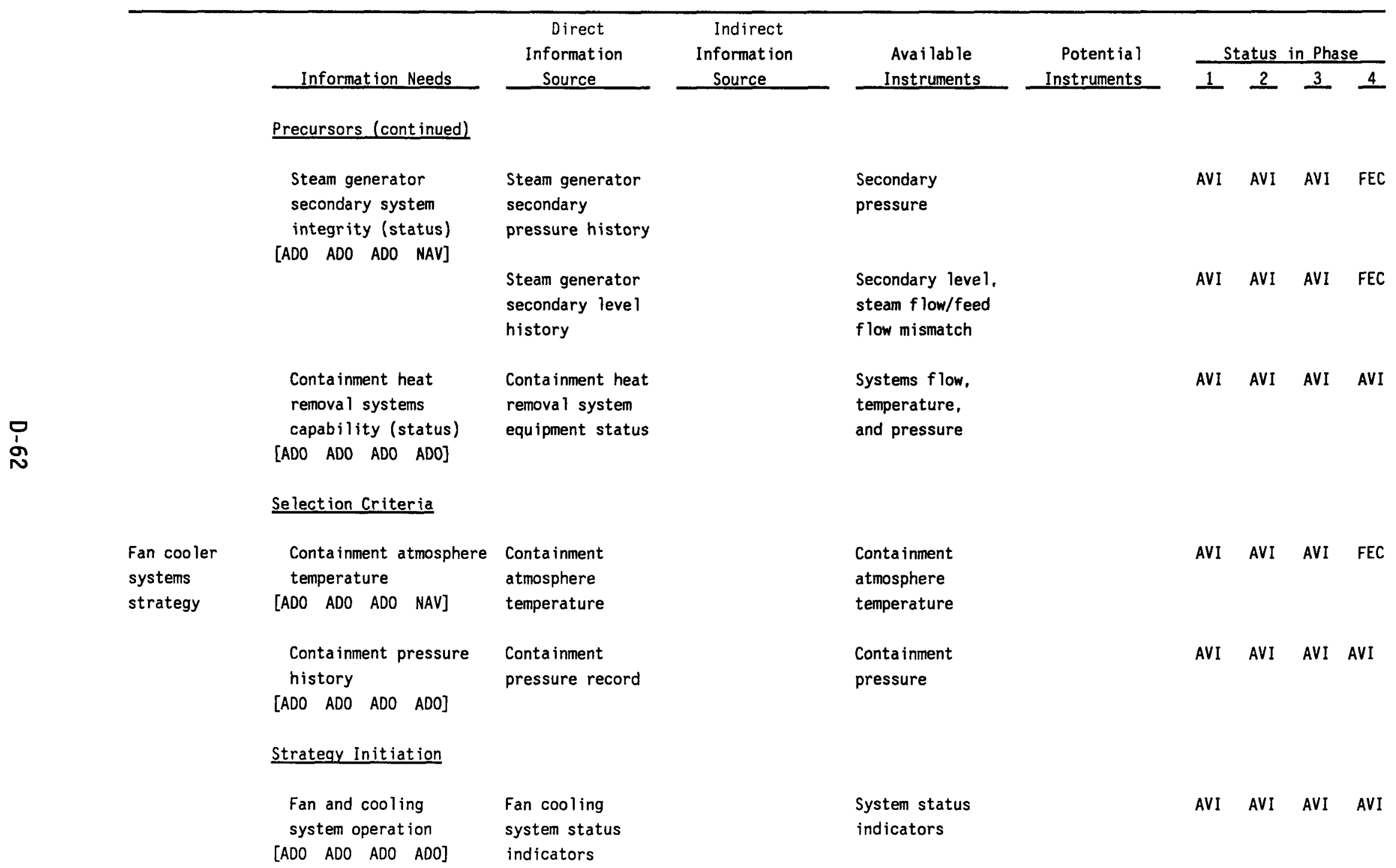


TABLE D2.1. (cont inued)

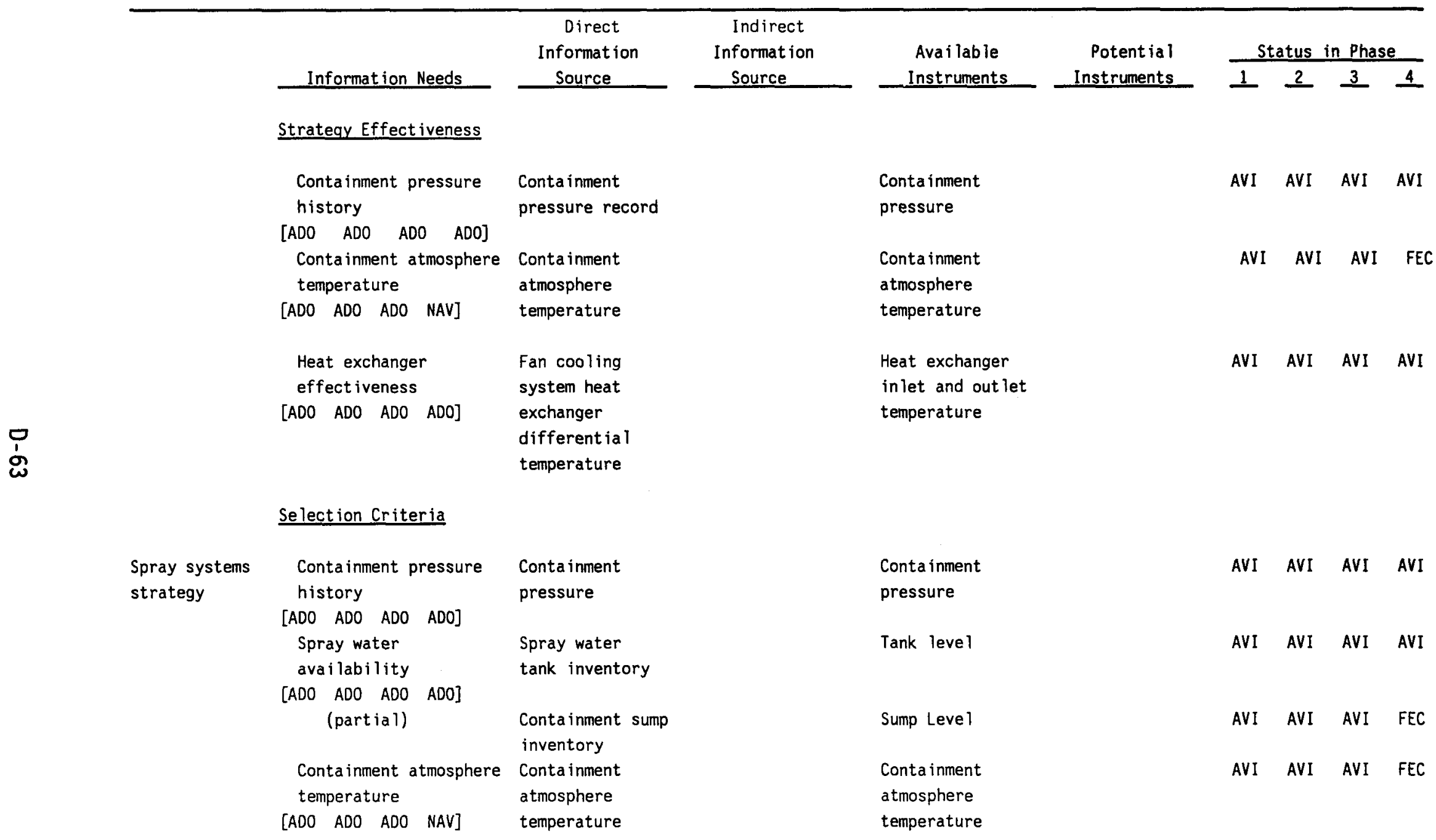


TABLE D2.1. (cont inued)

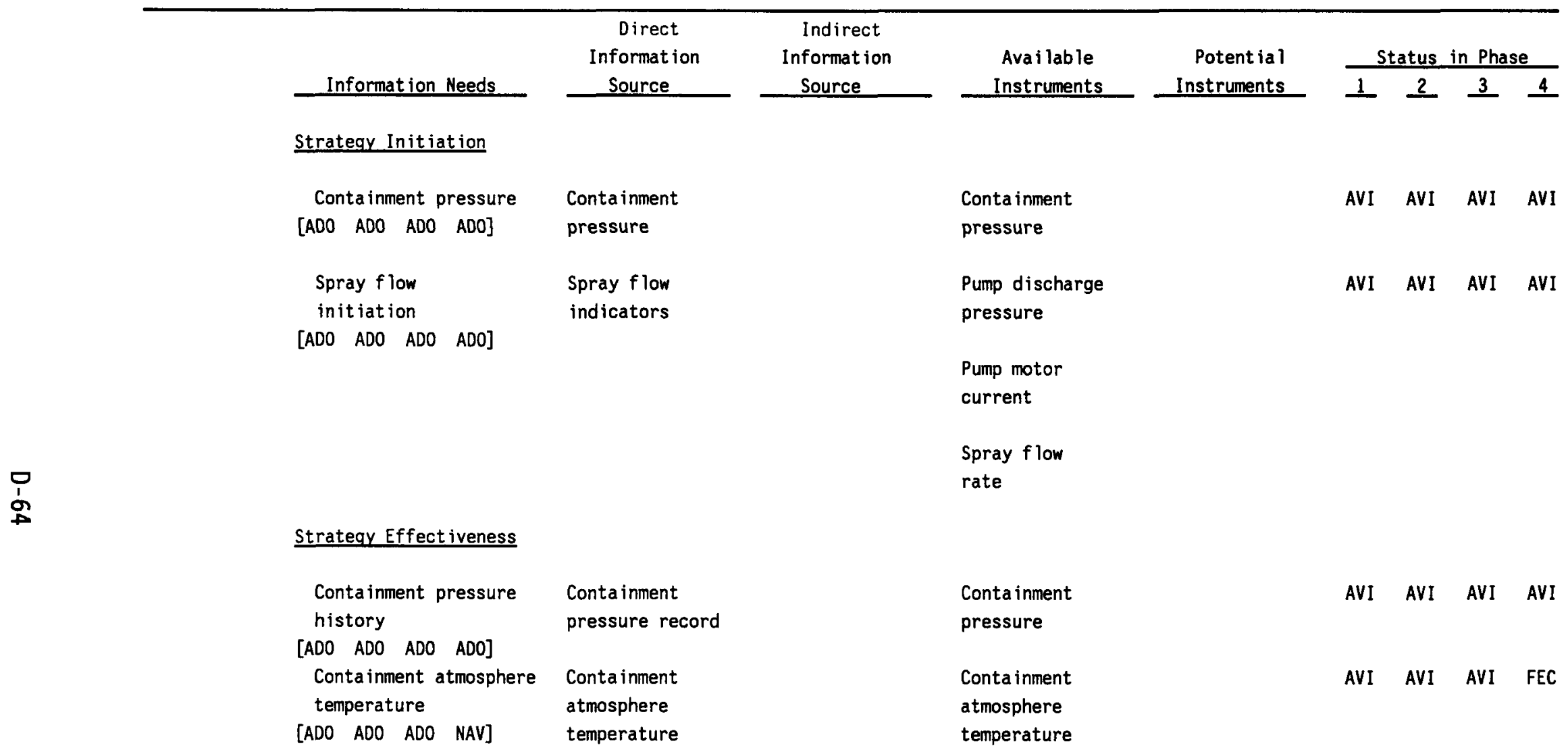


TABLE D2.1. (cont inued)

\begin{tabular}{|c|c|c|c|c|c|c|c|c|c|}
\hline & & $\begin{array}{c}\text { Direct } \\
\text { Information }\end{array}$ & $\begin{array}{c}\text { Indirect } \\
\text { Information }\end{array}$ & Available & Potential & $\underline{S}$ & atus & n Pho & \\
\hline & Information Needs & Source & Source & Instruments & Instruments & 1 & 2 & 3 & 4 \\
\hline & Selection Criteria & & & & & & & & \\
\hline \multirow[t]{13}{*}{ Vent strategy } & $\begin{array}{l}\text { Containment pressure } \\
\text { history }\end{array}$ & $\begin{array}{l}\text { Containment } \\
\text { pressure }\end{array}$ & & $\begin{array}{l}\text { Containment } \\
\text { pressure }\end{array}$ & & AVI & AVI & AVI & AVI \\
\hline & {$\left[\begin{array}{llll}A D O & A D O & A D O & A D 0\end{array}\right]$} & & & & & & & & \\
\hline & $\begin{array}{l}\text { Presence of radiation } \\
\text { in containment }\end{array}$ & $\begin{array}{l}\text { Conta inment } \\
\text { radiation level }\end{array}$ & & $\begin{array}{l}\text { Containment } \\
\text { radiation }\end{array}$ & & FSS & FSS & FSS & FSS \\
\hline & [NAV NAV NAV NAV] & & & monitors & & & & & \\
\hline & $\begin{array}{l}\text { Conta inment } \\
\text { humidity }\end{array}$ & $\begin{array}{l}\text { Containment } \\
\text { humidity }\end{array}$ & & $\begin{array}{l}\text { Humidity } \\
\text { detectors }\end{array}$ & & FEC & FEC & FEC & FEC \\
\hline & Strategy Initiation & & & & & & & & \\
\hline & $\begin{array}{l}\text { Vent system } \\
\text { initiation }\end{array}$ & $\begin{array}{l}\text { Vent system } \\
\text { flow rate }\end{array}$ & & & $\begin{array}{l}\text { Vent system } \\
\text { flow }\end{array}$ & NI & NI & NI & NI \\
\hline & [NAV NAV NAV NAV] & & & & measurement & & & & \\
\hline & Strategy Effectiveness & & & & & & & & \\
\hline & $\begin{array}{l}\text { Conta inment pressure } \\
\text { history }\end{array}$ & $\begin{array}{l}\text { Containment } \\
\text { pressure record }\end{array}$ & & $\begin{array}{l}\text { Containment } \\
\text { pressure }\end{array}$ & & AVI & AVI & AVI & AVI \\
\hline & {$\left[\begin{array}{llll}A D 0 & A D O & A D 0 & A D 0\end{array}\right]$} & & & & & & & & \\
\hline & $\begin{array}{l}\text { Conta inment atmosphere } \\
\text { temperature }\end{array}$ & $\begin{array}{l}\text { Conta inment } \\
\text { atmosphere }\end{array}$ & & $\begin{array}{l}\text { Containment } \\
\text { atmosphere }\end{array}$ & & AVI & AVI & AVI & AVI \\
\hline & {$\left[\begin{array}{llll}A D O & A D O & A D O & N A V\end{array}\right]$} & temperature & & temperature & & & & & \\
\hline
\end{tabular}


TABLE 02.2. PREVENT CONTAINMENT FAILURE (C)--NONCONDENSABLE GAS BUILDUP MECHANISM (C1A2)

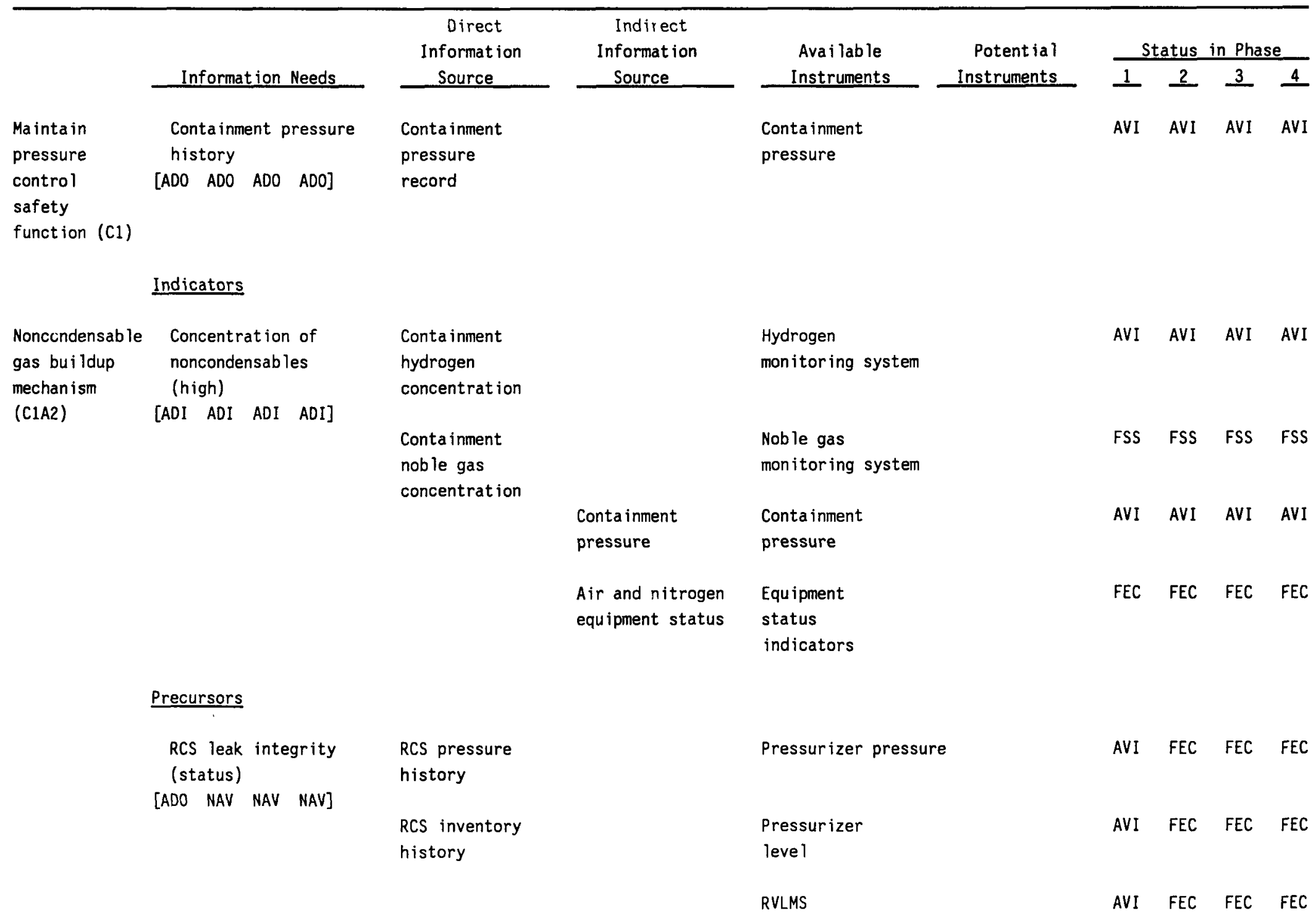


TABLE D2.2. (cont inued)

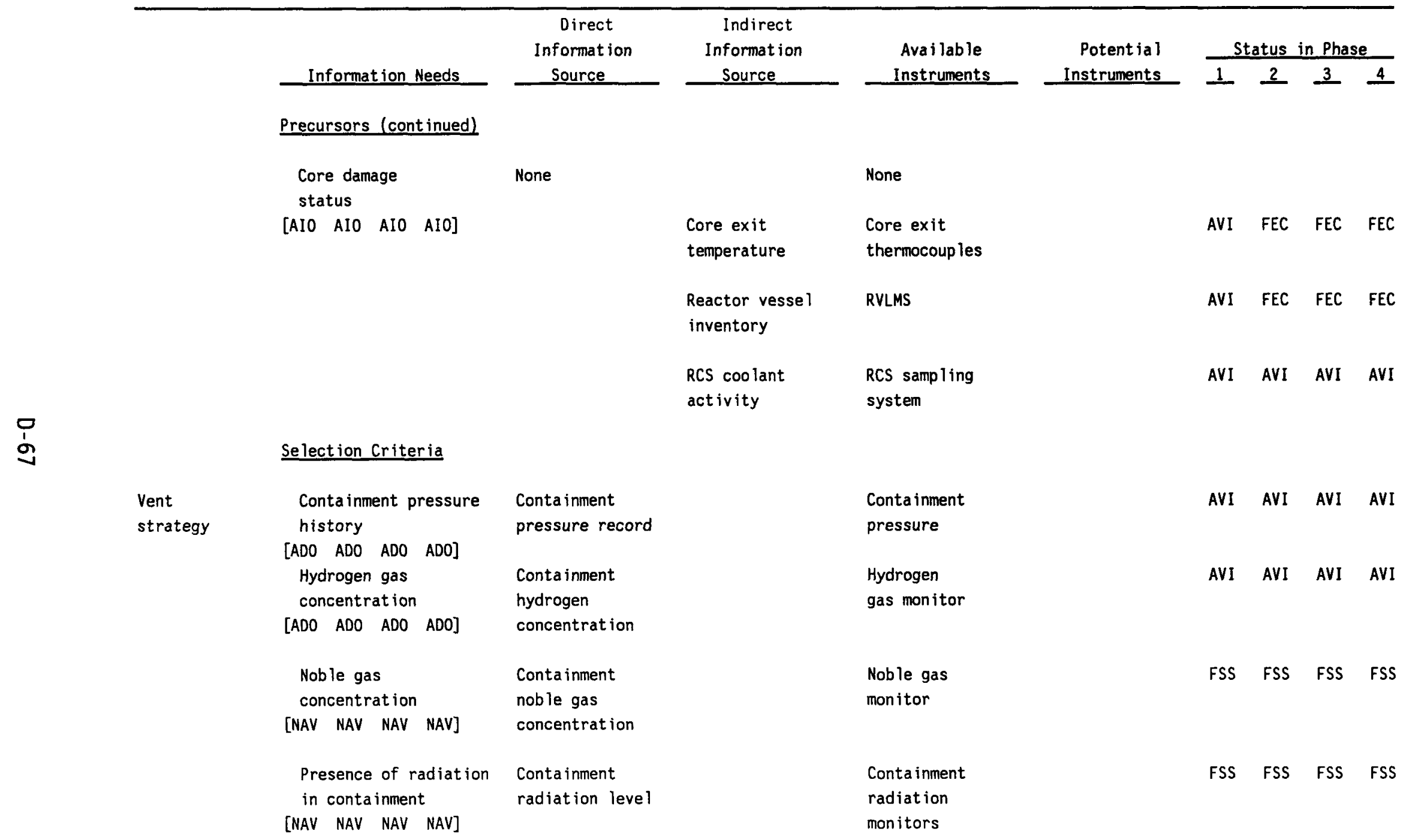


TABLE D2.2. (cont inued)

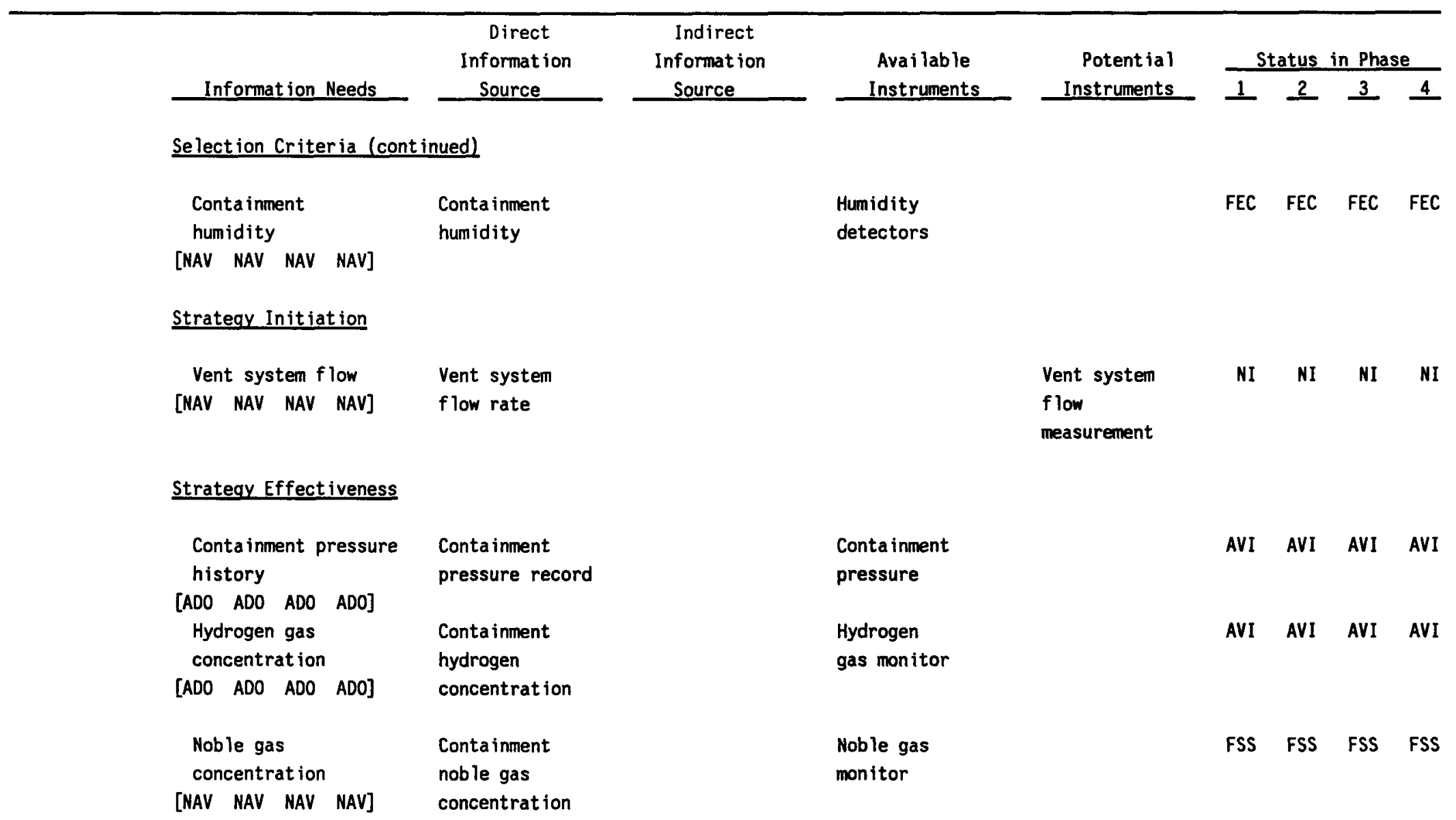


TABLE D2.2. (cont inued)

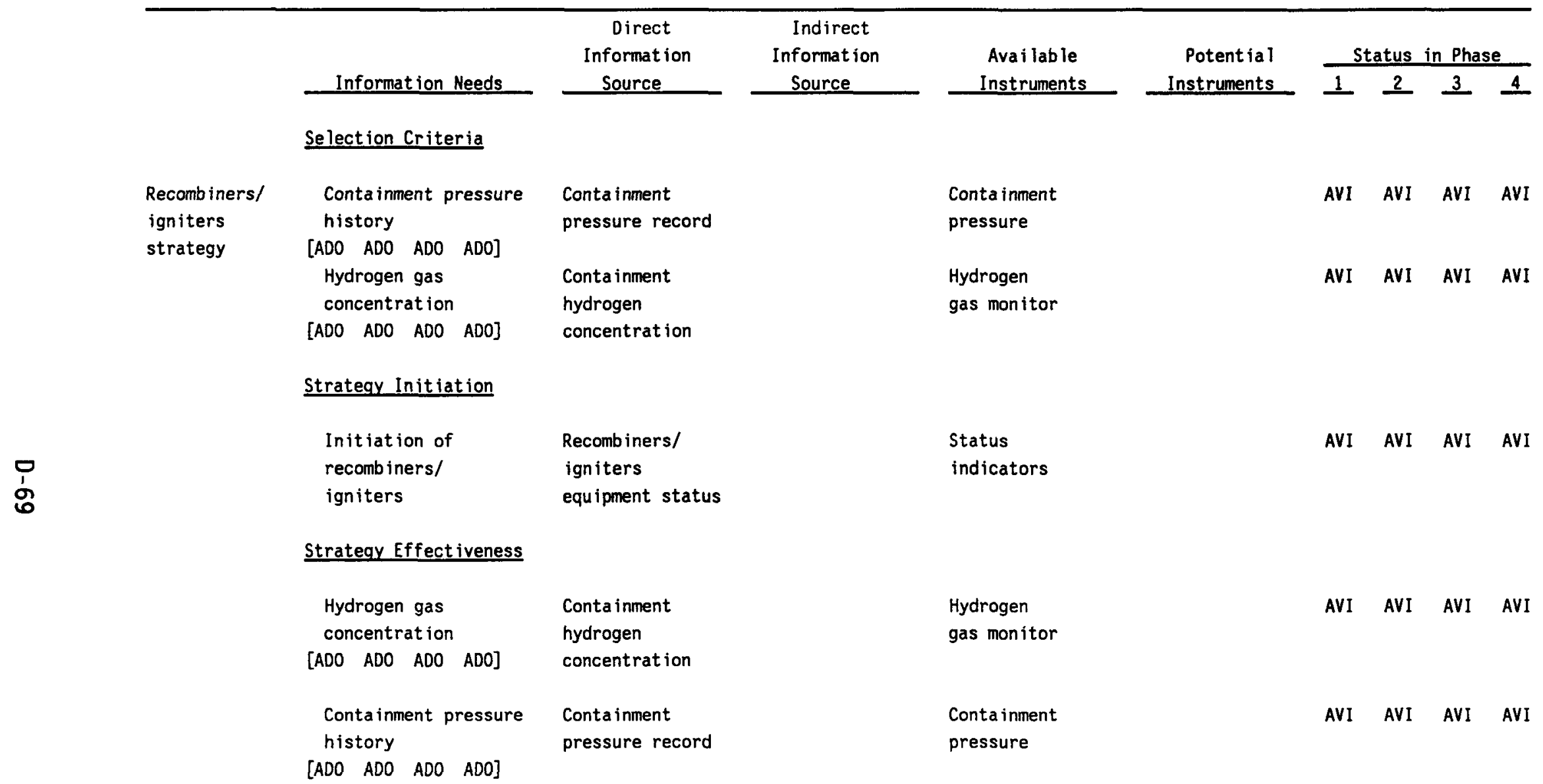


TABLE D2.3. PREVENT CONTAINMENT FAILURE (C)--DIRECT CONTAINMENT HEATING MECHANISM (C1B1)

\begin{tabular}{|c|c|c|c|c|c|c|c|c|c|}
\hline & \multirow[b]{2}{*}{ Information Needs } & \multirow{2}{*}{$\begin{array}{c}\text { Direct } \\
\text { Information } \\
\text { Source } \\
\end{array}$} & \multirow{2}{*}{$\begin{array}{c}\text { Indirect } \\
\text { Information } \\
\text { Source } \\
\end{array}$} & \multirow{2}{*}{$\begin{array}{l}\text { Available } \\
\text { Instruments }\end{array}$} & \multirow{2}{*}{$\begin{array}{r}\text { Potential } \\
\text { Instruments } \\
\end{array}$} & \multicolumn{4}{|c|}{ Status in Phase } \\
\hline & & & & & & 1 & 2 & 3 & 4 \\
\hline $\begin{array}{l}\text { Maintain } \\
\text { pressure } \\
\text { control } \\
\text { safety } \\
\text { function (C1) }\end{array}$ & $\begin{array}{l}\text { Containment pressure } \\
\text { history } \\
{\left[\begin{array}{llll}A D 0 & A D 0 & A D 0 & A D 0\end{array}\right]}\end{array}$ & $\begin{array}{l}\text { Containment } \\
\text { pressure }\end{array}$ & & $\begin{array}{l}\text { Containment } \\
\text { pressure }\end{array}$ & & AVI & AVI & AVI & AVI \\
\hline & Indicators & & & & & & & & \\
\hline $\begin{array}{l}\text { Direct } \\
\text { containment } \\
\text { heating }\end{array}$ & $\begin{array}{l}\text { Containment pressure } \\
\text { history } \\
{[A D O \quad A D O \quad A D O \quad A D 0]}\end{array}$ & $\begin{array}{l}\text { Containment } \\
\text { Pressure record }\end{array}$ & & $\begin{array}{l}\text { Containment } \\
\text { pressure }\end{array}$ & & AVI & AVI & AVI & AVI \\
\hline $\begin{array}{l}\text { mechan ism } \\
\text { (C1B1) }\end{array}$ & $\begin{array}{l}\text { Conta inment } \\
\text { temperature history }\end{array}$ & $\begin{array}{l}\text { Conta inment } \\
\text { atmosphere }\end{array}$ & & $\begin{array}{l}\text { Conta inment } \\
\text { thermocouples }\end{array}$ & & AVI & AVI & AVI & FEC \\
\hline & {$\left[\begin{array}{llll}A D O & A D O & A D O & N A V\end{array}\right]$} & temperature & & & & & & & \\
\hline & $\begin{array}{l}\text { Containment } \\
\text { radiation level }\end{array}$ & $\begin{array}{l}\text { Containment } \\
\text { radiation level }\end{array}$ & & $\begin{array}{l}\text { Containment } \\
\text { radiation monitor }\end{array}$ & & FSS & FSS & FSS & FSS \\
\hline & $\begin{array}{l}\text { [NAV NAV NAV NAV] } \\
\text { Precursors }\end{array}$ & & & & & & & & \\
\hline & $\begin{array}{l}\text { RCS pressure } \\
\text { (high) }\end{array}$ & RCS pressure & & Pressurizer pressure & & AVI & FEC & FEC & FEC \\
\hline & {$\left[\begin{array}{llll}A D O & \text { NAV } & \text { NAV } & \text { NAV}\end{array}\right]$} & & & & & & & & \\
\hline & $\begin{array}{l}\text { Approach to } \\
\text { vessel failure }\end{array}$ & $\begin{array}{l}\text { Vesse } 1 \text { visual } \\
\text { observation }\end{array}$ & & None & $\begin{array}{l}\text { Camera for } \\
\text { vesse } 1\end{array}$ & NI & NI & NI & NI \\
\hline & [AIO NAV NAV NAV] & & $\begin{array}{l}\text { Vessel wall } \\
\text { temperature }\end{array}$ & None & $\begin{array}{l}\text { observation } \\
\text { Vesse } 1 \\
\text { temperature } \\
\text { sensor }\end{array}$ & NI & NI & NI & NI \\
\hline
\end{tabular}


TABLE D2.3. (continued)

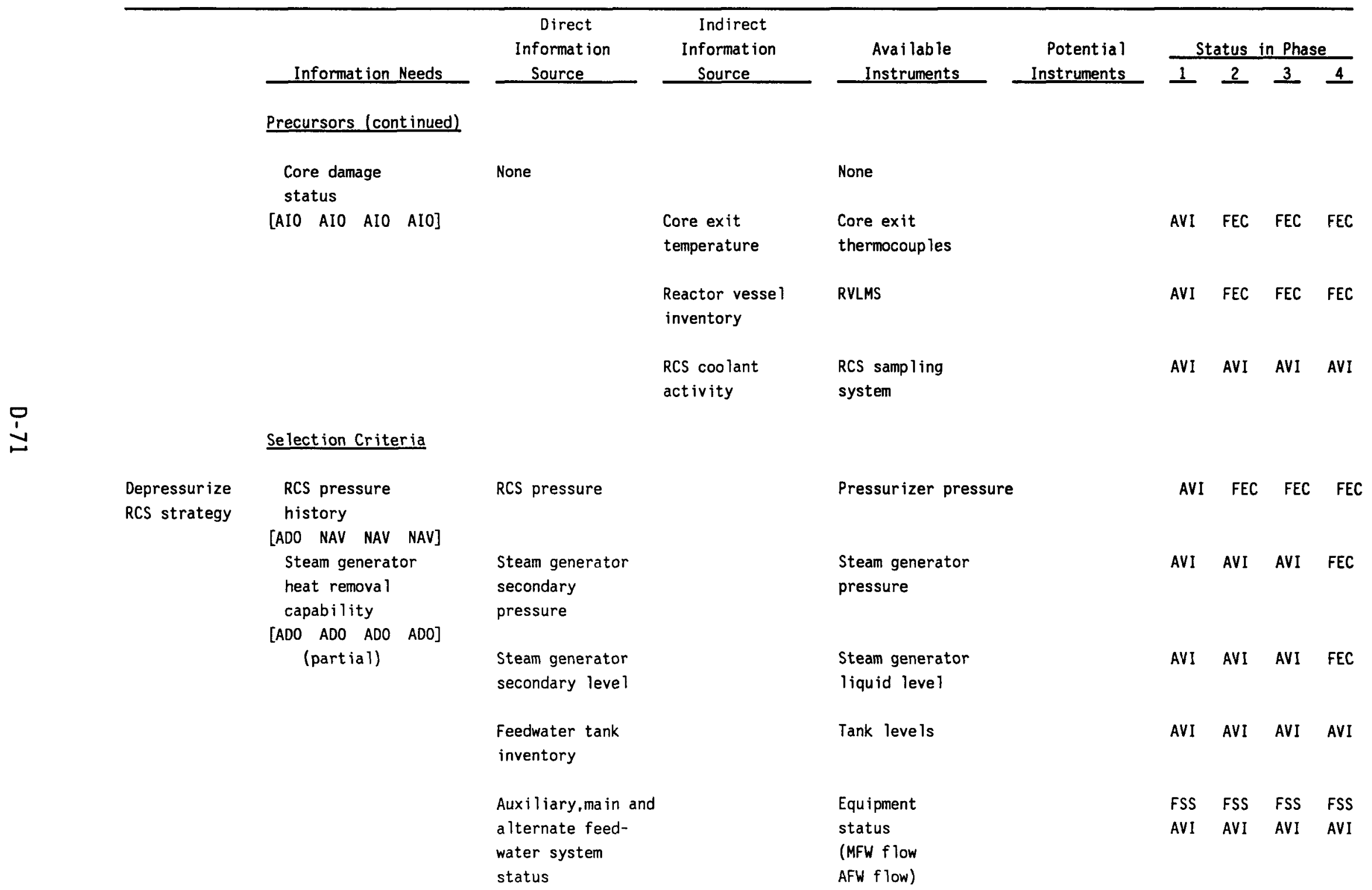


TABLE D2.3. (cont inued)

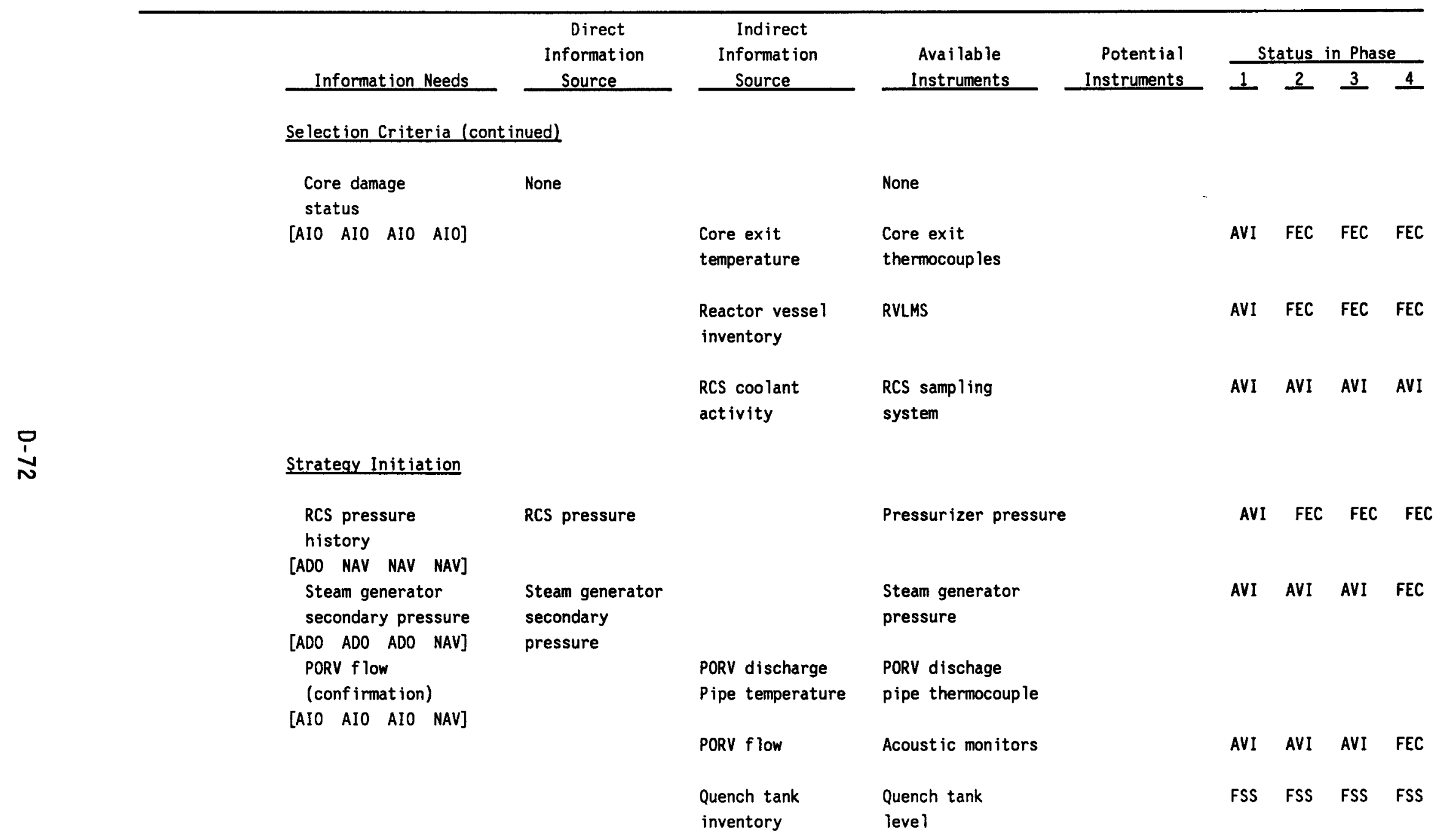


TABLE D2.3. (cont inued)

\begin{tabular}{|c|c|c|c|c|c|c|c|c|c|}
\hline & & $\begin{array}{c}\text { Direct } \\
\text { Information }\end{array}$ & $\begin{array}{l}\text { Indirect } \\
\text { Information }\end{array}$ & Available & Potential & & atus & P Phas & \\
\hline & Information Needs & Source & Source & Instruments & Instruments & 1 & 2 & 3 & 4 \\
\hline & Strategy Effect iveness & & & & & & & & \\
\hline & RCS pressure & RCS pressure & & Pressurizer pressure & & AVI & FEC & FEC & FEC \\
\hline & {$[$ ADO NAV NAV NAV] } & & & & & & & & \\
\hline & $\begin{array}{l}\text { Containment } \\
\text { pressure }\end{array}$ & $\begin{array}{l}\text { Conta inment } \\
\text { pressure }\end{array}$ & & $\begin{array}{l}\text { Conta inment } \\
\text { pressure }\end{array}$ & & AVI & AVI & AVI & AVI \\
\hline & {$\left[\begin{array}{llll}A D O & A D O & A D O & A D 0\end{array}\right]$} & & & & & & & & \\
\hline & Selection Criteria & & & & & & & & \\
\hline $\begin{array}{l}\text { Vent } \\
\text { strategy }\end{array}$ & $\begin{array}{l}\text { Conta inment } \\
\text { pressure }\end{array}$ & $\begin{array}{l}\text { Containment } \\
\text { pressure }\end{array}$ & & $\begin{array}{l}\text { Containment } \\
\text { pressure }\end{array}$ & & AVI & AVI & AVI & AVI \\
\hline & {$\left[\begin{array}{llll}A D O & A D O & A D O & A D 0\end{array}\right]$} & & & & & & & & \\
\hline & RCS pressure & RCS pressure & & Pressurizer pressure & & AVI & FEC & FEC & FEC \\
\hline & $\begin{array}{l}\text { [ADO NAV NAV NAV] } \\
\text { Core damage } \\
\text { status }\end{array}$ & None & & None & & & & & \\
\hline & {$[$ AIO AIO AIO AIO] } & & $\begin{array}{l}\text { Core exit } \\
\text { temperature }\end{array}$ & $\begin{array}{l}\text { Core exit } \\
\text { thermocouples }\end{array}$ & & AVI & FEC & FEC & FEC \\
\hline & & & $\begin{array}{l}\text { Reactor vesse } 1 \\
\text { inventory }\end{array}$ & RVLMS & & AVI & FEC & FEC & FEC \\
\hline & & & $\begin{array}{l}\text { RCS coolant } \\
\text { act ivity }\end{array}$ & $\begin{array}{l}\text { RCS sampling } \\
\text { system }\end{array}$ & & AVI & AVI & AVI & AVI \\
\hline & $\begin{array}{l}\text { Presence of radiation } \\
\text { in containment }\end{array}$ & $\begin{array}{l}\text { Containment } \\
\text { radiation level }\end{array}$ & & $\begin{array}{l}\text { Containment } \\
\text { radiation }\end{array}$ & & FSS & FSS & FSS & FSS \\
\hline & [NAV NAV NAV NAV] & & & monitors & & & & & \\
\hline
\end{tabular}


TABLE D2.3. (continued)

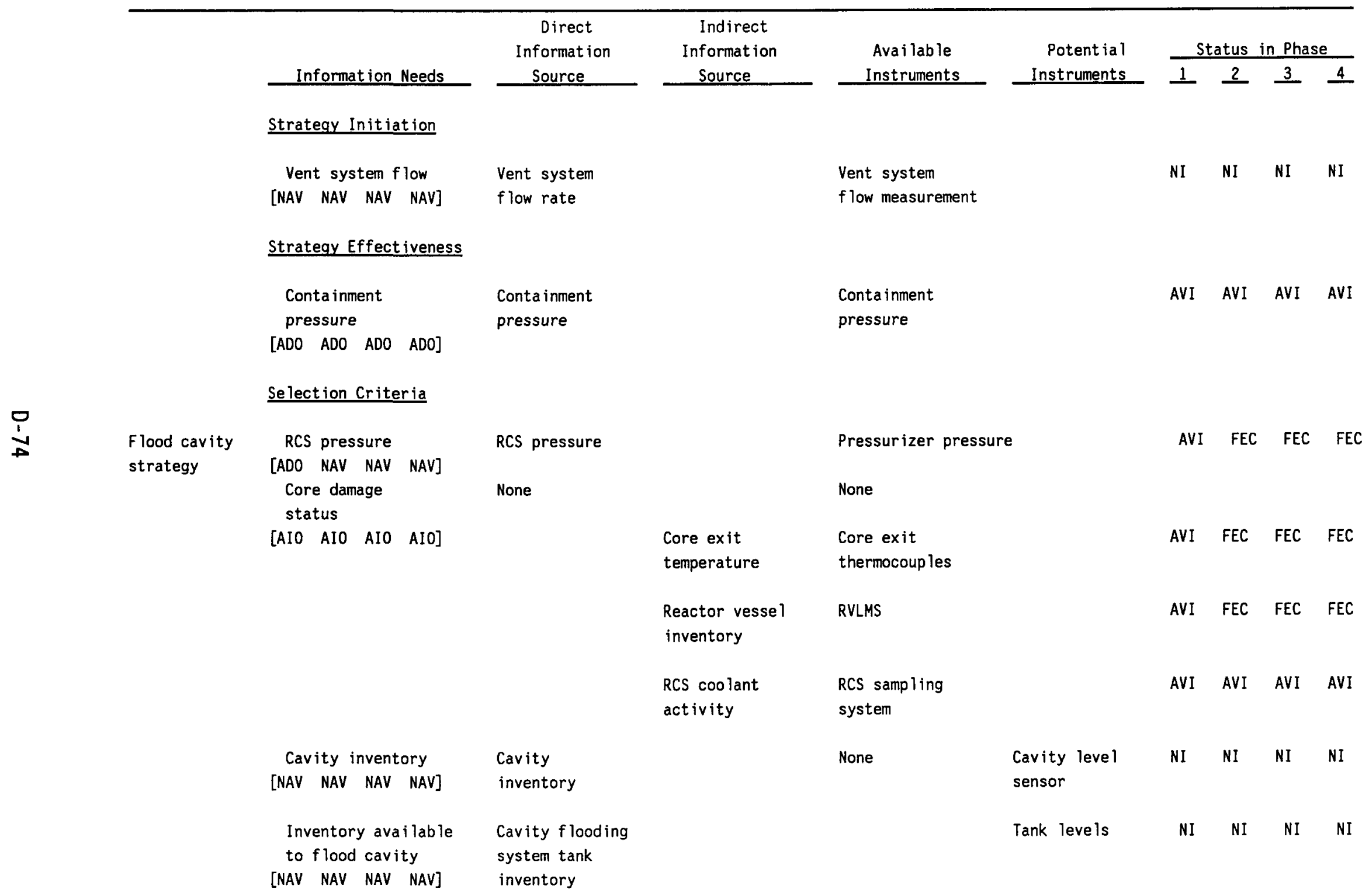


TABLE 02.3. (cont inued)

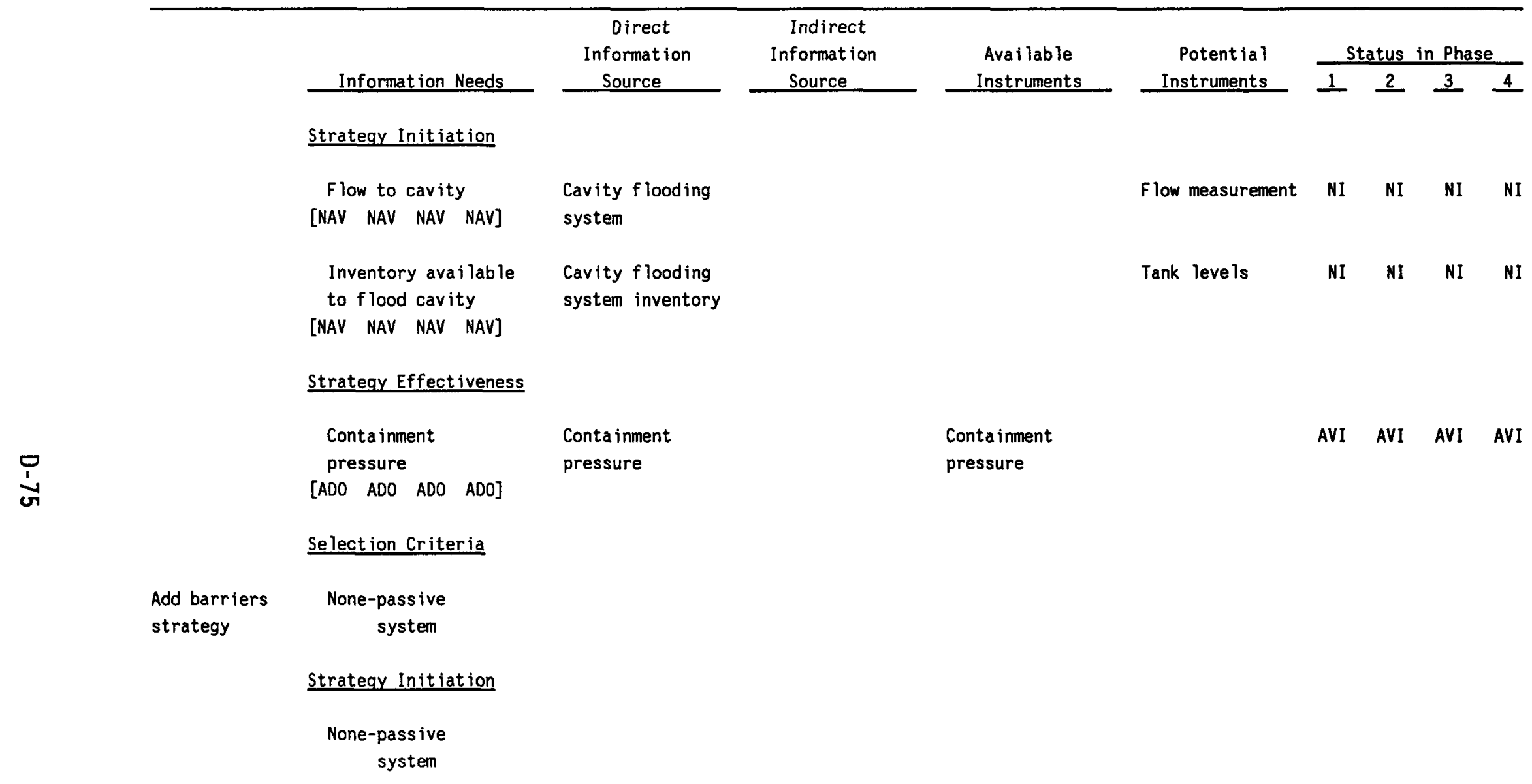


TABLE D2.4. PREVENT CONTAINMENT FAILURE (C)--COMBUSTABLE GAS DETONATION MECHANISM (C1B2)

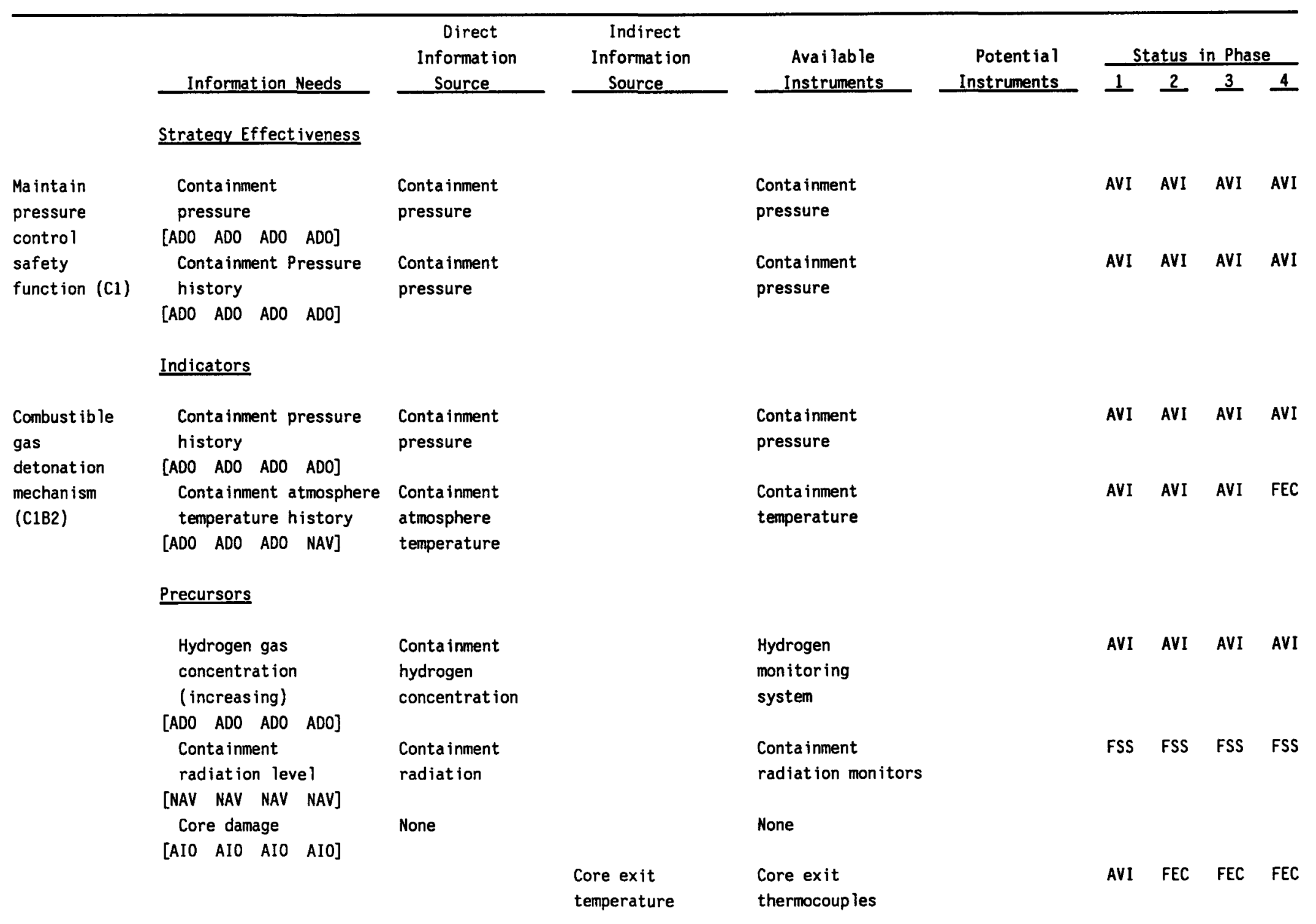


TABLE 02.4. (cont inued)

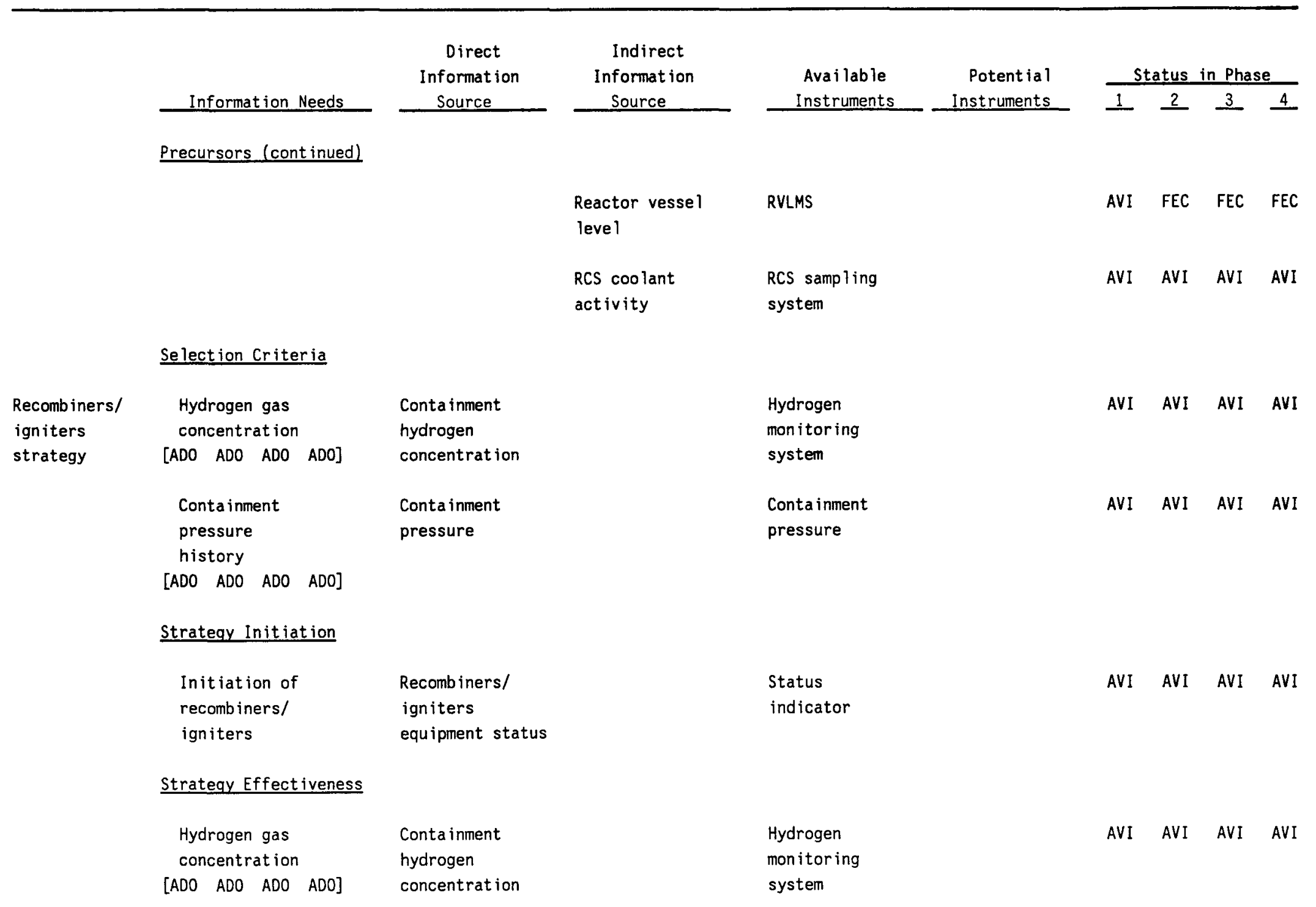


TABLE 02.4. (cont inued)

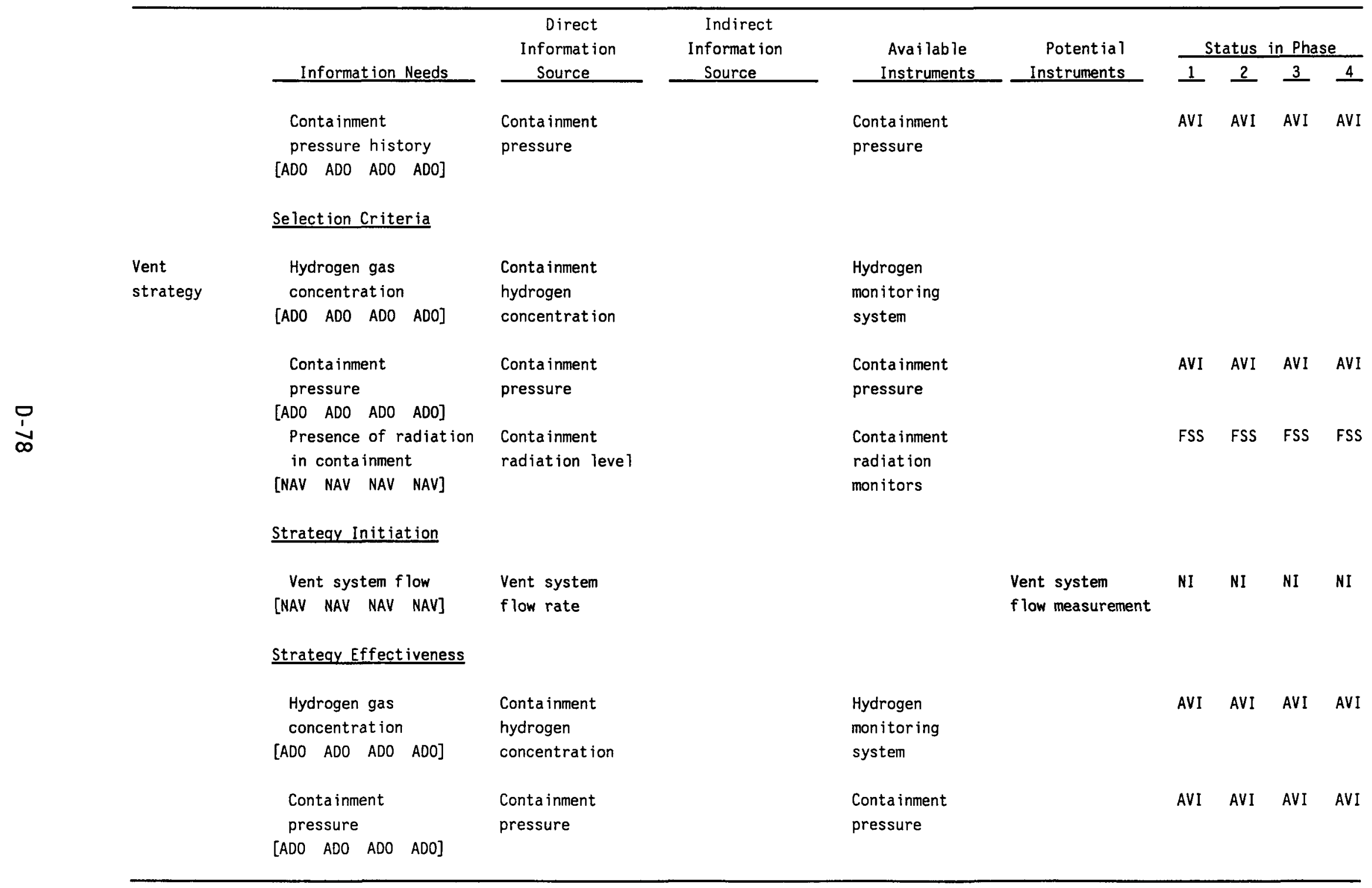


TABLE D2.5. PREVENT CONTAINMENT FAILURE (C)--STEAM EXPLOSION MECHANISM (C1B3)

\begin{tabular}{|c|c|c|c|c|c|c|c|c|c|}
\hline & & $\begin{array}{c}\text { Direct } \\
\text { Information }\end{array}$ & $\begin{array}{c}\text { Indirect } \\
\text { Information }\end{array}$ & Available & Potential & \multicolumn{4}{|c|}{ Status in Phase } \\
\hline & Information Needs & Source & Source & Instruments & Instruments & 1 & 2 & 3 & 4 \\
\hline $\begin{array}{l}\text { Maintain } \\
\text { pressure } \\
\text { control safety } \\
\text { function (C1) }\end{array}$ & $\begin{array}{l}\text { Containment pressure } \\
\text { history } \\
{\left[\begin{array}{llll}A D O & A D O & A D O & A D 0\end{array}\right]}\end{array}$ & $\begin{array}{l}\text { Containment } \\
\text { pressure }\end{array}$ & & $\begin{array}{l}\text { Containment } \\
\text { pressure }\end{array}$ & & AVI & AVI & AVI & AVI \\
\hline & Indicators & & & & & & & & \\
\hline $\begin{array}{l}\text { Steam } \\
\text { explosion } \\
\text { mechanism }\end{array}$ & $\begin{array}{l}\text { Containment pressure } \\
\text { history } \\
{[\text { AIO AIO AIO AI0] }}\end{array}$ & None & $\begin{array}{l}\text { Containment } \\
\text { pressure }\end{array}$ & $\begin{array}{l}\text { Containment } \\
\text { pressure }\end{array}$ & & AVI & AVI & AVI & AVI \\
\hline (C1B3) & $\begin{array}{l}\text { Containment atmosphere } \\
\text { temperature history } \\
{[\text { AIO AIO AIO AIO] }}\end{array}$ & None & $\begin{array}{l}\text { Containment } \\
\text { atmosphere } \\
\text { temperature }\end{array}$ & $\begin{array}{l}\text { Conta inment } \\
\text { thermocoup les }\end{array}$ & & AVI & AVI & AVI & FEC \\
\hline & $\begin{array}{l}\text { Containment } \\
\text { radiation level }\end{array}$ & None & $\begin{array}{l}\text { Containment } \\
\text { radiation level }\end{array}$ & $\begin{array}{l}\text { Containment } \\
\text { radiation }\end{array}$ & & FSS & FSS & FSS & FSS \\
\hline & [NAV NAV NAV NAV] & & & monitors & & & & & \\
\hline & Precursors & & & & & & & & \\
\hline & Vesse 1 integrity & Vessel visual & & None & Camera for & NI & NI & NI & NI \\
\hline & [NAV NAV NAV NAV] & observation & & & $\begin{array}{l}\text { vesse } 1 \\
\text { observation }\end{array}$ & & & & \\
\hline & & & $\begin{array}{l}\text { Vessel wall } \\
\text { temperature }\end{array}$ & None & $\begin{array}{l}\text { Vessel } \\
\text { temperature } \\
\text { sensor }\end{array}$ & NI & NI & NI & NI \\
\hline & & & $\begin{array}{l}\text { RCS (vessel) } \\
\text { pressure }\end{array}$ & $\begin{array}{l}\text { Pressurizer } \\
\text { pressure }\end{array}$ & & AVI & FEC & FEC & FEC \\
\hline
\end{tabular}


TABLE D2.5. (continued)

\begin{tabular}{|c|c|c|c|c|c|c|c|c|c|}
\hline & & $\begin{array}{c}\text { Direct } \\
\text { Information }\end{array}$ & $\begin{array}{c}\text { Indirect } \\
\text { Information }\end{array}$ & Available & Potential & \multicolumn{4}{|c|}{ Status in Phase } \\
\hline & Information Needs & Source & Source & Instruments & Instruments & 1 & 2 & 3 & 4 \\
\hline & \multicolumn{9}{|l|}{ Precursors (cont inued) } \\
\hline & $\begin{array}{l}\text { Core relocation } \\
\text { status }\end{array}$ & \multirow[t]{2}{*}{ None } & & None & & & & & \\
\hline & {$\left[\begin{array}{llll}\text { AIO NAV NAV NAV] } & \end{array}\right.$} & & $\begin{array}{l}\text { Radiation levels } \\
\text { outside core } \\
\text { region }\end{array}$ & $\begin{array}{l}\text { Source range } \\
\text { monitors }\end{array}$ & & AVI & FEC & FEC & FEC \\
\hline & $\begin{array}{l}\text { Core damage } \\
\text { status }\end{array}$ & \multirow[t]{2}{*}{ None } & & None & & & & & \\
\hline & {$[$ AIO AIO AIO AIO $]$} & & $\begin{array}{l}\text { Core exit } \\
\text { temperature }\end{array}$ & $\begin{array}{l}\text { Core exit } \\
\text { thermocouples }\end{array}$ & & AVI & FEC & FEC & FEC \\
\hline \multirow[t]{4}{*}{$\begin{array}{l}0 \\
1 \\
0\end{array}$} & & & $\begin{array}{l}\text { Reactor vesse } 1 \\
\text { level }\end{array}$ & RVLMS & & AVI & FEC & FEC & FEC \\
\hline & & & $\begin{array}{l}\text { RCS coolant } \\
\text { activity }\end{array}$ & RCS sampling & & AVI & AVI & AVI & AVI \\
\hline & $\begin{array}{l}\text { Water in Cavity } \\
\text { [NAV NAV NAV NAV] }\end{array}$ & $\begin{array}{l}\text { Cavity } \\
\text { inventory }\end{array}$ & None & None & $\begin{array}{l}\text { Cavity level } \\
\text { sensor }\end{array}$ & NI & NI & NI & NI \\
\hline & \multicolumn{2}{|l|}{ Selection Criteria } & & & & & & & \\
\hline $\begin{array}{l}\text { Eliminate } \\
\text { water strategy }\end{array}$ & $\begin{array}{l}\text { Cavity } \\
\text { inventory }\end{array}$ & \multicolumn{2}{|l|}{$\begin{array}{l}\text { Cavity } \\
\text { inventory }\end{array}$} & None & $\begin{array}{l}\text { Cavity level } \\
\text { sensor }\end{array}$ & NI & NI & NI & NI \\
\hline & $\begin{array}{l}\text { Core damage } \\
\text { status }\end{array}$ & None & & None & & & & & \\
\hline & {$[$ AIO AIO AIO AIO $]$} & & $\begin{array}{l}\text { Core exit } \\
\text { temperature }\end{array}$ & $\begin{array}{l}\text { Core exit } \\
\text { thermocouples }\end{array}$ & & AVI & FEC & FEC & FEC \\
\hline & & & $\begin{array}{l}\text { Reactor vessel } \\
\text { inventory }\end{array}$ & RVLMS & & AVI & FEC & FEC & FEC \\
\hline
\end{tabular}


TABLE 02.5. (continued)

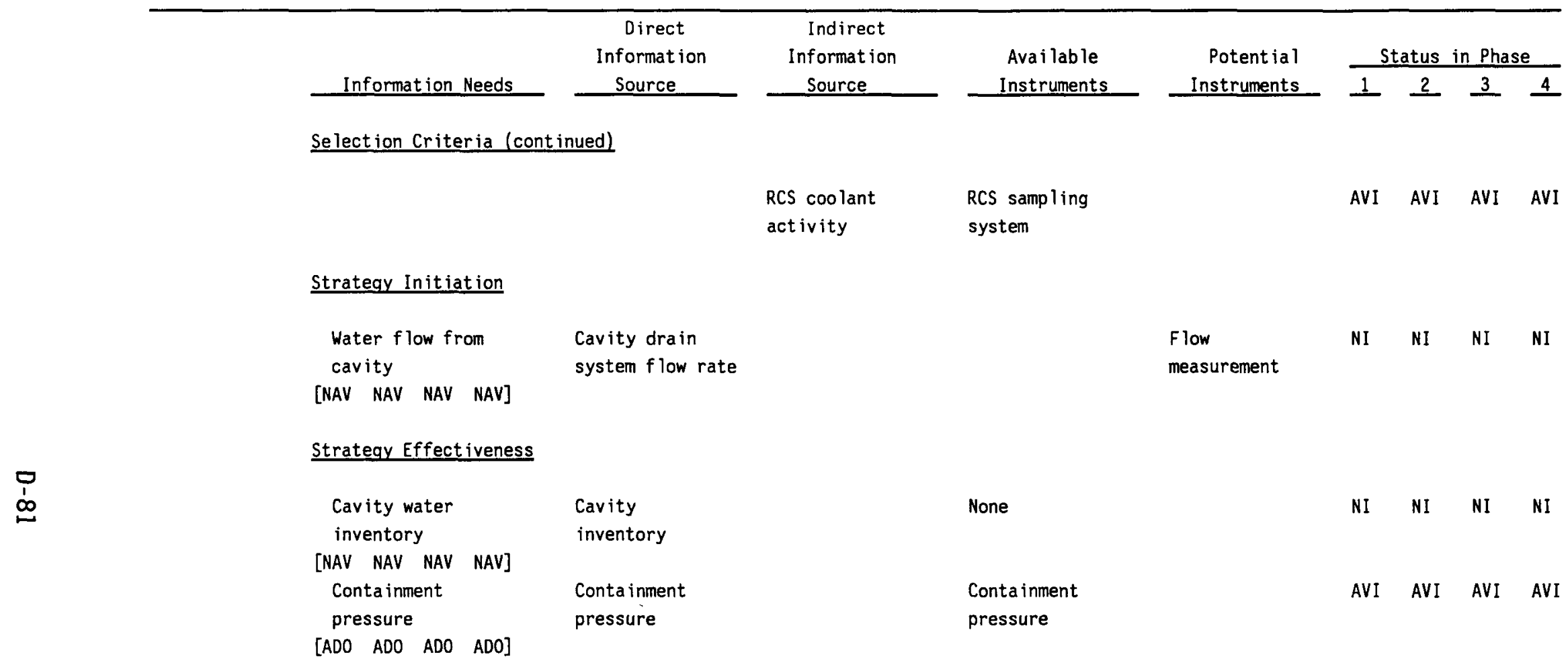


TABLE D2 5 (continued)

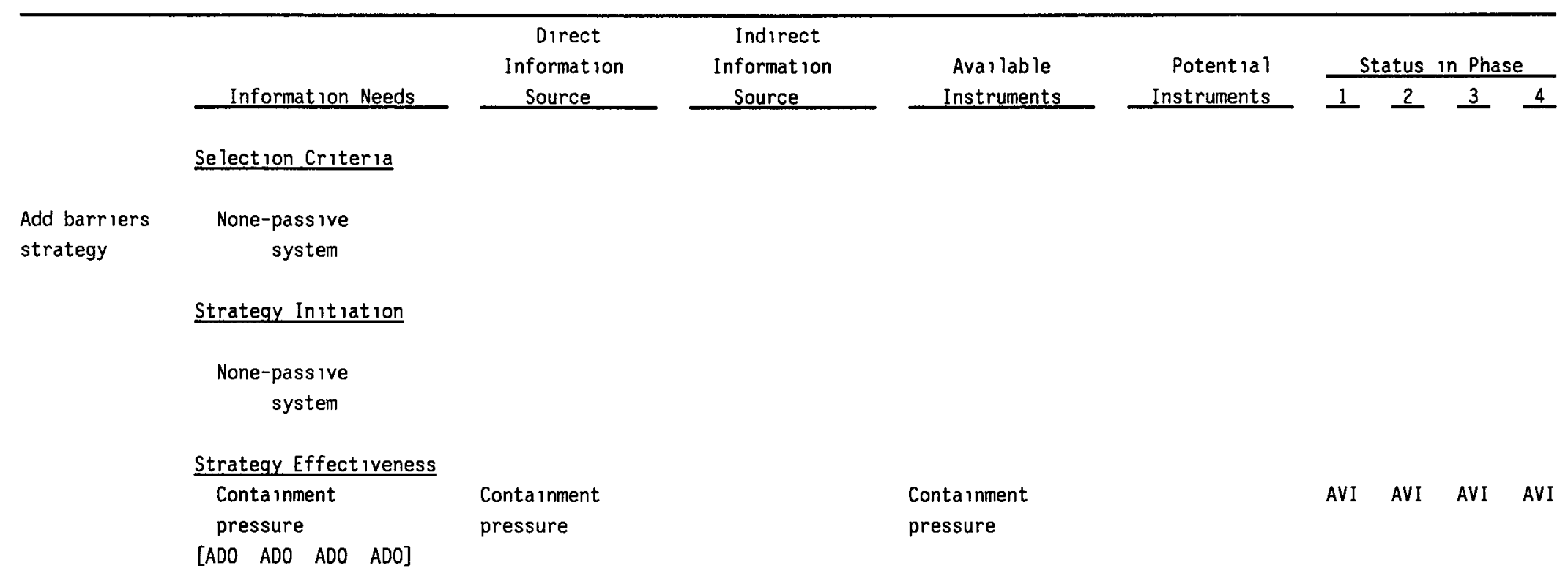




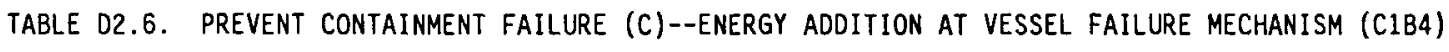

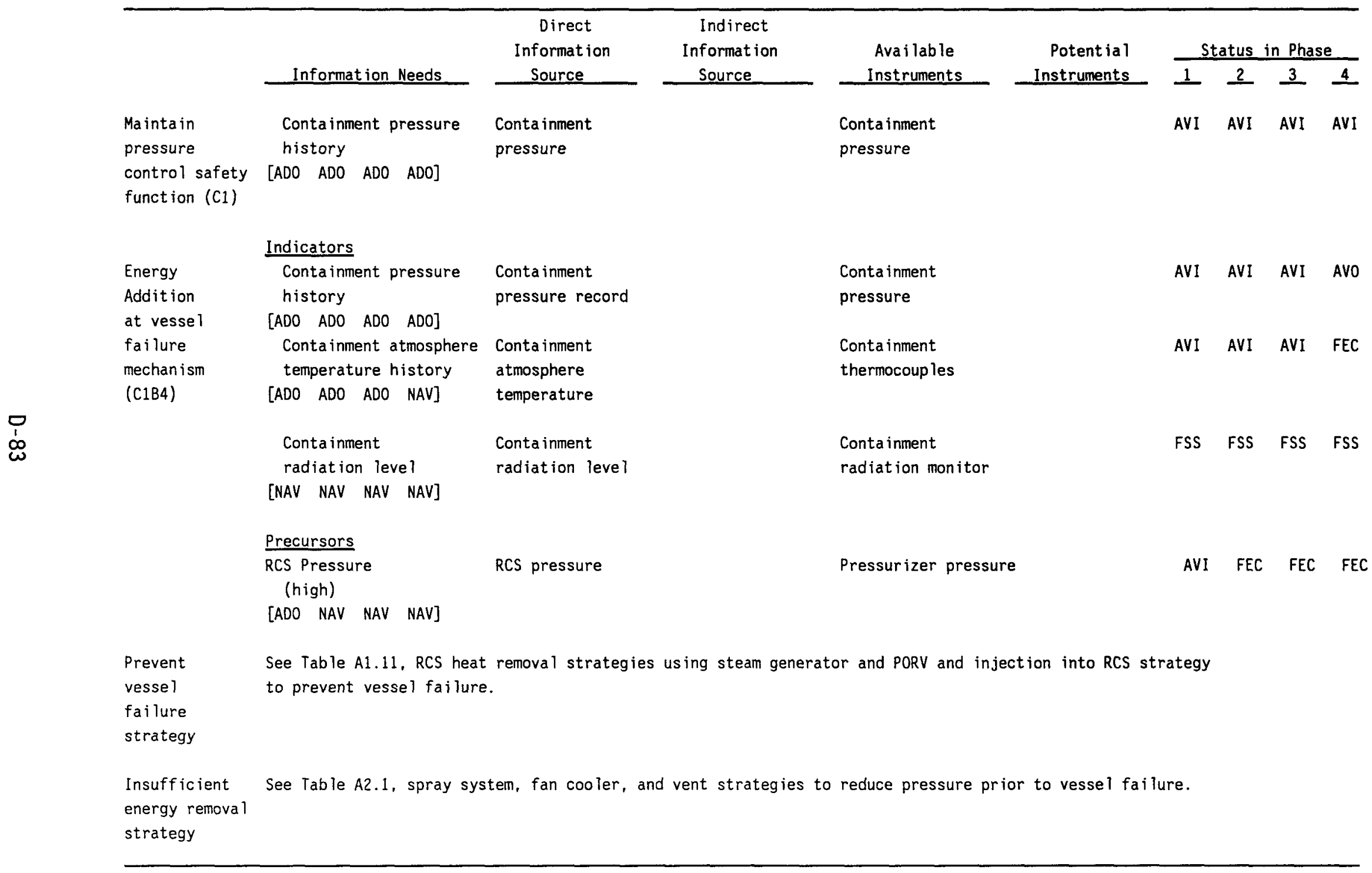


TABLE D2.7. PREVENT CONTAINMENT FAILURE (C)--TEMPERATURE INDUCED DEGRADATION MECHANISM (C2A1)

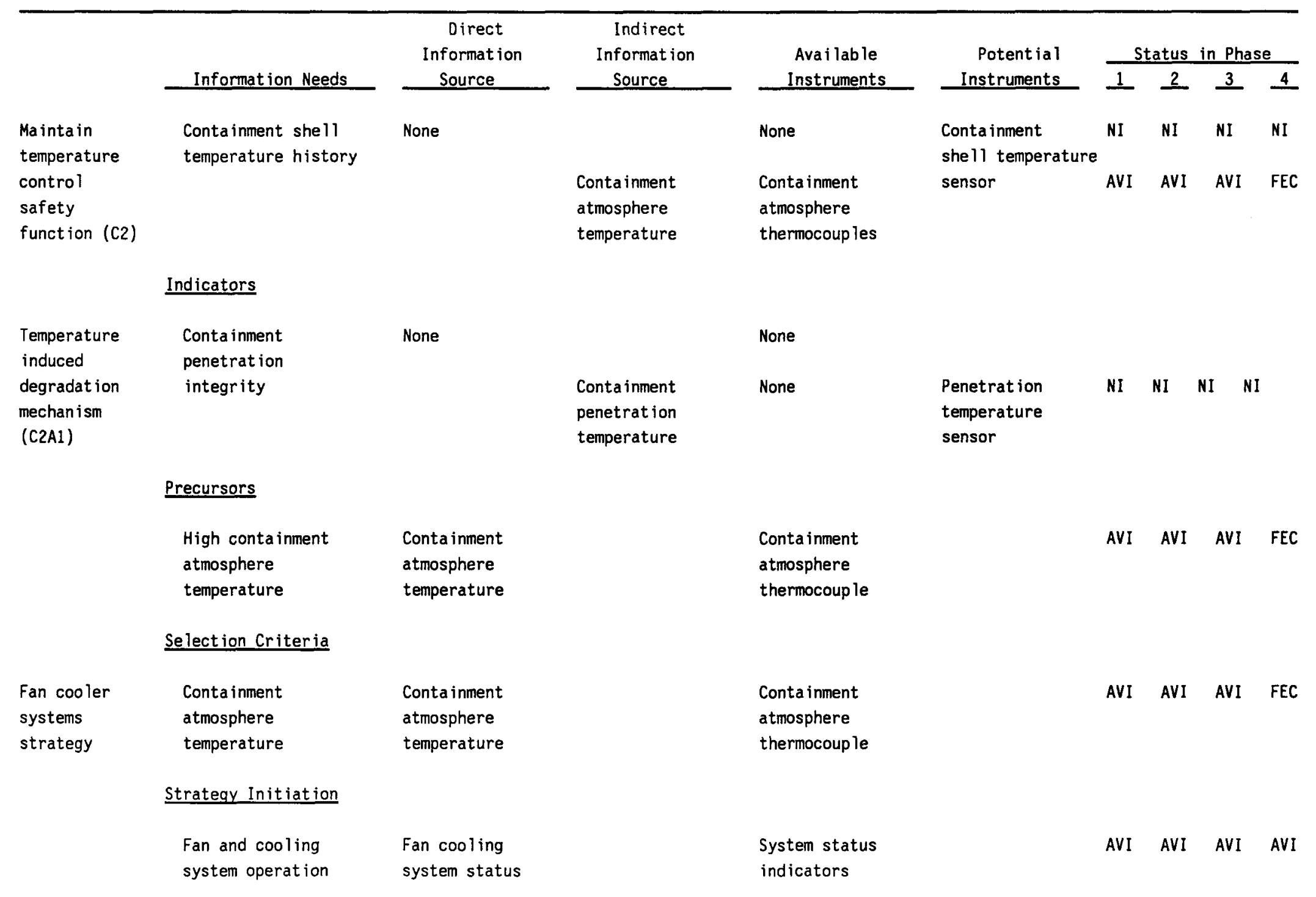


TABLE 02.7 (continued)

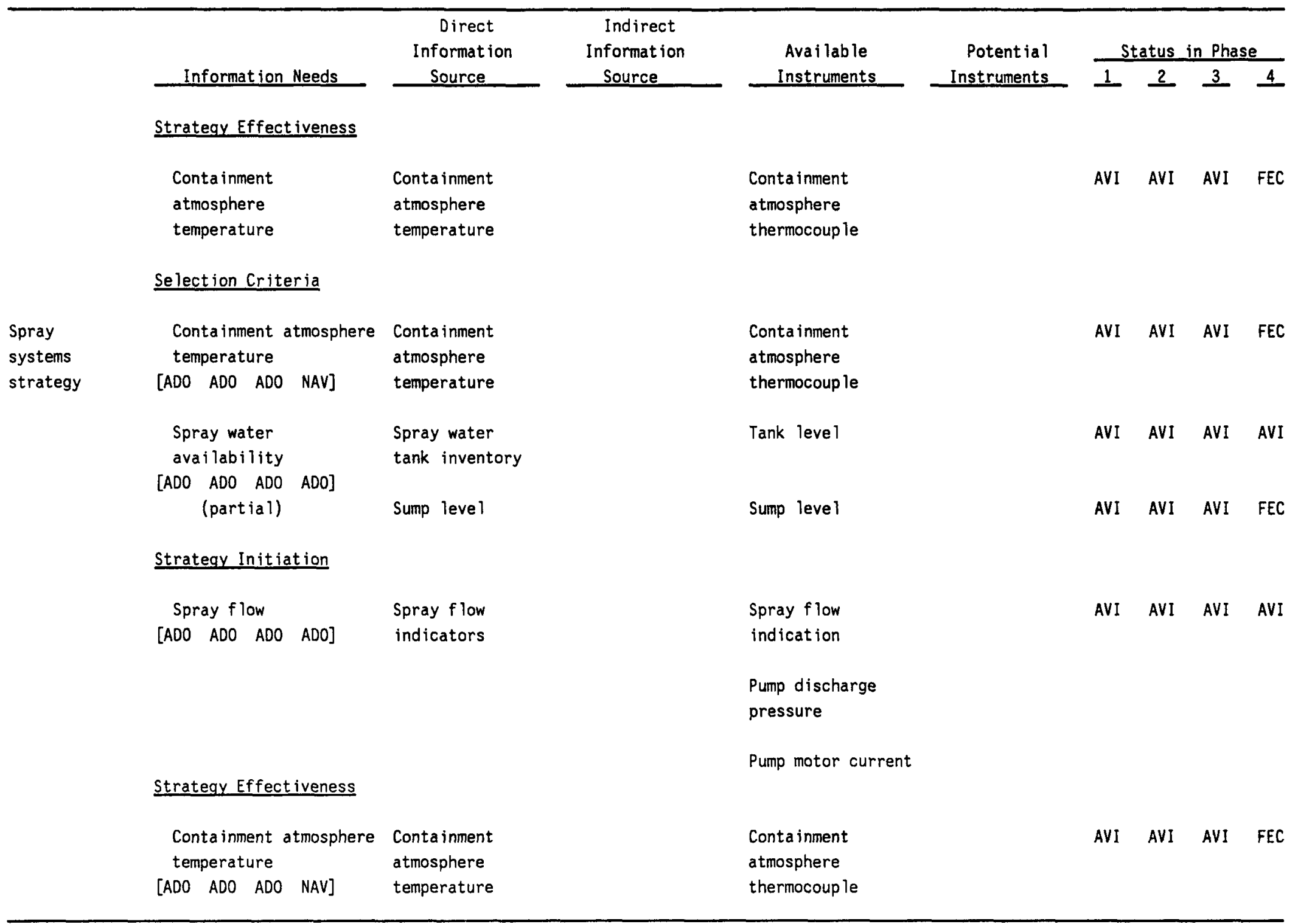


TABLE D2.8. PREVENT CONTAINMENT FAILURE (C)--CORE/CONCRETE INTERACTION MECHANISM (C2B1)

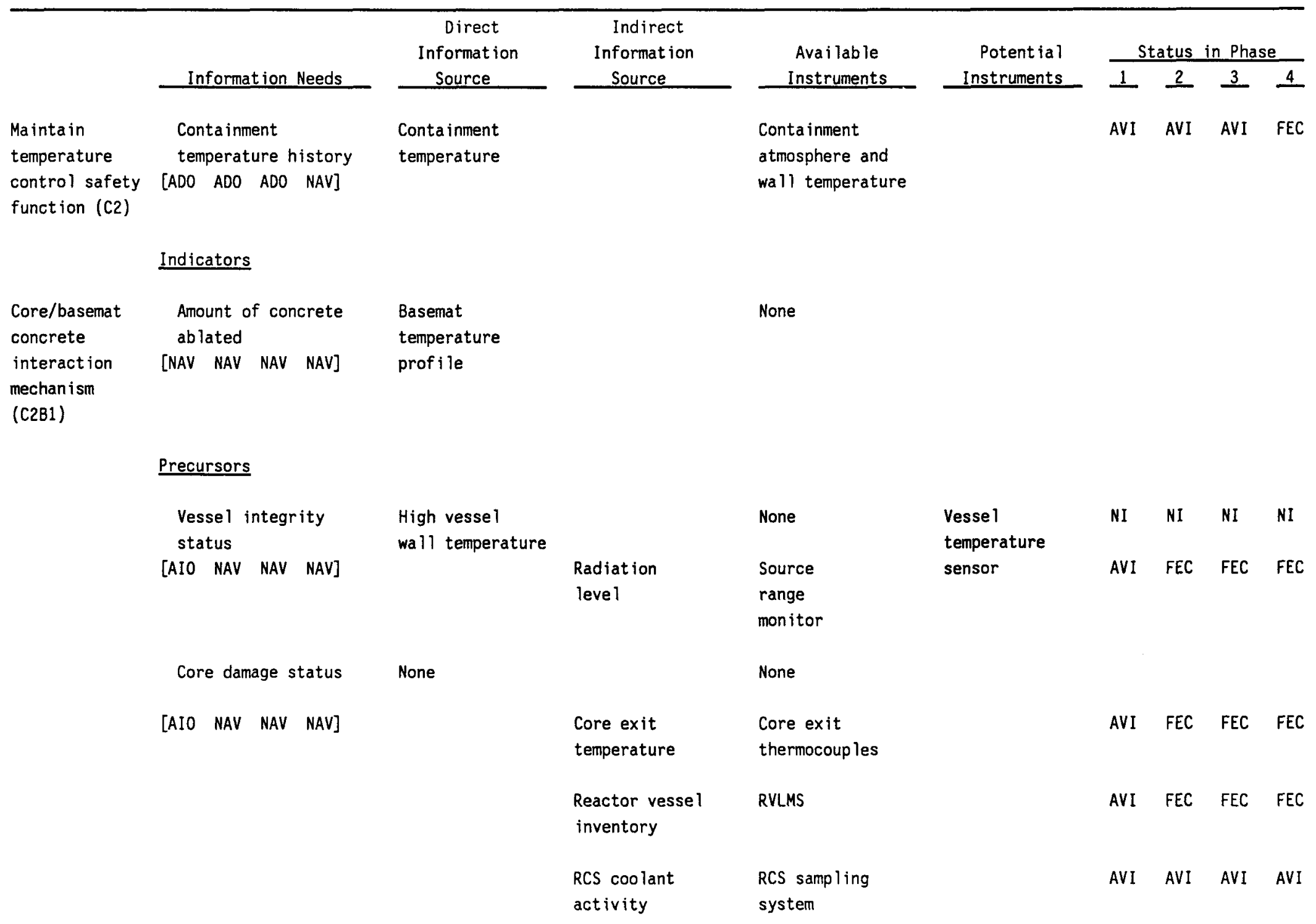


TABLE 02.8. (cont inued)

\begin{tabular}{|c|c|c|c|c|c|c|c|c|c|}
\hline & & $\begin{array}{c}\text { Direct } \\
\text { Information }\end{array}$ & $\begin{array}{c}\text { Indirect } \\
\text { Information }\end{array}$ & Available & Potential & $S$ & atus & Pha & \\
\hline & Information Needs & Source & Source & Instruments & Instruments & 1 & 2 & 3 & 4 \\
\hline & Selection Criteria & & & & & & & & \\
\hline \multirow{13}{*}{$\begin{array}{l}\text { Flood } \\
\text { cavity } \\
\text { strategy }\end{array}$} & Core damage status & None & & None & & & & & \\
\hline & {$\left[\begin{array}{llll}A I 0 & A I 0 & A I 0 & A I 0\end{array}\right]$} & & & & & & & & \\
\hline & & & $\begin{array}{l}\text { Core exit } \\
\text { temperature }\end{array}$ & $\begin{array}{l}\text { Core exit } \\
\text { thermocouples }\end{array}$ & & AVI & FEC & FEC & FEC \\
\hline & & & $\begin{array}{l}\text { Reactor vesse } 1 \\
\text { leve } 1\end{array}$ & RVLMS & & AVI & FEC & FEC & FEC \\
\hline & & & $\begin{array}{l}\text { RCS coolant } \\
\text { activity }\end{array}$ & RCS sampling & & AVI & AVI & AVI & AVI \\
\hline & $\begin{array}{l}\text { Cavity inventory } \\
\text { [NAV NAV NAV NAV] }\end{array}$ & Cavity inventory & & None & $\begin{array}{l}\text { Cavity level } \\
\text { sensor }\end{array}$ & NI & $N I$ & NI & NI \\
\hline & $\begin{array}{l}\text { Inventory available } \\
\text { to flood vacity }\end{array}$ & $\begin{array}{l}\text { Cavity flooding } \\
\text { system tank }\end{array}$ & & & Tank leve is & NI & NI & NI & NI \\
\hline & {$\left[\begin{array}{llll}\text { NAV } & \text { NAV } & \text { NAV } & \text { NAV }\end{array}\right]$} & inventory & & & & & & & \\
\hline & Strategy Initiation & & & & & & & & \\
\hline & Flow to cavity & Cavity flooding & & & F low measurement & NI & NI & NI & NI \\
\hline & {$\left[\begin{array}{llll}\text { NAV } & \text { NAV } & \text { NAV } & \text { NAV}\end{array}\right]$} & system flow rate & & & & & & & \\
\hline & $\begin{array}{l}\text { [NAV NAV NAV NAV] } \\
\text { Inventory available } \\
\text { to flood cavity }\end{array}$ & $\begin{array}{l}\text { Cavity flooding } \\
\text { system tank }\end{array}$ & & & Tank levels & NI & NI & NI & NI \\
\hline & [NAV NAV NAV NAV] & inventory & & & & & & & \\
\hline
\end{tabular}


TABLE D2.8. (cont inued)

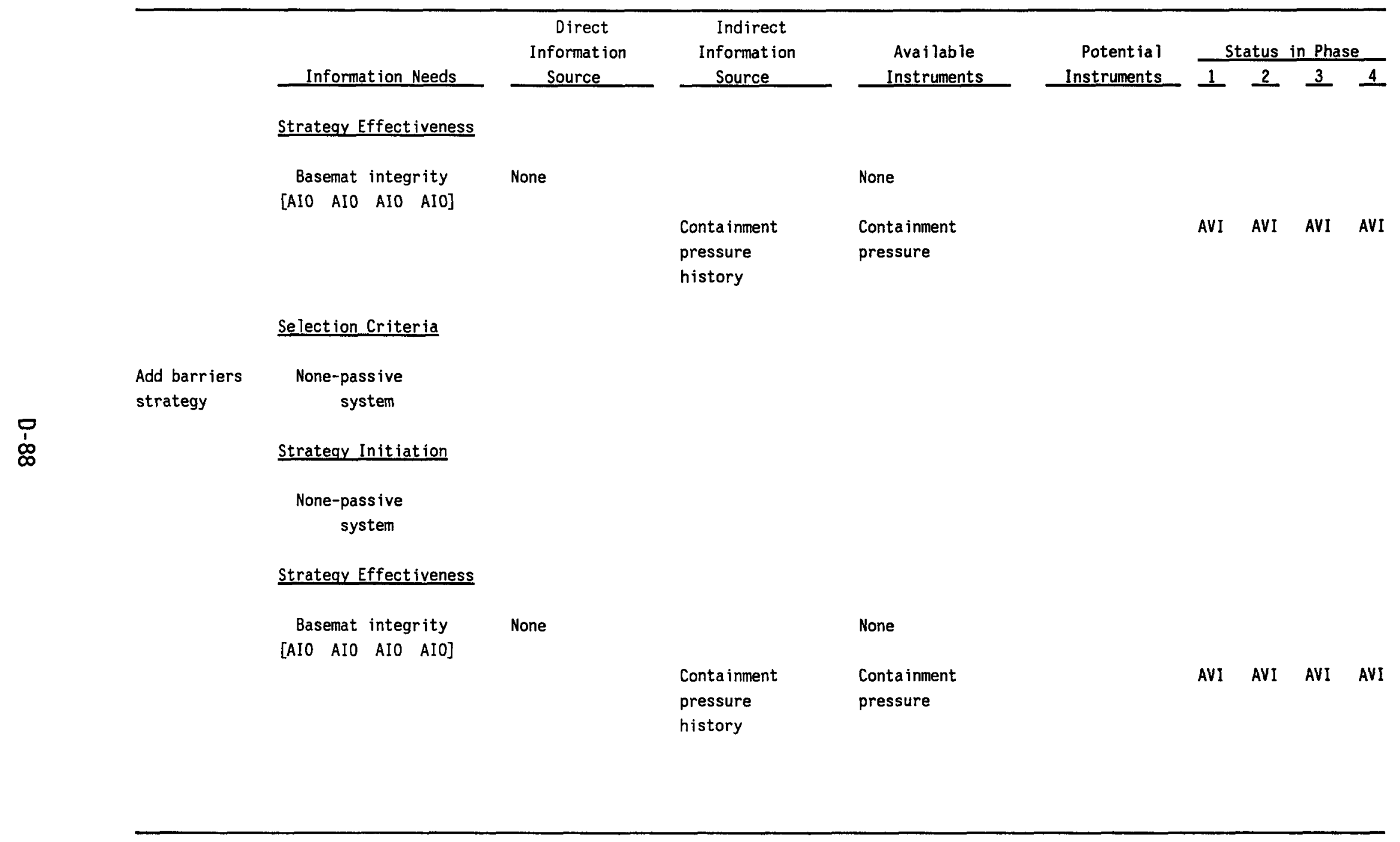


TABLE 02.9. PREVENT CONTAINMENT FAILURE (C)--FAILURE TO ISOLATE MECHANISM (C3A1)

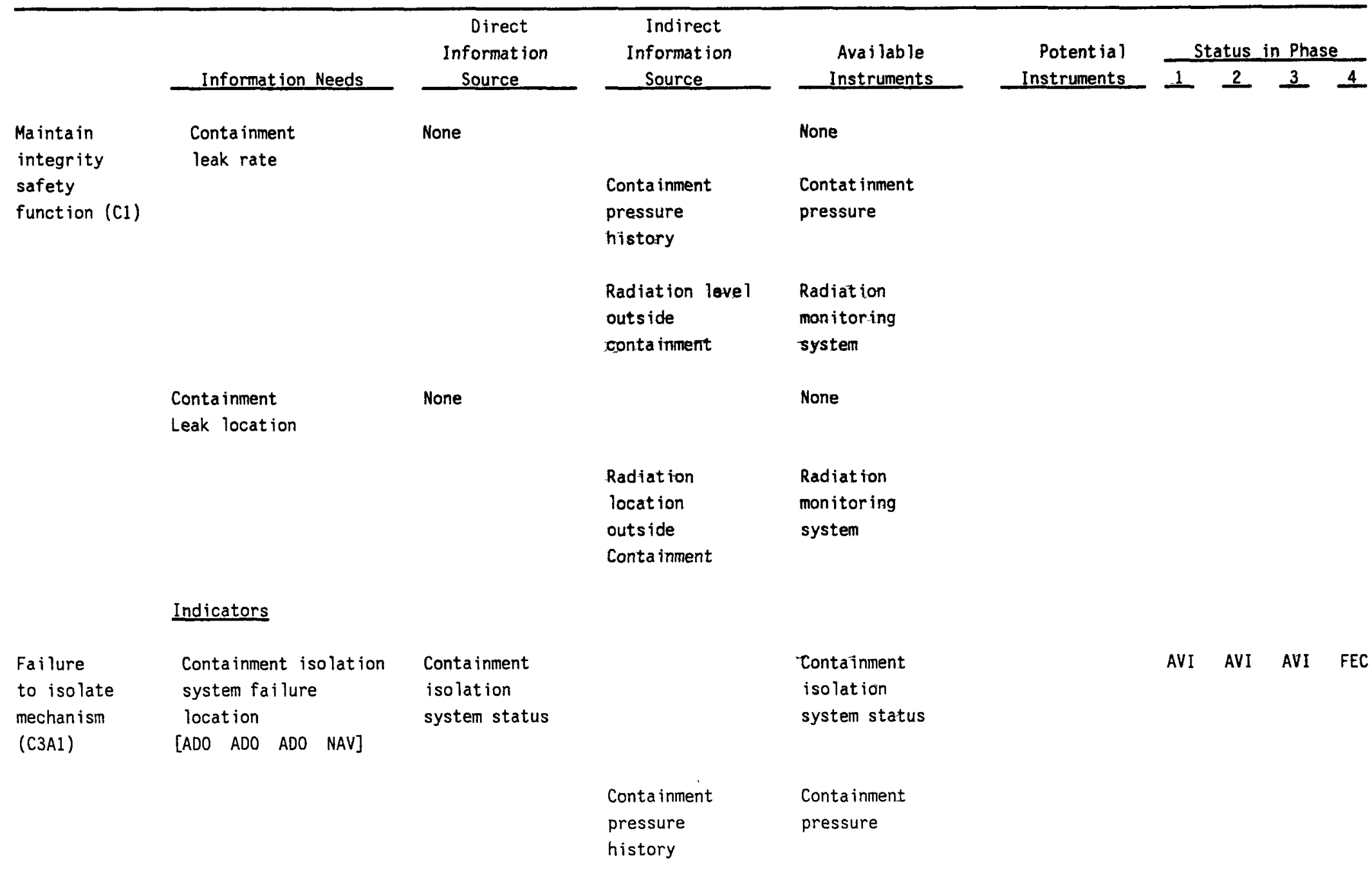


TABLE 02.9. (continued)

\begin{tabular}{|c|c|c|c|c|c|c|c|c|c|}
\hline & \multirow[b]{2}{*}{ Information Needs } & \multirow{2}{*}{$\begin{array}{c}\text { Direct } \\
\text { Information } \\
\text { Source } \\
\end{array}$} & $\begin{array}{c}\text { Indirect } \\
\text { Information }\end{array}$ & \multirow{2}{*}{$\begin{array}{c}\text { Avallable } \\
\text { Instruments }\end{array}$} & \multirow{2}{*}{$\begin{array}{r}\text { Potentia I } \\
\text { Instruments }\end{array}$} & \multicolumn{4}{|c|}{ Status in Phase } \\
\hline & & & Source & & & 1 & 2 & 3 & 4 \\
\hline & Indicators (cont inued) & & & & & & & & \\
\hline & & & $\begin{array}{l}\text { Radiation level } \\
\text { outside } \\
\text { containment }\end{array}$ & $\begin{array}{l}\text { Radiation } \\
\text { monitoring } \\
\text { system }\end{array}$ & & AVI & AVI & AVI & AVI \\
\hline & Precursors & & & & & & & & \\
\hline & $\begin{array}{l}\text { Core damage } \\
\text { status }\end{array}$ & None & & None & & & & & \\
\hline & {$[$ AIO AIO AIO AIO $]$} & & $\begin{array}{l}\text { RCS coolant } \\
\text { activity }\end{array}$ & Sampling system & & AVI & AVI & AVI & AVI \\
\hline & & & $\begin{array}{l}\text { Reactor vesse } 1 \\
\text { inventory }\end{array}$ & RVLMS & & AVI & FEC & FEC & FEC \\
\hline & $\begin{array}{l}\text { Containment } \\
\text { radiation } \\
\text { level }\end{array}$ & $\begin{array}{l}\text { Containment } \\
\text { radiation } \\
\text { level }\end{array}$ & & $\begin{array}{l}\text { Containment } \\
\text { radiation } \\
\text { monitor }\end{array}$ & & & & & \\
\hline $\begin{array}{l}\text { Plant specif ic } \\
\text { isolation } \\
\text { response } \\
\text { strategy }\end{array}$ & $\begin{array}{l}\text { Valve } \\
\text { position }\end{array}$ & $\begin{array}{l}\text { Plant } \\
\text { dependant }\end{array}$ & $\begin{array}{l}\text { Plant } \\
\text { dependant }\end{array}$ & $\begin{array}{l}\text { Plant } \\
\text { dependant }\end{array}$ & & & & & \\
\hline
\end{tabular}


TABLE 02.9. (cont inued)

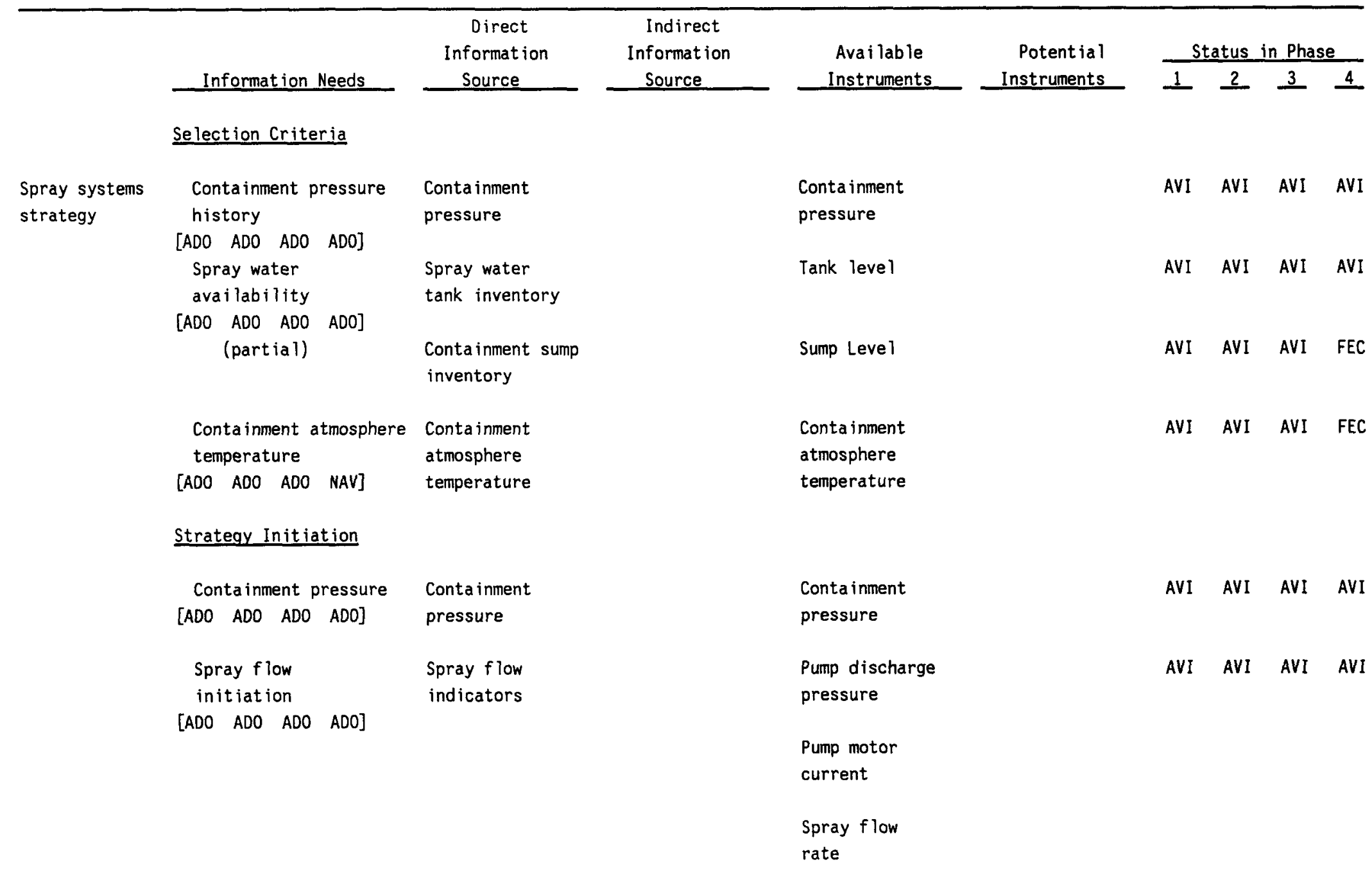


TABLE 02.9. (cont inued)

\begin{tabular}{|c|c|c|c|c|c|c|c|c|c|}
\hline & & $\begin{array}{c}\text { Direct } \\
\text { Information }\end{array}$ & $\begin{array}{l}\text { Indirect } \\
\text { Information }\end{array}$ & Available & Potential & & atus & $\mathrm{nPh}$ & \\
\hline & Information Needs & Source & Source & Instruments & Instruments & 1 & 2 & 3 & 4 \\
\hline & Strategy Effect iveness & & & & & & & & \\
\hline & $\begin{array}{l}\text { Containment pressure } \\
\text { history }\end{array}$ & $\begin{array}{l}\text { Containment } \\
\text { pressure record }\end{array}$ & & $\begin{array}{l}\text { Conta inment } \\
\text { pressure }\end{array}$ & & AVI & AVI & AVI & AVI \\
\hline & {$\left[\begin{array}{llll}A D O & A D O & A D O & A D 0\end{array}\right]$} & & & & & & & & \\
\hline & $\begin{array}{l}\text { Conta inment atmosphere } \\
\text { temperature }\end{array}$ & $\begin{array}{l}\text { Conta inment } \\
\text { atmosphere }\end{array}$ & & $\begin{array}{l}\text { Conta inment } \\
\text { atmosphere }\end{array}$ & & AVI & AVI & AVI & FEC \\
\hline & {$\left[\begin{array}{llll}A D D O & A D O & A D D & \text { NAV }\end{array}\right]$} & temperature & & temperature & & & & & \\
\hline & Selection Criteria & & & & & & & & \\
\hline Vent strategy & $\begin{array}{l}\text { Containment pressure } \\
\text { history }\end{array}$ & $\begin{array}{l}\text { Conta inment } \\
\text { pressure }\end{array}$ & & $\begin{array}{l}\text { Conta inment } \\
\text { pressure }\end{array}$ & & AVI & AVI & AVI & AVI \\
\hline & {$\left[\begin{array}{llll}A D O & A D O & A D O & A D 0\end{array}\right]$} & & & & & & & & \\
\hline & $\begin{array}{l}\text { Presence of radiation } \\
\text { in containment }\end{array}$ & $\begin{array}{l}\text { Containment } \\
\text { radiation level }\end{array}$ & & $\begin{array}{l}\text { Containment } \\
\text { radiation }\end{array}$ & & FSS & FSS & FSS & FSS \\
\hline & [NAV NAV NAV NAV] & & & monitors & & & & & \\
\hline & $\begin{array}{l}\text { Conta inment } \\
\text { humidity }\end{array}$ & $\begin{array}{l}\text { Containment } \\
\text { humidity }\end{array}$ & & $\begin{array}{l}\text { Humidity } \\
\text { detectors }\end{array}$ & & FEC & FEC & FEC & FEC \\
\hline & Strategy Initiation & & & & & & & & \\
\hline & $\begin{array}{l}\text { Vent system } \\
\text { initiation }\end{array}$ & $\begin{array}{l}\text { Vent system } \\
\text { flow rate }\end{array}$ & & & $\begin{array}{l}\text { Vent system } \\
\text { flow }\end{array}$ & NI & NI & NI & NI \\
\hline & [NAV NAV NAV NAV] & & & & measurement & & & & \\
\hline
\end{tabular}


TABLE D2.9. (cont inued)

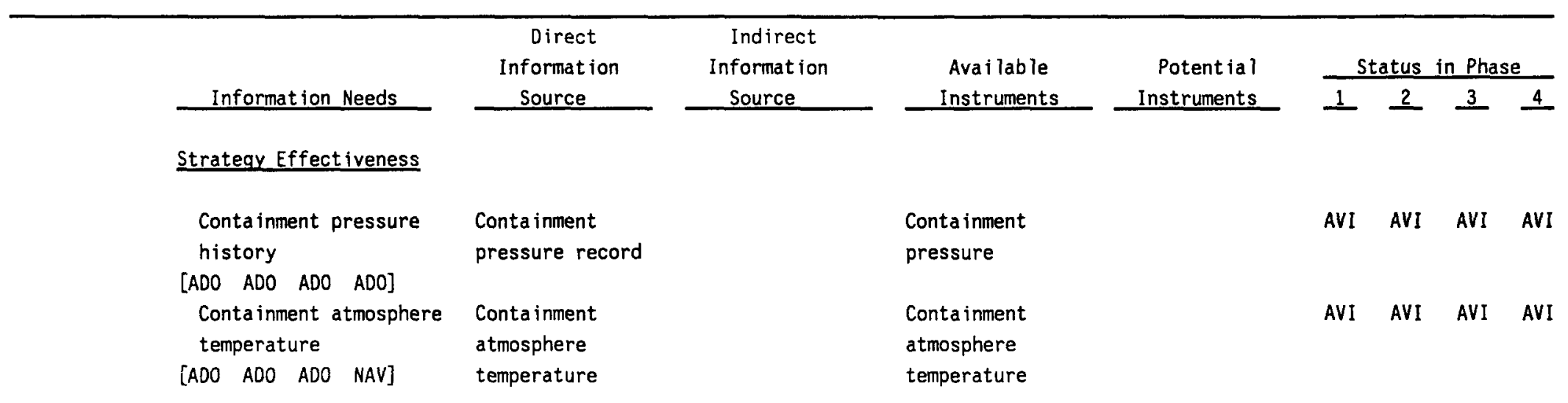


TABLE D2.10. PREVENT CONTAINMENT FAILURE (C)--FAILURE AFTER ISOLATION MECHANISM (C3A2)

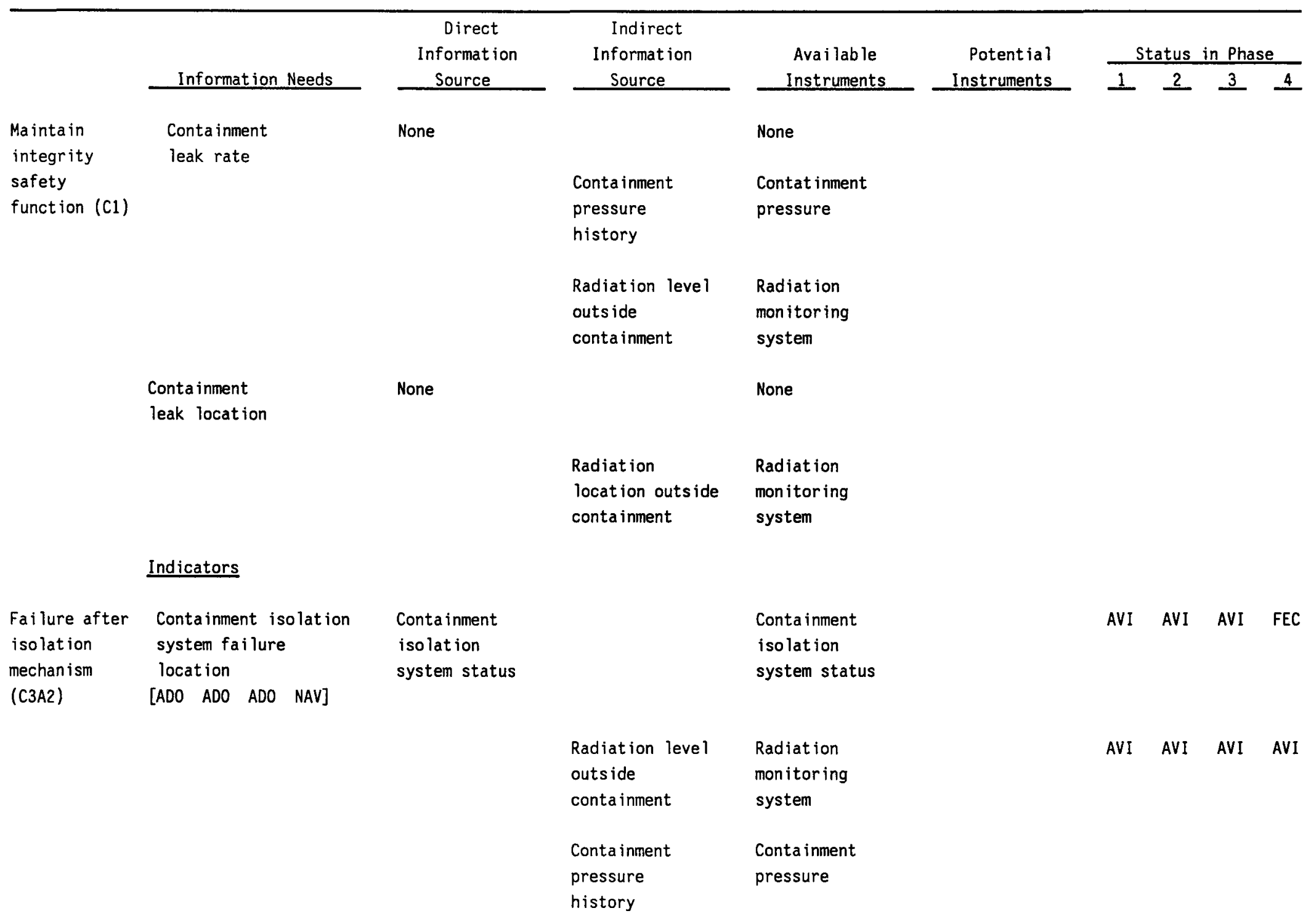


TABLE D2.10. (cont inued)

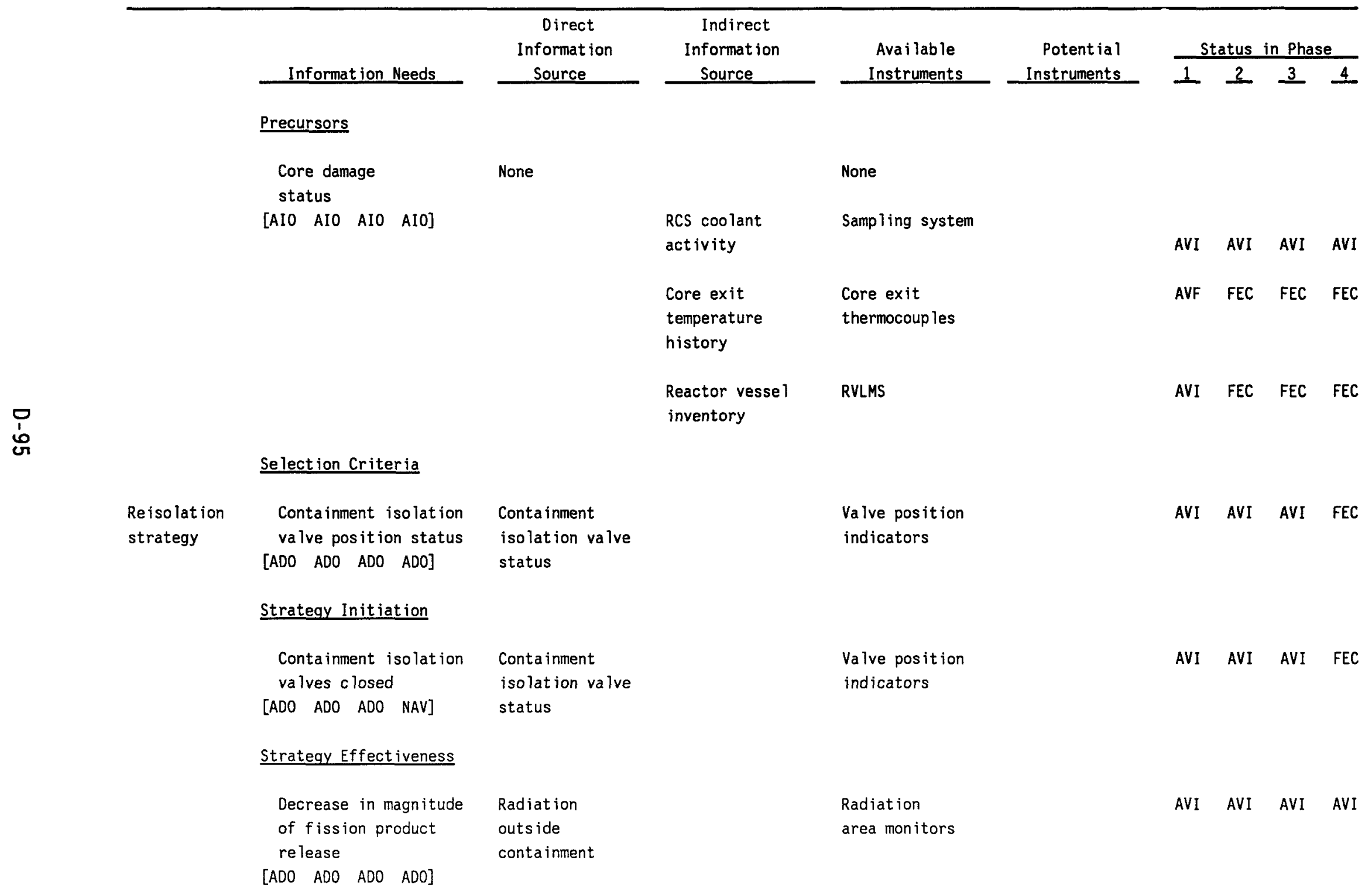


TABLE 02.10. (cont inued)

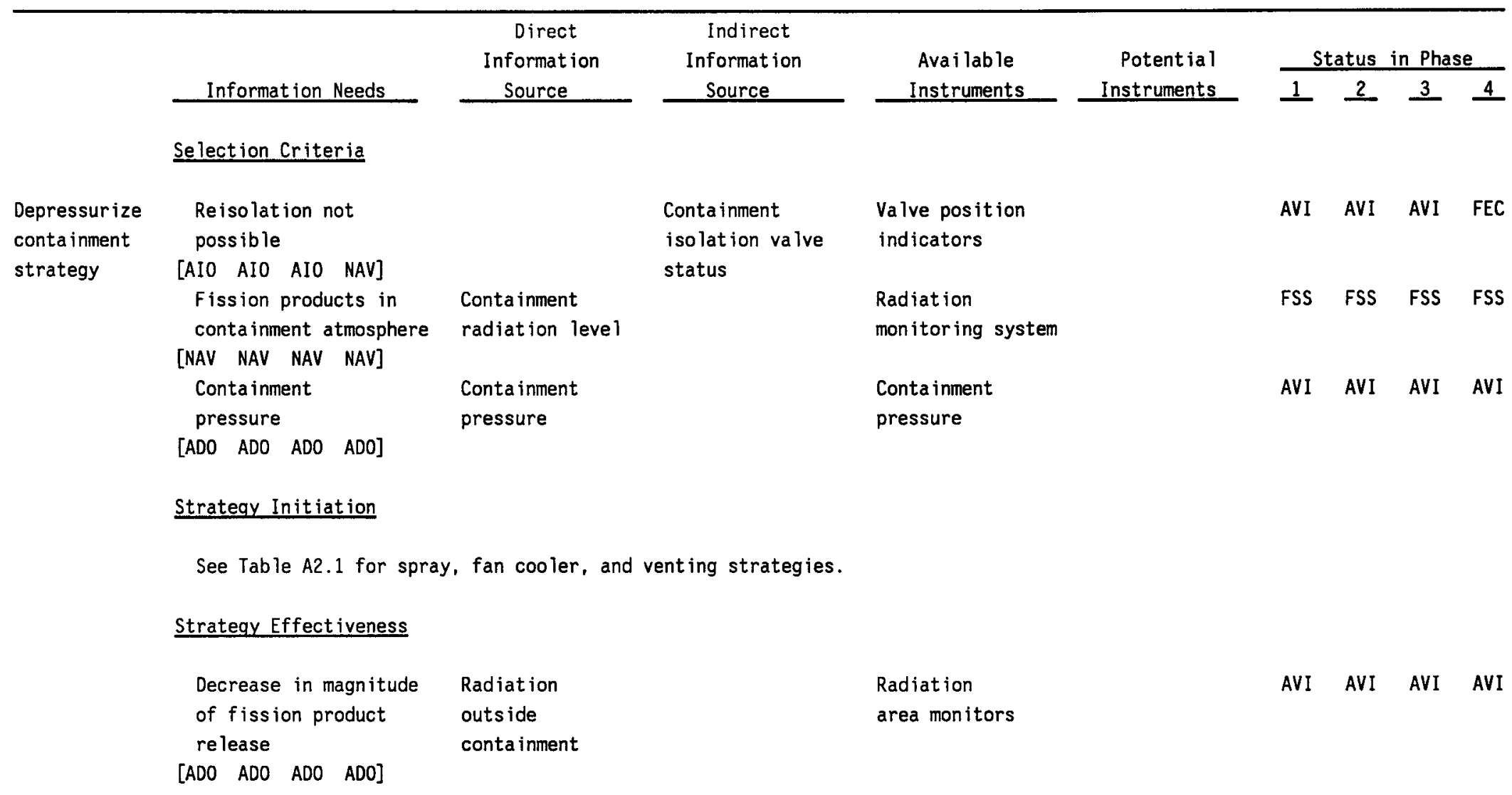


TABLE 02.10. (cont inued)

\begin{tabular}{|c|c|c|c|c|c|c|c|c|c|}
\hline & \multirow[b]{2}{*}{ Informat ion Needs } & $\begin{array}{c}\text { Direct } \\
\text { Information }\end{array}$ & $\begin{array}{c}\text { Indirect } \\
\text { Information }\end{array}$ & \multirow{2}{*}{$\begin{array}{c}\text { Ava i lab le } \\
\text { Instruments }\end{array}$} & \multirow{2}{*}{$\begin{array}{r}\text { Potential } \\
\text { Instruments } \\
\end{array}$} & \multicolumn{4}{|c|}{ Status in Phase } \\
\hline & & Source & Source & & & 1 & $\underline{2}$ & 3 & 4 \\
\hline & Selection Criteria & & & & & & & & \\
\hline \multirow{6}{*}{$\begin{array}{l}\text { Flood } \\
\text { conta inment } \\
\text { leak strategy }\end{array}$} & $\begin{array}{l}\text { Leak point can be } \\
\text { located and flooded }\end{array}$ & $\begin{array}{l}\text { Containment } \\
\text { leak location }\end{array}$ & & & $\begin{array}{l}\text { Visual or } \\
\text { camera }\end{array}$ & NI & NI & NI & NI \\
\hline & Strategy Initiation & & & & & & & & \\
\hline & $\begin{array}{l}\text { Flow to leak location } \\
\text { \{NAV NAV NAV NAV] }\end{array}$ & $\begin{array}{l}\text { Flow in leak } \\
\text { flooding system }\end{array}$ & & & Flow meter & NI & NI & NI & NI \\
\hline & Strategy effectiveness & & & & & & & & \\
\hline & $\begin{array}{l}\text { Decrease in magnitude } \\
\text { of fission product } \\
\text { release }\end{array}$ & $\begin{array}{l}\text { Radiation } \\
\text { outside } \\
\text { containment }\end{array}$ & & $\begin{array}{l}\text { Radiation } \\
\text { monitors }\end{array}$ & & AVI & AVI & AVI & AVI \\
\hline & {$\left[\begin{array}{lll}A D 0 & A D O & A D 0\end{array}\right]$} & & & & & & & & \\
\hline
\end{tabular}


TABLE 02.11. MITIGATE FISSION PRODUCT RELEASE (F)--STEAM GENERATOR TUBE RUPTURE FAILURE MECHANISM (C3B1)

\begin{tabular}{|c|c|c|c|c|c|c|c|c|c|}
\hline \multirow{2}{*}{\multicolumn{2}{|c|}{ Information Needs }} & $\begin{array}{c}\text { Direct } \\
\text { Information }\end{array}$ & $\begin{array}{c}\text { Indirect } \\
\text { Information }\end{array}$ & Available & Potential & \multicolumn{4}{|c|}{ Status in Phase } \\
\hline & & Source & Source & Instruments & Instruments & 1 & 2 & 3 & 4 \\
\hline \multirow{5}{*}{$\begin{array}{l}\text { Maintain } \\
\text { integrity } \\
\text { safety } \\
\text { function (C1) }\end{array}$} & $\begin{array}{l}\text { Containment } \\
\text { leak rate }\end{array}$ & None & & None & & & & & \\
\hline & & & $\begin{array}{l}\text { Conta inment } \\
\text { pressure } \\
\text { history }\end{array}$ & $\begin{array}{l}\text { Contatinment } \\
\text { pressure }\end{array}$ & & & & & \\
\hline & & & $\begin{array}{l}\text { Radiation level } \\
\text { outside } \\
\text { containment }\end{array}$ & $\begin{array}{l}\text { Radiation } \\
\text { monitoring } \\
\text { system }\end{array}$ & & & & & \\
\hline & $\begin{array}{l}\text { Containment } \\
\text { leak location }\end{array}$ & None & & None & & & & & \\
\hline & Indicators & & & & & & & & \\
\hline \multirow[t]{4}{*}{$\begin{array}{l}\text { SGTR } \\
\text { mechanism } \\
\text { (C3B1) }\end{array}$} & $\begin{array}{l}\text { Presence of fission } \\
\text { products in } \\
\text { steam line }\end{array}$ & $\begin{array}{l}\text { Main steam } \\
\text { line radiation } \\
\text { leve } 1\end{array}$ & & $\begin{array}{l}\text { Steam line } \\
\text { radiation } \\
\text { monitoring }\end{array}$ & & AVI & AVI & AVI & AVI \\
\hline & {$\left[\begin{array}{llll}A D O & A D O & A D O & A D 0\end{array}\right]$} & & & system & & & & & \\
\hline & & & $\begin{array}{l}\text { Condenser } \\
\text { off gas } \\
\text { radiation level }\end{array}$ & & $\begin{array}{l}\text { Condenser } \\
\text { off gas } \\
\text { radiation } \\
\text { monitoring } \\
\text { system }\end{array}$ & AVI & AVI & AVI & AVI \\
\hline & & & $\begin{array}{l}\text { Radiation } \\
\text { outside } \\
\text { containment }\end{array}$ & & $\begin{array}{l}\text { Radiation } \\
\text { surveys, constant } \\
\text { air monitor }\end{array}$ & AVI & AVI & AVI & AVI \\
\hline
\end{tabular}


TABLE D2.11. (cont inued)

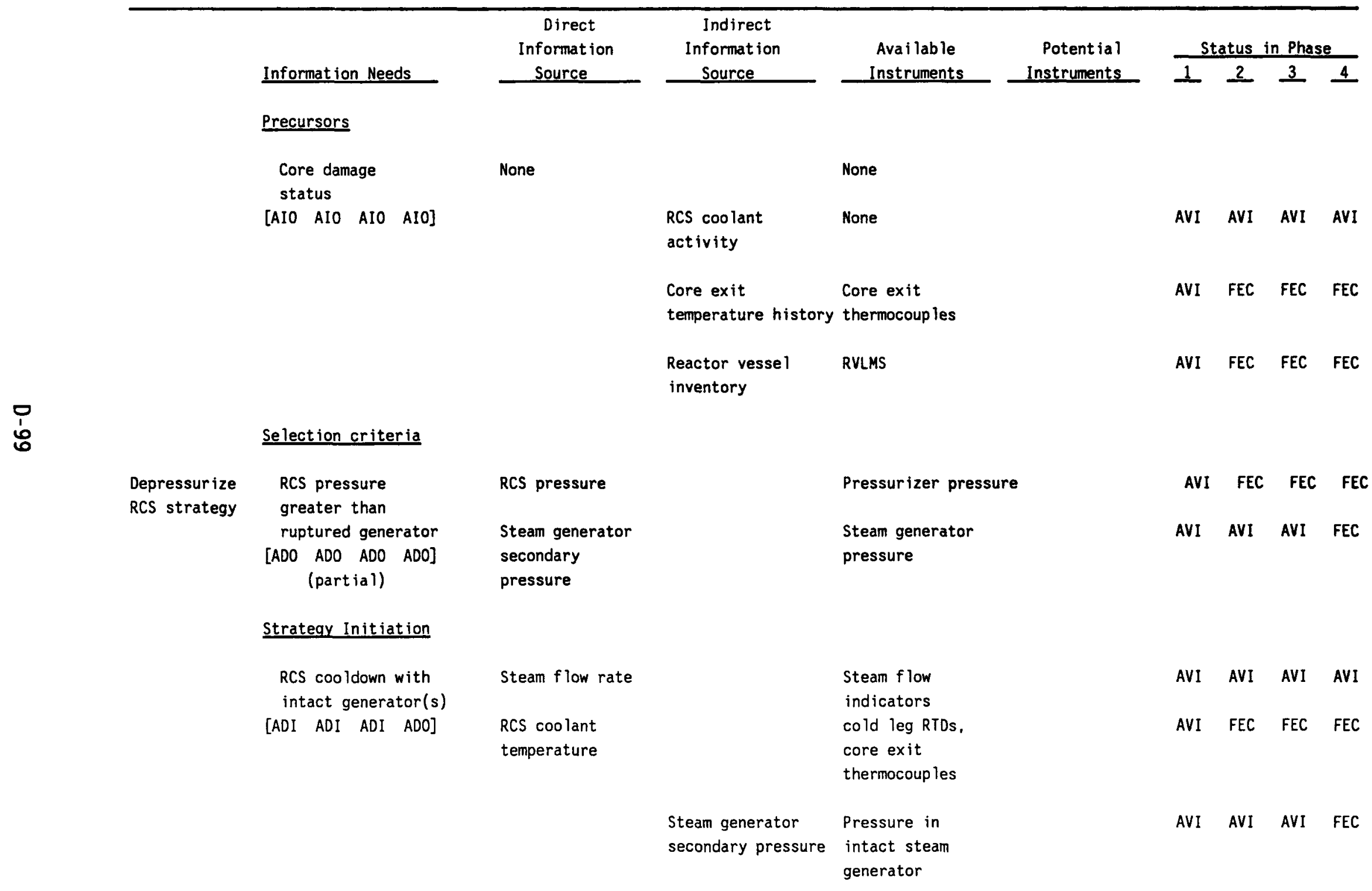


TABLE D2.11. (continued)

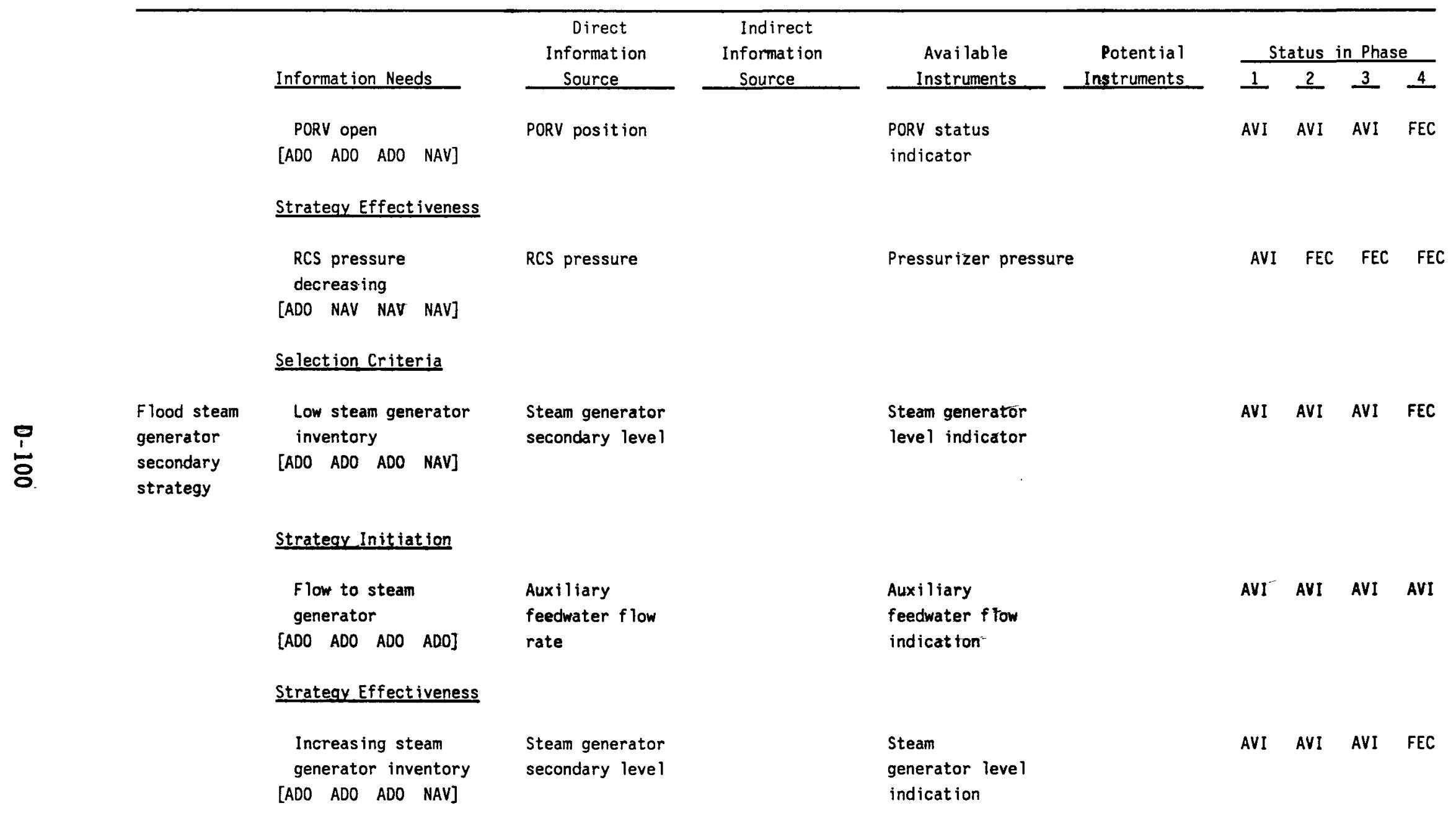


TABLE D2.12. PREVENT CONTAINMENT FAILURE (C)--ISLOCA MECHANISM (C3B2)

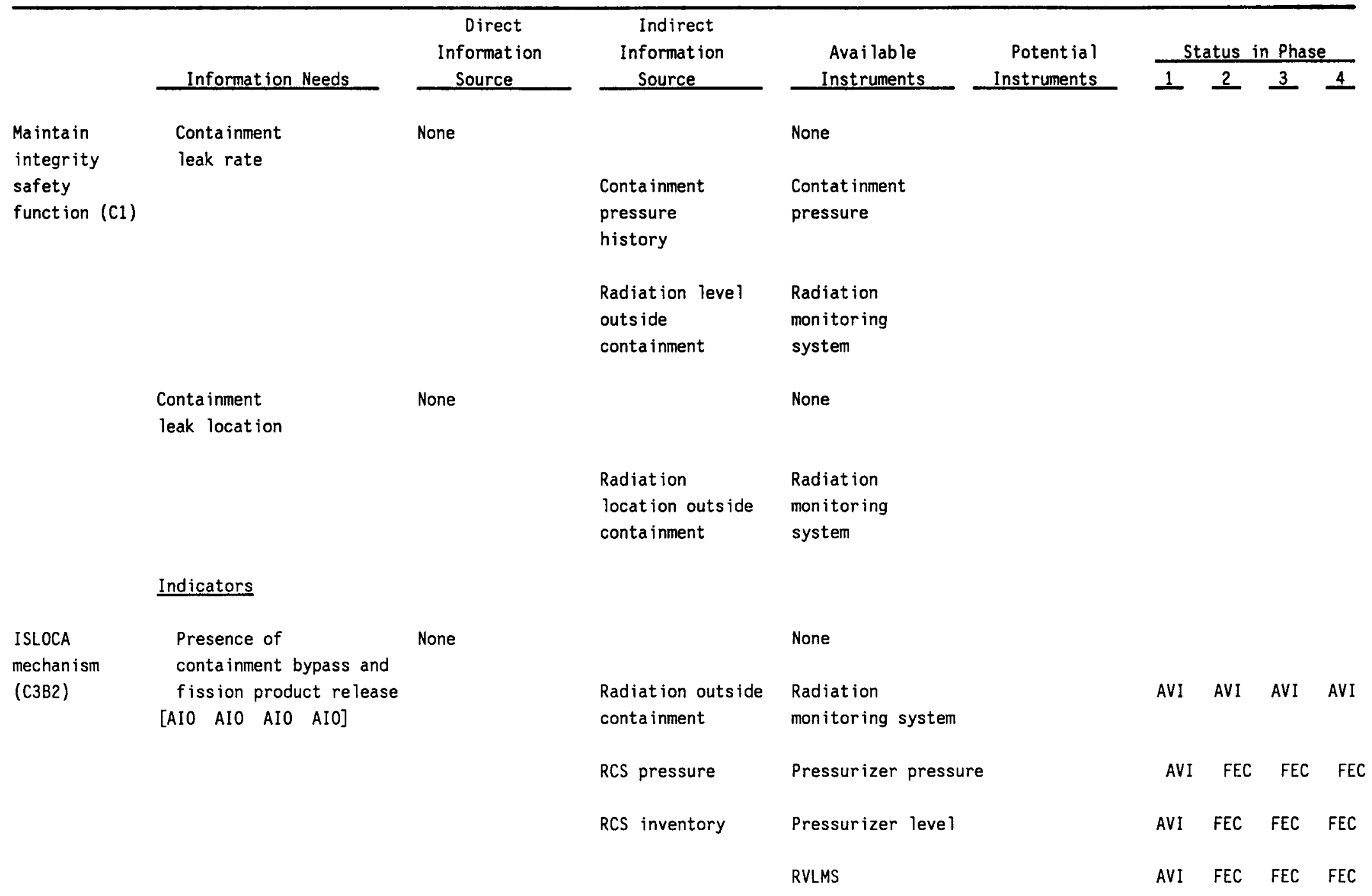


TABLE 02.12. (continued)

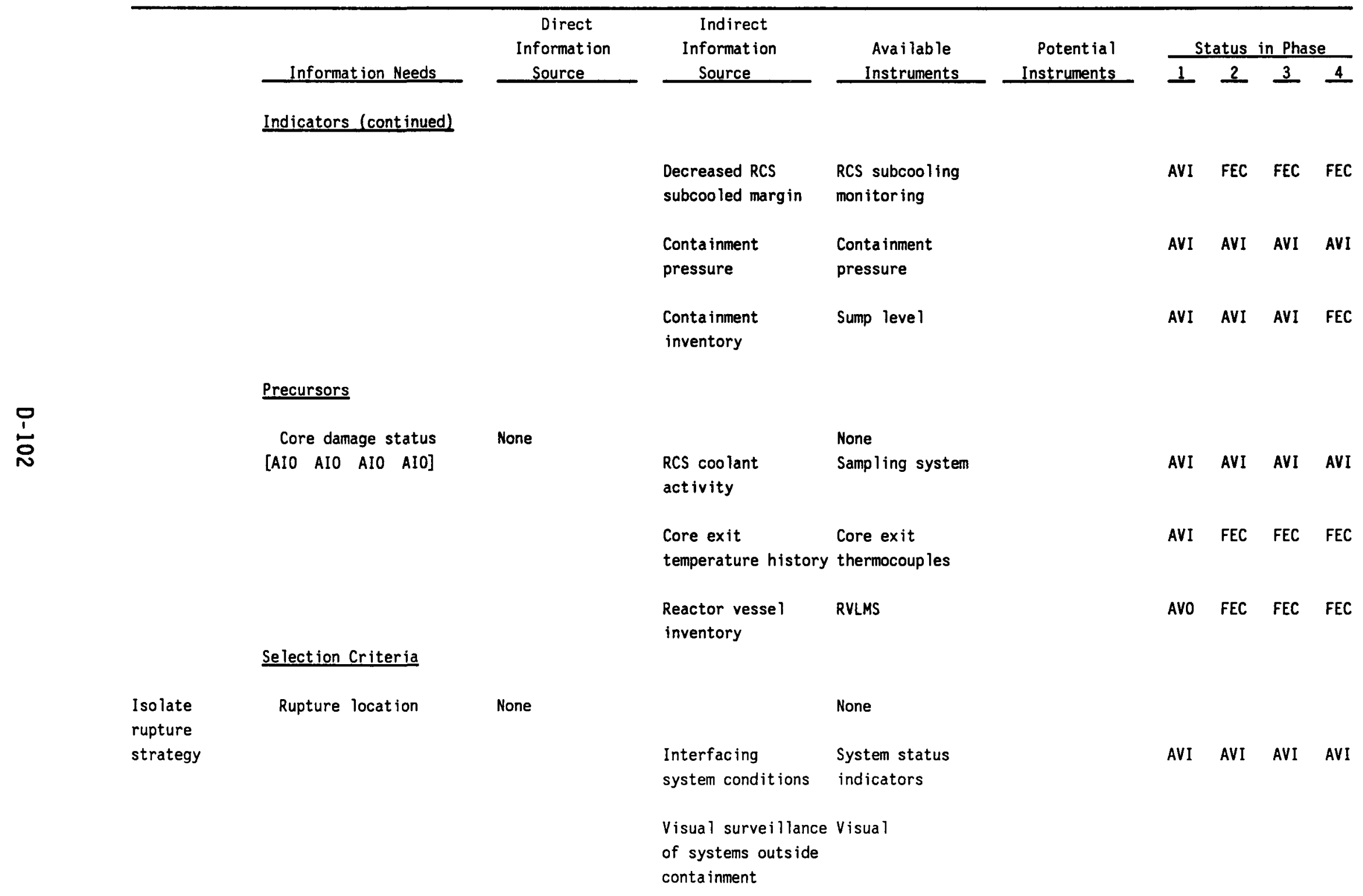


TABLE D2.12. (cont inued)

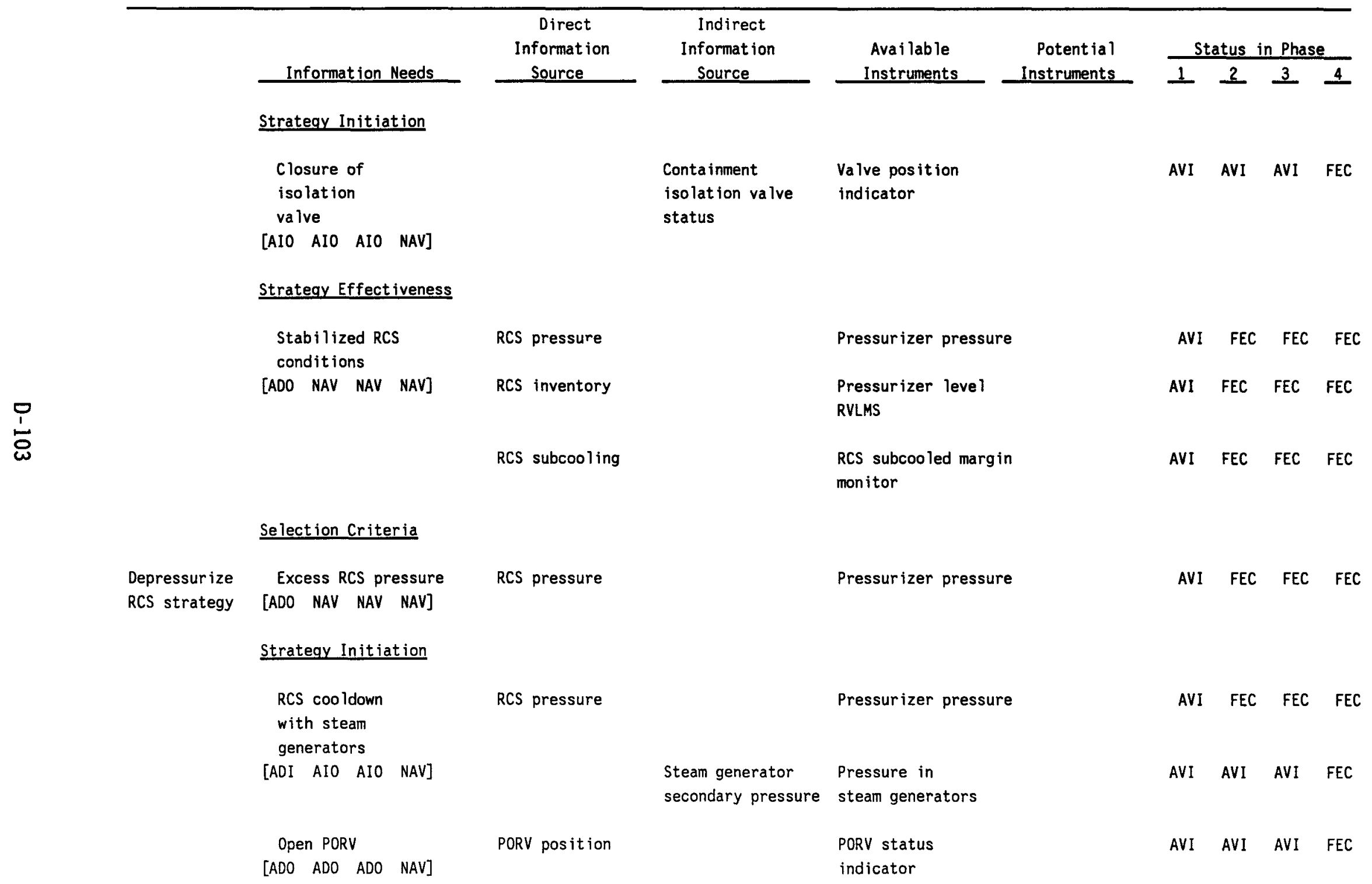


TABLE D2.12. (cont inued)

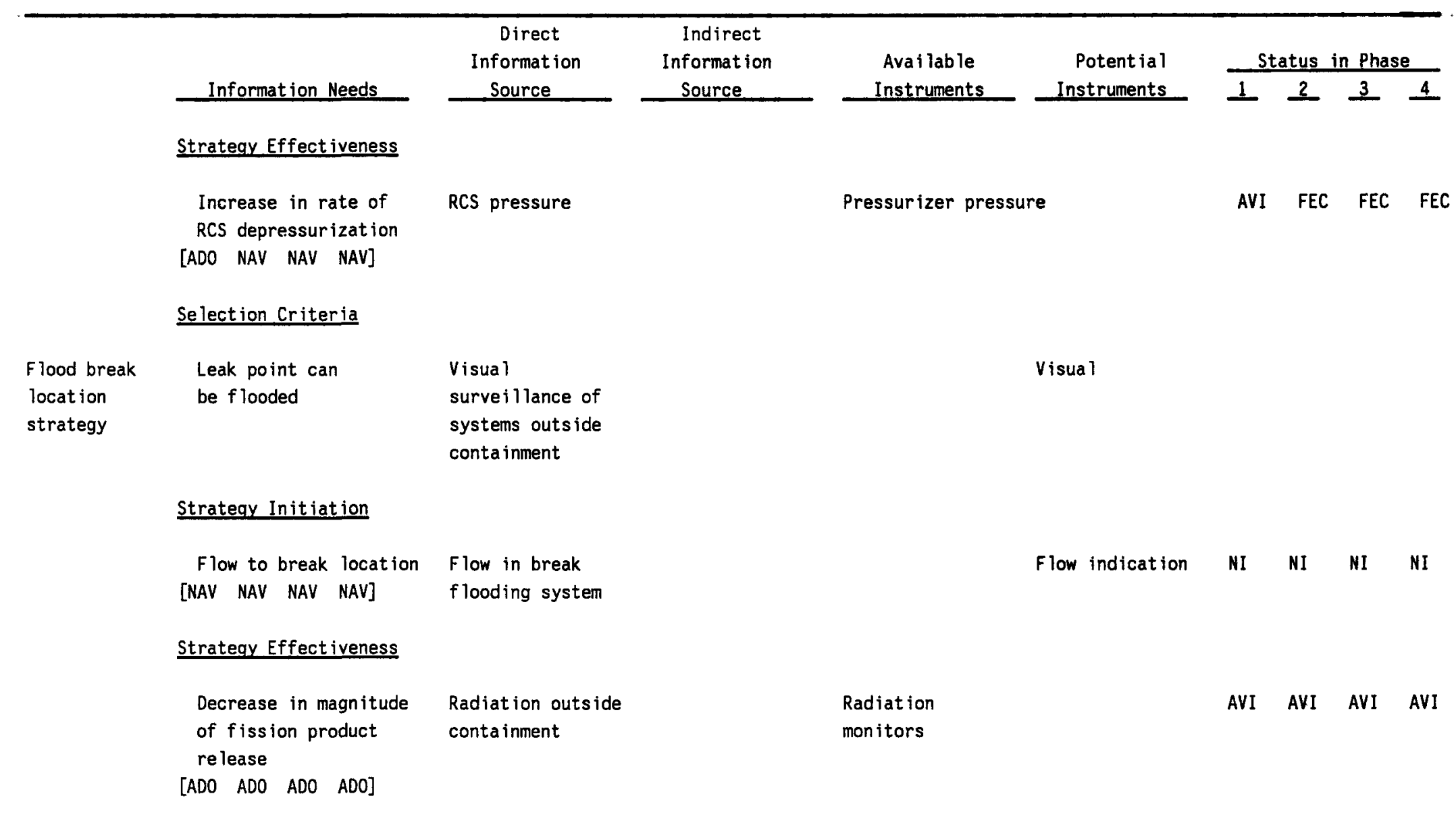


TABLE 02.13. PREVENT CONTAINMENT FAILURE (C)--STEAM EXPLOSION MISSILE MECHANISM (C3C1)

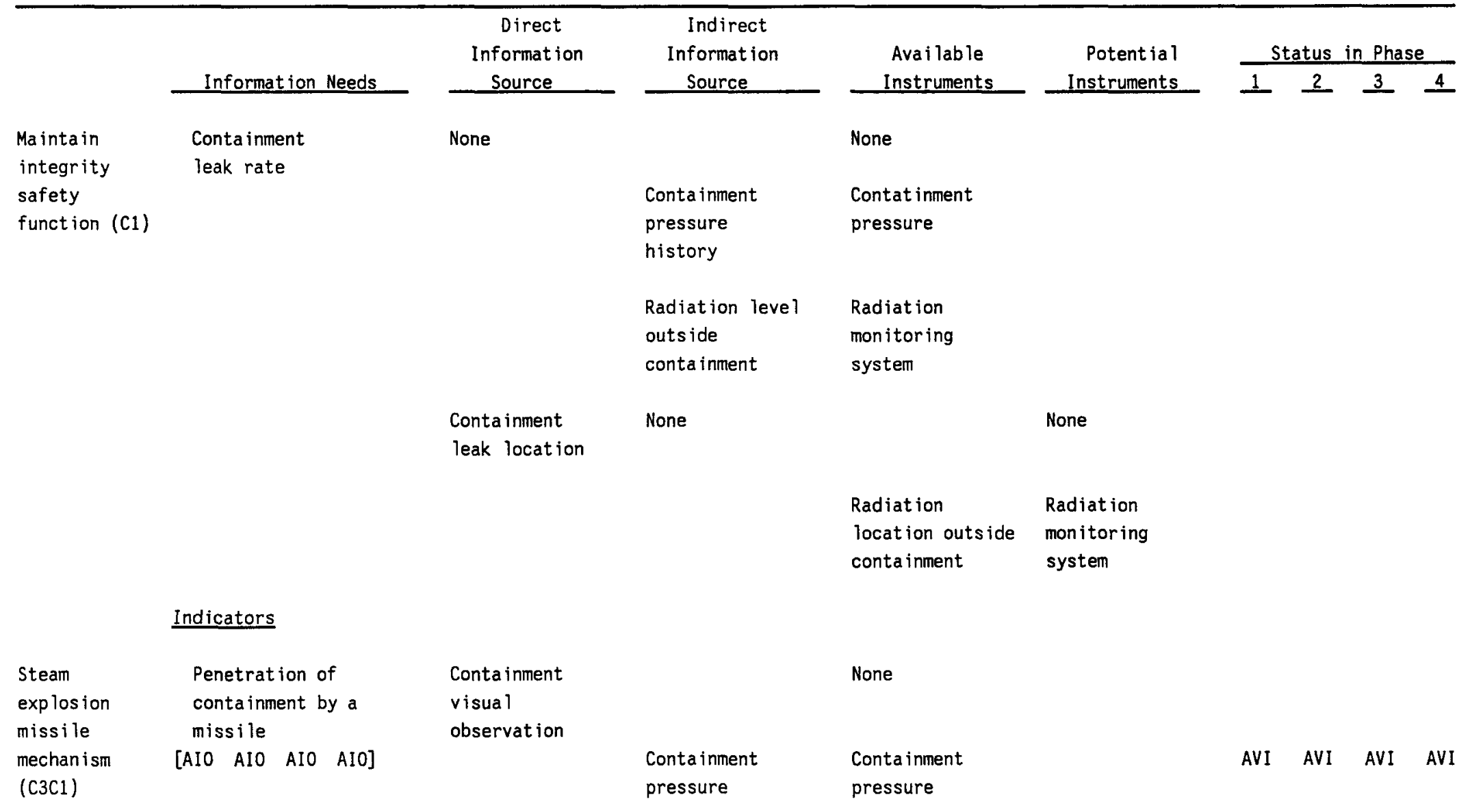


TABLE 02.13. (continued)

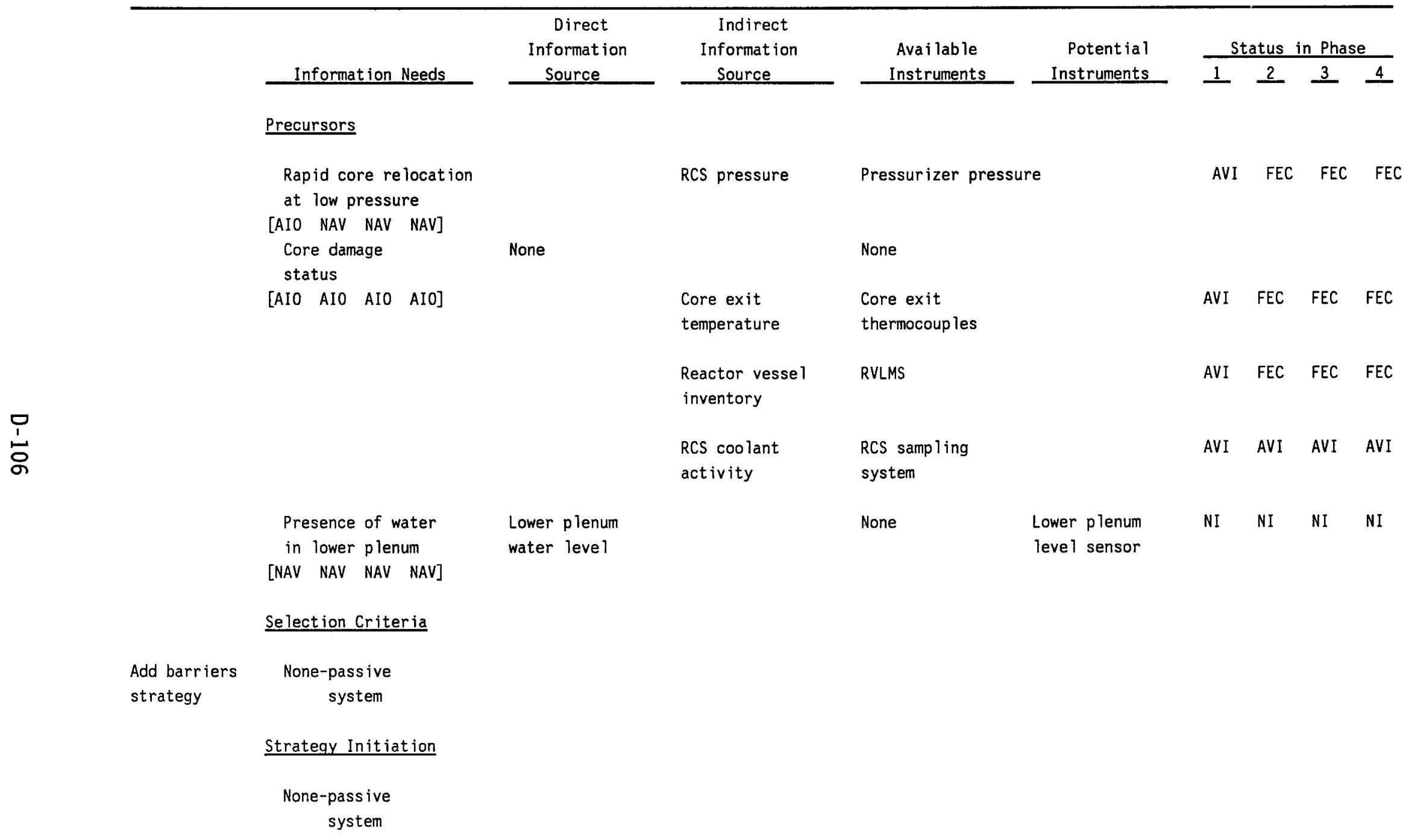


TABLE D2.13. (cont inued)

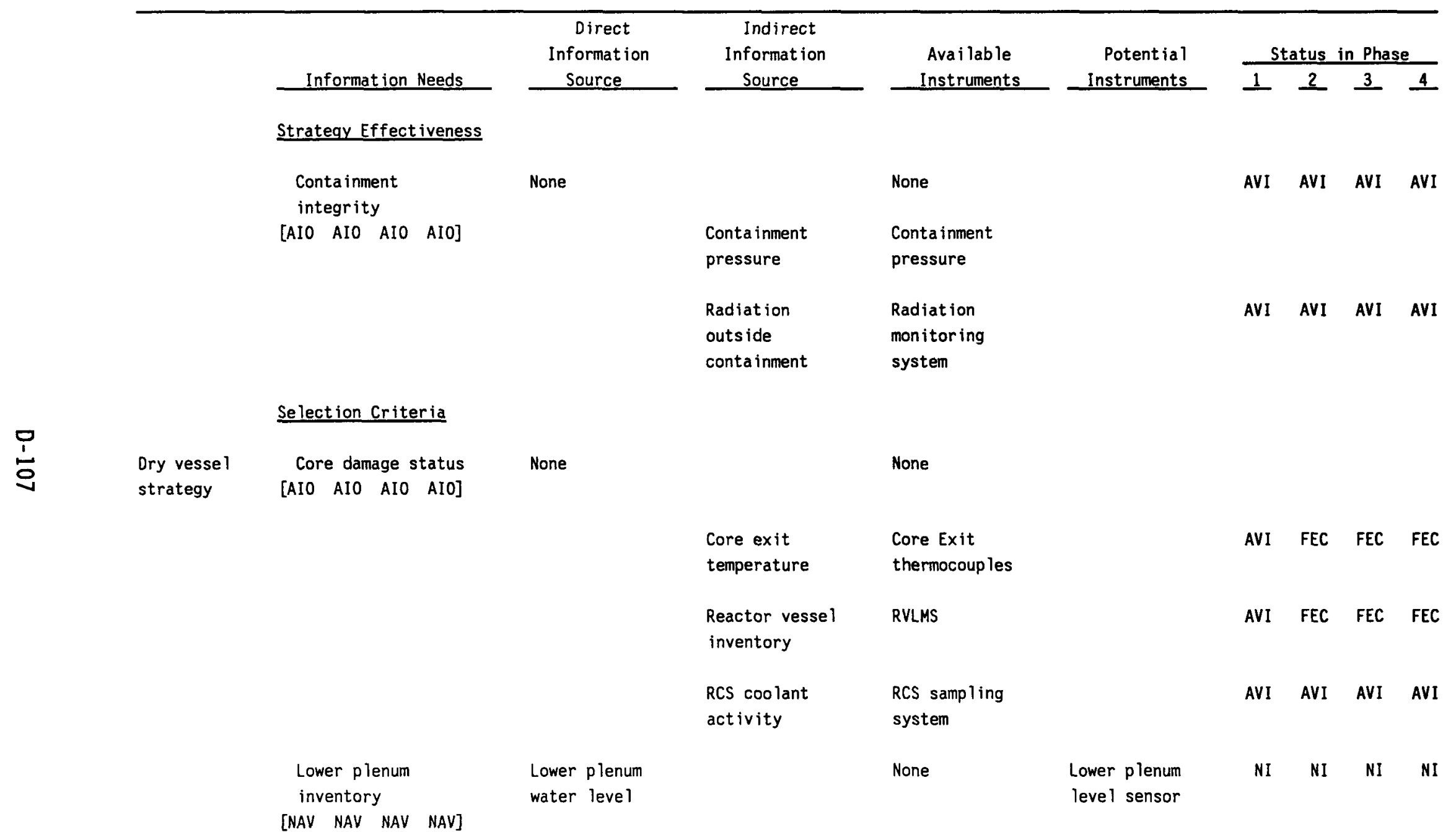


TABLE 02.13. (cont inued)

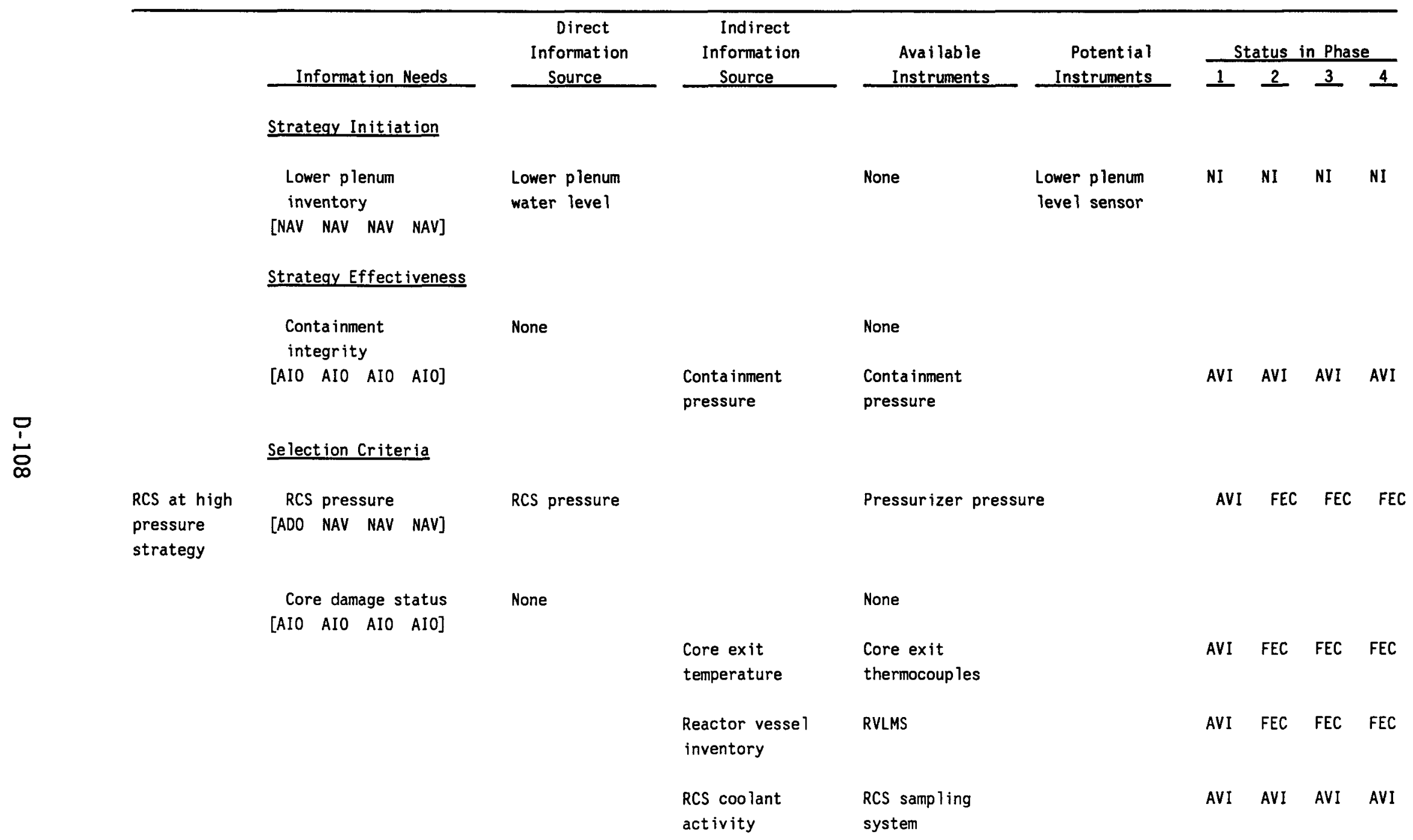


TABLE D2.13. (continued)

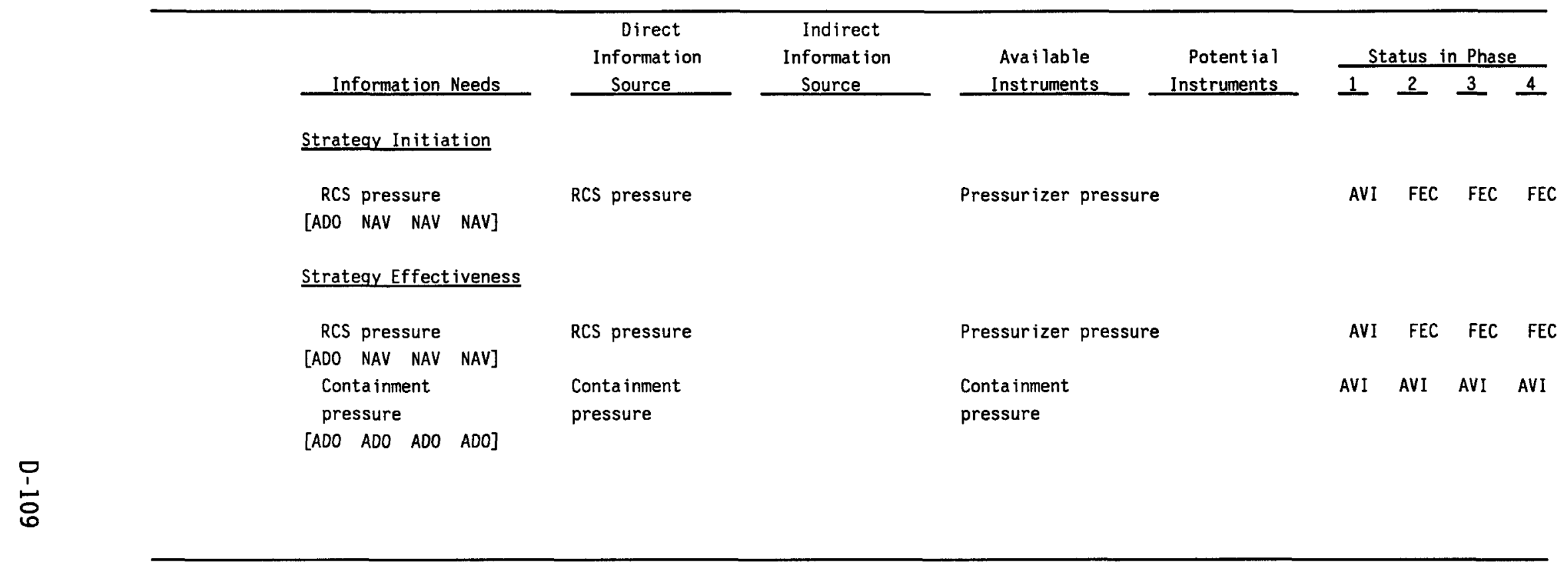


TABLE D2.14. PREVENT CONTAINMENT FAILURE (C)--HYDROGEN DETONATION MISSILE MECHANISM (C3C2)

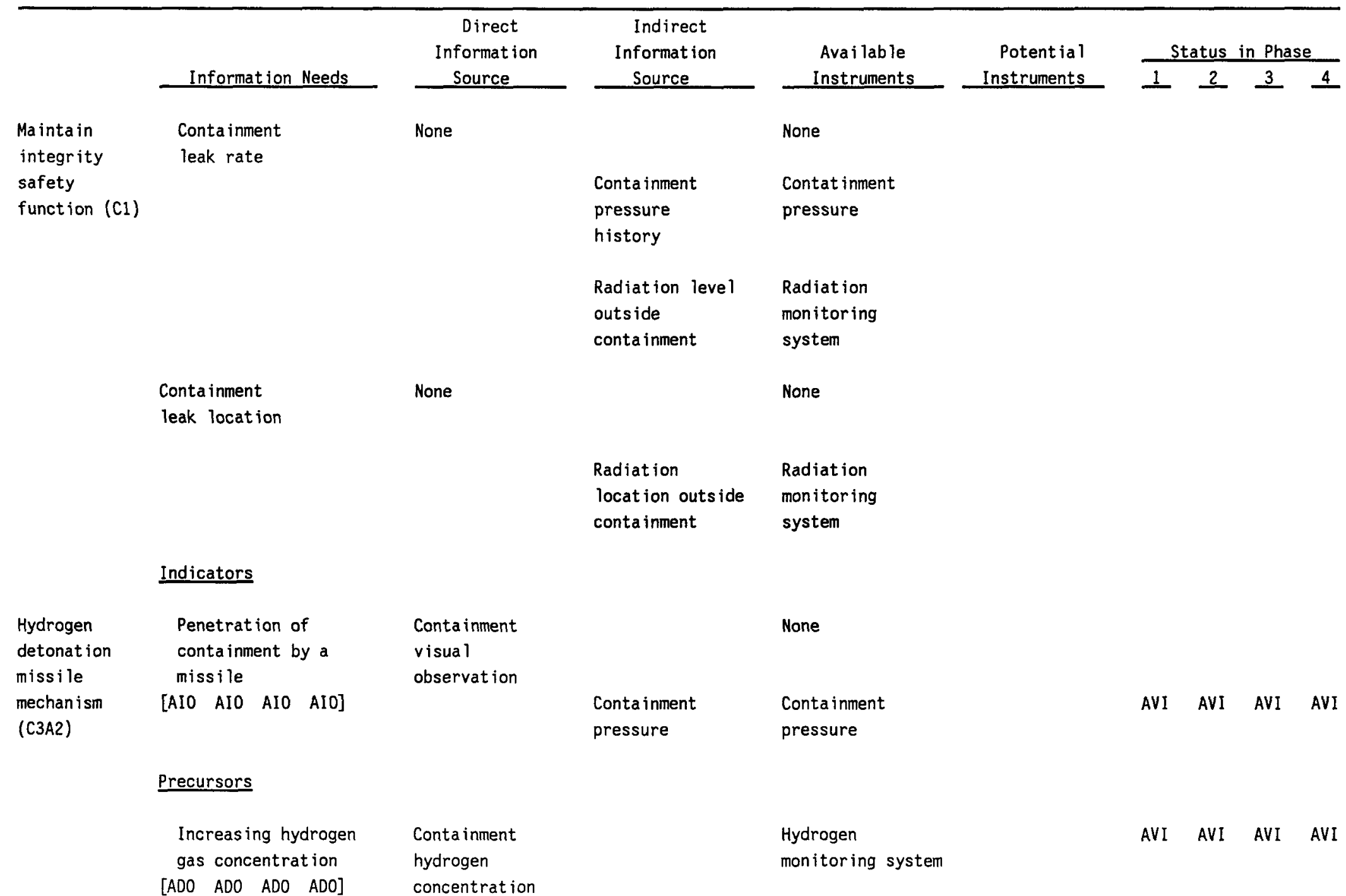


TABLE D2.14. (continued)

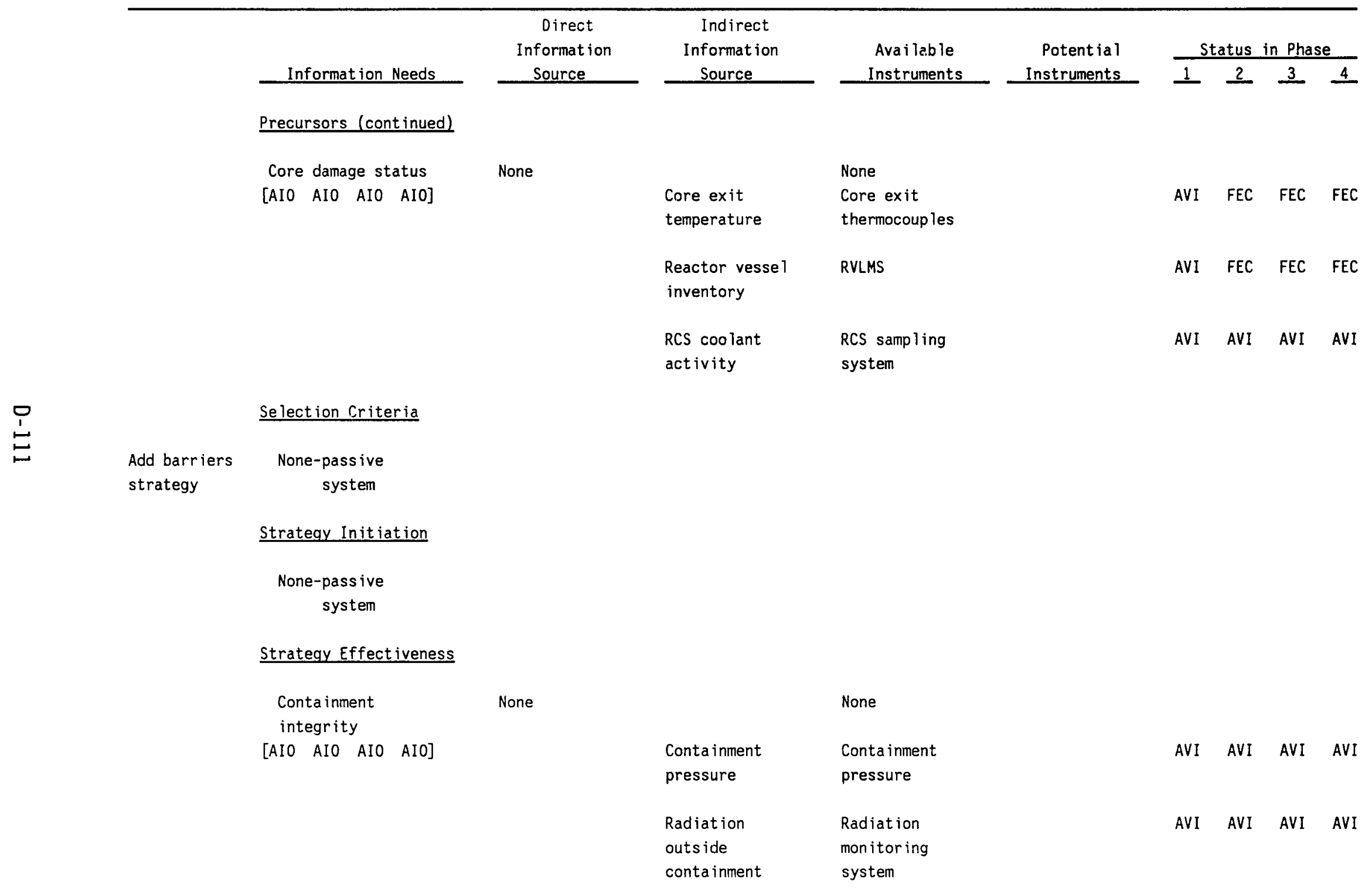


TABLE D2.14. (cont inued)

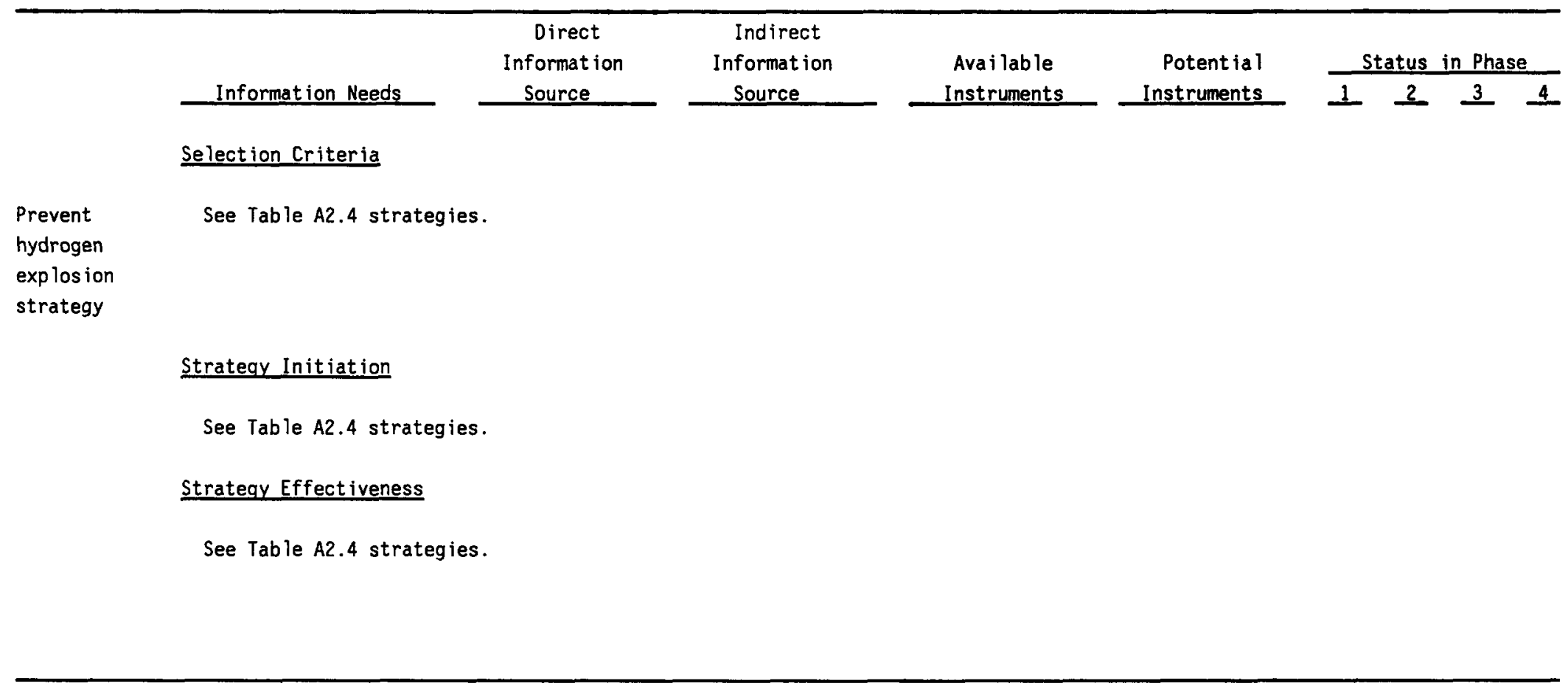


TABLE 03.1. MITIGATE FISSION PRODUCT RELEASE FROM CONTAINMENT (F)--AEROSOL DISPERSON MECHANISM (FIAI)

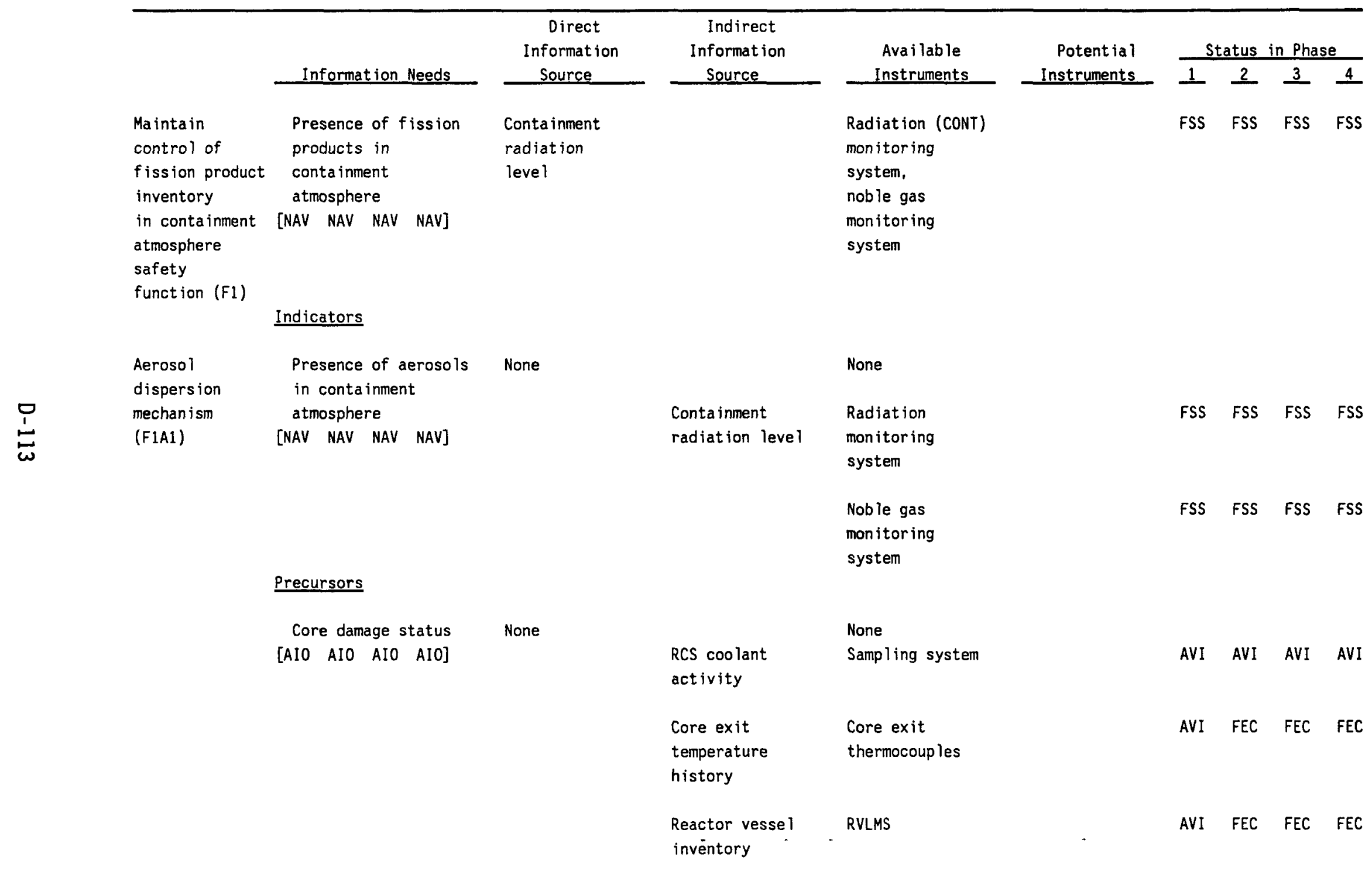


TABLE D3.1. (cont inued)

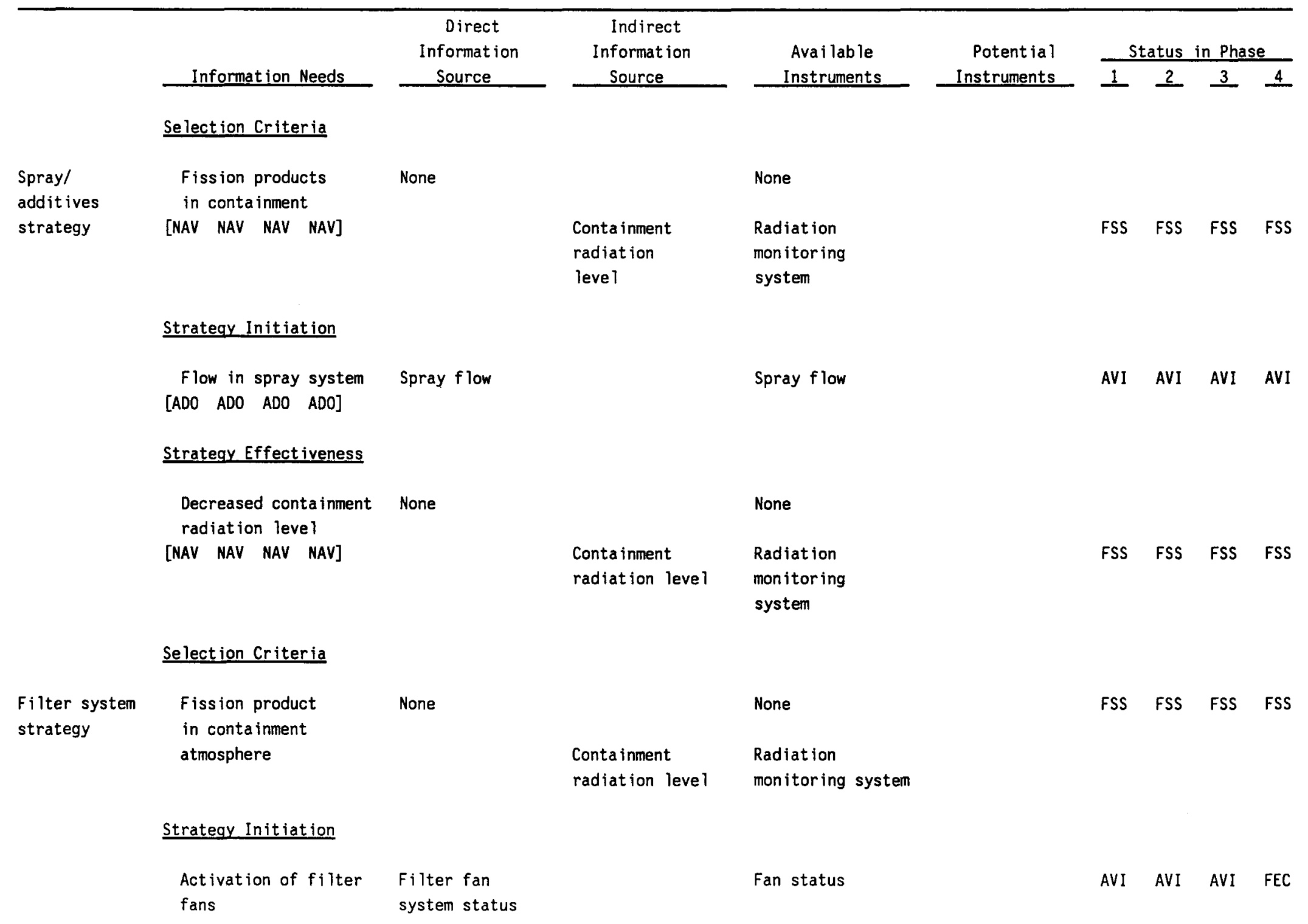


TABLE 03.1. (cont inued)

\begin{tabular}{|c|c|c|c|c|c|c|c|c|c|}
\hline & & $\begin{array}{c}\text { Direct } \\
\text { Information }\end{array}$ & $\begin{array}{c}\text { Indirect } \\
\text { Information }\end{array}$ & Available & Potential & & atus & Phe & \\
\hline & Information Needs & Source & Source & Instruments & Instruments & 1 & $\underline{2}$ & 3 & 4 \\
\hline & Strategy Effectiveness & & & & & & & & \\
\hline & $\begin{array}{l}\text { Decreased conta inment } \\
\text { radiation level }\end{array}$ & & $\begin{array}{l}\text { Containment } \\
\text { radiation leve } 1\end{array}$ & $\begin{array}{l}\text { Radiation monitoring } \\
\text { system }\end{array}$ & & FSS & FSS & FSS & FSS \\
\hline & [NAV NAV NAV NAV] & & & & & & & & \\
\hline & Selection Criteria & & & & & & & & \\
\hline $\begin{array}{l}\text { Chemical } \\
\text { reaction }\end{array}$ & $\begin{array}{c}\text { None-passive } \\
\text { system }\end{array}$ & & & & & & & & \\
\hline & Strategy Initiation & & & & & & & & \\
\hline & $\begin{array}{c}\text { None-passive } \\
\text { system }\end{array}$ & & & & & & & & \\
\hline & Strategy Effect iveness & & & & & & & & \\
\hline & $\begin{array}{l}\text { Low containment } \\
\text { radiation leve } 1\end{array}$ & None & & None & & & & & \\
\hline & [NAV NAV NAV NAV] & & $\begin{array}{l}\text { Conta inment } \\
\text { radiation level }\end{array}$ & $\begin{array}{l}\text { Radiation } \\
\text { monitoring } \\
\text { system }\end{array}$ & & FSS & FSS & FSS & FSS \\
\hline
\end{tabular}


TABLE 03.2. MITIGATE FISSION PRODUCT RELEASE FROM CONTAINMENT (F)--GASEOUS DISPERSION MECHANISM (FIA2)

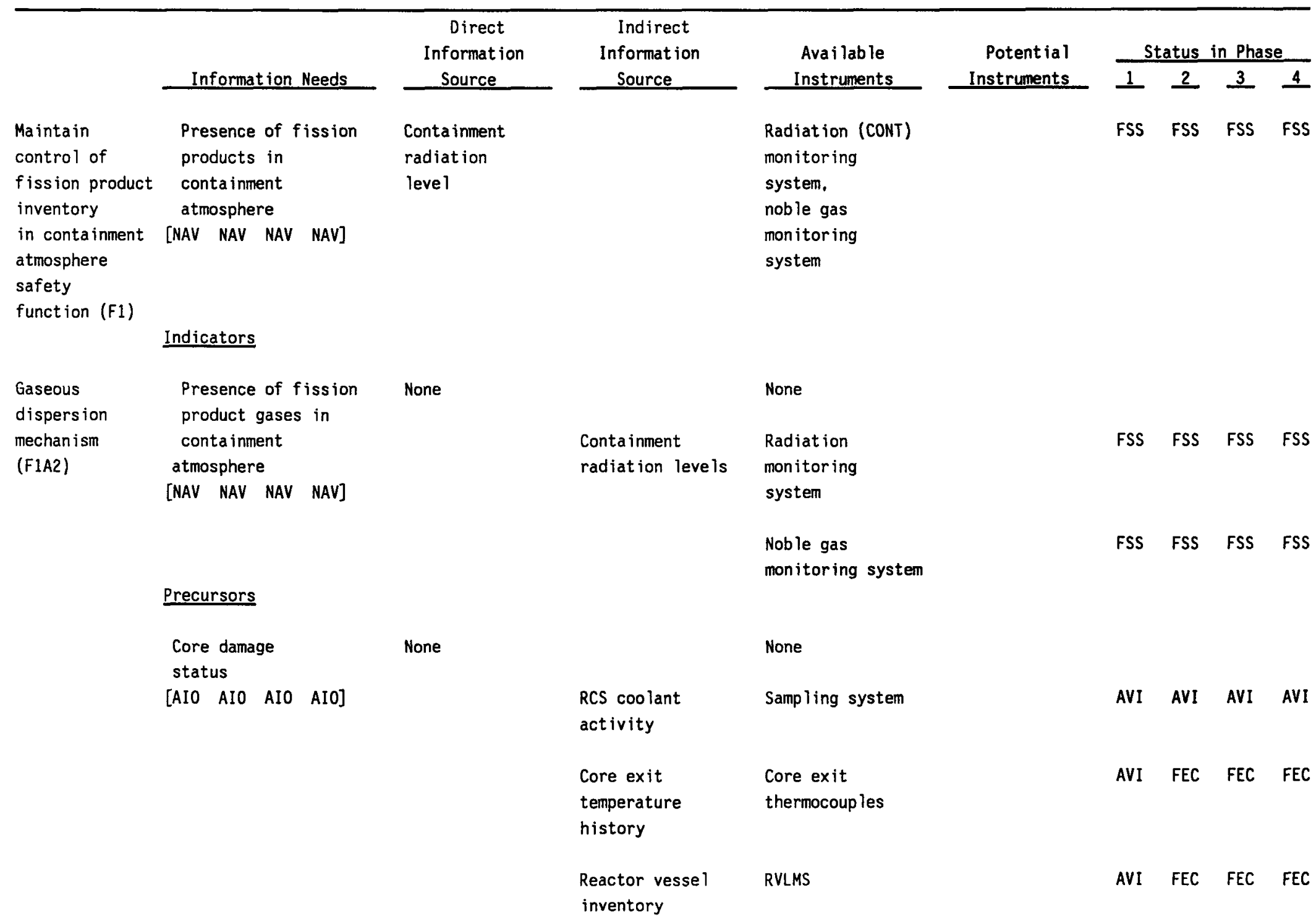


TABLE D3.2. (cont inued)

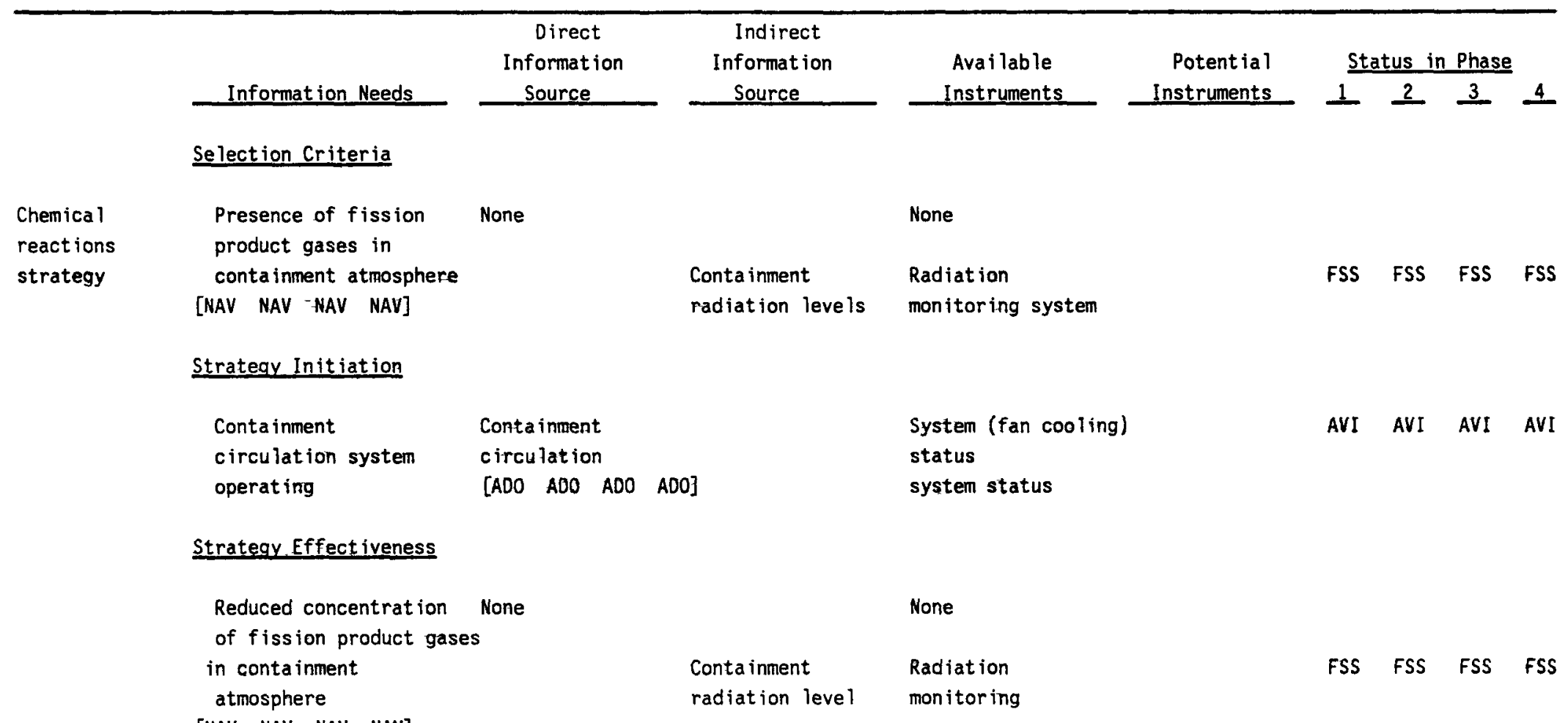


TABLE D3.2. (cont inued)

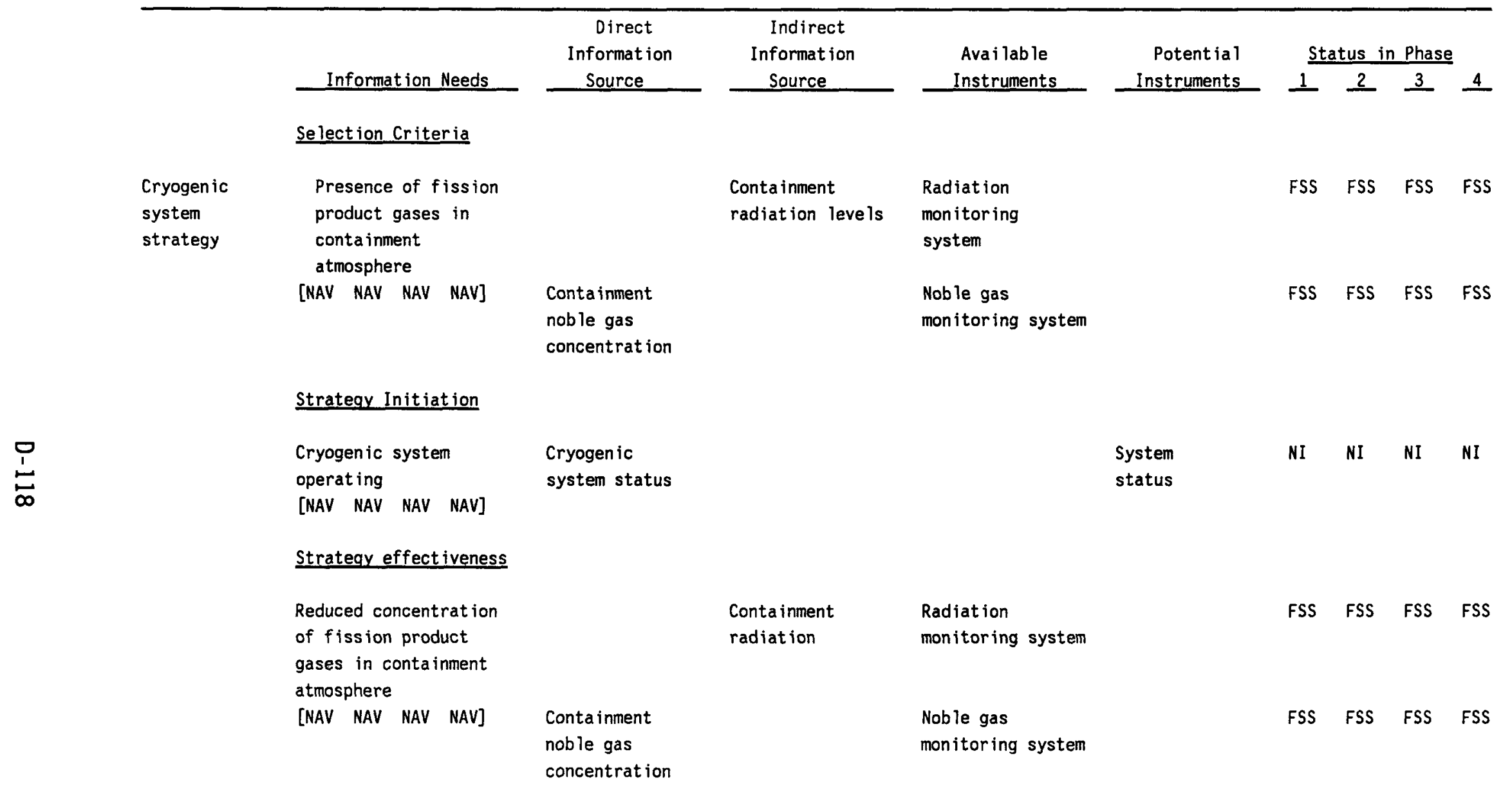


TABLE D3.3. MITIGATE FISSION PRODUCT RELEASE (F)--pH TOO-LOW MECHANISM (F2A1)

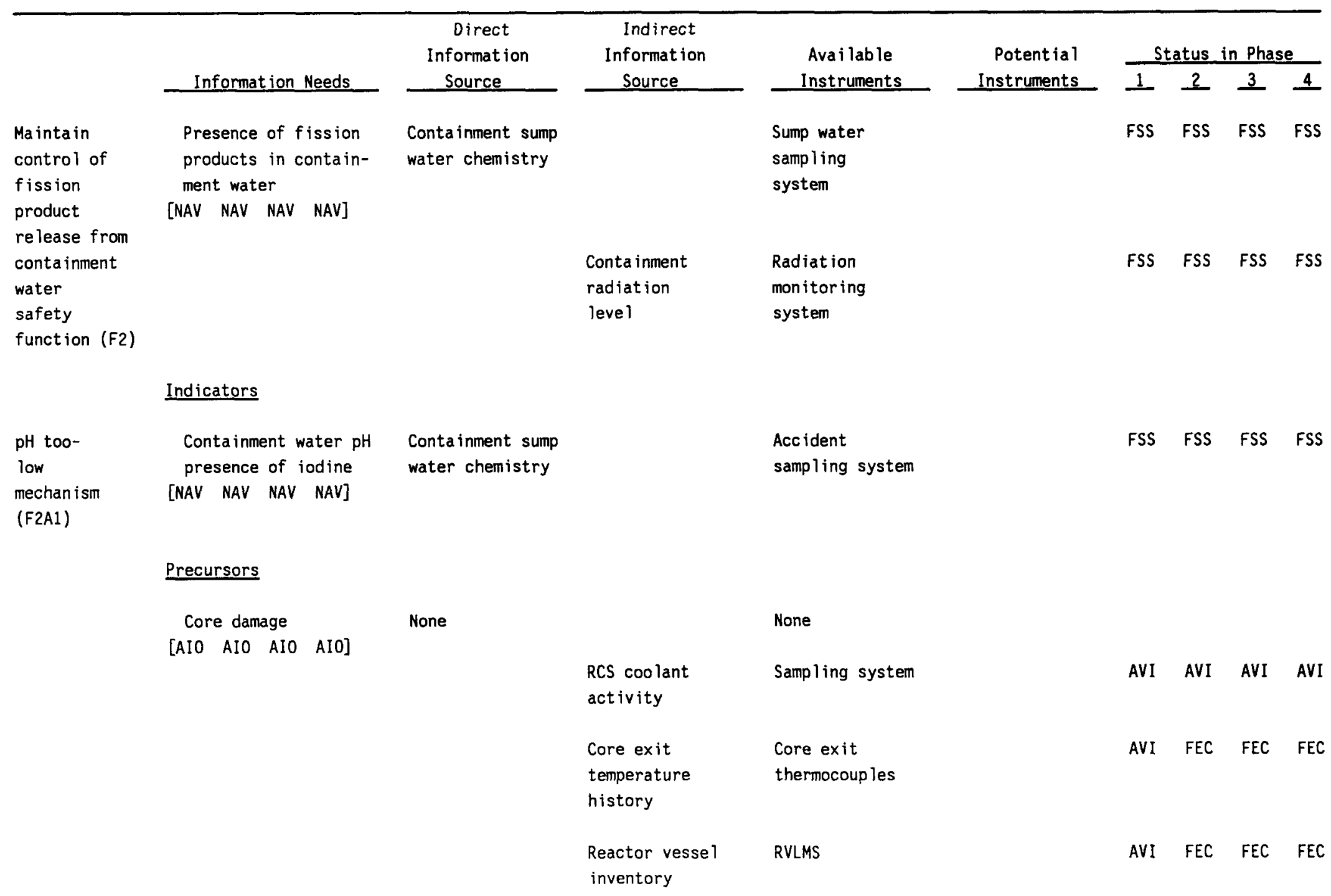


TABLE D3.3. (cont inued)

\begin{tabular}{|c|c|c|c|c|c|c|c|c|c|}
\hline & \multirow[b]{2}{*}{ Information, Needs } & \multirow{2}{*}{$\begin{array}{c}\text { Direct } \\
\text { Information } \\
\text { Source } \\
\end{array}$} & $\begin{array}{c}\text { Indirect } \\
\text { Information }\end{array}$ & \multirow{2}{*}{$\begin{array}{l}\text { Available } \\
\text { Instruments }\end{array}$} & \multirow{2}{*}{$\begin{array}{r}\text { Potential } \\
\text { Instruments } \\
\end{array}$} & \multicolumn{4}{|c|}{ Status in Phase } \\
\hline & & & Source & & & 1 & 2 & 3 & 4 \\
\hline & Se lection Criteria & & & & & & & & \\
\hline \multirow[t]{9}{*}{$\begin{array}{l}\text { Add base } \\
\text { strategy }\end{array}$} & $\begin{array}{l}\text { Amount of } \\
\text { contaminated }\end{array}$ & None & & None & & & & & \\
\hline & water & & Conta inment sump & Sump level & & AVI & AVI & AVI & FEC \\
\hline & {$\left[\begin{array}{llll}A & \text { AIO } & \text { AIO } & \text { NAV }\end{array}\right]$} & & inventory & indicator & & & & & \\
\hline & $\begin{array}{l}\text { Availability of } \\
\text { basic solution }\end{array}$ & None & & None & & & & & \\
\hline & [NAV NAV NAV NAV] & & $\begin{array}{l}\text { Supply of chemicals } \\
\text { for base solution }\end{array}$ & & Tank levels & NI & NI & NI & NI \\
\hline & Strategy Initiation & & & & & & & & \\
\hline & $\begin{array}{l}\text { F low of basic solution } \\
\text { [NAV NAV NAV NAV] }\end{array}$ & $\begin{array}{l}\text { Flow in system } \\
\text { for adding base } \\
\text { to containment } \\
\text { water }\end{array}$ & & & Flow indicator & NI & NI & MI & NI \\
\hline & Strategy Effect iveness & & & & & & & & \\
\hline & $\begin{array}{l}\text { Increase in containment } \\
\text { water pH } \\
\text { [NAV NAV NAV NAV] }\end{array}$ & $\begin{array}{l}\text { Conta inment sump } \\
\text { water chemistry }\end{array}$ & & $\begin{array}{l}\text { Accident } \\
\text { sampling } \\
\text { system }\end{array}$ & & FSS & FSS & FSS & FSS \\
\hline
\end{tabular}


TABLE 03.3. (cont inued)

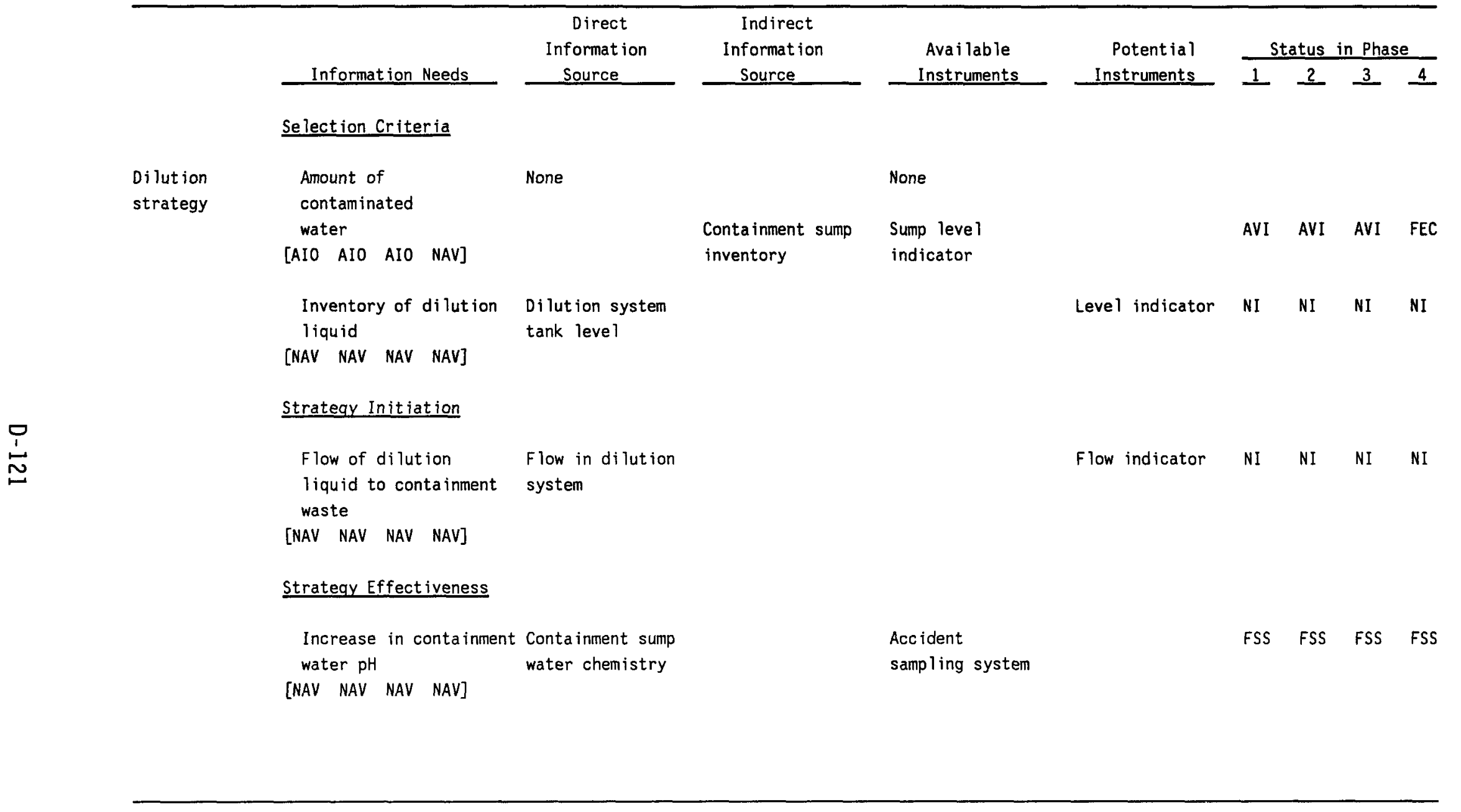


TABLE D3.4. MITIGATE FISSION PRODUCT RELEASE FROM CONTAINMENT (F)--RADIOLYSIS MECHANISM (F23A2)

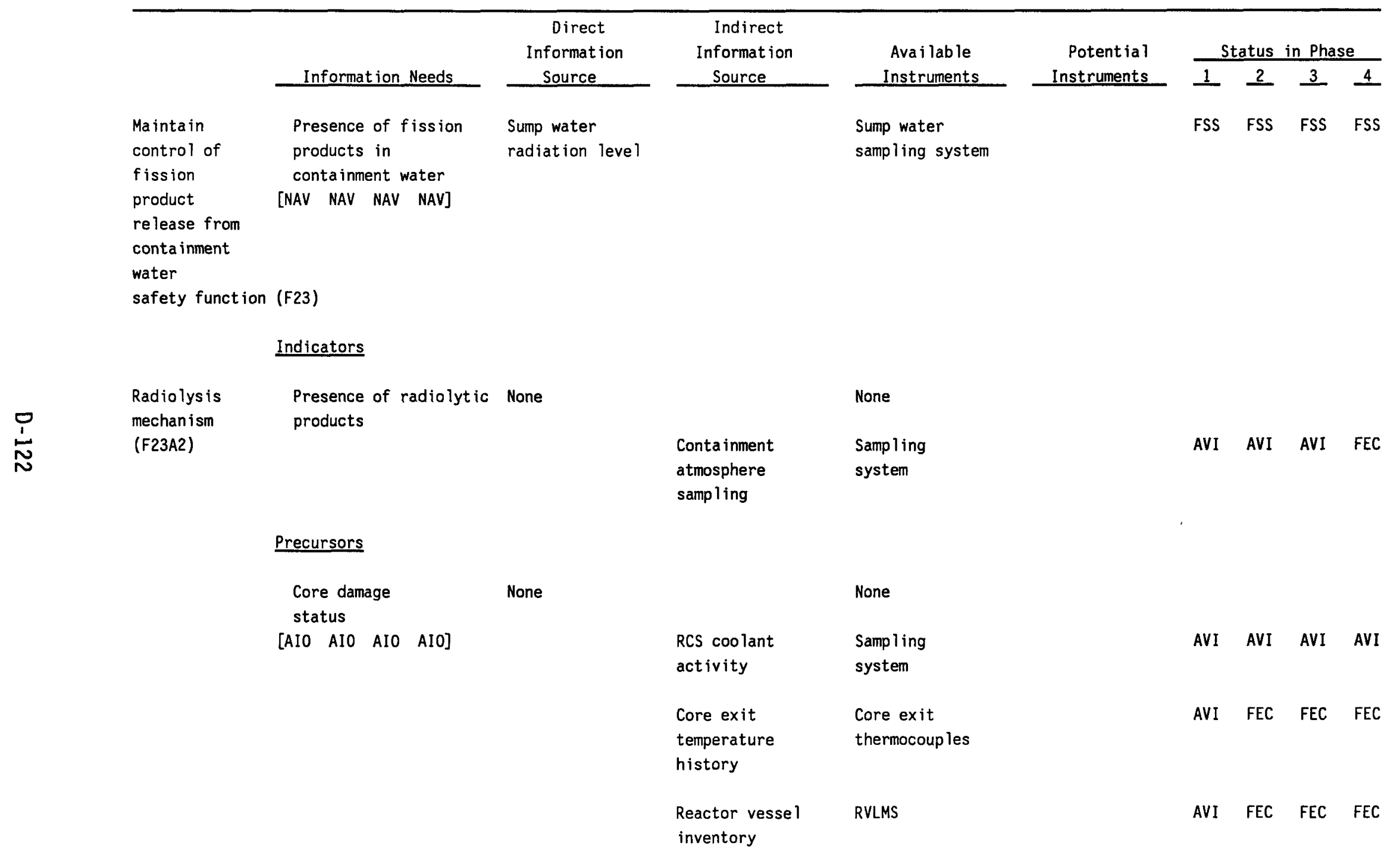


TABLE D3.4. (cont inued)

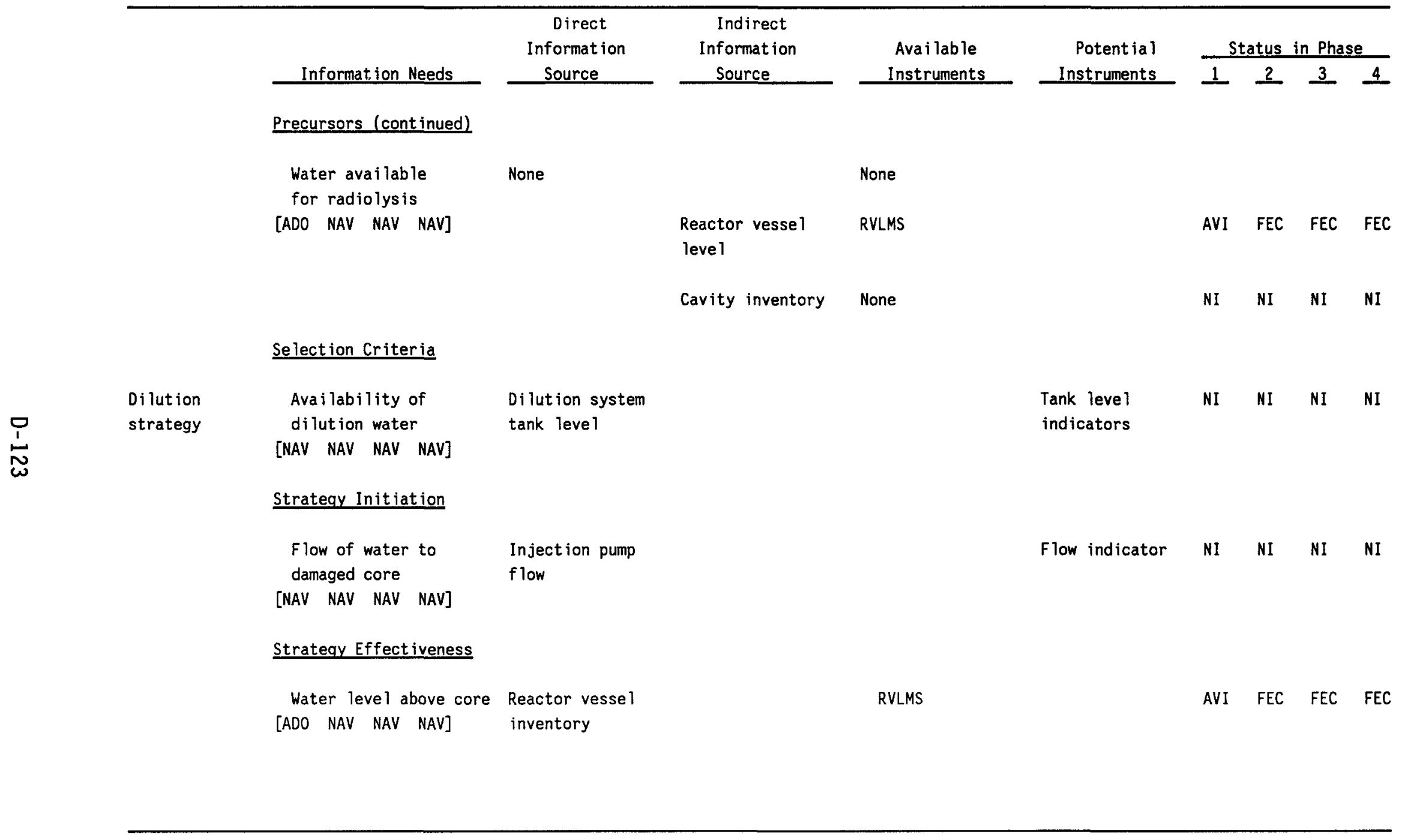


TABLE 03.5. MITIGATE FISSION PRODUCT RELEASE FROM CONTAINMENT (F)--EXCESSIVE WATER TEMPERATURE MECHANISM (F2A3)

\begin{tabular}{|c|c|c|c|c|c|c|c|c|c|}
\hline & & $\begin{array}{c}\text { Direct } \\
\text { Information }\end{array}$ & $\begin{array}{c}\text { Indirect } \\
\text { Information }\end{array}$ & Available & Potential & \multicolumn{3}{|c|}{ Status in Phase } & \\
\hline & Information Needs & Source & Source & Instruments & Instruments & 1 & 2 & 3 & 4 \\
\hline $\begin{array}{l}\text { Maintain } \\
\text { control of } \\
\text { fission } \\
\text { product } \\
\text { release from } \\
\text { containment } \\
\text { water } \\
\text { safety function } \\
\text { (F2) }\end{array}$ & $\begin{array}{l}\text { Presence of fission } \\
\text { products in } \\
\text { containment water } \\
\text { [NAV NAV NAV NAV] }\end{array}$ & $\begin{array}{l}\text { Sump water } \\
\text { radiation level }\end{array}$ & & $\begin{array}{l}\text { Sump water } \\
\text { sampling system }\end{array}$ & & FSS & FSS & FSS & FSS \\
\hline & Indicators & & & & & & & & \\
\hline $\begin{array}{l}\text { Excessive } \\
\text { water } \\
\text { temperature } \\
\text { mechanism } \\
\text { (F2A3) }\end{array}$ & $\begin{array}{l}\text { Sump water } \\
\text { temperature }\end{array}$ & $\begin{array}{l}\text { Sump water } \\
\text { temperature }\end{array}$ & & $\begin{array}{l}\text { Sump water } \\
\text { temperature }\end{array}$ & & NI & NI & NI & NI \\
\hline & Precursors & & & & & & & & \\
\hline & $\begin{array}{l}\text { Inadequate heat } \\
\text { removal from }\end{array}$ & None & & None & & & & & \\
\hline & sump water & & Sump water & Sump water & & NI & NI & NI & NI \\
\hline & {$\left[\begin{array}{llll}\mathrm{AIO} & \mathrm{AIO} & \mathrm{AIO} & \mathrm{AIO}\end{array}\right]$} & & temperature & $\begin{array}{l}\text { temperature } \\
\text { indicators }\end{array}$ & & & & & \\
\hline & & & $\begin{array}{l}\text { Sump water cooling } \\
\text { heat exchanger } \\
\text { out let temperature }\end{array}$ & $\begin{array}{l}\text { Temperature } \\
\text { indicators }\end{array}$ & & AVI & AVI & AVI & AVI \\
\hline
\end{tabular}


TABLE D3.5. (cont inued)

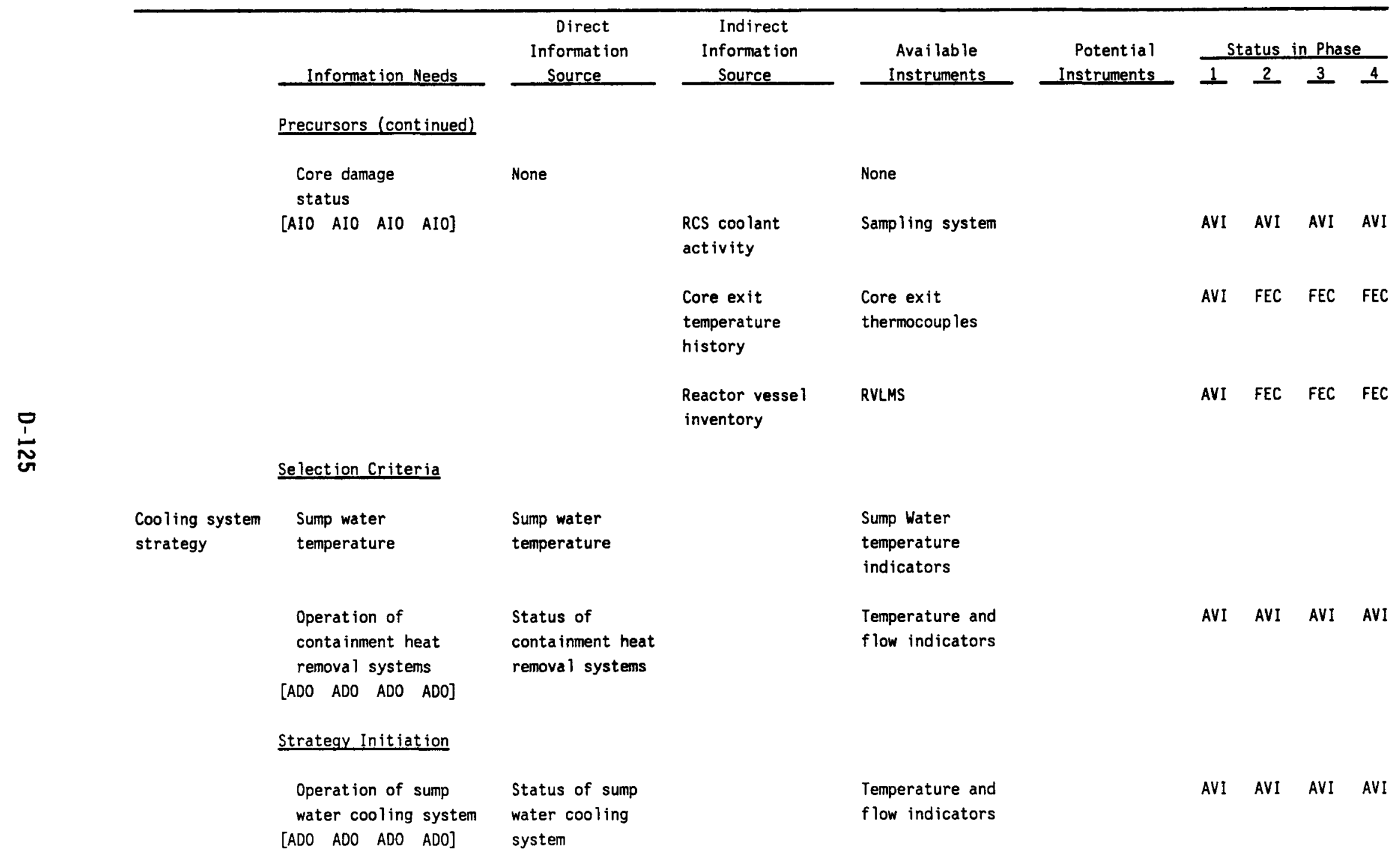


TABLE D3.5. (cont inued)

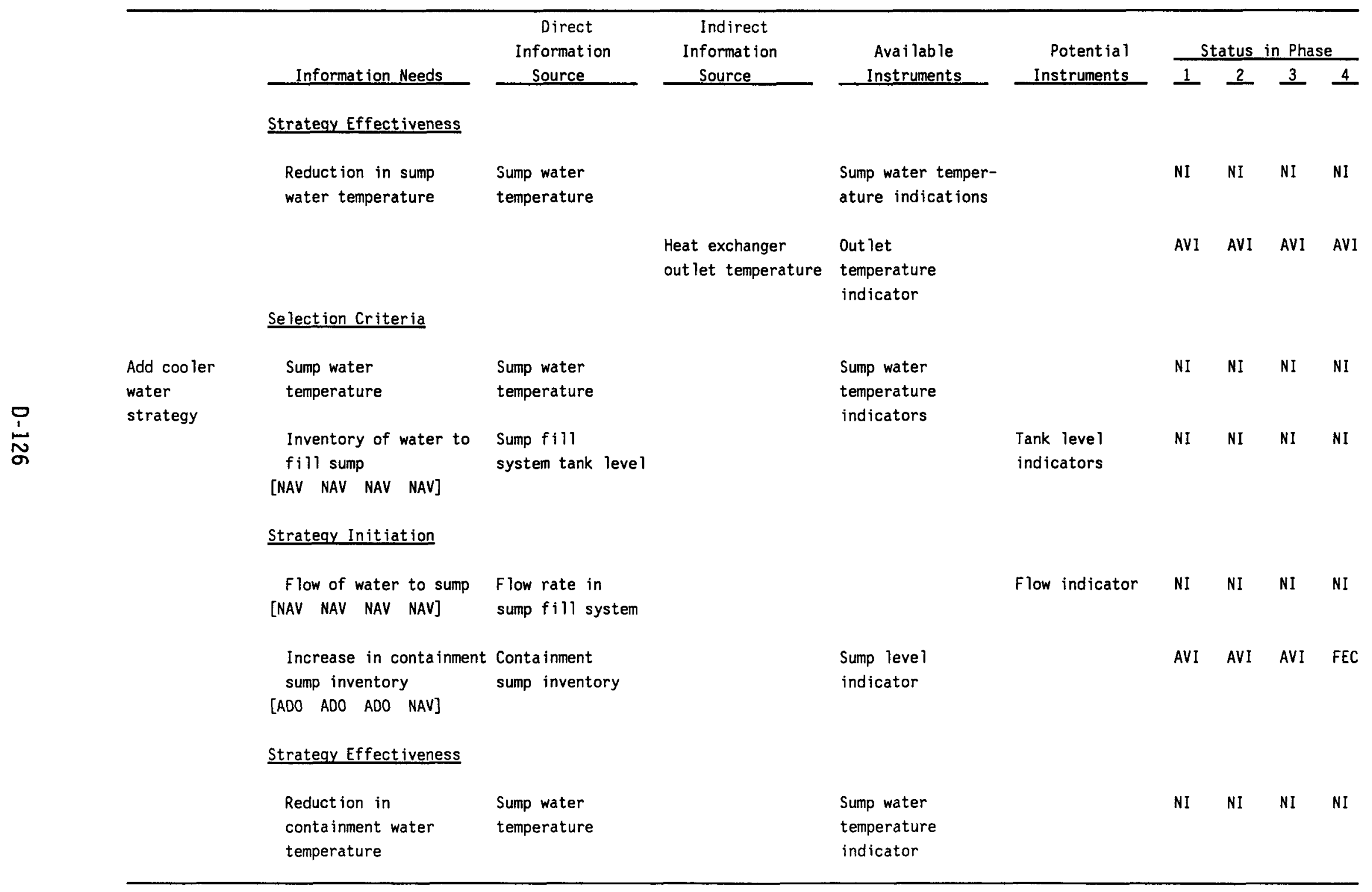




\section{APPENDIX E}

POTENTIAL MEASUREMENTS TO

FULFILL INFORMATION NEEDS

$$
E-1 / E-2
$$





\section{APPENDIX E \\ POTENTIAL MEASUREMENTS TO FULFILL INFORMATION NEEDS}

The tables contained in Appendix E contain the potential measurements identified to fulfill information needs for a plant represented by a Combustion Engineering $2700 \mathrm{MW}(\mathrm{t})$ plant such as Calvert Cliffs Units 1 and 2 or Millstone Unit 2. Table E1 lists proposed or potential measurements that could be used to supply information needs in cases where measurements are not currently available. Table E2 lists measurements that would be required for existing systems that could be used as part of a strategy for accident management. The columns that are contained in this table are as follows:

Potential Measurements Identified: the measurements identified from Tables Al through A3 that may be required for existing systems to be used for particular accident management strategies
Information Need: the information needs that would be provided by the potential measurement

Potential Severe Accident Measurement Range: the range of conditions the instrument might experience during a severe accident

Location of the Instrument: the general location of the instrument sensor.

Table E2 lists those measurements that could be used to monitor proposed systems that would be needed for new strategies for accident management. This table has columns to list the potential measurement, the information needs it would provide, the measurement range it would need to cover, and the location of the sensor. The last two columns were completed during the analysis of a specific severe accident presented in Section 4 of the main report. 
table E1. POTENTIAL MEASUREMENTS TO FULFILL INFORMATION NEEDS

\begin{tabular}{|c|c|c|c|}
\hline $\begin{array}{l}\text { Potent ial } \\
\text { Measurements } \\
\text { Ident if 1ed } \\
\end{array}$ & $\begin{array}{c}\text { Information } \\
\text { Need }\end{array}$ & $\begin{array}{l}\text { Potent ial } \\
\text { Severe Accident } \\
\text { Measurement } \\
\text { Range }\end{array}$ & $\begin{array}{c}\text { Location } \\
\text { of } \\
\text { Information } \\
\end{array}$ \\
\hline $\begin{array}{l}\text { Fuel rod internal } \\
\text { pressure }\end{array}$ & $\begin{array}{l}\text { Fuel rod geometry } \\
\text { Fuel rod cladding } \\
\text { condition }\end{array}$ & $\begin{array}{l}0 \text { to max } 1 \text { mum rupture } \\
\text { pressure }(-2000 \mathrm{ps} 1)\end{array}$ & Reactor \\
\hline Cladding temperature & $\begin{array}{l}\text { Cladding temperature } \\
\text { Core temperature } \\
\text { Core material temperature } \\
\text { and geometry } \\
\text { Fuel rod cladding } \\
\text { condition }\end{array}$ & 0 to $3500^{\circ} \mathrm{F}$ & Reactor \\
\hline Fuel location & Core mater $1 a 1$ geometry & To be determıned (TBD) & TBD \\
\hline Fuel temperature & $\begin{array}{l}\text { Core material temperature } \\
\text { and geometry } \\
\text { Cladding temperature }\end{array}$ & 0 to $5000^{\circ} \mathrm{F}$ & Reactor \\
\hline $\begin{array}{l}\text { Vesse } 1 \text { and lower } \\
\text { head visual } \\
\text { observation }\end{array}$ & $\begin{array}{l}\text { Vessel integrity } \\
\text { Approach to vessel } \\
\text { fallure } \\
\text { Lower head } \\
\text { temperature }\end{array}$ & & \\
\hline $\begin{array}{l}\text { Vessel wall } \\
\text { temperature }\end{array}$ & $\begin{array}{l}\text { Vessel integrity } \\
\text { Approach to vessel } \\
\text { fallure }\end{array}$ & $\begin{array}{l}0^{\circ} \mathrm{F} \text { to melting point } \\
\text { of lower head }\end{array}$ & Containment (cavity) \\
\hline $\begin{array}{l}\text { Basemat } \\
\text { temperature }\end{array}$ & $\begin{array}{l}\text { Vesse } 1 \text { wall temperature } \\
\text { Amount of concrete } \\
\text { ablated }\end{array}$ & $\begin{array}{l}0^{\circ} \mathrm{F} \text { to melting } \\
\text { point of concrete/ } \\
\text { basemat material }\end{array}$ & Containment (cavity) \\
\hline $\begin{array}{l}\text { Containment } \\
\text { visual observation }\end{array}$ & $\begin{array}{l}\text { Containment } \\
\text { penetration by } \\
\text { missile }\end{array}$ & & \\
\hline
\end{tabular}


TABLE E1. (cont inued)

\begin{tabular}{|c|c|c|c|}
\hline $\begin{array}{c}\text { Potential } \\
\text { Measurements } \\
\text { Identified } \\
\end{array}$ & $\begin{array}{c}\text { Information } \\
\text { Need }\end{array}$ & $\begin{array}{c}\text { Potential } \\
\text { Severe Accident } \\
\text { Measurement } \\
\text { Range }\end{array}$ & $\begin{array}{c}\text { Location } \\
\text { of } \\
\text { Information }\end{array}$ \\
\hline Cavity inventory & $\begin{array}{l}\text { Cavity inventory } \\
\text { Lower head failure } \\
\text { and fission product } \\
\text { release } \\
\text { Water available } \\
\text { for radiolysis }\end{array}$ & $\begin{array}{l}\text { Cavity leve } 1 \\
0 \text { to } 100 \%\end{array}$ & Containment (cavity) \\
\hline $\begin{array}{l}\text { Lower plenum } \\
\text { water leve } 1\end{array}$ & $\begin{array}{l}\text { Presence of water in } \\
\text { lower plenum } \\
\text { (Precursor to steam } \\
\text { explosion) } \\
\text { Lower plenum inventory } \\
\text { Potential for steam } \\
\text { explosion }\end{array}$ & $\begin{array}{l}\text { Lower head level } \\
0 \text { to } 100 \%\end{array}$ & Reactor vesse 1 \\
\hline $\begin{array}{l}\text { Quench tank } \\
\text { samples }\end{array}$ & $\begin{array}{l}\text { Presence of fission } \\
\text { products in relief } \\
\text { valve discharge flow } \\
\text { Presence of fission } \\
\text { products in quench tank } \\
\text { Fission product transport } \\
\text { from RCS }\end{array}$ & TBD & Containment \\
\hline $\begin{array}{l}\text { Flow in quench tank } \\
\text { fill system }\end{array}$ & Flow in quench tank & 0 to $110 \%$ design flow & Containment \\
\hline $\begin{array}{l}\text { Lower head } \\
\text { temperature }\end{array}$ & $\begin{array}{l}\text { Lower head failure and } \\
\text { fission product release }\end{array}$ & & \\
\hline & Lower head temperature & $\begin{array}{l}0^{\circ} \mathrm{F} \text { to melting } \\
\text { point of lower head }\end{array}$ & Containment (cavity) \\
\hline $\begin{array}{l}\text { Containment } \\
\text { penetration } \\
\text { temperature }\end{array}$ & $\begin{array}{l}\text { Containment } \\
\text { penetration } \\
\text { integrity }\end{array}$ & TBD & Conta inment \\
\hline
\end{tabular}


TABLE E2. MEASUREMENTS FOR SYSTEMS NEEDED FOR POTENTIAL STRATEGIES

\begin{tabular}{|c|c|c|c|}
\hline $\begin{array}{l}\text { Potential } \\
\text { Measurements } \\
\text { Ident if ied }\end{array}$ & $\begin{array}{l}\text { Information } \\
\text { Need }\end{array}$ & $\begin{array}{c}\text { Potential } \\
\text { Severe Accident } \\
\text { Measurement } \\
\text { Range }\end{array}$ & $\begin{array}{c}\text { Location } \\
\text { of } \\
\text { Information }\end{array}$ \\
\hline $\begin{array}{l}\text { Cavity flooding } \\
\text { system tank } \\
\text { inventory }\end{array}$ & $\begin{array}{l}\text { Inventory available } \\
\text { to flood cavity } \\
\text { Flooding initiation }\end{array}$ & TBD & TBD \\
\hline $\begin{array}{l}\text { Cavity flooding } \\
\text { system flow rate }\end{array}$ & $\begin{array}{l}\text { Flooding initiation } \\
\text { Water flow to cavity }\end{array}$ & $T B D$ & TBD \\
\hline $\begin{array}{l}\text { Cavity drain } \\
\text { system flow } \\
\text { rate }\end{array}$ & $\begin{array}{l}\text { Water flow from } \\
\text { cavity }\end{array}$ & TBD & TBD \\
\hline $\begin{array}{l}\text { Vent system } \\
\text { flow rate }\end{array}$ & $\begin{array}{l}\text { Vent system flow } \\
\text { initiation }\end{array}$ & TBD & TBD \\
\hline $\begin{array}{l}\text { Containment } \\
\text { leak location }\end{array}$ & $\begin{array}{l}\text { Leak point can be } \\
\text { located and flooded }\end{array}$ & TBD & TBD \\
\hline $\begin{array}{l}\text { Flow in leak } \\
\text { flooding system }\end{array}$ & Flow to leak location & TBD & TBD \\
\hline $\begin{array}{l}\text { Interfacing system } \\
\text { conditions }\end{array}$ & $\begin{array}{l}\text { Rupture location } \\
\text { (V-sequence) }\end{array}$ & TBD & $T B D$ \\
\hline $\begin{array}{l}\text { Visual surveillance } \\
\text { of systems outside } \\
\text { containment }\end{array}$ & $\begin{array}{l}\text { Rupture location } \\
\text { (V-sequence) }\end{array}$ & TBD & TBD \\
\hline & $\begin{array}{l}\text { Leak point can be } \\
\text { flooded }\end{array}$ & & \\
\hline $\begin{array}{l}\text { Flow in break } \\
\text { flooding system }\end{array}$ & $\begin{array}{l}\text { Flow to break location } \\
\text { outside containment } \\
\text { (V-sequence) }\end{array}$ & TBD & TBD \\
\hline $\begin{array}{l}\text { Cryogenic system } \\
\text { status }\end{array}$ & $\begin{array}{l}\text { Cryogenic system } \\
\text { operating }\end{array}$ & TBD & $T B D$ \\
\hline $\begin{array}{l}\text { Filter fan system } \\
\text { status }\end{array}$ & Activation of filter fans & TBD & TBD \\
\hline
\end{tabular}


TABLE E2. (continued)

\begin{tabular}{|c|c|c|c|}
\hline $\begin{array}{c}\text { Potential } \\
\text { Measurements } \\
\text { Identified } \\
\end{array}$ & $\begin{array}{l}\text { Information } \\
\text { Need }\end{array}$ & $\begin{array}{c}\text { Potential } \\
\text { Severe Accident } \\
\text { Measurement } \\
\text { Range } \\
\end{array}$ & $\begin{array}{c}\text { Location } \\
\text { of } \\
\text { Information }\end{array}$ \\
\hline $\begin{array}{l}\text { Supply of chemicals } \\
\text { for base solution }\end{array}$ & $\begin{array}{l}\text { Availability of basic } \\
\text { solution }\end{array}$ & TBD & TBD \\
\hline \multirow{2}{*}{$\begin{array}{l}\text { Flow in system for } \\
\text { adding base to } \\
\text { conta inment water }\end{array}$} & $\begin{array}{l}\text { Initiation of add base } \\
\text { strategy }\end{array}$ & TBD & TBD \\
\hline & Flow of basic solution & & \\
\hline \multirow[t]{2}{*}{$\begin{array}{l}\text { Dilution system } \\
\text { tank level }\end{array}$} & $\begin{array}{l}\text { Inventory of dilution } \\
\text { liquid }\end{array}$ & TBD & TBD \\
\hline & $\begin{array}{l}\text { Availability of dilution } \\
\text { water }\end{array}$ & & \\
\hline $\begin{array}{l}\text { Flow in dilution } \\
\text { system }\end{array}$ & $\begin{array}{l}\text { F low of dilution liquid } \\
\text { to conta inment water }\end{array}$ & TBD & TBD \\
\hline $\begin{array}{l}\text { Conta inment } \\
\text { atmosphere sampling }\end{array}$ & $\begin{array}{l}\text { Presence of radiolytic } \\
\text { products }\end{array}$ & TBD & TBD \\
\hline $\begin{array}{l}\text { Flow rate in sump } \\
\text { fill system }\end{array}$ & Flow of water to sump & TBD & TBD \\
\hline $\begin{array}{l}\text { Sump fill system } \\
\text { tank level }\end{array}$ & $\begin{array}{l}\text { Inventory of water to } \\
\text { fill sump }\end{array}$ & $\begin{array}{l}\text { See containment sump } \\
\text { leve ? }\end{array}$ & \\
\hline
\end{tabular}




\section{APPENDIX F}

ACCIDENT MANAGEMENT INFORMATION ASSESSMENT

$F-1 / F-2$ 


\section{APPENDIX F \\ ACCIDENT MANAGEMENT INFORMATION ASSESSMENT}

The tables contained in Appendix F describe the information needs and instruments available for each of the challenges and mechanisms under each safety function, starting with Inadequate Secondary Heat Removal for the Maintain RCS Heat Removal safety function. The strategies that may be available to combat a challenging mechanism are addressed immediately after addressing the individual mechanisms. The safety objective tree was reviewed from top-tobottom and left-to-right for this assessment of information needs and the results are tabulated accordingly in Tables F1 through F8. 
TABLE F1. ACCIDENT MANAGEMENT INFORMATION ASSESSMENT

Scenario SBLOCA/LOOP Plant

Safety Objective Prevent Core Dispersal from Vessel

Safety Function Maintain Core Boundary

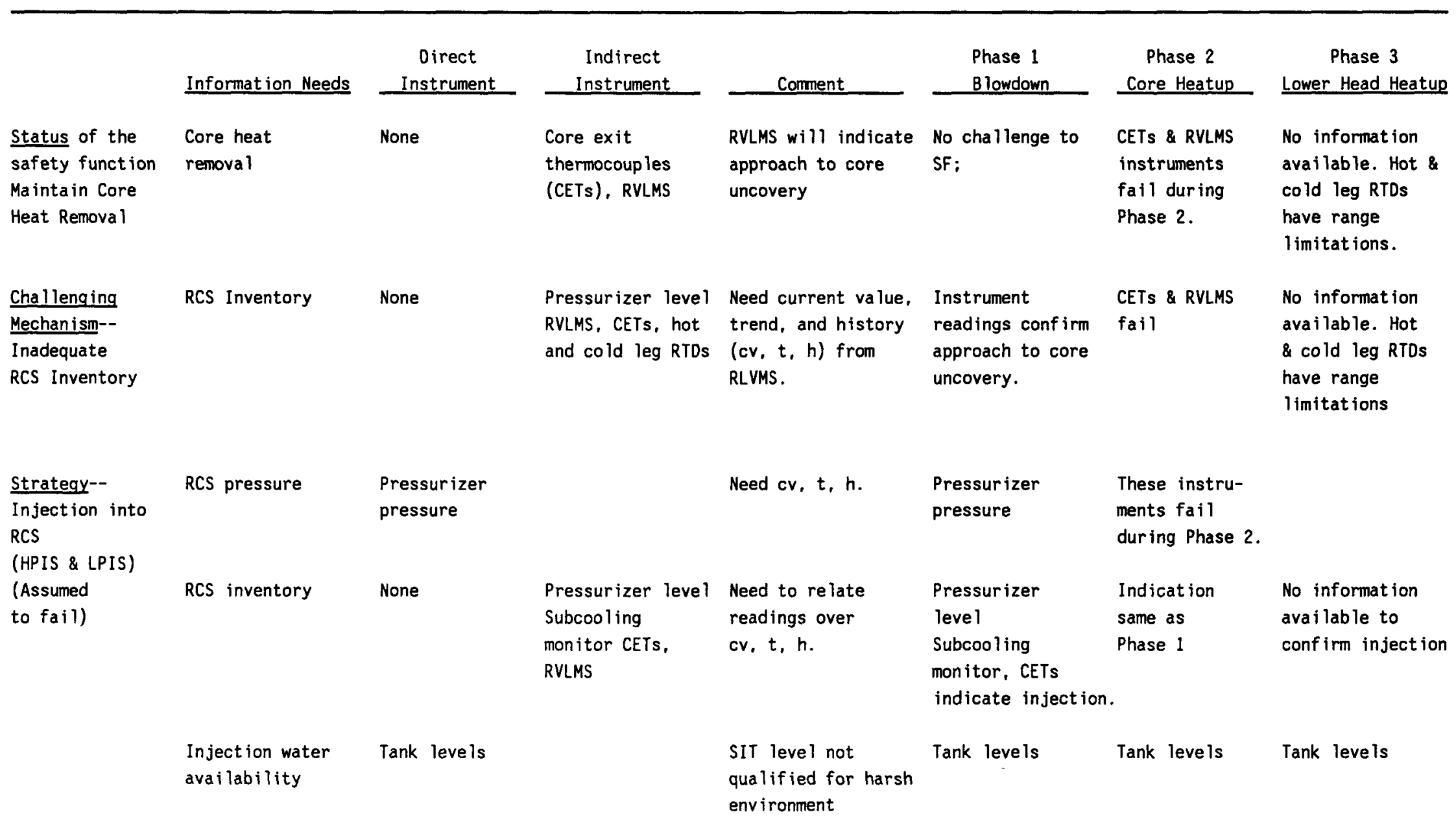


TABLE F1. (cont inued)

Scenario SBLOCA/LOOP Plant

Safety Objective Prevent Core Dispersal from Vessel

Safety Function Maintain Core Boundary

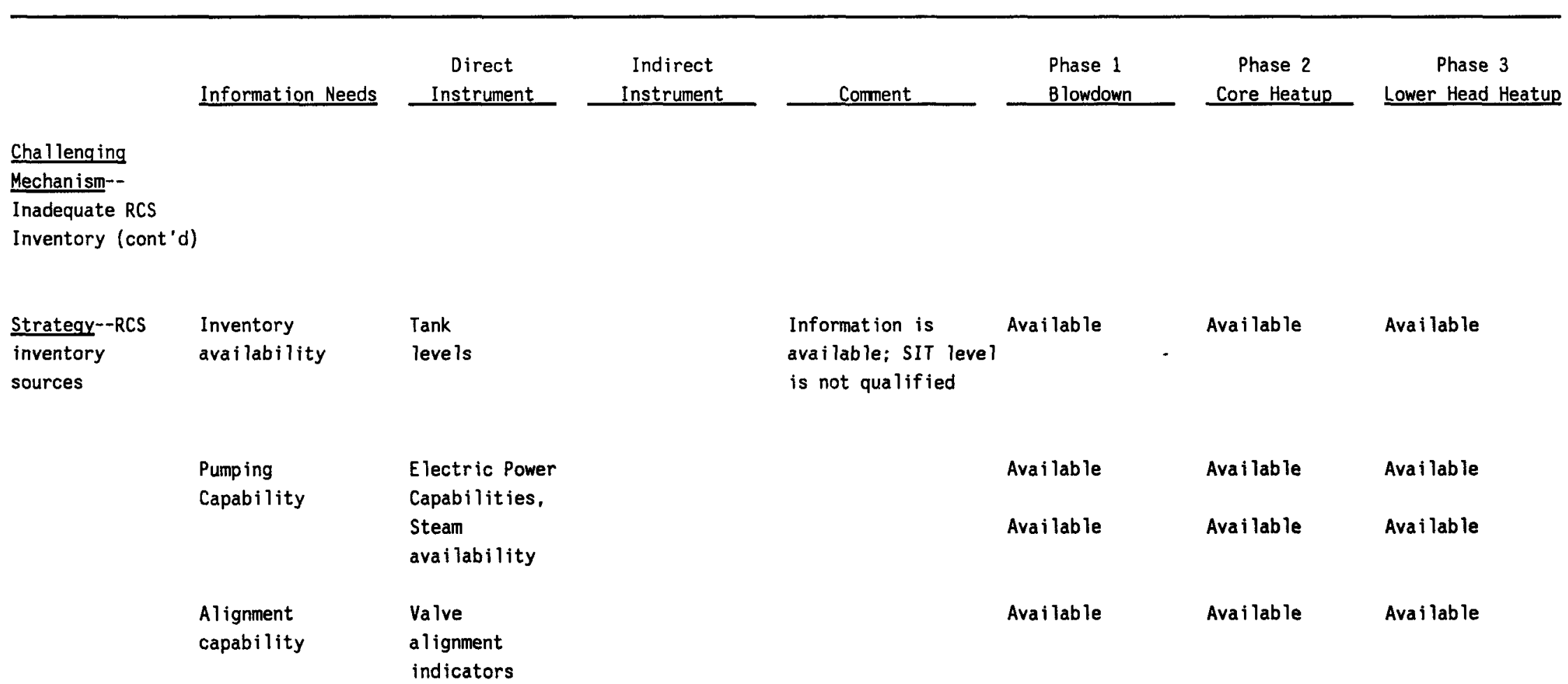


TABLE F1. (cont inued)

Scenar io SBLOCA/LOOP P lant

Safety Objective Prevent Core Dispersal from Vessel

Safety Function Mainta in Core Boundary

\begin{tabular}{|c|c|c|c|c|c|c|c|}
\hline & Information Needs & $\begin{array}{c}\text { Direct } \\
\text { Instrument }\end{array}$ & $\begin{array}{l}\text { Indirect } \\
\text { Instrument }\end{array}$ & Comment & $\begin{array}{l}\text { Phase } 1 \\
\text { B lowdown }\end{array}$ & $\begin{array}{c}\text { Phase } 2 \\
\text { Core Heatup } \\
\end{array}$ & $\begin{array}{c}\text { Phase } 3 \\
\text { Lower Head Heatup }\end{array}$ \\
\hline \multicolumn{8}{|l|}{ Challenging } \\
\hline $\begin{array}{l}\text { Change in } \\
\text { Core Geometry }\end{array}$ & $\begin{array}{l}\text { Core material } \\
\text { Temperature } \\
\text { and Geometry }\end{array}$ & None & $\begin{array}{l}\text { CETs, SPNDs, } \\
\text { SRNIs, Coolant } \\
\text { activity }\end{array}$ & $\begin{array}{l}\text { SRNIs will fail } \\
\text { since not qualified } \\
\text { for harsh environs; } \\
\text { SRNIs failed due } \\
\text { to environment in } \\
\text { in Phase } 2 \text {. }\end{array}$ & $\begin{array}{l}\text { No cha } 1 \text { lenge } \\
\text { present }\end{array}$ & $\begin{array}{l}\text { SPNDs \& CETs } \\
\text { will indicate } \\
\text { high core } \\
\text { temperature } \\
\text { prior to failure; } \\
\text { SRNIs will fail. }\end{array}$ & $\begin{array}{l}\text { No information } \\
\text { available } \\
\end{array}$ \\
\hline \multicolumn{8}{|c|}{$\begin{array}{l}\text { Strategy--RCS } \\
\text { Injection methods } \\
\text { and RCS Inventory } \\
\text { Sources (same as } \\
\text { above) }\end{array}$} \\
\hline \multirow[t]{2}{*}{$\frac{\text { Strategy--RCS }}{\text { Pump Restart }}$} & RCS flow status & $\begin{array}{l}\text { RCS flow rate, } \\
\text { RCS pump speed, } \\
\text { RCS pump } \\
\text { differential } \\
\text { pressure, RCS } \\
\text { pump motor } \\
\text { current }\end{array}$ & & $\begin{array}{l}\text { Information } \\
\text { available; however, } \\
\text { restart of pump } \\
\text { during voided } \\
\text { conditions could } \\
\text { promote damage. }\end{array}$ & No cha 1 lenge & $\begin{array}{l}\text { Information } \\
\text { available }\end{array}$ & $\begin{array}{l}\text { Information } \\
\text { available }\end{array}$ \\
\hline & $\begin{array}{l}\text { Fuel rod clad } \\
\text { temperature }\end{array}$ & None & CETS, SPNDS & & No chal lenge & $\begin{array}{l}\text { SPNDs and CETs } \\
\text { will indicate } \\
\text { high core temp. } \\
\text { prior to failure }\end{array}$ & $\begin{array}{l}\text { No information } \\
\text { available }\end{array}$ \\
\hline
\end{tabular}


TABLE F2. ACCIDENT MANAGEMENT INFORMATION ASSESSMENT

Scenario SBLOCA/LOOP P lant

Safety Objective Prevent Core Dispersal from Vesse 1

Safety Function Maintain Vessel Boundary

\begin{tabular}{|c|c|c|c|c|c|c|c|}
\hline E lement & Information Needs & $\begin{array}{c}\text { Direct } \\
\text { Instrument }\end{array}$ & $\begin{array}{c}\text { Indirect } \\
\text { Instrument } \\
\end{array}$ & Corment & $\begin{array}{c}\text { Phase } 2 \\
\text { Core Heatup }\end{array}$ & $\begin{array}{c}\text { Phase } 3 \\
\text { Lower } \\
\text { Head Heatup } \\
\end{array}$ & $\begin{array}{c}\text { Phase } 4 \\
\text { Containment } \\
\text { Heatup }\end{array}$ \\
\hline $\begin{array}{l}\text { Status of the } \\
\text { safety function } \\
\text { Maintain Vesse } 1 \\
\text { Boundary }\end{array}$ & Vessel integrity & None & $\begin{array}{l}\text { Pressurizer } \\
\text { pressure }\end{array}$ & Need $c v, t, h$. & \multicolumn{2}{|c|}{$\begin{array}{l}\text { Pressurizer pressure } \\
\text { (fails in later } \\
\text { stage of Phase } 2 \text { ). }\end{array}$} & \\
\hline $\begin{array}{l}\text { Chal lenging } \\
\text { Mechanism-- } \\
\text { Overtemperature } \\
\text { coolable }\end{array}$ & $\begin{array}{l}\text { Core relocation } \\
\text { status }\end{array}$ & None & $\begin{array}{l}\text { SRNIs, } \\
\text { Pressurizer } \\
\text { pressure, } \\
\text { Hot \& cold leg }\end{array}$ & Need $c v, t, h$. & \multicolumn{2}{|l|}{$\begin{array}{l}\text { These instruments } \\
\text { fail in later } \\
\text { stage of Phase } 2 .\end{array}$} & \\
\hline relocation & Core damage status & None & $\begin{array}{l}\text { RTDs, CETs, } \\
\text { RVLMS, } \\
\text { RCS sampling } \\
\text { system }\end{array}$ & & \multicolumn{2}{|c|}{$\begin{array}{l}\text { CET \& RVLMS instr. } \\
\text { fail in Phase 2. } \\
\text { RCS sampling } \quad \text { RCS sam } \\
\text { system }\end{array}$} & $\begin{array}{l}\text { RCS sampling } \\
\text { system }\end{array}$ \\
\hline \multirow{2}{*}{$\begin{array}{l}\text { Strategy-- } \\
\text { Injection into } \\
\text { RCS (HPIS and } \\
\text { LPIS failed) }\end{array}$} & $\begin{array}{l}\text { RCS pressure } \\
\text { RCS inventory }\end{array}$ & $\begin{array}{l}\text { Pzr, pressure } \\
\text { None }\end{array}$ & $\begin{array}{l}\text { Pressurizer } \\
\text { level. } \\
\text { RVLMS }\end{array}$ & Need $c v, t, h$. & $\begin{array}{l}\text { Instruments fail } \\
\text { during Phase } 2 .\end{array}$ & & \\
\hline & $\begin{array}{l}\text { Injection water } \\
\text { availability }\end{array}$ & $\begin{array}{l}\text { Tank levels } \\
\text { sump levels }\end{array}$ & & & $\begin{array}{l}\text { Tank levels } \\
\text { Sump level (fails } \\
\text { during Phase 2). }\end{array}$ & Tank levels & Tank levels \\
\hline
\end{tabular}


TABLE F2. (cont inued)

Scenario SBLOCA/LOOP Plant

Safety Objective Prevent Core Dispersal from Vesse]

Safety Function Maintain Vessel Boundary

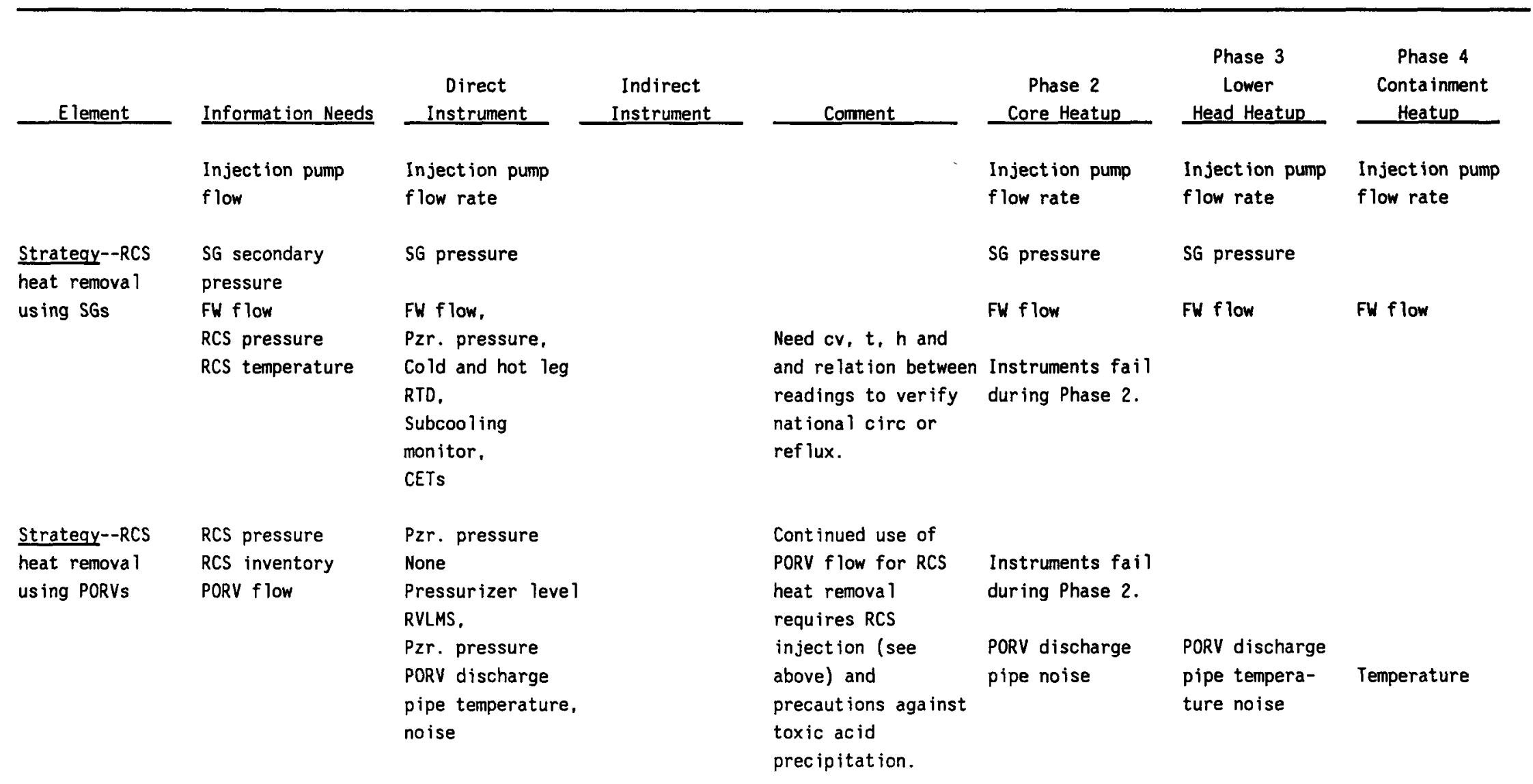

The strategy of using RCS injection methods may require depressurization of the RCS to allow lower pressure injection systems to operate and may involve use of steam generator atmospheric dump valve, bypass, or RCS PORVs. These strategies are included in the inadequate primary and secondary heat removal challenges to the Maintain RCS Heat Removal strategy and are included here since they may be necessary to provide alternate RCS injection methods. 
TABLE F2. (cont inued)

Scenario SBLOCA/LOOP Plant

Safety Objective Prevent Core Dispersal from Vesse 1

Safety Function Maintain Vessel Boundary

\begin{tabular}{|c|c|c|c|c|c|c|c|}
\hline Element & Information Needs & $\begin{array}{c}\text { Direct } \\
\text { Instrument }\end{array}$ & $\begin{array}{c}\text { Indirect } \\
\text { Instrument }\end{array}$ & Corment & $\begin{array}{c}\text { Phase } 2 \\
\text { Core Heatup }\end{array}$ & $\begin{array}{c}\text { Phase } 3 \\
\text { Lower } \\
\text { Head Heatup } \\
\end{array}$ & $\begin{array}{c}\text { Phase } 4 \\
\text { Containment } \\
\text { Heatup } \\
\end{array}$ \\
\hline \multirow{2}{*}{$\begin{array}{l}\frac{\text { Cha l lenging }}{\text { Mechanism-- }} \\
\text { Noncoo lable } \\
\text { re locat ion }\end{array}$} & $\begin{array}{l}\text { Core relocation } \\
\text { status }\end{array}$ & None & $\begin{array}{l}\text { SRNIs, } \\
\text { Pzr. pressure } \\
\text { Hot \& cold leg RTDs }\end{array}$ & & & & \\
\hline & Core damage status & None & $\begin{array}{l}\text { CETs, } \\
\text { RVLMS, } \\
\text { RCS sampling } \\
\text { system }\end{array}$ & & $\begin{array}{l}\text { RCS sampling } \\
\text { system }\end{array}$ & $\begin{array}{l}\text { RCS sampling } \\
\text { system }\end{array}$ & $\begin{array}{l}\text { RCS sampling } \\
\text { system }\end{array}$ \\
\hline$\frac{\text { Strategy--F lood }}{\text { cavity }}$ & & & & $\begin{array}{l}\text { No capability for } \\
\text { this strategy } \\
\text { is installed. }\end{array}$ & & & \\
\hline
\end{tabular}


TABLE F3. ACCIDENT MANAGEMENT INFORMATION ASSESSMENT

Scenario SBLOCA/LOOP Plant

Safety Objective Prevent Containment Failure

Safety Function Maintain Pressure Control

\begin{tabular}{|c|c|c|c|c|c|c|c|}
\hline E lement & Information Needs & $\begin{array}{c}\text { Direct } \\
\text { Instrument }\end{array}$ & $\begin{array}{c}\text { Indirect } \\
\text { Instrument } \\
\end{array}$ & Comment & $\begin{array}{c}\text { Phase } 2 \\
\text { Core Heatup } \\
\end{array}$ & $\begin{array}{c}\text { Phase } 3 \\
\text { Lower } \\
\text { Head Heatup } \\
\end{array}$ & $\begin{array}{c}\text { Phase } 4 \\
\text { Containment } \\
\text { Heatup } \\
\end{array}$ \\
\hline$\frac{\text { Status of safety }}{\text { function }}$ & $\begin{array}{l}\text { Containment } \\
\text { pressure }\end{array}$ & $\begin{array}{l}\text { Containment } \\
\text { pressure }\end{array}$ & & Need cr, $t, h$. & $\begin{array}{l}\text { Containment } \\
\text { pressure }\end{array}$ & $\begin{array}{l}\text { Containment } \\
\text { pressure }\end{array}$ & $\begin{array}{l}\text { Conta inment } \\
\text { pressure }\end{array}$ \\
\hline $\begin{array}{l}\text { Challenging } \\
\text { Mechan ism-- } \\
\text { Insuff ic ient } \\
\text { energy remova? }\end{array}$ & $\begin{array}{l}\text { Energy added to } \\
\text { the conta inment } \\
\text { atmosphere }\end{array}$ & None & $\begin{array}{l}\text { Containment } \\
\text { pressure } \\
\text { Containment } \\
\text { pressure } \\
\text { atmosphere } \\
\text { temperature }\end{array}$ & $\begin{array}{l}\text { Need } c v, t, h \text { and } \\
\text { parameters are } \\
\text { related. }\end{array}$ & $\begin{array}{l}\text { Containment } \\
\text { pressure } \\
\text { Containment } \\
\text { atmosphere } \\
\text { temperature }\end{array}$ & $\begin{array}{l}\text { Conta inment } \\
\text { pressure } \\
\text { Conta inment } \\
\text { atmosphere } \\
\text { temperature }\end{array}$ & $\begin{array}{l}\text { Containment } \\
\text { pressure }\end{array}$ \\
\hline & $\begin{array}{l}\text { RCS integrity } \\
\text { (LOCA information) }\end{array}$ & None & $\begin{array}{l}\text { Pzr. pressure } \\
\text { Pressurizer } \\
\text { level } \\
\text { RVLMS } \\
\text { Subcooling monitor }\end{array}$ & $\begin{array}{l}\text { Need } c v, t, h \text { and } \\
\text { parameters are } \\
\text { related. }\end{array}$ & $\begin{array}{l}\text { Instruments fail } \\
\text { during Phase } 2 \text {. }\end{array}$ & & \\
\hline & $\begin{array}{l}\text { SG secondary } \\
\text { integrity }\end{array}$ & None & $\begin{array}{l}\text { SG pressure } \\
\text { SG leve } 1 \\
\text { Steam flow/ } \\
\text { feed flow } \\
\text { mismatch }\end{array}$ & $\begin{array}{l}\text { Need } c v, t, h \text { and } \\
\text { parameters are } \\
\text { related. }\end{array}$ & $\begin{array}{l}\text { Need } c v, t, h \text { and } \\
\text { parameters are } \\
\text { related. }\end{array}$ & $\begin{array}{l}\text { Need } c v, t, h \\
\text { and parameters } \\
\text { are related. }\end{array}$ & $\begin{array}{l}\text { Need } c v, t, h \text { and } \\
\text { parameters are } \\
\text { related. }\end{array}$ \\
\hline
\end{tabular}


TABLE F3. (continued)

Scenario SBLOCA/LOOP P lant

Safety Objective Prevent Containment Failure

Safety Function Maintain Pressure Control

\begin{tabular}{|c|c|c|c|c|c|c|c|}
\hline Element & Informat ion Needs & $\begin{array}{c}\text { Direct } \\
\text { Instrument }\end{array}$ & $\begin{array}{c}\text { Indirect } \\
\text { Instrument }\end{array}$ & Comment & $\begin{array}{c}\text { Phase } 2 \\
\text { Core Heatup }\end{array}$ & $\begin{array}{c}\text { Phase } 3 \\
\text { Lower } \\
\text { Head Heatup } \\
\end{array}$ & $\begin{array}{c}\text { Phase } 4 \\
\text { Containment } \\
\text { Heatup } \\
\end{array}$ \\
\hline $\begin{array}{l}\text { Strateqy--Fan } \\
\text { coolers }\end{array}$ & $\begin{array}{l}\text { Fan cooling system } \\
\text { status }\end{array}$ & $\begin{array}{l}\text { System status } \\
\text { indicators }\end{array}$ & & $\begin{array}{l}\text { Includes heat } \\
\text { exchanger } \\
\text { effect iveness }\end{array}$ & $\begin{array}{l}\text { System status } \\
\text { indicators }\end{array}$ & $\begin{array}{l}\text { System status } \\
\text { indicators }\end{array}$ & $\begin{array}{l}\text { System status } \\
\text { indicators }\end{array}$ \\
\hline$\frac{\text { Strategy--Spray }}{\text { system }}$ & $\begin{array}{l}\text { Spray water } \\
\text { availability } \\
\text { Spray flow rate }\end{array}$ & $\begin{array}{l}\text { Tank leve } 1 \\
\text { Sump leve } 1 \\
\text { Spray flow rate } \\
\text { Pump discharge } \\
\text { pressure } \\
\text { Pump motor } \\
\text { current }\end{array}$ & & & $\begin{array}{l}\text { Tank leve } 1 \\
\text { Sump leve } 1 \\
\text { Spray flow rate } \\
\text { Pump discharge } \\
\text { pressure } \\
\text { Pump motor } \\
\text { current }\end{array}$ & $\begin{array}{l}\text { Tank leve } 1 \\
\text { Sump leve } 1 \\
\text { Spray flow rate } \\
\text { Pump discharge } \\
\text { pressure } \\
\text { Pump motor } \\
\text { current }\end{array}$ & $\begin{array}{l}\text { Tank leve } 1 \\
\text { Sump level } \\
\text { Spray f low rate } \\
\text { Pump discharge } \\
\text { pressure } \\
\text { Pump motor } \\
\text { current }\end{array}$ \\
\hline $\begin{array}{l}\text { Strategy-- } \\
\text { Containment } \\
\text { vent ing }\end{array}$ & $\begin{array}{l}\text { Conta inment } \\
\text { overpressure } \\
\text { threat }\end{array}$ & $\begin{array}{l}\text { Containment } \\
\text { pressure }\end{array}$ & & Need $c v, t, h$ & Need cv, $t, h$ & Need $c v, t, h$ & Need $c v, t, h$ \\
\hline & & $\begin{array}{l}\text { Conta inment } \\
\text { temperature }\end{array}$ & & Need $c v, t, h$ & Need $c v, t, h$ & Need $c v, t, h$ & \\
\hline & $\begin{array}{l}\text { Radioact ive } \\
\text { content of } \\
\text { containment } \\
\text { atmosphere }\end{array}$ & $\begin{array}{l}\text { Containment } \\
\text { radiation } \\
\text { monitors }\end{array}$ & & & & & \\
\hline
\end{tabular}


TABLE F3. (cont inued)

Scenario SBLOCA/LOOP Plant

Safety Objective Prevent Conta inment Fai lure

Safety Function Mainta in Pressure Control

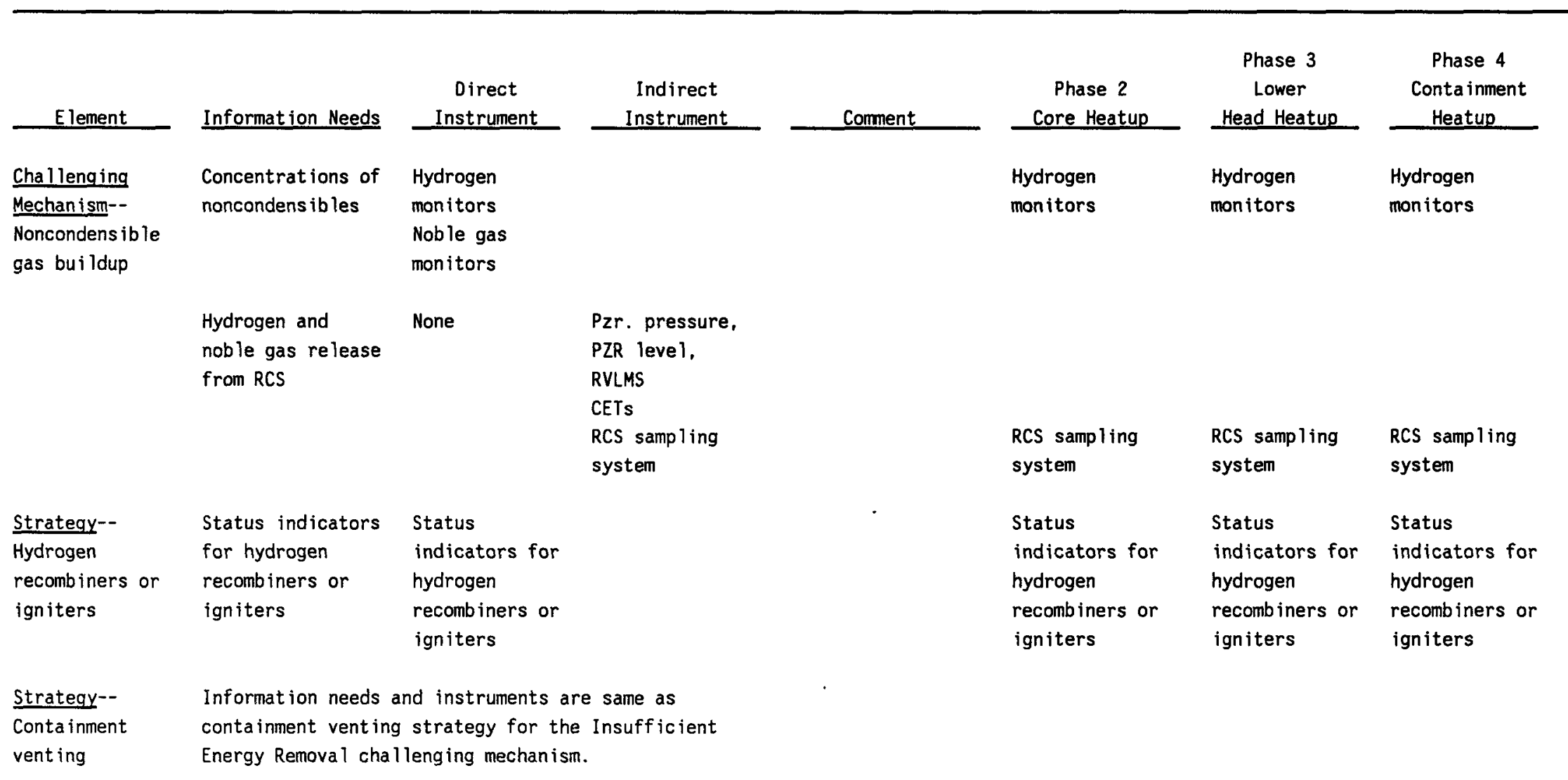


TABLE F3. (cont inued)

Scenario SBLOCA/LOOP Plant

Safety Objective Prevent Containment Failure

Safety Function Maintain Pressure Control

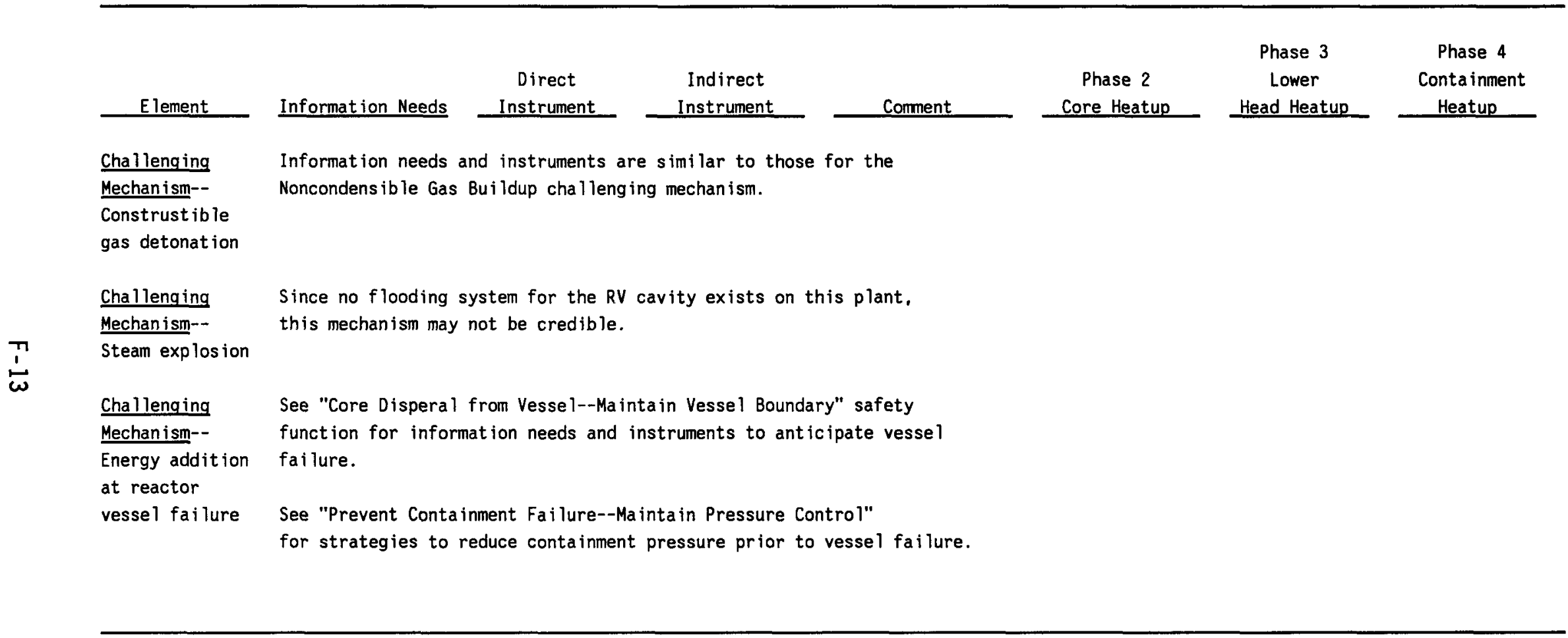


TABLE F4. ACCIDENT MANAGEMENT INFORMATION ASSESSMENT

Scenario SBLOCA/LOOP Plant

Safety objective Prevent Containment Failure

Safety Function Maintain Temperature Control

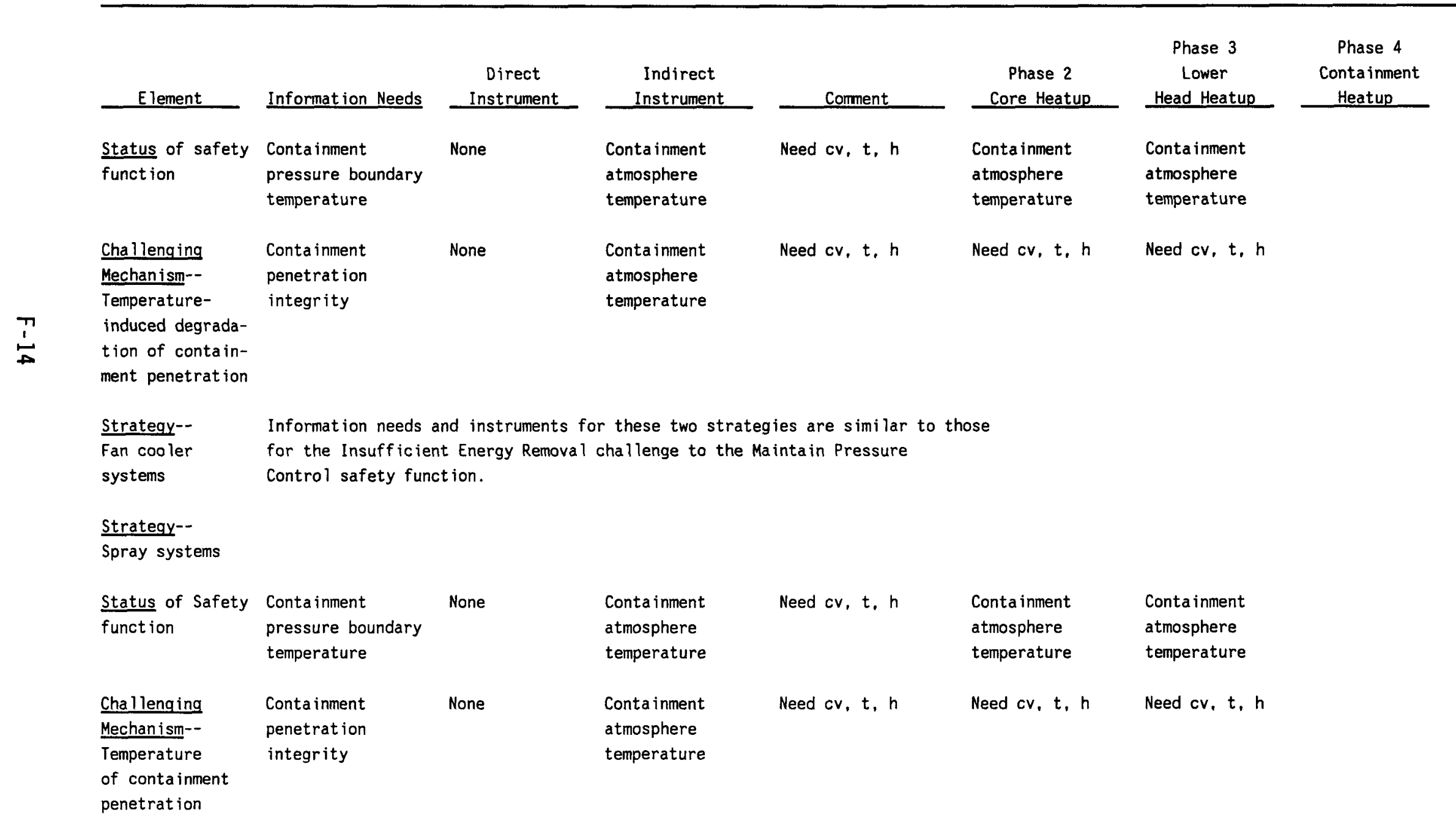


TABLE F4. (cont inued)

Scenario SBLOCA/LOOP Plant

Safety Objective Prevent Containment Failure

Safety Function Maintain Temperature Control

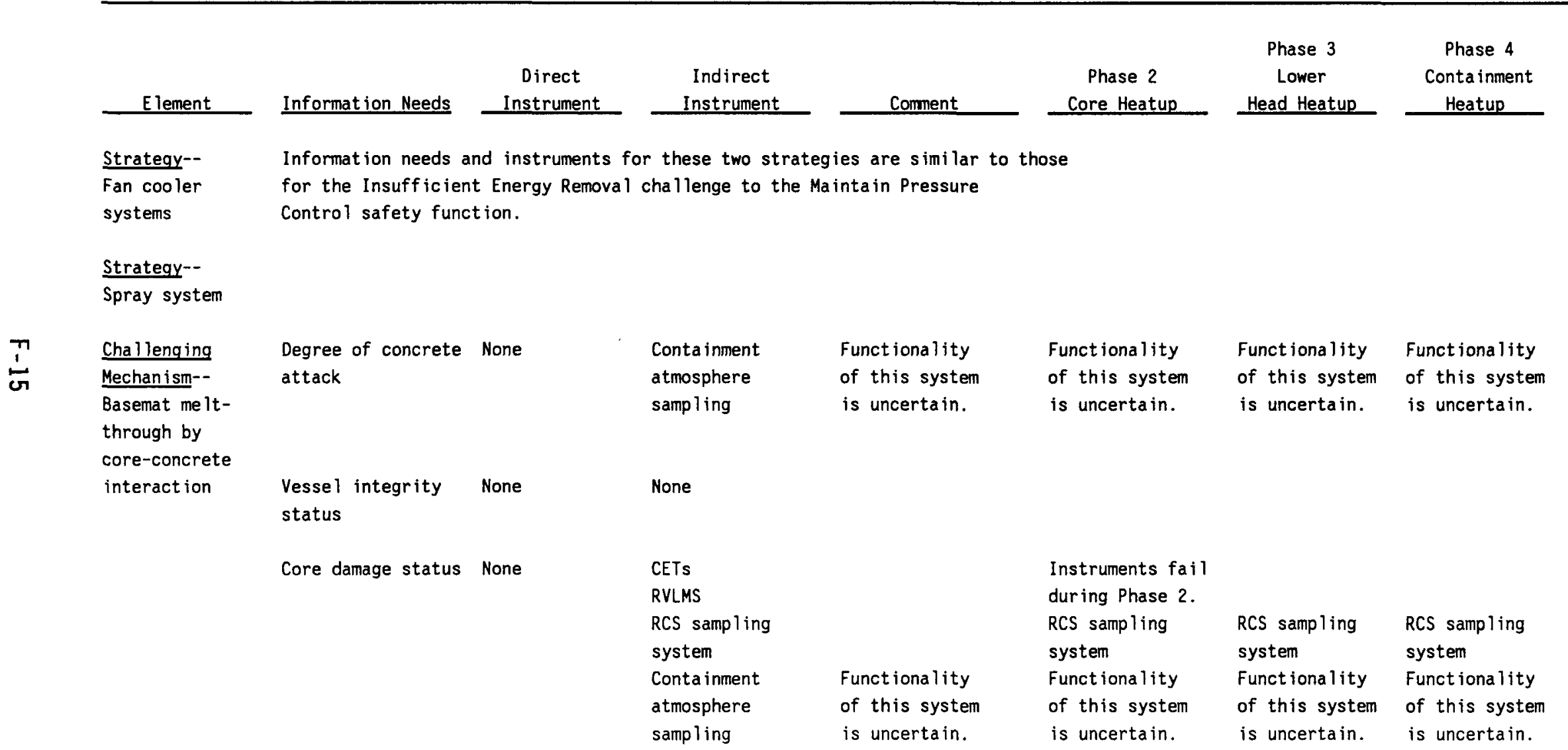


TABLE F5. ACCIDENT MANAGEMENT INFORMATION ASSESSMENT

Scenario SBLOCA/LOOP P Tant

Safety Objective Prevent Containment Failure

Safety Function Maintain Integrity

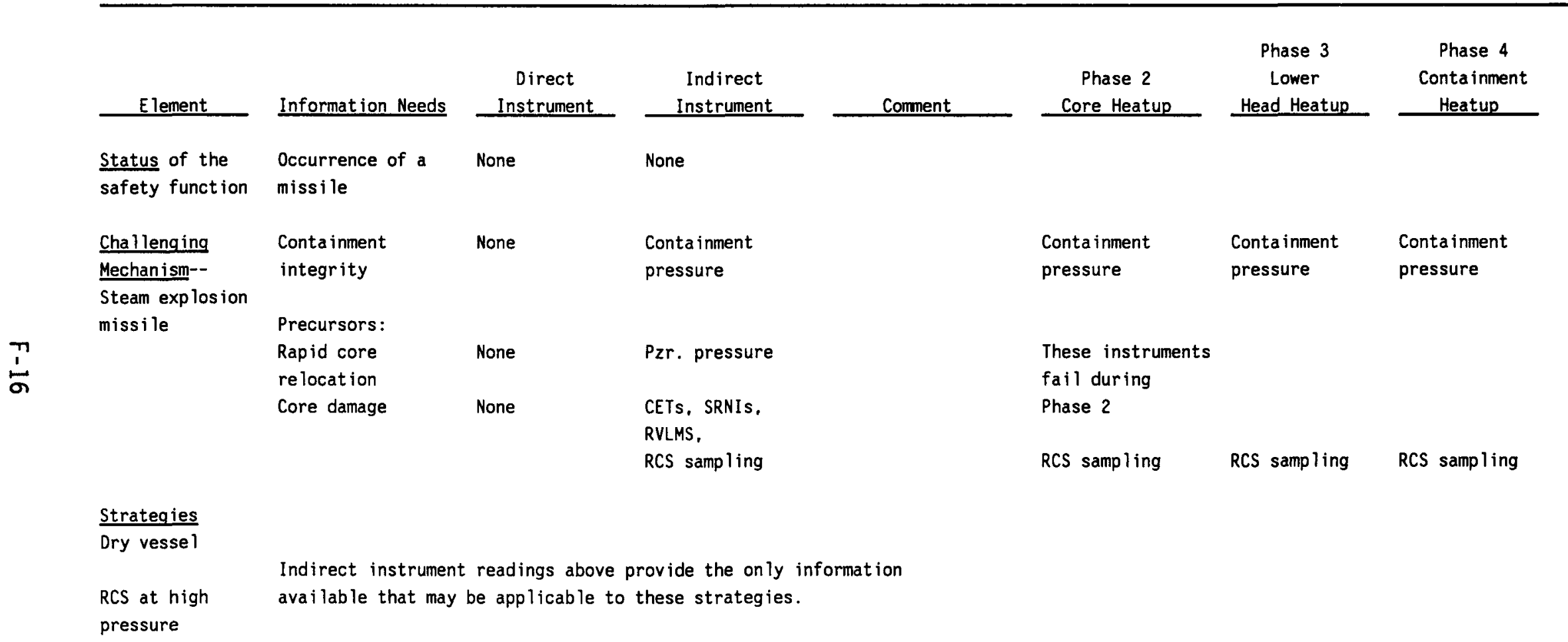


TABLE F5. (cont inued)

Scenario SBLOCA/LOOP P lant

Safety Objective Prevent Containment Failure

Safety Function Maintain Integrity

\begin{tabular}{|c|c|c|c|c|c|c|c|}
\hline E lement & Information Needs & $\begin{array}{c}\text { Direct } \\
\text { Instrument }\end{array}$ & $\begin{array}{c}\text { Indirect } \\
\text { Instrument }\end{array}$ & Comment & $\begin{array}{c}\text { Phase } 2 \\
\text { Core Heatup }\end{array}$ & $\begin{array}{c}\text { Phase } 3 \\
\text { Lower } \\
\text { Head Heatup } \\
\end{array}$ & $\begin{array}{c}\text { Phase } 4 \\
\text { Containment } \\
\text { Heatup } \\
\end{array}$ \\
\hline \multicolumn{8}{|l|}{ Challenging } \\
\hline \multicolumn{8}{|l|}{ Mechan ism-- } \\
\hline $\begin{array}{l}\text { Hydrogen } \\
\text { detonation } \\
\text { missile }\end{array}$ & $\begin{array}{l}\text { Hydrogen gas } \\
\text { concentration } \\
\left(\mathrm{H}_{2}: \mathrm{O}_{2}: \mathrm{H}_{2} \mathrm{O} \text { values }\right)\end{array}$ & $\begin{array}{l}\text { Hydrogen } \\
\text { monitoring }\end{array}$ & system & & $\begin{array}{l}\text { Hydrogen } \\
\text { monitoring }\end{array}$ & $\begin{array}{l}\text { Hydrogen } \\
\text { monitoring } \\
\text { system }\end{array}$ & $\begin{array}{l}\text { Hydrogen } \\
\text { monitoring } \\
\text { system system }\end{array}$ \\
\hline \multicolumn{8}{|l|}{ Strategy-- } \\
\hline $\begin{array}{l}\text { Prevent } \\
\text { hydrogen } \\
\text { explosion }\end{array}$ & $\begin{array}{l}\text { Similar to strateg } \\
\text { challenge to the } \\
\text { function. }\end{array}$ & $\begin{array}{l}\text { es for the Com } \\
\text { inta in Pressur }\end{array}$ & $\begin{array}{l}\text { tible Gas Deton } \\
\text { ontrol safety }\end{array}$ & & & & \\
\hline
\end{tabular}


TABLE F6. ACCIDENT MANAGEMENT INFORMATION ASSESSMENT

Scenario SBLOCA/LOOP Plant

Safety Objective Mitigate Fission Product Release from Containment

Safety Function Maintain Control of FP Inventory in Containment Atmosphere

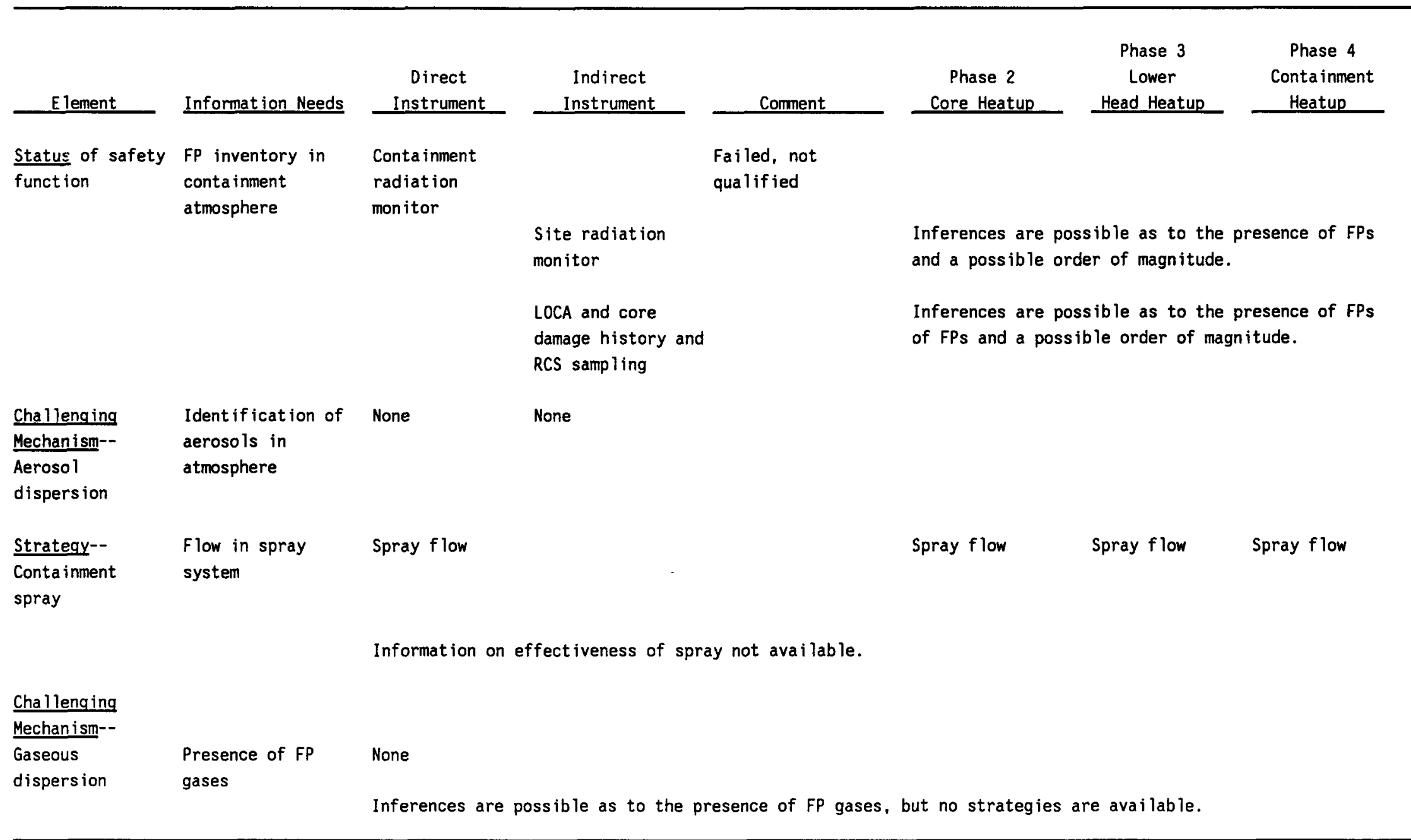


TABLE F7. ACCIDENT MANAGEMENT INFORMATION ASSESSMENT

Scenario SBLOCA/LOOP P lant

Safety Objective Mitigate Fission Product Release from Containment

Safety Function Maintain Control of FP Release from Containment Water

\begin{tabular}{|c|c|c|c|c|c|c|c|}
\hline Element & Information Needs & $\begin{array}{c}\text { Direct } \\
\text { Instrument }\end{array}$ & $\begin{array}{l}\text { Indirect } \\
\text { Instrument }\end{array}$ & Comment & $\begin{array}{c}\text { Phase } 2 \\
\text { Core Heatup }\end{array}$ & $\begin{array}{c}\text { Phase } 3 \\
\text { Lower } \\
\text { Head Heatup } \\
\end{array}$ & $\begin{array}{c}\text { Phase } 4 \\
\text { Conta inment } \\
\text { Heatup }\end{array}$ \\
\hline $\begin{array}{l}\text { Status of safety } \\
\text { function }\end{array}$ & $\begin{array}{l}\text { Presence of FP in } \\
\text { containment water }\end{array}$ & None & \multicolumn{5}{|c|}{$\begin{array}{l}\text { Inferences as to FP presence are possible, } \\
\text { but no quant ifiable }\end{array}$} \\
\hline \multicolumn{8}{|l|}{ Challenging } \\
\hline \multicolumn{8}{|l|}{ Mechan ism-- } \\
\hline $\mathrm{pH}$ too low & $\begin{array}{l}\text { Sump water } \\
\text { chemistry }\end{array}$ & None & None & $\begin{array}{l}\text { Failed, not } \\
\text { qualified }\end{array}$ & & & \\
\hline
\end{tabular}

Without the information shown above, the possible strategies are not viable.

Challenging

Mechan ism--

High temperature Sump temperature Sump temperature

of sump water

No instruments

Strategy--

Operation of

Status of sump

Status instruments

instru-

for sump water

containment

water cooling

cooling

Status instru-

ments for sump

water cooling
Status instru-

ments for sump

water cooling
Status instru- Status

ments for sump

water cooling systems

systems 
TABLE F8. ACCIDENT MANAGEMENT INFORMATION ASSESSMENT

Scenario SBLOCA/LOOP Plant

Safety Objective Prevent Containment Failure

Safety Function Maintain Integrity

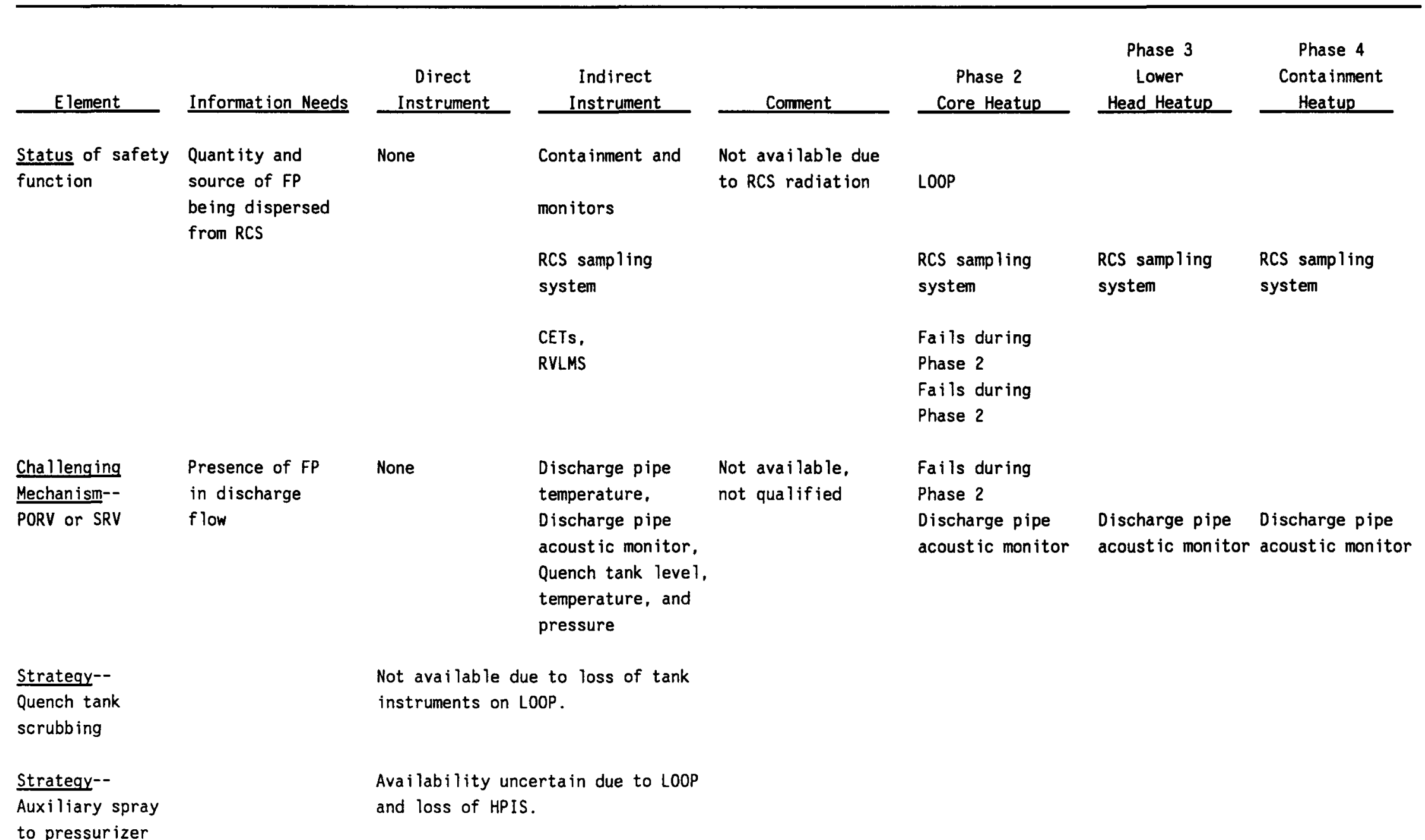


TABLE F8. (cont inued)

Scenario SBLOCA/LOOP P lant

Safety Objective Prevent Containment Failure

Safety Function Maintain Integrity

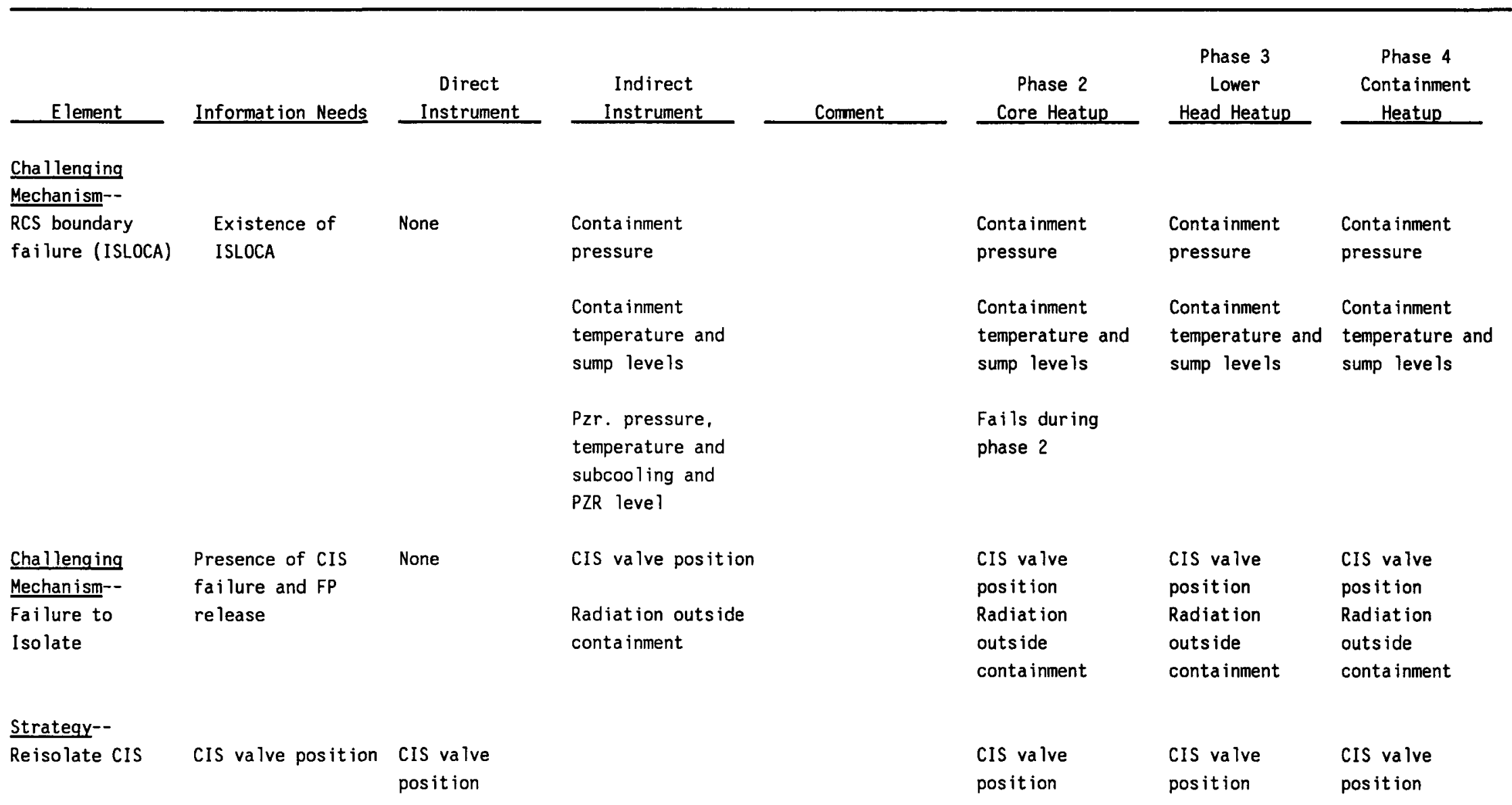


TABLE F8. (cont inued)

Scenario SBLOCA/LOOP Plant

Safety Objective Prevent Containment Failure

Safety Function Maintain Integrity

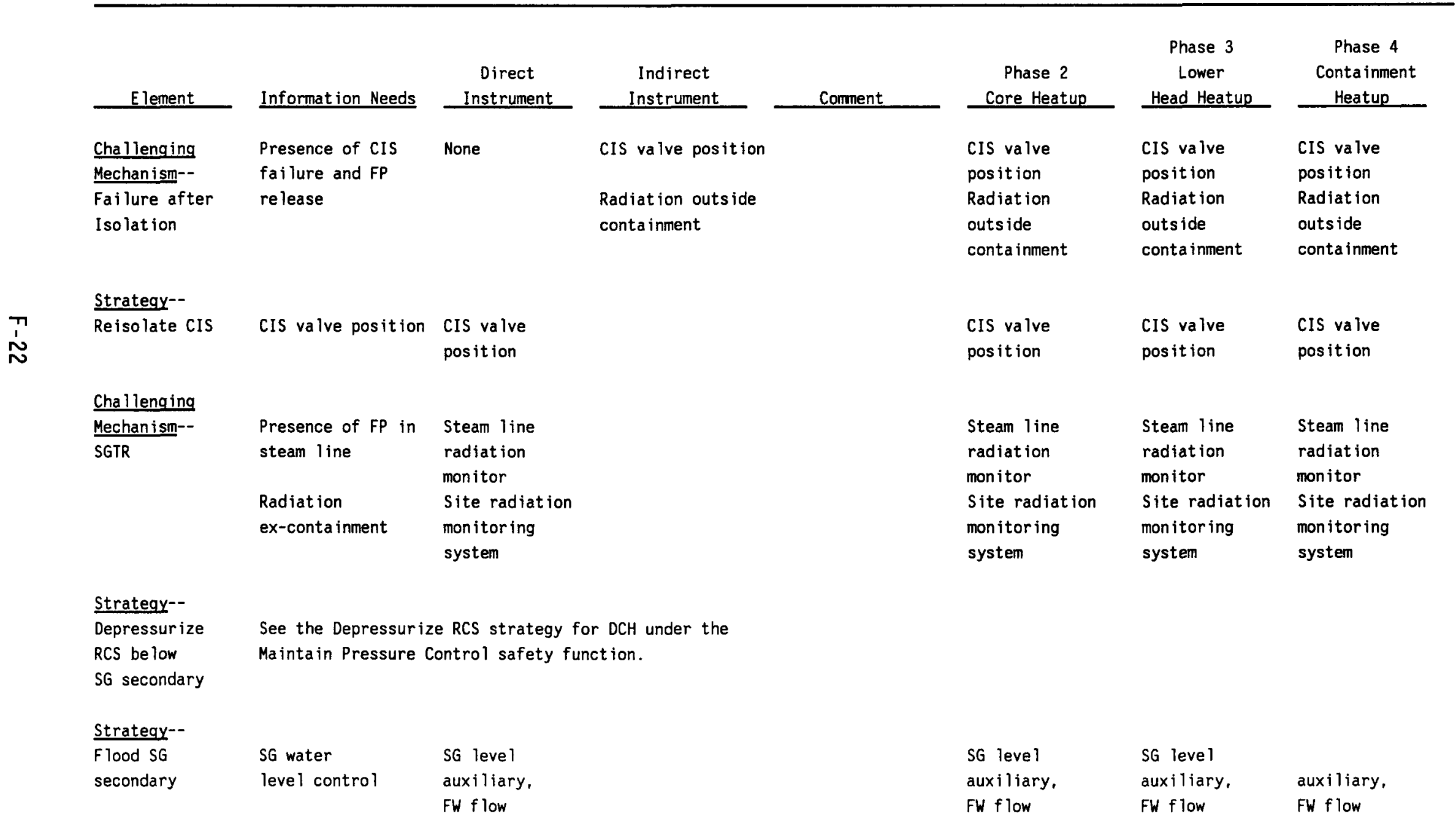


TABLE F8. (cont inued)

Scenario SBLOCA/LOOP Plant

Safety Objective Prevent Containment Failure

Safety Function Maintain Integrity

\begin{tabular}{|c|c|c|c|c|c|c|c|}
\hline E lement & Information Needs & $\begin{array}{c}\text { Direct } \\
\text { Instrument }\end{array}$ & $\begin{array}{l}\text { Indirect } \\
\text { Instrument }\end{array}$ & Comment & $\begin{array}{c}\text { Phase } 2 \\
\text { Core Heatup }\end{array}$ & $\begin{array}{c}\text { Phase } 3 \\
\text { Lower } \\
\text { Head Heatup }\end{array}$ & $\begin{array}{c}\text { Phase } 4 \\
\text { Containment } \\
\text { Heatup } \\
\end{array}$ \\
\hline \multicolumn{8}{|l|}{ Challenging } \\
\hline $\begin{array}{l}\text { LOCA with } \\
\text { conta inment } \\
\text { bypass }\end{array}$ & $\begin{array}{l}\text { Presence of LOCA } \\
\text { with bypass and FP } \\
\text { release }\end{array}$ & None & $\begin{array}{l}\text { Radiation outside } \\
\text { containment }\end{array}$ & & $\begin{array}{l}\text { Radiation } \\
\text { outs ide } \\
\text { conta inment }\end{array}$ & $\begin{array}{l}\text { Radiation } \\
\text { outside } \\
\text { containment }\end{array}$ & $\begin{array}{l}\text { Radiation } \\
\text { outside } \\
\text { containment }\end{array}$ \\
\hline & & & $\begin{array}{l}\text { Pzr. pressure, } \\
\text { temperature, } \\
\text { subcooling. RVLMS, } \\
\text { and Pzr. level }\end{array}$ & & $\begin{array}{l}\text { Fails during } \\
\text { Phase } 2\end{array}$ & & \\
\hline
\end{tabular}

Instruments are sufficient to determine that a challenge does not exist and that strategies are not required. 